\title{
Vital and Health Statistics
}

\section{Advance Data From Vital and Health Statistics: Numbers 261-270}

Series 16:

Compilations of Advance Data From Vital and Health Statistics No. 27

Data in this report from health and demographic surveys present statistics by age and other variables on injury-related visits to hospital emergency departments; office visits for glaucoma and office visits to neurologists; the relationship between cigarette smoking and other unhealthy behaviors among our Nation's youth; 1993 summaries of the National Hosplital Discharge Survey, National Hospital Ambulatory Medical Care Survey, and the National Ambulatory Medical Care Survey; urban and rural classification of national health providers; and characteristics of prepaid plan visits of office-based physicians. Estimates are based on the civilian noninstitutionallzed population of the United States. These reports were originally published in 1995.

U.S. DEPARTMENT OF HEALTH AND HUMAN SERVICES

Public Health Service

Centers for Disease Control and Prevention

National Center for Health Statistics

Hyattsville, Maryland

April 1996

DHHS Publication No. (PHS) 96-1886 


\section{Copyright Information}

All material appearing in this report is in the public domain and may be reproduced or copied without permission; citation as to source, however, is appreciated.

\section{Suggeated cltation}

National Center for Health Statistics. Advance data from vital and health statistics; numbers 261-270. National Center for Health Statistics. Vital Health Stat 16(27). 1996. 


\section{National Center for Health Statistics}

Jack R. Anderson, Acting Director

Jennifer H. Madans, Ph.D., Acting Deputy Director

Jacob J. Feldman, Ph.D., Associate Director for Analysis,

Epidemiology, and Health Promotion

Gail F. Fisher, Ph.D., Associate Director for Planning and Extramural Programs

Jack R. Anderson, Acting Associate Director for International Statistics

Stephen E. Nieberding, Associate Director for

Management

Charles J. Rothwell, Associate Director for Data

Processing and Services

Monroe G. Sirken, Ph.D., Associate Director for Research and Methodology 


\section{Contents}

Injury-Related Visits to Hospital Emergency Departments: United States, 1992 No. 261

Office Visits for Glaucoma: United States, 1991-92 No. 262 Relationship Between Cigarette Smoking and Other Unhealthy Behaviors Among Our Nation's Youth: United States, 1992

1993 Summary: National Hospital Discharge Survey No. 264

Underreporting of Race in the National Hospital Discharge Survey No. 265

Urban and Rural Classification of National Health Provider Inventory Providers: United States, 1991 No. 266

Office Visits to Neurologists: United States, 1991-92 No. 267

National Hospital Ambulatory Medical Care Survey: 1993 Outpatient Department Summary No. 268

Characteristics of Prepaid Plan Visits to Office-Based Physicians: United States, 1991 No. 269

National Ambulatory Medical Care Survey: 1993 Summary No. 270 


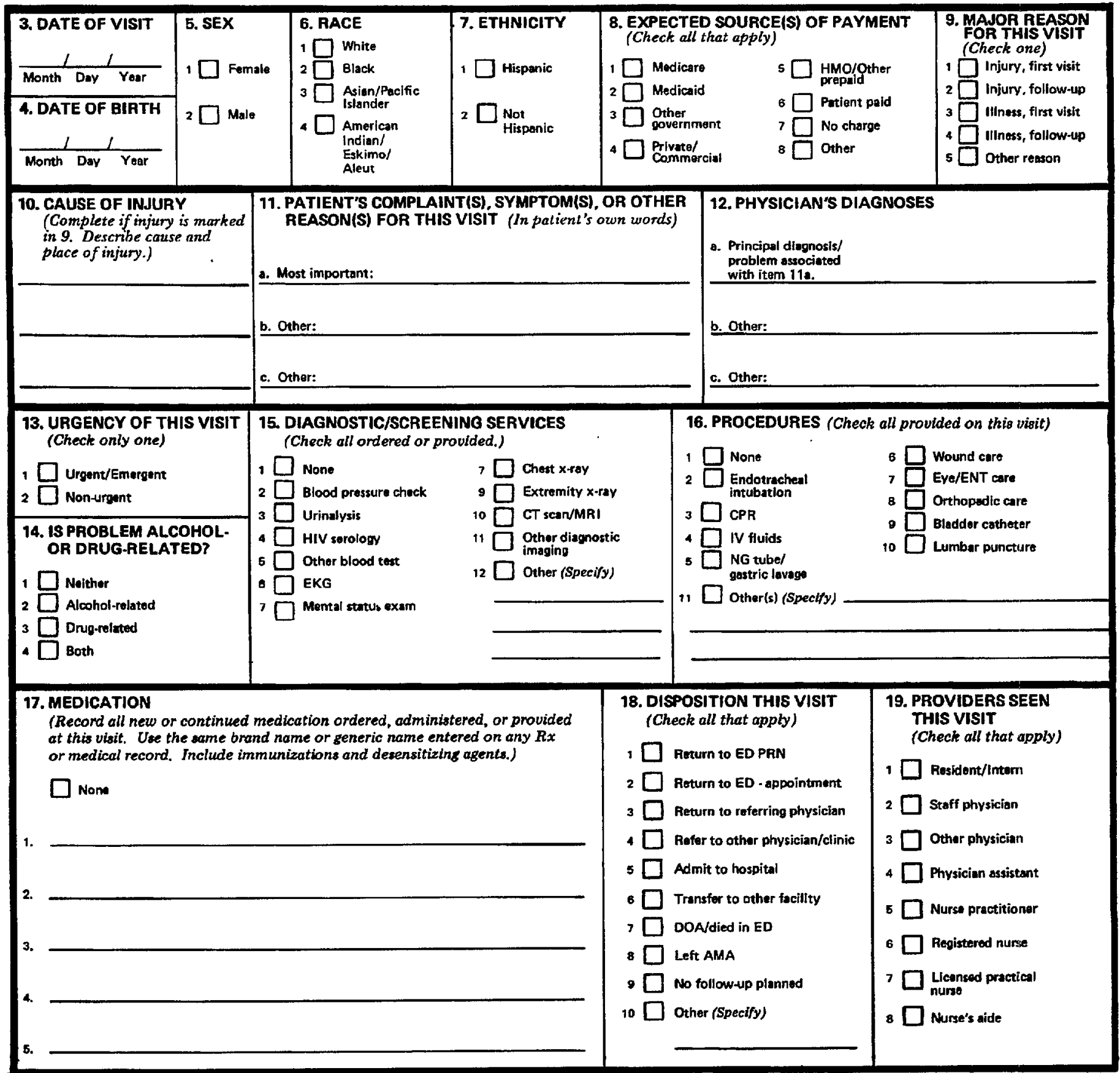

Flgure 1. National Hospital Ambulatory Medical Care Survey Emergency Department Patient Record

\section{Patient characteristics}

In 1992, an estimated 89.8 million visits were made to emergency departments of non-Federal, short-stay, and general hospitals in the United States-about 35.7 visits per 100 persons. Of these visits, 34.0 million (37.8 percent) were injury related. This results in an annual rate of 13.5 injury visits per 100 persons to a hospital emergency department. Injury-related visits by patient's age, sex, and race are shown in table 1 . Persons $15-24$ years of age had the highest ED injury visit rate (20.2 visits per 100 persons) of the six age categories analyzed. Males had a higher rate of injury-related visits than females did, using both crude and age-adjusted rates, and they accounted for 56.4 percent of all ED injury visits. This differs from ED visits in general, where there was no significant difference in the visit rates for males and females. Figure 2 shows the visit rates by age and sex. Males had significantly higher rates of injury-related visits compared with females for each age group under 45 years.

White persons made 82.9 percent of all injury-related ED visits, with black persons, Asian/Pacific Islanders, and American Indians/Eskimos/Aleuts accounting for 14.7 percent, 1.5 percent, and 0.8 percent, respectively. While the 
Table 1. Number, percent distribution, percent that are injury related, and annual rate of injury-related emergency department visits, by selected patient and visit characteristics: United States, 1992

\begin{tabular}{|c|c|c|c|c|}
\hline Patlent and visht characteristlc & $\begin{array}{l}\text { Number of } \\
\text { vists in } \\
\text { thousands }\end{array}$ & $\begin{array}{c}\text { Percent } \\
\text { distributlon }\end{array}$ & $\begin{array}{l}\text { Percent } \\
\text { injury } \\
\text { related }\end{array}$ & $\begin{array}{c}\text { Number of } \\
\text { vists per } \\
100 \text { persons } \\
\text { per year }\end{array}$ \\
\hline \multicolumn{5}{|l|}{ Patlent characteristlc } \\
\hline All injury-related visits $\ldots \ldots \ldots \ldots \ldots \ldots \ldots \ldots$ & 33,950 & 100.0 & 37.8 & 13.5 \\
\hline \multicolumn{5}{|l|}{ Age: } \\
\hline Under 15 yoars. $\ldots \ldots \ldots \ldots \ldots \ldots \ldots \ldots \ldots \ldots$ & $\mathbf{8 , 7 1 4}$ & 25.7 & 38.7 & 15.4 \\
\hline $15-24$ years. . . . . . . . . . . . . . . . . . & 6,937 & 20.4 & 46.7 & 20.2 \\
\hline $25-44$ years. $\ldots \ldots \ldots \ldots \ldots \ldots \ldots \ldots$ & 11,277 & 33.2 & 41.4 & 13.9 \\
\hline 45-64 years. . . . . . . . . . . . . . . . . & 3,959 & 11.7 & 31.6 & 8.2 \\
\hline $65-74$ years. . . . . . . . . . . . . . . . . & 1,458 & 4.3 & 25.1 & 7.9 \\
\hline 75 years and over $\ldots \ldots \ldots \ldots \ldots \ldots \ldots$ & 1,605 & 4.7 & 23.4 & 13.0 \\
\hline \multicolumn{5}{|l|}{ Sex and age: } \\
\hline Femalo $\ldots \ldots \ldots \ldots \ldots \ldots \ldots \ldots \ldots \ldots$ & 14,812 & 43.6 & 31.8 & 11.5 \\
\hline Under 15 years $\ldots \ldots \ldots \ldots \ldots \ldots \ldots \ldots$ & 3,567 & 10.5 & 35.0 & 12.9 \\
\hline $15-24$ years $\ldots \ldots \ldots \ldots \ldots \ldots \ldots \ldots \ldots$ & 2,670 & 7.9 & 33.2 & 15.4 \\
\hline $25-44$ years $\ldots \ldots \ldots \ldots \ldots \ldots \ldots \ldots$ & 4,714 & 13.9 & 33.6 & 11.4 \\
\hline $45-64$ years $\ldots \ldots \ldots \ldots \ldots \ldots \ldots \ldots \ldots$ & 1,796 & 5.3 & 27.1 & 7.1 \\
\hline $65-74$ years $\ldots \ldots \ldots \ldots \ldots \ldots \ldots \ldots \ldots$ & 940 & 2.8 & 28.1 & 9.2 \\
\hline 75 years and over. $\ldots \ldots \ldots \ldots \ldots \ldots \ldots$ & 1,124 & 3.3 & 25.9 & 14.6 \\
\hline Male $\ldots \ldots \ldots \ldots \ldots \ldots \ldots \ldots \ldots$ & 19,138 & 56.4 & 44.3 & 15.7 \\
\hline Under 15 years $\ldots \ldots \ldots \ldots \ldots \ldots \ldots \ldots$ & 5,147 & 15.2 & 41.8 & 17.8 \\
\hline $15-24$ years $\ldots \ldots \ldots \ldots \ldots \ldots \ldots \ldots \ldots \ldots$ & 4,267 & 12.6 & 62.8 & 25.0 \\
\hline $25-44$ years $\ldots \ldots \ldots \ldots \ldots \ldots \ldots \ldots$ & 6,564 & 19.3 & 49.7 & 16.4 \\
\hline $45-64$ years $\ldots \ldots \ldots \ldots \ldots \ldots \ldots \ldots \ldots$ & 2,163 & 6.4 & 36.8 & 9.3 \\
\hline $65-74$ yoars $\ldots \ldots \ldots \ldots \ldots \ldots \ldots \ldots$ & 518 & 1.5 & 21.1 & 6.3 \\
\hline 75 years and over. $\ldots \ldots \ldots \ldots \ldots \ldots \ldots$ & 480 & 1.4 & 19.0 & 10.4 \\
\hline \multicolumn{5}{|l|}{ Race and age: } \\
\hline White. . . . . . . . . . . . . . . . . & 28,154 & 82.9 & 39.9 & 13.4 \\
\hline Under 15 years $\ldots \ldots \ldots \ldots \ldots \ldots \ldots \ldots$ & 7,227 & 21.3 & 42.8 & 16.1 \\
\hline $15-24$ years $\ldots \ldots \ldots \ldots \ldots \ldots \ldots \ldots$ & 5,823 & 17.2 & 50.2 & 21.2 \\
\hline $25-44$ years $\ldots \ldots \ldots \ldots \ldots \ldots \ldots \ldots$ & 8,970 & 26.4 & 43.6 & 13.2 \\
\hline $45-64$ years $\ldots \ldots \ldots \ldots \ldots \ldots \ldots \ldots \ldots$ & 3,331 & 9.8 & 32.9 & 8.0 \\
\hline $65-74$ years $\ldots \ldots \ldots \ldots \ldots \ldots \ldots \ldots \ldots$ & 1,283 & 3.8 & 25.6 & 7.8 \\
\hline 75 years and over. $\ldots \ldots \ldots \ldots \ldots \ldots \ldots$ & 1,520 & 4.5 & 24.2 & 13.6 \\
\hline 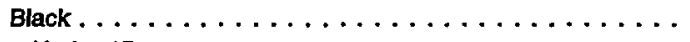 & 4,987 & 14.7 & 29.1 & 15.9 \\
\hline Under 15 years $\ldots \ldots \ldots \ldots \ldots \ldots \ldots \ldots$ & 1,311 & 3.9 & 25.5 & 14.6 \\
\hline $15-24$ years $\ldots \ldots \ldots \ldots \ldots \ldots \ldots \ldots \ldots$ & 972 & 2.9 & 33.8 & 19.1 \\
\hline $25-44$ years $\ldots \ldots \ldots \ldots \ldots \ldots \ldots \ldots \ldots$ & 1,974 & 5.8 & 33.8 & 20.2 \\
\hline $45-64$ years $\ldots \ldots \ldots \ldots \ldots \ldots \ldots \ldots \ldots$ & 524 & 1.5 & 24.8 & 10.5 \\
\hline $65-74$ years $\ldots \ldots \ldots \ldots \ldots \ldots \ldots \ldots \ldots \ldots$ & 138 & 0.4 & 20.1 & 8.4 \\
\hline 75 years and over. $\ldots \ldots \ldots \ldots \ldots \ldots \ldots$ & 67 & 0.2 & 13.3 & 6.9 \\
\hline All other races $\ldots \ldots \ldots \ldots \ldots \ldots \ldots \ldots \ldots$ & 808 & 2.4 & 37.3 & 7.7 \\
\hline Aslan/Pactic Islander. . . . . . . . . . . . & 529 & 1.5 & 37.8 & $\cdots$ \\
\hline American Indian/Eskimo/Aleut $\ldots \ldots \ldots \ldots \ldots \ldots$ & 279 & 0.8 & 35.3 & $\cdots$ \\
\hline \multicolumn{5}{|l|}{ Emergency department characteristlc } \\
\hline \multicolumn{5}{|l|}{ Goographic region: } \\
\hline Northeast $\ldots \ldots \ldots \ldots \ldots \ldots \ldots \ldots \ldots \ldots$ & 6,346 & 20.1 & 37.4 & 13.6 \\
\hline Midwest $\ldots \ldots \ldots \ldots \ldots \ldots \ldots \ldots \ldots \ldots$ & 9,268 & 29.4 & 35.9 & 16.1 \\
\hline South $\ldots \ldots \ldots \ldots \ldots \ldots \ldots \ldots \ldots \ldots$ & 9,692 & 30.7 & 32.8 & 12.4 \\
\hline 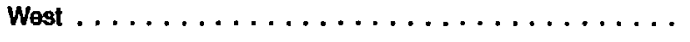 & 6,261 & 19.8 & 35.7 & 12.3 \\
\hline
\end{tabular}

Percent of all emergency department visits in each catogory that are injury releted.

2Basod on U.S. Buroau of the Consus cetimates of the oivilian, noninstitutionalized population of the United States as of July 1, 1902. 


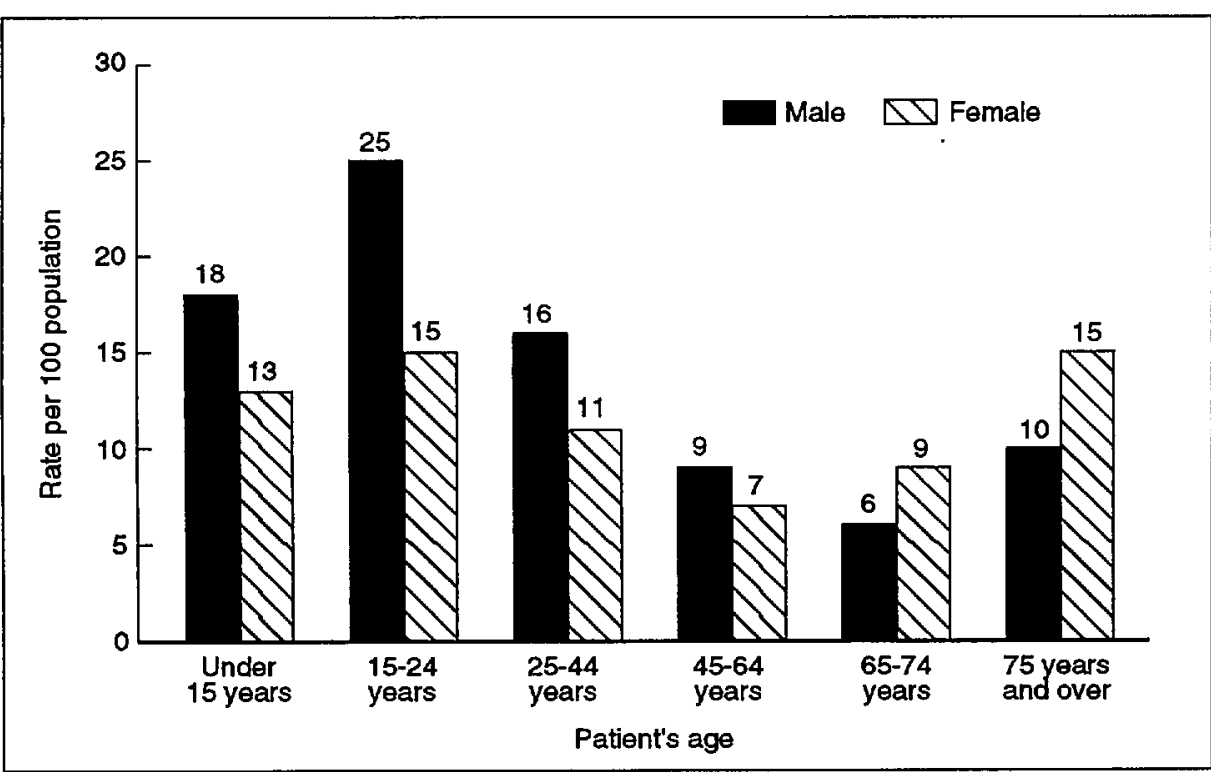

Flgure 2. Annual rate of injury-related visits to emergency departments by patient's age and sex: United States, 1993

Table 2. Number, percent distribution, and annual rate of visit to hospital emergency departments by major reason for visit: United States, 1992

\begin{tabular}{|c|c|c|c|}
\hline Visit characteristic & $\begin{array}{c}\text { Number } \\
\text { of visfls } \\
\text { In thousands }\end{array}$ & $\begin{array}{c}\text { Percent } \\
\text { d/stribution }\end{array}$ & $\begin{array}{c}\text { Number of } \\
\text { visits per } \\
100 \text { persons } \\
\text { per year }\end{array}$ \\
\hline All ED visits ${ }^{2}$ & 89,796 & 100.0 & 35.7 \\
\hline All Injury-related visits $\ldots \ldots \ldots \ldots \ldots \ldots \ldots$ & 33,950 & 37.8 & 13.5 \\
\hline Injury-first visit & 28,388 & 31.6 & 11.3 \\
\hline Injury-tollow-up visit . . . . . . . . . . . & 3,178 & 3.5 & 1.3 \\
\hline Stated cause of Injury ${ }^{3}$. & 2,383 & 2.7 & 0.9 \\
\hline Ilinese-first visit $\ldots \ldots \ldots \ldots \ldots \ldots \ldots$ & 1,467 & 1.6 & 0.6 \\
\hline Illness-follow-up visit $\ldots \ldots \ldots \ldots \ldots \ldots$ & 129 & 0.1 & 0.1 \\
\hline Other reason ${ }^{4} \ldots \ldots \ldots \ldots \ldots \ldots$ & 787 & 0.9 & 0.3 \\
\hline All other visits. & 55,846 & 62.2 & 22.2 \\
\hline
\end{tabular}

${ }^{9}$ Based on U.S. Bureau of the Census estimates of the civilian noninstitutionalized population of the United States as of July $1,1992$.

${ }^{2} E D$ is emergency department.

3 includes visits not recorded as injury related but had a cause of injury recorded in item 10.

Includes visits with blank or other major reasons recorded in item 9 , yet had a cause of injury in item 10.

overall ED visit rate for black persons was significantly higher than for white persons, there was no significant difference between the ED injury visit rates for white and black persons, whether using crude or age-adjusted rates. However, black persons between the ages of 25 and 44 did have a significantly higher injury-related visit rate than did white persons in that age category.

Examining the percent of ED visits that were injury related for each population subgroup in table 1 shows that while overall one-third of the ED visits were for injuries, almost twothirds of the ED visits for males aged 15-24 years were for injuries. White persons in all age groups tended to have a higher proportion of ED visits for injuries compared with black persons.

\section{Visit characteristics}

\section{Geographic region}

There were slight regional differences in the utilization of ED services for injuries. The Midwest had a higher injury visit rate (16 visits per 100 persons) than the South and West (12 visits per 100 persons) did (table 1 ).

\section{Prior-visit status}

The majority of injury-related visits (87.9 percent) were first-time visits. While just 5 percent of illness-related visits to ED's were classified as follow-up visits, 9.7 percent of the injury-related visits were follow-up visits from a previous injury visit. The majority of ED visits (62.2 percent) were made for illness and 37.8 percent were made for injury (table 2).

\section{Urgency of this visit}

Almost half of the first-visit injury cases were classified as urgent by hospital staff. In comparison, only 15.8 percent of follow-up injury visits were classified as urgent. Urgency of visit was defined as those visits in which the patient requires immediate attention for an acute illness or injury that threatens life or function and where delay would be harmful to the patient. Hospitals made slightly different interpretations about how they determined urgency for the survey. In some cases, the determination of urgency was based upon the severity of the patient's symptom(s); in other cases, it was based upon the patient's diagnosis or the nature of the treatment provided.

\section{Cause of injury}

Up to three external causes of injury were coded and classified according to the International Classification of Diseases, 9th Revision, Clinical Modification (ICD-9-CM) (3). Table 3 shows the number of annual injury-related ED visits for the firstlisted cause of injury, using the major cause of injury categories specified by the ICD-9-CM (E-codes) along with any subclassification codes that had reliable estimates. E-code data were reported for 84 percent of the injuryrelated visits. In visits where the place of occurrence was listed as the first cause, the second cause was used for purposes of this analysis. Almost one-third of the injury-related visits 
Table 3. Number and percent distribution of Injury-related emergency department visits by cause of injury: United States, 1992

Other accidents.

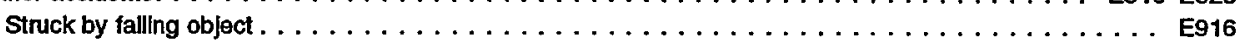

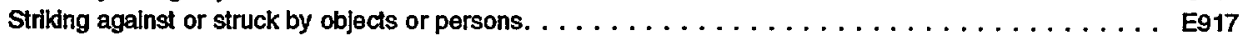

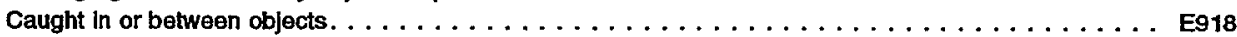

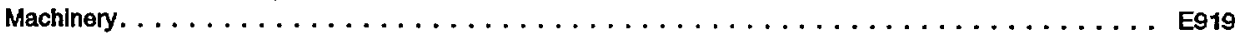

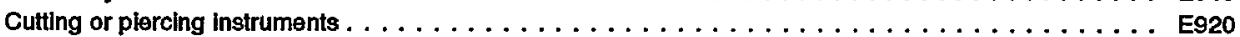

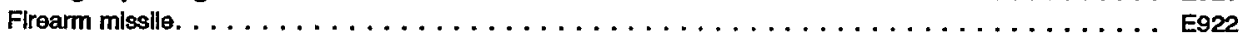

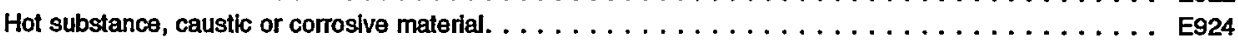

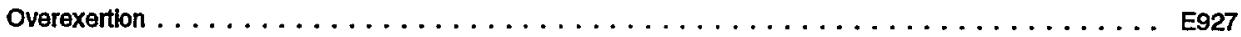

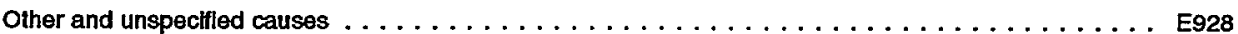

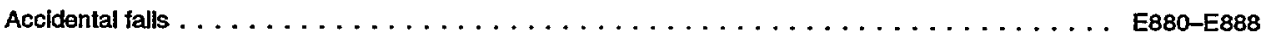

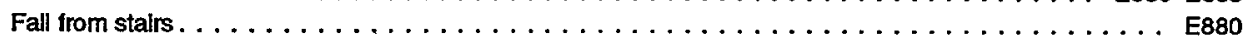

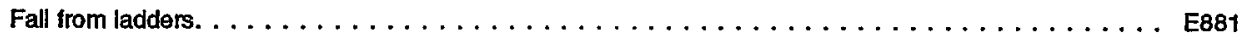

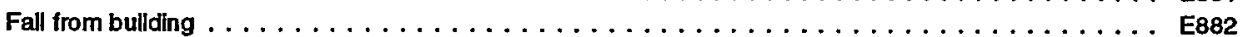

Fall into hole $\ldots \ldots \ldots \ldots \ldots \ldots \ldots \ldots \ldots \ldots \ldots \ldots \ldots \ldots \ldots \ldots \ldots \ldots \ldots$

Other fall trom one level to another $\ldots \ldots \ldots \ldots \ldots \ldots \ldots \ldots \ldots \ldots \ldots \ldots \ldots$ E884

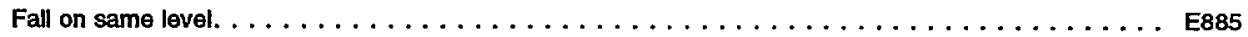

Other and unspecffied falls $\ldots \ldots \ldots \ldots \ldots \ldots \ldots \ldots \ldots \ldots \ldots \ldots \ldots \ldots$

10,368

00.0

639

3,018

670

488

3,077

87

473

1,587

195

30.5

Motor vehicle accidents, traffic and non-traffic . . . . . . . . . . . . . . . E810-E825

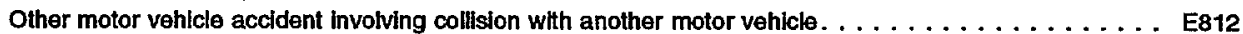
Molor vehicle accident involving colliston with other vehicle $\ldots \ldots \ldots \ldots \ldots \ldots \ldots \ldots \ldots$ Motor vehicte collsion with pedestrian $\ldots \ldots \ldots \ldots \ldots \ldots \ldots \ldots \ldots \ldots \ldots \ldots \ldots$ E814

Other motor vehicle accident involving collision on highway $\ldots \ldots \ldots \ldots \ldots \ldots \ldots \ldots \ldots$

Motor vehicle accident due to loss of control without collision on highway $\ldots \ldots \ldots \ldots \ldots \ldots \ldots$ E816

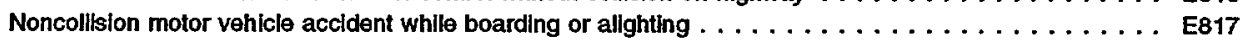

Other noncollston motor vehicle accldent $\ldots \ldots \ldots \ldots \ldots \ldots \ldots \ldots \ldots \ldots \ldots \ldots \ldots \ldots$ E818

Unspecifled motor vehicle accident $\ldots \ldots \ldots \ldots \ldots \ldots \ldots \ldots \ldots \ldots \ldots \ldots \ldots \ldots \ldots \ldots$

Other motor vehicle nontraffic accident . . . . . . . . . . . . . . . . . . E824

Homiclde and injury purposely inflicted by other persons . . . . . . . . . . . . . E960-E969

Fight, brawi, rape . . . . . . . . . . . . . . . . . . . . . . . . E960

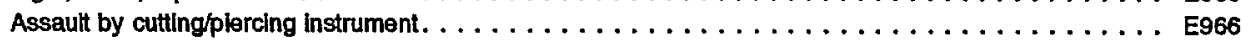

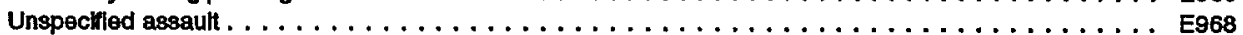

Accidents due to natural and environmental factors. . . . . . . . . . . . . . E E90-E909

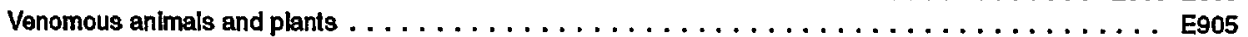

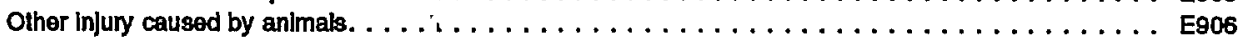

Accidents caused by submersion, suftocation, and forelgn bodies. . . . . . . . . . . . . E910-E915

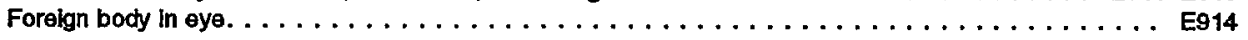

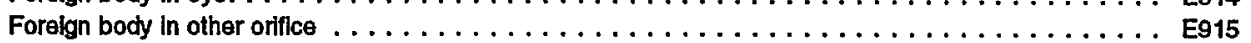

Other road vehicle accidents. . . . . . . . . . . . . . . . . . . . . . E826-E829

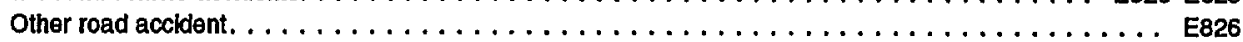

Accident involing animal being rldden . . . . . . . . . . . . . . . . . . E

4,130

403

62

178

101

114

73

333

2,694

86

1.9

0.6

0.5

0.5

2.9

0.4

12.4

12.2

1.2

0.2

0.5

0.3

0.3

0.2

1.0

7.9

0.3

1,554

588

4.6

173

1.7

731

Surgical and medical procedures as the cause of abnormal reactlon of pallent or later complicalion

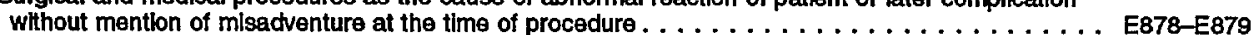

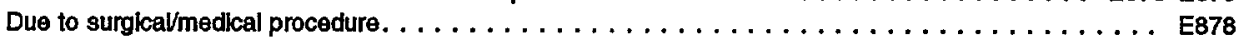

Due to olher medical procedure . . . . . . . . . . . . . . . . . . . . . . E879

Drugs, medicinal and blological substances causing adverse effects in therapeutic use . . . . . . E930-E949 Due to unspecified drugs $\ldots \ldots \ldots \ldots \ldots \ldots \ldots \ldots \ldots \ldots \ldots \ldots \ldots \ldots \ldots$. . . . . . . . . . . . . . . .

Accidental poisoning by drugs, medicinal substances, and biologlcals . . . . . . . . . . E850-E858

Polsoning by analgesics, antpyrelics, and antirheumatics $\ldots \ldots \ldots \ldots \ldots \ldots \ldots \ldots \ldots \ldots \ldots \ldots$ E850

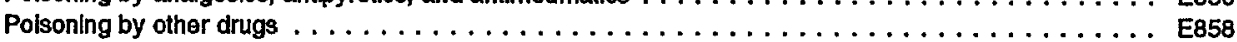

Accidental polsoning by other solld and liquld substances, gases, and vapors . . . . . . . . . . E860-E869

73

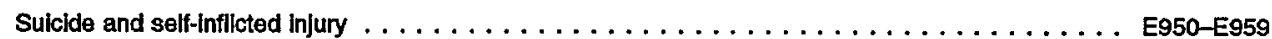

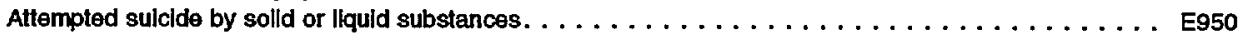

Accldents caused by fire and flames. . . . . . . . . . . . . . . . . . . . . . E890-E899

Unspecified fires. . . . . . . . . . . . . . . . . . . . . . . . . E Es99

Other $^{2}$

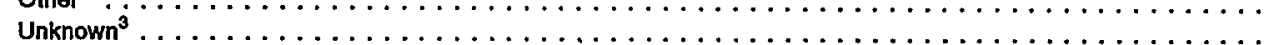




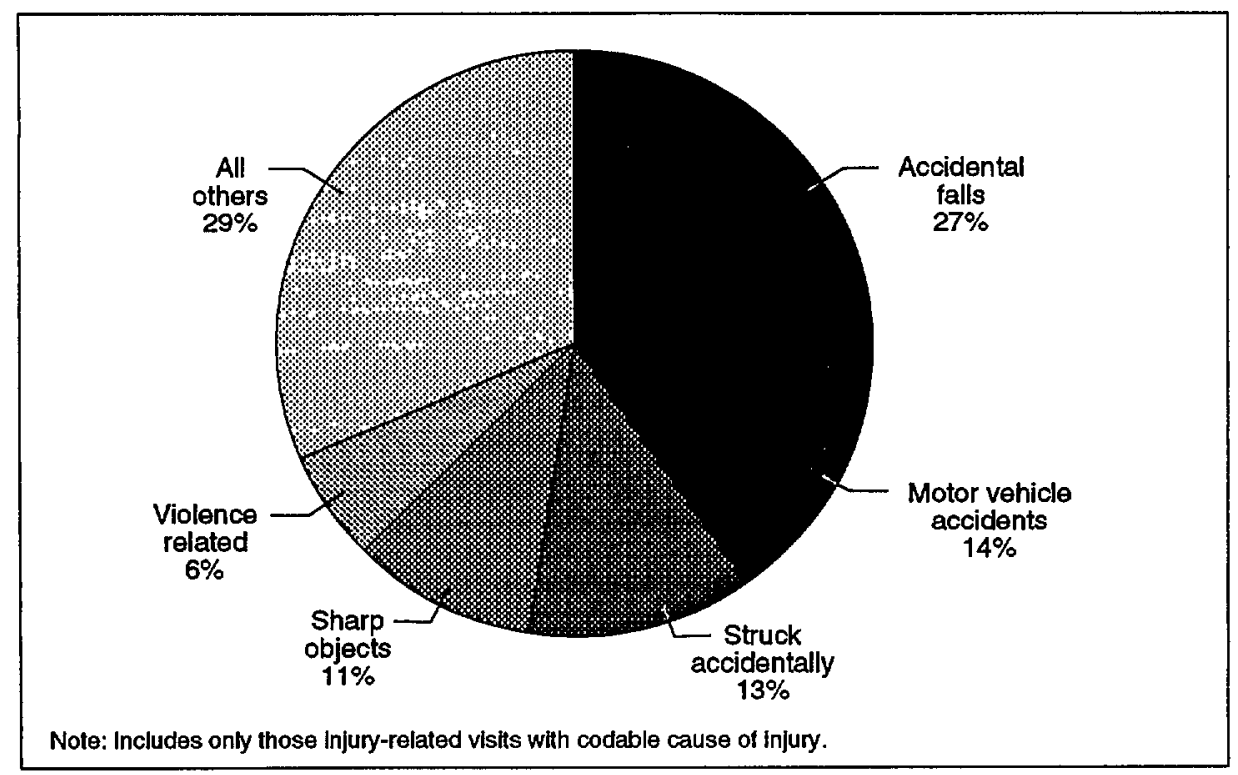

Figure 3. Percent dlstribution of leading causes of injury treated at emergency department visits: United States, 1992

were under the general category of "Other accidents" (E916-E928) (30.5 percent). "Accidental falls" (E880-E888) (22.7 percent) and "motor vehicle accidents" (E810-E825) (12.2 percent) were the second and third largest categories. Table 3 also presents the estimates for individual 3-digit codes within the larger categories. The codes listed were those for which the numbers of visits were large enough to provide a reliable estimate. The most frequently occurring 3-digit E-code was "unspecified fall" (E888) (12.4 percent of total). The category "other road vehicle accidents" (E826), which includes bicycle accidents, accounted for over half a million ED injury visits. "Unspecified assault" (E968), "fight, brawl, rape" (E960), and "assault by cutting or piercing instruments" (E966) have reliable estimates within the broader category of "homicide and injury purposely inflicted by other persons." Together these 3 codes accounted for 96 percent of the specific causes within the larger category.

Combining various E-code categories at and above the 3-digit level yields interesting results for summarization purposes. The major groupings in table 3 might be combined in a better way because some of the individual E-codes within "other accidents" have a greater frequency of occurrence than do some of the major categories. Figure 3 presents the top five causes of injury if E-codes are categorized in a slightly different manner. Still, "accidental falls" and "motor vehicle accidents," as defined above, accounted for 41 percent of the injury-related visits with E-codes. However, combining "accidentally struck by persons or objects" (E916) with "struck by falling objects" (E917) places this category, labeled "struck accidentally" in figure 3 , among the leading causes. "Accidents caused by cutting or piercing instruments or objects" (E920) alone accounted for 11 percent of the causes of injury-related visits. Combining "homicide and injury purposely inflicted" (E960-E969) with "suicide and self-inflicted injury" (E950-E959), as a measure of injury caused by violence, places it among the top five causes of injury in visits to emergency departments. These five causes accounted for over 70 percent of injury-related visits in 1992 where a cause was specified. It should be noted that although the E-code classification of injury causes includes the terms "homicide" and "suicide," very few visits with these causes ended in death in the emergency department. In fact, there were so few cases observed in the sample that reliable population estimates could not be made. The term "intentional injury" (either by self or others) is perhaps a better label when using the classification for morbidity purposes.

Table 4 shows the annual visit rates and percent distribution of these top five injury causes by selected patient characteristics. The data show that persons in the age categories under 15 years and 65 years and over had higher rates of visits for accidental falls than the other age categories. Not surprisingly, persons between 15 and 24 years had the highest rate of visits for motor vehicle accidents. The rates of visits related to intentional injuries were highest among persons between 15 and 44 years. The data also show that black persons were more likely than white persons to make an ED visit because of violence (3:1) and motor vehicle accidents (5:3). On the other hand, white persons were more likely to make a visit due to accidental falls than were black persons (3:2). Figure 4 displays ED injury-related visit rates by race for the top five causes of injury.

Using the E-code data at the fourth digit level, the estimates may be combined to provide reliable estimates of certain categories of injuries. For example, table 5 shows the number of visits to ED's related to firearms, pedal cyclists, pedestrians, and motorcyclists.

Place of occurrence information was provided for less than 15 percent of the injury-related visits with appropriate causes of injury (e.g., E850-E869 or E880-E928) and is therefore not discussed in further detail. A separate item for place of occurrence was added to the 1993-94 Patient Record form to improve reporting of this information.

\section{Alcohol- or drug-related problem}

The proportion of visits that were alcohol related was higher for injuryrelated ED visits ( 3.6 percent) compared with noninjury related visits (2.3 percent). Nelson and Stussman (4) examined E-code data for different responses to item 14, "Is problem alcohol or drug related?", on the ED Patient Record and found that an injury was three times as likely to be classified as "homicide and injury purposely 
Table 4. Number, annual rate, and percent distribution of injury-related emergency department visits by selected patient characteristics, according to the top five causes of injury: United States, 1992

\begin{tabular}{|c|c|c|c|c|c|c|c|}
\hline \multirow[b]{2}{*}{ Selected characteristic } & \multirow[b]{2}{*}{ Total } & \multicolumn{6}{|c|}{ Cause of injury } \\
\hline & & $\begin{array}{l}\text { Accidental } \\
\text { falls }\end{array}$ & $\begin{array}{c}\text { Motor } \\
\text { vehicle } \\
\text { accidents }\end{array}$ & $\begin{array}{c}\text { Struck } \\
\text { accidentally }\end{array}$ & $\begin{array}{l}\text { Cut by } \\
\text { shapp } \\
\text { objects }\end{array}$ & Volence & Other ${ }^{2}$ \\
\hline & \multicolumn{7}{|c|}{ Number of visits in thousands } \\
\hline \multirow[t]{2}{*}{ All injury-related visits $\ldots \ldots \ldots \ldots \ldots \ldots$} & 33,950 & 7,706 & 4,130 & 3,657 & 3,077 & 1,714 & 13,666 \\
\hline & \multicolumn{7}{|c|}{ Rate per 1,000 persons $^{3}$} \\
\hline All injuny-related visits $\ldots \ldots \ldots \ldots \ldots \ldots$ & 135.0 & 30.6 & 16.4 & 14.5 & 12.2 & 6.8 & 54.3 \\
\hline \multicolumn{8}{|l|}{ Age } \\
\hline Under 15 years. . . . . . . . . . . . . . & 154.4 & 44.8 & 9.4 & 20.0 & 12.7 & 2.9 & 64.7 \\
\hline $15-24$ years. $\ldots \ldots \ldots \ldots \ldots \ldots \ldots \ldots$ & 201.7 & 27.4 & 36.1 & 26.0 & 19.3 & 15.9 & 77.0 \\
\hline $25-44$ years. . . . . . . . . . . . . . . & 138.7 & 21.5 & 19.1 & 13.4 & 14.3 & 10.4 & 59.9 \\
\hline $45-64$ years. . . . . . . . . . . . . . . & 81.6 & 20.5 & 10.2 & 7.6 & 8.1 & 2.9 & 32.4 \\
\hline 65 years and over $\ldots \ldots \ldots \ldots \ldots \ldots \ldots$ & 99.5 & 48.6 & 10.1 & 5.8 & 4.6 & ${ }^{\star 0} 0.6$ & 29.7 \\
\hline \multicolumn{8}{|l|}{ Sex } \\
\hline Female. . . . . . . . . . . . . . . . & 114.6 & 30.8 & 15.3 & 10.2 & 8.1 & 5.3 & 44.7 \\
\hline Male $\ldots \ldots \ldots \ldots \ldots \ldots \ldots \ldots$ & 156.6 & 30.5 & 17.6 & 19.1 & 16.6 & 8.4 & 64.5 \\
\hline \multicolumn{8}{|l|}{ Race } \\
\hline 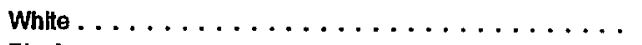 & 134.4 & 32.2 & 15.2 & 14.8 & 12.1 & 5.3 & 54.7 \\
\hline Black $\ldots \ldots \ldots \ldots \ldots \ldots \ldots \ldots \ldots$ & 158.5 & 24.7 & 25.5 & 15.5 & 13.5 & 17.7 & 61.6 \\
\hline Other $\ldots \ldots \ldots \ldots \ldots \ldots \ldots \ldots \ldots \ldots$ & 76.8 & 17.5 & 13.1 & 5.9 & 10.6 & $" 4.1$ & 25.6 \\
\hline & \multicolumn{7}{|c|}{ Percent distroution } \\
\hline All injury-related visits $\ldots \ldots \ldots \ldots \ldots \ldots \ldots$ & 100.0 & 100.0 & 100.0 & 100.0 & 100.0 & 100.0 & 100.0 \\
\hline \multicolumn{8}{|l|}{ Age } \\
\hline Under 15 years. . . . . . . . . . . . . . & 25.7 & 32.8 & 12.8 & 30.8 & 23.2 & 9.5 & 26.7 \\
\hline 15-24 years. . . . . . . . . . . . . . & 20.4 & 12.2 & 30.1 & 24.4 & 21.6 & 32.0 & 19.4 \\
\hline 25-44 years. . . . . . . . . . . . . . & 33.2 & 22.7 & 37.6 & 29.9 & 37.8 & 49.3 & 35.7 \\
\hline 45-64 years. . . . . . . . . . . . . . . & 11.7 & 12.9 & 11.9 & 10.1 & 12.8 & 8.1 & 11.5 \\
\hline 65 years and over $\ldots \ldots \ldots \ldots \ldots \ldots \ldots$ & 9.0 & 19.4 & 7.5 & 4.9 & 4.6 & $" 1.1$ & 6.7 \\
\hline \multicolumn{8}{|l|}{ Sex } \\
\hline Female. . . . . . . . . . . . . . . . . & 43.6 & 51.7 & 48.0 & 36.1 & 34.1 & 40.3 & 42.3 \\
\hline Male $\ldots \ldots \ldots \ldots \ldots \ldots \ldots \ldots \ldots$ & 56.4 & 48.3 & 52.0 & 63.9 & 65.9 & 59.7 & 57.7 \\
\hline \multicolumn{8}{|l|}{ Race } \\
\hline 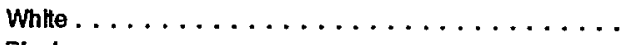 & 82.9 & 87.5 & $\pi 7.2$ & 85.0 & 82.6 & 65.0 & 83.8 \\
\hline Black . . . . . . . . . . . . . . & 14.7 & 10.1 & 19.4 & 13.3 & 13.8 & 32.5 & 14.2 \\
\hline Other $\ldots \ldots \ldots \ldots \ldots \ldots \ldots \ldots \ldots$ & 2.4 & 2.4 & 3.3 & 1.7 & 3.6 & $* 2.5$ & 2.0 \\
\hline
\end{tabular}

inflicted" in an alcohol- and drug-related visit in comparison with all other visits. Alcohol and drug use was determined by the hospital staff if the patient indicated or staff suspected that alcohol or drugs played a part in the injury, whether by the patient or another person. Patient's use of alcohol or drugs was not necessarily verified by blood or urine tests. These data undoubtedly underestimate the role of alcohol and drugs in ED injury visits.

Notwithstanding, visits classified as related to alcohol and/or drugs had a greater likelihood (6:1) of being caused by violence than were visits not so classified.

\section{Reason for visit}

In item 11 of the ED Patient Record, the patient's (or patient surrogate's) "complaint(s), symptom(s), or other reason(s) for this visit in the patient's own words" is recorded. Up to three reasons for visit are classified and coded according to A Reason for Visit Classification for Ambulatory Care (RVC) (5). The principal reason is the problem, complaint, or reason listed in item 11a of the ED Patient Record.

The RVC is divided into the eight modules or groups of reasons displayed in table 6. Half of all injury-related visits were made for reasons classified in the injuries and adverse effects module. About 43.2 percent were in the symptoms module with the largest being symptoms referable to the 


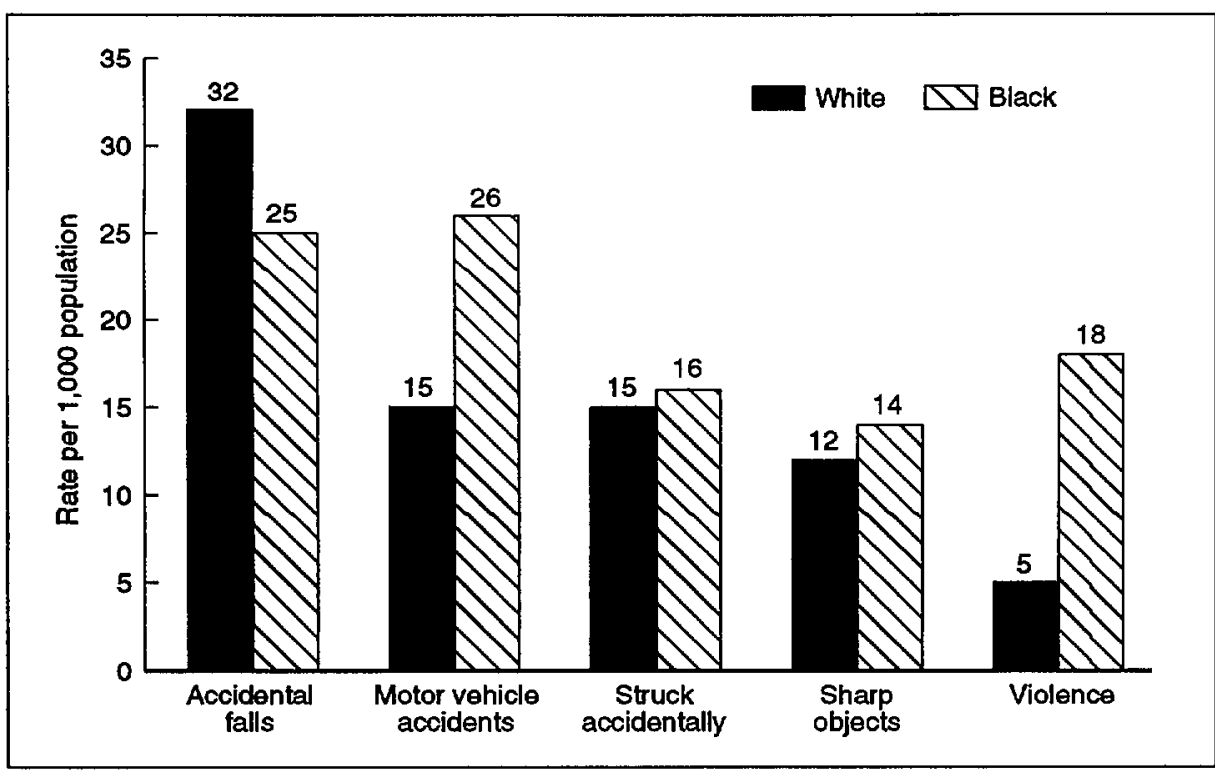

Figure 4. Annual rate of injury-related visits to emergency department by patient's race and leading causes of injury: United States, 1992

Table 5. Number, percent, and rate of visits to emergency departments by selected causes of Injury: United States, 1992

\begin{tabular}{|c|c|c|c|}
\hline Selected cause ${ }^{1}$ & $\begin{array}{l}\text { Number of } \\
\text { visits in } \\
\text { thousands }\end{array}$ & $\begin{array}{c}\text { Percent of } \\
\text { Injuny } \\
\text { visits }\end{array}$ & $\begin{array}{c}\text { Rate per } \\
1,000 \\
\text { persons }^{2}\end{array}$ \\
\hline Firearms . . . . . . . . . . . . . & 112 & 0.3 & 0.4 \\
\hline Pedal cyclists . . . . . . . . . . . . . . . . & 321 & 0.9 & 1.3 \\
\hline Pedestrians . . . . . . . . . . . . . . . & 74 & 0.2 & 0.3 \\
\hline Motorcyclists . . . . . . . . . . . . . . . & 62 & 0.2 & 0.2 \\
\hline
\end{tabular}

'Based on the Intemational Classification of Diseases, 9th Revision, Clinical Modification (3): Firearms (E922, E955.0-.4, E965.0-.4, E970, and E985.0-.4); Pedal cyclists (E800-E807(.3), E810-E825(.6), E826-E829(.1)); Pedestrians in motor vehicle accidents (E810-E825(.7)); Motorcyclists (E810-E825(.2)).

${ }^{2}$ Based on U.S. Bureau of the Census estimates of the civilian noninstitutionalized population of the United States as of July 1 , 1992.

musculoskeletal system, accounting for 27.4 percent of the visits.

The 20 most frequently mentioned principal reasons for visit, representing 52.6 percent of all visits, are shown in table 7. It is important to note that the rank ordering presented in this and other tables may not always be reliable because near estimates may not differ from each other due to sampling variability. "Upper extremity lacerations" was the most frequently mentioned specific reason for visit (6.8 percent).

\section{Principal diagnosis}

The principal diagnosis or problem associated with the patient's most important reason for visit and any other significant current diagnoses are recorded in item 12. Up to three diagnoses are coded and classified according to the ICD-9-CM (3). As expected, injury and poisoning (ICD-9-CM codes 800-999) accounted for 81 percent of all visits, and diseases of the musculoskeletal system (710-739) accounted for 5 percent. Supplementary classification diagnoses (those unrelated to injury or illness such as general examination) were made for 4 percent of the injury-related visits. The remaining 10 percent were distributed over all the other major categories.

Within the main ICD-9-CM injury category (N-codes), most of the principal diagnoses were "open wounds" (870-897) (24.1 percent), "sprains and strains of joints and adjacent muscles" (840-848)

(14.4 percent), "contusions" (920-924)
(14.0 percent), and "fractures" (800829) (11.2 percent). There were no sex differences for the principal diagnoses except that males tended to have a higher percent of their diagnoses as "open wounds" compared to females (28.2 and 18.7 percent respectively). There were few race differences in diagnoses although white persons tended to have higher rates of "fractures" and "crushing injuries" (925-929) than black persons had. There were 1.8 million visits that had an injury diagnosis $(\mathrm{N}$-code) that were not indicated by hospital staff to be injury related or to have a cause of injury recorded in item 10 of the Patient Record. These visits are not included in this report as injury visits.

The type of $\mathrm{N}$-code most frequently found for the principal diagnosis differed, as expected, by age of patient and cause of injury. Visits for patients 65 years and over were twice as likely to have a principal diagnosis of "fracture" compared to younger patients. Patients between the ages of 15 and 44 years were twice as likely to have a principal diagnosis of "sprains and strains" compared to other age groups. Children under 15 years were one and a half times more likely to have an open wound diagnosis than were older patients. Similarly, of the top five causes of injury visits to emergency departments, visits resulting from accidental falls were more likely to result in a "fracture" diagnosis than were other causes (2:1). Visits due to motor vehicle accidents more likely resulted in a principal diagnosis of "sprains and strains" compared to other causes (2:1). "Open wounds" was found most often for visits caused by cuts from sharp objects (86.7 percent). "Open wounds" was also the leading principal diagnosis for patients struck accidentally or who were victims of intentional injuries (about 28 percent each).

The 20 most frequently reported principal diagnoses are shown in table 8 . These are categorized at the three-digit coding level of the ICD-9-CM and account for 55.5 percent of all injuryrelated ED visits. The most commonly recorded diagnosis was "open wound of head other than eye or ear" (873), 
Table 6. Number and percent dletribution of injury-related emergency department visits by patient's principal reaseon for vieit: United States, 1992

\begin{tabular}{|c|c|c|}
\hline Princlpal reason for vist and $A V C$ code' & $\begin{array}{l}\text { Number of } \\
\text { visits in } \\
\text { thousands }\end{array}$ & $\begin{array}{c}\text { Percent } \\
\text { dfstribution }\end{array}$ \\
\hline 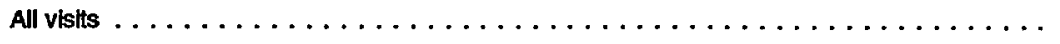 & 33,950 & 100.0 \\
\hline Symplom module. . . & 14,663 & 43.2 \\
\hline General symploms $\ldots \ldots \ldots \ldots \ldots \ldots \ldots \ldots \ldots \ldots \ldots \ldots$ so01-S099 & 1,949 & 5.7 \\
\hline Symptoms referable to psychological/mental dlsorders . . . . . . . . S100-S199 & 197 & 0.6 \\
\hline Symptoms referable to the nervous system (excluding sense organs) . . . . S S200-S259 & 1,016 & 3.0 \\
\hline Symptoms referable to the cardlovascularhymphatic system. . . . . . . . S260-\$299 & 42 & 0.1 \\
\hline Symptoms referable to the eyes and ears . . . . . . . . . . . . . s300-s399 & 704 & 2.1 \\
\hline Symptoms referable to the resplratory system . . . . . . . . . . . . . $\$ 400-\$ 499$ & 403 & 1.2 \\
\hline Symptoms referable to the digestlve system. . . . . . . . . . . . $\$ 500-\$ 639$ & 606 & 1.8 \\
\hline Symptoms referable to the genitourinary system . . . . . . . . . . . . S640-\$829 & 120 & 0.4 \\
\hline Symptoms referable to the skin, hair, and nalls . . . . . . . . . . . . . . . . . s830-s899 & 327 & 1.0 \\
\hline Symptoms referable to the musculoskeletal system . . . . . . . . . . . . . . . . s900-s999 & $\mathbf{9 , 2 9 8}$ & 27.4 \\
\hline 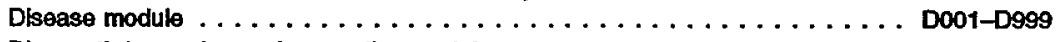 & 84 & 0.2 \\
\hline Diagnostic/screening and preventive madule. . . . . . . . . . . . . . . . . . . X100-X599 & 151 & 0.4 \\
\hline Treatment module . . . . . . . . . . . . . . . . . . . . . . . . . . . . . . . . . . . . . . . T100-T899 & 1,276 & 3.8 \\
\hline Injurles and actverse effects module . . . . . . . . . . . . . . . . . . . . . . J001-J999 & 17,061 & 50.3 \\
\hline Injury by type and/or location . . . . . . . . . . . . . . . . . . . . . . . . . . . . . . J001-J799 & 14,691 & 43.3 \\
\hline Injury, NOS . . . . . . . . . . . . . . . . . . . . . . . . . . . . . . . . . . . . . J800-J899 & 1,965 & 5.8 \\
\hline Polsoning and adverse effects. . . . . . . . . . . . . . . . . . . . . . . J J900-1999 & 405 & 1.2 \\
\hline$\ldots \ldots \ldots \ldots \ldots \ldots \ldots \ldots \ldots \ldots \ldots \ldots$ R100-8700 & 24 & 0.1 \\
\hline Administrative module. $\ldots \ldots \ldots \ldots \ldots \ldots \ldots \ldots \ldots \ldots \ldots \ldots \ldots \ldots$ A100-A140 & *24 & 0.1 \\
\hline Other $^{2} \ldots \ldots \ldots \ldots \ldots \ldots \ldots \ldots \ldots \ldots \ldots \ldots \ldots$ U990-U999 & 666 & 2.0 \\
\hline
\end{tabular}

'Based on A Rasson for Visit Classification for Ambulatory Care (RVC) (5).

includes problems and complaints not elsewhere classified, entries of "None," blanks, and illegible entries.

Table 7. Number, percent distribution, and cumulative percent of Injury-related emergency department visits by the 20 principal reasens for vlsit most frequently mentloned by patients: United States, 1992

\begin{tabular}{|c|c|c|c|}
\hline Principal reason for vish and RVC code' & $\begin{array}{l}\text { Number of } \\
\text { visits in } \\
\text { thousands }\end{array}$ & $\begin{array}{c}\text { Percent } \\
\text { clstutbution }\end{array}$ & $\begin{array}{c}\text { Cumulatve } \\
\text { percent }\end{array}$ \\
\hline 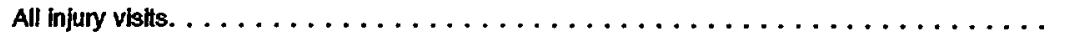 & 33,950 & 100.0 & $\cdots$ \\
\hline 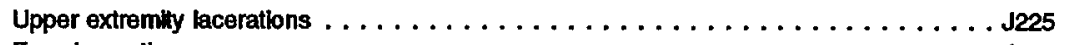 & 2,321 & 6.8 & 6.8 \\
\hline 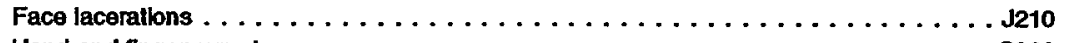 & 1,483 & 4.4 & 11.2 \\
\hline Hand and finger symptoms. . . . . . . . . . . . . . . . . . . . . . . . . . s960 & 1,225 & 3.6 & 14.8 \\
\hline 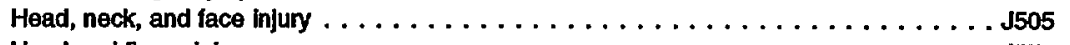 & 1,065 & 3.1 & 18.0 \\
\hline 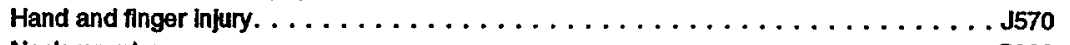 & 993 & 2.9 & 20.9 \\
\hline 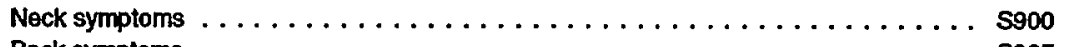 & 933 & 2.7 & 23.6 \\
\hline Back symploms. . . . . . . . . . . . . . . . . . . . . . . . . . . . . s905 & 913 & 2.7 & 26.3 \\
\hline 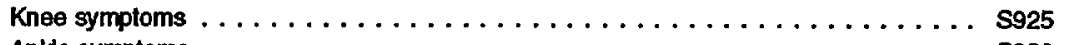 & 880 & 2.6 & 28.9 \\
\hline 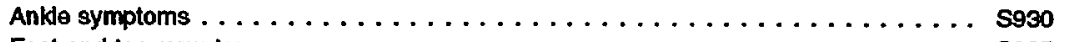 & 838 & 2.5 & 31.4 \\
\hline Foot and tos symptoms. . . . . . . . . . . . . . . . . . . . . . . . s935 & 780 & 2.3 & 33.7 \\
\hline Head and neck laceratlons. . . . . . . . . . . . . . . . . . . . . . J205 & 728 & 2.1 & 35.8 \\
\hline Suture-insertion/removal . . . . . . . . . . . . . . . . . . . . . . . . T555 & 693 & 2.0 & 37.9 \\
\hline Accident Nos. . . . . . . . . . . . . . . . . . . . . . . . . . J810 & 687 & 2.0 & 39.9 \\
\hline Shoulder symptoms . . . . . . . . . . . . . . . . . . . . . . . . . . s940 & 642 & 1.9 & 41.8 \\
\hline Arm symptoms . . . . . . . . . . . . . . . . . . . . . . . . . . . . s945 & 635 & 1.9 & 43.6 \\
\hline Pah, spectfled stte not reterable . . . . . . . . . . . . . . . . . . . . . . so5s & 621 & 1.8 & 45.5 \\
\hline Headache, pah in head . . . . . . . . . . . . . . . . . . . . . . . s210 & 621 & 1.8 & 47.3 \\
\hline Leg symptoms . . . . . . . . . . . . . . . . . . . . . . . . . . . . . . . . . . . s920 & 601 & 1.8 & 49.1 \\
\hline Low back symptoms. . . . . . . . . . . . . . . . . . . . . . . . . . . . s910 & 601 & 1.8 & 50.8 \\
\hline Wrist symptoms . . . . . . . . . . . . . . . . . . . . . . . . . . ssss & 591 & 1.7 & 52.6 \\
\hline 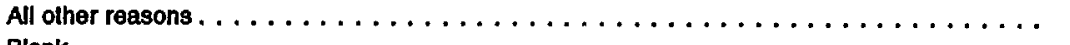 & 15,634 & 48.0 & 89.6 \\
\hline 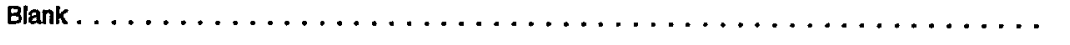 & 466 & 1.4 & 100.0 \\
\hline
\end{tabular}

\footnotetext{
${ }^{1}$ Based on A Roason for Visit Classification for Ambulatory Caro (AVC) (5).
} 
Table 8. Number, percent distribution, and cumulative percent of Injury-related emergency department visits by the 20 princlpal diagnoses most frequently rendered by hospital staft: United States, 1992

\begin{tabular}{|c|c|c|c|}
\hline Principal dlagnosis and $1 C D-9-C M$ code ${ }^{1}$ & $\begin{array}{c}\text { Number of } \\
\text { visits in } \\
\text { thousands }\end{array}$ & $\begin{array}{c}\text { Percent } \\
\text { distribution }\end{array}$ & $\begin{array}{c}\text { Cumulatwe } \\
\text { percent }\end{array}$ \\
\hline 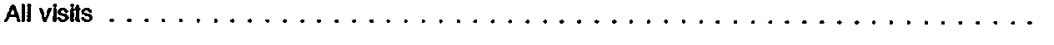 & 33,950 & 100.0 & $\cdots$ \\
\hline Open wound of head (other than eye or ear) $\ldots \ldots \ldots \ldots \ldots \ldots$ & 2,561 & 7.5 & 7.5 \\
\hline Contusion of lower llmb and other unspecified sites . . . . . . . . . . . . . . . . 924 & 1,755 & 5.2 & 12.7 \\
\hline Open wound of finger $\ldots \ldots \ldots \ldots \ldots \ldots \ldots \ldots \ldots \ldots$ & 1,604 & 4.7 & 17.4 \\
\hline 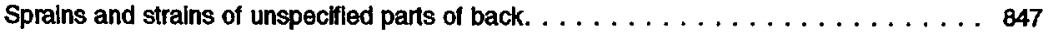 & 1,597 & 4.7 & 22.1 \\
\hline Spralns and strains of ankle and foot $\ldots \ldots \ldots \ldots \ldots \ldots \ldots$ & 1,311 & 3.9 & 26.0 \\
\hline Open wound of other and unspecifled sites, except limbs $\ldots \ldots \ldots \ldots \ldots$. . . . . 879 & 1,257 & 3.7 & 29.7 \\
\hline Contusion of upper $\| m b \ldots \ldots \ldots \ldots \ldots \ldots$ & 1,255 & 3.7 & 33.4 \\
\hline Contusion of face, scaip, and neck except eyes. . . . . . . . . . . . . . . . . 920 & 863 & 2.5 & 35.9 \\
\hline 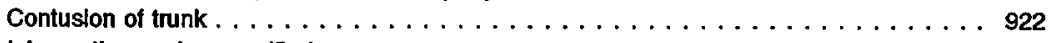 & 744 & 2.2 & 38.1 \\
\hline 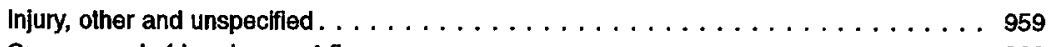 & 720 & 2.1 & 40.3 \\
\hline Open wound of hand, except finger $\ldots \ldots \ldots \ldots \ldots \ldots \ldots \ldots \ldots$ & 675 & 2.0 & 42.2 \\
\hline 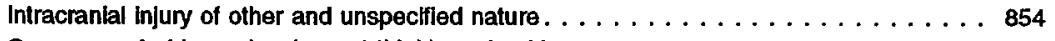 & 564 & 1.7 & 43.9 \\
\hline Open wound of knee, leg (except thigh), and ankle. . . . . . . . . . . . . . 891 & 548 & 1.6 & 45.5 \\
\hline Encounter for other and unspectfled procedures and aftercare $\ldots \ldots \ldots \ldots \ldots \ldots$ & 540 & 1.6 & 47.1 \\
\hline Fracture of radius and ulna $\ldots \ldots \ldots \ldots \ldots \ldots \ldots \ldots \ldots \ldots \ldots$ & 518 & 1.5 & 48.6 \\
\hline Sprains and stralns of wrlst and hand $\ldots \ldots \ldots \ldots \ldots$ & 518 & 1.5 & 50.2 \\
\hline 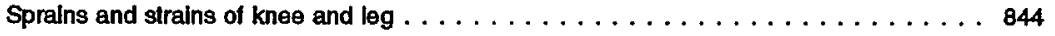 & 509 & 1.5 & 51.7 \\
\hline Fracture of one or more phalanges of hand $\ldots \ldots \ldots \ldots \ldots \ldots \ldots$ & 482 & 1.4 & 53.1 \\
\hline Superficlal injury of eye and adnexa $\ldots \ldots \ldots \ldots \ldots \ldots \ldots \ldots$ & 442 & 1.3 & 54.4 \\
\hline 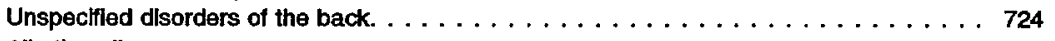 & 392 & 1.2 & 55.5 \\
\hline 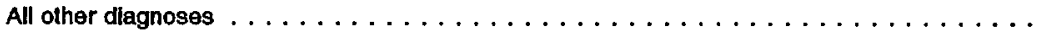 & 15,095 & 44.5 & 100.0 \\
\hline
\end{tabular}

'Based on the Intemational Classification of Disoases, 9th Revision, Clinical Modification (ICD-9-CM) (3).

Table 9. Number and percent distrlbution of emergency department visits and percent of total visits that are injury related, by selected dlagnostic and/or screening services: United States, 1992

\begin{tabular}{|c|c|c|c|}
\hline Dlagnostic and/or screening senvice ordered or provided by physiclan ${ }^{1}$ & $\begin{array}{c}\text { Number of } \\
\text { visits in } \\
\text { thousands }\end{array}$ & $\begin{array}{c}\text { Percent } \\
\text { distribution }\end{array}$ & $\begin{array}{c}\text { Percent } \\
\text { Injuny } \\
\text { related }\end{array}$ \\
\hline 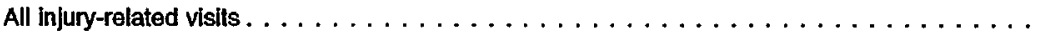 & 33,950 & 100.0 & 37.8 \\
\hline 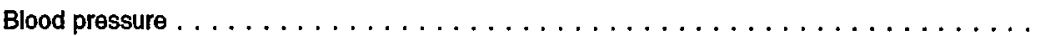 & 25,202 & 74.2 & 38.1 \\
\hline 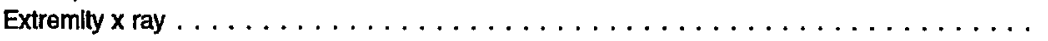 & 12,091 & 35.6 & 89.3 \\
\hline 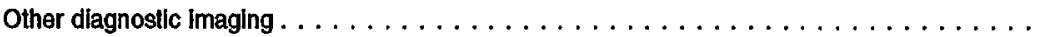 & 4,147 & 12.2 & 44.3 \\
\hline 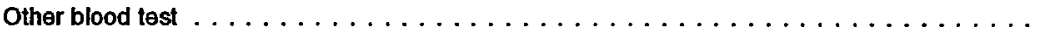 & 3,397 & 10.0 & 13.2 \\
\hline 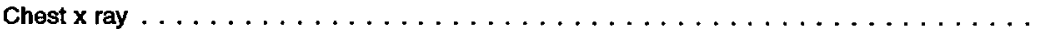 & 2,657 & 7.8 & 17.6 \\
\hline 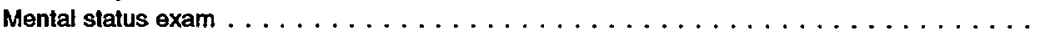 & 2,044 & 6.0 & 38.7 \\
\hline 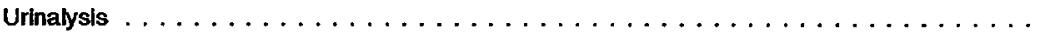 & 2,063 & 6.1 & 15.1 \\
\hline Electrocardiogram (EKG) $\ldots \ldots \ldots \ldots \ldots \ldots \ldots \ldots \ldots \ldots \ldots \ldots \ldots$ & 1,495 & 4.4 & 12.6 \\
\hline 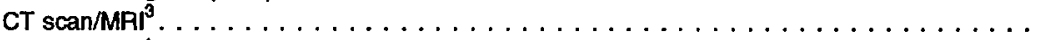 & 860 & 2.5 & 99.8 \\
\hline 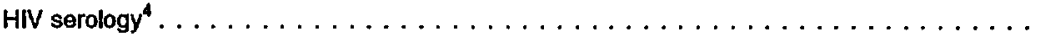 & 92 & 0.3 & 94.2 \\
\hline 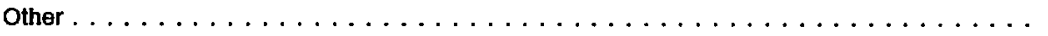 & 4,584 & 13.5 & 25.9 \\
\hline None . . . . . . . . . . . . . . . . . . . . . . . . . . . & 3,819 & 11.2 & 35.2 \\
\hline
\end{tabular}

${ }^{1}$ Total may exceed total number of visits because more than one service may be reported per visit.

2Percent of all emergency department visits in each calegory that are injury related.

${ }^{3} \mathrm{CT}$ is computerized tomography. MRI is magnetic resonanco imaging.

${ }^{4} \mathrm{HIV}$ is human immunodeficiency virus.

occurring at 7.5 percent of all injury visits.

\section{Diagnostic and screening services}

Statistics on various diagnostic and screening services ordered or provided by hospital staff during an injury-related ED visit are displayed in table 9.
Approximately 88.8 percent of all injury-related ED visits included one or more diagnostic or screening service. The most frequently mentioned diagnostic service was blood pressure check, recorded at 74.2 percent of visits. Extremity $x$ ray ( 35.6 percent) was the second most frequent diagnostic procedure. About 9 of every $10 \mathrm{ED}$ visits with extremity $x$ rays were injury related. Half of all injury-related visits for accidental falls involved an extremity $\mathrm{x}$ ray. Visits due to motor vehicle accidents or violence were more likely to have blood tests and urinalysis performed compared to injury visits for other causes.

Readers should note that for items $8,15,16,18$, and 19 , hospital staff were asked to check all of the applicable 
Table 10. Number and percent distribution of Injury-related emergency department visits and percent that are injury related, by selected procedures: United States, 1992

\begin{tabular}{|c|c|c|c|}
\hline Procedure provided by hospltal staff ${ }^{1}$ & $\begin{array}{c}\text { Number of } \\
\text { visits in } \\
\text { thousands }\end{array}$ & $\begin{array}{l}\text { Percent } \\
\text { distribution }\end{array}$ & $\begin{array}{c}\text { Percent } \\
\text { injury } \\
\text { related }\end{array}$ \\
\hline All vists $\ldots \ldots \ldots \ldots \ldots \ldots \ldots \ldots \ldots \ldots \ldots \ldots \ldots$ & 33,950 & 100.0 & 37.8 \\
\hline 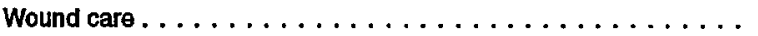 & 10,757 & 31.7 & 93.1 \\
\hline Orthopedic care $\ldots \ldots \ldots \ldots \ldots \ldots \ldots \ldots$ & 6,706 & 19.8 & 94.8 \\
\hline 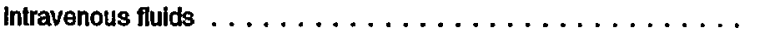 & 2,075 & 6.1 & 16.0 \\
\hline Eye and/or ear, nose, and throat care . . . . . . . . . . . & 1,241 & 3.7 & 49.9 \\
\hline 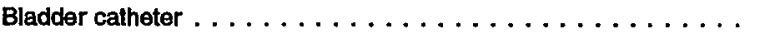 & 415 & 1.2 & 17.9 \\
\hline Nasogastric tube and/or gastric lavage $\ldots \ldots \ldots \ldots \ldots$ & 372 & 1.1 & 42.4 \\
\hline Endotracheal Intubation. . . . . . . . . . . . . . . . & 110 & 0.3 & 27.0 \\
\hline 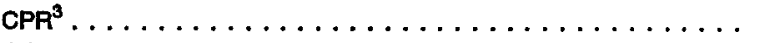 & 63 & 0.2 & 21.6 \\
\hline 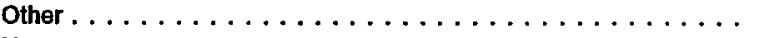 & 2,470 & 7.3 & 36.9 \\
\hline None . . . . . . . . . . . . . . . . . . . . . . . . & 13,296 & 39.2 & 25.7 \\
\hline
\end{tabular}

Total may exceed total number of visits because more than one procedure may be reported per visit.

2Percent of all emergency department visits in each category that are injury related.

${ }^{3} \mathrm{CPR}$ is cardiopulmonary resuscitation.

Table 11. Number and percent distribution of injury-related emergency department visits and percent that are injury related, by number of medications provided or prescribed: Unlted States, 1992

\begin{tabular}{|c|c|c|c|}
\hline Number of medications & $\begin{array}{l}\text { Number of } \\
\text { Vsits in } \\
\text { thousands }\end{array}$ & $\begin{array}{l}\text { Percent } \\
\text { distribution }\end{array}$ & $\begin{array}{c}\text { Percent } \\
\text { injuy } \\
\text { relateds }\end{array}$ \\
\hline All injury-related visits $\ldots \ldots \ldots \ldots \ldots \ldots \ldots \ldots$ & 33,950 & 100.0 & 37.8 \\
\hline None $\ldots \ldots \ldots \ldots \ldots$ & 12,812 & 37.7 & 46.2 \\
\hline 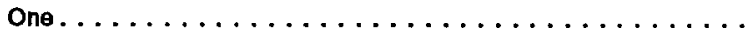 & 12,244 & 36.1 & 41.8 \\
\hline 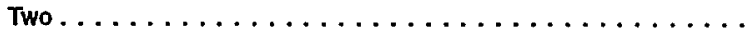 & 5,691 & 16.8 & 30.2 \\
\hline 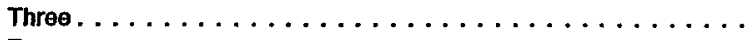 & 2,004 & 5.9 & 24.8 \\
\hline 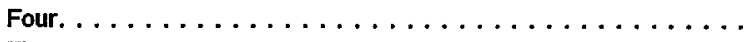 & 723 & 2.1 & 22.4 \\
\hline Flve or more $\ldots \ldots \ldots \ldots \ldots \ldots \ldots \ldots \ldots \ldots$ & 476 & 1.4 & 17.9 \\
\hline
\end{tabular}

Percent of all omergency department visits in each category that are injury related.

Table 12. Number, percent distribution, cumulative percent, and therapeutic classification of the 15 drugs most frequently provided or prescribed in injury-rolated emergency department visits by ontry name of drug: United States, 1992

\begin{tabular}{|c|c|c|c|c|}
\hline Entry name of drug ${ }^{1}$ & $\begin{array}{l}\text { Number of } \\
\text { mentlons in } \\
\text { thousands }\end{array}$ & $\begin{array}{c}\text { Percent } \\
\text { distribution }\end{array}$ & $\begin{array}{c}\text { Cumulative } \\
\text { percent }\end{array}$ & $\begin{array}{l}\text { Therapeutlc } \\
\text { classification }\end{array}$ \\
\hline$\ldots \ldots \ldots \ldots \ldots \ldots$ & 34,910 & 100.0 & $\cdots$ & $\cdots$ \\
\hline Tetanus-relaled biologicals . . . . . . . . . . . . . & 3,311 & 9.5 & 9.5 & Vaccines and antiserums \\
\hline Tylenot . . . . . . . . . . . . . . . . . & 2,592 & 7.4 & 16.9 & General analgesics \\
\hline Motrn. . . . . . . . . . . . . . & 2,216 & 6.3 & 23.3 & General analgesics \\
\hline Tylenol with codeine. . . . . . . . . . . . . . & 1,790 & 5.1 & 28.4 & General analgesics \\
\hline Toradol . . . . . . . . . . . . . . . . . . & 1,079 & 3.1 & 31.5 & General analgesics \\
\hline Demerol . . . . . . . . . . . . . . . . . . . & 1,017 & 2.9 & 34.4 & General analgesics \\
\hline Advill $\ldots \ldots \ldots \ldots \ldots \ldots \ldots \ldots \ldots \ldots$ & 873 & 2.5 & 36.9 & General anaigesics \\
\hline Vicodin $\ldots \ldots \ldots \ldots \ldots \ldots \ldots \ldots \ldots$ & 794 & 2.3 & 39.2 & General analgesics \\
\hline Kaflex. . . . . . . . . . . . . . . . . . . & 701 & 2.0 & 41.2 & Cephalosporins \\
\hline Darvocet-N $\ldots \ldots \ldots \ldots \ldots \ldots \ldots \ldots \ldots$ & 669 & 1.9 & 43.1 & General analgesics \\
\hline Lidocaine. . . . . . . . . . . . . . . . . & 635 & 1.8 & 44.9 & Local anesthetics \\
\hline Ibuprofen, $\ldots \ldots \ldots \ldots \ldots \ldots \ldots \ldots \ldots$ & 627 & 1.8 & 46.7 & General analgesics \\
\hline Neosporin $\ldots \ldots \ldots \ldots \ldots \ldots \ldots \ldots \ldots$ & 607 & 1.7 & 48.4 & Antbacterial agents \\
\hline Flexeril $\ldots \ldots \ldots \ldots \ldots \ldots \ldots \ldots \ldots \ldots$ & 546 & 1.6 & 50.0 & Muscle relaxants \\
\hline Benadryl . . . . . . . . . . . . . . . & 507 & 1.5 & 51.5 & Anthistamines \\
\hline All other mentions $\ldots \ldots \ldots \ldots \ldots \ldots \ldots \ldots$ & 16,948 & 48.5 & 100.0 & $\cdots$ \\
\hline
\end{tabular}

The entry made by the hospital staff on the prescription or other medical reoords. This may be a trade name, generic name, or deesired therapeutic effect.

2Therapeutic classification is based on the National Drug Coda Directory, 1985 Edition (6). 
Table 13. Number, percent distribution, and cumulative percent of drug mentions for the 15 most frequently used generic substances in Injury-related emergency department visits: United States, 1992

\begin{tabular}{|c|c|c|c|}
\hline Generic substance & $\begin{array}{l}\text { Number of } \\
\text { mentions in } \\
\text { thousands }\end{array}$ & $\begin{array}{c}\text { Percent } \\
\text { dlstrlbution }\end{array}$ & $\begin{array}{c}\text { Cumulathe } \\
\text { percent }\end{array}$ \\
\hline 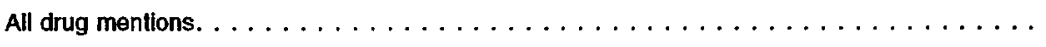 & 45,207 & 100.0 & $\cdots$ \\
\hline 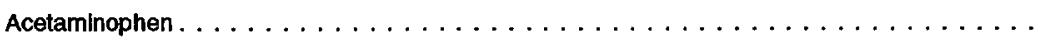 & 6,759 & 15.0 & 15.0 \\
\hline 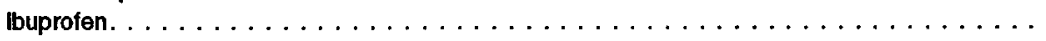 & 3,869 & 8.6 & 23.5 \\
\hline 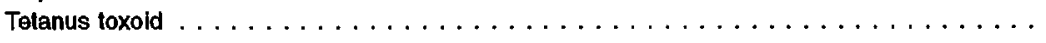 & 2,793 & 6.2 & 29.7 \\
\hline 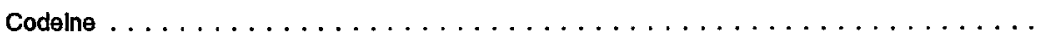 & 2,008 & 4.4 & 34.1 \\
\hline 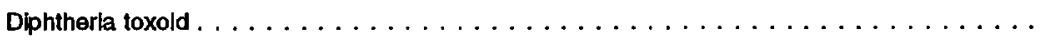 & 1,873 & 4.1 & 38.3 \\
\hline 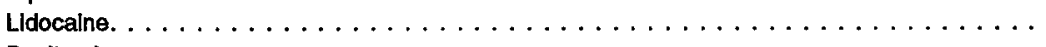 & 1,230 & 2.7 & 41.0 \\
\hline 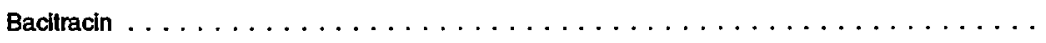 & 1,195 & 2.6 & 43.6 \\
\hline 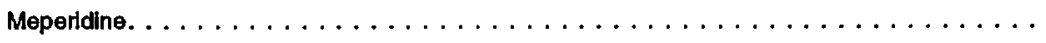 & 1,086 & 2.4 & 46.0 \\
\hline 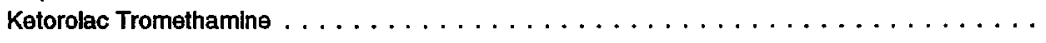 & 1,079 & 2.4 & 48.4 \\
\hline 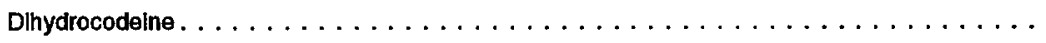 & 924 & 2.0 & 50.5 \\
\hline 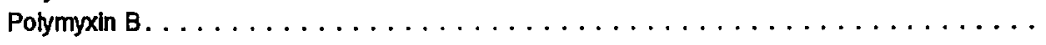 & 848 & 1.9 & 52.3 \\
\hline 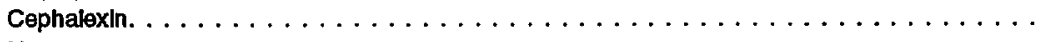 & 792 & 1.8 & 54.1 \\
\hline 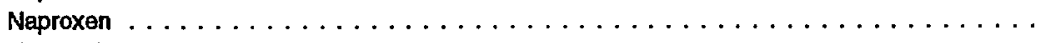 & 741 & 1.6 & 55.7 \\
\hline 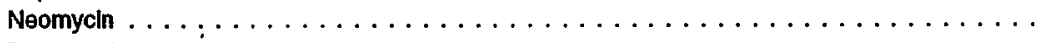 & 734 & 1.6 & 57.4 \\
\hline 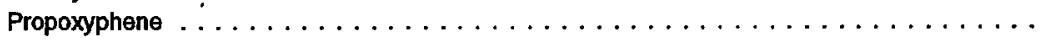 & 715 & 1.6 & 58.9 \\
\hline 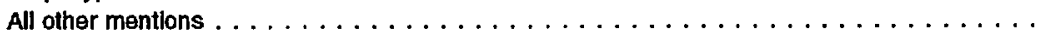 & 18,561 & 41.1 & 100.0 \\
\hline
\end{tabular}

${ }^{1}$ Frequency of mention combines single-ingredient agents with mentions of the agent as an ingredient in a combination drug.

Table 14. Number and percent distribution of emergency department visits and percent of total visits that are injury related, by patient's expected source of payment: United States, 1992

\begin{tabular}{|c|c|c|c|}
\hline Expected source of payment ${ }^{1}$ & $\begin{array}{c}\text { Number of } \\
\text { vists In } \\
\text { thousands }\end{array}$ & $\begin{array}{c}\text { Percent } \\
\text { distribution }\end{array}$ & $\begin{array}{c}\text { Percent } \\
\text { injury } \\
\text { related }\end{array}$ \\
\hline 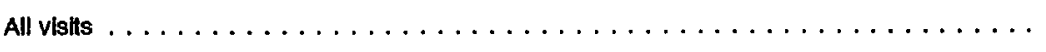 & 33,950 & 100.0 & 37.8 \\
\hline 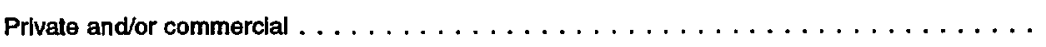 & 13,869 & 40.9 & 42.9 \\
\hline 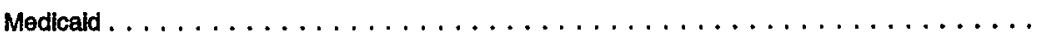 & 5,072 & 14.9 & 24.9 \\
\hline 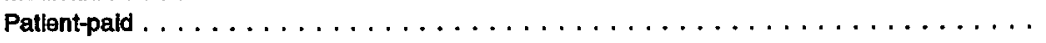 & 5,006 & 14.7 & 40.4 \\
\hline Medicare . . . . . . . . . . . . . . . . . . . . . . . . . . & 3,129 & 9.2 & 23.0 \\
\hline 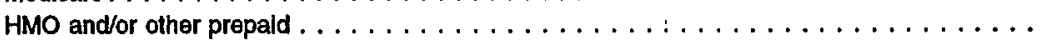 & 2,701 & 8.0 & 41.1 \\
\hline 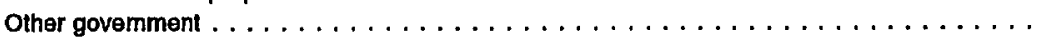 & 1,759 & 5.2 & 43.6 \\
\hline 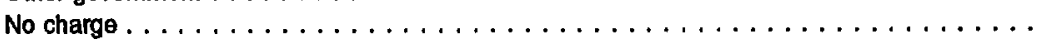 & 213 & 0.6 & 27.4 \\
\hline 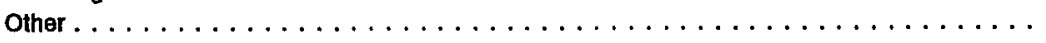 & 3,677 & 10.8 & 60.1 \\
\hline 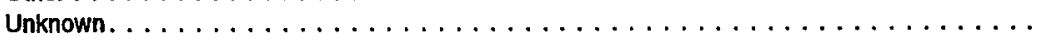 & 484 & 1.4 & 32.2 \\
\hline
\end{tabular}

${ }^{1}$ Total may oxceed total number of visits because more than one pay sourco may be coded for each visit.

${ }^{2}$ Percent of all emergency department visits in each category that are injury rolated.

Table 15. Number and percent dlstribution of emergency department visits and percent of total visits that are injury related, by type of provider seen: Unlted States, 1992

\begin{tabular}{|c|c|c|c|}
\hline Type of provider ${ }^{1}$ & $\begin{array}{c}\text { Number of } \\
\text { vists in } \\
\text { thousands }\end{array}$ & $\begin{array}{c}\text { Percent } \\
\text { distribution }\end{array}$ & $\begin{array}{c}\text { Percent } \\
\text { infury } \\
\text { related }\end{array}$ \\
\hline 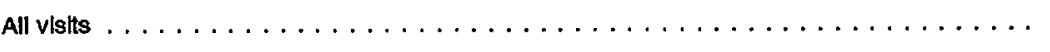 & 33,950 & 100.0 & 37.8 \\
\hline 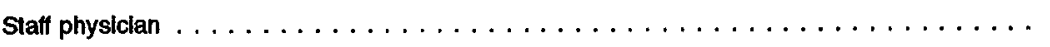 & 28,466 & 83.8 & 38.4 \\
\hline 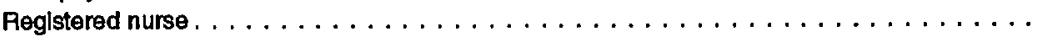 & 28,350 & 83.5 & 38.0 \\
\hline 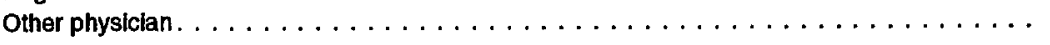 & 3,799 & 11.2 & 36.1 \\
\hline 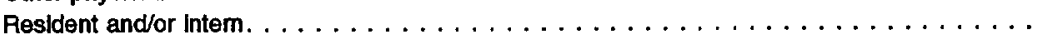 & 3,750 & 11.0 & 30.5 \\
\hline Nurse's alde. . . . . . . . . . . . . . . . . . . . . . . . . . & 3,210 & 9.5 & 37.8 \\
\hline 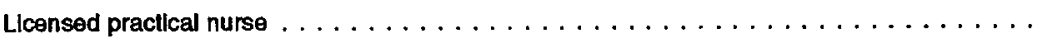 & 2,039 & 6.0 & 34.9 \\
\hline 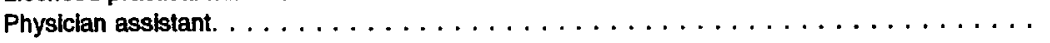 & 864 & 2.5 & 49.2 \\
\hline 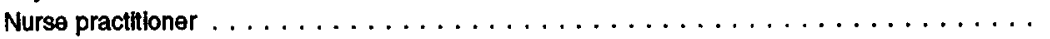 & 554 & 1.6 & 31.7 \\
\hline
\end{tabular}

\footnotetext{
${ }^{1}$ Total may exceed total number of visits because more than one provider may be reported per visit

${ }^{2}$ Percent of all emergency department visits in each calegory that are injury related.
} 
Table 16. Number, percent dlstribution of Injury-related emergency department visits and percent that are injury related, by disposition of visit: United States, 1992

\begin{tabular}{|c|c|c|c|}
\hline Disposition ${ }^{1}$ & $\begin{array}{c}\text { Number of } \\
\text { visits in } \\
\text { thousands }\end{array}$ & $\begin{array}{c}\text { Percent } \\
\text { distribution }\end{array}$ & $\begin{array}{c}\text { Percent } \\
\text { Inluy } \\
\text { related }\end{array}$ \\
\hline 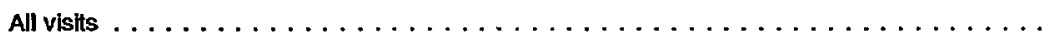 & 33,950 & 100.0 & 37.8 \\
\hline 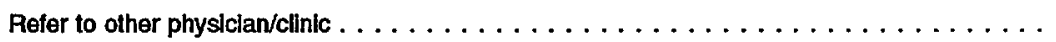 & 13,548 & 39.9 & 40.8 \\
\hline Return to emergency department as needed. . . . . . . . . . . . . . . . . & 8,928 & 26.3 & 39.8 \\
\hline 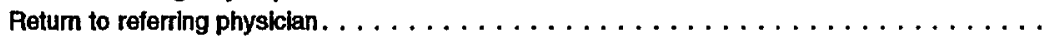 & 6,957 & 20.5 & 36.6 \\
\hline 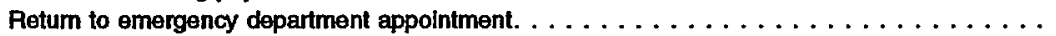 & 2,891 & 8.5 & 66.9 \\
\hline 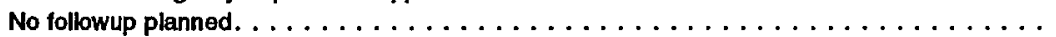 & 2,325 & 6.8 & 43.6 \\
\hline 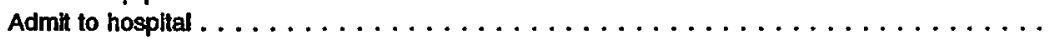 & 2,072 & 6.1 & 17.1 \\
\hline 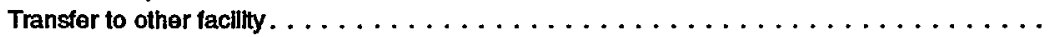 & 340 & 1.0 & 31.1 \\
\hline 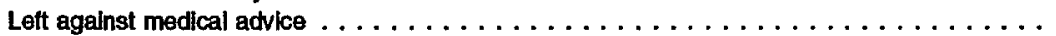 & 316 & 0.9 & 30.2 \\
\hline Dead on arrval or dled in emergency department. . . . . . . . . . . . . . . . & $\star 51$ & $* 0.2$ & ${ }^{*} 18.2$ \\
\hline 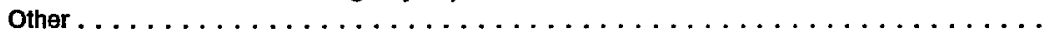 & 1,650 & 4.9 & 36.0 \\
\hline
\end{tabular}

Total may exceed tolal number of visits because more than one disposition may be reported per visit.

2percont of all emergency department visits in each calegory that are injury related.

categories for that item, with the result that multiple responses could be coded for each visit.

\section{Procedures}

Procedures were performed at 60.8 percent of injury-related ED visits (table 10), which was twice the percent for illness-related visits. The most frequently mentioned procedure was wound care, recorded at 31.7 percent of the visits. Orthopedic care was the procedure with the second highest frequency, occurring at one-fifth of the visits. Roughly 94 percent of all visits with wound care or orthopedic care were identified as related to an injury. Injury visits were less likely to require the use of intravenous fluids compared with illness-related visits (6.1 and 19.5 percent respectively).

\section{Medication therapy}

Medication was used at 62.3 percent of the injury-related visits. Hospital staff were instructed to record all new or continued medications ordered or provided at the visit, including prescription and nonprescription preparations, and immunizing and desensitizing agents. As many as five medications or drug mentions could be coded per visit. Visits with one or more drug mentions are termed "drug visits" for this report. Table 11 shows the frequency and percent of numbers of medications administered or prescribed during the visit. There was an average

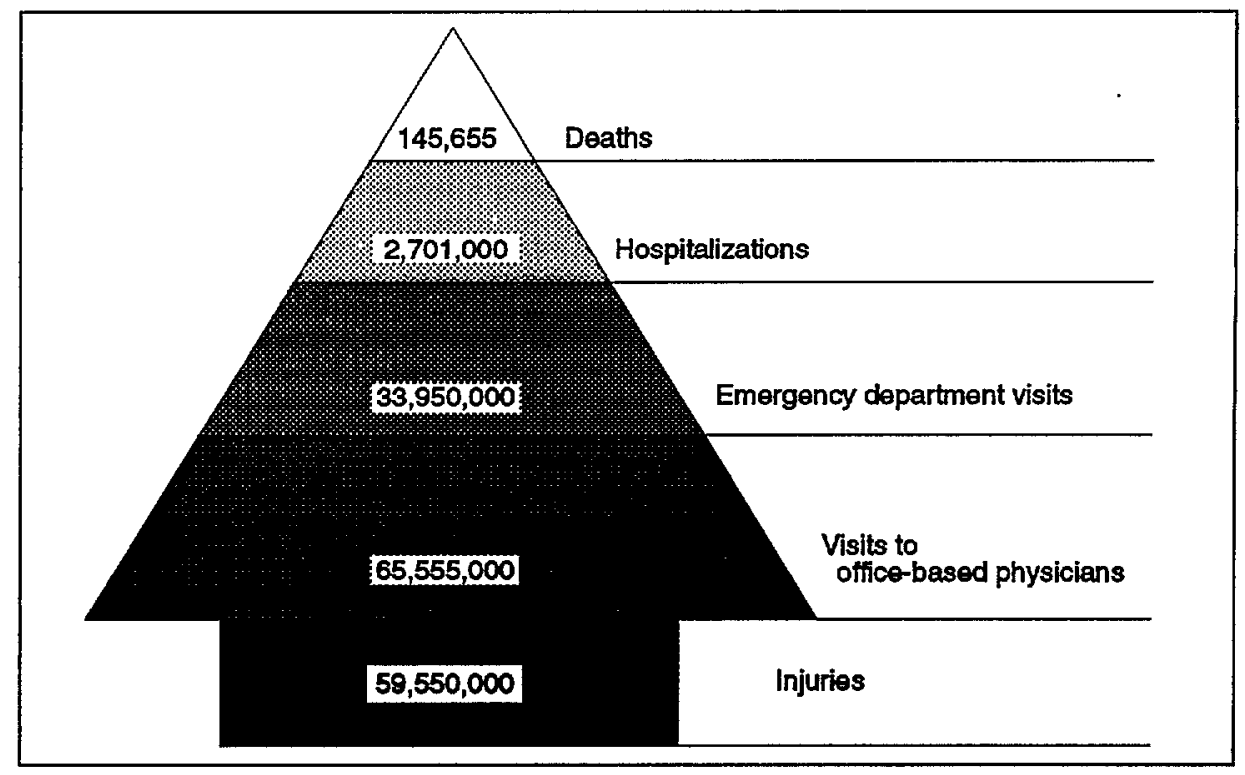

Figure 5. The Injury-yramid: United States, 1992

of 1.1 drug mentions per injury-related ED visit or 1.8 mentions per injuryrelated drug visit. Only one drug mention was recorded at 36.1 percent of the injury-related visits. Medications were administered or prescribed less frequently for injury-related visits compared with illness-related visits (62.3 and 73.3 percent respectively).

The 15 most frequently mentioned medications in injury-related ED visits are presented in table 12 according to the name written on the ED Patient Record by the health care provider regardless of whether it is a brand name, generic name, or therapeutic effect. Tetanus-related biologicals, Tylenol, and Motrin were the three drugs or immunizing agents most frequently provided or prescribed during injuryrelated ED visits. They accounted for about one-quarter of all drug mentions. Of the top drug names mentioned, most are classified as general analgesics. This is based on the therapeutic categories used in the National Drug Code Directory, 1985 edition (NDC) (6).

The 15 most frequently used generic substances for 1992 injuryrelated ED visits are shown in table 13. Drug products containing more than one ingredient (combination products) are included in the data for each ingredient. For example, acetaminophen with codeine is included in both the count for acetaminophen and the count for 
codeine. Acetaminophen was the generic ingredient most frequently used in drugs ordered or provided by hospital staff, occurring in 15 percent of drug mentions. The top 15 generic substances accounted for almost 60 percent of all drug mentions. As expected, the top generic substances in injury-related visits are different from those for illness-related ED visits. Only acetaminophen, ibuprofen, and codeine appear on both lists of top 15 generic substances.

\section{Expected source of payment}

Expected source of payment for injury-related visits (table 14) was most often private/commercial insurance (40.9 percent). "Medicaid" and "patient-paid" each accounted for about 15 percent of the injury-related visits. "HMO/other prepaid" and "Medicare" were each mentioned at about 8 percent of injury-related $\mathrm{ED}$ visits. The patient-paid category includes the patient's contribution toward "copayments" and "deductibles." While injuries made up 37.5 percent of the ED workload, they accounted for 60 percent of the visits whose payment source was categorized as "other" on the form. It is possible that visits paid by worker compensation were recorded under "other." A separate category for worker compensation was placed on the 1995-96 Patient Record to provide better information for future analyses. About 40 percent of the "Other Government," "HMO/Other prepaid," "Private commercial," and "Patientpaid" visits are injury related. Only about 24 percent of the Medicare and Medicaid visits are injury related.

\section{Providers seen this visit}

A registered nurse or staff physician was seen at 84 percent of injury-related ED visits (table 15). These percents are not significantly different from those corresponding to all ED visits.

\section{Disposition of this visit}

The most frequent disposition was to refer the patient to another physician or clinic (39.9 percent). Only 6.1 percent of injury-related $\mathrm{ED}$ visits resulted in hospital admission (table 16). This is lower than the 18 percent of illnessrelated visits that ended in hospitalization. Roughly one-quarter of the injury-related visits had a disposition of "Return to ED as needed." This means no followup is planned, but if the condition worsens, the patient should return. Patients in injury-related visits were more likely to be scheduled for another ED appointment compared with illness-related visits ( 8.5 and 2.6 percent, respectively). Visits resulting from accidental falls and motor vehicle accidents were more likely to result in a hospital admission for the patient $\mathbf{( 9 . 2}$ and 11.2 percent respectively) than were injury visits for other causes (5.2 percent).

\section{Impact of data}

The NHAMCS data present a better picture of the impact of injuries on health care utilization. Figure 5 presents the injury pyramid indicating the national estimates from which relative rates of various health care events and death may be determined. The following rates are based on 59.6 million reported injuries that were obtained from the 1992 National Health Interview Survey (7). For every 100 injuries requiring medical attention or resulting in the loss of at least one-half day from usual activities, there were 110 physician office visits (8); 57 emergency department visits; 4.5 hospitalizations (9); and 0.24 deaths (10). Another way of comparing the relative impact of injuries is using the number of deaths as the base. For each death in 1992 resulting from an injury, there were 19 hospitalizations, $233 \mathrm{ED}$ visits, and 450 physician office visits.

The impact of injuries comprises a significant portion of health care expenditures in the United States. The average cost of an ED visit in 1987 was $\$ 166$ (11). After adjusting for changes in the consumer price index between 1987 and 1992, the cost in 1992 dollars would be $\$ 271$. Based on this cost, the annual national cost of visits to ED's alone for injury-related purposes is over 9.2 billion dollars. The 1992 NHAMCS data revealed that the rates of injuries resulting in ED visits are highest among males 15-24 years of age. Close to two-thirds of all ED visits for this population were for injuries. Continued reliance on injury prevention programs, especially targeted to this population, should help to reduce resources spent in health care, loss of productivity, loss of life, and loss of quality of life as a result of personal injuries. These data support the injury prevention programs that emphasize nonviolent solutions to conflicts, elimination of alcohol and drug abuse, and increased education in home, school, recreational, workplace, and transportation safety procedures and practices. The 1992 NHAMCS data provide the first national data on nonfatal causes of injury resulting in emergency medical care. Analysis of data by E-codes supports the prevention efforts that promote individual practices and behaviors that reduce a person's risk of injury, such as proper storage of firearms in the home, avoiding drinking and driving, using safety restraints while riding in an automobile, and wearing a helmet when riding a bicycle or motorcycle.

Additional reports that utilize the 1992 NHAMCS data will be published. In addition, a computer tape containing both the emergency and outpatient department data is available at a nominal cost from the National Technical Information Service. These data will also be available on CD-ROM and diskettes. Questions regarding this report may be directed to the Ambulatory Care Statistics Branch by calling (301) 436-7132.

\section{References}

1. McCaig LF. National Hospital Ambulatory Medical Care Survey: 1992 emergency department summary. Advance data from vital and health statistics; no 245. Hyattsville, Maryland: National Center for Health Statistics. 1994.

2. McCaig LF, McLemore T. Plan and operation of the National Hospital Ambulatory Medical Care Survey. National Center for Health Statistics. Vital Health Stat 1(34). 1994. 
3. Public Health Service and Health Care Financing Administration. International classification of diseases, 9th revision, clinical modification. Washington, D.C.: Public Health Service. 1991.

4. Nelson CR, Stussman BJ. Alcoholand drug-related visits to hospital emergency departments: 1992 National Hospital Ambulatory Medical Care Survey. Advance data from vital and health statistics; no 251. Hyattsville, Maryland: National Center for Health Statistics. 1994.

5. Schneider D, Appleton L, McLemore, T. A reason for visit classification for ambulatory care. National Center for Health Statistics. Vital Health Stat 2(78). 1979.
6. Food and Drug Administration. National Drug Code directory, 1985 ed. Washington: Public Health Service. 1985.

7. Benson V, Marano MA. Current estimates from the National Health Interview Survey. National Center for Health Statistics. Vital Health Stat 10(189). 1994.

8. Schappert SM. National Ambulatory Medical Care Survey: 1992 summary. Advance data from vital and health statistics; no 253. Hyattsville, Maryland: National Center for Health Statistics. 1994.

9. Graves EJ. 1992 Summary: National Hospital Discharge Survey. Advance data from vital and health statistics; no 249. Hyattsville, Maryland:
National Center for Health Statistics. 1994.

10. Kochanek KD, Hudson BL. Advance report of final mortality statistics, 1992. Monthly vital statistics report; vol. 43 , no. 6 . suppl. Hyattsville, Maryland: National Center for Health Statistics. 1994.

11. 1987 National Medical Expenditure Survey: Public Use Tape 14.5; Household Survey; Ambulatory Medical Visit Data, Calendar Year 1987.

12. Shah BV, Barnwell BG, Hunt PN, LaVange LM. SUDAAN user's manual, release 5.50. Research Triangle Park, North Carolina: Research Triangle Institute. 1991. 


\section{Technical notes}

\section{Source of data and sample design}

The information presented in this report is based on data collected in the 1992 National Hospital Ambulatory Medical Care Survey (NHAMCS) from December 2, 1991 through December 27,1992 . The data were adjusted to produce annual estimates. The target universe of NHAMCS includes visits made in the United States by patients to emergency departments (ED's) and outpatient departments (OPD's) of non-Federal, short-stay, and general hospitals. Telephone contacts are excluded.

A four-stage probability sample design is used in NHAMCS. It involves samples of primary sampling units (PSU's), hospitals with ED's and/or OPD's within PSU's, ED's within hospitals and/or clinics within OPD's, and patient visits within ED's and/or clinics. For 1992, a sample of 524 non-Federal, short-stay, and general hospitals was selected from the SMG Hospital Market Database. Of this group, 474 hospitals were in scope, or eligible to participate in the survey. The hospital response rate for the NHAMCS during this period was 93 percent. Hospital staff were asked to complete a Patient Record (figure 1) for a systematic random sample of patient visits occurring during a randomly assigned 4-week reporting period. The number of Patient Record forms completed for ED's was 36,271.

Characteristics of the hospital, such as ownership and expected number of ED visits, were obtained from the hospital administrator during an induction interview. The U.S. Bureau of the Census, Housing Surveys Branch, was responsible for the survey's data collection. Data processing operations and medical coding were performed by the National Center for Health Statistics, Health Care Surveys Section, Research Triangle Park, North Carolina.

\section{Sampling errors}

The standard error is primarily a measure of the sampling variability that occurs by chance when only a sample, rather than an entire universe, is surveyed. The standard error also reflects part of the measurement error, but does not measure any systematic biases in the data. The chances are 95 out of 100 that an estimate from the sample differs from the value that would be obtained from a complete census by less than twice the standard error.

The standard errors that were used in tests of significance for this report were calculated using generalized linear models for predicting the relative standard error for estimates based on the linear relationship between the actual standard error, as approximated using SUDAAN software, and the size of the estimate. SUDAAN computes standard errors by using a first-order Taylor approximation of the deviation of estimates from their expected values. A description of the software and the approach it uses has been published (12). The relative standard error (RSE) of an estimate is obtained by dividing the standard error by the estimate itself. The result is then expressed as a percent of the estimate.

Relative standard errors for emergency department estimates are shown in tables I and II. Standard errors for estimates in percents of visits and drug mentions are shown in tables III and IV. Multiplying the estimate by the RSE will provide an estimate of the standard error for the estimate.

Alternatively, relative standard errors for aggregate estimates may be calculated using the following general formula: where $x$ is the aggregate of interest in thousands, and $A$ and $B$ are the appropriate coefficients from table V.

$$
\operatorname{RSE}(x)=\sqrt{A+\frac{B}{x}} \mathrm{v} 100
$$

Similarly, relative standard errors for an estimate of a percent may be calculated using the following general formula:

$$
R S E(x)=\sqrt{\frac{B \vee(1-p)}{p \vee x}} \vee 100
$$

where $p$ is the percent of interest, expressed as a proportion, and $x$ is the denominator of the percent in thousands,
Table I. Approximate relative standard errors for estimated numbers of emergency department visits: National Hospltal

\begin{tabular}{|c|c|}
\hline $\begin{array}{l}\text { Estimated number of } \\
\text { emergency department } \\
\text { visits in thousands }\end{array}$ & $\begin{array}{l}\text { Relative } \\
\text { standard } \\
\text { error in } \\
\text { percent }\end{array}$ \\
\hline $10 \ldots \ldots \ldots \ldots$ & 71.1 \\
\hline $20 \ldots \ldots \ldots \ldots \ldots$ & 50.4 \\
\hline $50 \ldots \ldots \ldots \ldots$ & 32.0 \\
\hline $58 \ldots \ldots \ldots \ldots \ldots$ & 29.7 \\
\hline $100 \ldots \ldots \ldots \ldots$ & 22.8 \\
\hline $200 \ldots \ldots \ldots \ldots$ & 16.4 \\
\hline $500 \ldots \ldots \ldots$ & 10.8 \\
\hline $1,000 \ldots \ldots \ldots \ldots$ & 8.1 \\
\hline $2,000 \ldots \ldots \ldots \ldots$ & 6.4 \\
\hline $5,000 \ldots \ldots \ldots \ldots$ & 5.1 \\
\hline $10,000 \ldots \ldots \ldots$ & 4.6 \\
\hline $20,000 \ldots \ldots \ldots \ldots$ & 4.3 \\
\hline $50,000 \ldots \ldots \ldots$ & 4.1 \\
\hline $100,000 \ldots \ldots \ldots$ & 4.0 \\
\hline
\end{tabular}
Ambulatory Medical Care Survey, 1992

NOTE: The smallest reliable estimate for visits to hospital emergency departments is 58,000 . Estimates below this figure have a relative standard error greater than 30 percent and are deemed unreliable by by NCHS standards.

Example of use of table: An aggregate estimate of 20 million visits has a relative standard orror of 4.3 percent or a standard error of 860,000 visits (4.3 percent of 20 million).

Table II. Approximate relative standard errors for estimated numbers of drug mentions at emergency department visits: Natlonal Hospltal Ambulatory Medical Care

\begin{tabular}{|c|c|}
\hline $\begin{array}{l}\text { Estimated number } \\
\text { of divg mentions } \\
\text { In thousands }\end{array}$ & $\begin{array}{l}\text { Relative } \\
\text { standard } \\
\text { error in } \\
\text { percent }\end{array}$ \\
\hline $10 \ldots \ldots \ldots \ldots$ & 71.9 \\
\hline $20 \ldots \ldots \ldots \ldots$ & 50.9 \\
\hline $50 \ldots \ldots \ldots \ldots$ & 32.4 \\
\hline $59 \ldots \ldots \ldots \ldots$ & 29.9 \\
\hline $100 \ldots \ldots \ldots \ldots$ & 23.1 \\
\hline $200 \ldots \ldots \ldots \ldots$ & 16.6 \\
\hline $500 \ldots \ldots \ldots \ldots$ & 11.1 \\
\hline $1,000 \ldots \ldots \ldots \ldots$ & 8.5 \\
\hline $2,000 \ldots \ldots \ldots \ldots$ & 6.8 \\
\hline $5,000 \ldots \ldots \ldots \ldots$ & 5.5 \\
\hline $10,000 \ldots \ldots \ldots$ & 5.0 \\
\hline $20,000 \ldots \ldots \ldots \ldots$ & 4.8 \\
\hline $50,000 \ldots \ldots \ldots \ldots$ & 4.6 \\
\hline $100,000 \ldots \ldots \ldots$ & 4.5 \\
\hline $200,000 \ldots \ldots \ldots \ldots$ & 4.5 \\
\hline \multicolumn{2}{|c|}{$\begin{array}{l}\text { NOTE: The smallest roliable estimale of drug mentions at } \\
\text { visits to hospital emergency departments is } 59 \text { million. } \\
\text { Estimates below this figure have a relative standard error } \\
\text { greater than } 30 \text { percent and are deemed unreliable by NCHS } \\
\text { standards. } \\
\text { Example of use of table: An aggregate estimate of } 10 \text { million } \\
\text { drug mentions has a relative standard error of } 5.5 \text { percent or } \\
\text { a standard error of } 550,000 \text { drug mentions ( } 5.5 \text { percent of } 10 \\
\text { million). }\end{array}$} \\
\hline
\end{tabular}
Survey, 1992

using the appropriate coefficients from table V.

\section{Adjustments for hospital nonresponse}

Estimates from NHAMCS data were adjusted to account for sample 
Table III. Approximate standard errors of percents of estimated numbers of emergency department visits: National Hospital Ambulatory Medlcal Care Survey, 1992

\begin{tabular}{|c|c|c|c|c|c|c|c|}
\hline \multirow{2}{*}{$\begin{array}{l}\text { Base of percent } \\
\text { (visits in thousands) }\end{array}$} & \multicolumn{7}{|c|}{ Estimated percent } \\
\hline & 1 or 99 & 5 or 95 & 10 or 90 & 20 or 80 & 30 or 70 & 40 or 60 & 50 \\
\hline & \multicolumn{7}{|c|}{ Standard error in percentage points } \\
\hline $10 \ldots \ldots \ldots \ldots \ldots \ldots \ldots$ & 7.1 & 15.5 & 21.3 & 28.4 & 32.5 & 34.8 & 35.5 \\
\hline$\cdots \ldots \ldots \ldots \ldots$ & 5.0 & 10.9 & 15.1 & 20.1 & 23.0 & 24.6 & 25.1 \\
\hline $50 \ldots \ldots \ldots \ldots \ldots \ldots \ldots$ & 3.2 & 6.9 & 9.5 & 12.7 & 14.6 & 15.6 & 15.9 \\
\hline $100 \ldots \ldots \ldots \ldots \ldots \ldots$ & 2.2 & 4.9 & 6.7 & 9.0 & 10.3 & 11.0 & 11.2 \\
\hline $200 \ldots \ldots \ldots \ldots \ldots \ldots \ldots$ & 1.6 & 3.5 & 4.8 & 6.4 & 7.3 & 7.8 & 7.9 \\
\hline $500 \ldots \ldots \ldots \ldots \ldots \ldots$ & 1.0 & 2.2 & $\mathbf{3 . 0}$ & 4.0 & 4.6 & 5.9 & 5.0 \\
\hline $1,000 \ldots \ldots \ldots \ldots \ldots \ldots$ & 0.7 & 1.5 & 2.1 & 2.8 & 3.3 & 3.5 & 3.6 \\
\hline $2,000 \ldots \ldots \ldots \ldots \ldots \ldots$ & 0.5 & 1.1 & 1.5 & 2.0 & 2.3 & 2.5 & 2.5 \\
\hline $5,000 \ldots \ldots \ldots \ldots \ldots \ldots$ & 0.3 & 0.7 & 1.0 & 1.3 & 1.5 & 1.6 & 1.6 \\
\hline $10,000 \ldots \ldots \ldots \ldots \ldots$ & 0.2 & 0.5 & 0.7 & 0.9 & 1.0 & 1.1 & 1.1 \\
\hline $20,000 \ldots \ldots \ldots \ldots \ldots \ldots$ & 0.2 & 0.3 & 0.5 & 0.6 & 0.7 & 0.8 & 0.8 \\
\hline $50,000 \ldots \ldots \ldots \ldots \ldots \ldots$ & 0.1 & 0.2 & 0.3 & 0.4 & 0.5 & 0.5 & 0.5 \\
\hline $100,000 \ldots \ldots \ldots \ldots \ldots \ldots$ & 0.1 & 0.2 & 0.2 & 0.3 & 0.3 & 0.3 & 0.4 \\
\hline
\end{tabular}

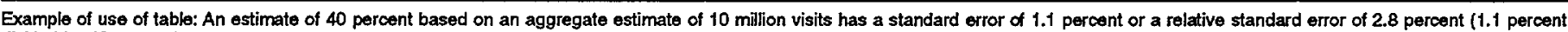
divided by 40 percent).

Table IV. Approximate standard errors of percents of estimated numbers of drug mentions at emergency department visits: National Hospital Ambulatory Medical Care Survey, 1992

\begin{tabular}{|c|c|c|c|c|c|c|c|}
\hline \multirow{2}{*}{$\begin{array}{c}\text { Base of percent } \\
\text { (drug mentions in thousands) }\end{array}$} & \multicolumn{7}{|c|}{ Estimated percent } \\
\hline & 1 or 99 & 5 or 95 & 10 or 90 & 20 or 80 & 30 or 70 & 40 or 60 & 50 \\
\hline & \multicolumn{7}{|c|}{ Standard error in percentage points } \\
\hline $10 \ldots \ldots \ldots \ldots \ldots \ldots$ & 7.1 & 15.6 & 21.5 & 28.7 & 32.9 & 35.1 & 35.9 \\
\hline $20 \ldots \ldots \ldots \ldots \ldots \ldots$ & 5.0 & 11.1 & 15.2 & 20.3 & 23.2 & 24.8 & 25.4 \\
\hline $50 \ldots \ldots \ldots \ldots \ldots$ & 3.2 & 7.0 & 9.6 & 12.8 & 14.7 & 15.7 & 16.0 \\
\hline $100 \ldots \ldots \ldots \ldots \ldots$ & 2.3 & 4.9 & 6.8 & 9.1 & 10.4 & $\$ 1.1$ & 11.3 \\
\hline $200 \ldots \ldots \ldots \ldots \ldots \ldots$ & 1.6 & 3.5 & 4.8 & 6.4 & 7.4 & 7.9 & 8.0 \\
\hline $500 \ldots \ldots \ldots \ldots \ldots \ldots$ & 1.0 & 2.2 & $\mathbf{3 . 0}$ & 4.1 & 4.6 & 5.0 & 5.1 \\
\hline $1,000 \ldots \ldots \ldots \ldots \ldots \ldots$ & 0.7 & 1.6 & 2.2 & 2.9 & $\mathbf{3 . 3}$ & 3.5 & 3.6 \\
\hline $2,000 \ldots \ldots \ldots \ldots \ldots \ldots$ & 0.5 & 1.1 & 1.5 & 2.0 & 2.3 & 2.5 & 2.5 \\
\hline $5,000 \ldots \ldots \ldots \ldots \ldots$ & 0.3 & 0.7 & 1.0 & 1.3 & 1.5 & 1.6 & 1.6 \\
\hline $10,000 \ldots \ldots \ldots \ldots$ & 0.2 & 0.5 & 0.7 & 0.9 & 1.0 & 1.1 & 1.1 \\
\hline $20,000 \ldots \ldots \ldots \ldots \ldots$ & 0.2 & 0.3 & 0.5 & 0.6 & 0.7 & 0.8 & 0.8 \\
\hline $50,000 \ldots \ldots \ldots \ldots \ldots$ & 0.1 & 0.2 & 0.3 & 0.4 & 0.5 & 0.5 & 0.5 \\
\hline $100,000 \ldots \ldots \ldots \ldots$ & 0.1 & 0.2 & 0.2 & 0.3 & 0.3 & 0.3 & 0.4 \\
\hline $200,000 \ldots \ldots \ldots \ldots$ & 0.1 & 0.1 & 0.2 & 0.2 & 0.2 & 0.2 & 0.3 \\
\hline
\end{tabular}

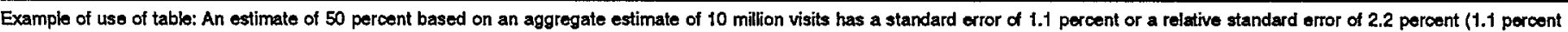
divided by 50 percent).

hospitals that were in scope but did not participate in the study. This adjustment was calculated to minimize the impact of response on final estimates by imputing to nonresponding hospitals data from visits to similar hospitals. For this purpose, hospitals were judged similar if they were in the same region, ownership control group, and metropolitan statistical area control group.

\section{Adjustments for ED and/or clinic nonresponse}

Estimates from NHAMCS data were adjusted to account for ED's and sample clinics that were in scope but did not participate in the study. This adjustment was calculated to minimize the impact of response on final estimates by imputing to nonresponding ED's or clinics' data from visits to similar ED's or clinics. For this purpose, ED's or clinics were judged similar if they were in the same ED or clinic group.

\section{Test of significance and rounding}

The determination of statistical inference is based on the $t$-test. The Bonferroni inequality was used to establish the critical value for statistically significant differences ( 0.05 level of significance over all analyses performed on estimates in a table). Terms relating to differences such as "higher than" indicate that the difference is statistically significant. A lack of comment regarding the difference between any two estimates does not mean that the difference was tested and found to be not significant.

In the tables, estimates of ED visits have been rounded to the nearest thousand. Consequently, estimates will not always add to totals. Rates and percents were calculated from original unrounded figures and do not 
necessarily agree with percents calculated from rounded data.

\section{Definition of terms}

Patient-An individual seeking personal health services who is not currently admitted to any health care institution on the premises.

Hospital-All hospitals with an average length of stay for all patients of less than 30 days (short-stay) or hospital whose specialty is general (medical or surgical) or children's general. Federal hospitals, hospital units of institutions, and hospitals with fewer than six beds staffed for patient use are excluded.

Emergency department-Hospital facility for the provision of unscheduled outpatient services to patients whose conditions require immediate care and which is staffed 24 hours a day. If an ED provided emergency services in different areas of the hospital, then all these areas were selected with certainty into the sample. Off-site emergency

Table V. Coefficients appropriate for determining relative standard error by type of estimate for hospltal emergency departments: National Hospltal Ambulatory Medical Care Survey, 1992

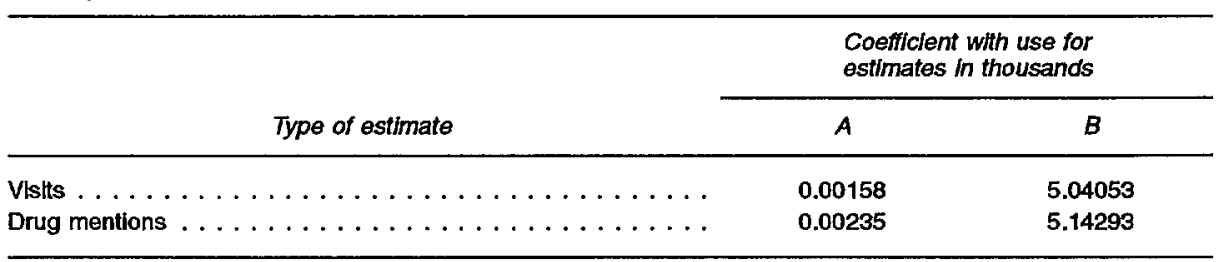

departments open less than 24 hours are included if staffed by the hospital's emergency department.

Visit-A direct personal exchange between a patient and a physician or other health care provider working under the physician's supervision, for the purpose of seeking care and receiving personal health services.

Urgent/emergent-A visit wherein the patient requires immediate attention for an acute illness or injury that threatens life or function and where delay would be harmful to the patient.
Non-urgent-A visit wherein the patient does not require attention immediately or within a few hours.

Injury-related visit-A visit during which hospital staff indicated that the visit was a result of any kind of accident or injury including but not limited to falls; lacerations; burns; intentional injuries; unintentional poisonings by drugs, medicinal substances, biologicals, gases, or vapors; adverse reaction to drugs; complications of surgical and medical procedures; and insect and animal bites. 
Symbols

- - Data not available

... Category not applicable

- Quantity zero

0.0 Quantity more than zero but less than 0.05

Z Quantity more than zero but less than 500 where numbers are rounded to thousands

* Figure does not meet standard of reliability or precision 


\section{Suggested citation}

Burt CW. Injury-related visits to hospital emergency departments: United States, 1992. Advance data from vital and health statistics; no 261. Hyattsville, Maryland: National Center for Health Statistics. 1995.

\section{Trade name dlsclaimer}

The use of trade names is for identification only and does not imply endorsement by the Public Health Service, U.S. Department of Health and Human Services.

\section{Copyright Information}

All material appearing in this report is in the public domain and may be reproduced or copied without permission; citation as to source, however, is appreciated.
National Center for Health Statistics

Director

Manning Feinleib, M.D., Dr. P.H.

Deputy Director Jack R. Anderson

\section{U.S. DEPARTMENT OF HEALTH AND} HUMAN SERVICES

Public Health Service

Centers for Disease Control and Prevention

National Center for Health Statistics

6525 Belcrest Road

Hyattsville, Maryland 20782

OFFICIAL BUSINESS

PENALTY FOR PRIVATE USE, $\$ 300$

To receive this publication regularly, contact the National Center for Health Statistics by calling 301-436-8500

DHHS Publication No. (PHS) 95-1250

5-0411 (2/95) 


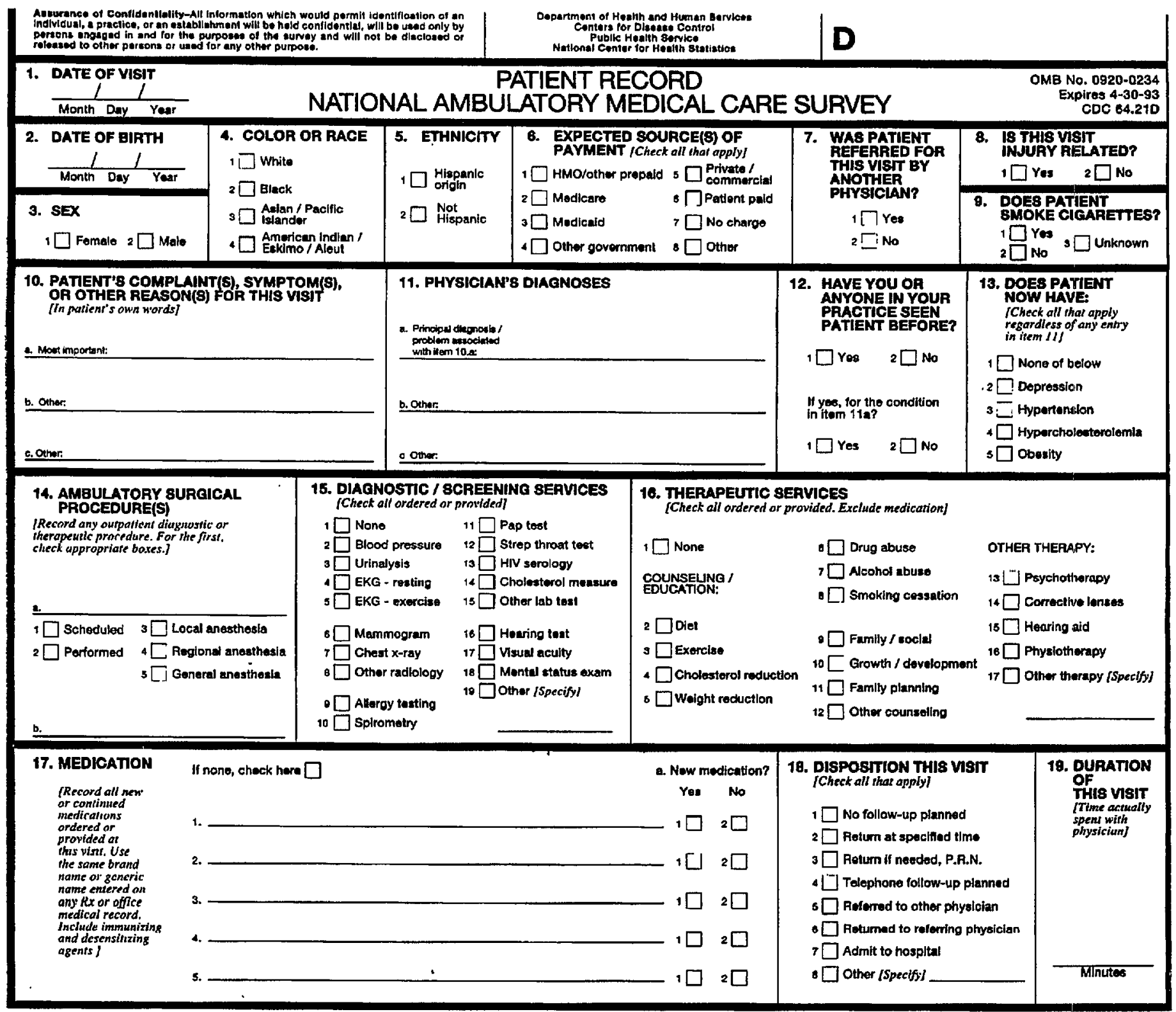

Figure 1. Patient Record form

females making an average of 4.2 visits per 100 for each year compared with 2.8 visits per 100 males. While increasing rates by age were observed for both females and males, age-specific rates were not found to be significantly different by sex in any age category.

The rate of visits with a principal diagnosis of glaucoma was not significantly different for white persons than for black persons. White persons made an average of 3.7 visits per 100 persons per year compared with 3.0 visits per 100 black persons. For persons ages 45 years and over, the rates for white persons and black persons were 10.6 and 10.9 visits per 100 , respectively. Further analysis of age-specific visit rates by race was hampered by the fact that visit estimates for black persons in several of the age groups were too low to ensure statistical reliability. Aggregation of the estimates into broader categories (for example, 65 years and over and 75 years and over) showed rates for black persons that appeared to be substantially larger than for white persons in these age groups, but none of the apparent differences were statistically significant because of the high standard errors associated with the low estimates.

The lack of difference in racespecific visit rates for glaucoma is noteworthy because it has been found that black persons tend to have higher intraocular pressure, the main determinant and risk factor for glaucoma, than white persons (5), that glaucoma is the most common cause of irreversible blindness among black Americans (6), and that black Americans are at a higher risk of primary openangle glaucoma than are their white counterparts (7). Javitt et al. have noted that glaucoma is six to eight times more prevalent among black persons in this country, but that black persons are not receiving care for open-angle glaucoma at the same rate as older white Americans (8). 
Table 1. Number, percent dlstribution, and annual rate of office visits with a principal diagnosls of glaucoma by patient's age, sex, race, and geographic region of the visit, averaged over a 2-year period: United State8, 1991-92

\begin{tabular}{|c|c|c|c|}
\hline $\begin{array}{l}\text { Selected patient and } \\
\text { visit characteristics }\end{array}$ & $\begin{array}{l}\text { Number } \\
\text { of vishls in } \\
\text { thousands }\end{array}$ & $\begin{array}{c}\text { Percent } \\
\text { distribution }\end{array}$ & $\begin{array}{l}\text { Visit } \\
\text { rate } \\
\text { per } 100 \\
\text { persons }\end{array}$ \\
\hline $\begin{array}{c}\text { All visits } \ldots \ldots \ldots \ldots \ldots \ldots \ldots \\
\qquad g g e\end{array}$ & 8,742 & 100.0 & 3.5 \\
\hline Under 25 years . . . . . . . . . . . . . . & $" 58$ & 0.7 & "0.1 \\
\hline $25-44$ years. . . . . . . . . . . . . & 564 & 6.5 & 0.7 \\
\hline $45-54$ years. . . . . . . . . . . . & 720 & 8.2 & 2.7 \\
\hline $55-64$ years. . . . . . . . . . . . . & 1,315 & 15.0 & 6.2 \\
\hline $65-74$ years. . . . . . . . . . . . . & 2,831 & 32.4 & 15.4 \\
\hline $\begin{array}{l}75 \text { years and over } \ldots \ldots \ldots \ldots \\
\qquad \text { Sex }\end{array}$ & 3,254 & 37.2 & 26.8 \\
\hline 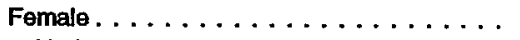 & 5,359 & 61.3 & 4.2 \\
\hline Under 25 years . . . . . . . . . . & $\star 45$ & $\star 0.5$ & *0.1 \\
\hline $25-44$ years . . . . . . . . . . . & $* 265$ & $* 3.0$ & "0.6 \\
\hline $45-54$ years . . . . . . . . . . . & 414 & 4.7 & 3.0 \\
\hline $55-64$ years $\ldots \ldots \ldots \ldots \ldots \ldots$ & 697 & 8.0 & 6.3 \\
\hline $65-74$ years . . . . . . . . . . & 1,809 & 20.7 & 17.8 \\
\hline 75 years and over $\ldots \ldots \ldots \ldots$ & 2,128 & 24.3 & 27.9 \\
\hline Male . . . . . . . . . . . . . & 3,382 & 38.7 & 2.8 \\
\hline Under 25 years $\ldots \ldots \ldots \ldots \ldots$ & $* 12$ & ${ }^{*} 0.1$ & 0.0 \\
\hline $25-44$ years $\ldots \ldots \ldots \ldots \ldots$ & "299 & *3.4 & ${ }^{\star} 0.7$ \\
\hline $45-54$ years . . . . . . . . . . . & *306 & ${ }^{*} 3.5$ & $" 1.6$ \\
\hline $55-64$ yoars . . . . . . . . . . . & 618 & 7.1 & 6.2 \\
\hline $65-74$ years . . . . . . . . . . . . & 1,021 & 11.7 & 12.4 \\
\hline $\begin{array}{l}75 \text { years and over } \ldots \ldots \ldots \ldots \ldots \ldots \\
\text { Race }\end{array}$ & 1,126 & 12.9 & 24.8 \\
\hline White . . . . . . . . . . . . . . . & 7,721 & 88.3 & 3.7 \\
\hline Black . . . . . . . . . . . . . . . & 934 & 10.7 & $\mathbf{3 . 0}$ \\
\hline $\begin{array}{c}\text { Other } \ldots \ldots \ldots \ldots \ldots \ldots \ldots \ldots \ldots \\
\text { Geographic region }\end{array}$ & "87 & 1.0 & ${ }^{*} 0.9$ \\
\hline Northeast $\ldots \ldots \ldots \ldots \ldots \ldots$ & 1,662 & 19.0 & 3.3 \\
\hline Midwest . . . . . . . . . . . . . . & 1,724 & 19.7 & 2.8 \\
\hline South . . . . . . . . . . . . & 3,644 & 41.7 & 4.3 \\
\hline West $\ldots \ldots \ldots \ldots \ldots \ldots \ldots$ & 1,711 & 19.6 & 2.1 \\
\hline
\end{tabular}
'Based on U.S. Bureau of the Census estimates of the civilian noninstitutionalized population for July 1, 1981, and July 1, 1992,
averaged over the 2-year period.

\section{Comparative data from the National} Hospital Ambulatory Medical Care Survey show that black persons accounted for about one-third (36.6 percent) of the glaucoma visits made to hospital outpatient departments (OPD's) in 1992 compared with white persons ( 61.3 percent).

However, the estimated number of OPD visits with this principal diagnosis was only 278,000 overall, resulting in estimates that were too low to permit meaningful analysis by race and age.

Office visit rates did not differ statistically by geographic region of the country, except that the rate was higher in the South (4.3 visits per 100 persons) than in the West (2.1 visits per 100 persons). both in the AMA masterfile and in the NAMCS.)

Glaucoma was the second most frequently reported principal diagnosis at office visits to ophthalmologists after cataract, accounting for 15.3 percent of the visits to this specialty (table 2). It should be noted that the ranked order presented in this and other tables in this report may not always be reliable because some estimates may not be statistically different from other near estimates due to sampling variability.

\section{Visit characteristics}

\section{Referral status and prior-visit status}

Data pertaining to patient's referral status and prior-visit status are shown in table 3 . Only 6.8 percent of all glaucoma visits during 1991-92 were the result of a referral by another physician.

However, of all visits made by new patients (that is, patients who had not seen the physician previously), about two-thirds (68.1 percent) were recorded as referrals from another physician. In contrast, about one-third (31.6 percent) of all nonglaucoma visits made by new patients (that is, visits with a principal diagnosis other than glaucoma) were the result of referrals from other physicians.

The majority ( 89.1 percent) of glaucoma visits were made by patients who were making return visits to the physician for care of their condition. Ten percent of the visits were made by new patients. However, by age group, 17.3 percent of the visits by persons 45-64 years were made for new problems, compared with 9.0 percent of those 65 years of age and over. "New problem" visits include those made as a new patient or as a continuing patient.

The chronic nature of glaucoma is highlighted by the fact that among all return visits for the care of previously treated problems, glaucoma was the fifth most frequently recorded principal diagnosis related to illness or injury. Among visits with this principal diagnosis, there were 4.1 return visits recorded during the 2-year period for glaucoma specialists, such physicians were classified as "other" specialists

\section{Physician practice}

About three-quarters (76.8 percent) remainder (23.2 percent) were made to other specialists, including physicians specialists. (Because the American Medical Association's (AMA) master file, upon which the determination of physician specialty for NAMCS purposes is based, did not have a separate specialty code for physicians reporting themselves to the AMA as were classified as "other" specialists 


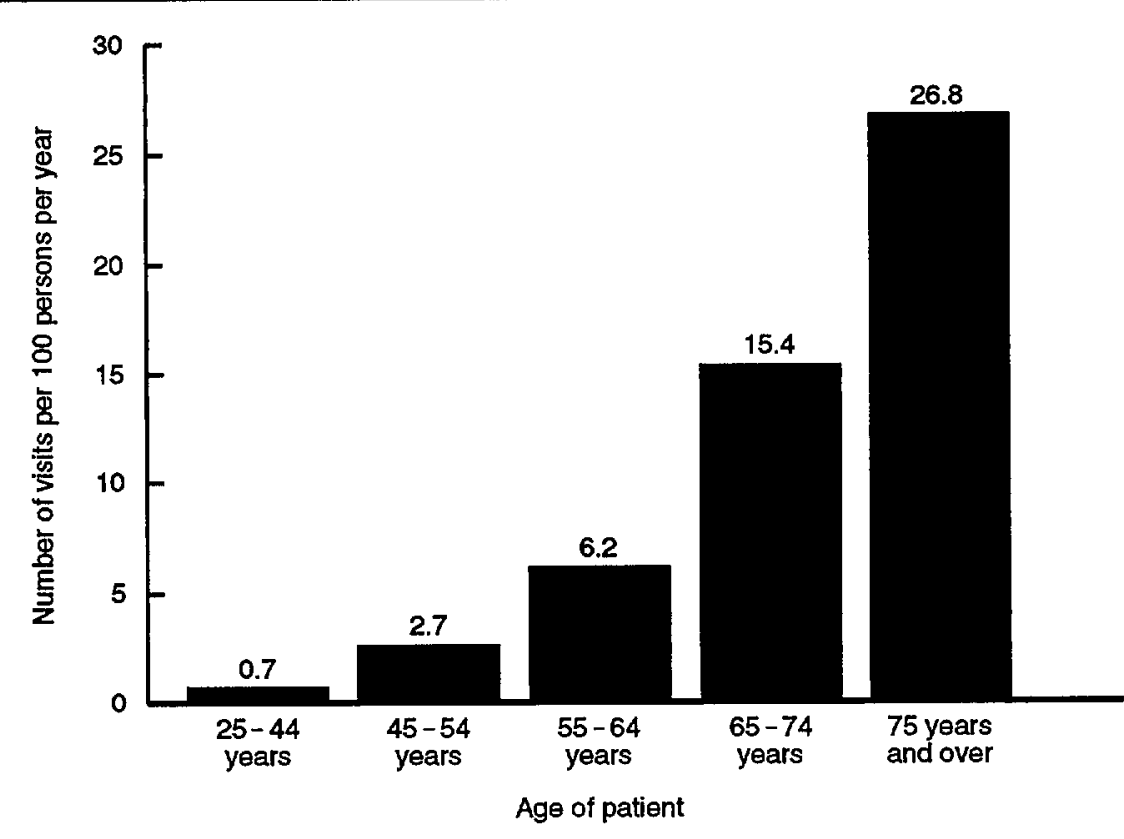

NOTE: Estimates for persons under 25 years of age were statistically unreliable.

Figure 2. Annual rate of office visits with a princlpal diagnosis of glaucoma by age of patient, averaged over a 2-year period: United States, 1991-92

Table 2. Annual number and percent distribution of office visits to ofince-based ophthaimologists by the 10 most frequently mentioned principal dlagnoses, averaged over a 2-year perlod: United States, 1991-92

\begin{tabular}{|c|c|c|c|}
\hline Principal dlagnosis and ICD-g-CM code ${ }^{1}$ & $\begin{array}{c}\text { Number of } \\
\text { visits in } \\
\text { thousands }\end{array}$ & $\begin{array}{c}\text { Percent } \\
\text { distribution }\end{array}$ & $\begin{array}{c}\text { Cumulathe } \\
\text { percent }\end{array}$ \\
\hline All vists $\ldots \ldots \ldots \ldots \ldots \ldots \ldots$ & 43,884 & 100.0 & $\cdots$ \\
\hline Cataract. . . . . . . . . . . . . . . .366 & 7,196 & 16.4 & 16.4 \\
\hline Glaucoma. . . . . . . . . . . . . . . .365 & 6,715 & 15.3 & 31.7 \\
\hline Disorders of refraction and accommodation . . 367 & 5,871 & 13.4 & 45.1 \\
\hline Organ or tlssue replaced by other means . . .V43 & 2,731 & 6.2 & 51.3 \\
\hline Other retinal dlsorders . . . . . . . . . . . . .362 & 2,214 & 5.0 & 56.3 \\
\hline Other disorders of eye . . . . . . . . . . . . 379 & 1,961 & 4.5 & 60.8 \\
\hline Special investigations and examinations . . . V72 & 1,838 & 4.2 & 65.0 \\
\hline Disorders of conjunctiva . . . . . . . . . . . . 372 & 1,605 & 3.7 & 68.7 \\
\hline Dlabetes mellitus . . . . . . . . . . . . . 250 & 1,335 & 3.0 & 71.7 \\
\hline Inflammation of eyellds. . . . . . . . . . . 373 & 1,296 & 3.0 & 74.7 \\
\hline All other dlagnoses $\ldots \ldots \ldots \ldots \ldots \ldots$ & 11,121 & 25.3 & 100.0 \\
\hline
\end{tabular}

'Based on the Intemational Classification of Diseases, 9th Revision, Clinical Modification (ICD-9-CM) (1).

every visit that was recorded as a new problem encounter (table 4).

\section{Expected source of payment}

In item 6 of the Patient Record form the physician is asked to list the expected source of payment for the visit; more than one source may be listed by the physician for each visit. Medicare was the expected source of payment at 61.9 percent of visits with a principal diagnosis of glaucoma, followed by private insurance
(36.6 percent), payment made by the patient (18.8 percent), Medicaid ( 8.0 percent), and HMO/prepaid plan (7.1 percent) (table 5).

\section{Reason for visit}

In item 10a of the Patient Record form, the physician is asked to record the patient's most important complaint, symptom, or other reason for the visit using the patient's (or patient surrogate's) own words. These responses have been classified and coded using the
Reason for Visit Classification for Ambulatory Care (RVC) (9). This classification is divided into eight modules, or groups of reasons. These are shown in table 6 . The disease module accounted for the highest percent of visits with a first-listed diagnosis of glaucoma ( 46.9 percent), indicating that the majority of visits were made by persons whose condition had been diagnosed previously and was known to them. This finding corresponds with the high return visit ratio found among glaucoma visits that was discussed earlier. The disease module was followed by the diagnostic, screening, and preventive module (27.2 percent), the treatment module (9.7 percent), and the symptom module ( 9.4 percent).

\section{Diagnostic and screening services}

The majority ( 82.3 percent) of glaucoma visits included a visual acuity examination ordered or provided by the physician, compared with 4.8 percent of all other office visits (that is, visits that did not list glaucoma as a principal diagnosis). Overall, 82.5 million office visits included a visual acuity exam during 1991-92, and glaucoma was the most frequently recorded principal diagnosis at these visits, accounting for 17.4 percent of the total.

About one-third (32.7 percent) of glaucoma visits included one diagnostic service ordered or provided by the physician; about half (52.4 percent) included two diagnostic services. With the exception of visual acuity, none of the specified categories was reported at frequencies high enough to yield reliable estimates, and 54.2 percent of the visits reported "other" diagnostic services that were unspecified as to type. Data on diagnostic services are shown in table 7.

\section{Principal diagnosis}

Glaucoma is classified into more specific diagnoses according to the International Classification of Diseases, 9th Revision, Clinical Modification (ICD-9-CM) (1). Of the total number of glaucoma visits made during 1991-92, the majority (63.2 percent) were coded as unspecified glaucoma (ICD-9-CM code 365.9); 20.7 percent were openangle glaucoma (ICD-9-CM code 365.1 ); and 14.0 percent were coded as 
Table 3. Number and percent distribution of office visits with a princlpal diagnosis of glaucoma by roferral status and prior-visit status, averaged over a 2-year perlod: United State8, 1991-92

\begin{tabular}{|c|c|c|}
\hline Vsit characteristic & $\begin{array}{l}\text { Number of } \\
\text { visits in } \\
\text { thousands }\end{array}$ & $\begin{array}{c}\text { Percent } \\
\text { distribution }\end{array}$ \\
\hline All visits ... & 8,742 & 100.0 \\
\hline \multicolumn{3}{|l|}{ Referral status } \\
\hline Patlent was referred by another physictan & 597 & 6.8 \\
\hline $\begin{array}{l}\text { Patlent was not referred by another physiclan . . } \\
\text { Prior-vislt status }\end{array}$ & 8,144 & 93.2 \\
\hline New pallent . . . . . . . . . . . . . . . & 877 & 10.0 \\
\hline Old patlent. . . . . . . . . . . . . . & 7,864 & 90.0 \\
\hline New problem. . . . . . . . . . . . . . & 74 & 0.9 \\
\hline Old problem $\ldots \ldots \ldots \ldots \ldots \ldots \ldots$ & 7,790 & 89.1 \\
\hline
\end{tabular}

Table 4. Number and percent of office visits and return visit ratio for the 10 most frequent principal diagnoses among return visits for the care of proviously treated probloms, averaged over a 2-year period: United States, 1991-92

\begin{tabular}{|c|c|c|c|}
\hline Princoal diagnosis and ICD-9-CM code ${ }^{1}$ & $\begin{array}{l}\text { Number of } \\
\text { visits in } \\
\text { thousands }\end{array}$ & Percent & $\begin{array}{l}\text { Return } \\
\text { visit ratio }\end{array}$ \\
\hline All return visits $\ldots \ldots \ldots \ldots \ldots \ldots$ & 443,996 & 100.0 & .. \\
\hline Essential hypertension . . . . . . . . . .401 & 23,552 & 5.3 & 4.0 \\
\hline Normal pregnancy . . . . . . . . . . v22 & 20,655 & 4.7 & 2.4 \\
\hline Health supervision of infant or child. . . . . V20 & 12,643 & 2.8 & 2.1 \\
\hline Suppurative and unspeciffed otitls media. . . .382 & 12,067 & 2.7 & 0.9 \\
\hline Dlabetes mellitus $\ldots \ldots \ldots \ldots \ldots \ldots .250$ & 11,810 & 2.7 & 3.4 \\
\hline General medical examination. . . . . . . . . v70 & 9,346 & 2.1 & 0.5 \\
\hline Acute upper resplratory infections . . . . . .465 & 8,774 & 2.0 & 0.4 \\
\hline Glaucoma. . . . . . . . . . . . . 365 & 7,790 & 1.8 & 4.1 \\
\hline Asthma $\ldots \ldots \ldots \ldots \ldots \ldots \ldots \ldots . \ldots .493$ & 7,678 & 1.7 & 2.4 \\
\hline Allergic rhinitis . . . . . . . . . . . . . 4777 & 6,737 & 1.5 & 1.9 \\
\hline
\end{tabular}

1Based on the Intermational Classification of Diseasos, Sth Revision, Clinical Modification (ICD-9-CM) (1).

${ }^{2}$ Fetum visit ratio is the ratio of visits made by previously seen patients for the care of previously treated problems to visits made for the treatment of new problems. "New problem" visits may be made by oither new or old patients.

Table 5. Number and percent distribution of office visits with a principal diagnosis of glaucoma by expected source(s) of payment, averaged over a 2-year period: United States, 1991-92

\begin{tabular}{|c|c|c|}
\hline Expected source(s) of payment & $\begin{array}{l}\text { Number of } \\
\text { vistts in } \\
\text { thousands }\end{array}$ & $\begin{array}{c}\text { Percent } \\
\text { distrbution }\end{array}$ \\
\hline$\ldots \ldots \ldots \ldots \ldots \ldots$ & 8,742 & 100.0 \\
\hline Medicare. . . . . . . . . . . . . . & 5,409 & 61.9 \\
\hline Private/commerclal Insurance . . . . . . . . . & 3,196 & 36.6 \\
\hline Patient-paid . . . . . . . . . . . . & 1,641 & 18.8 \\
\hline Medicald . . . . . . . . . . . . . & 700 & 8.0 \\
\hline HMO/other prepaid plan ${ }^{2} \ldots \ldots \ldots \ldots \ldots$ & 624 & 7.1 \\
\hline Other govemment $\ldots \ldots \ldots \ldots \ldots \ldots \ldots$ & 412 & 4.7 \\
\hline Other $\ldots \ldots \ldots \ldots \ldots \ldots \ldots \ldots$ & $" 249$ & $\star^{*} 2.9$ \\
\hline No charge $\ldots \ldots \ldots \ldots \ldots \ldots \ldots$ & *105 & $* 1.2$ \\
\hline Unknown. . . . . . . . . . . . . . . & "66 & ${ }^{*} 0.8$ \\
\hline
\end{tabular}

${ }^{1}$ Numbers may not add to totals because more than one expected source of payment may be reported per visit. HMO is health maintenance organization.

borderline glaucoma (ICD-9-CM code 365.0). Visits for glaucoma are described by specific diagnosis in table 8.

The prominence of glaucoma as a principal diagnosis among office visits by older adults is underscored by the finding that for persons in the age groups 65-74 and 75 years and over, it was the third most frequently reported principal diagnosis, accounting for 3.2 percent of the diagnoses among those 65-74 years and 4.4 percent of the diagnoses among those 75 years and over. For visits by all age groups, glaucoma was the 10th most frequently reported morbidity-related principal diagnosis and the 13th most frequent principal diagnosis during 1991-92. (Morbidity-related diagnoses are defined here as those classifiable to disease or injury, in contrast to nonillness- or noninjury-related visits. Examples of visits with diagnoses that are not morbidity related would include visits for routine pregnancy examination or general medical examination.)

\section{Concomitant diagnoses}

About one-quarter (26.4 percent) of glaucoma visits had a second diagnosis listed on the Patient Record form, and 9.1 percent included a third diagnosis. Cataract was the most frequently reported second- or third-listed diagnosis, showing up at about 12.5 percent of all visits with a principal diagnosis of glaucoma.

\section{Physician's checklist of selected conditions}

In item 13 , which was added to the Patient Record form for 1991, physicians were requested to report if the patient had any of four medical conditions-hypertension, hypercholesterolemia, obesity, and depression-regardless of what was coded as the first, second, or third diagnosis in item 11 of the Patient Record form. At 11.2 percent of glaucoma visits, physicians checked hypertension as an accompanying condition. However, virtually none of the glaucoma visits during 1991-92 included a second or third diagnosis of hypertension in item 11 of the Patient Record form. This suggests that physicians tend to underreport existing chronic conditions as a diagnosis in item 11.

\section{Therapeutic services}

Therapeutic services ordered or provided at glaucoma visits are shown in tables 9-11. Medication therapy was the most frequently mentioned therapeutic service at glaucoma visits, 
Table 6. Number and percent distribution of offlce visits with a princlpal dlagnosis of glaucoma by patient's principal reason for visit, averaged over a 2-year perlod: Unlted States, 1991-92

\begin{tabular}{|c|c|c|}
\hline Principal reason for visit and AVC code ${ }^{1}$ & $\begin{array}{c}\text { Number of visits } \\
\text { in thousands }\end{array}$ & $\begin{array}{c}\text { Percent } \\
\text { distribution }\end{array}$ \\
\hline 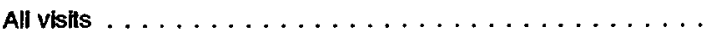 & 8,742 & 100.0 \\
\hline symptom module. . . . . . . . . . . . . . . . . . . s001-s999 & 825 & 9.4 \\
\hline Vision dysfunctions $\ldots \ldots \ldots \ldots$. . . . . . . . . . . . . . . . & 536 & 6.1 \\
\hline All other $\ldots \ldots \ldots \ldots \ldots \ldots \ldots \ldots$ & ${ }^{*} 289$ & *3.3 \\
\hline Disease module . . . . . . . . . . . . . . . . . D001-D999 & 4,096 & 46.9 \\
\hline 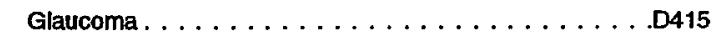 & 4,041 & 46.2 \\
\hline All other $\ldots \ldots \ldots \ldots \ldots \ldots \ldots \ldots$ & *55 & ${ }^{\star 0.7}$ \\
\hline Diagnostic, screening, and preventive module . . . X100-X599 & 2,380 & 27.2 \\
\hline Other and unspecified diagnostic tests $\ldots \ldots \ldots \ldots \ldots$. . . . . & 1,972 & 22.6 \\
\hline Eye examination . . . . . . . . . . . . . . . . . x230 & -315 & $* 3.6$ \\
\hline All other $\ldots \ldots \ldots \ldots \ldots \ldots \ldots$ & "93 & $* 1.0$ \\
\hline Treatment module . . . . . . . . . . . . . . . . . T100-T899 & 846 & 9.7 \\
\hline Progress vlsit, not otherwise specifled. . . . . . . . . . . . T800 & 542 & 6.2 \\
\hline All other $\ldots \ldots \ldots \ldots \ldots \ldots \ldots$ & "304 & ${ }^{*} 3.5$ \\
\hline Test results module . . . . . . . . . . . . . . . . . . R100-R700 & "84 & $" 1.0$ \\
\hline Other $^{2} \ldots \ldots \ldots \ldots \ldots$. . . . . . . . . . . . U990-U999 & 511 & 5.8 \\
\hline
\end{tabular}

"Based on "A Reason for Visit Classification for Ambulatory Care" (RVC) (9).

Includes problems and complaints not elsewhere classified, entries of "none," blanks, and illegible entries. None of the visits had reasons coded in the injuries and adverse offeets module (J001-J998) or the administrative module (A100-A140).

Table 7. Number and percent dlstribution of office visits with a princlpal diagnosis of glaucoma by diagnostlc and screening services, averaged over a 2-year perlod: United States, 1991-92

\begin{tabular}{|c|c|c|}
\hline $\begin{array}{l}\text { Dlagnostic and screening services } \\
\text { ordered or performed at the vistt }\end{array}$ & $\begin{array}{l}\text { Number of } \\
\text { wislts in } \\
\text { thousands }\end{array}$ & $\begin{array}{c}\text { Percent } \\
\text { distribution }\end{array}$ \\
\hline All vistts $\ldots \ldots \ldots \ldots \ldots \ldots$ & 8,742 & 100.0 \\
\hline None . . . . . . . . . . . . . . . . & 1,208 & 13.8 \\
\hline Visual acuity $\ldots \ldots \ldots \ldots \ldots \ldots$ & 7,196 & 82.3 \\
\hline other $\ldots \ldots \ldots \ldots \ldots \ldots$ & 5,110 & 58.5 \\
\hline \multicolumn{3}{|l|}{$\begin{array}{l}\text { Number of diagnostic and screening senvices } \\
\text { ordered or performed at the visit }\end{array}$} \\
\hline 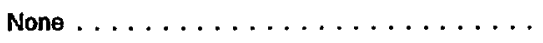 & 1,208 & 13.8 \\
\hline 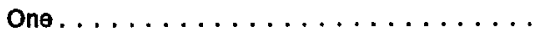 & 2,862 & 32.7 \\
\hline Two $\ldots \ldots \ldots \ldots \ldots \ldots \ldots$ & 4,579 & 52.4 \\
\hline Three or more $\ldots \ldots \ldots \ldots \ldots \ldots \ldots$ & *93 & -1.1 \\
\hline
\end{tabular}

Numbers may not add to tolals because more than one category may be reported per visit.

254.2 percent of glaucoma visits included unspecified diagnostic services; none of the specific diagnostic services listed on the Patient Record form (with the exception of the visual acuity examination) were recorded at frequencies large enough to provide estimates that were statistically reliable.

Table 8. Number and percent distribution of office visits with a princlpal diagnosis of glaucoma by detailed diagnosis, averaged over a 2-year period: United States, 1991-92

\begin{tabular}{|c|c|c|}
\hline Principal diagnos/s and $/ C D-9-C M \operatorname{cod}^{1}$ & $\begin{array}{c}\text { Number of } \\
\text { vislts in } \\
\text { thousands }\end{array}$ & $\begin{array}{c}\text { Percent } \\
\text { distribution }\end{array}$ \\
\hline All visits $\ldots \ldots \ldots \ldots \ldots \ldots$ & 8,742 & 100.0 \\
\hline Borderline glaucoma . . . . . . . . . 365.0 & 1,222 & 14.0 \\
\hline Preglaucoma, unspecifled . . . . . . 365.00 & 792 & 9.1 \\
\hline Other borderline glaucoma . . . .365.01,365.02 & *39 & "0.5 \\
\hline Ocular hypertension . . . . . . . . . 365.04 & 391 & 4.5 \\
\hline Open-angle glaucoma . . . . . . . . . . . 365.1 & 1,809 & 20.7 \\
\hline Open-angle glaucoma, unspeclfed . . . 365.10 & 808 & 9.2 \\
\hline Primary open-angle glaucoma . . . . . 365.11 & 932 & 10.7 \\
\hline Other open-angle glaucoma . . $365.12,365.13$ & *69 & ${ }^{*} 0.8$ \\
\hline Primary angle-closure glaucoma. . . . . . 365.2 & $* 186$ & $* 2.1$ \\
\hline Unspecified glaucoma . . . . . . . . . . . . 365.9 & 5,525 & 63.2 \\
\hline
\end{tabular}

Based on the Intemational Classification of Diseases, 9th Revision, Clinical Modification (ICD-9-CM) (1). recorded at 79.6 percent of visits (table 9). This is significantly higher than the 63.3 percent of all other visits at which medication therapy was mentioned. Nonmedication therapy was mentioned at 12.4 percent of glaucoma visits, with counseling ( 4.9 percent), corrective lenses ( 4.4 percent), and other therapy ( 5.4 percent) recorded by the physician as either ordered or provided at the visit.

As used in the NAMCS, the term "drug" is interchangeable with the term "medication" and includes all new or continued medications ordered or provided at the visit, including both prescription and nonprescription preparations, immunizing agents, and desensitizing agents. The term "drug mention" refers to each mention of medication on the Patient Record form. Because doctors can record more than one drug per visit, the total number of drug mentions will generally be higher than the number of visits. The term "drug visit" refers to any visit in which at least one drug is ordered or provided by the physician. An earlier report is available that describes the method and instruments used in collecting and processing NAMCS drug data (10).

There were about 27.7 million drug mentions at glaucoma visits during $1991-92$, an average of 13.8 million mentions per year. This yields an average of 2.0 drug mentions per drug visit or 1.6 drugs ordered or provided per visit overall.

About one-third of glaucoma visits included a single medication (33.6 percent), while approximately one-fifth (21.6 percent) listed two medications and one-quarter (24.4 percent) listed three or more medications.

As expected, most of the drugs prescribed were classified as ophthalmic drugs, specifically agents used to treat glaucoma (59.6 percent) and ocular anti-infective and anti-inflammatory agents (9.6 percent). Drug mentions at glaucoma visits are listed in table 10 by therapeutic classification, based on the National Drug Code Directory, 1985 edition (11).

The majority of drugs mentioned at glaucoma visits were single-ingredient preparations (91.3 percent), were prescribed as trade names rather than generics ( 71.4 percent), and were 
Table 9. Number and percent dletribution of ofice visits with a princlpal dlagnosis of glaucoma by therapeutic services, averaged over a 2-year period: United States, 1991-92

\begin{tabular}{|c|c|c|}
\hline $\begin{array}{l}\text { Therapeutic senices } \\
\text { ordered or provided at the visit }\end{array}$ & $\begin{array}{l}\text { Number of } \\
\text { visits in } \\
\text { thousands }\end{array}$ & $\begin{array}{l}\text { Percent } \\
\text { distrlbution }\end{array}$ \\
\hline $\begin{array}{c}\text { All visits } \ldots \ldots \ldots \ldots \ldots \ldots \ldots \ldots \ldots \\
\text { Medication therapy }\end{array}$ & 8,742 & 100.0 \\
\hline $\begin{array}{l}\text { New or continuing medicatlon } \ldots \ldots \ldots \ldots \ldots \\
\text { Visits without mention of medlcation } \ldots \ldots \ldots\end{array}$ & $\begin{array}{l}6,962 \\
1,779\end{array}$ & $\begin{array}{l}79.6 \\
20.4\end{array}$ \\
\hline $\begin{array}{l}\text { Number of new or } \\
\text { continued medications }\end{array}$ & & \\
\hline None . . . . . . . . . . . . . . . . . . . . & 1,779 & 20.4 \\
\hline 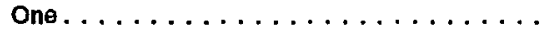 & 2,938 & 33.6 \\
\hline Two $\ldots \ldots \ldots \ldots \ldots \ldots \ldots$ & 1,889 & 21.6 \\
\hline Three.................... & 1,589 & 18.2 \\
\hline $\begin{array}{l}\text { Four or more } \ldots \ldots \ldots \ldots \ldots \ldots \ldots \ldots \\
\qquad \text { Nonmedication therapy }\end{array}$ & \multicolumn{2}{|c|}{ Nonmedication therapy } \\
\hline None . . . . . . . . . . . . . . . . & 7,659 & 87.6 \\
\hline Other counseling ${ }^{2} \ldots \ldots \ldots \ldots \ldots$ & 425 & 4.9 \\
\hline Corrective lenses. . . . . . . . . . . . . & 386 & 4.4 \\
\hline $\begin{array}{l}\text { Other therapy } \ldots \ldots \ldots \ldots \ldots \ldots \ldots \ldots \ldots \\
\text { Ambulatory surgery }\end{array}$ & 470 & 5.4 \\
\hline None . . . . . . . . . . . . . . . . . . . . & 8,125 & 92.9 \\
\hline One or more procedures . . . . . . . . . . . & 616 & 7.1 \\
\hline
\end{tabular}

Numbers may not acd to totals because more than one category may be reported per visit.

${ }^{2}$ Counseling other than the specified categories of diet, exercise, weight reduction, alcohol abuse, smoking cessation, and family/social.

Table 10. Number and percent distribution of drug mentions by therapeutic classification for office visits with a principal diagnosis of glaucoma, averaged over a 2-year period: United States, 1991-92

\begin{tabular}{|c|c|c|}
\hline Therapeutic classification ${ }^{1}$ & $\begin{array}{l}\text { Number of } \\
\text { drug mentions } \\
\text { in thousands }\end{array}$ & $\begin{array}{c}\text { Percent } \\
\text { dlstribution }\end{array}$ \\
\hline All drug mentions. $\ldots \ldots \ldots \ldots \ldots \ldots \ldots$ & 13,835 & 100.0 \\
\hline Ophthalmic drugs & 10,930 & 79.0 \\
\hline $\begin{array}{l}\text { Agents used to treal glaucoma } \ldots . \ldots \ldots \ldots \\
\text { Ocular anti-infective and anti-inflammatory }\end{array}$ & 8,241 & 59.6 \\
\hline agents $\ldots \ldots \ldots \ldots \ldots \ldots \ldots \ldots$ & 1,330 & 9.6 \\
\hline Mlscellaneous ophthalmic preparations. . . . . & 1,199 & 8.7 \\
\hline Mydrlatics and cycloplegics. . . . . . . & -160 & $* 1.2$ \\
\hline Cardiovascular-renal drugs. $\ldots \ldots \ldots \ldots \ldots$ & 1,138 & 8.2 \\
\hline Diuretics. $\ldots \ldots \ldots \ldots \ldots \ldots \ldots$ & 807 & 5.8 \\
\hline Other. . . . . . . . . . . . & $* 330$ & *2.4 \\
\hline Other ${ }^{2}$ & 1,767 & 12.8 \\
\hline Unclassified/miscellaneous. . . . . . . . . . . . . & *511 & "3.7 \\
\hline
\end{tabular}

${ }^{1}$ Therapeutic class is based on the standard drug classification used in the Nationa/ Drug Coob Dinectory, 1985 Edition (11). includes the following classifications: anesthetic drugs, antimicrobial agents, psychopharmacologic drugs, gastrointestinal agents, metabolic and nutrient agents, hormones and agents affocting hormonal mechanisms, immunologic agents, skin/mucous membrane, oncolytics, drugs used for pain relief, and respiratory tract drugs.

available only by prescription

(92.8 percent).

Drug mentions at glaucoma visits are displayed in table 11 according to their most frequently occurring generic ingredients. Timolol was the generic ingredient that appeared most frequently, showing up in 21.4 percent of all glaucoma drug mentions. Pilocarpine was also prominent, occurring in 16.6 percent of drug mentions at glaucoma visits.

\section{Ambulatory surgical procedures}

The 1991 NAMCS added a new item pertaining to whether ambulatory surgery was scheduled or performed at the current visit. Physicians were asked to record up to two ambulatory surgical procedures per visit. These were coded according to the International Classification of Diseases, 9th Revision, Clinical Modification, Volume 3 (ICD-9-CM) (1).

Ambulatory surgery was recorded at an estimated 1.2 million glaucoma visits over the 2-year period (an average of 616,000 visits per year), and a total of 1.3 million procedures were scheduled or performed. The proportion of glaucoma visits with mention of ambulatory surgery (7.1 percent) is not significantly different than the 6.0 percent of visits with principal diagnoses other than glaucoma that included ambulatory surgery in 1991-92.

While no specific ambulatory procedures were recorded at frequencies large enough to obtain reliable estimates, all of the surgical procedures mentioned were related to the eye and included operations on the iris, ciliary body, sclera, and anterior chamber; iridotomy and simple iridectomy; operations on the lens; operations on the retina, choroid, vitreous, and posterior chamber; and operations on the orbit and eyeball (ICD-9-CM, Volume 3, codes 12-14, 16).

\section{Disposition of visit}

Nine of ten glaucoma visits (93.3 percent) resulted in a scheduled return visit. In contrast, 62.0 percent of all other visits included a scheduled return visit. The predominance of this type of disposition among glaucoma visits is mirrored in the correspondingly high return visit ratio that was discussed previously. Data on disposition of visit are shown in table 12 .

\section{Duration of visit}

The mean duration of physicianpatient contact for glaucoma visits was 21.7 minutes, compared with 17.3 minutes for office visits in general. Mean duration does not include visits in which no face-to-face contact with the physician occurred. Physician-patient contact only includes the time spent in actual face-to-face contact between physician and patient. Data on duration 
Table 11. Number, percent distribution, and therapeutic classification for the five most trequently occurring generic ingredlents in drug mentions at office visits with a principal diagnosis of glaucoma, averaged over a 2-year period: United States, 1991-92

\begin{tabular}{|c|c|c|c|}
\hline Genenc ingredjent ${ }^{t}$ & $\begin{array}{l}\text { Number of } \\
\text { drug mentions } \\
\text { in thousands }\end{array}$ & $\begin{array}{c}\text { Percent } \\
\text { distribution }\end{array}$ & $\begin{array}{l}\text { Therapoutic } \\
\text { classification }\end{array}$ \\
\hline All mentions . . . . . . . . & 13,835 & 100.0 & $\ldots$ \\
\hline Timolol . . . . . . . . . . & 2,957 & 21.4 & Agents used to treat glaucoma \\
\hline Pilocarpine. . . . . . . . . & 2,295 & 16.6 & Agents used to treat glaucoma \\
\hline Betaxolol hydrochloride. . . . & 1,284 & 9.3 & Agents used to treat glaucoma \\
\hline Diplverrin. . . . . . . . & 1,055 & 7.6 & Agents used to treat glaucoma \\
\hline Levobunolol hydrochloride . . & 911 & 6.6 & Miscellaneous ophthalmic preparations \\
\hline
\end{tabular}

${ }^{1}$ Frequency of mention combines single-ingredient agents with mentions of the agent as an ingredient in a combination drug.

${ }^{2}$ Therapeutic classification is based on the National Drug Code Directory, 1985 Edition (11). In cases where a generic ingredient had more than one therapeutic classification, it was listed in the category which occurred with the greatest frequency.

Table 12. Number and percent distribution of office visits with a principal diagnosis of glaucoma by disposition and duration of visit, averaged over a 2-year period:

United State8, 1991-92

\begin{tabular}{|c|c|c|}
\hline Vsit charactenistic & $\begin{array}{l}\text { Number of } \\
\text { visits in } \\
\text { thousands }\end{array}$ & $\begin{array}{c}\text { Percent } \\
\text { distribution }\end{array}$ \\
\hline $\begin{array}{c}\text { All vists } \ldots \ldots \ldots \ldots \ldots \ldots \ldots \\
\text { Disposittion of visit }{ }^{1}\end{array}$ & 8,742 & 100.0 \\
\hline Retum at specified time. . . . . . . . . & 8,154 & 93.3 \\
\hline $\begin{array}{l}\text { Other }^{2} \ldots \ldots \ldots \ldots \\
\text { Duration of visit }\end{array}$ & 814 & 9.3 \\
\hline 0 minutes $^{3} \ldots \ldots \ldots \ldots \ldots$ & *39 & 0.4 \\
\hline $1-5$ minutes $\ldots \ldots \ldots \ldots \ldots$ & 754 & 8.6 \\
\hline $6-10$ minutes $\ldots \ldots \ldots \ldots \ldots$ & 1,657 & 19.0 \\
\hline $11-15$ minutes $\ldots \ldots \ldots \ldots \ldots$ & 1,936 & 22.1 \\
\hline $16-30$ minutes $\ldots \ldots \ldots \ldots \ldots$ & 1,809 & 20.7 \\
\hline More than $\mathbf{3 0}$ minutes. . . . . . . . & 2,547 & 29.1 \\
\hline
\end{tabular}

Numbers may not add to totals because more than one disposition may be reported per visit.

${ }^{2}$ None of the other specific disposition categories had frequencies large enough to provide estimates that were statistically reliablo.

3isits at which there was no face-to-face contact between the physıcian and the patient.

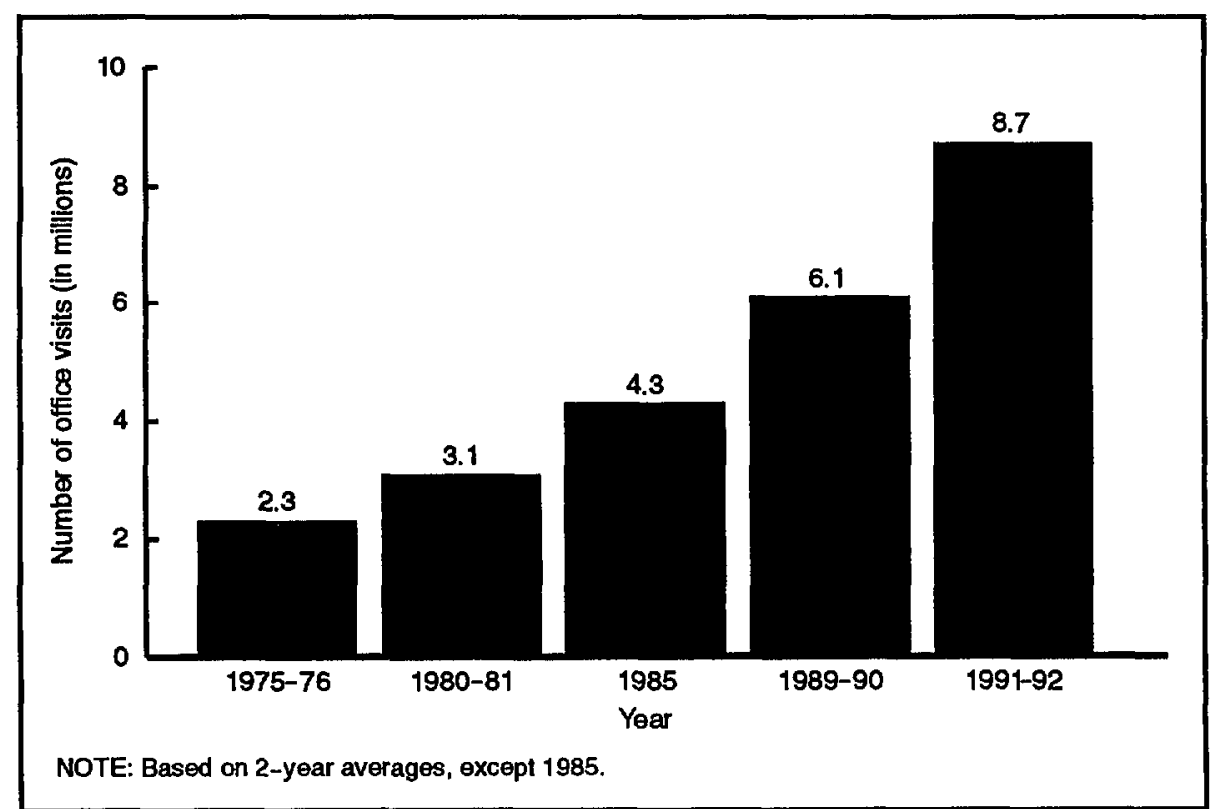

Figure 3. Office visits with a principal diagnosis of glaucoma: United States, 1975-92 of glaucoma visits are shown in table 12.

\section{Visits with a second or third diagnosis of glaucoma}

In addition to the estimated total of 17.5 million office visits with a first-listed diagnosis of glaucoma during 1991-92, there were 3.2 million office visits at which a second or third diagnosis was listed as glaucoma. Visits in which the second or third diagnosis was glaucoma were not found to differ significantly from visits in which the principal diagnosis was glaucoma in terms of the age, sex, or race of patients.

At office visits in which glaucoma was the second- or third-listed diagnosis, the principal diagnosis was listed within the major ICD-9-CM coding class of disorders of the eye and adnexa (ICD-9-CM codes 360-379) 62.9 percent of the time. No specific diagnosis was recorded at frequencies high enough to provide reliable estimates, although the frequency of visits with a principal diagnosis of cataract approached statistical reliability.

\section{Glaucoma visits between 1975 and 1992}

In 1975 , glaucoma was the ninth most frequently mentioned morbidityrelated principal diagnosis among persons 65 years of age and older; by 1992, it was the fifth. Overall, glaucoma visits were estimated at 4.5 million during 1975-76, an average of 2.3 million per year. However, the average for 1991 and 1992 was 8.7 million-an increase of 284.6 percent (figure 3 ). Visits for glaucoma by age and sex of patients between 1975 and 1992 are shown in table 13. Race data have been omitted from the table because glaucoma visit estimates for the black population prior to 1989 were statistically unreliable when using NAMCS data.

Annual rates of glaucoma visits between 1975 and 1992 for the U.S. population in general are shown in figure 4, using both crude and ageadjusted rates. Both the crude and the age-adjusted rates for 1991-92 were 
Table 13. Number, percent distribution, and annual rate of office visits with a principal diagnosis of glaucoma by patient's age and sex: United States, 1975-92

\begin{tabular}{|c|c|c|c|c|c|}
\hline \multirow[b]{2}{*}{ Patient characteristic } & \multicolumn{5}{|c|}{ Year } \\
\hline & $1975-76$ & $1980-81$ & 1985 & $1989-90$ & $1991-92$ \\
\hline & \multicolumn{5}{|c|}{ Number of vislts in thousands ${ }^{1}$} \\
\hline$\ldots \ldots \ldots \ldots \ldots$ & 2,273 & 3,080 & 4,304 & 6,093 & 8,742 \\
\hline \multicolumn{6}{|l|}{ Age } \\
\hline Under 25 years. . . . . . . . . . . . . . . & ${ }^{* 75}$ & "45 & ${ }^{* 62}$ & -27 & "58 \\
\hline $25-44$ years. . . . . . . . . . . . & $* 138$ & 233 & ${ }^{\star} 214$ & $" 234$ & 564 \\
\hline 45-64 years. . . . . . . . . . . . & 827 & 994 & 1,218 & 1,537 & 2,035 \\
\hline $65-74$ years. $\ldots \ldots \ldots \ldots \ldots \ldots$ & 706 & 897 & 1,356 & 1,891 & 2,831 \\
\hline 75 years and over $\ldots \ldots \ldots \ldots \ldots$ & 527 & 910 & 1,454 & 2,405 & 3,254 \\
\hline \multicolumn{6}{|l|}{ Sex } \\
\hline Female. . . . . . . . . . . . . . & 1,398 & 1,864 & 2,610 & 3,847 & 5,359 \\
\hline Male $\ldots \ldots \ldots \ldots \ldots \ldots \ldots$ & 875 & 1,215 & 1,695 & 2,246 & 3,382 \\
\hline & \multicolumn{5}{|c|}{ Percent distribution } \\
\hline All visits $\ldots \ldots \ldots \ldots \ldots \ldots \ldots$ & 100.0 & 100.0 & 100.0 & 100.0 & 100.0 \\
\hline \multicolumn{6}{|l|}{ Age } \\
\hline Under 25 years. . . . . . . . . . . . & $" 3.3$ & $* 1.5$ & $\star 1.4$ & $" 0.4$ & $" 0.7$ \\
\hline 25-44 years. . . . . . . . . . . . . & "6.1 & 7.6 & $\star 5.0$ & $* 3.8$ & 6.5 \\
\hline 45-64 years. . . . . . . . . . . . & 36.4 & 32.3 & 28.3 & 25.2 & 23.3 \\
\hline 65-74 years. . . . . . . . . . . . & 31.0 & 29.1 & 31.5 & 31.0 & 32.4 \\
\hline 75 years and over $\ldots \ldots \ldots \ldots \ldots \ldots$ & 23.2 & 29.6 & 33.8 & 39.5 & 37.2 \\
\hline \multicolumn{6}{|l|}{ Sex } \\
\hline Female. . . . . . . . . . . . . . & 61.5 & 60.5 & 60.6 & 63.1 & 61.3 \\
\hline Male $\ldots \ldots \ldots \ldots \ldots \ldots \ldots$ & 38.5 & 39.5 & 39.4 & 36.9 & 38.7 \\
\hline & \multicolumn{5}{|c|}{ Vlsit rale per 100 persons ${ }^{2}$} \\
\hline All vistts $\ldots \ldots \ldots \ldots \ldots \ldots \ldots$ & 1.1 & 1.4 & 1.8 & 2.5 & 3.5 \\
\hline \multicolumn{6}{|l|}{ Age } \\
\hline Under 25 years. . . . . . . . . . . . . & ${ }^{*} 0.1$ & -0.0 & ${ }^{*} 0.1$ & 0.0 & *0.1 \\
\hline 25-44 years. . . . . . . . . . . . & "0.3 & 0.4 & 0.3 & 0.3 & 0.7 \\
\hline 45-64 years. . . . . . . . . . . . & 1.9 & 2.3 & 2.7 & 3.3 & 4.3 \\
\hline 65-74 years. . . . . . . . . . . . . & 5.2 & 5.8 & 8.2 & 10.5 & 15.4 \\
\hline 75 years and over $\ldots \ldots \ldots \ldots \ldots \ldots$ & 6.7 & 10.2 & 14.1 & 20.8 & 26.8 \\
\hline \multicolumn{6}{|l|}{ Sex } \\
\hline Female. . . . . . . . . . . . . . . & 1.3 & 1.6 & 2.2 & 3.1 & 4.2 \\
\hline Male . . . . . . . . . . . . . . & 0.9 & 1.1 & 1.5 & 1.9 & 2.8 \\
\hline
\end{tabular}

${ }^{1}$ Figures are shown as 2-year averages, except for 1885.

ZBased on Bureau of the Census estimates of the civilian noninstitutionalized population for July 1 of each survey year. Fates for combinad yoars are based on an average of the population estimates for July 1 of each year of the 2-year period. Survey years from 1975-95 did not include Alaska or Hawaii.

significantly higher than those reported in 1975-76.

Visit rates increased for the age groups 45-64 years, 65-74 years, and 75 years and over between 1975 and 1992 (figure 5). Among persons 65 years of age and over, the rate of glaucoma visits went from 5.7 visits per 100 persons in 1975 to 19.9 visits per 100 persons in 1992. Visit rates increased for both sexes between 1975 and 1992.

Significant differences were noted in the overall glaucoma visit rates for males
65-74 was not found to differ significantly between 1975 and 1992 .

Reasons for the substantial increase in rates of glaucoma-related office visits during 1975-92 are unclear. Data from the National Health Interview Survey (NHIS) show an increase in the overall rate of persons reporting a glaucomatous condition, from 5.7 conditions per 1,000 persons in 1977 to 10.4 conditions per 1,000 persons in $1991(12,13)$. Agespecific rates for glaucoma were not available from the NHIS during the 1970 's, but an increase in glaucomatous conditions was noted among persons 65 years of age and over between 1982 and 1991, from 41.8 conditions per 1,000 persons to 57.0 conditions per 1,000 persons (14).

In 1991, the National Eye Institute of the National Institutes of Health issued new government guidelines for glaucoma testing that advise all Americans ages 60 and older and black Americans ages 40-59 to receive glaucoma screening tests at least once every 2 years. This heightened awareness of the need for early detection of glaucoma, in combination with new diagnostic procedures such as laser tomographic scanners and Fourier ellipsometry that yield more precise measurements than are possible with photography and ophthalmoscopes (15), may result in even higher visit rates for glaucoma than are seen in the 1991-92 NAMCS survey data.

\section{References}

1. Public Health Service and Health Care Financing Administration. International Classification of Diseases, 9th Revision, Clinical Modification. Washington: Public Health Service. 1980.

2. Schappert SM. National Ambulatory Medical Care Survey: 1991 summary. Advance data from vital and health statistics; no. 230. Hyattsville, Maryland: National Center for Health Statistics. 1993.

3. Schappert SM. National Ambulatory Medical Care Survey: 1991 summary. National Center for Health Statistics. Vital and Health Stat 13(116).

4. Schappert SM. National Ambulatory Medical Care Survey: 1992 summary. Advance data from vital 


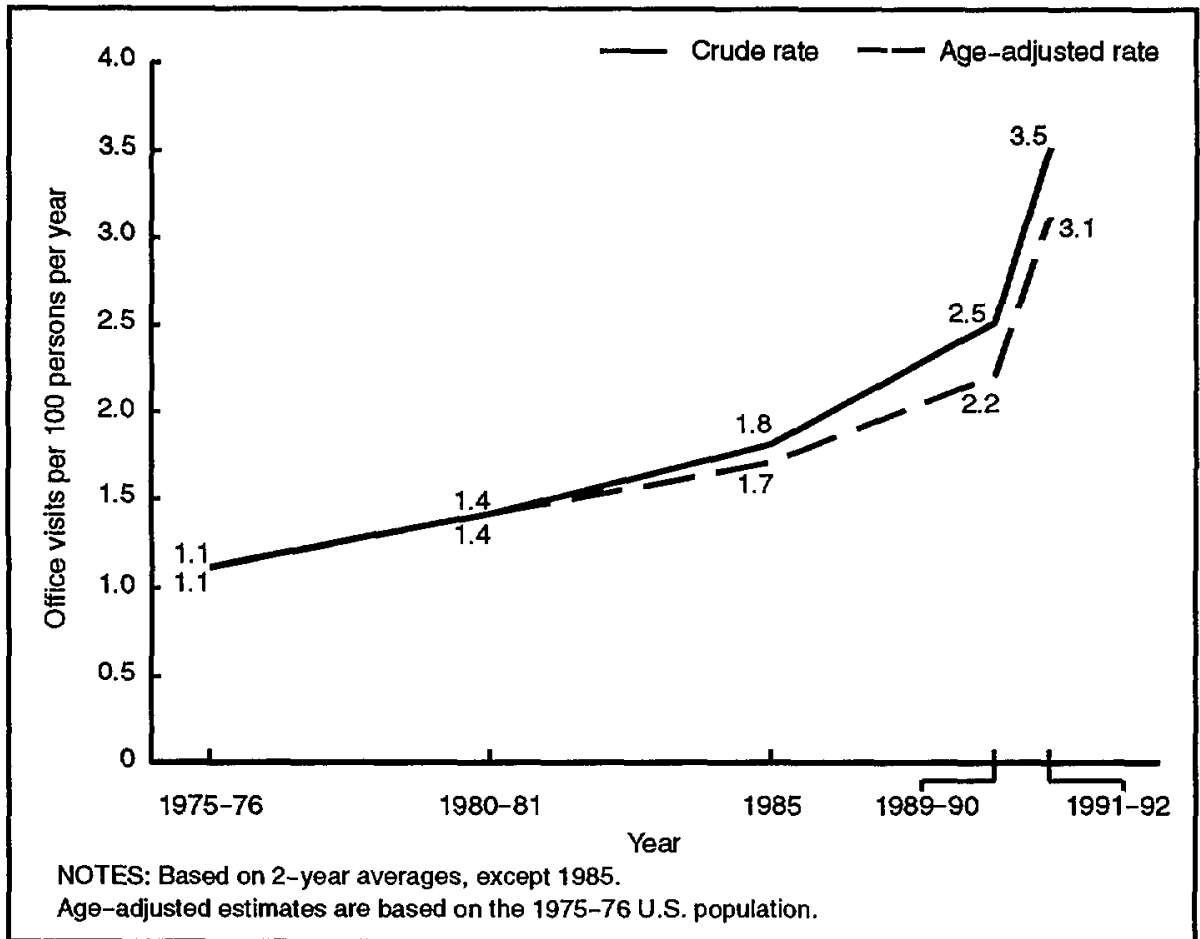

Figure 4. Annual rate of office visits with a principal diagnosis of glaucoma: United States, 1975-92

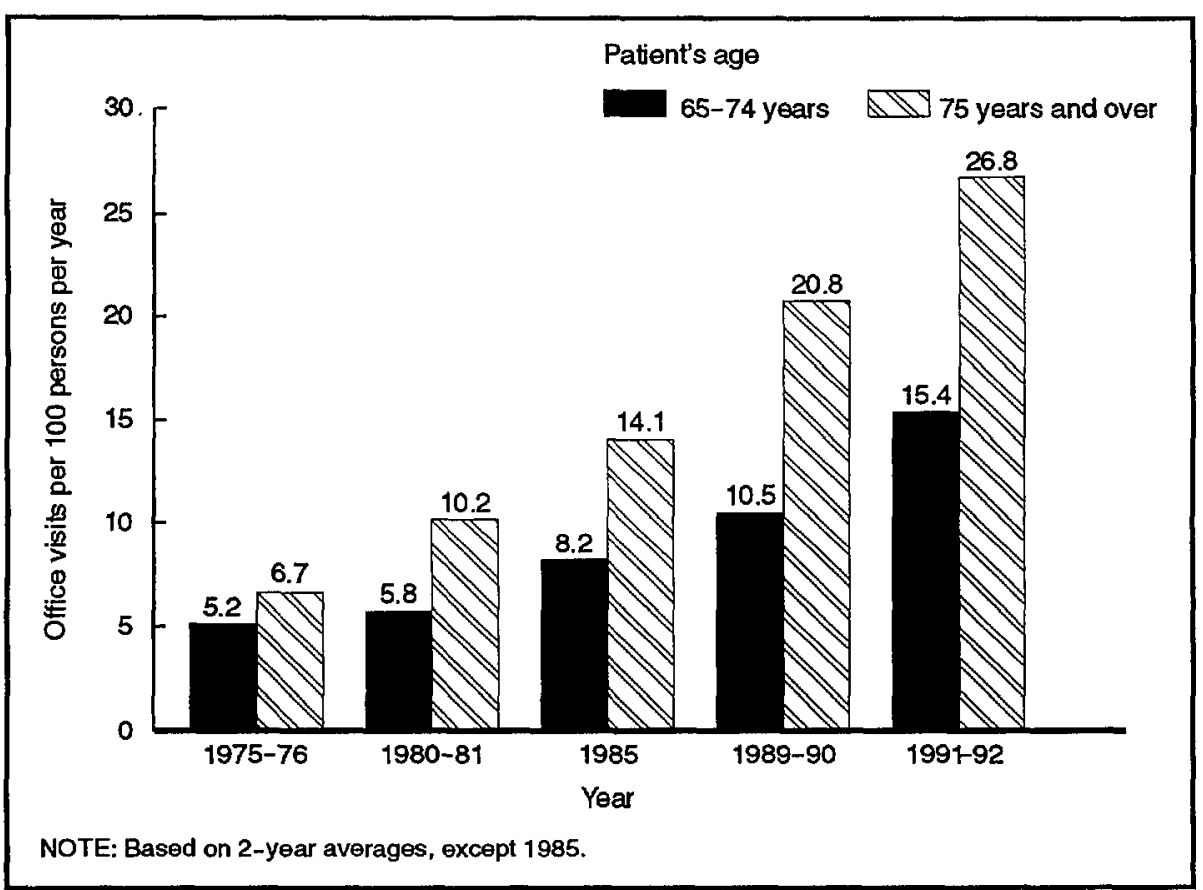

Figure 5. Annual rate of office visits with a principal diagnosis of glaucoma by patients 65-74 years and 75 years and over: United States, 1975-92. and health statistics; no. 253. Hyattsville, Maryland: National Center for Health Statistics. 1994.

5. Hoskins HD, Kass MA (eds). Becker-Shaffer's Diagnosis and
Therapy of the Glaucomas, 6th ed. St. Louis: C.V. Mosby. 1989.

6. Danyluk AW, Paton D. Diagnosis and Management of Glaucoma. Clinical Symposia. 1991. Vol. 43. No. 4. 2-2.
7. Tielsch JM, Sommer A, Katz J, Royall RM et al. Racial Variations in the Prevalence of Primary Openangle Glaucoma. Journal of the American Medical Association. 1991. 226:369-74.

8. Javitt JC, McBean AM, Nicholson GA, Babish JD et al. Undertreatment of Glaucoma among Black Americans. New England Journal of Medicine. 1991. 325:1418-22.

9. Schneider D, Appleton L, McLemore T. A reason for visit classification for ambulatory care. National Center for Health Statistics. Vital and Health Stat 2(78). 1979.

10. Koch H, Campbell W. The collection and processing of drug information. National Ambulatory Medical Care Survey, 1980. National Center for Health Statistics. Vital and Health Stat 2(90). 1982.

11. Food and Drug Administration. National Drug Code Directory, 1985 Edition. Washington: Public Health Service. 1985.

12. Feller, Barbara A. Prevalence of selected impairments, United States, 1977. National Center for Health Statistics. Vital and Health Stat 10(134). 1981.

13. Adams PF, Benson V. Current estimates from the National Health Interview Survey, 1991. National Center for Health Statistics. Vital and Health Stat 10(184). 1992.

14. National Center for Health Statistics. Current estimates from the National Health Interview Survey: United States, 1982. Vital and Heallh Stat 10(150). 1985.

15. Cotton P. Glaucoma: Detection before Damage, Fewer Side Effects May Be Possible. Journal of the American Medical Association. 1990. $264: 1793$ 


\section{Technical notes}

\section{Source of data and sample design}

The information presented in this report is based on data collected in the National Ambulatory Medical Care Survey (NAMCS) over the 2-year period from January 1991 through December 1992. The target universe of NAMCS includes office visits made in the United States by ambulatory patients to nonfederally employed physicians who are principally engaged in office practice, but not in the specialties of anesthesiology, pathology, or radiology. Telephone contacts and nonoffice visits are excluded.

A multistage probability sample design is used in NAMCS, involving samples of primary sampling units (PSU's), physician practices within PSU's, and patient visits within physician practices. The PSU's are counties, groups of counties, county equivalents (such as parishes or independent cities), or towns and townships (for some PSU's in New England). For 1991, a sample of 2,540 nonfederal, office-based physicians was selected from master files maintained by the American Medical Association and American Osteopathic Association. Physicians were screened at the time of the survey to ensure that they were eligible for survey participation. Of those screened, 1,887 physicians were eligible (in-scope) to participate in the survey. The remaining 653 physicians were ineligible (out-of-scope) due to reasons of being retired, employed primarily in teaching, research, or administration, or other reasons. The physician response rate for the 1991 NAMCS was 72 percent.

For 1992, a sample of 3,000 nonfederal, office-based physicians was selected from master files maintained by the American Medical Association and American Osteopathic Association. Of those screened, 858 physicians were ruled ineligible (out-of-scope); 2,142 were in-scope for the survey. The physician response rate for the 1992 NAMCS was 71 percent.

Sample physicians were asked to complete Patient Record forms (figure 1) for a systematic random sample of office visits occurring during a randomly assigned 1-week reporting period. Responding physicians completed 33,795 Patient Record forms in 1991 and 34,606 Patient Record forms in 1992.

Characteristics of the physician's practice, such as primary specialty and type of practice, were obtained from the physicians during an induction interview. The U.S. Bureau of the Census, Housing Surveys Branch, was responsible for the survey's data collection. Processing operations and medical coding were performed by the National Center for Health Statistics, Health Care Survey Section, Research Triangle Park, North Carolina.

For 1992, several changes were made in the sample design of the NAMCS that should be considered in the interpretation of the survey results. In an effort to even the precision of estimates across each of the physician specialty strata in the sample design, the decision was made to increase the proportion in the sample of specialists in general surgery, psychiatry, otolaryngology, and neurology. Although this would result in a corresponding decrease in the sample of the larger physician specialties, most notably general and family practice, internal medicine, and pediatrics, the precision of these estimates tended to be much higher relative to the smaller specialties, and it was expected that the end result would be an acceptable balance of precision levels across all strata.

However, the reduced number of general practitioners, internists, and pediatricians sampled in 1992, coupled with the high percents of sampled physicians in these specialties who were determined to be ineligible (out-ofscope) for survey participation, resulted in low numbers of survey respondents in these categories and a lowering of the precision of these estimates relative to other survey years, especially when disaggregated by other variables such as race. Because visits made by black patients were often found to be clustered among the sampled physicians and were more likely to be made to general and family practitioners, which were undersampled in 1992, it is recommended that caution be exercised when interpreting differences in race data and individual physician specialties.

Despite the difference in sample sizes, the 1991 and 1992 surveys were identical in terms of survey instruments, definitions, and procedures. The resulting 2 years of data have been combined to provide more reliable estimates. All estimates, percent distributions, and rates presented here, unless otherwise noted, reflect 1991 and 1992 data that were averaged over the 2-year period.

\section{Sampling errors}

The standard error is primarily a measure of the sampling varitsitity that occurs by chance when only atrample, rather than an entire universe, is surveyed. The relative standard error of an estimate is obtained by dividing the standard error by the estimate itself; the result is then expressed as a percent of the estimate.

Relative standard errors (RSE's) for estimated numbers of office visits, expressed as 2-year averages for the period 1991-92, are shown in table 1 . Relative standard errors for estimated numbers of drug mentions, also expressed as 2-year averages, are

\begin{tabular}{|c|c|}
\hline $\begin{array}{l}\text { Esthmated number } \\
\text { of office vists } \\
\text { (expressed as } \\
\text { annual averages) } \\
\text { in thousands }\end{array}$ & $\begin{array}{l}\text { Relative standard } \\
\text { error in percent }\end{array}$ \\
\hline $50 \ldots \ldots \ldots \ldots$ & 78.4 \\
\hline $100 \ldots \ldots \ldots \ldots \ldots$ & 55.5 \\
\hline $250 \ldots \ldots \ldots \ldots \ldots$ & 35.2 \\
\hline $346 \ldots \ldots \ldots \ldots$ & 30.0 \\
\hline $500 \ldots \ldots \ldots \ldots \ldots$ & 25.0 \\
\hline $1,000 \ldots \ldots \ldots \ldots \ldots$ & 17.8 \\
\hline $2,500 \ldots \ldots \ldots \ldots \ldots$ & 11.6 \\
\hline $5,000 \ldots \ldots \ldots \ldots$ & 8.5 \\
\hline $10,000 \ldots \ldots \ldots \ldots \ldots$ & 6.5 \\
\hline $25,000 \ldots \ldots \ldots \ldots$ & 4.9 \\
\hline $50,000 \ldots \ldots \ldots \ldots \ldots$ & 4.2 \\
\hline $100,000 \ldots \ldots \ldots \ldots$ & 3.8 \\
\hline $250,000 \ldots \ldots \ldots \ldots$ & 3.6 \\
\hline $500,000 \ldots \ldots \ldots \ldots$ & 3.5 \\
\hline
\end{tabular}

NOTE: The smallest reliable estimate for visits to aggregated specialties is 346,000 visits per yoar (or a 2-year total of 691,000 visits). Estimates below this figure have a rolative standard error greater than 30 percent and are deemed unreliable by NCHS standards.

Example of use of table: An aggregrate estimate of 10 million visits per year has a relative standard error of 6.5 percent or a standard error of 650,000 visits (6.5 percent of 10 million). 
Table II. Approximate relative standard errors for estimated numbers of drug mentions: National Ambulatory Medical Care Survey, 1991-92

\begin{tabular}{|c|c|}
\hline $\begin{array}{l}\text { Estimated number } \\
\text { of drug mentions } \\
\text { (expressed as } \\
\text { annual averages) } \\
\text { in thousands }\end{array}$ & $\begin{array}{l}\text { Relative standard } \\
\text { error in percent }\end{array}$ \\
\hline $50 \ldots \ldots \ldots \ldots \ldots$ & 109.0 \\
\hline $100 \ldots \ldots \ldots \ldots \ldots$ & 77.2 \\
\hline $250 \ldots \ldots \ldots \ldots \ldots$ & 48.9 \\
\hline $500 \ldots \ldots \ldots \ldots \ldots$ & 34.7 \\
\hline $674 \ldots \ldots \ldots \ldots \ldots$ & 30.0 \\
\hline $1,000 \ldots \ldots \ldots \ldots \ldots$ & 24.7 \\
\hline $2,500 \ldots \ldots \ldots \ldots \ldots$ & 16.0 \\
\hline $5,000 \ldots \ldots \ldots \ldots \ldots \ldots$ & 11.7 \\
\hline $10,000 \ldots \ldots \ldots \ldots$ & 8.8 \\
\hline $25,000 \ldots \ldots \ldots \ldots \ldots$ & 6.5 \\
\hline $50,000 \ldots \ldots \ldots \ldots \ldots$ & 5.5 \\
\hline $100,000 \ldots \ldots \ldots \ldots$ & 4.9 \\
\hline $250,000 \ldots \ldots \ldots \ldots$ & 4.6 \\
\hline $500,000 \ldots \ldots \ldots \ldots$ & 4.4 \\
\hline
\end{tabular}

NOTE: The smallest raliable estimate of drug mentions to aggregatod specialties is 674,000 drug mentions per year (or a 2-year fotal of 1,347,000 mentions). Estimates below this figure have a relative standard error greater than 30 percent and are deemed unreliable by NCHS standards.

Example of use of table: An aggregrate estimate of 25 million drug mentions per year has a relative standard error of 6.5 percent or a standard error of $1,625,000$ drug mentions $(6.5$ porcent of 25 million).

presented in table II. Standard errors for estimated percents of visits and drug mentions are displayed in tables III and IV.

Alternatively, relative standard errors for 2-year averages may be calculated using the following general formula, where $x$ is the average of interest in thousands multiplied by 2 to obtain the 2-year total, and $A$ and $B$ are
Table IV. Approximate standard errors of percents for estimated numbers of drug mentions: Natlonal Ambulatory Medical Care Survey, 1991-92

\begin{tabular}{|c|c|c|c|c|c|c|c|}
\hline \multirow{2}{*}{$\begin{array}{l}\text { Base of percent } \\
\text { (visits, expressed as } \\
\text { annual averages, } \\
\text { in thousands) }\end{array}$} & \multicolumn{7}{|c|}{ Estimated percent } \\
\hline & $\begin{array}{c}1 \text { or } \\
99\end{array}$ & $\begin{array}{c}5 \text { or } \\
95\end{array}$ & $\begin{array}{c}10 \text { or } \\
90\end{array}$ & $\begin{array}{c}20 \text { or } \\
80\end{array}$ & $\begin{array}{c}30 \text { or } \\
70\end{array}$ & $\begin{array}{c}40 \text { or } \\
60\end{array}$ & 50 \\
\hline & \multicolumn{7}{|c|}{ Standard error in percentage points } \\
\hline 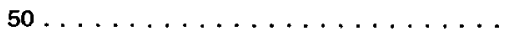 & 10.8 & 23.7 & 32.7 & 43.6 & 49.9 & 53.4 & 54.5 \\
\hline 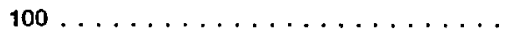 & 7.7 & 16.8 & 23.1 & 30.8 & 35.3 & 37.7 & 38.5 \\
\hline 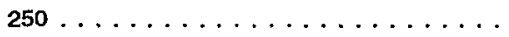 & 4.9 & 10.6 & 14.6 & 19.5 & 22.3 & 23.9 & 24.4 \\
\hline 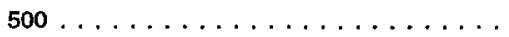 & 3.4 & 7.5 & 10.3 & 13.8 & 15.8 & 16.9 & 17.2 \\
\hline $1,000 \ldots \ldots \ldots \ldots \ldots \ldots \ldots$ & 2.4 & 5.3 & 7.3 & 9.7 & 11.2 & 11.9 & 12.2 \\
\hline $2,500 \ldots \ldots \ldots \ldots \ldots \ldots \ldots$ & 1.5 & 3.4 & 4.6 & 6.2 & 7.1 & 7.6 & 7.7 \\
\hline $5,000 \ldots \ldots \ldots \ldots \ldots \ldots \ldots$ & 1.1 & 2.4 & 3.3 & 4.4 & 5.0 & 5.3 & 5.5 \\
\hline $10,000 \ldots \ldots \ldots \ldots \ldots$ & 0.8 & 1.7 & 2.3 & 3.1 & 3.5 & 3.8 & 3.9 \\
\hline $25,000 \ldots \ldots \ldots \ldots \ldots \ldots \ldots$ & 0.5 & 1.1 & 1.5 & 2.0 & 2.2 & 2.4 & 2.4 \\
\hline $50,000 \ldots \ldots \ldots \ldots \ldots \ldots$ & 0.3 & 0.8 & 1.0 & 1.4 & 1.6 & 1.7 & 1.7 \\
\hline $100,000 \ldots \ldots \ldots \ldots \ldots \ldots$ & 0.2 & 0.5 & 0.7 & 1.0 & 1.0 & 1.2 & 1.2 \\
\hline $250,000 \ldots \ldots \ldots \ldots \ldots$ & 0.2 & 0.3 & 0.5 & 0.6 & 0.7 & 0.8 & 0.8 \\
\hline $500,000 \ldots \ldots \ldots \ldots \ldots \ldots$ & 0.1 & 0.2 & 0.3 & 0.4 & 0.5 & 0.5 & 0.6 \\
\hline
\end{tabular}

Example of use of table: An estimate of 20 percent based on an estimate of 10 million drug mentions per year has a standard error of 3.1 percent or a relative standard efror of 15.5 percent $(3.1$ percent divided by 20 percent). the appropriate coefficients from table V. The relative standard error obtained in this way applies to both the 2-year total and the 2-year average.

$$
R S E(x)=\sqrt{A+\frac{B}{x}} \cdot 100
$$

Similarly, relative standard errors for percents may be calculated using the following general formula, where $p$ is the percent of interest and $x$ is the denominator of the percent in thousands (and the denominator is the 2-year aggregate estimate rather than the average), using the appropriate
Table III. Approximate standard errors of percents of estimated numbers of office visits: National Ambulatory Medical Care Survey, 1991-92

\begin{tabular}{|c|c|c|c|c|c|c|c|}
\hline \multirow{2}{*}{$\begin{array}{l}\text { Base of percent } \\
\text { (visits, expressed as } \\
\text { annual averages, } \\
\text { in thousands) }\end{array}$} & \multicolumn{7}{|c|}{ Estimated percent } \\
\hline & $\begin{array}{l}1 \text { or } \\
99\end{array}$ & $\begin{array}{c}5 \text { or } \\
95\end{array}$ & $\begin{array}{c}10 \text { or } \\
90\end{array}$ & $\begin{array}{c}20 \text { or } \\
80\end{array}$ & $\begin{array}{c}30 \text { or } \\
70\end{array}$ & $\begin{array}{c}40 \text { or } \\
60\end{array}$ & 50 \\
\hline & \multicolumn{7}{|c|}{ Standard error in percentage points } \\
\hline $50 \ldots \ldots \ldots \ldots$ & 7.8 & 17.1 & 23.5 & 31.3 & 35.9 & 38.4 & 39.2 \\
\hline $100 \ldots \ldots \ldots$ & 5.5 & 12.1 & 16.6 & 22.2 & 25.4 & 27.1 & 27.7 \\
\hline $250 \ldots \ldots \ldots \ldots$ & 3.5 & 7.6 & 10.5 & 14.0 & 16.1 & 17.2 & 17.5 \\
\hline $500 \ldots \ldots \ldots$ & 2.5 & 5.4 & 7.4 & 9.9 & 11.4 & 12.1 & 12.4 \\
\hline $1,000 \ldots \ldots \ldots \ldots$ & 1.7 & 3.8 & 5.3 & 7.0 & 8.0 & 8.6 & 8.8 \\
\hline $2,500 \ldots \ldots \ldots$ & 1.1 & 2.4 & 3.3 & 4.4 & 5.1 & 5.4 & 5.5 \\
\hline $5,000 \ldots \ldots \ldots$ & 0.8 & 1.7 & 2.4 & 3.1 & 3.6 & 3.8 & 3.9 \\
\hline $10,000 \ldots \ldots \ldots$ & 0.6 & 1.2 & 1.7 & 2.2 & 2.5 & 2.7 & 2.8 \\
\hline $25,000 \ldots \ldots \ldots$ & 0.4 & 0.8 & 1.1 & 1.4 & 1.6 & 1.7 & 1.8 \\
\hline $50,000 \ldots \ldots \ldots$ & 0.3 & 0.5 & 0.7 & 1.0 & 1.1 & 1.2 & 1.2 \\
\hline $100,000 \ldots \ldots \ldots$ & 0.2 & 0.4 & 0.5 & 0.7 & 0.8 & 0.9 & 0.9 \\
\hline $250,000 \ldots \ldots \ldots$ & 0.1 & 0.2 & 0.3 & 0.4 & 0.5 & 0.6 & 0.6 \\
\hline $500,000 \ldots \ldots \ldots$ & 0.1 & 0.2 & 0.2 & 0.3 & 0.4 & 0.4 & 0.4 \\
\hline
\end{tabular}

Example of use of table: An estimate of 20 percent based on an estimate of 25 million visits per year has a standard error of 1.4 percent or a relative standard error of 7.0 percent $(1,4$ percent divided by 20 percent). coefficient from table V. (The 2-year aggregate is obtained by multiplying the average estimate by 2 .)

$$
R S E(p)=\frac{\sqrt{B \cdot(1-p)}}{p \cdot x} \cdot 100
$$

\section{Adjustments for nonresponse}

Estimates from NAMCS data were adjusted to account for sample physicians who were in-scope but did not participate in the study. This adjustment was calculated to minimize the impact of response on final estimates by imputing to nonresponding physicians data from visits to similar physicians. For this purpose, physicians were judged similar if they had the same specialty designation and practiced in the same PSU.

\section{Test of significance and rounding}

In this report, the determination of statistical inference is based on the two-tailed $t$-test. The Bonferroni inequality was used to establish the critical value for statistically significant differences ( 0.05 level of significance) based on the number of possible comparisons within a particular variable or (combination of variables) of interest. Terms relating to differences such as "greater than" or "less than" indicate that the difference is statistically 
Table V. Coefficients appropriate for determining relative standard errors by type of estimate and physiclan groups: National Ambulatory Medical Care Survey, 1991-92

\begin{tabular}{|c|c|c|}
\hline \multirow[b]{2}{*}{ Type of estimate and physiclan specialty } & \multicolumn{2}{|c|}{ Coefficlent for use with estimates in thousands } \\
\hline & $A$ & $\boldsymbol{B}$ \\
\hline \multicolumn{3}{|l|}{ Visits } \\
\hline Overall totals $\ldots \ldots \ldots \ldots \ldots \ldots$ & 0.001157131 & 61.31199989 \\
\hline General and family practlce $\ldots \ldots \ldots \ldots \ldots$ & 0.007330504 & 54.54704362 \\
\hline Osteopathy . . . . . . . . . . . . . . & 0.01402452 & 18.13642054 \\
\hline Intemal medicine $\ldots \ldots \ldots \ldots \ldots \ldots \ldots$ & 0.008718567 & 55.2168744 \\
\hline Pedlatrics $\ldots \ldots \ldots \ldots \ldots \ldots \ldots \ldots \ldots \ldots$ & 0.007994386 & 35.33091768 \\
\hline General surgery $\ldots \ldots \ldots \ldots \ldots \ldots \ldots$ & 0.006685247 & 10.65103125 \\
\hline Obstetrics and gynecology $\ldots \ldots \ldots \ldots \ldots \ldots$ & 0.00919584 & 25.59962011 \\
\hline Orthopedic surgery. . . . . . . . . . . . . . & 0.005641337 & 24.20372144 \\
\hline Cardlovescular dlseases $\ldots \ldots \ldots \ldots \ldots \ldots$ & 0.01383253 & 12.58489271 \\
\hline Dermalology. . . . . . . . . . . . . . . . . & 0.01275351 & 10.28901849 \\
\hline Urological surgery $\ldots \ldots \ldots \ldots \ldots \ldots$ & 0.008000282 & 11.92853664 \\
\hline Psychiatry . . . . . . . . . . . . . . & 0.009414736 & 12.88530675 \\
\hline Neurology $\ldots \ldots \ldots \ldots \ldots \ldots \ldots \ldots \ldots$ & 0.01314774 & 5.36720816 \\
\hline Ophthalmology . . . . . . . . . . . . . . . & 0.007938148 & 23.84517495 \\
\hline Otolaryngology $\ldots \ldots \ldots \ldots \ldots \ldots \ldots$ & 0.007549396 & 8.0936265 \\
\hline All other specialties $\ldots \ldots \ldots \ldots \ldots \ldots$ & 0.01537018 & 35.00317779 \\
\hline \multicolumn{3}{|l|}{ Drug mentions } \\
\hline Overall totals $\ldots \ldots \ldots \ldots \ldots \ldots$ & 0.001853163 & 118.69462 \\
\hline General and famlly practice $\ldots \ldots \ldots \ldots \ldots$ & 0.009085669 & 100.96778 \\
\hline Osteopathy $\ldots \ldots \ldots \ldots \ldots \ldots$ & 0.01658477 & 23.4739982 \\
\hline Intemal medicine $\ldots \ldots \ldots \ldots \ldots \ldots \ldots$ & 0.01148498 & 103.21387 \\
\hline 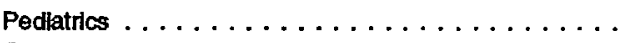 & 0.01245118 & 26.73517786 \\
\hline 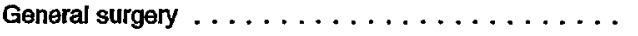 & 0.03935224 & 8.06806796 \\
\hline Obstetrics and gynecology . . . . . . . . . . . . . & 0.01454044 & 31.24058408 \\
\hline Orthopedic surgery. . . . . . . . . . . . . . & 0.01568053 & 23.3833057 \\
\hline Cardlovascular diseases . . . . . . . . . . . & 0.01575914 & 24.23751806 \\
\hline Dermatology. . . . . . . . . . . . . . . & 0.01299377 & 15.94507357 \\
\hline Urologlcal surgery $\ldots \ldots \ldots \ldots \ldots \ldots \ldots \ldots$ & 0.01867719 & 10.6886669 \\
\hline Psychiatry $\ldots \ldots \ldots \ldots \ldots \ldots \ldots \ldots$ & 0.01430555 & 15.99374434 \\
\hline Neurology . . . . . . . . . . . . . . . . & 0.01593433 & 6.67244993 \\
\hline Ophthalmology . . . . . . . . . . . . . . . & 0.0251486 & 25.1381195 \\
\hline Otolaryngology . . . . . . . . . . . . . . & 0.008374063 & 12.25916054 \\
\hline All other speclalties $\ldots \ldots \ldots \ldots \ldots \ldots$ & 0.0226229 & 57.79950436 \\
\hline
\end{tabular}

significant. A lack of comment regarding the difference between any two estimates does not mean that the difference was tested and found to be not significant.

In the tables, estimates of office visits have been rounded to the nearest thousand. Consequently, estimates will not always add to totals. Rates and percents were calculated from original unrounded figures and do not necessarily agree with percents calculated from rounded data.

\section{Definition of terms}

Ambulatory patient-An ambulatory patient is an individual seeking personal health services who is not currently admitted to any health care institution on the premises.
Drug mention-A drug mention is the physician's entry on the Patient Record form of a pharmaceutical agent - by any route of administration - for prevention, diagnosis, or treatment. Generic as well as brand-name drugs are included, as are nonprescription and prescription drugs. Along with all new drugs, the physician also records continued medications if the patient was specifically instructed during the visit to continue the medication. Physicians may report up to five medications per visit.

Drug visit-A drug visit is a visit at which medication was prescribed or provided by the physician.

Office-An office is the space identified by a physician as a location for his or her ambulatory practice. Offices customarily include consultation, examination, or treatment spaces that patients associate with the particular physician.

Physician-A physician is a duly licensed doctor of medicine (M.D.) or doctor of osteopathy (D.O.) who is currently in office-based practice and who spends some time caring for ambulatory patients. Excluded from the NAMCS are physicians who are hospital based; who specialize in anesthesiology, pathology, or radiology; who are federally employed; who treat only institutionalized patients; or who are employed full time by an institution and spend no time seeing ambulatory patients.

Visit-A visit is a direct personal exchange between an ambulatory patient and a physician or a staff member working under the physician's supervision, for the purpose of seeking care and rendering personal health services. Excluded from the NAMCS are visits where medical care was not provided, such as visits made to drop off specimens, pay bills, make appointments, and walk-outs. 


\section{Symbols}

.. Data not available

... Category not applicable

- Quantity zero

0.0 Quantity more than zero but less than 0.05

Z Quantity more than zero but less than $\mathbf{5 0 0}$ where numbers are rounded to thousands

- Figure does not meet standard of reliability or precision 


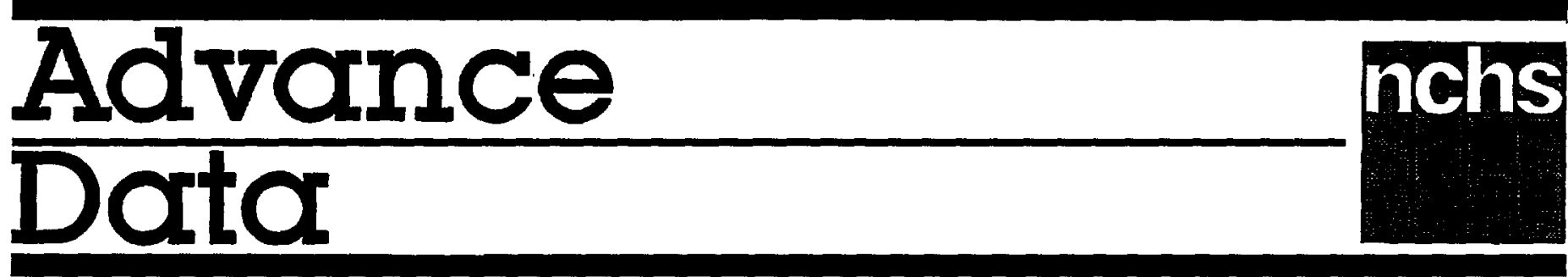

\title{
Relationship Between Cigarette Smoking and Other Unhealthy Behaviors Among our Nation's Youth: United States, 1992
}

\author{
by Jean C. Willard and Charlotte A. Schoenborn, M.P.H., Division of Health Interview Statistics
}

\section{Introduction}

The transitional period between childhood and adulthood is a time in which youth experience many physical changes, as well as a developing sense of self and increasing emotional independence (1). During this time, adolescents often develop behaviors that extend into adulthood (2). Young people may experiment with risky health behaviors, some of which have long term health consequences (3). Some adolescents use these behaviors to bond with peers, improve their social image, and appear independent and mature (1). Recently, health risk behaviors of adolescents have been the focus of considerable study (4-13).

Cigarette smoking almost always begins in the adolescent years $(5,14)$ and smoking at early ages increases the risk of becoming ill or dying from causes attributable to smoking (1). Reduction in smoking prevalence among adolescents is one of the objectives established in the National Health Objectives for the Year 2000 (2). These objectives incompass 22 priority areas, including tobacco, alcohol and other drugs, shysical activity and fitness, nutrition, violent and abusive behavior, and family slanning. Each priority area contains numerous specific, measurable health objectives. Many of the objectives specifically target health-threatening behaviors among adolescents and young adults.

Progress toward achieving the National Health Objectives for the Year 2000 is monitored closely at the Federal level. Much research was devoted to establishing baseline prevalence estimates of high risk behaviors and developing objectives based on both the baseline estimates and a realistic appraisal of what can be accomplished by the end of the decade. While the objectives set targets for individual behaviors, a large body of research suggests that many high risk behaviors are interrelated. The recent Surgeon General's Report, Preventing Tobacco Use Among Young People (1), summarized studies that have shown relationships between smoking and other health-threatening behaviors such as drinking alcohol, using illicit drugs, using smokeless tobacco products, carrying weapons, engaging in physical fights, ever having had sexual intercourse, and failure to wear seat belts. Research has also shown that adolescents who participated in interscholastic sports were less likely than youth who did not participate to be regular or heavy smokers (6). Much of this earlier research was based on samples of youth who were in school, with data collected in a classroom setting. This report expands upon earlier research by delineating the relationship between cigarette smoking and other high risk behaviors among adolescents in the general household population of the United States, including youth who have left school either prematurely or by graduating. Examining the relationships between smoking and other high risk behaviors may provide clues on how to reduce smoking and other unhealthy behaviors among adolescents, thereby furthering progress toward achieving the National Health Objectives for the Year 2000.

This report uses data from the 1992 National Health Interview Survey of Youth Risk Behavior (NHIS-YRBS) and presents prevalence estimates for selected unhealthy behaviors among adolescents in the United States according to smoking status. These unhealthy behaviors, consistent with earlier studies, include drinking alcohol; consuming more than five alcoholic beverages in a row; using marijuana, cocaine, and smokeless tobacco;

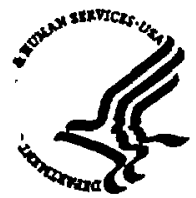

\section{U.S. DEPARTMENT OF HEALTH AND HUMAN SERVICES \\ Public Health Service \\ Centers for Disease Control and Prevention \\ National Center for Health Statistics}

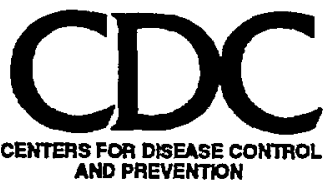


carrying weapons; physical fighting; sexual intercourse; failure to use a seat belt; lack of exercise; and consumption of fewer than five servings of fruits and vegetables daily. These behaviors are of significant public health concern as evidenced by their inclusion in the National Health Objectives for the Year 2000.

The data presented in this report provide an overview of unhealthy behaviors that are recognized as important for the current future health of our Nation's youth. However, these data do not provide specific tracking information for the objectives because of the age specificity of most objectives.

\section{Data and methods}

The NHIS-YRBS was developed to provide estimates of health risk behaviors for the noninstitutionalized, household population of youth aged 12-21 years. The NHIS is a continuous, nationwide, household interview survey of the civilian noninstitutionalized population of the United States, conducted by the National Center for Health Statistics (NCHS)(15). Interviews are conducted for NCHS by the interviewing staff of the U.S. Bureau of the Census. Information is obtained about the health and sociodemographic characteristics of each member of the household. Each year, special topic surveys are included in conjunction with the basic NHIS. These topics change annually. In 1992, the NHIS-YRBS was one of the special topics.

Within each NHIS sample family, one youth who was attending school and up to two youth who were not in school or whose in-school status was unknown were selected for the NHIS-YRBS interview. Youth in all NHIS sample families, including emancipated youth (married youth and/or those not living with a parent or guardian), were eligible for selection. The youth were followed back approximately two months after the initial household interview. NHISYRBS interviews were conducted in person from April 1992 through March 1993. NHIS-YRBS interviews were completed for 10,645 youth, representing an overall response rate of 73.9 percent.

Rather than using the traditional face-to-face interview or selfadministered questionnaires, the NHIS-YRBS used a unique audiocassette technology, developed in collaboration with researchers at University of Michigan's Survey Research Center. This technology allowed the youth to listen to the questions, using a personal headset and to record answers on an answer sheet that contained only answer categories. The answer sheet did not contain any information that would allow parents or others in the household to know what questions the youth was answering. The voice on the interview tape matched the sex of the respondent; males heard a male voice and females heard a female voice. This data collection method ensured greater privacy and increased data quality for youth with poor reading skills.

Using data from the 1992 NHISYRBS, this report presents prevalence estimates for selected unhealthy behaviors among male and female adolescents 12-21 years old and examines the relationship between cigarette smoking and each of the other behaviors, using age-adjusted statistics. Table 1 contains percents and standard errors for each of the selected unhealthy behaviors by smoking status. These unadjusted statistics (table 1) are used for discussions of overall prevalence. Table 2 contains percents, age-adjusted to the full NHIS-YRBS sample, and the associated standard errors. For data on sexual intercourse, age adjustment was limited to the NHIS-YRBS sample aged 14-21 years because only youth 14 years and older were asked the questions related to sexual experiences. Age-adjusted statistics are used in all discussions of findings concerning relationships between smoking and other unhealthy behaviors. Age-adjusted statistics control for variations in the age distributions of the various smoking status groups. Statistics for all youth ages 12-21 years are shown in order to provide an overall view of the relationships between smoking and a variety of other behaviors during the adolescent and young adult years. Age-specific analyses (not shown) revealed that the relationships between smoking and other unhealthy behaviors were stronger at the younger ages, but remained consistent, if somewhat attenuated, among youth 18-21 years of age.

\section{Definition of smoking terms}

Definitions for smoking status in the national health objectives are aimed at adults. For adults, current smoker is a person who has ever smoked 100 cigarettes in his or her lifetime and smokes "now" - "now" being defined by the respondent. Recent measures of smoking status assess regularity of smoking in adults by distinguishing between "everyday" and "some day" smokers. These definitions may not be appropriate for adolescents.

Consistent with other studies of adolescents, current smokers are defined as youth who have smoked at least one cigarette in the past 30 days. Former smokers are youth who had smoked at least one cigarette every day for 30 days at some time in their lives, but had not smoked cigarettes in the past month. Experimenters are youth who had smoked at least one or two puffs of a cigarette, but had never smoked cigarettes every day for 30 days and had not used cigarettes in the past 30 days. "Never smokers" are youth who had never had even one or two puffs of a cigarette. Definitions for other terms used in this report are in the technical notes.

\section{Findings}

Figure 1 shows prevalence of smoking among youth aged 12-21 years. About 29 percent of male youth and 26 percent of female youth were current smokers in 1992 and about 3 percent of both sexes were former smokers. About 28 percent of male youth and about 30 percent of female youth had experimented with cigarettes, but had never smoked regularly. About 40 percent of youth had never taken a puff of a cigarette. 


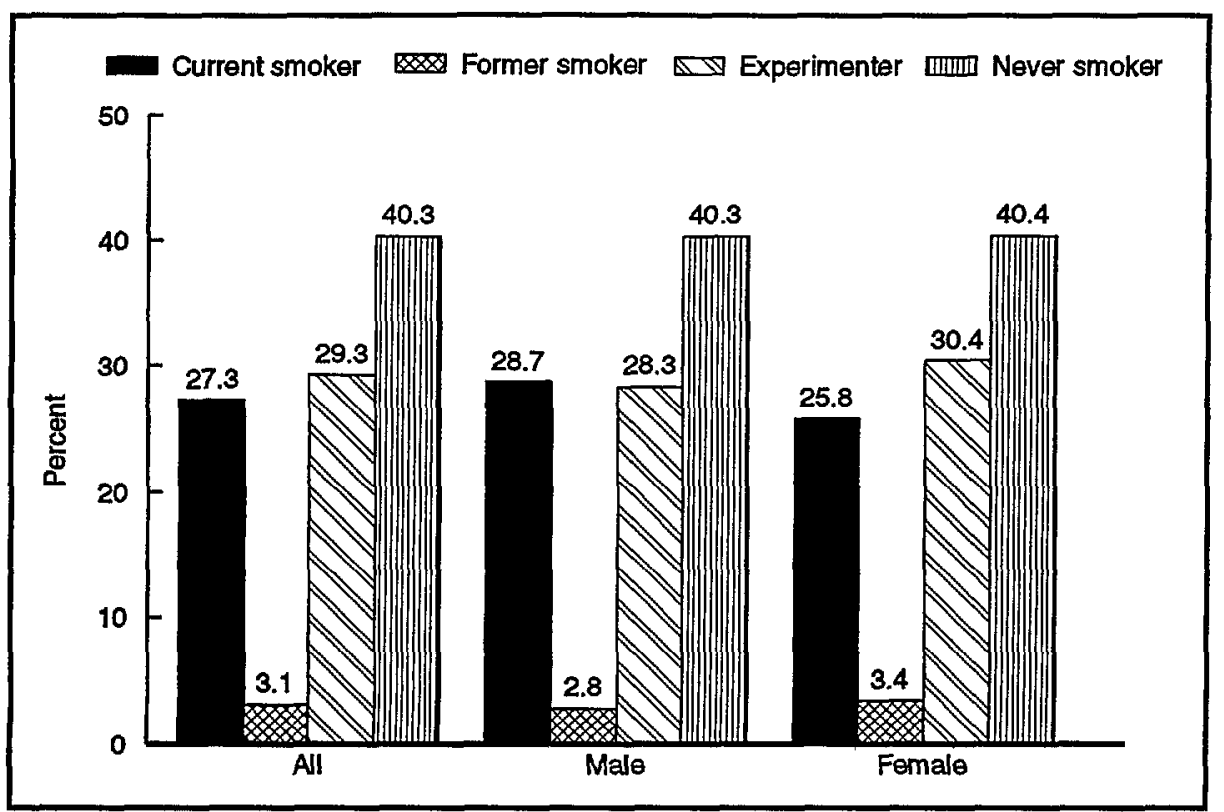

Figure 1. Percent of youth 12-21 years of age by smoking status and sex: United States, 1992

Table 1 shows the prevalence of selected unhealthy behaviors among male and female adolescents by smoking status. Figures 2 and 3 display overall prevalence of these behaviors for males and females, respectively. The data in figures 2 and 3 correspond to column 1 of table 1 . Together these data show the overall pattern of unhealthy behaviors among U.S. adolescents. In general, the patterns appear similar for adolescent males and females. For example, among both male and female youth, failure to eat at least five servings of fruits and vegetables daily had the highest prevalence of the unhealthy behaviors examined (86.1 percent and 87.9 percent, respectively), followed by failure to always use seat belts (70.2 percent and 61.4 percent of males

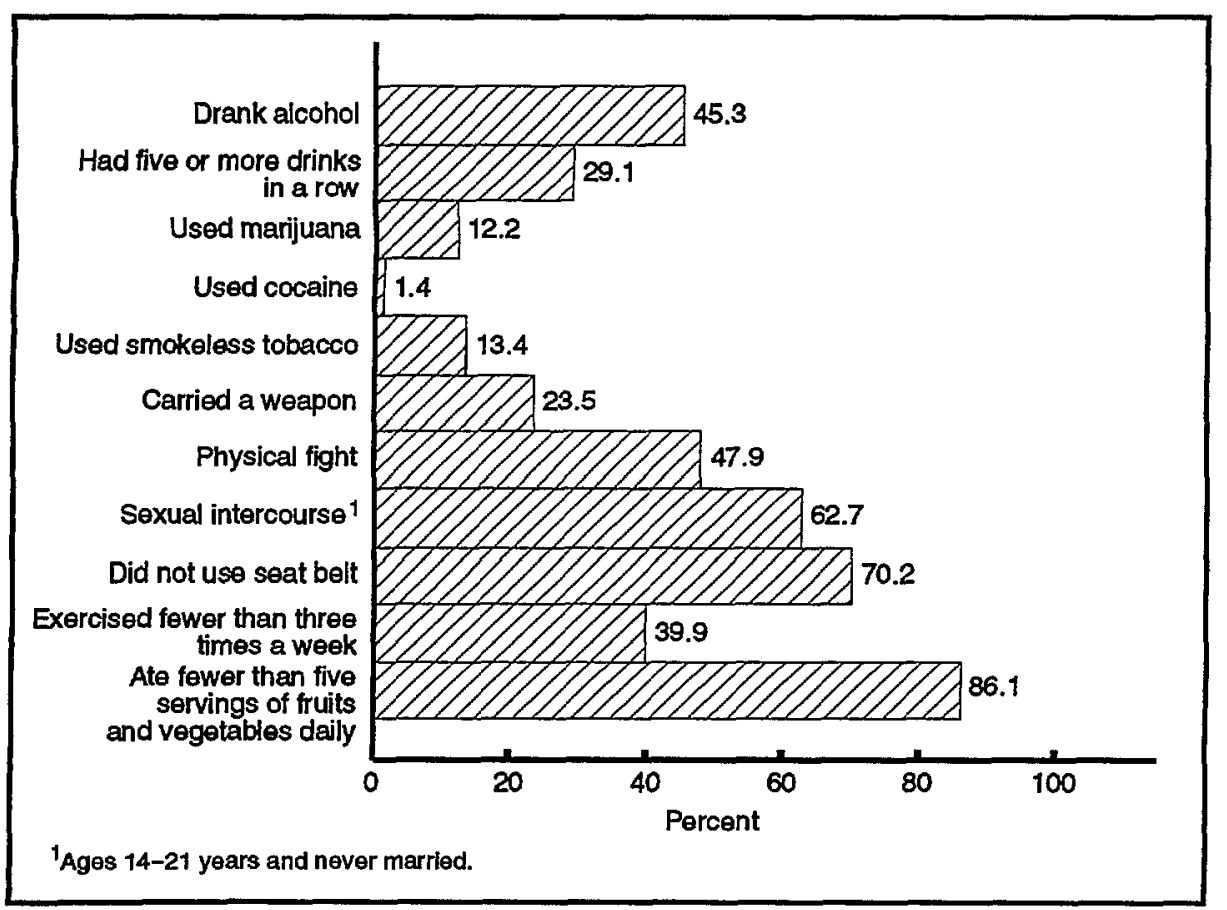

Figure 2. Prevalence of selected unhealthy behavlors among adolescent males: United States, 1992 and females, respectively), and engaging in sexual intercourse (62.7 percent and 58.7 percent of males and females, respectively). Prevalence of cocaine use was only about 1 percent for both groups.

Although the patterns among the behaviors appear similar for males and females, prevalence estimates for some behaviors differed markedly between the sexes. Among the more noteworthy differences are: male youth were more likely than female youth to have engaged in a physical fight in the past year (47.9 percent versus 29.2 percent); to have carried a weapon in the past month ( 23.5 percent versus 5.6 percent); to have used smokeless tobacco (13.4 percent versus 1.5 percent); and to have used marijuana (12.2 percent versus 9.2 percent). Female youth (52.7 percent) were more likely than male youth (39.9 percent) to get inadequate exercise (defined as exercising less than three times per week). Prevalence of consumption of any alcohol was about the same for male ( 45.3 percent) and female youth (44.0 percent), although males

(29.1 percent) were somewhat more likely than females ( 22.0 percent) to have had five or more drinks in a row.

Figures 1-3 and table 1 show prevalence estimates of unhealthy behaviors among U.S. adolescents. Using these estimates and the population table in the technical notes of this report, the reader can estimate the numbers of youth who are both smoking and engaging in other high risk behaviors. Analysis of interrelationships between smoking and other unhealthy behaviors should be restricted to the data in table 2.

Table 2 presents data on the relationship between smoking and selected other unhealthy behaviors among adolescents, adjusted for differences in the age composition of the various smoking status groups. Generally, youth who had never smoked were significantly less likely to have engaged in each unhealthy behavior studied than were current smokers. With a few exceptions, "never smokers" were also less likely than former smokers or experimenters to participate in unhealthy behaviors. 


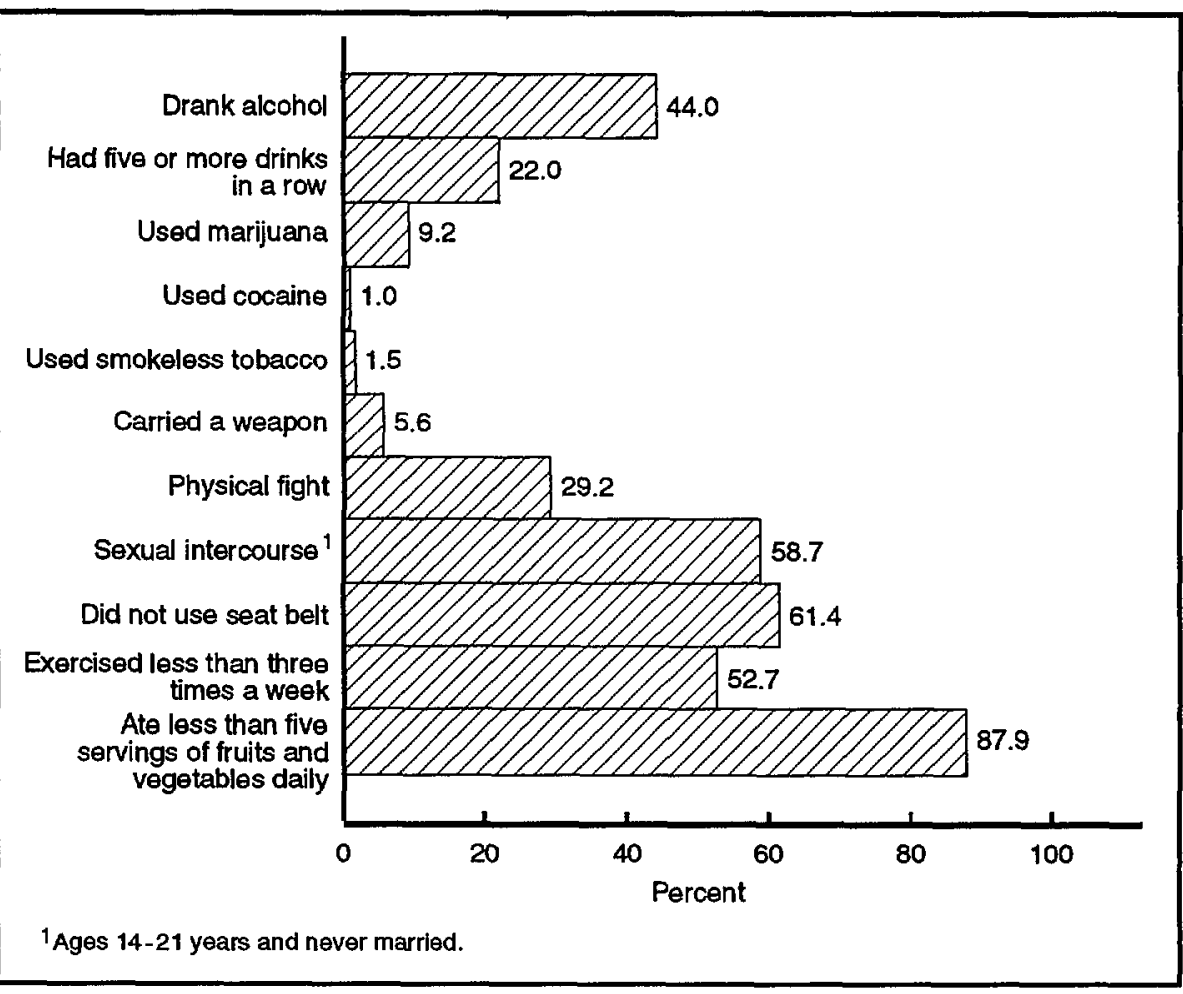

Figure 3. Prevalence of selected unhealthy behaviors among adolescent females: United Staes, 1992

\section{Drinking}

Alcohol consumption among youth has serious short term and long term health consequences. Prevention efforts are directed at encouraging young people to abstain from alcohol and to

avoid episodic heavy drinking (sometimes called binge drinking) if they do drink. Table 2 shows that after controlling for differences in age composition, about three-quarters (74.4 percent) of current smokers aged 12-21 years old had consumed alcohol

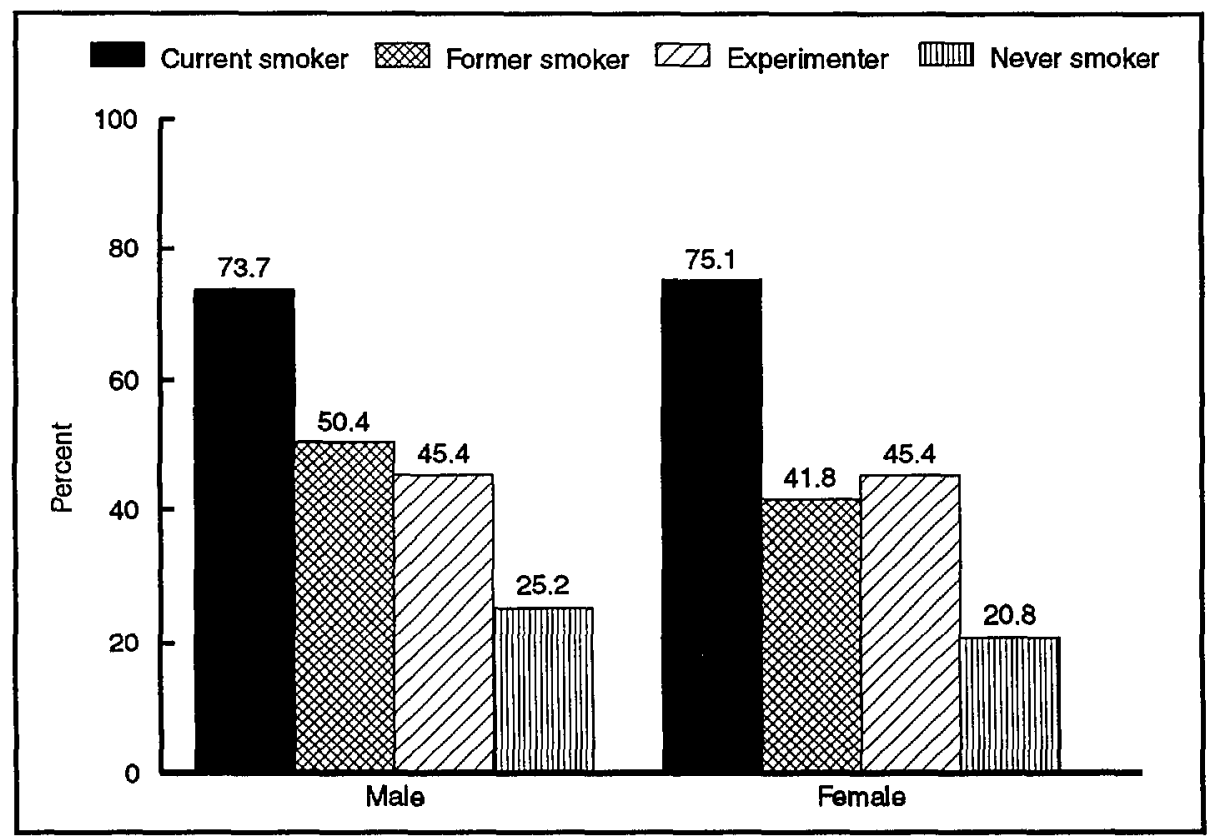

Figure 4. Percent of youth who drank alcohol in the past month, by smoking status and sex: United States, 1992 in the past 30 days, compared with 23.0 percent of "never smokers." Figure 4 shows the percent of youth who drank alcohol in the past month by smoking status and sex. Among current smokers, prevalence of alcohol consumption in the past month was about the same for males and females. Among those who have never smoked, males were slightly more likely than females to have consumed alcohol in the past month. Table 2 also shows that current smokers (50.3 percent) were considerably more likely than former smokers (25.5 percent), experimenters (21.3 percent), and "never smokers" (9.5 percent) to have had five or more drinks in a row in the past month. Rates of episodic heavy drinking were somewhat lower for females than for males across all categories of smoking status, but the pattern remained the same.

\section{Marijuana and cocaine use}

As with alcohol, drug use among our Nation's young people is a major problem and the focus of extensive prevention efforts. Table 2 shows past month use of each of two drugsmarijuana and cocaine-by smoking status. Among youth 12-21 years of age, controlling for differences in age composition, 26.5 percent of adolescent current smokers reported marijuana use in the previous 30 days compared with 10.3 percent of former smokers, 6.2 percent of experimenters, and only 1.5 percent of youth who had never smoked. Among both males and females, current marijuana use was considerably more prevalent among current smokers than among youth who were not currently smoking. Table 2 also shows that 3.5 percent of current smokers 12-21 years old had used cocaine in the previous month. Although data for former smokers, experimenters, and "never smokers" do not meet reliability standards, patterns indicate that prevalence may be lower in these groups.

\section{Smokeless tobacco}

Smokeless tobacco use among our Nation's youth is a serious public health concern. Like so many other behaviors, use of smokeless tobacco (chewing 
tobacco and snuff) is frequently taken up during the adolescent years. Table 2 shows that after controlling for differences in age composition, male adolescents who were current smokers (28.1 percent) and former smokers (27.5 percent) were almost seven times more likely to have used smokeless tobacco in the past month than were male youth who had never smoked (4.1 percent). Although the small number of female adolescents reporting use of smokeless tobacco makes estimates of this behavior unreliable for most subgroups, the data suggest that currently smoking females may be more likely to be users of smokeless tobacco than other adolescent females.

\section{Carrying woapons and physical fights}

Table 2 also provides important insights into the extent to which youth engage in two types of violent behavior-carrying weapons and engaging in physical fights-according to smoking status. After controlling for differences in age composition, about one-quarter of youth who were current smokers reported carrying a weapon such as a gun, knife, or club during the

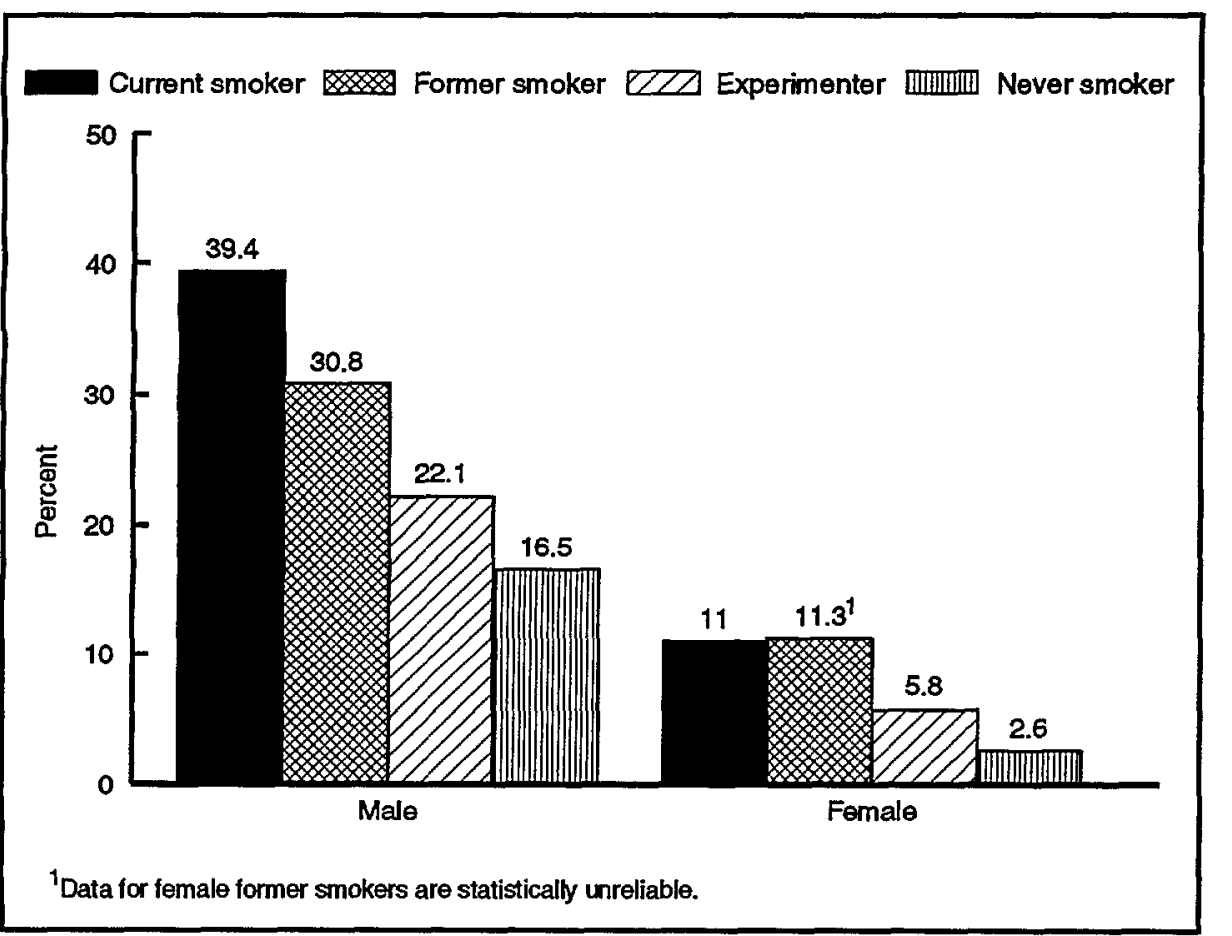

Figure 5. Percent of youth who carried weapons in the past month, by smoking status and sex: United States, 1992 previous 30 days, compared with about one-tenth of youth who had never smoked. Figure 5 shows that adolescent male smokers were more than twice as likely (39.4 percent) as males who had never smoked (16.5 percent) to have carried a weapon. Rates of carrying weapons among male former smokers (30.8 percent) and experimenters (22.1 percent) ranked between the other two groups. Adolescent female current smokers were more than four times as likely (11.0 percent) as female youth who had never smoked ( 2.6 percent) to have carried a weapon during the previous month. As with males, female experimenters ( 5.8 percent) ranked between those of current and "never smokers" on the rate of those who carried weapons. Due to the small number of female former smokers who carried weapons, data for this group were unreliable.

Table 2 shows that male current smokers (64.1 percent) were more likely than males who had experimented with cigarettes (47.1 percent) and those who had never smoked ( 38.4 percent) to have been involved in a physical fight in the past year. Among female adolescents, current smokers (44.3 percent) were more than twice as likely as "never smokers" (19.8 percent) to have engaged in physical fighting in the past year.

\section{Sexual intercourse}

The NHIS-YRBS includes data on sexual intercourse for adolescents 14-21 years of age. For this report, analysis was restricted to youth who had never been married.

Prevalence of sexual intercourse among never married adolescents was high across all smoking status groups. Table 1 indicates that 6 of 10 never married adolescents 14 years old and older ( 60.8 percent) had engaged in sexual intercourse at some time in their lives. There appears to be a relationship between cigarette smoking and sexual intercourse. Current smokers (80.0 percent) and former smokers ( 80.4 percent) were most likely to have engaged in sexual intercourse compared with 60.6 percent of youth who had only experimented with cigarettes and 41.4 percent of youth who had never smoked at all (table 2).

\section{Seat belts}

Injuries are one of the leading causes of death among adolescents and young adults. Injury deaths among those 15-24 years of age are largely attributable to motor vehicle accidents (2). Overall, 65.8 percent of adolescents did not always use seat belts when they rode in a car (table 1). Table 2 shows that over three-quarters (76.6 percent) of current smokers did not always wear a seat belt compared with about one-half (55.7 percent) of adolescents who had never smoked, with the other two groups falling in between.

\section{Vigorous exercise}

Regular participation in vigorous exercise is recognized as having important health benefits for people of all ages, including youth. In the NHIS-YRBS, youth were asked how often in the past week they engaged in any activities that made them sweat or breathe hard. The percent of youth who reported participating in such vigorous activities 3 or more days in the week preceding the interview is shown in table 2, according to smoking status. 
Overall, prevalence of regular, vigorous exercise among adolescents was low (46.2 percent), regardless of smoking status (table 1). Table 2 shows that 49.6 percent of adolescent current smokers exercised less than 3 times during the week preceding the survey; 44.8 percent of "never smokers" exercised this infrequently.

\section{Eating habits}

Guidelines for healthy eating generally recommend eating five or more servings of fruits and vegetables daily. The National Health Objectives for the Year 2000 address this issue for adults, but not for children or youth: Nevertheless, the NHIS-YRBS asked youth about fruit and vegetable consumption during the day preceding the interview. Overall, 87.0 percent of all adolescents consumed less than five servings of fruits and vegetables (table 1). Table 2 shows that consumption of fewer than the recommended minimum quantities of fruits and vegetables was somewhat more common among adolescents who currently smoked (89.6 percent) than among those who had never smoked (83.6 percent).

\section{Discussion and conclusions}

This report provides a broad overview of the links between smoking and other high risk behaviors. Although it does not establish causal links, it does show a consistent association between smoking and other unhealthy behaviors among adolescents, further strengthening the evidence that unhealthy behaviors among adolescents are interrelated. In almost all cases, current smokers had the highest and "never smokers" the lowest rates of other risk behaviors. The differences were particularly striking for use of other addictive substances such as alcohol, marijuana, and smokeless tobacco: current smokers were 3-17 times more likely than adolescents who had never smoked to have used these other substances in the past 30 days. (Data for cocaine were suggestive of a similar relationship, but were not statistically reliable due to small numbers of youth reporting cocaine use.) Although not quite as dramatic, differences between smoking and nonsmoking teenagers were also noteworthy for carrying weapons, physical fighting, sexual intercourse, and failure to use seat belts. For two risk behaviors-exercising less than three times per week and eating fewer than five servings of fruits and vegetables-smokers similarly had higher prevalence rates than those who had never smoked, but the differences were less striking.

The interrelationships between smoking and other unhealthy behaviors are undoubtedly complex. Multivariate analyses are needed to delineate the nature of these interrelationships. The data presented here suggest that high risk behaviors may cluster. That is, youth who engage in some high risk behaviors are likely to be engaging in others. Interventions that target multiple high risk behaviors may be more effective in getting youth to adopt healthy behaviors than programs that target a single behavior.

\section{References}

1. Centers for Disease Control. Preventing tobacco use among young people: a report of the Surgeon General. Atlanta: National Center for Chronic Disease Prevention and Health Promotion, Office on Smoking and Health, 1994.

2. Public Health Service. Healthy people 2000: national health promotion and disease prevention objectives. Washington: U.S. Department of Health and Human Services, 1991.

3. Designing health promotion approaches to high-risk adolescents through formative research with youth and parents. Public Health Rep 108(supp. 1): 68-7. 1993.

4. Marcus SE, Giovino GA, Pierce JP, Harel Y. Measuring tobacco use among adolescents. Public Health Rep 108(1):20-24. 1993.

5. Escabedo LG, Marcus SD, Holtzman D, Giovino GA. Sports participation, age at smoking initiation, and the risk of smoking among US high school students. JAMA 269(11): 1391-95. 1993.

6. Bachman JG, Wallace JM, O'Malley $\mathrm{PM}$, et al. Racial/ethnic differences in smoking, drinking, and illicit drug use among American high school seniors, 1976-89. Am J Public Health 81(3):372-77. 1991.

7. Centers for Disease Control. Tobacco, alcohol, and other drug use among high school students--United States, 1991. MMWR 41(37):698-03. 1992.

8. Webster DW, Gainer PS, Champion HR. Weapon carrying among inner-city junior high school students: defensive behavior vs aggressive delinquency. Am J Public Health 83:1604-8. 1993.

9. Vanderschmidt HF, Lang JM, Knight-Williams V, Vanderschmidt GF. Risks among inner-city young teens: the prevalence of sexual activity, violence, drugs, and smoking. Journal of Adolescent Health 14:282-88. 1993.

10. Centers for Disease Control. Physical fighting among high school students-United States, 1990. MMWR 41(6):91-94. 1992.

11. Centers for Disease Control. Sexual behavior among high school students-United States, 1990. MMWR 40(51). 1992.

12. Centers for Disease Control. Vigorous physical activity among high school students-United States, 1990. MMWR 41(3). 1992.

13. Centers for Disease Control. Selected tobacco-use behaviors and dietary patterns among high school students-United States, 1991. MMWR 41(24). 1992.

14. Centers for Disease Control. Reducing the health consequences of smoking: 25 years of progress, a report of the Surgeon General. Washington: Public Health Service. DHHS Publication No (CDC) 89-8411. 1989.

15. Benson V, Marano MA. Current estimates from the National Health Interview Survey, 1992. National Center for Health Statistics. Vital Health Stat 10(189). 1994.

16. Kolbe LJ, Kann L, Collins JL. Overview of the Youth Risk Behavior Surveillance System. Public Health Rep 108(supp. 1): 2-10. 1993

17. Centers for Disease Control. Health risk behaviors among adolescents who do and do not attend schoolUnited States, 1992. MMWR 43(8). 1994. 
Table 1. Percent of youth ages 12-21 years who engaged in selected unhealthy behaviors by type of behavior, sex, and smoking status: United States, 1992

\begin{tabular}{|c|c|c|c|c|c|c|c|c|c|c|}
\hline \multirow[b]{2}{*}{ Unhealthy behavior' and sex } & \multicolumn{2}{|c|}{$\begin{array}{l}\text { All smoking } \\
\text { statuses }\end{array}$} & \multicolumn{2}{|c|}{$\begin{array}{l}\text { Current } \\
\text { smoker }\end{array}$} & \multicolumn{2}{|c|}{$\begin{array}{l}\text { Former } \\
\text { smoker }\end{array}$} & \multicolumn{2}{|c|}{ Experimenter } & \multicolumn{2}{|c|}{$\begin{array}{l}\text { Never } \\
\text { smoker }\end{array}$} \\
\hline & Percent & $\begin{array}{l}\text { Standard } \\
\text { enror }\end{array}$ & Percent & $\begin{array}{l}\text { Standard } \\
\text { error }\end{array}$ & Percent & $\begin{array}{l}\text { Standard } \\
\text { error }\end{array}$ & Percent & $\begin{array}{l}\text { Standard } \\
\text { error }\end{array}$ & Percent & $\begin{array}{l}\text { Standard } \\
\text { entor }\end{array}$ \\
\hline \multicolumn{11}{|l|}{ Drank alcohol } \\
\hline Both sexes. . . . . . . . . . . . . . . . & 44.6 & 0.69 & 77.9 & 0.93 & 55.5 & 3.40 & 48.7 & 1.12 & 17.6 & 0.79 \\
\hline 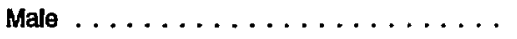 & 45.3 & 0.86 & 78.2 & 1.31 & 56.4 & 5.69 & 48.0 & 1.64 & 18.8 & 1.00 \\
\hline Female. . . . . . . . . . . . . . . & 44.0 & 0.94 & 77.7 & 1.29 & 54.9 & 4.01 & 49.4 & 1.57 & 16.5 & 1.07 \\
\hline \multicolumn{11}{|l|}{ Had five or more dinks in a row } \\
\hline Both sexes. . . . . . . . . . . . . . . & 25.6 & 0.58 & 54.5 & 1.07 & 28.8 & 2.80 & 23.4 & 1.00 & 6.8 & 0.51 \\
\hline Male $\ldots \ldots \ldots \ldots \ldots \ldots \ldots$ & 29.1 & 0.78 & 59.5 & 1.42 & 34.6 & 4.92 & 26.7 & 1.49 & 8.2 & 0.76 \\
\hline Female................... & 22.0 & 0.75 & 48.9 & 1.57 & 24.0 & 3.10 & 20.2 & 1.23 & 5.4 & 0.61 \\
\hline \multicolumn{11}{|l|}{ Used marijuana } \\
\hline Both sexes. . . . . . . . . . . . . . . . & 10.7 & 0.39 & 29.1 & 1.04 & 11.7 & 2.13 & 6.8 & 0.56 & 1.1 & 0.18 \\
\hline Male $\ldots \ldots \ldots \ldots \ldots \ldots \ldots$ & 12.2 & 0.59 & 31.1 & 1.49 & 16.4 & 3.97 & 7.8 & 0.84 & 1.6 & 0.30 \\
\hline Female. . . . . . . . . . . . . . & 9.2 & 0.46 & 26.8 & 1.35 & 7.8 & 1.99 & 5.8 & 0.70 & 0.6 & 0.18 \\
\hline \multicolumn{11}{|l|}{ Used cocaine } \\
\hline Both sexes. . . . . . . . . . . . . . . & 1.2 & 0.11 & 3.9 & 0.37 & $* 1.5$ & 0.75 & ${ }^{*} 0.3$ & 0.09 & $" 0.1$ & 0.06 \\
\hline 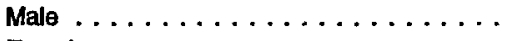 & 1.4 & 0.18 & 4.2 & 0.57 & $* 1.6$ & 1.15 & ${ }^{\star} 0.4$ & 0.15 & ${ }^{\star} 0.2$ & 0.08 \\
\hline Female . . . . . . . . . . . . . . & 1.0 & 0.15 & 3.5 & 0.55 & ${ }^{* 1.5}$ & 1.00 & ${ }^{*} 0.2$ & 0.11 & ${ }^{*} 0.1$ & 0.07 \\
\hline \multicolumn{11}{|l|}{ Used smokeless tobacco } \\
\hline Both sexes. . . . . . . . . . . . . . & 7.5 & 0.37 & 16.1 & 0.90 & 14.2 & $\mathbf{3 . 0 7}$ & 6.2 & 0.51 & 2.1 & 0.25 \\
\hline 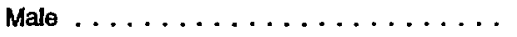 & 13.4 & 0.65 & 27.9 & 1.53 & 29.4 & 5.72 & 11.2 & 0.95 & 3.6 & 0.44 \\
\hline Female $\ldots \ldots \ldots \ldots \ldots \ldots \ldots$ & 1.5 & 0.20 & 2.9 & 0.52 & $" 1.4$ & 0.84 & 1.4 & 0.36 & ${ }^{*} 0.6$ & 0.19 \\
\hline \multicolumn{11}{|l|}{ Carrled a weepon } \\
\hline Both sexes. . . . . . . . . . . . . . & 14.5 & 0.42 & 23.2 & 0.91 & 18.9 & 3.13 & 13.3 & 0.76 & 9.2 & 0.54 \\
\hline 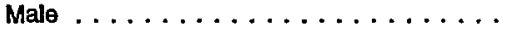 & 23.5 & 0.72 & 35.0 & 1.43 & 29.9 & 5.88 & 21.5 & 1.35 & 16.2 & 0.92 \\
\hline 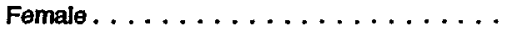 & 5.6 & 0.39 & 10.1 & 0.93 & 9.9 & 2.79 & 5.7 & 0.66 & 2.4 & 0.39 \\
\hline \multicolumn{11}{|l|}{ Engaged in physical fight in past year } \\
\hline Both sexes. . . . . . . . . . . . . . & 38.6 & 0.62 & 48.7 & 1.09 & 42.3 & 2.99 & 36.2 & 1.06 & 33.2 & 0.89 \\
\hline Male ... . . . . . . . . . . . . . & 47.9 & 0.86 & 57.7 & 1.52 & 49.5 & 5.21 & 44.9 & 1.51 & 42.9 & 1.23 \\
\hline 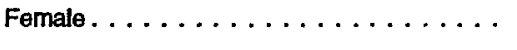 & 29.2 & 0.78 & 38.7 & 1.54 & 36.4 & 3.98 & 28.1 & 1.37 & 23.4 & 1.11 \\
\hline \multicolumn{11}{|l|}{ Ever had sexual intercourse ${ }^{2}$} \\
\hline Both sexes. . . . . . . . . . . . . & 60.8 & 0.77 & 81.7 & 0.98 & 82.4 & 2.70 & 61.8 & 1.28 & 37.9 & 1.29 \\
\hline Male ...................... & 62.7 & 1.02 & 81.8 & 1.34 & $" 80.8$ & 4.12 & 61.8 & 1.86 & 42.5 & 1.65 \\
\hline Fomale. . . . . . . . . . . . . . & 58.7 & 1.07 & 81.6 & 1.41 & $* 84.1$ & 3.20 & 61.9 & 1.76 & 33.2 & 1.77 \\
\hline \multicolumn{11}{|l|}{ Did not always use seat bett } \\
\hline Both sexes. . . . . . . . . . . . . . & 65.8 & 0.69 & 75.5 & 1.05 & 67.2 & 3.13 & 66.9 & 1.13 & 58.4 & 1.00 \\
\hline Male $\ldots \ldots \ldots \ldots \ldots \ldots \ldots \ldots$ & 70.2 & 0.88 & 79.7 & 1.27 & $\pi 7.7$ & 4.21 & 70.4 & 1.47 & 62.9 & 1.33 \\
\hline 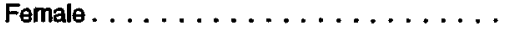 & 61.4 & 0.94 & 70.8 & 1.63 & 58.4 & 4.13 & 63.7 & 1.60 & 53.8 & 1.36 \\
\hline \multicolumn{11}{|l|}{$\begin{array}{l}\text { Exercised vigorously fewer } \\
\text { than } 3 \text { times in past week }\end{array}$} \\
\hline Both sexes. . . . . . . . . . . . . . . & 46.2 & 0.59 & 54.4 & 1.06 & 55.3 & 3.17 & 46.1 & 1.03 & 40.1 & 0.94 \\
\hline 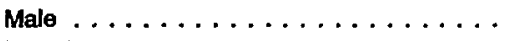 & 39.9 & 0.80 & 50.2 & 1.49 & 55.0 & 5.17 & 37.2 & 1.46 & 33.3 & 1.19 \\
\hline 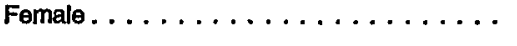 & 52.7 & 0.85 & 59.2 & 1.49 & 55.5 & 4.01 & 54.4 & 1.40 & 46.9 & 1.39 \\
\hline \multicolumn{11}{|l|}{$\begin{array}{c}\text { Ate fewer than flve servings of frults } \\
\text { and vegetables yesterday }\end{array}$} \\
\hline Both sexes. . . . . . . . . . . . . & 87.0 & 0.42 & 89.8 & 0.71 & 91.3 & 1.66 & 89.2 & 0.64 & 83.1 & 0.73 \\
\hline Male . . . . . . . . . . . . . . . & 86.1 & 0.58 & 89.4 & 0.95 & 93.3 & 2.42 & 87.6 & 1.03 & 82.2 & 1.01 \\
\hline Female...................... & 87.9 & 0.57 & 90.3 & 0.93 & 89.7 & 2.25 & 90.8 & 0.77 & 84.0 & 0.95 \\
\hline
\end{tabular}

Treforence poriod is past 30 days unless otherwise specified.

${ }^{2}$ Ages 14-21 years and never married.

3igorous is defined as exercise that made the youth sweat and breathe hard. 
Table 2. Age-adjusted percent of youth ages 12-21 years who engaged in selected unhealthy behaviors by type of behavior, sex, and smoking status: United States, 1992

\begin{tabular}{|c|c|c|c|c|c|c|c|c|c|c|}
\hline \multirow[b]{2}{*}{ Unhealthy behavior and sex } & \multicolumn{2}{|c|}{$\begin{array}{l}\text { All smoking } \\
\text { statuses }\end{array}$} & \multicolumn{2}{|c|}{$\begin{array}{l}\text { Current } \\
\text { smoker }\end{array}$} & \multicolumn{2}{|c|}{$\begin{array}{l}\text { Former } \\
\text { smoker }\end{array}$} & \multicolumn{2}{|c|}{ Experimenter } & \multicolumn{2}{|c|}{$\begin{array}{l}\text { Never } \\
\text { smoker }\end{array}$} \\
\hline & Percent & $\begin{array}{l}\text { Standard } \\
\text { error }\end{array}$ & Percent & $\begin{array}{c}\text { Standard } \\
\text { error }\end{array}$ & Percent & $\begin{array}{l}\text { Standard } \\
\text { error }\end{array}$ & Percent & $\begin{array}{l}\text { Standard } \\
\text { emor }\end{array}$ & Percent & $\begin{array}{l}\text { Standard } \\
\text { emor }\end{array}$ \\
\hline \multicolumn{11}{|l|}{ Drank alcohol } \\
\hline Both sexes. . . . . . . . . . . . . . & 44.3 & 0.61 & 74.4 & 1.11 & 47.0 & 3.92 & 45.4 & 1.01 & 23.0 & 1.02 \\
\hline 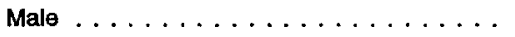 & 45.3 & 0.80 & 73.7 & 1.60 & 50.4 & 6.23 & 45.4 & 1.49 & 25.2 & 1.34 \\
\hline Female . . . . . . . . . . . . . . . & 43.4 & 0.85 & 75.1 & 1.56 & 41.8 & 3.25 & 45.4 & 1.42 & 20.8 & 1.29 \\
\hline \multicolumn{11}{|l|}{ Had flve or more drinks in a row } \\
\hline Both sexes. . . . . . . . . . . . . & 25.4 & 0.53 & 50.3 & 1.22 & 25.5 & 2.93 & 21.3 & 0.88 & 9.5 & 0.69 \\
\hline Male ................ & 29.2 & 0.72 & 53.7 & 1.61 & 31.5 & 4.57 & 24.7 & 1.27 & 12.0 & 1.07 \\
\hline Female ................... & 21.7 & 0.71 & 46.4 & 1.80 & 18.8 & 2.69 & 18.2 & 1.11 & 7.2 & 0.81 \\
\hline \multicolumn{11}{|l|}{ Used marl|uana } \\
\hline Both sexes. . . . . . . . . . . . . . . & 10.7 & 0.38 & 26.5 & 1.02 & 10.3 & 2.19 & 6.2 & 0.52 & 1.5 & 0.25 \\
\hline 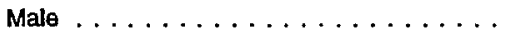 & 12.3 & 0.58 & 28.0 & 1.48 & 15.2 & 3.98 & 7.4 & 0.79 & 2.3 & 0.43 \\
\hline Female. . . . . . . . . . . . . . & 9.1 & 0.45 & 24.7 & 1.34 & 5.4 & 1.30 & 5.2 & 0.62 & $* 0.8$ & 0.25 \\
\hline \multicolumn{11}{|l|}{ Used cocaine } \\
\hline Both sexes. . . . . . . . . . . . . . & 1.2 & 0.11 & 3.5 & 0.46 & *1.1 & 0.55 & $* 0.3$ & 0.09 & ${ }^{*} 0.2$ & 0.10 \\
\hline 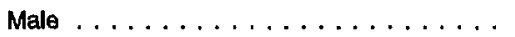 & 1.5 & 0.18 & 3.5 & 0.47 & -1.4 & 0.99 & "0.4 & 0.14 & ${ }^{\infty} 0.3$ & 0.13 \\
\hline Fomale. . . . . . . . . . . . . . & 1.0 & 0.15 & 3.6 & 0.79 & $* 1.0$ & 0.62 & "0.2 & 0.10 & ${ }^{*} 0.2$ & 0.12 \\
\hline \multicolumn{11}{|l|}{ Used smokeless tobacco } \\
\hline Both sexes. . . . . . . . . . . . . & 7.5 & 0.37 & 16.1 & 1.02 & 13.7 & 3.50 & 6.1 & 0.49 & 2.4 & 0.28 \\
\hline Male ................. & 13.5 & 0.65 & 28.1 & 1.76 & 27.5 & 6.48 & 11.1 & 0.92 & 4.1 & 0.52 \\
\hline Female. . . . . . . . . . . . . & 1.5 & 0.21 & 3.0 & 0.60 & 1.5 & 0.92 & 1.5 & 0.36 & -0.6 & 0.20 \\
\hline \multicolumn{11}{|l|}{ Carried a weapon } \\
\hline Both sexes. . . . . . . . . . . . . . . . & 14.5 & 0.41 & 25.6 & 1.12 & 20.2 & 3.42 & 13.8 & 0.78 & 9.5 & 0.59 \\
\hline 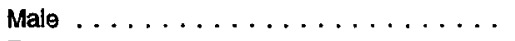 & 23.4 & 0.71 & 39.4 & 1.74 & 30.8 & 6.40 & 22.1 & 1.37 & 16.5 & 1.02 \\
\hline 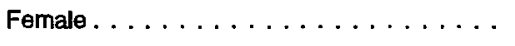 & 5.6 & 0.38 & 11.0 & 1.10 & $* 11.3$ & 4.29 & 5.8 & 0.70 & 2.6 & 0.43 \\
\hline \multicolumn{11}{|l|}{ Engaged in physical fight in past year } \\
\hline Both sexes. . . . . . . . . . . . . & 38.6 & 0.59 & 54.7 & 1.09 & 48.6 & 3.50 & 38.3 & 1.05 & 29.0 & 0.86 \\
\hline Male ... . . . . . . . . . . . & 47.7 & 0.81 & 64.1 & 1.40 & 53.9 & 5.42 & 47.1 & 1.39 & 38.4 & 1.32 \\
\hline 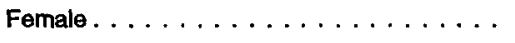 & 29.3 & 0.77 & 44.3 & 1.70 & 41.8 & 5.20 & 29.6 & 1.45 & 19.8 & 0.95 \\
\hline \multicolumn{11}{|l|}{ Ever had sexual intercourse ${ }^{2}$} \\
\hline Both sexes. . . . . . . . . . . . . & 60.8 & 0.72 & 80.0 & 0.99 & 80.4 & 3.27 & 60.6 & 1.13 & 41.4 & 1.40 \\
\hline Male . . . . . . . . . . . . . . & 62.4 & 0.96 & 79.9 & 1.38 & 77.7 & 5.58 & 60.0 & 1.61 & 45.9 & 1.85 \\
\hline 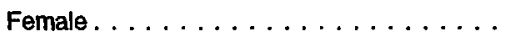 & 59.1 & 1.05 & 80.0 & 1.42 & 82.9 & 3.43 & 61.0 & 1.64 & 36.6 & 1.89 \\
\hline \multicolumn{11}{|l|}{ Did not always use seat belt } \\
\hline Both sexes. . . . . . . . . . . . . . . & 65.8 & 0.68 & 76.6 & 1.03 & 71.6 & 3.05 & 67.7 & 1.11 & 55.7 & 1.11 \\
\hline 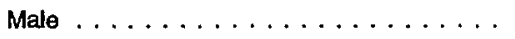 & 70.2 & 0.87 & 80.6 & 1.38 & 79.0 & 4.34 & 70.9 & 1.44 & 60.3 & 1.56 \\
\hline Female . . . . . . . . . . . . . & 61.4 & 0.93 & 72.2 & 1.61 & 66.3 & 3.61 & 64.9 & 1.56 & 51.2 & 1.47 \\
\hline \multicolumn{11}{|l|}{$\begin{array}{l}\text { Exercised vigorously fewer than } \\
3 \text { times in past week }\end{array}$} \\
\hline Both sexes. . . . . . . . . . . . . . & 46.2 & 0.58 & 49.6 & 1.17 & 48.9 & 3.78 & 44.1 & 1.00 & 44.8 & 1.00 \\
\hline 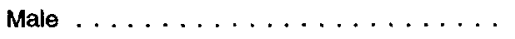 & 40.1 & 0.79 & 45.6 & 1.62 & 51.5 & 6.27 & 36.1 & 1.42 & 37.7 & 1.39 \\
\hline Female . . . . . . . . . . . . . & 52.4 & 0.83 & 54.0 & 1.64 & 45.6 & 4.44 & 51.6 & 1.40 & 51.7 & 1.43 \\
\hline \multicolumn{11}{|l|}{$\begin{array}{c}\text { Ate fewer than the servings of frults and } \\
\text { vegetables yesterday }\end{array}$} \\
\hline Both sexes. . . . . . . . . . . . . . & 87.0 & 0.42 & 89.6 & 0.80 & 91.2 & 1.94 & 89.0 & 0.65 & 83.6 & 0.74 \\
\hline Male $\ldots \ldots \ldots \ldots \ldots \ldots$ & 86.1 & 0.58 & 88.4 & 1.18 & 92.8 & 2.61 & 87.5 & 1.04 & 82.7 & 1.05 \\
\hline Fernale. . . . . . . . . . . . . & 87.9 & 0.57 & 90.7 & 1.00 & 90.1 & 2.91 & 90.5 & 0.82 & 84.5 & 1.01 \\
\hline
\end{tabular}

${ }^{1}$ Peference period is past 30 days unless otherwise specified.

${ }^{2}$ Ages 14-21 years and never married.

3 Vigorous is defined as exercise that made the youth sweat and breathe hard.

NOTE: Total age-adjusted percents may differ slightly from total percents shown in table 1 due to minor variations in item nonresponse among youth in the various smoking status groups. 


\section{Technical notes}

\section{Target population}

The estimates presented in this report are based on data from the 1992 National Health Interview Survey of Youth Risk Behavior (NHIS-YRBS). The National Health Interview Survey (NHIS) is a continuous, nationwide, household interview survey of the civilian noninstitutionalized population of the United States, conducted by the National Center for Health Statistics (NCHS), Centers for Disease Control and Prevention (CDC).

The NHIS-YRBS was a followback survey of a subsample of youth ages 12-21 years who were identified at the time of the 1992 NHIS household interviews. Within each NHIS sample family, one youth attending school and up to two youth not in school or whose in-school status was unknown were randomly selected for the NHIS-YRBS interview. Youth in all NHIS sample families, including emancipated youth (married youth and/or those not living with a parent or guardian), were eligible for selection. The youth were interviewed approximately 2 months after the initial NHIS interview. NHIS-YRBS interviews were conducted in person from April 1992 through March 1993. The ages shown in this report represent the youths' ages at the time of the initial NHIS interview, which may not be the same as their ages at the time of the NHIS-YRBS interview because of the 2-month lag between the two data collection points. The questions on sexual intercourse were asked of all youth who were 14 years old or older by the time of the NHIS-YRBS interview. However, for consistency with other data presented in this report, the data on sexual intercourse shown in tables 1 and 2 are limited to youth who were 14 years old at the time of the initial NHIS household interview.

\section{Jescription of the survey}

The NHIS-YRBS provides estimates of health risk behaviors for the noninstitutionalized household population of youth ages 12-21 years. Topics covered in the YRBS include: tobacco use including cigarettes, chewing tobacco, and snuff; alcohol consumption; illegal drug use and perceptions of risks associated with their use; nutrition, including weight control; physical activity; injury control, including helmet use when riding bicycles and motorcycles; violence, including frequency of physical fighting and carrying weapons; overnight stays away from home and without permission; sexual history and practices; and AIDS education at home and in school. This survey is part of the Youth Risk Behavior Surveillance System (YRBSS). The YRBSS was developed by the Division of Adolescent and School Health of the National Center for Chronic Disease Prevention and Health Promotion, Centers for Disease Control and Prevention (CDC), to monitor the major health risk behaviors of American youth. The surveillance system is described elsewhere (16). The majority of the YRBSS is school-based and has been tracking behaviors of in-school youth since 1990. The 1992 NHISYRBS added a new dimension to the study of health risk behaviors among American youth by providing estimates of risk behaviors for out-of-school youth (17). Out-of-school youth were oversampled in the NHIS-YRBS to achieve reliable estimates for this hard-to-reach group; a special question was added to the basic NHIS questionnaire in 1992 to determine school status for the YRBS sample.

\section{Data collection methods}

Interviews are conducted for NCHS by staff of the U.S. Bureau of the Census. The basic health and demographic questionnaire of the NHIS is administered in a personal interview, with telephone follow-up permitted for hard-to-reach people. Basic health information is collected for every member of the family residing in the household. Based on the roster of family members listed at the time of interview, a subsample of youth was selected for the NHIS-YRBS.

Because the sampling frame was the 1992 NHIS and three-quarters of the data were collected in 1992, for ease of reading, findings from the NHIS-YRBS are referred to as 1992 results although the data were collected from April 1992 through March 1993.

In collaboration with researchers at University of Michigan's Survey

Research Center, extensive methodological testing was conducted during the development of the NHISYRBS to determine the optimal mode of data collection from adolescents. Results indicated that, due to the sensitive nature of the questions on the NHISYRBS, privacy and confidentiality would be of paramount concern to teens during the interview. Youth indicated that they would be more likely to answer questions honestly if the questions could not be heard by others in the household. Further, younger teens and those with less developed reading skills found a written questionnaire to be difficult to complete. For these reasons, questions were asked of teens using a portable audio headset. They recorded their answers on an answer sheet that included only answer categories, not questions. In addition to providing privacy and being easier for less advanced readers, this mode of data collection had the added benefit of providing standardization in asking questions that eliminated the normal variations that occur when an interviewer asks the questions.

\section{Response rates}

Of the 13,789 persons $12-21$ years of age identified as eligible in the basic NHIS interview, NHIS-YRBS interviews were completed for 10,645 youths, representing a response rate of 77.2 percent of eligible respondents and an overall response rate of 73.9 percent (the product of the YRBS response rate (77.2 percent) and the response rate for the basic NHIS household interview (95.7 percent)).

School status was ascertained in two ways. At the time of the initial NHIS questionnaire, 8,062 youth were currently in school or on vacation from school, 1,886 youth were not in school, and the school status of the remaining 697 adolescents was unknown. At the time of the NHIS-YRBS interview, 8,203 were currently in school or on vacation from school, 2,384 were not in 
school, and the school status of the remaining 58 youth was not ascertained. Due to some field difficulties in rostering eligible youth, the number of teenagers selected for the NHIS-YRBS was somewhat smaller than the number of youth ultimately identified as having been eligible for interview. Hence, the response rate for the NHIS-YRBS was somewhat lower than it might have been had all eligible youth been given the opportunity to respond. Another factor that may have contributed to the YRBS nonresponse rate was the requirement that interviews be done in person. Because of the portable audio headset method of data collection, telephone follow-up was not permitted for the NHIS-YRBS. Comparison of respondents and nonrespondents indicated that the two groups were not substantially different in terms of their sociodemographic profiles. Item nonresponse ranged from 0.15 to 7.86 percent for the questions discussed in this report.

\section{Sample design and statistical testing}

The NHIS sample is selected so that a national probability sample of households is interviewed each week throughout the year. A detailed discussion of the sample design is available in Current Estimates from the National Health Interview Survey, 1992 (15). Because the estimates shown in this report are based on a sample, they are subject to sampling error. The standard error is a measure of sampling error. The standard errors shown in tables 1 and 2 of this report were calculated using SUDAAN (SUrvey DAta ANalysis), developed by the Research Triangle Institute for analysis of complex sample surveys. The unadjusted percents were calculated using PROC CROSSTABS. The age-adjusted percents were calculated using PROC DESCRIPT. The entire NHIS-YRBS sample (age groups: 12-13 years, 14-17 years, and 18-21 years) was used as the standard population. For the data on sexual intercourse, age adjustment was limited to the two older groups because the question was asked only of youth 14 years and older. Data for tables 1 and 2 were tabulated using WOR (without replacement) design. All estimates in this report are based on data that have been weighted to represent the U.S. population of youth 12-21 years old. Table I shows the numbers of youth in the total U.S. population and in each of the four smoking status subgroups. This table can be used to estimate numbers of youth engaging in combinations of smoking and other health risk behaviors. Population estimates derived by using table I may be slightly different from those that would be obtained had the exact denominators for each individual variable been provided. However, the differences will be small and of no statistical consequence.

All differences cited in this report are statistically significant at the .05 level. The $t$-test, with a critical value of 1.96 , was used to test all comparisons that are discussed. Lack of comment regarding the difference between any two estimates does not mean that the difference was tested and found not to be statistically significant.

\section{Definition of terms}

Current smokers-Youth who had smoked at least one cigarette in the past 30 days.
Former smokers-Youth who had at one time smoked at least one cigarette per day for 30 days, but had not smoked cigarettes in the past month.

Experimenters-Youth who had smoked at least one or two puffs of a cigarette, but had never smoked cigarettes every day for 30 days and had not used cigarettes in the last 30 days.

Never smokers - Youth who had never had even one or two puffs of a cigarette.

Drank alcohol-Consumed at least one alcoholic drink, including beer, wine, wine coolers, and liquor on at least one of the past 30 days.

Episodic heavy drinkingConsumed at least 5 drinks within a couple of hours in the past month.

Smokeless tobacco-Snuff such as Skoal, Skoal Bandits, or Copenhagen, or chewing tobacco such as Redman, Levi Garrett, or Beechnut.

Marijuana-Marijuana, grass, or pot.

Cocaine-Any form of cocaine, including powder, crack, or freebase.

Carried a weapon-Gun, knife, or club, carried at least once in the past 30 days.

Physical fight-Had been involved in at least one physical fight in the past year.

Vigorous exercise-Exercise that caused sweating and heavy breathing.

Fewer than five servings of fruits and vegetables-Adolescents were asked about their consumption of selected foods the day before the interview and could respond that they had consumed the food once, twice or more, or not at all. The foods were fruit juice, fruit, green salad, and cooked vegetables. The sum of the youth's fruit and vegetable intake was obtained by

Table I. Number of youth by cigarette smoking status and age, United States, 1992

\begin{tabular}{|c|c|c|c|c|c|c|}
\hline \multirow[b]{2}{*}{ Smoking status } & \multicolumn{3}{|c|}{ Ages 12-21 years } & \multicolumn{3}{|c|}{ Ages 14-21 years' } \\
\hline & Total & Males & Females & Total & Males & Fomales \\
\hline & \multicolumn{6}{|c|}{ Number in thousands } \\
\hline All youth ${ }^{2}$. & 33,518 & 16,816 & 16,702 & 23,412 & 12,152 & 11,260 \\
\hline Current smokers $\ldots \ldots \ldots \ldots \ldots \ldots \ldots$ & 9,132 & 4,818 & 4,314 & 7,336 & 4,042 & 3,295 \\
\hline Former smokers $\ldots \ldots \ldots \ldots \ldots \ldots$ & 1,033 & 468 & 565 & 739 & 372 & 367 \\
\hline Experimenters $\ldots \ldots \ldots \ldots \ldots \ldots \ldots$ & 9,835 & 4,759 & 5,076 & 7,478 & 3,734 & 3,744 \\
\hline Never smokers . . . . . . . . . . . . . . . . . & 13,518 & 6,771 & 6,747 & 7,859 & 4,005 & 3,854 \\
\hline
\end{tabular}

${ }^{1}$ Never married and 14-21 years of age at time of NHIS initial household interview.

${ }^{2}$ Excludes youth for whom smoking status is unknown. 
adding 1 for each time the youth said "once" and 2 for each time the youth said " 2 or more." Thus, the prevalence of fruit and vegetable intake is a conservative estimate because youth who had more than 2 servings of a food were counted as having had only 2 servings.

\section{Availability of data and related data sources}

The NHIS-YRBS is available on data tape from the Division of Health Interview Statistics. The NHIS-YRBS public use data tape includes data for all questions included in the youth risk behavior questionnaire as well as all other health and demographic information gathered during the initial household interview. For some youth ages 18-21 years, data from the NHIS-YRBS can be linked to other special topics that were part of the 1992 NHIS, including AIDS Knowledge and Attitudes, Cancer Control, Cancer Epidemiology, and Family Resources. The NHIS-YRBS is also available on CD-ROM from the U.S. Government Printing Office and from the National Technical Information Service. Contact the National Center for Health Statistics' Data Dissemination Branch for ordering information.

\section{Symbols}

- - Data not available

... Category not applicable

- Quantity zero

* Figure does not meet standard of reliability or precision (more than 30-percent relative standard error in numerator of percent or rate)

* Figure does not meet standard of reliability and quantity zero 


\section{Suggested citation}

Willard JC, Schoenborn CA. Relationship between cigarette smoking and other unhealthy behaviors among our Nation's youth: United States, 1992. Advance data from vital and health statistics; no 263. Hyattsville, Maryland: National Center for Health Statistics. 1995.

\section{Copyright information}

All material appearing in this report is in the public domain and may be reproduced or copied without permission; citation as to source, however, is appreciated.
National Center for Health Statistics

$$
\begin{aligned}
& \text { Acting Director } \\
& \text { Jack R. Anderson }
\end{aligned}
$$

Acting Deputy Director Jennifer H. Madans, Ph.D.

\section{U.S. DEPARTMENT OF HEALTH AND HUMAN SERVICES \\ Public Health Service \\ Centers for Disease Control and Prevention \\ National Center for Health Statistics \\ 6525 Belcrest Road \\ Hyattsville, Maryland 20782}

OFFICIAL BUSINESS

PENALTY FOR PRIVATE USE, $\$ 300$

To receive this publication regularly, contact the National Center for Health Statistics by calling 301-436-8500

DHHS Publication No. (PHS) 95-1250

5-0740 (4/95) 


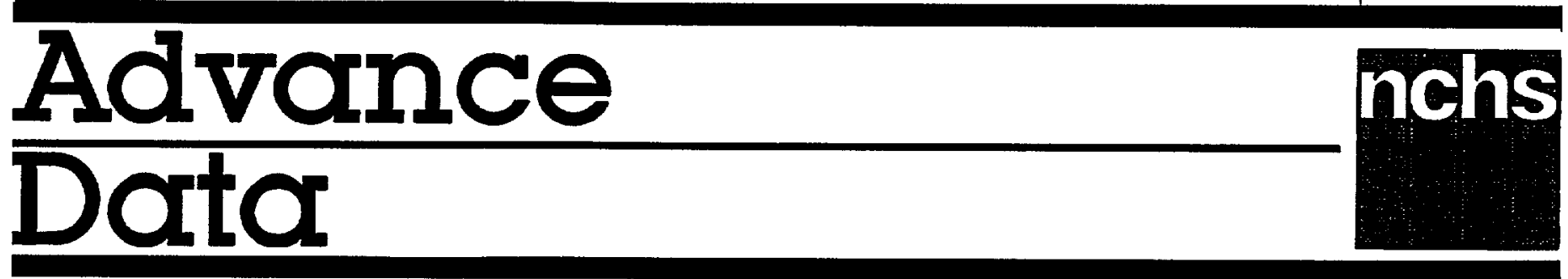

\title{
1993 Summary: National Hospital Discharge Survey
}

\author{
by Edmund J. Graves, Division of Health Care Statistics
}

\section{Introduction}

During 1993, there were an estimated 30.8 million discharges of inpatients, excluding newborn infants, from short-stay non-Federal hospitals in the United States. These discharges accounted for 184.6 million days of inpatient hospital care. The discharge rate was 120.2 per 1,000 population and the average length of stay was 6.0 days.

These and other statistics presented in this report are based on data collected by means of the National Hospital Discharge Survey (NHDS), a continuous survey that has been conducted by the National Center for Health Statistics (NCHS) since 1965. In 1993, data were abstracted from the medical records of approximately 235,000 discharges from 466 short-stay non-Federal hospitals. Beginning in 1988, a new three-stage stratified sample design was put in operation. A brief description of the new design, data collection procedures, estimation process, and definitions of terms used in this report are in the section entitled "Technical notes." A description of the development and design of the original NHDS, which was in operation from 1965-1987, has been published (1). Differences may exist between data for 1988-93 and earlier years because of the redesign of the survey.

Medical data were coded according to the International Classification of Diseases, 9th Revision, Clinical Modification (ICD-9-CM) (2). Up to seven diagnoses and four procedures were coded for each discharge. Although diagnoses included in the ICD-9-CM section entitled "Supplementary classification of external causes of injury and poisoning" (codes E800-E999) were used in the NHDS, these diagnoses are excluded from this report.

Beginning in 1991, all ICD-9-CM procedure codes were used in the NHDS. In previous years, selected codes were excluded. These were primarily codes for certain miscellaneous diagnostic and therapeutic procedures.

Starting in 1985, some hospitals participating in the NHDS have been submitting machine-readable data tapes. In 1993, approximately 32 percent of the hospitals used this method to submit data. More detailed analyses of NHDS data are published in Series 13 of the NCHS Vital and Health Statistics reports.

\section{Data highlights}

\section{Utilization by patient and hospital characteristics}

The number, rate, and average length of stay of discharges from short-stay non-Federal hospitals are shown by age, geographic region, and sex in tables 1-3. Of the 30.8 million discharges from short-stay hospitals during 1993, an estimated 12.3 million were for males and 18.6 million were for females. The discharge rate per 1,000 population for females was 141 , which was 44 percent higher than the rate of 98 for males. The number and rate of discharges were higher for females than for males largely because women $15-44$ years of age were

\section{Acknowledgments}

This report was prepared in the Division of Health Care Statistics. Jean Kozak of the Hospital Care Statistics Branch provided technical assistance in developing the style and content of this report. Elaine Wood of the Hospital Care Statistics Branch verified the data. Maria Owings, also of the Hospital Care Statistics Branch, and George Wolfe of the Technical Services Branch, produced estimated parameters for relative standard error equations. Charles Adams and Malcolm Graham of the Technical Services Branch did the computer programming for the report. This report was edited by Klaudia Cox and typeset by Annette F. Facemire of the Publications Branch, Division of Data Services.

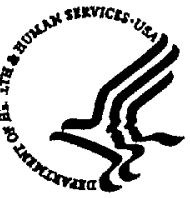

\section{U.S. DEPARTMENT OF HEALTH AND HUMAN SERVICES \\ Public Health Service \\ Centers for Disease Control and Prevention \\ National Center for Health Statistics}

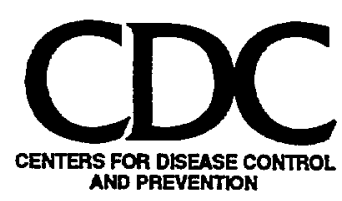


Table 1. Number of discharges from short-stay hospitals by selected characteristics: United State8, 1993

[Dlscharges of inpatients from non-Federal hospltals. Excludes newborn infants]

\begin{tabular}{|c|c|c|c|}
\hline Selected characteristic & $\begin{array}{l}\text { Both } \\
\text { sexes }\end{array}$ & Male & Female \\
\hline & \multicolumn{3}{|c|}{ Number in thousands } \\
\hline Total. . . . . . . . . . . . . . . . & 30,825 & 12,262 & 18,563 \\
\hline \multicolumn{4}{|l|}{ Age } \\
\hline Under 15 years . . . . . . . . . . . . & 2,141 & 1,193 & 948 \\
\hline $15-44$ years. . . . . . . . . . . . & 11,200 & 3,179 & 8,021 \\
\hline $45-64$ years. . . . . . . . . . . . . & 6,283 & 3,143 & 3,141 \\
\hline 65 years and over . . . . . . . . . . & 11,201 & 4,748 & 6,453 \\
\hline \multicolumn{4}{|l|}{ Region } \\
\hline Northeast . . . . . . . . . . . . . . & 6,965 & 2,931 & 4,033 \\
\hline Midwest . . . . . . . . . . . . . & 7,097 & 2,900 & 4,197 \\
\hline South . . . . . . . . . . . . & 11,580 & 4,448 & 7,132 \\
\hline West ..................... & 5,183 & 1,983 & 3,200 \\
\hline
\end{tabular}

Table 2. Rate of discharges from short-stay hospitals by selected characteristics: Unlted States, 1993

[Discharges of inpatients from non-Federal hospitals. Excludes newborn infants]

\begin{tabular}{|c|c|c|c|}
\hline Selected characterlstic & $\begin{array}{c}\text { Both } \\
\text { sexes }\end{array}$ & Male & Female \\
\hline & \multicolumn{3}{|c|}{ Number per 1,000 population } \\
\hline Total. . . . . . . . . & 120.2 & 98.4 & 140.8 \\
\hline \multicolumn{4}{|l|}{ Age } \\
\hline Under 15 years . . . . . . . . . . . . . & 37.7 & 41.1 & 34.2 \\
\hline $15-44$ years. . . . . . . . . . . . & 95.4 & 54.5 & 136.0 \\
\hline $45-64$ years. . . . . . . . . . . . . & 126.8 & 131.5 & 122.5 \\
\hline 65 years and over . . . . . . . . . . & 341.6 & 357.2 & 330.9 \\
\hline \multicolumn{4}{|l|}{ Reglon } \\
\hline Northeast $\ldots \ldots \ldots \ldots \ldots \ldots$ & 135.8 & 118.8 & 151.6 \\
\hline Micwwest $\ldots \ldots \ldots \ldots \ldots \ldots \ldots$ & 116.4 & 97.9 & 133.9 \\
\hline South . . . . . . . . . . . . & 130.6 & 104.0 & 155.5 \\
\hline West ... . . . . . . . . . . . & 93.3 & 72.0 & 114.2 \\
\hline
\end{tabular}

Table 3. Average length of stay for discharges from short-stay hospltals by selected characterlstics: United States, 1993

[Discharges of inpatients from non-Federal hospitals. Excludes newborn Inlants]

\begin{tabular}{|c|c|c|c|}
\hline Selected characteristlc & $\begin{array}{l}\text { Both } \\
\text { sexes }\end{array}$ & Male & Female \\
\hline & \multicolumn{3}{|c|}{ Number of days } \\
\hline$\ldots \ldots \ldots \ldots$ & 6.0 & 6.5 & 5.6 \\
\hline \multicolumn{4}{|l|}{ Age } \\
\hline Under 15 years $\ldots \ldots \ldots \ldots \ldots \ldots \ldots$ & 5.2 & 5.0 & 5.4 \\
\hline $15-44$ years. . . . . . . . . . . & 4.2 & 5.8 & 3.5 \\
\hline $45-64$ years. . . . . . . . . . . . & 6.2 & 6.3 & 6.1 \\
\hline 65 years and over $\ldots \ldots \ldots \ldots \ldots$ & 7.8 & 7.5 & 8.1 \\
\hline \multicolumn{4}{|l|}{ Region } \\
\hline$\ldots \ldots \ldots \ldots$ & 7.0 & 7.4 & 6.7 \\
\hline Mldwest $\ldots \ldots \ldots \ldots \ldots \ldots \ldots$ & 6.1 & 6.5 & 5.8 \\
\hline South . . . . . . . . . . . & 5.7 & 6.3 & 5.4 \\
\hline West $\ldots \ldots \ldots \ldots \ldots \ldots \ldots \ldots$ & 5.1 & 5.8 & 4.6 \\
\hline
\end{tabular}

frequently hospitalized for deliveries and pregnancy-related conditions.

The average length of stay was 6.5 days for males and 5.6 days for females during 1993. The average length of stay of the 4.0 million women who were hospitalized for deliveries was 2.4 days. The average length of stay was 5.2 days for children under 15 years of age, 4.2 days for the 15-44 years age group, 6.2 days for those 45-64 years of age, and 7.8 days for the group 65 years of age and over.

The number of discharges from short-stay hospitals by geographic region during 1993 ranged from 11.6 million in the South to 5.2 million in the West. Regional differences in the number of discharges were accounted for in part by variations in the population sizes. The discharge rates per 1,000 population ranged from 136 in the Northeast region to 93 in the West. Average lengths of stay by geographic region were 5.1 days in the West, 5.7 days in the South, 6.1 days in the Midwest, and 7.0 days in the Northeast.

\section{Utilization by diagnosis}

The number and rates of discharges and average length of stay for each ICD-9-CM diagnostic chapter and selected categories within chapters are shown by sex and age in tables 4-6. In 1993, 5.6 million discharges had a principal or first-listed diagnosis in the ICD-9-CM diagnostic chapter of diseases of the circulatory system. Other leading ICD-9-CM diagnostic chapters were supplementary classifications (including females with deliveries) (4.4 million discharges), diseases of the respiratory system (3.1 million discharges), and diseases of the digestive system (3.1 million discharges). Approximately 53 percent of the discharges from non-Federal short-stay hospitals were included in these four ICD-9-CM diagnostic chapters.

Within the chapters, the common diagnostic categories were deliveries, heart disease, malignant neoplasms, pneumonia, psychoses, and fractures. Excluding deliveries, these last five diagnostic categories were leading 
first-listed diagnoses for both males and females.

Common diagnoses for children under 15 years of age were acute respiratory infections, pneumonia, and asthma. For the age group 15-44 years of age, frequent diagnoses were deliveries, psychoses, and fractures. For those 45-64 years of age and 65 years of age and over, heart disease and malignant neoplasms were major causes of hospitalization. Average lengths of stay ranged from 1.3 days for chronic disease of tonsils and adenoids to 12.0 for psychosis.

\section{Utilization by procedure}

One or more surgical or nonsurgical procedures were performed during an estimated 20.0 million of the 30.8 million hospitalizations in 1993. A total of 41.6 million procedures, or an average of 2.1 per discharge where at least one procedure was performed, were recorded in 1993.

The number and rate of all-listed procedures in 1993 for each ICD-9-CM procedure chapter and selected categories are shown by sex and age in tables 7 and 8. More than three-fourths of all the surgical and nonsurgical procedures performed during 1993 were in 5 of the $16 \mathrm{ICD}-9-\mathrm{CM}$ procedure chapters. These chapters were miscellaneous diagnostic and therapeutic procedures (13.6 million), obstetrical procedures (6.8 million), operations on the digestive system (5.1 million), operations on the cardiovascular system (4.4 million), and operations on the musculoskeletal system (3.2 million).

Within the chapters, frequent procedures for males were arteriography and angiocardiography, cardiac catheterization, diagnostic ultrasound, and computerized axial tomography. Procedures commonly performed on females were episiotomy, fetal EKG and fetal monitoring, cesarean section, and repair of obstetric laceration.

Commonly performed procedures for children under 15 years of age were respiratory therapy and spinal tap; for the age group 15-44 years, episiotomy, fetal EKG and fetal monitoring, and cesarean section; for those 45-64 years of age, arteriography and angiocardiography, cardiac catheterization, and diagnostic ultrasound; and for the group 65 years of age and over, arteriography and angiocardiography, diagnostic ultrasound, and computerized axial tomography.

\section{References}

1. Simmons WR. Development of the design of the NCHS Hospital Discharge Survey. National Center for Health Statistics. Vital Health Stat 2(39). 1970.

2. Public Health Service and Health Care Financing Administration. International Classification of Diseases, 9th Revision, Clinical Modification. Washington: Public Health Service. 3rd ed. 1980.

3. SMG Marketing Group, Inc. Hospital Market Database. Chicago: Healthcare Information Specialists. 1987.

4. SMG Marketing Group, Inc. Hospital Market Database. Chicago: Healthcare Information Specialists. April 1991.

5. Shah BV. SESUDAAN: Standard errors program for computing of standardized rates from sample survey data. Research Triangle Park, North Carolina: Research Triangle Institute. 1981. 
Table 4. Number of discharges from short-stay hospitals, by first-llsted diagnosis, sex, and age: United States, 1993

[Dlscharges of inpatients from non-Federal hospitals. Excludes newborn infants. Diagnostlc groupings and code numbers are based on the International Classificatlon of Diseases, 9th Revision, Clinical Modifications (CD-9-CM)]

\begin{tabular}{|c|c|c|c|c|c|c|c|}
\hline \multirow[b]{2}{*}{ Category of first-listed diagnosis and ICD-9-CM code } & \multirow[b]{2}{*}{ Total } & \multicolumn{2}{|c|}{ Sex } & \multicolumn{4}{|c|}{ Age } \\
\hline & & Male & Female & $\begin{array}{c}\text { Under } 15 \\
\text { years }\end{array}$ & $\begin{array}{l}15-44 \\
\text { years }\end{array}$ & $\begin{array}{l}45-64 \\
\text { years }\end{array}$ & $\begin{array}{l}65 \text { years } \\
\text { and over }\end{array}$ \\
\hline & \multicolumn{7}{|c|}{ Number in thousands } \\
\hline 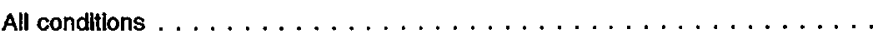 & 30,825 & 12,262 & 18,563 & 2,141 & 11,200 & 6,283 & 11,201 \\
\hline Infectious and parasitic diseases. . . . . . . . . . . . . . . . .001-139 & 797 & 390 & 407 & 168 & 231 & 120 & 278 \\
\hline Septlcemia . . . . . . . . . . . . . . . . . . . . . . . 038 & 270 & 118 & 152 & 18 & 28 & 44 & 180 \\
\hline Neoplasms . . . . . . . . . . . . . . . . . . . . . . . . . . . . . . . . 140-239 & 1,855 & 759 & 1,096 & 39 & 346 & 584 & 885 \\
\hline Malignant neoplasms . . . . . . . . . . . . . . . . . . . . 140-208,230-234 & 1,482 & 690 & 792 & 31 & 180 & 459 & 811 \\
\hline Malignant neoplasm of large Intestine and rectum $\ldots \ldots \ldots$. . . . 153-197.5 & 157 & 73 & 84 & * & $" 7$ & 46 & 103 \\
\hline Mallgnant neoplasm of trachea, bronchus, and lung $\ldots \ldots 162,197.0,197.3$ & 194 & 114 & 80 & $*$ & 7 & 75 & 111 \\
\hline Mallgnant neoplasm of breast . . . . . . . . . . . . . .174-175,198.81 & 168 & $*$ & 167 & $*$ & 36 & 62 & 71 \\
\hline \multicolumn{8}{|l|}{ Benign neoplasms and neoplasms of uncertain } \\
\hline behavlor and unspecifled nature . . . . . . . . . . . . . 210-229,235-239 & 373 & 69 & 304 & ॠB & 166 & 125 & 74 \\
\hline Endocrine, nutritional and metabolic diseases, & & & & & & & \\
\hline and immunity disorders. . . . . . . . . . . . . . . . . . . 240-279 & 1,210 & 480 & 730 & 90 & 256 & 300 & 564 \\
\hline Dlabetes mellitus . . . . . . . . . . . . . . . . . . . 250 & 464 & 212 & 252 & 15 & 120 & 147 & 182 \\
\hline Volume depletion . . . . . . . . . . . . . . . . . . . . . 276.5 & 347 & 129 & 218 & 57 & 50 & 50 & 189 \\
\hline Diseases of the blood and blood-forming organs . . . . . . . . . . 280-289 & 327 & 149 & 178 & 50 & 100 & 63 & 113 \\
\hline Mental dlsorders. . . . . . . . . . . . . . . . . . . . . . . . . . . . . . . 290-319 & 1,827 & 959 & 868 & 75 & 1,089 & 375 & 288 \\
\hline Psychoses . . . . . . . . . . . . . . . . . . . . . . . . . . 290-299 & 1,054 & 500 & 554 & 30 & 564 & 237 & 222 \\
\hline Alcohol dependence syndrome . . . . . . . . . . . . . . 303 & 252 & 193 & 59 & $*$ & 175 & 62 & 13 \\
\hline Diseases of the nervous system and sense organs . . . . . . . . . 320-389 & 681 & 312 & 369 & 95 & 179 & 154 & 252 \\
\hline Dlseases of the central nervous system . . . . . . . . 320-336,340-349 & 278 & 119 & 159 & 29 & 98 & 61 & 90 \\
\hline Diseases of the ear and mastoid process . . . . . . . . . . . . .380-389 & 118 & 59 & 59 & 52 & 17 & 20 & 29 \\
\hline Diseases of the circulatory system. . . . . . . . . . . . . . . . . 390-459 & 5,633 & 2,885 & 2,747 & 25 & 421 & 1,599 & 3,587 \\
\hline Heart dlsease . . . . . . . . . 391-392.0,393-398,402,404,410-416,420-429 & 3,951 & 2,078 & 1,873 & 13 & 242 & 1,167 & 2,529 \\
\hline Acute myocardlal Infarction $\ldots \ldots \ldots \ldots \ldots$ & 745 & 435 & 310 & 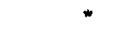 & 47 & 250 & 446 \\
\hline Goronary atherosclerosis. . . . . . . . . . . . . . . 414.0 & 492 & 322 & 170 & 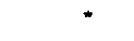 & 25 & 208 & 258 \\
\hline Other ischemic heart dlsease . . . . . . . . . . . . . 411-413,414.1-414.9 & 842 & 447 & 395 & 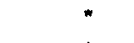 & 45 & 299 & 499 \\
\hline Cardlac dysthythmlas . . . . . . . . . . . . . . . . . . . . . . . . .427 & 549 & 267 & 282 & $\star$ & 42 & 123 & 380 \\
\hline 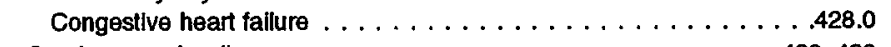 & 875 & 394 & 481 & $*$ & 21 & 169 & 681 \\
\hline Cerebrovascular dlsease . . . . . . . . . . . . . . . . . 430-438 & 841 & 385 & 456 & $*$ & 38 & 172 & 629 \\
\hline Diseases of the respiratory system $\ldots \ldots \ldots \ldots \ldots \ldots \ldots \ldots$. . . . . . . . . . . . . & 3,142 & 1,528 & 1,614 & 667 & 468 & 576 & 1,430 \\
\hline Acute respiratory infections. . . . . . . . . . . . . . . . 460-466 & 400 & 204 & 196 & 222 & 62 & 41 & 75 \\
\hline Chronic disease of tonsils and adenolds . . . . . . . . . . . . . . . .474 & 37 & 17 & 20 & 26 & 9 & $\star$ & - \\
\hline Pneumonia. . . . . . . . . . . . . . . . . . . . . . 480-486 & 1,184 & 598 & 586 & 209 & 142 & 191 & 642 \\
\hline Asthma . . . . . . . . . . . . . . . . . . . . . . . & 468 & 191 & 278 & 159 & 128 & 94 & 87 \\
\hline Diseases of the digestlve system . . . . . . . . . . . . . . . 520-579 & 3,079 & 1,358 & 1,721 & 206 & 878 & 810 & 1,185 \\
\hline Ulcers of the stomach and small Intestine. . . . . . . . . . .531-534 & 216 & 114 & 102 & $*$ & 34 & 61 & 120 \\
\hline Appendicltis . . . . . . . . . . . . . . . . . . . .540-543 & 223 & 131 & 92 & 47 & 131 & 29 & 16 \\
\hline Inguinal hernia. . . . . . . . . . . . . . . . . . . . . . .550 & 83 & 76 & 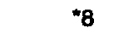 & $" 8$ & 17 & 19 & 40 \\
\hline 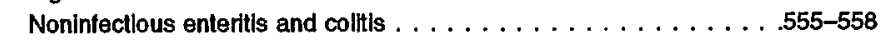 & 350 & 139 & 211 & 87 & 107 & 61 & 95 \\
\hline Cholelithiasis. . . . . . . . . . . . . . . . . . . . . . . 574 & 476 & 134 & 342 & 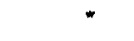 & 168 & 146 & 161 \\
\hline Dlseases of the genitourinary system . . . . . . . . . . . . . . . .580-629 & 1,915 & 663 & 1,252 & 62 & 746 & 446 & 662 \\
\hline Calculus of kidney and ureter . . . . . . . . . . . . . . . . . . . 592 & 225 & 143 & 82 & * & 104 & 84 & 36 \\
\hline 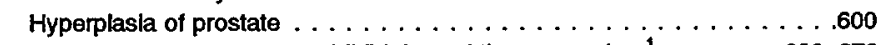 & 185 & 185 & $\ldots$ & * & " & 44 & 140 \\
\hline Complications of pregnancy, childbirth, and the puerperium ${ }^{1} \ldots \ldots$. . .630-676 & 594 & $\ldots$ & 594 & $*$ & 592 & * & $\cdots$ \\
\hline Abortions and ectoplc and molar pregnancies . . . . . . . . . .630-639 & 133 & $\ldots$ & 133 & $*$ & 132 & $*$ & $\cdots$ \\
\hline Diseases of the skin and subcutaneous tlssue . . . . . . . . . . . .680-709 & 451 & 214 & 237 & 37 & 129 & 105 & 180 \\
\hline Mellulitis and abscess . . . . . . . . . . . . . . . . . . . . . . . . .681-682 & 304 & 148 & 155 & 23 & 94 & 76 & 111 \\
\hline Diseases of the musculoskeletal system and connective tissue $\ldots . .710-739$ & 1,561 & 687 & 874 & 37 & 481 & 433 & 611 \\
\hline Arthropathies and related disorders. . . . . . . . . . . .710-719 & 541 & 215 & 325 & 11 & 111 & 114 & 305 \\
\hline Intervertebral disc disorders . . . . . . . . . . . . . . . . . . .722 & 391 & 219 & 172 & * & 206 & 131 & 55 \\
\hline Congenital anomalies. . . . . . . . . . . . . . . . . .740-759 & 150 & 82 & 69 & 105 & 28 & 10 & $" 8$ \\
\hline Certain conditions orlginating in the perinatal period. . . . . . . .760-779 & 139 & 81 & 58 & 134 & $*$ & $*$ & 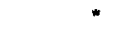 \\
\hline Symploms, signs, and ill-defined conditions . . . . . . . . . . .780-799 & 327 & 153 & 174 & 53 & 134 & 87 & 53 \\
\hline 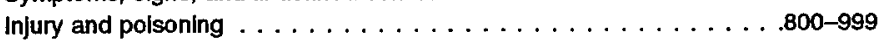 & 2,718 & 1,395 & 1,323 & 238 & 1,007 & 515 & 959 \\
\hline Fractures, all sites . . . . . . . . . . . . . . . . . . . . . . 800-829 & 1,017 & 440 & 577 & 77 & 303 & 144 & 494 \\
\hline Fracture of neck of temur $\ldots \ldots \ldots \ldots \ldots \ldots \ldots$ & 307 & 72 & 235 & $*$ & $" B$ & 20 & 276 \\
\hline Intracranlal injuries (excluding those with skull fracture) $\ldots . . . . .850-854$ & 160 & 102 & 58 & 30 & 72 & 23 & 35 \\
\hline Lacerations and open wounds. . . . . . . . . . . . . . . . . . . . 870-904 & 171 & 129 & 42 & 20 & 107 & 25 & 20 \\
\hline Supplementary classifications. . . . . . . . . . . . . . . . . V01-V82 & 4,419 & 168 & 4,251 & 57 & 4,110 & 105 & 147 \\
\hline Females with delveries $\ldots \ldots \ldots \ldots \ldots \ldots \ldots \ldots$ & 4,015 & $\ldots$ & 4,015 & 11 & 4,001 & $\star$ & $\cdots$ \\
\hline
\end{tabular}

"The first-listed diagnoses for females with deliveries is coded V27, shown under "Supplementary classifications." 
Table 5. Rate of discharges from short-8tay hospitals, by first-listed diagnosis, sex, and age: United States, 1993

[Discharges of inpatients from non-Federal hospitals. Excludes newborn infants. Dlagnostic groupings and code numbers are based on the Imternational Classiffcation of Diseases, gth Revision, Clinical Modffications (ICD-9-CM)]

\begin{tabular}{|c|c|c|c|c|c|c|c|}
\hline \multirow[b]{2}{*}{ Category of first-listed dlagnosis and ICD-9-CM code } & \multirow[b]{2}{*}{ Total } & \multicolumn{2}{|c|}{ Sex } & \multicolumn{4}{|c|}{ Age } \\
\hline & & Male & Female & $\begin{array}{l}\text { Under } 15 \\
\text { years }\end{array}$ & $\begin{array}{l}15-44 \\
\text { years }\end{array}$ & $\begin{array}{l}45-64 \\
\text { years }\end{array}$ & $\begin{array}{l}65 \text { years } \\
\text { and over }\end{array}$ \\
\hline
\end{tabular}

All conditions $\ldots \ldots \ldots \ldots$

Infectlous and parasitic diseases. ................. 001-139

Septicemla . . . . . . . . . . . . . . . . . . . . . . . . 038

Neoplasms . . . . . . . . . . . . . . . . . 140-239

Mallgnant neoplasms . . . . . . . . . . . . . . . . . . . 140-208,230-234

Malgnant neoplasm of large Intestine and rectum . . . . . 153-154,197.5

Mallgnant neoplasm of trachea, bronchus, and lung . . . . 162,197.0,197.3

Malignant neoplasm of breast . . . . . . . . . . . . . . 174-175,198.81 Benign neoplasms and neoplasms of uncertain

behavior and unspecified nature . . . . . . . . . . . . . 210-229,235-239

Endocrine, nutritonal and metabollc diseases,

and immunty disorders. . . . . . . . . . . . . . . . . . . . . 240-279

Dlabetes mellitus . . . . . . . . . . . . . . . . . . . . . 250

Volume depletion . . . . . . . . . . . . . . . . . . . . . . . . 276.5

Diseases of the blood and blood-forming organs . . . . . . . . . . 280-289

Mental dlsorders. . . . . . . . . . . . . . . . . . . . . . . . . .290-319

Psychoses . . . . . . . . . . . . . . . . . . . . . . . . . . . 290-299

Alcohol dependence syndrome . . . . . . . . . . . . . . . . . 303

Diseases of the nervous system and sense organs . . . . . . . . . . .320-389

Dlseases of the central nervous system . . . . . . . . . . . .320-336,340-349

Diseases of the ear and mastold process . . . . . . . . . . . . . . .380-389

Diseases of the clrculatory system. . . . . . . . . . . . . . . . . . . . .390-459

Heart disease . . . . . . . . 391-392.0,393-398,402,404,410-416,420-429

Acute myocardial infarction . . . . . . . . . . . . . . . . . . .410

Coronary atherosclerosis. . . . . . . . . . . . . . . . . . . 414.0

Other ischemic heart disease . . . . . . . . . . . . .411-413,414.1-414.9

Cardlac dysrhythmlas . . . . . . . . . . . . . . . . . . . . . .427

Congestlve heart fallure . . . . . . . . . . . . . . . . .428.0

Cerebrovascular disease . . . . . . . . . . . . . . . . . . . . 430-438

Dkseases of the respiralony system . . . . . . . . . . . . . . . . 460-519

Acute respiratory infections. . . . . . . . . . . . . . . . . . . . 460-466

Chronic disease of tonslls and adenoids. . . . . . . . . . . . . . . . 474

Pneumonia. . . . . . . . . . . . . . . . . . . . . . . . . . 480-486

Asthma ... . . . . . . . . . . . . . . . . . . . . . . .493

Diseases of the digestlve system . . . . . . . . . . . . . . . . . . . .520-579

Ulcers of the stomach and small intestine. . . . . . . . . . . . . . 531-534

Appondlcitls . . . . . . . . . . . . . . . . . . 540-543

Inguinal hemla. . . . . . . . . . . . . . . . . . . . . . . . . . . . . .550

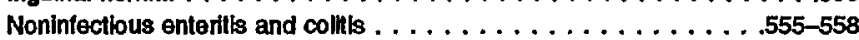

Cholellthlasis. . . . . . . . . . . . . . . . . . . . . . . . . . . . . . . . . .574

Dkseases of the genltourinary system. . . . . . . . . . . . . . .580-629

Calculus of kidney and ureter . . . . . . . . . . . . . . . . . . . . 592

Hyperplasla of prostate . . . . . . . . . . . . . . . . . .600

Compllcations of pregnancy, chllobirth, and the pueperium' . . . . . . . 630-676

Abottions and ectoplc and molar pregnancies . . . . . . . . . . . 630-639

Diseases of the skin and subcutaneous tlssue . . . . . . . . . . . . .680-709

Cellultis and abscess . . . . . . . . . . . . . . . . . . . . . . . . 681-682

Diseases of the musculoskeletal system and connectlve tissue . . . . . 710-739

Arthropathles and related disorders. . . . . . . . . . . . . . .710-719

Intervertebral disc dlsorders . . . . . . . . . . . . . . . . . . .722

Congentlal anomalles. . . . . . . . . . . . . . . . . . . . . . . .740-759

Certain conditions ortginating in the perinatal period. . . . . . . . . . 760-779

Symptoms, signs, and ill-defined condlilons . . . . . . . . . . . . . 780-799

Injury and poisoning . . . . . . . . . . . . . . . . . . . . . 800-999

Fractures, all stes . . . . . . . . . . . . . . . . . . . . . . 800-829

Fracture of neck of femur . . . . . . . . . . . . . . . . . . . 820 Intracranial injuries (excluding those wilh skull fracture) . . . . . . . 850-854 Lacerations and open wounds. . . . . . . . . . . . . . . . . . . 870-904

Supplementary classlfications. . . . . . . . . . . . . . . . . . V01-V82

Females with delverles . . . . . . . . . . . . . . . . . . V27
$1,202.1$

31.1

10.5

72.3

57.8

6.1

7.6

6.6

14.5

47.2

18.1

13.5

12.7

71.3

41.1

9.8

26.5

10.8

4.6

219.6

154.1

29.0

19.2

32.8

21.4

34.1

32.8

122.5

15.6

1.4

46.2

18.3

120.1

8.4

8.7

3.3

13.7

18.6

74.7

8.8

7.2

23.2

5.2

17.6

11.8

60.9

21.1

15.3

5.9

5.4

12.7

106.0

39.7

12.0

62

6.7

172.3

156.6

Number per 10,000 population

\section{2}

31.3

9.5

60.9

55.3

5.9

9.2

5.5

38.5

17.0

10.4

11.9

77.0

40.1

15.5

25.0

9.6

4.7

231.6

166.8

34.9

25.9

35.9

21.5

31.6

30.9

122.6

16.4

1.3

48.0

15.3

109.0

9.1

10.5

6.1

11.1

10.8

53.2

11.5

14.8

...

…

17.2

11.9

55.1

17.3

17.6

6.5

6.5

12.3

112.0

35.3

5.8

8.2

10.4

13.5

...
$1,407.9$

30.9

11.5

83.2

60.1

6.4

6.1

12.7

23.1

\section{4}

19.1

16.5

13.5

65.9

42.0

4.5

28.0

12.1

4.5

208.4

142.1

23.5

12.9

29.9

21.4

36.5

34.6

122.4

14.9

1.5

44.5

21.1

130.5

7.7

7.0

"0.6

16.0

25.9

95.0

6.2

...

45.1

10.1

18.0

11.8

66.3

24.7

13.0

5.2

4.4

13.2
100.4

43.8

17.8

4.4

3.2

304.5

$\begin{array}{rr}377.3 & 945.5 \\ 29.6 & 19.7 \\ 3.2 & 2.4 \\ 7.0 & 29.5 \\ 5.5 & 15.4 \\ * & * 0.6 \\ * & * 0.6 \\ * & 3.0\end{array}$

$1,268.1$

8.9

117.8

92.6

9.3

12.4

$\star 1.4$

14.2

25.2

15.9

21.8

2.6
10.0

8.9

13.2

5.3

16.8

16.8
5.2

9.1

4.4

2.4

2.

"

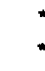

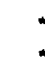

117.6

39.2

4.5

36.9

28.0

36.3

8.3

$\star 1.4$

15.3

10.9

*

:

.

6.5

4.1

6.5

1.9

18.5

23.6

9.3

41.9 
Table 6. Average length of stay for dlscharges from short-stay hospltals, by first-listed diagnosis, sex, and age: United States, 1993 [Discharges of Inpatients from non-Federal hospitals. Excludes newborn Infants. Dlagnostlc groupings and cade numbers are based on the International Classfflcation of Diseases, 9th Revision, Clinical Modifications (ICD-9-CM)]

\begin{tabular}{|c|c|c|c|c|c|c|c|}
\hline \multirow[b]{2}{*}{ Category of first-llsted diagnosis and ICD-9-CM code } & \multirow[b]{2}{*}{ Total } & \multicolumn{2}{|c|}{ Sex } & \multicolumn{4}{|c|}{ Age } \\
\hline & & Male & Female & $\begin{array}{c}\text { Under } 15 \\
\text { years }\end{array}$ & $\begin{array}{l}15-44 \\
\text { years }\end{array}$ & $\begin{array}{l}45-64 \\
\text { years }\end{array}$ & $\begin{array}{l}65 \text { years } \\
\text { and over }\end{array}$ \\
\hline & \multicolumn{7}{|c|}{ Number of days } \\
\hline$\ldots \ldots \ldots \ldots \ldots \ldots \ldots$ & 6.0 & 6.5 & 5.6 & 5.2 & 4.2 & 6.2 & 7.8 \\
\hline Infectious and parasitic diseases. . . . . . . . . . . . . . . .001-139 & 7.9 & 8.4 & 7.5 & 4.4 & 7.7 & 9.5 & 9.6 \\
\hline Septicemia . . . . . . . . . . . . . . . . . . . . . . . . . .038 & 10.6 & 10.9 & 10.3 & 7.7 & 11.7 & 12.1 & 10.3 \\
\hline Neoplasms . . . . . . . . . . . . . . . . . . . . . . . . . . 140-239 & 7.4 & 8.3 & 6.7 & 7.2 & 4.8 & 7.3 & 8.4 \\
\hline Malignant neoplasms . . . . . . . . . . . . . . . . . 140-208,230-234 & 8.1 & 8.5 & 7.7 & 7.0 & 6.1 & 8.0 & 8.6 \\
\hline Mallgnant neoplasm of large Intestine and rectum . . . . 153-154,197.5 & 11.0 & 10.5 & 11.4 & * & $" 9.3$ & 9.8 & 11.7 \\
\hline Mallgnant neoplasm of trachea, bronchus, and lung $\ldots \ldots .162,197.0,197.3$ & 8.7 & 8.4 & 9.0 & * & "6.5 & 8.6 & 8.9 \\
\hline Mallgnant neoplasm of breast . . . . . . . . . . . . 174-175,198.81 & 3.7 & * & 3.7 & * & 3.2 & 3.9 & 3.8 \\
\hline Benign neoplasms and neoplasms of uncertain & & & & & & & \\
\hline behavior and unspecifled nature $\ldots \ldots \ldots \ldots \ldots \ldots .210-229,235-239$ & 4.5 & 5.9 & 4.1 & ${ }^{* 8.0}$ & 3.5 & 4.5 & 6.1 \\
\hline Endocrine, nutrtional and metabollc diseases, & & & & & & & \\
\hline and immunity dlsorders. . . . . . . . . . . . . . . . . . . . 240-279 & 6.6 & 6.4 & 6.7 & 4.1 & 5.2 & 6.1 & 7.8 \\
\hline Dlabetes mellitus . . . . . . . . . . . . . . . . . . . . .250 & 7.5 & 7.2 & 7.7 & 4.5 & 5.0 & 7.3 & 9.6 \\
\hline Volume depletion . . . . . . . . . . . . . . . . . . . . . 276.5 & 5.9 & 5.5 & 6.2 & 2.7 & 6.2 & 5.2 & 7.0 \\
\hline Diseases of the blood and blood-forming organs $\ldots \ldots \ldots \ldots \ldots .280-289$ & 5.8 & 5.7 & 5.9 & 4.5 & 5.3 & 5.8 & 6.8 \\
\hline Mental disorders. . . . . . . . . . . . . . . . . . 290-319 & 10.3 & 9.8 & 10.8 & 14.5 & 9.3 & 10.5 & 12.5 \\
\hline Psychoses . . . . . . . . . . . . . . . . . . . . . . . . . . 290-299 & 12.0 & 11.6 & 12.3 & 16.1 & 10.9 & 12.4 & 13.7 \\
\hline Alcohol dependence syndrome . . . . . . . . . . . . . . 303 & 8.5 & 8.2 & 9.3 & $*$ & 8.4 & 8.5 & 9.2 \\
\hline Diseases of the nervous system and sense organs $\ldots \ldots \ldots \ldots \ldots .320-389$ & 5.4 & 5.7 & 5.3 & 4.2 & 5.1 & 5.2 & 6.3 \\
\hline Dlseases of the central nervous system . . . . . . . . . .320-336,340-349 & 8.5 & 9.1 & 8.1 & 6.2 & 6.7 & 8.1 & 11.5 \\
\hline Dlseases of the ear and mastoid process $\ldots \ldots \ldots \ldots \ldots \ldots .380-389$ & 3.1 & 2.5 & 3.7 & 3.4 & 2.0 & 2.9 & 3.5 \\
\hline Diseases of the circulatory system. . . . . . . . . . . . . . . . . .390-459 & 6.7 & 6.3 & 7.0 & 5.2 & 5.3 & 5.9 & 7.2 \\
\hline Heart disease $\ldots \ldots \ldots \ldots . .391-392.0,393-398,402,404,410-416,420-429$ & 6.3 & 6.0 & 6.6 & 5.6 & 4.7 & 5.5 & 6.8 \\
\hline Acute myocardlal infarction $\ldots \ldots \ldots \ldots \ldots \ldots \ldots \ldots . \ldots \ldots$ & 7.4 & 7.0 & 7.8 & $*$ & 5.5 & 6.6 & 8.0 \\
\hline Coronary atherosclerosis. . . . . . . . . . . . . . 414.0 & 6.0 & 5.6 & 6.7 & * & 4.1 & 5.2 & 6.9 \\
\hline Other ischemic heart disease $\ldots \ldots \ldots \ldots \ldots$. . . . .11-413,414.1-414.9 & 4.5 & 4.5 & 4.6 & * & 3.2 & 4.1 & 4.9 \\
\hline Cardlac dysrhythmlas . . . . . . . . . . . . . . . . . .427 & 4.8 & 5.0 & 4.7 & * & 2.7 & 4.4 & 5.2 \\
\hline Congestive heart fallure . . . . . . . . . . . . . . . . .428.0 & 7.5 & 7.0 & 7.9 & * & 6.2 & 6.5 & 7.8 \\
\hline Cerebrovascular disease . . . . . . . . . . . . . . . .430-438 & 8.4 & 8.1 & 8.7 & * & 9.0 & 8.3 & 8.4 \\
\hline Diseases of the respiratory system . . . . . . . . . . . . .460-519 & 6.7 & 6.5 & 7.0 & 3.8 & 4.9 & 6.9 & 8.6 \\
\hline Acute respiratory infections. . . . . . . . . . . . . . . .460-466 & 4.0 & 3.6 & 4.4 & 3.2 & 3.6 & 4.4 & 6.4 \\
\hline Chronlc disease of tonsils and adenoids. . . . . . . . . . . .474 & 1.3 & 1.2 & 1.3 & 1.3 & 1.2 & $*$ & - \\
\hline neumonia. . . . . . . . . . . . . . . . . . . 480-486 & 7.8 & 7.6 & 8.0 & 4.8 & 6.6 & 7.4 & 9.2 \\
\hline Asthma . . . . . . . . . . . . . . . . . . . . . . . . . .493 & 4.4 & 3.8 & 4.9 & 3.4 & 3.5 & 5.4 & 6.7 \\
\hline Diseases of the digestlve system . . . . . . . . . . . . . . . . .520-579 & 5.7 & 5.6 & 5.7 & 4.0 & 4.4 & 5.4 & 7.1 \\
\hline Ulcers of the stomach and small intestine. . . . . . . . . .531-534 & 6.7 & 6.5 & 6.8 & * & 4.9 & 6.1 & 7.5 \\
\hline Appendicttls . . . . . . . . . . . . . . . . . . . . . . . 540-543 & 4.5 & 4.6 & 4.3 & 5.0 & 3.6 & 5.1 & 8.7 \\
\hline 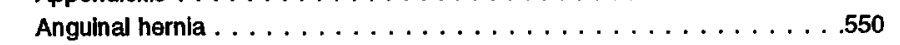 & 2.7 & 2.6 & *4.6 & "2.5 & 1.6 & 1.9 & 3.7 \\
\hline 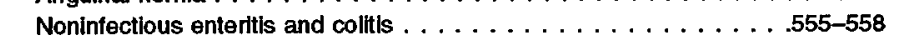 & 4.8 & 4.6 & 5.0 & 2.5 & 4.0 & 5.5 & 7.6 \\
\hline Cholellthiasis. . . . . . . . . . . . . . . . . . . . . . 574 & 4.2 & 4.6 & 4.1 & $*$ & 3.3 & 3.2 & 6.1 \\
\hline Dlseases of the genitourinary system . . . . . . . . . . . . . .580-629 & 4.5 & 4.7 & 4.4 & 4.1 & 3.4 & 4.0 & 6.1 \\
\hline Calculus of kidney and ureter . . . . . . . . . . . . . . .592 & 2.9 & 2.8 & 3.2 & $*$ & 2.8 & 2.6 & 4.0 \\
\hline Hyperplasta of prostate $\ldots \ldots \ldots \ldots \ldots \ldots \ldots \ldots \ldots$ & 3.8 & 3.8 & $\ldots$ & * & $*$ & 3.3 & 3.9 \\
\hline Complications of pregnancy, childbirth, and the puerperlum ${ }^{4} \ldots \ldots$. . .630-676 & 2.6 & $\ldots$ & 2.6 & * & 2.6 & * & $\ldots$ \\
\hline Abortlons and ectopic and molar pregnancles . . . . . . . . .630-639 & 2.0 & $\ldots$ & 2.0 & " & 2.0 & $*$ & $\ldots$ \\
\hline Diseases of the skin and subcutaneous tlssue . . . . . . . . . .680-709 & 7.6 & 7.9 & 7.3 & 3.9 & 6.5 & 7.5 & 9.2 \\
\hline Cellulitls and abscess . . . . . . . . . . . . . . . .681-682 & 6.6 & 6.7 & 6.4 & 3.8 & 5.8 & 6.8 & 7.7 \\
\hline Diseases of the musculoskeletal system and connective tissue . . . . .710-739 & 5.8 & 5.1 & 6.4 & 4.7 & 3.7 & 4.8 & 8.2 \\
\hline Arthropathies and related dlsorders. . . . . . . . . . . . .710-719 & 6.8 & 6.0 & 7.4 & 4.2 & 3.3 & 6.1 & 8.5 \\
\hline Intervertebral dlsc disorders $\ldots \ldots \ldots \ldots \ldots \ldots \ldots \ldots . . \ldots 22$ & 4.0 & 3.6 & 4.5 & * & 3.5 & 3.9 & 5.9 \\
\hline Congenital anomalles. . . . . . . . . . . . . . . . .740-759 & 6.7 & 6.1 & 7.3 & 7.2 & 4.8 & 4.8 & ${ }^{*} 8.0$ \\
\hline Certain conditions originating in the perinatal period. . . . . . . .760-779 & 11.3 & 11.3 & 11.3 & 11.5 & $\cdot$ & * & $*$ \\
\hline Symptoms, signs, and ill-defined conditions . . . . . . . . . . .780-799 & 2.8 & 2.7 & 2.8 & 3.1 & 2.4 & 2.3 & 4.1 \\
\hline Injury and polsoning $\ldots \ldots \ldots \ldots \ldots \ldots \ldots \ldots \ldots$. . . . . . . . . . . . . & 6.4 & 5.8 & 6.9 & 4.6 & 4.8 & 6.3 & 8.5 \\
\hline Fractures, all sttes . . . . . . . . . . . . . . . . . 800-829 & 7.5 & 6.7 & 8.0 & 4.3 & 5.6 & 6.4 & 9.4 \\
\hline Fracture of neck of femur $\ldots \ldots \ldots \ldots \ldots \ldots \ldots \ldots \ldots$ & 10.3 & 10.5 & 10.2 & * & $* 10.1$ & 10.4 & 10.3 \\
\hline Intracranial Injurles (excluding those with skull fracture) $\ldots \ldots \ldots .850-854$ & 7.3 & 7.1 & 7.5 & 2.9 & 7.5 & 7.5 & 10.5 \\
\hline Lacerations and open wounds. . . . . . . . . . . . . . . . . . .870-904 & 3.7 & 3.5 & 4.2 & 3.3 & 3.0 & 4.5 & 6.3 \\
\hline Supplementary classlifications. . . . . . . . . . . . . . . v01-V82 & 2.9 & 8.3 & 2.7 & 6.1 & 2.5 & 6.0 & 11.9 \\
\hline Females with delveries $\ldots \ldots \ldots \ldots \ldots \ldots \ldots \ldots \ldots$. . . . . . . . . . & 2.4 & $\ldots$ & 2.4 & 2.6 & 2.4 & $*$ & $\cdots$ \\
\hline
\end{tabular}

\footnotetext{
"The first-listod diagnoses for fomales with doliveries is coded V27, shown undor "Supplementary classifications."
} 
Table 7. Number of all-listed procedures for discharges from short-stay hospltals, by procedure category, sex, and age: United States, 1993

[Discharges of inpatients from non-Federal hospitals. Excludes newborn Infants. Procedure groupings and code numbers are based on the International Classification of Diseases, 9th Revision, Clinical Modfications (ICD-9-CM)]

\begin{tabular}{|c|c|c|c|c|c|c|c|}
\hline \multirow[b]{2}{*}{ Procedure category and $1 C D-9-C M$ code } & \multirow[b]{2}{*}{ Total } & \multicolumn{2}{|c|}{ Sex } & \multicolumn{4}{|c|}{ Age } \\
\hline & & Male & Female & $\begin{array}{l}\text { Under } 15 \\
\text { years }\end{array}$ & $\begin{array}{l}15-44 \\
\text { years }\end{array}$ & $\begin{array}{l}45-64 \\
\text { years }\end{array}$ & $\begin{array}{l}65 \text { years } \\
\text { and over }\end{array}$ \\
\hline
\end{tabular}

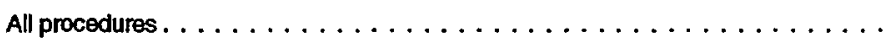

Operations on the nervous system . . . . . . . . . . . . . . . . . .

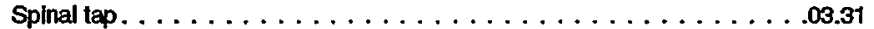

Operations on the endocrine system. . . . . . . . . . . . .06-07

Operations on the eye .................. .

Operations on the ear. . . . . . . . . . . . . . . . . . 18-20

Operations on the nose, mouth, and pharynx. . . . . . . . . 21-29

Tonsillectomy with or whout adenoldectomy . . . . . . . .28.2-28.3

Operations on the respiralory system . . . . . . . . . . . .30-34

Bronchoscopy with or without biopsy . . . . . . . . . . . .33.21-33.24,33.27

Operations on the cardlovascular system . . . . . . . . . . . .35-39

Removal of coronary artery obstructon . . . . . . . . . . . . .

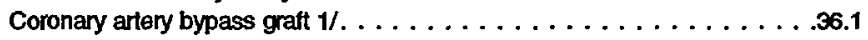

Cardlac catheterization . . . . . . . . . . . . . . .37.21-37.23

Insertion, replacement, removal, and revision

of pacomaker teads or device. . . . . . . . . . . . . . . .37.7-37.8

Shunt or vascular bypass . . . . . . . . . . . . . . 39.0-39.2

Hemodlalysis. . . . . . . . . . . . . . . . . . . . 39.95

Operations on the hemic and hymphatic system . . . . . . . 40-41

Operations on the digestive system. . . . . . . . . . . . . . . .42-54

Endoscopy of small Intestine with or without blopsy . . . . . 45.11-45.14,45.16

Endoscopy of large intestine with or without blopsy . . . . . . . 45.21-45.25

Partial exclston of large intestine $\ldots \ldots \ldots \ldots \ldots \ldots \ldots \ldots .45 .7$

Appendectomy, excluding incldental . . . . . . . . . . . .47.0

Cholecystectomy . . . . . . . . . . . . . . . . .51.2

Repair of Inguinal hemia . . . . . . . . . . . . . . . .53.0-53.1

Lysis of peritoneal achesions . . . . . . . . . . . . . . . . .54.5

Operations on the urinary system. . . . . . . . . . . . . . . .55-59

Cystoscopy with or whthout blopsy . . . . . . . . . . . 57.31-57.33

Operations on the male genltal organs. . . . . . . . . . . . . . . .60-64

Prostatectomy. . . . . . . . . . . . . . . . . .60.2-60.6

Operations on the female genttal organs. . . . . . . . . . 65-71

Oophorectomy and sapingo-oophorectomy . . . . . . . . .65.3-65.6

Bllateral destruction or occluston of falloptan tubes . . . . . . . . .66.2-66.3

Hysterectormy . . . . . . . . . . . . . . . . . . . . . . .68.3-68.7,68.9

Dilation and curettage of uterus. . . . . . . . . .

Pepalr of cystocele and rectocele $\ldots \ldots \ldots \ldots \ldots \ldots \ldots \ldots .70 .5$

Obstetrical procedures . . . . . . . . . . . . . . . .72-75

Eplstotomy with or without forceps or

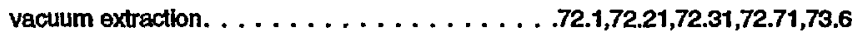

Artifictal rupture of membranes . . . . . . . . . . . . . . . . . . .73.0

Cesarean section . . . . . . . . . . . . . . .74.0-74.2,74.4,74.99

Fetal EKG (scalp) and fetal monltoring, not otherwise specifted . . .75.32,75.34

Repair of current obstetric laceration . . . . . . . . . . . 75.5-75.6

Operations on the musculoskeletal system . . . . . . . . . . . .76-84

Partlal exctston of bone . . . . . . . . . . . .76.2-76.3,77.6-77.8

Open reduction of fracture with internal flxation $\ldots \ldots \ldots \ldots \ldots \ldots .79 .3$

Exciston or destruction of intervertebral disc . . . . . . . . . .80.5

Total hip replacement . . . . . . . . . . . . . . . . . . . .81.51

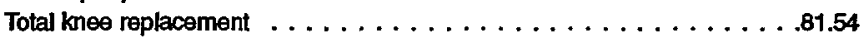

Operatlons on the Integumentary system $\ldots \ldots \ldots \ldots \ldots \ldots \ldots . .85-86$

Mastectomy . . . . . . . . . . . . . . . . . . . . .85.4

Debridement of wound, infection, or burn . . . . . . . . . .86.22,86.28

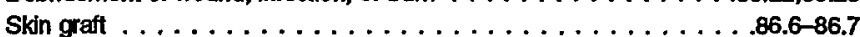

Miscellaneous dlagnostic and therapeutlc procedures. . . . . . . . .87-99

Computerized axial tomography. . . . . . . . . .87.03,87.41,87.71,88.01,88.38

Pyelogram . . . . . . . . . . . . . . . . . . . . . . . .87.73-87.75

Arterlography and anglocardlography using contrast material. . . . . .88.4-88.5

Diegnostic ultrasound. $\ldots \ldots \ldots \ldots \ldots \ldots \ldots \ldots \ldots \ldots \ldots \ldots . .86 .7$

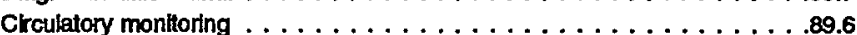

Radiolsolope scan . . . . . . . . . . . . . . . . . . . .

Resplratory therapy. . . . . . . . . . . . . . . . . . . . .93.9

\begin{tabular}{|c|c|c|c|c|c|c|}
\hline \multirow[b]{2}{*}{41,608} & & \multirow[b]{2}{*}{14,546} \\
\hline & 16,142 & 25,466 & 1,863 & 16,021 & 9,178 & \\
\hline 909 & 449 & 460 & 188 & 327 & 183 & 211 \\
\hline 334 & 168 & 166 & 140 & 88 & 47 & 58 \\
\hline 90 & 25 & 64 & " & 30 & 35 & 22 \\
\hline 391 & 188 & 203 & 19 & 83 & 81 & 208 \\
\hline 83 & 48 & 35 & 40 & 22 & 13 & "8 \\
\hline 390 & 213 & 177 & 77 & 163 & 84 & 66 \\
\hline 50 & 24 & 26 & 31 & 16 & $*$ & " \\
\hline 986 & 554 & 432 & 46 & 187 & 277 & 475 \\
\hline 301 & 173 & 128 & 13 & 58 & 88 & 142 \\
\hline 4,410 & 2,619 & 1,791 & 131 & 486 & 1,560 & 2,234 \\
\hline 398 & 268 & 130 & " & 30 & 185 & 183 \\
\hline 485 & 353 & 133 & - & 21 & 205 & 260 \\
\hline 1,010 & 613 & 397 & 14 & 90 & 420 & 485 \\
\hline 281 & 141 & 141 & $*$ & * & 42 & 232 \\
\hline 173 & 99 & 74 & $"$ & 19 & 58 & 94 \\
\hline 328 & 168 & 161 & * & 73 & 117 & 137 \\
\hline 377 & 197 & 181 & 22 & 63 & 117 & 176 \\
\hline 5,096 & 2,106 & 2,990 & 169 & 1,376 & 1,342 & 2,210 \\
\hline 832 & 367 & 465 & 11 & 143 & 207 & 471 \\
\hline 517 & 211 & 306 & " & 74 & 133 & 307 \\
\hline 207 & 93 & 113 & " & 24 & 60 & 120 \\
\hline 250 & 135 & 115 & 49 & 148 & 35 & 17 \\
\hline 502 & 148 & 354 & " & 182 & 150 & 168 \\
\hline 109 & 96 & 13 & 10 & 19 & 27 & 54 \\
\hline 347 & 58 & 289 & " & 171 & 84 & 90 \\
\hline 1,263 & 653 & 610 & 35 & 317 & 341 & 570 \\
\hline 329 & 211 & 118 & " & 60 & 83 & 181 \\
\hline 468 & 468 & $\cdots$ & 30 & 30 & 96 & 312 \\
\hline 317 & 317 & $\ldots$ & $\ldots$ & " & 66 & 250 \\
\hline 2,197 & $\cdots$ & 2,197 & ${ }^{* 6}$ & 1,454 & 492 & 245 \\
\hline 443 & $\cdots$ & 443 & " & 225 & 164 & 52 \\
\hline 384 & $\ldots$ & 384 & " & 383 & * & $\ldots$ \\
\hline 562 & $\ldots$ & 562 & " & 326 & 172 & 63 \\
\hline 127 & $\cdots$ & 127 & * & 99 & 19 & 9 \\
\hline 159 & $\cdots$ & 159 & - & 41 & 60 & 58 \\
\hline 6,763 & $\cdots$ & 6,763 & 19 & 6,740 & " & $\cdots$ \\
\hline 1,562 & $\ldots$ & 1,562 & " & 1,555 & * & $\ldots$ \\
\hline 744 & $\ldots$ & 744 & " & 742 & " & $\cdots$ \\
\hline 917 & $\cdots$ & 917 & " & 915 & " & $\cdots$ \\
\hline 1,142 & $\cdots$ & 1,142 & " & 1,139 & " & $\cdots$ \\
\hline 860 & $\cdots$ & 860 & " & 857 & " & $\cdots$ \\
\hline 3,223 & 1,600 & 1,623 & 151 & 1,231 & 798 & 1,043 \\
\hline 227 & 123 & 104 & $"$ & 96 & 76 & 50 \\
\hline 423 & 175 & 247 & 13 & 142 & 79 & 189 \\
\hline 333 & 183 & 150 & - & 175 & 115 & 44 \\
\hline 125 & 51 & 74 & " & 9 & 31 & 83 \\
\hline 179 & 62 & 117 & * & $\star$ & 42 & 131 \\
\hline 1,364 & 565 & 799 & 74 & 475 & 364 & 450 \\
\hline 124 & " & 123 & * & 24 & 43 & 57 \\
\hline 334 & 184 & 150 & 20 & 108 & 88 & 118 \\
\hline 120 & 69 & 51 & 11 & 46 & 28 & 34 \\
\hline 13,599 & 6,455 & 7,143 & 854 & 3,038 & 3,391 & 6,315 \\
\hline 1,158 & 565 & 594 & 59 & 272 & 251 & 576 \\
\hline 197 & 108 & 89 & $"$ & 73 & 59 & 61 \\
\hline 1,731 & 1,024 & 706 & 19 & 183 & 699 & 830 \\
\hline 1,420 & 572 & 848 & 60 & 354 & 318 & 688 \\
\hline 505 & 239 & 266 & 23 & 89 & 122 & 271 \\
\hline 412 & 173 & 239 & 12 & 69 & 109 & 222 \\
\hline 876 & 427 & 449 & 185 & 117 & 165 & 409 \\
\hline
\end{tabular}


Table 8. Rate of all-listed procedures for discharges from short-stay hospitals, by procedure category, sex, and age: United States, 1993 [Discharges of inpatients from non-Federal hospitals. Excludes newborn infants. Procedure groupings and code numbers are based on the International Classification of Diseases, 9th Revision, Clinical Modifications (ICD-9-CM)]

\begin{tabular}{|c|c|c|c|c|c|c|c|}
\hline \multirow[b]{2}{*}{ Procedure category and ICD-9-CM code } & \multirow[b]{2}{*}{ Total } & \multicolumn{2}{|c|}{ Sex } & \multicolumn{4}{|c|}{ Age } \\
\hline & & Male & Female & $\begin{array}{l}\text { Under } 15 \\
\text { years }\end{array}$ & $\begin{array}{l}15-44 \\
\text { years }\end{array}$ & $\begin{array}{l}45-64 \\
\text { years }\end{array}$ & $\begin{array}{l}65 \text { years } \\
\text { and over }\end{array}$ \\
\hline & \multicolumn{7}{|c|}{ Number per 100,000 population } \\
\hline All procedures & $16,225.6$ & $12,955.9$ & $19,315.6$ & 3,283.4 & $13,653.6$ & $18,521.8$ & $44,360.0$ \\
\hline Operations on the nervous system. & 354.5 & 360.7 & 348.6 & 330.9 & 278.5 & 369.4 & 644.6 \\
\hline Spinal tap $\ldots \ldots \ldots \ldots \ldots \ldots \ldots \ldots \ldots \ldots \ldots \ldots \ldots \ldots . . \ldots \ldots$ & 130.1 & 134.6 & 125.8 & 247.0 & 75.3 & 93.8 & 178.4 \\
\hline 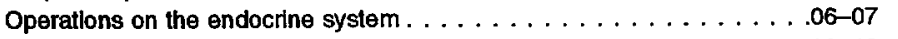 & 35.0 & 20.2 & 48.9 & $*$ & 25.2 & 70.9 & 65.8 \\
\hline Operations on the eye. $\ldots \ldots \ldots \ldots \ldots \ldots \ldots \ldots \ldots .0 .16$ & 152.4 & 151.1 & 153.7 & 34.1 & 70.4 & 163.2 & 634.5 \\
\hline Operations on the ear . . . . . . . . . . . . . . . . .18-20 & 32.5 & 38.4 & 26.8 & 69.9 & 19.1 & 25.9 & ${ }^{\star} 25.8$ \\
\hline Operallons on the nose, mouth, and pharynx $\ldots \ldots \ldots \ldots \ldots \ldots .21-29$ & 152.2 & 171.3 & 134.1 & 135.7 & 138.7 & 170.3 & 201.6 \\
\hline Tonsillectomy with or without adenoidectomy $\ldots \ldots \ldots \ldots \ldots .28 .2-28.3$ & 19.6 & 19.1 & 20.0 & 54.3 & 13.6 & * & " \\
\hline Operations on the respiratory system $\ldots \ldots \ldots \ldots \ldots \ldots \ldots \ldots$.30-34 & 384.4 & 444.7 & 327.4 & 80.7 & 159.7 & 559.3 & $1,449.9$ \\
\hline Bronchoscopy wllh or without biopsy. . . . . . . . . .33.21-33.24,33.27 & 117.3 & 138.6 & 97.2 & 23.7 & 49.5 & 176.8 & 432.2 \\
\hline Operations on the cardlovascular system . . . . . . . . . . . .35-39 & $1,719.8$ & $2,102.0$ & $1,358.7$ & 230.0 & 414.2 & $3,148.5$ & $6,811.7$ \\
\hline Removal of coronary artery obstructon $\ldots \ldots \ldots \ldots \ldots \ldots .36 .0$ & 155.3 & 215.2 & 98.7 & * & 25.6 & 372.9 & 558.4 \\
\hline Coronary antery bypass graft $1 / \ldots \ldots \ldots \ldots \ldots \ldots \ldots .1$ & 189.3 & 283.2 & 100.5 & - & 17.9 & 413.2 & 791.8 \\
\hline $\begin{array}{l}\text { Cardlac catheterization } \ldots \ldots \ldots \ldots \ldots \ldots \ldots \ldots \ldots \ldots .37 .21-37.23 \\
\text { Insertlon, replacement, removal, and revision }\end{array}$ & 393.8 & 491.6 & 301.3 & 24.9 & 76.7 & 848.2 & $1,479.8$ \\
\hline of pacemaker leads or device. . . . . . . . . . . . .37.7-37.8 & 109.6 & 112.8 & 106.6 & * & * & 85.0 & 708.2 \\
\hline 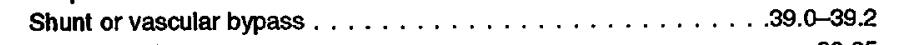 & 67.5 & 79.4 & 56.2 & $*$ & 16.4 & 118.0 & 285.5 \\
\hline Hemodialysis . . . . . . . . . . . . . . . . . . . . . 39.95 & 128.1 & 134.5 & 122.1 & * & 62.3 & 235.9 & 419.1 \\
\hline Operatlons on the hemic and lymphatic system . . . . . . . .40-41 & 147.1 & 157.7 & 137.0 & 38.4 & 53.5 & 235.6 & 536.2 \\
\hline Operatlons on the dlgesthe system . . . . . . . . . . . .42-54 & $1,987.4$ & $1,690.7$ & $2,267.7$ & 298.6 & $1,172.3$ & $2,707.5$ & 6.738 .7 \\
\hline Endoscopy of small intestine with or wlthout blopsy. . . . .45.11-45.14,45.16 & 324.5 & 294.3 & 353.0 & 18.9 & 121.8 & 418.1 & $1,437.1$ \\
\hline Endoscopy of large intestine with or without biopsy $\ldots \ldots \ldots .45 .21-45.25$ & 201.7 & 169.6 & 232.0 & * & 62.7 & 268.0 & 936.0 \\
\hline Partial excision of large intestine . . . . . . . . . . . . . . . 45.7 & 80.6 & 74.8 & 86.0 & * & 20.8 & 120.6 & 367.3 \\
\hline 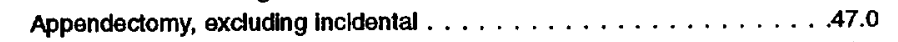 & 97.5 & 108.5 & 87.1 & 87.2 & 126.4 & 71.2 & 52.0 \\
\hline Cholecystectomy. . . . . . . . . . . . . . . . . . . . . 51.2 & 195.6 & 118.6 & 268.4 & * & 155.4 & 302.2 & 512.8 \\
\hline Repalr of inguinal hemia $\ldots \ldots \ldots \ldots \ldots \ldots \ldots \ldots \ldots .5 \ldots \ldots 3.1$ & 42.6 & 77.3 & 9.9 & 18.0 & 16.0 & 53.7 & 163.7 \\
\hline 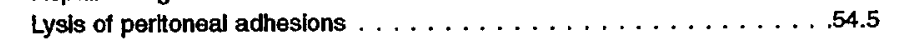 & 135.5 & 46.7 & 219.3 & * & 145.3 & 169.7 & 273.6 \\
\hline Operations on the urinary system. $\ldots \ldots \ldots \ldots \ldots \ldots \ldots \ldots 5-59$ & 492.4 & 524.0 & 462.5 & 61.1 & 270.4 & 688.1 & $1,737.3$ \\
\hline Cystoscopy with or without blopsy . . . . . . . . . . 57.31-57.33 & 128.2 & 169.2 & 89.4 & * & 51.3 & 166.8 & 552.8 \\
\hline 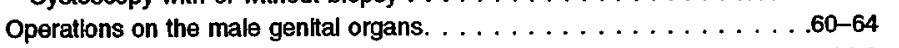 & 182.6 & 375.8 & $\ldots$ & 53.3 & 25.4 & 193.4 & 952.2 \\
\hline 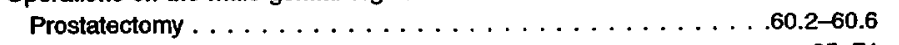 & 123.7 & 254.6 & $\ldots$ & $\ldots$ & • & 133.5 & 761.3 \\
\hline Operations on the female genital organs $\ldots \ldots \ldots \ldots \ldots \ldots \ldots 5-71$ & 856.6 & $\ldots$ & $1,666.0$ & $" 10.0$ & $1,238.9$ & 993.0 & 747.6 \\
\hline Oophorectomy and salpingo-oophorectomy $\ldots \ldots \ldots \ldots \ldots .65 .3-65.6$ & 172.6 & $\ldots$ & 335.6 & * & 191.7 & 331.3 & 159.7 \\
\hline Bllateral destruction or occlusion of fallopian tubes $\ldots \ldots \ldots \ldots .66 .2-66.3$ & 149.6 & $\ldots$ & 291.0 & * & 326.4 & * & $\ldots$ \\
\hline Hysterectomy . . . . . . . . . . . . . . . .68.3-68.7,68.9 & 219.0 & $\ldots$ & 426.0 & " & 278.2 & 347.1 & 191.5 \\
\hline 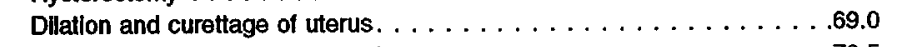 & 49.6 & $\ldots$ & 96.4 & * & 84.6 & 38.7 & "26.1 \\
\hline Repair of cystocele and rectocele $\ldots \ldots \ldots \ldots \ldots \ldots \ldots \ldots .70 .5$ & 62.1 & $\ldots$ & 120.8 & - & 35.1 & 121.5 & 176.5 \\
\hline Obstetrical procedures $\ldots \ldots \ldots \ldots \ldots \ldots \ldots \ldots \ldots \ldots \ldots \ldots \ldots \ldots \ldots .72-75$ & $2,637.3$ & $\ldots$ & $5,129.6$ & 33.9 & $5,743.7$ & * & $\cdots$ \\
\hline Eplsiotomy with or without forceps or & & & & & & $*$ & \\
\hline vacuum extractlon $\ldots \ldots \ldots \ldots \ldots \ldots \ldots .72 .1,72.21,72.31,72.71,73.6$ & 608.9 & $\cdots$ & $1,184.4$ & " & $1,325.6$ & 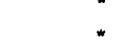 & $\cdots$ \\
\hline Artificlal rupture of membranes $\ldots \ldots \ldots \ldots \ldots \ldots \ldots \ldots .73 .0$ & 290.3 & $\ldots$ & 564.6 & $"$ & 631.9 & $"$ & $\cdots$ \\
\hline Cesarean section $\ldots \ldots \ldots \ldots \ldots \ldots \ldots \ldots \ldots .74 .0-74.2,74.4,74.99$ & 357.7 & $\ldots$ & 695.8 & * & 780.0 & * & $\ldots$ \\
\hline Fetal EKG (scap) and fetal monitoring, not otherwise spectfled . .75.32,75.34 & 445.5 & $\ldots$ & 866.4 & * & 970.4 & * & $\cdots$ \\
\hline 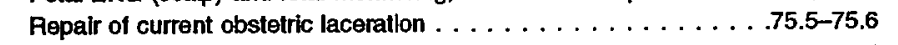 & 335.3 & ... & 652.2 & * & 730.0 & * & $\cdots$ \\
\hline Operations on the musculoskeletal system . . . . . . . . . . . . .76-84 & $1,256.9$ & $1,284.5$ & $1,230.9$ & 265.6 & $1,049.2$ & $1,610.2$ & $3,182.2$ \\
\hline Pantial excision of bone . . . . . . . . . . . .76.2-76.3,77.6-77.8 & 88.6 & 98.8 & 78.9 & $*$ & 82.1 & 152.8 & 153.1 \\
\hline Open reduction of fracture with internal fixation $\ldots \ldots \ldots \ldots \ldots .79 .3$ & 164.8 & 140.8 & 187.4 & 23.0 & 120.8 & 159.7 & 575.0 \\
\hline 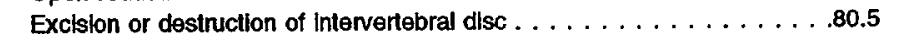 & 130.0 & 147.2 & 113.7 & $\star$ & 148.8 & 231.5 & 133.7 \\
\hline Total hip replacement $\ldots \ldots \ldots \ldots \ldots \ldots \ldots \ldots . .6 \ldots$ & 48.8 & 41.1 & 56.1 & * & 7.8 & 63.4 & 252.5 \\
\hline Total knee replacement . . . . . . . . . . . . . . . . . . 81.54 & 69.7 & 49.8 & 88.5 & • & " & 85.5 & 400.0 \\
\hline Operations on the integumentary system $\ldots \ldots \ldots \ldots \ldots \ldots .85-86$ & 531.7 & 453.5 & 605.7 & 129.8 & 405.2 & 735.2 & $1,372.7$ \\
\hline Mastectomy . . . . . . . . . . . . . . . . . . . . . & 48.3 & * & 93.1 & * & 20.2 & 86.8 & 173.1 \\
\hline 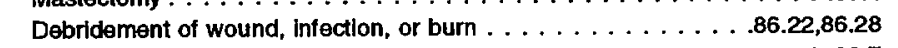 & 130.2 & 147.9 & 113.5 & 35.1 & 91.9 & 176.8 & 361.3 \\
\hline 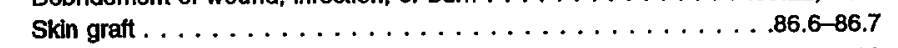 & 46.7 & 55.4 & 38.4 & 18.9 & 39.6 & 57.2 & 104.0 \\
\hline Miscellaneous diagnostic and therapeutic procedures $\ldots \ldots \ldots \ldots .87-99$ & $5,302.9$ & $5,181.3$ & $5,417.9$ & $1,505.4$ & $2,589.2$ & $6,843.1$ & $19,259.2$ \\
\hline Computerized axtal tomography $\ldots \ldots \ldots .87 .03,87.41,87.71,88.01,88.38$ & 451.8 & 453.3 & 450.3 & 104.4 & 231.9 & 505.9 & $1,757.9$ \\
\hline Pyelogram. . . . . . . . . . . . . . . . . .87.73-87.75 & 76.8 & 86.4 & 67.8 & $*$ & 62.1 & 118.4 & 187.2 \\
\hline Arteriography and angiocardlography using contrast materlal $\ldots$. .88.4-88.5 & 674.9 & 822.2 & 535.7 & 33.2 & 156.0 & $1,410.4$ & $2,531.1$ \\
\hline Dlagnosttc ultrasound $\ldots \ldots \ldots \ldots \ldots \ldots \ldots \ldots \ldots \ldots . .8 \ldots$ & 553.9 & 459.3 & 643.2 & 105.9 & 302.0 & 641.7 & $2,097.6$ \\
\hline Circulatory monitoring. . . . . & 196.9 & 192.0 & 201.5 & 40.3 & 75.6 & 246.7 & 826.7 \\
\hline Radiolsotope scan . . . . . . & 160.6 & 139.1 & 181.0 & 21.3 & 59.0 & 219.8 & 676.1 \\
\hline Respiratory therapy . . . . . . . . . . . . . . . . . . . . .93.9 & 341.7 & 342.6 & 340.8 & 326.2 & 99.4 & 333.1 & $1,248.2$ \\
\hline
\end{tabular}

${ }^{1}$ The rate por 100,000 population of discharges with a coronary artery bypass graft was 120.6 . 


\section{Technical notes}

\section{Survey methodology}

\section{Source of data}

The National Hospital Discharge Survey covers discharges from noninstitutional hospitals, exclusive of Federal, military, and Department of Veterans Affairs hospitals, located in the 50 States and the District of Columbia. Only short-stay hospitals (hospitals with an average length of stay for all patients of fewer than 30 days) or those whose specialty is general (medical or surgical) or children's general are included in the survey. These hospitals must also have six beds or more staffed for patient use.

From 1988 through 1990, the NHDS sampling frame consisted of hospitals that were listed in the April 1987 SMG Hospital Market Database (3), met the above criteria, and began accepting patients by August 1987. In 1991 the sampling frame was updated to include hospitals from the 1991 SMG Hospital Database (4). For 1993, the sample consisted of 528 hospitals. Of the 528 hospitals, 15 were found to be out of scope (ineligible) because they went out of business or otherwise failed to meet the criteria for the NHDS universe. Of the 513 in-scope (eligible) hospitals, 466 responded to the survey.

\section{Sample design and data collection}

The NCHS has conducted the NHDS continuously since 1965 . The original sample was selected in 1964 from a frame of short-stay hospitals listed in the National Master Facility Inventory. That sample was updated periodically with samples of newly opened hospitals. Sample hospitals were selected with probabilities ranging from certainty for the largest hospitals to 1 in 40 for the smallest hospitals. Within each sample hospital, a systematic random sample of discharges was selected. A report on the design and development of the original NHDS has been published (1).

Beginning in 1988, the NHDS sample includes with certainty all hospitals with 1,000 beds or more or 40,000 discharges or more annually. The remaining sample of hospitals is based on a stratified three-stage design. The first stage consists of a selection of 112 primary sampling units (PSU's) that comprise a probability subsample of PSU's to be used in the 1985-94 National Health Interview Survey. The second stage consists of a selection of noncertainty hospitals from the sample PSU's. At the third stage, a sample of discharges was selected by a systematic random sampling technique.

Two data collection procedures were used for the survey. The first was a manual system of sample selection and data abstraction. The second was an automated method, used for approximately 32 percent of the respondent hospitals in 1993, that involved the purchase of data tapes from abstracting service organizations, State data systems, or hospitals.

In the manual system, the sample selection and the transcription of information from the hospital records to abstract forms were performed at the hospitals. The completed forms, along with sample selection control sheets, were forwarded to NCHS for coding, editing, and weighting. Of the hospitals using the manual system in 1993, about 55 percent had the work performed by their own medical records staff. In the remaining hospitals using the manual system, personnel of the U.S. Bureau of the Census did the work on behalf of NCHS.

For the automated system, NCHS purchased tapes containing machinereadable medical record data that were systematically sampled by NCHS.

The medical abstract form and the automated data tapes contain items relating to the personal characteristics of the patient, including birth date, sex, race, and marital status, but not name and address; administrative information, including admission and discharge dates, discharge status, and medical record number; and medical information, including diagnoses and surgical and nonsurgical operations or procedures. Since 1977, patient ZIP Code, expected source of payment, and dates of surgery have also been collected. (The medical record number and patient ZIP Code are confidential information and are not available to the public.)

\section{Presentation of estimates}

The relative standard error of the estimate and the number of sample records on which the estimate is based (referred to as the sample size) are used to identify estimates with relatively low reliability.

Because of the complex sample design of the NHDS, estimates of less than 5,000 are not presented; only an asterisk $\left(^{*}\right)$ appears in the tables. These estimates generally have a relative standard error of more than 30 percent or are based on a sample of fewer than 30 cases. Estimates of 5,000 to 9,000 are preceded by an asterisk $\left(^{*}\right)$ to indicate that they should not be assumed to be reliable. These estimates are generally based on fewer than 60 cases.

\section{Sampling errors and rounding of numbers}

The standard error is primarily a measure of sampling variability that occurs by chance because only a sample rather than the entire universe is surveyed. The relative standard error of the estimate is obtained by dividing the standard error by the estimate itself and is expressed as a percent of the estimate. The resulting value is multiplied by 100 , so the relative standard error is expressed as a percent of the estimate.

Estimates of sampling variability were calculated with SESUDAAN software, which computes standard errors by using a first-order Taylor approximation of the deviation of estimates from their expected values. A description of the software and the approach it uses has been published (5).

The constants for relative standard error curves for the 1993 National Hospital Discharge Survey are presented in table I. The relative standard error $\operatorname{RSE}(X)$ of an estimate $X$ may be estimated from the formula:

$$
\operatorname{RSE}(X)=100 \sqrt{a+b / X}
$$

where $X, a$, and $b$ are as defined in table I. 
Table I. Estimated parameters tor relative standard error equations for National Hospltal Discharge Survey statistics by selected characteristics: Unlted States, 1993

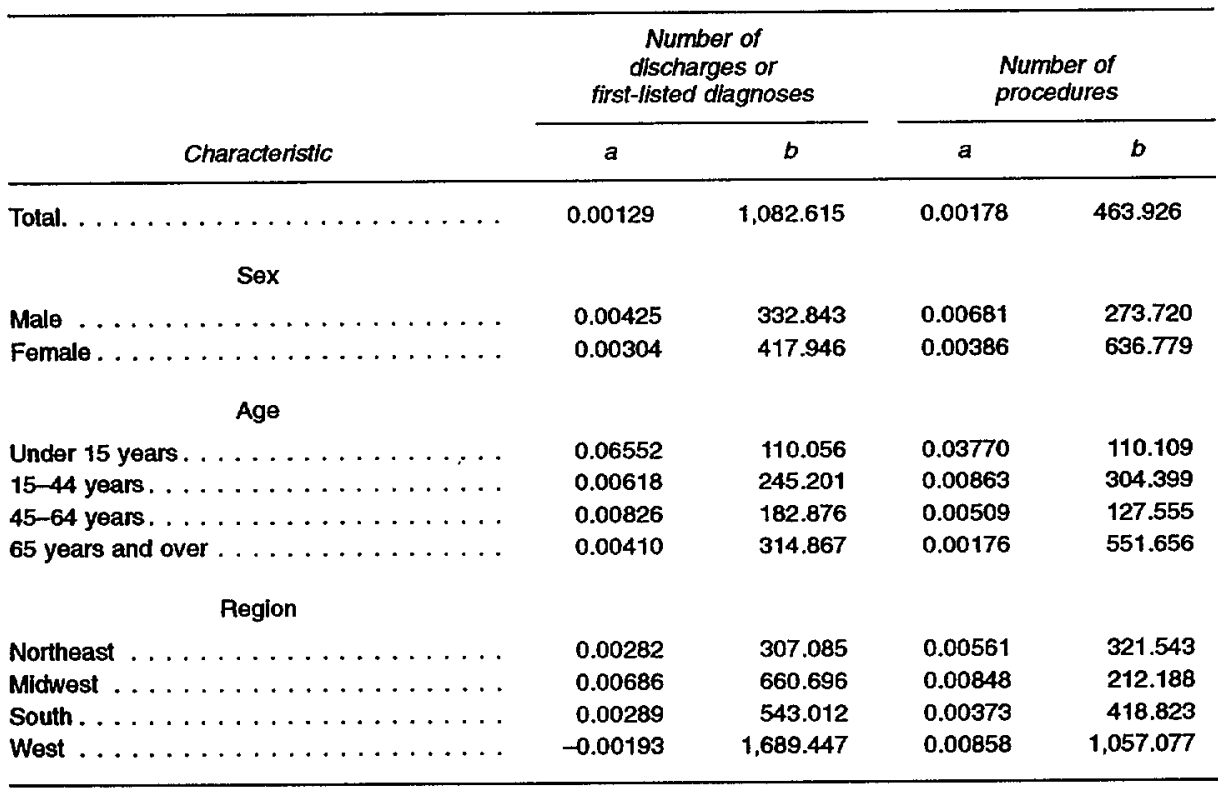

Estimates have been rounded to the nearest thousandth. For this reason, figures within tables do not always add to the totals. Rates and average lengths of stay were calculated from original, unrounded figures and will not necessarily agree precisely with rates or average lengths of stay calculated from rounded data.

\section{Tests of significance}

In this report, statistical inference is based on the two-tailed t-test with a critical value of $1.96(0.05$ level of significance). Terms such as "higher" and "less" indicate that differences are statistically significant. Terms such as "similar" or "no difference" mean that no statistically significant difference exists between the estimates being compared. A lack of comment on the difference between any two estimates does not mean that the difference was tested and found not to be significant.

\section{Terms relating to hospitalization}

Hospitals-All hospitals with an average length of stay for all patients of fewer than 30 days or hospitals whose specialty is general (medical or surgical) or children's general are eligible for inclusion in the National Hospital Discharge Survey, except Federal hospitals, hospital units of institutions, and hospitals with fewer than six beds staffed for patients' use.

Patient-A person who is formally admitted to the inpatient service of a short-stay hospital for observation, care, diagnosis, or treatment. The terms "patient" and "inpatient" are used synonymously.

Newborn infant-A patient admitted by birth to a hospital.

Discharge-The formal release of a patient by a hospital; that is, the termination of a period of hospitalization by death or by disposition to place of residence, nursing home, or another hospital. The terms "discharges" and "patients discharged" are used synonymously.

Discharge rate-The ratio of the number of hospital discharges during a year to the number of persons in the civilian population on July 1 of that year.

Days of care-The number of patient days accumulated by a patient at time of discharge. A stay of less than 1 day (patient admission and discharge on the same day) is counted as 1 day in the summation of total days of care. For patients admitted and discharged on different days, the number of days of care is computed by counting all days from (and including) the date of admission to (but not including) the date of discharge.

Average length of stay-The number of days of care accumulated by patients discharged during the year divided by the number of these patients.

\section{Terms relating to diagnioses}

Diagnosis-A disease or injury (or factor that influences health status and contact with health services that is not itself a current illness or injury) on the medical record of a patient.

Principal diagnosis-The condition established after study to be chiefly responsible for occasioning the admission of the patient to the hospital for care.

First-listed diagnosis-The coded diagnosis identified as the principal diagnosis or listed first on the face sheet or discharge summary of the medical record if the principal diagnosis cannot be identified. The number of first-listed diagnoses is equivalent to the number of discharges.

\section{Terms relating to procedures}

Procedure-A surgical or nonsurgical operation, diagnostic procedure, or special treatment reported on the medical record of a patient. Beginning with the 1991 data, all ICD-9-CM procedure codes are used in the NHDS. Previously selected codes, primarily codes for miscellaneous diagnostic and therapeutic procedures, were not used.

All-listed procedures-The number of procedures on the face sheet of the medical record. In the NHDS a maximum of four procedures are coded.

Rate of procedures-The ratio of the number of procedures during a year to the number of persons in the civilian population on July 1 of that year determines the rate of procedures.

\section{Demographic terms}

Population-The U.S. resident population excluding members of the Armed Forces.

Age-Patient's age at birthday prior to admission to the hospital. 
Geographic region-Hospitals are classified by location in one of the four geographic regions of the United States that correspond to those used by the U.S. Bureau of the Census.

\section{Region States included}

Northeast. . . Maine, New Hampshire, Vermont, Massachusetts, Rhode Island, Connecticut, New York, New Jersey, and Pennsylvania

Midwest. .... Michigan, Ohio, Illinois, Indiana, Wisconsin, Minnesota, Iowa, Missouri, North Dakota, South Dakota, Nebraska, and Kansas

South...... Delaware, Maryland, District of Columbia, Virginia, West Virginia, North Carolina, South Carolina, Georgia, Florida, Kentucky, Tennessee, Alabama, Mississippi, Arkansas, Louisiana, Oklahoma, and Texas

West. ...... Montana, Idaho, Wyoming, Colorado, New Mexico, Arizona, Utah, Nevada, Washington, Oregon, California, Hawaii, and Alaska

\section{Symbols}

-. Data not available

... Category not applicable

- Quantity zero

0.0 Quantity more than zero but less than 0.05

Z Quantity more than zero but less than $\mathbf{5 0 0}$ where numbers are rounded to thousands

* Figure does not meet standard of reliability or precision (more than 30-percent relative standard error in numerator of percent or rate)

\# $\quad$ Figure suppressed to comply with confidentiality requirements 


\section{Suggested citation}

Graves EJ. 1993 Summary: National Hospital Discharge Survey. Advance data from vital and health statistics; no 264. Hyattsville, Maryland: National Center for Health Statistics. 1995.

\section{Copyright information}

All material appearing in this report is in the public domain and may be reproduced or copied without permission; citation as to source, however, is appreciated.
National Center for Health Statistics

Acting Director

Jack R. Anderson

Acting Deputy Director Jennifer H. Madans, Ph.D.

\section{U.S. DEPARTMENT OF HEALTH AND HUMAN SERVICES \\ Public Health Service \\ Centers for Disease Control and Prevention \\ National Center for Health Statistics \\ 6525 Belcrest Road \\ Hyattsville, Maryland 20782}

OFFICIAL BUSINESS

PENALTY FOR PRIVATE USE, $\$ 300$

To receive this publication regularly, contact the National Center for Health Statistics by calling 301-436-8500

DHHS Publication No. (PHS) 95-1250

5-1237 (5/95) 


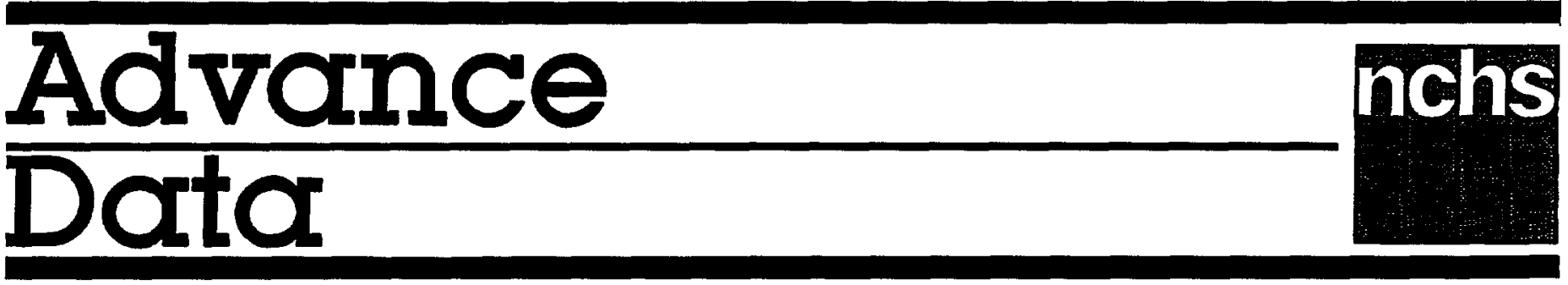

\title{
Underreporting of Race in the National Hospital Discharge Survey
}

\author{
by Lola Jean Kozak, Ph.D., Division of Health Care Statistics
}

\section{Introduction}

Race data from the National Hospital Discharge Survey (NHDS) has become increasingly incomplete in recent years. This report examines factors related to the underreporting of race and explores the effects of the underreporting on NHDS estimates of hospital use by race. A major concern is whether discharges for each racial group are equally likely to be underestimated. If underreporting of race is a general phenomenon, then the NHDS estimates of hospital use for each racial group are too low. However, if discharges for some racial groups are more likely to be underestimated than others in the NHDS, using the data to make comparisons across racial groups could be misleading.

The NHDS has been conducted continuously by the National Center for Health Statistics (NCHS) since 1965. The data for the survey come from a sample of inpatient records that are obtained from a national sample of
non-Federal general and short-stay specialty hospitals located in the United States. In 1990, data were abstracted from medical records of 266,000 discharges from 474 hospitals. In 1991, 484 hospitals provided data from 274,000 medical records, and 494 hospitals provided data from 274,000 medical records in 1992.

Beginning in 1988, a 3-stage stratified sample design was put into operation for the NHDS. For the first stage, primary sampling units (PSU's) were sampled; in the second, hospitals were sampled from the PSU's, and the third stage consisted of sampling discharges within the selected hospitals. In addition, hospitals with 1,000 beds or more or 40,000 discharges or more per year were selected with certainty.

Since 1985 , two data collection procedures have been used for the NHDS. One is a manual system in which data are abstracted from the face sheet or discharge summary of the medical record for each sampled discharge at the hospital, either by hospital staff or personnel of the U.S. Bureau of the Census, on behalf of NCHS. The other, an automated method, involves the purchase of data tapes from abstracting service organizations, State data systems, or hospitals.

Further information about the survey design, data collection procedures, sampling errors, and definition of terms used in this report can be found in the section entitled "Technical notes."

Data from the NHDS have been used to examine racial differences in patterns of hospital use that may reflect differences in access to care or in the distribution of health problems. Recent studies that have used race data from the NHDS have investigated a variety of topics, including hysterectomy (1), HIV (2), stroke (3), children's asthma (4), preeclampsia and eclampsia (5), appendicitis and appendectomy (6), coronary arteriography and coronary bypass surgery (7), hip fractures (8), and idiopathic cardiomyopathy (9).

\section{Acknowledgments}

This report was prepared in the Division of Health Care Statistics. Linda Lawrence and Chuck Dennison of the Hospital Care Statistics Branch provided operational data and assisted with the description of the survey. Michael J. Monsour, formerly of the Technical Services Branch produced the parameters for relative standard error equations. The report was edited by Klaudia M. Cox and typeset by Annette F. Facemire of the Publications Branch, Division of Data Services.

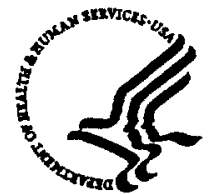

\section{U.S. DEPARTMENT OF HEALTH AND HUMAN SERVICES \\ Public Health Service \\ Centers for Disease Control and Prevention National Center for Health Statistics}

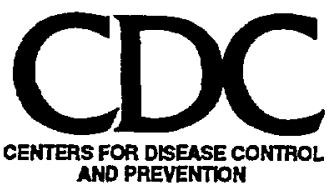
AND PREVENTIOH 


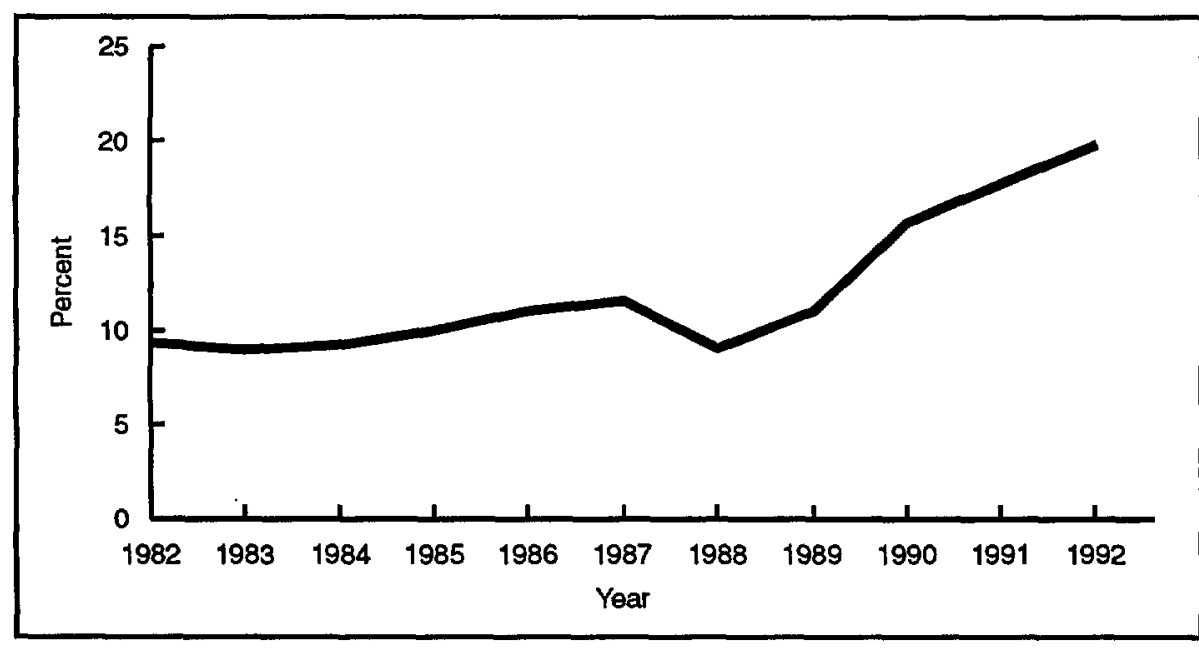

Flgure 1. Percent of dlscharges with race not stated: United States, 1982-92

The NHDS race data have never been 100 percent complete. From 1982 through 1989, discharges with race not stated ranged from 8.9 to 11.5 percent of total discharges. Then, in 1990, the proportion of discharges with race not stated increased to 15.7 percent. In 1991, 17.8 percent of discharges were not identified by race, and in 1992 , 19.8 percent of discharges were in the race-not-stated category (figure 1 ).

Two main factors were found to be related to the increasing underreporting of race in the NHDS. First, a growing number of the hospitals that participated in the NHDS in 1990-92 did not report race for any of their patients. In 1989, 17 hospitals did not report race, but in 1992 the number had increased to 63. Nonreporting hospitals made up only 12.8 percent of the hospitals participating in the survey in 1992 , but accounted for 70.7 percent of the discharges with race not stated. Second, race was not reported for the majority of patients that were identified as Hispanic. In 1992, 16.0 percent of the discharges with race not stated were identified as Hispanic.

These two main problems will be discussed further in the following sections of this report. Approaches to estimating the racial distribution of patients not reported by race will also be examined. To further evaluate the effects of the underreporting of race, the NHDS race data will be compared with data from other sources, including hospital use data from National Health Interview Survey, Medicare data from the Health Care Financing

Administration, and natality data from the Division of Vital Statistics. Finally, adjustment of NHDS race data for underreporting will be discussed.

\section{Highlights}

- The number of hospitals in the NHDS that reported race for less than 3 percent of discharges increased from 17 in 1989 to 63 in 1992.

- Most of the hospitals that did not report race in the 1990-92 period used the automated data collection method.

- Based on data from previous years and county populations, hospitals that did not report race in 1990-92 were likely to have a higher proportion of white discharges than hospitals that reported race.

- A specific race was reported for only 25-35 percent of Hispanic patients in the 1990-92 period; most Hispanic patients were probably white.

- In comparison with the number of discharges estimated from the 1990-92 National Health Interview Survey, NHDS estimates of discharges were significantly lower for white patients, but not significantly different for black patients.

- Proportional adjustment of NHDS race data may produce more accurate estimates of white discharges, but it does not improve comparisons between racial groups.

\section{Hospital reporting patterns}

The majority of hospitals that participate in the National Hospital Discharge Survey (NHDS) report race for all or almost all discharges. In 1992, for example, 296 hospitals (59.9 percent) reported race on $97-100$ percent of their sample records (table 1 ). The discharges not identified by race come primarily from a small group of hospitals that do not report race for any or almost any of their discharges. In 1992, 63 hospitals (12.8 percent) reported race on less than 3 percent of sampled records. These 63 hospitals accounted for 83.4 percent of the sampled records with race not stated in the $1992 \mathrm{NHDS}$, and 70.7 percent of the estimated number of discharges with race not stated.

Nonreporting hospitals, which are defined in this report as those providing race data for less than 3 percent of discharges, have increased in number in recent years (figure 2). In 1989 there were only 17 nonreporting hospitals, but the number jumped to 45 in 1990 , increased to 50 in 1991, and to 63 in 1992. Nonreporting hospitals accounted for 50 percent of the sampled records with race not stated in 1989 , but for 81-84 percent in 1990-92.

Most of the hospitals that did not report race in the 1990-92 period used the automated data collection method. As described earlier, the automated method refers to the purchase of medical record data in electronic form from abstracting service organizations, State data systems, or hospitals. In contrast, data collected via the manual method were transcribed from the medical record to abstract forms specifically for the NHDS.

As shown in figure 2, only 5 hospitals that did not report race used the automated data collection method in 1989 , but in 1990, 31 nonreporting hospitals used the automated method. In 1991, 35 nonreporting hospitals used the automated data collection method, as did 48 nonreporting hospitals in 1992 . The number of nonreporting hospitals using the manual method only increased from 12 in 1989 to 15 in 1992.

A concerted effort was made to improve the NHDS response rate in the 1990 's, which resulted in a substantial 
Table 1. Number and percent distrlbution of hospitals by proportion of sample records with race reported: United State8, 1992

[Short-stay non-Federal hospitals that particlpated in the National Hospltal Discharge Survey. Excludes newborn infants]

\begin{tabular}{|c|c|c|}
\hline \multirow{2}{*}{$\begin{array}{l}\text { Proportion of records } \\
\text { with race reported }\end{array}$} & \multicolumn{2}{|c|}{ Hospitals } \\
\hline & Number & Percent \\
\hline All records . . . . . . . . . . & 494 & 100.0 \\
\hline $0.0-2.9$ percent. . . . . . . . . & 63 & 12.8 \\
\hline 3.0-49.9 percent . . . . . . . . . & 15 & 3.0 \\
\hline $50.0-74.9$ percent $\ldots \ldots \ldots \ldots$ & 21 & 4.3 \\
\hline $75.0-89.9$ percent $\ldots \ldots \ldots \ldots . .$. & 41 & 8.3 \\
\hline $90.0-96.9$ percent $\ldots \ldots \ldots \ldots \ldots$ & 58 & 11.7 \\
\hline 97.0-100.0 percent . . . . . . . . . & 296 & 59.9 \\
\hline
\end{tabular}

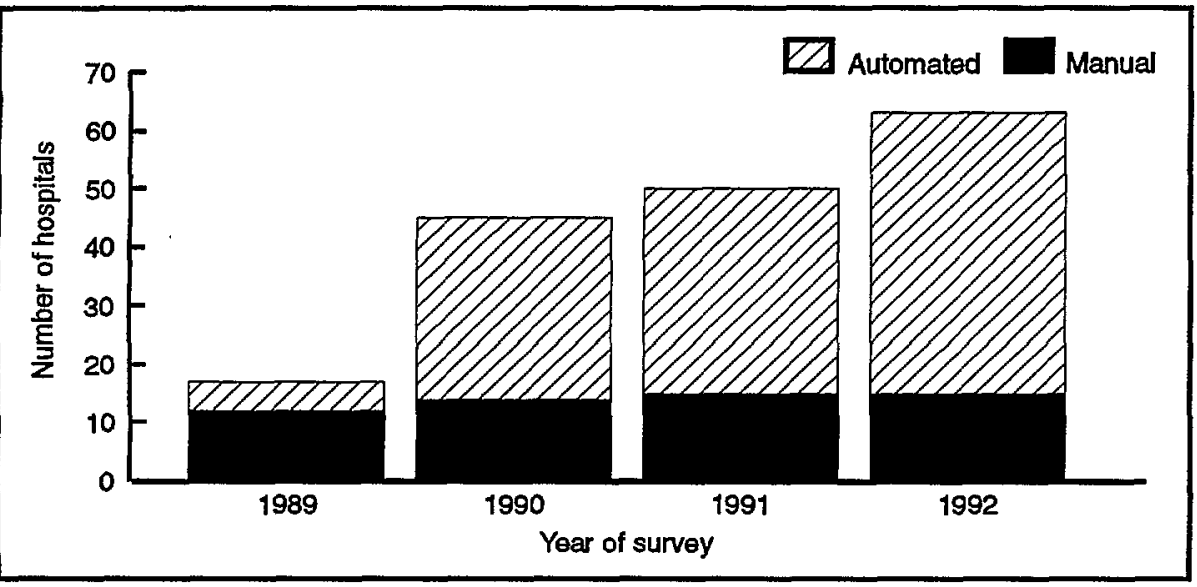

Figure 2. Number of hospltals not reporting race that used the automated and manual methods of data collection: 1989-92

Table 2. Percent distribution of discharges by race, for 26 nonreporting hospitals based on provlous data and for reporting hospitals: United States, 1992

[Discharges of inpatients from non-Federal short-stay haspitals. Excludes newborn infants]

\begin{tabular}{ccc}
26 non- & reporting \\
nosphtals & Reporting \\
Race & hospta/s \\
\hline
\end{tabular}

\begin{tabular}{|c|c|c|}
\hline \multirow[b]{2}{*}{ All races $\ldots \ldots \ldots \ldots \ldots \ldots \ldots$} & \multicolumn{2}{|c|}{ Percent distribution } \\
\hline & 100.0 & 100.0 \\
\hline White . . . . . . . . . . . . . & 87.3 & 75.2 \\
\hline Black ................... & 8.3 & 13.9 \\
\hline All other races $\ldots \ldots \ldots \ldots \ldots \ldots \ldots$ & 2.1 & 4.2 \\
\hline Face not stated. $\ldots \ldots \ldots \ldots \ldots \ldots$ & 2.3 & 6.7 \\
\hline
\end{tabular}

increase in the number of hospitals participating in the survey, from 408 in 1989 to 474 in 1990, 484 in 1991, and 494 in 1992. Many hospitals that had not participated in previous years agreed to participate in the NHDS through the automated systems. Much of the data obtained using the automated method were from State data systems or other systems that used the National Uniform Bill, or UB-82 (and its successor, UB-92) for data collection. The National Uniform Bill was established for processing hospital bills and does not include race as a required item. Thus, the race of discharges was often not included in the data from such systems purchased for the NHDS.

\section{Race for nonreporting hospitals}

An important question to investigate is whether nonreporting hospitals have the same racial distribution of discharges as reporting hospitals. Two approaches were taken to answer this question. First, data from previous years were reviewed. The NHDS was redesigned in 1988, and with some exceptions (some hospitals went out of business, a few new hospitals were added to the sample in 1991), the same hospitals were in the sample from 1988 through 1992. Among the 63 hospitals that did not report race in 1992 were 26 that had reported race in at least 1 year from 1988-91. For each of these 26 hospitals, the percent distribution of the weighted number of discharges by race was obtained for the most recent year in which race was reported. This percent distribution was multiplied by the weighted number of discharges from the hospital in 1992.

The resulting distribution of discharges by race for the 26 hospitals combined is shown in table 2 . The estimated proportion of patients who were white was significantly larger in the 26 nonreporting hospitals (87.3 percent) than in hospitals that reported race in 1992 (75.2 percent). The reporting hospitals had higher proportions of patients who were black, all other races, and race not stated.

Because the 26 hospitals that previously reported race may or may not be representative of all the hospitals that did not report race, a second approach to estimate the racial distribution of discharges from nonreporting hospitals was used. This involved an examination of the populations they served. The 1990 Census provided data on the racial distributions of counties (10) that can be used to approximate service areas of hospitals despite the fact that some hospitals may serve only part of a county or multiple counties.

A preliminary test was conducted to investigate whether it would be reasonable to use county population distributions by race as proxy measures for the distribution of discharges by race. A 20-percent stratified random sample was taken of the hospitals that 
reported race for $97-100$ percent of their discharges in 1992. The county in which each sampled hospital was located was identified and the percent distribution of the population of the county by race was obtained. This percent distribution was multiplied by the weighted number of discharges from the hospital in the county.

The resulting distribution of discharges by race for the sample hospitals was 76.8 percent white patients, $\mathbf{1 7 . 5}$ percent black patients, and 5.6 percent patients of other races. In comparison, the distribution reported for these sample hospitals was 80.0 percent white discharges, 15.2 percent black discharges, and 4.1 percent discharges of other races.

Although not exact, the population distribution of a county appeared useful as a general indicator of the racial distribution of discharges from a hospital in the county. Therefore, the procedure used in the test was applied to the nonreporting hospitals. The county in which each nonreporting hospital was located was identified and the percent distribution of the population of the county by race was obtained. This percent distribution was multiplied by the weighted number of discharges from the nonreporting hospital in the county.

The resulting distributions of discharges by race for nonreporting hospitals are shown in table 3 . For each year 1990 through 1992, the proportion of discharges that were white calculated for nonreporting hospitals was significantly higher than the proportion in reporting hospitals. Conversely, the proportions of discharges that were black calculated for nonreporting hospitals were significantly lower than the proportions in reporting hospitals. The "all other races" category accounted for larger proportions of discharges for the nonreporting than for the reporting hospitals, but these proportions may have been somewhat overestimated, as in the preliminary test.

These findings are not definitive, but along with the data on racial distributions in previous years, they suggest that nonreporting hospitals may have a higher proportion of white discharges and a lower proportion of black discharges than reporting hospitals.

\section{Hispanic patients}

Race and ethnicity are separate data items for the NHDS. On the ethnicity variable, patients are identified as being of Hispanic origin, non-Hispanic origin, or not stated. Ethnicity, in general, is not well reported. For example, in 1992, only 24.6 percent of all NHDS discharges were identified as Hispanic or non-Hispanic. Because ethnicity data are not reliable, these data are not released from the NHDS.

Data on Hispanic origin are discussed here because patients identified as Hispanic usually have missing race data. As shown in table 4, more than half of Hispanic patients were in the race-not-stated category in 1990-92. Another 13-17 percent were reported in the "other" race category, so only $25-35$ percent were identified as a specific race during this 3-year period.

The lack of race data for Hispanic patients is separate from the problem of . hospitals not reporting race. Hospitals that do not report race almost never report ethnicity. However, certain other hospitals report all or almost all patients identified as Hispanic in the race not stated category. In 1992, for example, 112 hospitals reported race for less than 3 percent of their Hispanic discharges, and these hospitals accounted for two-thirds of the Hispanic discharges not identified by race.

These 112 hospitals were more likely to provide data through the manual data collection system than the automated system. In the manual system, NCHS staff are instructed to code records as "race not stated" when Hispanic is written in as a race. Automated system data are not coded by NCHS staff and do not necessarily follow this practice. In 1992, a total of 32 NHDS hospitals were found in which all Hispanic discharges were assigned to the "other" race category. These were predominantly hospitals using the automated data collection system.

Some of the hospitals that have all their Hispanic discharges assigned to the "race not stated" or "other" race categories are known to be using data collection forms that do not separate race and ethnicity. Other hospitals probably also use combined categories. The Federal standards for reporting race and ethnic statistics (11) allow race and ethnicity to be collected in a combined format in which Hispanics are not identified by race.

If identified by race, the evidence indicates that most Hispanics in the "race not stated" and "other" categories would be classified as white. Among

Table 3. Percent distribution of discharges by race for nonreporting hospitals based on county population and for reporting hospitals, according to year: United States, 1990-92

[Discharges of inpatients from non-Federal short-stay hospitals. Excludes newborn infants]

\begin{tabular}{|c|c|c|c|c|c|c|}
\hline \multirow[b]{2}{*}{ Race } & \multicolumn{2}{|c|}{1990} & \multicolumn{2}{|c|}{1991} & \multicolumn{2}{|c|}{1992} \\
\hline & $\begin{array}{l}\text { Nonroporting } \\
\text { hospittals }^{1}\end{array}$ & $\begin{array}{l}\text { Reporting } \\
\text { hospltals }\end{array}$ & $\begin{array}{l}\text { Nonroporting } \\
\text { hospitals }\end{array}$ & $\begin{array}{l}\text { Reporting } \\
\text { hospltals }\end{array}$ & $\begin{array}{l}\text { Nonroporting } \\
\text { hospitals }^{1}\end{array}$ & $\begin{array}{l}\text { Reporting } \\
\text { hospitals }\end{array}$ \\
\hline & \multicolumn{6}{|c|}{ Percent distribution } \\
\hline All races $\ldots \ldots \ldots \ldots \ldots \ldots \ldots$ & 100.0 & 100.0 & 100.0 & 100.0 & 100.0 & 100.0 \\
\hline White . . . . . . . . . & 83.0 & 77.7 & 83.8 & 76.4 & 83.2 & 75.2 \\
\hline Black $\ldots \ldots \ldots \ldots \ldots \ldots \ldots$ & 11.4 & 13.1 & 10.5 & 13.6 & 10.6 & 13.9 \\
\hline All other races $\ldots \ldots \ldots \ldots \ldots \ldots \ldots$ & 5.6 & 3.5 & 5.7 & 3.8 & 6.2 & 4.2 \\
\hline Race not stated. $\ldots \ldots \ldots \ldots \ldots \ldots$ & - & 5.7 & - & 6.2 & - & 6.7 \\
\hline
\end{tabular}

${ }_{1}^{1}$ Nonreporting hospitals reported race for less than 3 percent of discharges. Race distribution was based on the population of the county where the hospital was located.

${ }^{2}$ Hospitals that reported race for 3-100 percent of discharges. 
Table 4. Percent distribution of discharges by race for patients Identifled as Hispanic, according to year: United States, 1990-92

[Dlscharges of Inpatlents from non-Federal short-stay hospitals. Excludes newbom Infants]

\begin{tabular}{|c|c|c|c|}
\hline Race & 1990 & 1991 & 1992 \\
\hline & \multicolumn{3}{|c|}{ Percent distrbution } \\
\hline 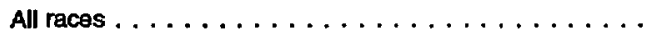 & 100.0 & 100.0 & 100.0 \\
\hline 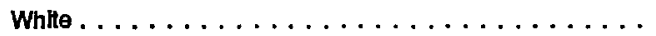 & 33.3 & 28.0 & 23.1 \\
\hline Black . . . . . . . . . . . . . . . & 0.8 & ${ }^{\star} 0.6$ & 0.8 \\
\hline American Indlan/Eskimo/Aleut or Asian/Paclfic Islander . . & 0.9 & 3.5 & 1.7 \\
\hline Other $\ldots \ldots \ldots \ldots \ldots \ldots \ldots \ldots$ & 13.3 & 14.4 & 17.2 \\
\hline Race not stated. . . . . . . . . . . . . . & 51.8 & 53.4 & 57.3 \\
\hline
\end{tabular}

Hispanic discharges reported as a specific race in 1992, 90.4 percent were identified as white, 3.1 percent as black, and 6.5 percent as American Indian/ Eskimo/Aleut or Asian/Pacific Islander. In addition, the U.S. Bureau of the Census estimated that in 1990 the Hispanic population was 91.3 percent white, 5.4 percent black, and 3.3 percent American Indian/Eskimo/Aleut or Asian/Pacific Islander (12). Thus, the lack of information on the race of the majority of discharges identified as Hispanic is likely to affect NHDS estimates of white discharges disproportionately.

\section{Comparisons}

\section{National Health Interview Survey}

If discharges of white patients are underestimated to a greater extent than discharges of patients of other races in the NHDS, this should be evident in comparisons of estimates from the NHDS to data from other sources. A comparison was made of NHDS data with data from the National Health Interview Survey (NHIS), which also produces estimates of hospital use by race (13-15).

The NHIS is based on a different universe and uses different definitions and data collection procedures than the NHDS. The estimates of hospitalizations are obtained from interview questions about overnight stays in short-stay hospitals during the previous 6 months. Hospitalizations of persons who died or were institutionalized during the reference period are excluded, as are hospitalizations of healthy newborn infants.
Before comparing NHIS estimates with NHOS estimates of hospital use, the NHDS estimates were adjusted. Patients hospitalized for less than 1 day were excluded because the NHIS data were only for overnight stays. Persons discharged dead and those transferred to long-term care institutions were excluded, although the NHDS data would probably still include some persons who died or were institutionalized during a 6-month period. All newborn infants were excluded, as is usual for NHDS estimates, although some sick newborn infants may be included in the NHIS estimates. The adjustments do not make the NHDS and NHIS data completely alike, but they should be more comparable.

The adjusted NHDS estimates and the NHIS estimates of discharges from short-stay hospitals are shown in table 5 for 1990 through 1992. In each of the 3 years, the NHIS estimate of total discharges was not significantly different from the adjusted NHDS estimate. However, the estimated number of discharges for white patients from the NHIS was significantly higher than the adjusted NHDS estimate each year. The NHIS estimate was 22 percent higher in 1990, 26 percent higher in 1991, and 30 percent higher in 1992. The estimated number of discharges for black patients from the NHIS was not significantly different from the adjusted NHDS estimate of black discharges in any of the years. NHIS estimates of discharges for other racial groups were not available.

\section{Medicare}

Another source of information on the race of hospital discharges is the Health Care Financing Administration (HCFA), which obtains data on the hospitalizations of Medicare beneficiaries. In 1990, HCFA reported $10,522,000$ discharges from short-stay hospitals for Medicare beneficiaries (16). Of these, $9,037,000$ ( 85.9 percent) were identified as white, $1,185,000$ (11.3 percent) were other than white, and 300,000 ( 2.9 percent) were not identified by race.

The NHDS estimate of discharges with Medicare as the principal expected
Table 5. Number of discharges estimated from the National Hospltal Discharge Survey and the National Health Interview Survey, by year and race: United States, 1990-92 [Discharges of inpatients from short-stay hospitak]

\begin{tabular}{|c|c|c|}
\hline Year and race & $\begin{array}{l}\text { National Hosphal } \\
\text { Dlscharge Survey }\end{array}$ & $\begin{array}{l}\text { Natlonal Health } \\
\text { Interview Survey }\end{array}$ \\
\hline 1990 & \multicolumn{2}{|c|}{ Number in thousands } \\
\hline All races ${ }^{2} \ldots \ldots \ldots \ldots \ldots$ & 27,250 & 27,058 \\
\hline White . . . . . . . . . . . & 18,713 & 22,821 \\
\hline Black . . . . . . . . . . . & 3,300 & 3,692 \\
\hline \multicolumn{3}{|l|}{1991} \\
\hline$\ldots \ldots \ldots \ldots$ & 27,275 & 26,873 \\
\hline White $\ldots \ldots \ldots \ldots \ldots \ldots$ & 18,084 & 22,778 \\
\hline Black ............... & 3,395 & 3,420 \\
\hline \multicolumn{3}{|l|}{1992} \\
\hline All races ${ }^{2}$. & 27,289 & 27,039 \\
\hline White $\ldots \ldots \ldots \ldots \ldots \ldots$ & 17,429 & 22,607 \\
\hline Black .... & 3,363 & 3,654 \\
\hline
\end{tabular}

'Discharges from non-Federal hospitals. Excludes newbom infants, discharges to long-term care institutions, patients diachargad doad, and discharges with inpationt stays of loss than 1 day.

Includes pationts of all races and patients whose race was not stated. 
Table 6. Number of dlscharges for women with dellveries and number of live births, by year and race: United States, 1990-92

\begin{tabular}{|c|c|c|}
\hline Year and race & $\begin{array}{c}\text { Discharges for } \\
\text { women with } \\
\text { dellveries }\end{array}$ & $\underset{\text { births }}{\text { Live }}$ \\
\hline 1990 & \multicolumn{2}{|c|}{ Number in thousands } \\
\hline All races $\ldots \ldots \ldots \ldots \ldots \ldots \ldots \ldots \ldots \ldots \ldots \ldots$ & 4,025 & 4,158 \\
\hline White $\ldots \ldots \ldots \ldots \ldots \ldots \ldots \ldots$ & 2,431 & 3,290 \\
\hline Black . . . . . . . . . . . . . . . . & 584 & 684 \\
\hline All other races $\ldots \ldots \ldots \ldots \ldots \ldots$ & 262 & 184 \\
\hline Race not stated. . . . . . . . . . . . . . & 748 & - \\
\hline \multicolumn{3}{|l|}{1991} \\
\hline All races $\ldots \ldots \ldots \ldots \ldots \ldots \ldots$ & 3,973 & 4,111 \\
\hline White $\ldots \ldots \ldots \ldots \ldots \ldots \ldots$ & 2,244 & 3,241 \\
\hline Black ..................... & 557 & 683 \\
\hline All other races $\ldots \ldots \ldots \ldots \ldots \ldots$ & 289 & 187 \\
\hline Race not stated. . . . . . . . . . . . . & 883 & - \\
\hline \multicolumn{3}{|l|}{1992} \\
\hline All races $\ldots \ldots \ldots \ldots \ldots \ldots \ldots \ldots$ & 3,910 & 4,065 \\
\hline White . . . . . . . . . . . . . . . . & 2,148 & 3,202 \\
\hline Black . . . . . . . . . . . . . . & 511 & 674 \\
\hline All other races $\ldots \ldots \ldots \ldots \ldots \ldots$ & 334 & 190 \\
\hline Race not stated. . . . . . . . . . . . . . & 916 & - \\
\hline
\end{tabular}

${ }^{1}$ Discharges of inpationts from non-Federal short-stay hospitals estimated from the National Hospital Discharge Survey.

${ }^{2}$ Data from bith certificates. Race of mother assigned to child.

source of payment was $10,625,000$ discharges in 1990. Of these, $8,135,000$ (76.6 percent) were identified as white, and $1,037,000$ ( 9.8 percent) were black and other races. The remaining $1,452,000$ ( 13.7 percent) were in the race-not-stated category.

The estimates of total Medicare discharges from these two sources were not significantly different. Likewise, the estimate of discharges for Medicare patients of black and other races from HCFA was not significantly different from the NHDS estimate for this group. However, the HCFA estimate of white Medicare discharges was significantly higher than the NHDS estimate. Thus, these findings also suggest that white patients are more likely to be underestimated in the NHDS than patients of other races.

\section{Birth certificates}

Information about race from birth certificates was also compared to NHDS data on the race of women hospitalized for deliveries. Beginning with 1989, the data from birth certificates have been tabulated by the race of the mother. The number of births would be expected to be somewhat higher than the number of women with deliveries estimated from the NHDS because not all births take place in non-Federal short-stay hospitals, and because one delivery can result in multiple births. However, the differences in total number of deliveries and births were not significantly different in the 1990-92 period.

The racial distribution of live births reported from birth certificates (17) is compared to the racial distribution of women with deliveries from the NHDS in table 6 . The number of live births identified as white was 35 percent higher than the number of white women with deliveries in 1990, 44 percent higher in 1991, and 49 percent higher in 1992. In 1990, the number of black live births was 17 percent higher than the NHDS estimate of black women with deliveries; it was 22 percent higher in 1991, and 32 percent higher in 1992.

The number of live births that were other races was not significantly different from the estimate of women with deliveries of other races in 1990. However, in 1991 and 1992, there were more women with deliveries in the "other races" category than live births. This was due to a large number of women with deliveries in the NHDS who were reported as an unspecific other race. These data suggest problems with NHDS estimates for all the racial categories of women with deliveries, but also support the thesis that white patients are markedly underestimated in the NHDS.

\section{Adjustments for underreporting}

Table 7 shows the number and rate of discharges by race as estimated from the NHDS in 1990-92. These estimates are compared with estimates produced by two types of adjustments. The first is proportional adjustment, a strategy used by researchers to compensate for the underreporting of race in the NHDS (1, 4,8 ). In this approach, the discharges in the race-not-stated category are assigned to specific race categories based on the distribution of the discharges whose race is known.

For example, in the 1992 NHDS, $24,838,000$ of the estimated $30,951,000$ discharges were identified by race. Among the discharges identified by race, 80.6 percent were white, 14.9 percent were black, and 4.5 percent were other races. Distributing the $6,113,000$ discharges in the race-not-stated category in the same proportions, $4,927,000$ were added to the white category, 909,000 to the black category, and 278,000 to the other races category.

Proportional adjustment would be appropriate if the evidence suggested that patients of all races were equally underreported. Because white patients appear to be underreported to a greater extent than patients of other races in the NHDS, proportional adjustment would not be expected to correct accurately for nonresponse. Using this adjustment, white discharges would still be underestimated, and discharges of patients in the other race categories would be overestimated. The relative differences in discharge rates between racial groups are not affected by proportional adjustment. Thus, comparisons of proportionally adjusted rates across racial categories are no different than comparisons of unadjusted rates.

Numbers and rates of discharges resulting from a population-lbased adjustment are also shown in table 7. The population-based adjustment used the populations of the counties in which nonreporting hospitals were located to estimate the racial distribution of 
Table 7. Number and rate of dlscharges, by year, race, and type of estimate: United States, 1990-92

[Discharges of inpatlents from non-Federal short-stay hospltals. Excludes newbom infants]

\begin{tabular}{|c|c|c|c|c|c|c|}
\hline \multirow[b]{2}{*}{ Year and race } & \multicolumn{3}{|c|}{ Discharges in thousands } & \multicolumn{3}{|c|}{$\begin{array}{c}\text { Discharge rate } \\
\text { per } 1,000 \text { population }\end{array}$} \\
\hline & $\begin{array}{l}\text { NHDS } \\
\text { estimate }\end{array}$ & $\begin{array}{l}\text { Proportional } \\
\text { adjustment }^{2}\end{array}$ & $\begin{array}{l}\text { Population-based } \\
\text { adjustment }^{3}\end{array}$ & $\begin{array}{l}\text { NHDS } \\
\text { estimate }\end{array}$ & $\begin{array}{l}\text { Proportional } \\
\text { adjustment }^{2}\end{array}$ & $\begin{array}{l}\text { Population-based } \\
\text { adjustment }\end{array}$ \\
\hline \multicolumn{7}{|l|}{1990} \\
\hline White . . . . . . . . . . . & 21,376 & 25,366 & 24,995 & 102.8 & 122.0 & 120.2 \\
\hline Black $\ldots \ldots \ldots \ldots \ldots \ldots \ldots$ & 3,611 & 4,285 & 4,038 & 119.2 & 141.4 & 133.3 \\
\hline All other races $\ldots \ldots \ldots \ldots \ldots \ldots$ & 958 & 1,137 & 974 & 99.8 & 118.5 & 101.5 \\
\hline Race not stated. $\ldots \ldots \ldots \ldots \ldots \ldots$ & 4,843 & - & 781 & $\cdots$ & $\cdots$ & $\cdots$ \\
\hline \multicolumn{7}{|l|}{1991} \\
\hline White. . . . . . . . . . . . & 20,816 & 25,317 & 25,078 & 99.3 & 120.8 & 119.6 \\
\hline 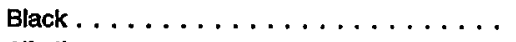 & 3,717 & 4,521 & 4,184 & 120.5 & 146.5 & 135.6 \\
\hline All other races $\ldots \ldots \ldots \ldots \ldots \ldots$ & 1,036 & 1,260 & 1,046 & 103.0 & 125.2 & 104.0 \\
\hline Race not stated. $\ldots \ldots \ldots \ldots \ldots \ldots$ & 5,528 & - & 790 & $\cdots$ & $\cdots$ & $\cdots$ \\
\hline \multicolumn{7}{|l|}{1992} \\
\hline White $\ldots \ldots \ldots \ldots \ldots \ldots \ldots$ & 20,018 & 24,945 & 24,778 & 94.6 & 117.8 & 117.1 \\
\hline Black ................... & 3,692 & 4,601 & 4,219 & 117.9 & 146.9 & 134.7 \\
\hline All other races $\ldots \ldots \ldots \ldots \ldots \ldots$ & 1,128 & 1,405 & 1,142 & 107.5 & 134.0 & 108.9 \\
\hline Race not stated. . . . . . . . . . . . & 6,113 & - & 811 & $\cdots$ & ... & $\ldots$ \\
\hline
\end{tabular}

Unadjusted race data from National Hospital Discharge Survey (NHDS).

2NHDS race data with discharges in race not stated category distributed to raco catogories in proportions of discharges with known race.

WHDS race data with discharges from nonreporting hospitals distributed to race categories based on county populations and Hispanic discharges nof identified as specific race diatributed to race categories in the proportions of the Hispanio population.

discharges in those hospitals. This procedure is described in the section, "Race for nonreporting hospitals." In addition, Hispanic discharges in the race-not-stated category and the "other race" category were assigned a race using the racial distribution of the Hispanic population as estimated by the U.S. Bureau of the Census.

The population-based adjustment assumes that patients are hospitalized in the same proportions that they are in the population, which is probably not the case (13-15). In addition, the population-based adjustment does not result in an assignment of race to all discharges, only to those from the nonreporting hospitals and Hispanic patients. In 1990-92, 781,000 to 811,000 discharges remained in the race-not-stated category after the population-based adjustment. Thus, the numbers and rates of discharges for specific race categories, while probably more accurate, remain underestimated.

Using either method, adjusted numbers and rates of discharges for white patients were significantly higher than unadjusted estimates. Although calculated differently, the numbers and rates of white discharges produced by proportional adjustment and population- based adjustment were similar. It should be noted, however, that both of these adjustments are expected to underestimate white discharges to some extent. The adjusted estimates of black discharges and discharges of all other races were not significantly different from the unadjusted rates.

\section{Discussion}

Race data from the NHDS became increasingly incomplete in recent years primarily because a growing number of hospitals that participated in the survey did not provide racial data on any of their patients. Most of these hospitals used the automated data collection method. They submitted tapes of data that were usually collected for other purposes to the NHDS. These data were often collected using the National Uniform Bill (UB-82 and UB-92), which does not include an item on race.

One solution to this problem would be for the NHDS to stop using automated data collection. However, even before 1985, when all data were collected manually using NHDS abstracts, the proportion of discharges with no race reported was a concern, ranging from 9-14 percent. In addition, the automated data collection system has become an integral part of the NHDS survey design. Approximately one third of the hospitals that participate in the survey now provide data through the automated method, and many are unwilling to participate in a manual system.

Another solution would be to add race to the UB-92 form. This could greatly increase the amount of race data reported through the automated method. However, the NHDS is only a secondary user of UB-92 data. The principal users, insurance companies and the Health Care Financing Administration, do not view a billing form as the best place to collect race data. They have enrollment forms that provide information on the race of beneficiaries (18).

The other main problem with the NHDS race data, lack of racial information for Hispanic patients, is also related to data collection forms. Hospitals and data systems that use a combined race/ethnicity item cannot supply the NHDS with information on the race of Hispanic patients. The Federal standards for reporting racial and ethnic statistics have been undergoing a wide-ranging review (19). It is uncertain whether one standard approach to reporting will be 
established, or whether it will continue to be acceptable to report race and ethnicity either separately or in a combined format.

In 1990-92, the hospitals that did not report race to the NHDS apparently had a larger proportion of white patients than the reporting hospitals. Estimates based on racial distributions of discharges in previous years and on racial distributions of county populations both suggested that white patients made up a larger share of discharges in nonreporting hospitals than in reporting hospitals. The Hispanic patients not reported by race were also likely to be white in larger proportions than all patients, based on the distribution of those with a reported race and on the racial distribution of the Hispanic population. Therefore, discharges of white patients were probably underestimated to a greater extent than discharges of other racial groups.

Comparisons of NHDS data with data from other sources supported the hypothesis that white patients were disproportionately underestimated. The National Health Interview Survey estimated significantly larger numbers of white discharges than the NHDS, but similar numbers of black discharges. A larger number of Medicare discharges were white according to data from the Health Care Financing Administration than estimated by NHDS. However, the number of Medicare discharges of other races reported by these two sources were not significantly different. The number of live births that were white or black were larger than the NHDS estimates of white or black women hospitalized for deliveries, but differences were greater for the white category.

Because white patients are probably underreported to a greater extent than patients of other races, proportional adjustment of NHDS data would not be expected to produce completely accurate estimates of the number of discharges in each race group. This adjustment would probably produce a more accurate estimate of white discharges, but it would overestimate discharges of other races and would not affect comparisons between racial groups. The populationbased adjustment may also produce a more accurate estimate of white discharges, but because it is based on the assumption that all racial groups have the same discharge rates, the estimates of racial groups with higher rates would be underestimated to some extent, and comparisons between racial groups could be distorted.

At present, no ideal solution exists to eliminate the problem of underreporting of race in the NHDS. Therefore, the NHDS race data need to be used cautiously and not overinterpreted. The data can still be useful for some types of analyses. General inferences can be drawn if the differences between racial groups are large. For example, the rate of HIV hospitalizations for black patients was so much larger than the rate for white patients that even if all the patients in the race-not-stated category were added to the white category, the difference would remain highly significant (2).

When white patients have a higher rate than other racial groups despite the underestimate, such as for coronary artery bypass grafts (20), it is reasonable to conclude that the rate for white patients is higher. Research can also be done on hospital use patterns within racial groups, such as investigation of major diagnostic categories for black patients or sex differences in discharge rates for white patients. In all these areas, though, it must be recognized that the numbers and rates produced from the NHDS for specific racial groups will be underestimated to an unknown extent.

\section{References}

1. Wilcox LS, Koonin LM, Pokras R, et al. Hysterectomy in the United States, 1988-1990. Obstet-Gynecol. 83(4): 549-55. 1994.

2. Kozak LJ, McCarthy E, Moien M. Patterns of hospital use by patients with diagnoses related to HIV infection. Public Health Rep 108(5): 571-81. 1993.

3. Modan B, Wagener DK. Some epidemiological aspects of stroke: Mortality/morbidity trends, age, sex, race, socioeconomic status. Stroke 23(9): 1230-36. 1992.

4. Gergen PJ, Weiss KB. Changing patterns of asthma hospitalization among children: 1979 to 1987. JAMA 264(13): 1688-92. 1990.

5. Saftlas AF, Olson DR, Franks AL, et al. Epidemiology of preeclampsia and eclampsia in the United States, 1979-1986. Am J Obstet Gynecol 163(2): 460-65. 1990.

6. Addiss DG, Shaffer N, Fowler BS, Tauxe RV. The epidemiology of appendicitis and appendectomy in the United States. Am J Epidemiol 132(5): 910-25. 1990.

7. Ford E, Cooper R, Castaner A, et al. Coronary arteriography and coronary bypass survey among whites and other racial groups relative to hospital-based incidence rates for coronary artery disease: Findings from NHDS. Am J Public Health 79(4): 437-40. 1989.

8. Rodriguez JG, Sattin RW, Waxweiler RJ. Incidence of hip fractures, United States, 1970-83. Am J Prev Med 5(3): 175-81. 1989.

9. Gillum RF. Idiopathic cardiomyopathy in the United States, 1970-1982. Am Heart J. 111(4): 752-55. 1986.

10. U.S. Bureau of the Census. General population characteristics. 1990 Census of Population. vol CP 1-2-CP 1-52. Washington. U.S. Department of Commerce. 1992.

11. Office of Management and Budget. Directive no. 15: Race and ethnic standards for Federal statistics and administrative reporting. Statistical Policy Handbook. U.S. Department of Commerce, Office of Federal Statistical Policy and Standards. 1978.

12. U.S. Bureau of the Census. U.S. population estimates, by age, sex, race, and Hispanic origin: 1980 to 1991. Current population reports. series P-25, no. 1095. Washington: U.S. Department of Commerce. 1993.

13. Adams PF, Benson V. Current estimates from the National Health Interview Survey, 1990. National Center for Health Statistics. Vital Health Stat 10(181). 1991.

14. Adams PF, Benson V. Current estimates from the National Health Interview Survey, 1991. National Center for Health Statistics. Vital Health Stat 10(184). 1992.

15. Benson V, Marano MA. Current estimates from the National Health Interview Survey, 1992. National Center for Health Statistics. Vital Health Stat 10(189). 1994. 
16. Helbing C. Hospital insurance short-stay hospital benefits. Health Care Finan Rev. 1992 annual supplement. 55-96. 1993.

17. Ventura SJ, Martin JA, Taffel SM, et al. Advance report of final natality statistics, 1992. Monthly vital statistics report; vol 43 no. 5, suppl. Hyattsville, Maryland: National Center for Health Statistics. 1994.

18. Moore R. Report on HCFA data. Presented to the Subcommittee on Health Statistics for Minority and Other Special Populations, National Committee on Vital and Health Statistics. Sept 14. Washington, D.C. 1994.

19. Office of Management and Budget. Standards for classification of Federal data on race and ethnicity. No. 59. Federal Register. 29831. 1994.

20. Graves EJ. National Hospital Discharge Survey: annual summary, 1992. National Center for Health Statistics. Vital Health Stat 13(119). 1994.

21. SMG Marketing Group, Inc. Hospital Market Database. Chicago, Illinois: Healthcare Information Specialists. 1987.

22. SMG Marketing Group, Inc. Hospital Market Database. Chicago, nlinois: Healthcare Information Specialists. 1991.

23. Simmons WR, Schnack GA. Development of the design of the NCHS Hospital Discharge Survey. National Center for Health Statistics. Vital Health Stat 2(39). 1977.

24. Haupt BJ, Kozak LJ. Estimates from two survey designs: National Hospital Discharge Survey. National Center for Health Statistics. Vital Health Stat 13(111). 1992.

25. Shah BV. SESUDAAN: Standard errors program for computing of standardized rates from sample survey data. Research Triangle Park, North Carolina: Research Triangle Institute. 1981. 


\section{Technical notes}

\section{Survey methodology}

\section{Source of data}

The National Hospital Discharge Survey covers discharges from noninstitutional hospitals, except Federal, military, and Department of Veterans Affairs hospitals located in the 50 States and the District of Columbia. Only short-stay hospitals (hospitals with an average length of stay for all patients of fewer than 30 days) or those whose specialty is general (medical or surgical) or children's general are included in the survey. These hospitals must also have six beds or more staffed for patient use.

From 1988 through 1990, the NHDS sampling frame consisted of hospitals that were listed in the April 1987 SMG Hospital Market Database (21), met the above criteria, and began accepting patients by August 1987. In 1991 the sampling frame was updated to include hospitals from the 1991 SMG Hospital Database (22). The sample consisted of 542 hospitals in 1990 and 528 hospitals in 1991 and 1992. In 1990,23 of the sample hospitals were found to be out of scope (ineligible) because they went out of business or otherwise failed to meet the criteria for the NHDS universe. Seven hospitals were out of scope in 1991, and 14 were out of scope in 1992. In 1990, 474 of the 519 in-scope (eligible) hospitals responded to the survey. In 1991, 484 of 521 in-scope hospitals responded, and 494 of 514 in-scope hospitals responded in 1992.

\section{Sample design and data collection}

The NCHS has conducted the NHDS continuously since 1965 . The original sample was selected in 1964 from a frame of short-stay hospitals listed in the National Master Facility Inventory. That sample was updated periodically with samples of newly opened hospitals. Sample hospitals were selected with probabilities ranging from certainty for the largest hospitals to 1 in 40 for the smallest hospitals. Within each sample hospital, a systematic random sample of discharges was selected. A report on the design and development of the original NHDS has been published (23).

Beginning in 1988, the NHDS sample included with certainty all hospitals with 1,000 beds or more or 40,000 discharges or more annually. The remaining sample of hospitals is based on a stratified three-stage design. The first stage consists of a selection of 112 primary sampling units (PSU's) that comprise a probability subsample of PSU's used in the 1985-94 National Health Interview Survey. The second stage consists of a selection of noncertainty hospitals from the sample PSU's. At the third stage, a sample of discharges was selected by a systematic random sampling technique. A detailed comparison of the old and new survey designs has been published (24).

Two data collection procedures are used for the survey. The first is a manual system of sample selection and data abstraction. The second is an automated method that involves the purchase of data tapes from abstracting service organizations, State data systems, or hospitals. Approximately one third of the respondent hospitals used the automated method in 1990 through 1992.

In the manual system, the sample selection and the transcription of information from the hospital records to abstract forms are performed at the hospitals. The completed forms, along with sample selection control sheets, are forwarded to NCHS for coding, editing, and weighting. Of the hospitals using the manual system, about two-thirds had the work performed by their own medical records staff in 1990 and 1991 and 58 percent in 1992. In the remaining hospitals using the manual system, personnel of the U.S. Bureau of the Census do the work on behalf of NCHS. For the automated system, NCHS purchases tapes containing machinereadable medical record data that are systematically sampled by NCHS.

The medical abstract form and the automated data tapes contain items relating to the personal characteristics of the patients, including birth date, sex, race, and marital status, but not name and address; administrative information, including admission and discharge dates, discharge status, and medical record number; and medical information, including diagnoses and surgical and nonsurgical operations or procedures. Since 1977, patient ZIP Code, expected source of payment, and dates of surgery have also been collected. (The medical record number, birth date, and patient ZIP Code are confidential information and are not available to the public.)

\section{Presentation of estimates}

The relative standard error of the estimate and the number of sample records on which the estimate is based (referred to as the sample size) are used to identify estimates with relatively low reliability.

Because of the complex sample design of the NHDS, estimates of less than 5,000 are not presented; only an asterisk (*) appears in the tables. These estimates generally have a relative standard error of more than 30 percent or are based on a sample of fewer than 30 cases. Estimates based on fewer than 60 cases are preceded by an asterisk (*) to indicate that they should not be assumed to be reliable. These estimates are generally 5,000 to 9,000 .

\section{Sampling errors and rounding of numbers}

The standard error is primarily a measure of sampling variability that occurs by chance because only a sample rather than the entire universe is surveyed. The relative standard error of the estimate is obtained by dividing the standard error by the estimate itself and is expressed as a percent of the estimate. The resulting value is multiplied by 100 , so the relative standard error is expressed as a percent of the estimate.

Estimates of sampling variability were calculated with SESUDAAN software, which computes standard errors by using a first-order Taylor approximation of the deviation of estimates from their expected values. A description of the software and the approach has been published (25).

The constants for relative standard error curves for estimates of discharges by race from the 1990-92 NHDS are presented in table $I$. The relative 
Table I. Estimated parameters for relative standard error equations for number of discharges, by race: National Hospltal Discharge Survey, 1991-92

\begin{tabular}{|c|c|c|c|c|c|c|}
\hline \multirow[b]{2}{*}{ Race } & \multicolumn{2}{|c|}{1990} & \multicolumn{2}{|c|}{1991} & \multicolumn{2}{|c|}{1992} \\
\hline & $a$ & $b$ & $a$ & $b$ & $a$ & $\boldsymbol{b}$ \\
\hline All races $\ldots \ldots \ldots \ldots \ldots \ldots \ldots$ & 0.00213 & 228.834 & 0.00101 & 546.321 & 0.00097 & 449.059 \\
\hline White . . . . . . . . . . . . & 0.00212 & 298.564 & 0.00234 & 927.094 & 0.00241 & 419.274 \\
\hline Black . . . . . . . . . . . . & 0.00537 & 264.999 & 0.00569 & 273.368 & 0.00740 & 363.901 \\
\hline All other races $\ldots \ldots \ldots \ldots \ldots \ldots$ & 0.02899 & 119.661 & 0.02889 & 280.075 & 0.02271 & 182.649 \\
\hline Race not stated. . . . . . . . . . . . & 0.02252 & 226.201 & 0.01666 & 427.619 & 0.01496 & 301.892 \\
\hline
\end{tabular}

standard error $[\operatorname{RSE}(X)]$ of an estimate $X$ may be estimated from the formula:

$$
\operatorname{RSE}(X)=100 \sqrt{a+b / X}
$$

where $X, a$ and $b$ are defined in table I.

Estimates have been rounded to the nearest thousand. For this reason, figures within tables do not always add to the totals. Rates and percents were calculated from original, unrounded figures and will not necessarily agree precisely with rates or percents calculated from rounded data.

\section{Tests of significance}

In general, statistical inference was based on the two-tailed $t$-test using the Bonferroni critical values for post-hoc multiple comparisons ( 0.05 level of significance). Critical values were determined for each set of comparisons, that is, within each table. For comparisons of NHDS estimates with the Medicare data from the Health Care Financing Administration (HCFA) and numbers of live births from birth certificates, confidence intervals at the 95 percent level (plus and minus 1.96 times the standard error) were constructed around the NHDS estimates. If the number of HCFA Medicare discharges or live births fell outside the confidence interval, it was reported as significantly different from the NHDS estimate.

In this report, terms such as "higher" and "less" indicate that differences are statistically significant. Terms such as "similar" or "no difference" mean that no statistically significant difference exits between the estimates being compared. A lack of comment on the difference between any two estimates does not mean that the difference was tested and found to not be significant.

\section{Definitions of terms}

Discharge - The formal release of a patient by a hospital; that is the termination of a period of hospitalization by death or by disposition to place of residence, nursing home, or another hospital. The terms "discharges" and "patients discharged" are used synonymously.

Discharge rate-The ratio of the number of hospital discharges during a year to the number of persons in the civilian population on July 1 of that year.

Ethnicity-In the NHDS, the ethnicity of discharges can be reported in three categories, which are Hispanic origin, non-Hispanic, and not stated.

Hospital-All hospitals with an average length of stay for all patients of less than 30 days or hospitals whose specialty is general (medical or surgical) or children's general are eligible for inclusion in the National Hospital Discharge Survey except Federal hospitals, hospital units of institutions, and hospitals with fewer than six beds staffed for patients' use.

- Reporting hospital -In this report, a reporting hospital is one that reported race for 3-100 percent of discharges.

- Nonreporting hospital-In this report, a nonreporting hospital is one that reported race for less than 3 percent of discharges.

Live birth-A live birth is the complete expulsion or extraction from its mother of a product of conception, irrespective of the duration of the pregnancy, which, after separation, breathes or shows any evidence of life.

Newborn infant-A newborn infant is a patient admitted by birth to the hospital.
Patient-A person who is formally admitted to the inpatient service of a short-stay hospital for observation, care, diagnosis, or treatment is a patient. The terms "patient" and "inpatient" are used synonymously.

Population-The U.S. civilian population, which is the resident population of the United States, excluding members of the Armed Forces, was used to compute rates. The U.S. resident population was used to make population-based adjustments in estimates of discharges by race.

Race - In the NHDS, the race of discharges can be reported in six categories, which are white, black, American Indian/Eskimo/Aleut, Asian/Pacific Islander, other, and not stated.

\section{Symbols}

-. Data not available

... Category not applicable

- Quantity zero

0.0 Quantity more than zero but less than 0.05

Z Quantity more than zero but less than $\mathbf{5 0 0}$ where numbers are rounded to thousands

* Figure does not meet standard of reliability or precision (see Technical notes)

\# $\quad$ Figure suppressed to comply with confidentiality requirements 


\section{Suggested cltation}

Kozak LJ. Underreporting of race in the National Hospital Discharge Survey. Advance data from vital and health statistics; no 265. Hyattsville, Maryland: National Center for Health Statistics. 1995.

\section{Copyright In formation}

All material appearing in this report is in the public domain and may be reproduced or copied without permission; citation as to source, however, is appreciated.
Natlonal Center for Health Statistics

Acting Director

Jack R. Anderson

Acting Deputy Director Jennifer H. Madans, Ph.D.

\section{DEPARTMENT OF \\ HEALTH \& HUMAN SERVICES}

Public Health Service

Centers for Disease Control and Prevention

National Center for Health Statistics

6525 Belcrest Road

Hyattsville, Maryland 20782

OFFICIAL BUSINESS

PENALTY FOR PRIVATE USE, $\$ 300$

To receive this publication regularly, contact the National Center for Health Statistics by calling 301-436-8500

DHHS Publication No. (PHS) 95-1250

5-1327 (6/95)
BULK RATE

POSTAGE \& FEES PAID

PHS/NCHS

PERMIT NO. G-281 


\title{
Urban and Rural Classification of National Health Provider Inventory Providers: United States, 1991
}

\author{
by Renee Delfosse, M.A., Division of Health Care Statistics
}

\section{Highlights}

This report presents information from the National Health Provider Inventory (NHPI) on the numbers of home health care agencies, hospices, nursing homes, and board and care homes classified according to their location. These data are provided to aid in studies of the differential availability and use of health care. Table 1 compares the total numbers of providers according to an urban-rural continuum. Subsequent tables compare the total numbers of providers in urban and rural locations within Cenșus Divisions (table 2) and within States (tables 3-5).

Previous inventories conducted by the National Center for Health Statistics (NCHS) included health providers such as hospitals, homes for the blind, deaf, mentally retarded, and emotionally disturbed in addition to nursing homes and board and care homes. The 1991 inventory includes nursing homes, board and care homes, plus two providers, home health care agencies, and hospices, the large majority of whom care for patients in an outpatient setting, the home (1).

Table 1. Number and percent of home health agencies, hospices, nursing homes, and board and care homes by rural-urban continuum codes: United States, 1991

\begin{tabular}{|c|c|c|c|c|c|c|c|c|c|c|}
\hline \multirow{2}{*}{$\begin{array}{c}\text { Ruraturban continuum codes for motropolttan and } \\
\text { nonmetropolltan counties }\end{array}$} & \multicolumn{2}{|c|}{ Total } & \multicolumn{2}{|c|}{ Home heath care agencles } & \multicolumn{2}{|c|}{ Hosplces } & \multicolumn{2}{|c|}{ Nursing homes } & \multicolumn{2}{|c|}{ Baard and care homes } \\
\hline & Numbór & Porcont & Number & Percent & Number & Percent & Number & Percent & Number & Percent \\
\hline alted States $\ldots \ldots \ldots \ldots \ldots \ldots$ & 41,492 & 100.0 & 6,846 & 16.5 & 946 & 2.3 & 15,487 & 37.3 & 18,213 & 43.9 \\
\hline \multicolumn{11}{|l|}{ Metropolitan countles: } \\
\hline Central countles of 1 million population or more. & 14,403 & 100.0 & 2,098 & 14.6 & 253 & 1.8 & 4,791 & 33.3 & 7,261 & 50.4 \\
\hline Eringe countles of 1 million population or more. & 1,503 & 100.0 & 218 & 14.5 & 43 & 2.9 & 621 & 41.3 & 621 & 41.3 \\
\hline Sountles of 250,000 to 1 million population. . . . . . . & 9,027 & 100.0 & 1,409 & 15.6 & 176 & 1.9 & 3,071 & 34.0 & 4,371 & 48.4 \\
\hline Sounties of fewer then 250,000 population . . . . . . & 3,615 & 100.0 & 613 & 17.0 & 106 & 2.9 & 1,374 & 38.0 & 1,522 & 42.1 \\
\hline \multicolumn{11}{|l|}{ Nonmetropolitan countles: } \\
\hline Jiban population of 20,000 or more, adjacent to a & & & & & & & & & & \\
\hline metropolitan area $\ldots \ldots \ldots \ldots \ldots \ldots \ldots \ldots \ldots \ldots$ or more, not adjacent to a & 1,943 & 100.0 & 315 & 16.2 & 60 & 3.1 & 762 & 39.2 & 806 & 41.5 \\
\hline metropolitan area $\ldots \ldots \ldots \ldots \ldots \ldots \ldots \ldots$ & 1,345 & 100.0 & 245 & 18.2 & 66 & 4.9 & 539 & 40.1 & 495 & 36.8 \\
\hline $\begin{array}{l}\text { metropolitan area } \ldots \ldots \ldots \ldots \ldots \ldots \\
\text { iban population of } 2,500 \quad 19,000, \text { not adjacent to a }\end{array}$ & 4,013 & 100.0 & 719 & 17.9 & 100 & 2.5 & 1,767 & 44.0 & 1,427 & 35.6 \\
\hline metropolltan area $\ldots \ldots \ldots \ldots \ldots \ldots \ldots \ldots$. $\ldots \ldots \ldots$. & 3,663 & 100.0 & 781 & 21.3 & 107 & 2.9 & 1,620 & 44.2 & 1,155 & 31.5 \\
\hline 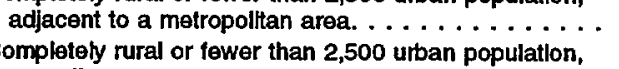 & 702 & 100.0 & 139 & 19.8 & 6 & 0.9 & 338 & 48.1 & 219 & 31.2 \\
\hline not adjacent to a metropolitan area . . . . . . . . . & 1,278 & 100.0 & 309 & 24.2 & 29 & 2.3 & 604 & 47.3 & 336 & 26.3 \\
\hline
\end{tabular}

NOTES: Excludes board and care homes for the mentally retarded. Excludes nonresponding board and care homes. A total of 85 places could not be coded urbanirural.

\section{U.S. DEPARTMENT OF HEALTH AND HUMAN SERVICES \\ Public Health Service \\ Centers for Disease Control and Prevention National Center for Health Statistics}

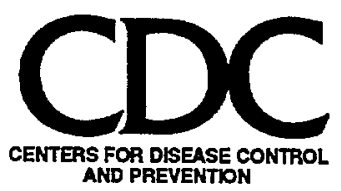


Table 2. Number and percent distribution of home health agencies, hospices, nursing homes, and board and care homes in urban and rural locations by Census Division: United States, 1991

\begin{tabular}{|c|c|c|c|c|c|c|c|c|c|c|}
\hline \multirow[b]{2}{*}{ Divlslon } & \multicolumn{2}{|r|}{ Total } & \multicolumn{2}{|c|}{ Home health care agencles } & \multicolumn{2}{|c|}{ Hospices } & \multicolumn{2}{|c|}{ Nursing homes } & \multicolumn{2}{|c|}{ Board and care homes } \\
\hline & Number & Percent distribution & Number & Percent & Number & Percent & Number & Percent & Number & Percent \\
\hline All locations $\ldots \ldots \ldots \ldots \ldots$ & 41,577 & 100.0 & 6,853 & 16.5 & 951 & 2.3 & 15,511 & 37.3 & 18,262 & 43.9 \\
\hline New England . . . . . . . . . . & 2,666 & 100.0 & 433 & 16.2 & 75 & 2.8 & 1,188 & 44.6 & 970 & 36.4 \\
\hline Middle Atlantic $\ldots \ldots \ldots \ldots \ldots$ & 4,734 & 100.0 & 838 & 17.7 & 82 & 1.7 & 1,604 & 33.9 & 2,210 & 46.7 \\
\hline East North Central . . . . . . . . . . . & 7,209 & 100.0 & 1,062 & 14.7 & 182 & 2.5 & 3,147 & 43.7 & 2,818 & 39.1 \\
\hline West North Central . . . . . . . . & 4,469 & 100.0 & 902 & 20.2 & 111 & 2.5 & 2,269 & 50.8 & 1,187 & 26.6 \\
\hline South Atlantic . . . . . . . . . & 6,819 & 100.0 & 1,129 & 16.6 & 184 & 2.7 & 1,968 & 28.9 & 3,538 & 51.9 \\
\hline East South Central. . . . . . . . . . & 2,465 & 100.0 & 617 & 25.0 & 50 & 2.0 & 946 & 38.4 & 852 & 34.6 \\
\hline West South Central . . . . . . . . . & 3,389 & 100.0 & 945 & 27.9 & 77 & 2.3 & 1,966 & 58.0 & 401 & 11.8 \\
\hline Mountain . . . . . . . . . . & 2,035 & 100.0 & 414 & 20.3 & 69 & 3.4 & 703 & 34.5 & 849 & 41.7 \\
\hline Pacific $\ldots \ldots \ldots \ldots \ldots$ & 7,791 & 100.0 & 513 & 6.6 & 121 & 1.6 & 1,720 & 22.1 & 5,437 & 69.8 \\
\hline Urban localions. . . . . . . . . & 31,836 & 100.0 & 4,898 & 15.4 & 704 & 2.2 & 11,158 & 35.0 & 15,076 & 47.4 \\
\hline New England $\ldots \ldots \ldots \ldots \ldots$ & 2,271 & 100.0 & 390 & 17.2 & 56 & 2.5 & 1,068 & 47.0 & 757 & 33.3 \\
\hline Middle Atlantic . . . . . . . . . & 4,269 & 100.0 & 776 & 18.2 & 76 & 1.8 & 1,469 & 34.4 & 1,948 & 45.6 \\
\hline East North Central . . . . . . . . . & 5,437 & 100.0 & 802 & 14.8 & 126 & 2.3 & 2,364 & 43.5 & 2,145 & 39.5 \\
\hline West North Central . . . . . . . . & 2,128 & 100.0 & 377 & 17.7 & 58 & 2.7 & 1,004 & 47.2 & 689 & 32.4 \\
\hline South Allantic . . . . . . . . . . . & 5,244 & 100.0 & 854 & 16.3 & 144 & 2.7 & 1,479 & 28.2 & 2,767 & 52.8 \\
\hline East South Central. . . . . . . . . & 1,430 & 100.0 & 333 & 23.3 & 35 & 2.4 & 512 & 35.8 & 550 & 38.5 \\
\hline West South Central . . . . . . . . & 2,202 & 100.0 & 637 & 28.9 & 61 & 2.8 & 1,186 & 59.9 & 318 & 14.4 \\
\hline Mountain . . . . . . . . . . . & 1,445 & 100.0 & 263 & 18.2 & 40 & 2.8 & 457 & 31.6 & 685 & 47.4 \\
\hline Paclfic $\ldots \ldots \ldots \ldots \ldots$ & 7,410 & 100.0 & 466 & 6.3 & 108 & 1.5 & 1,619 & 21.8 & 5,217 & 70.4 \\
\hline Rural locallons $\ldots \ldots \ldots \ldots \ldots$ & 9,656 & 100.0 & 1,948 & 20.2 & 242 & 2.5 & 4,329 & 44.8 & 3,137 & 32.5 \\
\hline Now England . . . . . . . . . . & 382 & 100.0 & 43 & 11.3 & 19 & 5.0 & 116 & 30.4 & 204 & 53.4 \\
\hline Middle Atlantic . . . . . . . . . . & 452 & 100.0 & 62 & 13.7 & 6 & 1.3 & 131 & 29.0 & 253 & $\mathbf{5 6 . 0}$ \\
\hline East North Central . . . . . . . . . & 1,766 & 100.0 & 259 & 14.7 & 56 & 3.2 & 782 & 44.3 & 669 & 37.9 \\
\hline West North Central . . . . . . . . & 2,334 & 100.0 & 524 & 22.5 & 53 & 2.3 & 1,263 & 54.1 & 494 & 21.2 \\
\hline South Atlantic . . . . . . . . . . & 1,562 & 100.0 & 273 & 17.5 & $\mathbf{3 7}$ & 2.4 & 487 & 31.2 & 765 & 49.0 \\
\hline East South Central. . . . . . . . . . & 1,027 & 100.0 & 282 & 27.5 & 15 & 1.5 & 431 & 42.0 & 299 & 29.1 \\
\hline West South Central . . . . . . . & 1,184 & 100.0 & 308 & 26.0 & 16 & 1.4 & 777 & 65.6 & 83 & 7.0 \\
\hline Mountaln. . . . . . . . . . & 587 & 100.0 & 151 & 25.7 & 29 & 4.9 & 244 & 41.6 & 163 & 27.8 \\
\hline 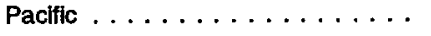 & 362 & 100.0 & 46 & 12.7 & 11 & 3.0 & 98 & 27.1 & 207 & 57.2 \\
\hline
\end{tabular}

NOTES: Excludes board and care homes for the mentally retarded. Excludes nonresponding board and care homes. A total of 85 places could not be coded urbanirural.

There were 18,262 board and care homes, 15,511 nursing homes, 6,853 home health care agencies, and 951 hospices in the United States in 1991 (table 2). Of the 31,836 providers in urban locations, 47 percent were board and care homes, 35 percent were nursing homes, 15 percent were home health care agencies, and 2 percent were hospices. In rural locations the distribution differed: 45 percent of the 9,656 providers were nursing homes, 33 percent were board and care homes, 20 percent were home health care agencies, and 3 percent were hospices.

The majority of patients served by the NHPI providers were elderly. Approximately 75 percent of home health care agency and hospice clients, 92 percent of nursing home patients, and 68 percent of board and care home residents were 65 years and over $(2,3)$. These percentages were found to hold on the divisional level for all providers (4), and on the State level for most nursing homes and board and care homes (tables 6-7).

\section{References}

1. Delfosse R. Hospice and home health agency characteristics: 1991 National Health Provider Inventory. National Center for Health Statistics. Vital Health Stat. In preparation.

2. Strahan G. Overview of home health and bospice care patients:

Preliminary data from the 1992

National Home and Hospice Care Survey. Advance data from vital and health statistics; no 235 . Hyattsville, Maryland: National Center for Health Statistics. 1993.

3. Sirrocco A. Nursing homes and board and care homes. Advance data from vital and health statistics; no 244. Hyattsville, Maryland: National Center for Health Statistics. 1994.
4. National Center for Health Statistics. National Home Health and Hospice Care Survey. Hyattsville, Maryland. 1992.

5. National Center for Health Statistics. The Agency Reporting System for maintaining the national inventory of hospitals and institutions. Vital Health Stat 1(6). 1968.

6. Institute of Medicine. Toward a national health care survey, a data system for the 21st century. Washington: National Academy Press. 1992.

7. Urban-rural continuum codes for metro and nonmetro counties, 1993. USDA, ERS. 
Table 3. Number and percent distribution of home health care agencies, hospices, nureing homes, and board and care homes by State: United States, 1991

\begin{tabular}{|c|c|c|c|c|c|c|c|c|c|c|}
\hline \multirow[b]{2}{*}{ State } & \multicolumn{2}{|r|}{ Total } & \multicolumn{2}{|c|}{ Home health care agencies } & \multicolumn{2}{|c|}{ Hospices } & \multicolumn{2}{|c|}{ Nursing homes } & \multicolumn{2}{|c|}{ Board and care homes } \\
\hline & Number & Percent distribution & Number & Percent & Number & Percent & Number & Percent & Number & Percent \\
\hline Uniled States . . . . . . . & 41,577 & 100.0 & 6,853 & 16.5 & 951 & 2.3 & 15,511 & 37.3 & 18,262 & 43.9 \\
\hline Alabama . . . . . . . . & 561 & 100.0 & 127 & 22.6 & 19 & 3.4 & 214 & 38.1 & 201 & 35.8 \\
\hline Alaska . . . . . . . . & 48 & 100.0 & 10 & 20.8 & 5 & 10.4 & 18 & 37.5 & 15 & $\mathbf{3 1 . 3}$ \\
\hline Arizona. . . . . . . . . & 417 & 100.0 & 71 & 17.0 & 9 & 2.2 & 120 & 28.8 & 217 & 52.0 \\
\hline Arkansas. . . . . . . . & 446 & 100.0 & 133 & 29.8 & 11 & 2.5 & 225 & 50.4 & 77 & 17.3 \\
\hline Callornia. . . . . . . . & 5,316 & 100.0 & 393 & 6.3 & 83 & 1.6 & 1,178 & 22.2 & 3,722 & 70.0 \\
\hline Colorado. . . . . . . . & 515 & 100.0 & 101 & 19.6 & 19 & 3.7 & 191 & 37.1 & 204 & 39.6 \\
\hline Connecticut . . . . . . & 482 & 100.0 & 110 & 22.8 & 9 & 1.9 & 244 & 50.6 & 119 & 24.7 \\
\hline Delaware. . . . . . . . & 141 & 100.0 & 19 & 13.5 & 3 & 2.1 & 47 & 39.3 & 72 & 51.1 \\
\hline District of Columbla . . . & 144 & 100.0 & 18 & 12.5 & 3 & 2.1 & 18 & 12.5 & 105 & 72.9 \\
\hline Florida $\ldots \ldots \ldots \ldots$ & 2,103 & 100.0 & 413 & 19.6 & 34 & 1.6 & 552 & 26.2 & 1,104 & 52.5 \\
\hline Georgla. . . . . . . . . & 935 & 100.0 & 70 & 7.5 & 18 & 1.9 & 342 & 36.6 & 505 & 54.0 \\
\hline Hawall . . . . . . . . & 380 & 100.0 & 18 & 4.7 & 8 & 2.1 & 54 & 14.2 & 300 & 78.9 \\
\hline Idaho ............ & 199 & 100.0 & 26 & 13.1 & 14 & 7.0 & 71 & 35.7 & 88 & 44.2 \\
\hline llilinok. ........... & 1,267 & 100.0 & 309 & 24.4 & 49 & 3.9 & 813 & 64.2 & 96 & 7.6 \\
\hline Indlana . . . . . . . . & 744 & 100.0 & 150 & 20.2 & 13 & 1.7 & 531 & 71.4 & 50 & 6.7 \\
\hline lowa. . . . . . . . . & 767 & 100.0 & 155 & 20.2 & 25 & 3.3 & 459 & 59.8 & 128 & 16.7 \\
\hline Kansas............ & 698 & 100.0 & 153 & 21.9 & 23 & 3.3 & 386 & 55.3 & 136 & 19.5 \\
\hline Kentucky. . . . . . . . & 728 & 100.0 & 112 & 15.4 & 19 & 2.6 & 287 & 39.4 & 310 & 42.6 \\
\hline Loulslana. . . . . . . . . & 561 & 100.0 & 180 & 32.1 & 11 & 2.0 & 319 & 56.9 & 51 & 9.1 \\
\hline Malne. . . . . . . . . & 403 & 100.0 & 35 & 8.7 & 15 & 3.7 & 138 & 34.2 & 215 & 53.3 \\
\hline Maryland. . . . . . . . & 580 & 100.0 & 96 & 16.6 & 25 & 4.3 & 219 & 37.8 & 240 & 41.4 \\
\hline Massachusetts . . . . . . & 1,063 & 100.0 & 166 & 15.6 & 26 & 2.4 & 560 & 52.7 & 311 & 29.3 \\
\hline Mlchigan . . . . . . . & 2,497 & 100.0 & 196 & 7.8 & 57 & 2.3 & 495 & 19.8 & 1,749 & 70.0 \\
\hline Minnesota . . . . . . . & 1,022 & 100.0 & 196 & 19.2 & 33 & 3.2 & 451 & 44.1 & 342 & 33.5 \\
\hline Mlssiksippl. . . . . . . . . & 355 & 100.0 & 116 & 32.7 & 1 & 0.3 & 160 & 45.1 & 78 & 22.0 \\
\hline Mlssourd . . . . . . . . & 1,115 & 100.0 & 173 & 15.5 & 15 & 1.3 & 535 & 48.0 & 392 & 35.2 \\
\hline Montana . . . . . . . & 225 & 100.0 & 49 & 21.8 & 12 & 5.3 & 98 & 43.6 & 66 & 29.3 \\
\hline Nebraska. . . . . . . . . & 395 & 100.0 & 75 & 19.0 & 6 & 1.5 & 229 & 58.0 & 85 & 21.5 \\
\hline Nevada. . . . . . . . . & 138 & 100.0 & 28 & 20.3 & 2 & 1.4 & 34 & 24.6 & 74 & 53.6 \\
\hline New Hampshire . . . . . & 295 & 100.0 & 60 & 20.3 & 13 & 4.4 & 87 & 29.5 & 135 & 45.8 \\
\hline Now Jersey . . . . . . . . & 762 & 100.0 & 111 & 14.6 & 19 & 2.5 & 319 & 41.9 & 313 & 41.1 \\
\hline Now Mexico . . . . . . . . & 250 & 100.0 & 45 & 18.0 & 3 & 1.2 & 68 & 27.2 & 134 & 53.6 \\
\hline Now York. . . . . . . . . & 1,904 & 100.0 & 410 & 21.5 & 35 & 1.8 & 595 & 31.3 & 864 & 45.4 \\
\hline North Carolina . . . . . & 1,210 & 100.0 & 155 & 12.8 & 48 & 4.0 & 309 & 25.5 & 698 & 57.7 \\
\hline North Dakota . . . . . . . & 200 & 100.0 & 70 & 35.0 & 2 & 1.0 & 85 & 42.5 & 43 & 21.5 \\
\hline Ohb. ............ & 1,570 & 100.0 & 252 & 16.1 & 39 & 2.5 & 877 & 55.9 & 402 & 25.6 \\
\hline OWahoma . . . . . . . & 532 & 100.0 & 75 & 14.1 & 13 & 2.4 & 389 & 73.1 & 55 & 10.3 \\
\hline Oregon............ & 1,203 & 100.0 & 71 & 5.9 & 14 & 1.2 & 191 & 15.9 & 927 & 77.1 \\
\hline Pennsytvania . . . . . . & 2,068 & 100.0 & 317 & 15.3 & 28 & 1.4 & 690 & 39.4 & 1,030 & 50.0 \\
\hline Rhode Island . . . . . . & 204 & 100.0 & 43 & 21.1 & 5 & 2.5 & 104 & 51.0 & 52 & 25.5 \\
\hline South Carollna . . . . . . & 506 & 100.0 & 101 & 20.0 & 13 & 2.6 & 140 & 27.7 & 252 & 49.8 \\
\hline South Dakota . . . . . . . & 272 & 100.0 & 80 & 29.4 & 7 & 2.6 & 124 & 45.6 & 61 & 22.4 \\
\hline Tenneseses. . . . . . . . . & 821 & 100.0 & 262 & 31.9 & 11 & 1.3 & 285 & 34.7 & 263 & 32.0 \\
\hline Taxas . . . . . . . . . . & 1,850 & 100.0 & 557 & 30.1 & 42 & 2.3 & 1,033 & 55.8 & 218 & 11.8 \\
\hline Utah. . . . . . . . . & 193 & 100.0 & 53 & 27.5 & 4 & 2.1 & 90 & 46.6 & 46 & 23.8 \\
\hline Vermont . . . . . . . . & 219 & 100.0 & 19 & 8.7 & 7 & 3.2 & 55 & 25.1 & 138 & 63.0 \\
\hline Virginia . . . . . . . . & 788 & 100.0 & 193 & 24.5 & 28 & 3.6 & 227 & 28.8 & 340 & 43.1 \\
\hline Washington . . . . . . . & 844 & 100.0 & 81 & 9.6 & 11 & 1.3 & 279 & 33.1 & 473 & 56.0 \\
\hline West Virginla . . . . . . & 412 & 100.0 & 64 & 15.5 & 12 & 2.9 & 114 & 27.7 & 222 & 53.9 \\
\hline Wlsconsin ......... & 1,131 & 100.0 & 155 & 13.7 & 24 & 2.1 & 431 & 38.1 & 521 & 46.1 \\
\hline Wyoming. . . . . . . . & 98 & 100.0 & 41 & 41.8 & 6 & 6.1 & 31 & 31.6 & 20 & 20.4 \\
\hline
\end{tabular}

NOTES: Exeludes board and care homes for the mentally retarded. Excludes a percentage of nonresponding board and care homes. 
Table 4. Number and percent of home health agencies, hosplces, nursing homes, and board and care homes In urban areas: United States, 1991

\begin{tabular}{|c|c|c|c|c|c|c|c|c|c|c|}
\hline \multirow[b]{2}{*}{ State } & \multicolumn{2}{|r|}{ Total } & \multicolumn{2}{|c|}{ Home health care agencles } & \multicolumn{2}{|c|}{ Hosplces } & \multicolumn{2}{|c|}{ Nursing homes } & \multicolumn{2}{|c|}{ Board and care homes } \\
\hline & Number & Percent dlstribution & Number & Percent & Number & Percent & Number & Percent & Number & Percent \\
\hline United States . . . . . . & 31,836 & 100.0 & 4,898 & 15.4 & 704 & 2.2 & 11,158 & 35.0 & 15,076 & 47.4 \\
\hline Alabama . . . . . . . & 386 & 100.0 & 72 & 18.7 & 14 & $\mathbf{3 . 6}$ & 141 & 36.5 & 159 & 41.2 \\
\hline Alaska ........... & 27 & 100.0 & 6 & 22.2 & 2 & 7.4 & 9 & 33.3 & 10 & 37.0 \\
\hline Arlzona . . . . . . . . . & 411 & 100.0 & 70 & 17.0 & 9 & 2.2 & 117 & 28.5 & 215 & 52.3 \\
\hline Arkansas. . . . . . . & 200 & 100.0 & 39 & 19.5 & 6 & 3.0 & 104 & 52.0 & 51 & 25.5 \\
\hline California. . . . . . . . & 5,187 & 100.0 & 320 & 6.2 & 75 & 1.4 & 1,151 & 22.2 & 3,641 & 70.2 \\
\hline Colorado . . . . . . . . & 374 & 100.0 & 69 & 18.4 & 10 & 2.7 & 129 & 34.5 & 166 & 44.4 \\
\hline Connectlcut . . . . . . & 480 & 100.0 & 110 & 22.9 & 9 & 1.9 & 244 & 50.8 & 117 & 24.4 \\
\hline Delaware. . . . . . . . . & 104 & 100.0 & 12 & 11.5 & 2 & 1.9 & 33 & 31.7 & 57 & 54.8 \\
\hline District of Columbla . . . . & 144 & 100.0 & 18 & 12.5 & 3 & 2.1 & 18 & 12.5 & 105 & 72.9 \\
\hline Florlda . . . . . . . . & 1,963 & 100.0 & 377 & 19.2 & 33 & 1.7 & 515 & 26.2 & 1,038 & 52.9 \\
\hline Georgla. . . . . . . . . & 582 & 100.0 & 41 & 7.0 & 16 & 2.7 & 186 & 32.0 & 339 & 58.2 \\
\hline Hawail . . . . . . . . & 377 & 100.0 & 18 & 4.8 & 8 & 2.1 & 54 & 14.3 & 297 & 78.8 \\
\hline Idaho ............ & 111 & 100.0 & 15 & 13.5 & 8 & 7.2 & 31 & 27.9 & 57 & 51.4 \\
\hline Illinois. . . . . . . . . . & 962 & 100.0 & 250 & 26 & 39 & 4.1 & 593 & 61.6 & 80 & 8.3 \\
\hline Indlana . . . . . . . . & 527 & 100.0 & 103 & 19.5 & 12 & 2.3 & 373 & 70.8 & 39 & 7.4 \\
\hline lowa. ........... & 298 & 100.0 & 56 & 18.8 & 9 & 3.0 & 177 & 59.4 & 56 & 18.8 \\
\hline Kansas . . . . . . . . . & 351 & 100.0 & 74 & 21.1 & 14 & 4.0 & 159 & 45.3 & 104 & 29.6 \\
\hline Kentucky. . . . . . . . . . & 328 & 100.0 & 48 & 14.6 & 11 & 3.4 & 125 & 38.1 & 144 & 43.9 \\
\hline Loutsiana. . . . . . . . & 412 & 100.0 & 134 & 32.5 & 9 & 2.2 & 223 & 54.1 & 46 & 11.2 \\
\hline Maine. . . . . . . . . & 275 & 100.0 & 25 & 9.1 & 9 & $\mathbf{3 . 3}$ & 89 & 32.4 & 152 & 55.3 \\
\hline Maryland . . . . . . . . & 532 & 100.0 & 84 & 15.8 & 23 & 4.3 & 199 & 37.4 & 226 & 42.5 \\
\hline Massachusetts . . . . . & 1,053 & 100.0 & 164 & 15.6 & 24 & 2.3 & 557 & 52.9 & 308 & 29.2 \\
\hline Michigan . . . . . . . . & 1,810 & 100.0 & 135 & 7.5 & 27 & 1.5 & 378 & 20.9 & 1,270 & 70.2 \\
\hline Minnesota . . . . . . . & 578 & 100.0 & 98 & 17.0 & 13 & 2.2 & 242 & 41.9 & 225 & 38.9 \\
\hline Mississippi . . . . . . . . . . & 172 & 100.0 & 42 & 24.4 & 1 & 0.6 & 68 & 39.5 & 61 & 35.5 \\
\hline Missourl . . . . . . . . & 615 & 100.0 & 95 & 15.4 & 11 & 1.8 & 291 & 47.3 & 218 & 35.4 \\
\hline Montana . . . . . . . . & 88 & 100.0 & 12 & 13.6 & 4 & 4.5 & 30 & 34.1 & 42 & 47.7 \\
\hline Nebraska. . . . . . . . . & 175 & 100.0 & 31 & 17.7 & 5 & 2.9 & 85 & 48.6 & 54 & 30.9 \\
\hline Nevada. . . . . . . . . & 117 & 100.0 & 21 & 17.9 & 2 & 1.7 & 25 & 21.4 & 69 & 59.0 \\
\hline New Hampshire ...... & 217 & 100.0 & 45 & 20.7 & 9 & 4.1 & 66 & 30.4 & 97 & 44.7 \\
\hline New Jersey . . . . . . . . & 750 & 100.0 & 111 & 14.8 & 19 & 2.5 & 315 & 42.0 & 305 & 40.7 \\
\hline Now Mexico . . . . . . . & 168 & 100.0 & 30 & 17.9 & 3 & 1.8 & 47 & 28.0 & 88 & 52.4 \\
\hline New York. . . . . . . . . & 1,751 & 100.0 & 383 & 21.9 & 33 & 1.9 & 555 & 31.7 & 780 & 44.5 \\
\hline North Carollna . . . . . . & 786 & 100.0 & 95 & 12.1 & 28 & 3.6 & 208 & 26.5 & 455 & 57.9 \\
\hline North Dakota . . . . . . . . & 49 & 100.0 & 13 & 26.5 & 2 & 4.1 & 20 & 40.8 & 14 & 28.6 \\
\hline Ohlo.............. & 1,367 & 100.0 & 214 & 15.7 & 31 & 2.3 & 750 & 54.9 & 372 & 27.2 \\
\hline Okahoma ......... & 277 & 100.0 & 43 & 15.5 & 11 & 4.0 & 197 & 71.1 & 26 & 9.4 \\
\hline Oregon . . . . . . . . & 1,056 & 100.0 & 49 & 4.6 & 13 & 1.2 & 163 & 15.4 & 831 & 78.7 \\
\hline Pennsylvania . . . . . . & 1,768 & 100.0 & 282 & 16.0 & 24 & 1.4 & 599 & 33.9 & 863 & 48.8 \\
\hline Rhode Island . . . . . . . & 199 & 100.0 & 43 & 21.6 & 5 & 2.5 & 101 & 50.8 & 50 & 25.1 \\
\hline South Carollna . . . . . . & 377 & 100.0 & 67 & 17.8 & 9 & 2.4 & 102 & 27.1 & 199 & 52.8 \\
\hline South Dakota . . . . . . . & 62 & 100.0 & 10 & 16.1 & 4 & 6.5 & 30 & 48.4 & 18 & 29.0 \\
\hline Tennessee . . . . . . . . & 544 & 100.0 & 171 & 31.4 & 9 & 1.7 & 178 & 32.7 & 186 & 34.2 \\
\hline Texas . . . . . . . . . . & 1,313 & 100.0 & 421 & 32.1 & 35 & 2.7 & 662 & 50.4 & 195 & 14.9 \\
\hline Utah. . . . . . . . . . & 146 & 100.0 & 32 & 21.9 & 2 & 1.4 & 70 & 47.9 & 42 & 28.8 \\
\hline Vermont . . . . . . . . & 47 & 100.0 & 3 & 6.4 & 11 & 23.4 & 33 & 70.2 & - & - \\
\hline Virginia . . . . . . . & 547 & 100.0 & 126 & 23.0 & 21 & 3.8 & 159 & 29.1 & 241 & 44.1 \\
\hline Washington ........ & 763 & 100.0 & 73 & 9.6 & 10 & 1.3 & 242 & 31.7 & 438 & 57.4 \\
\hline West Virglnla . . . . . . & 209 & 100.0 & 34 & 16.3 & 9 & 4.3 & 59 & 28.2 & 107 & 51.2 \\
\hline Wisconsin . . . . . . . & 771 & 100.0 & 100 & 13.0 & 17 & 2.2 & 270 & 35.0 & 384 & 49.8 \\
\hline Wyoming. . . . . . . . & 30 & 100.0 & 14 & 46.7 & 2 & 6.7 & 8 & 26.7 & 6 & 20.0 \\
\hline
\end{tabular}

NOTES: Exeludes board and care homes for the mentally retarded. Excludes nonresponding board and care homes. A total of 85 places could not be coded urbanirural. 
Table 5. Number and percent of home health agencles, hospices, nursing homes, and board and care homes in rural areas: United State8, 1991

\begin{tabular}{|c|c|c|c|c|c|c|c|c|c|c|}
\hline \multirow[b]{2}{*}{ State } & \multicolumn{2}{|r|}{ Total } & \multicolumn{2}{|c|}{ Home heahth care agencies } & \multicolumn{2}{|c|}{ Hosplces } & \multicolumn{2}{|c|}{ Nursing homes } & \multicolumn{2}{|c|}{ Board and care homes } \\
\hline & Number & Percent dlstribution & Number & Percent & Number & Percent & Number & Percent & Number & Percent \\
\hline United States . . . . . . . & 9,656 & 100.0 & 1,948 & 20.2 & 242 & 2.5 & 4,329 & 44.8 & 3,137 & 32.5 \\
\hline Alabama . . . . . . . . & 167 & 100.0 & 53 & 31.7 & 5 & $\mathbf{3 . 0}$ & 70 & 41.9 & 39 & 23.4 \\
\hline Alaska . . . . . . . . & 17 & 100.0 & 3 & 17.6 & 1 & 5.9 & 9 & 52.9 & 4 & 23.5 \\
\hline 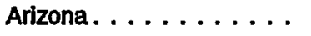 & 5 & 100.0 & 1 & 20.0 & 2 & 40.0 & 2 & 40.0 & - & - \\
\hline Arkansas. . . . . . . & 246 & 100.0 & 94 & 38.2 & 5 & 2.0 & 121 & 49.2 & 26 & 10.6 \\
\hline California. . . . . . . . . & 124 & 100.0 & 13 & 10.5 & 8 & 6.5 & 25 & 20.2 & 78 & 62.9 \\
\hline Colorado .......... & 140 & 100.0 & 32 & 22.9 & 9 & 6.4 & 62 & 44.3 & 37 & 26.4 \\
\hline Connecticut . . . . . . . & $\cdots$ & $\cdots$ & $\cdots$ & & & $\cdots$ & & .. & $\cdots$ & $\ldots$ \\
\hline Delaware. . . . . . . . . . & 36 & 100.0 & 7 & 19.4 & 1 & 2.8 & 13 & 36.1 & 15 & 41.7 \\
\hline District of Columbla . . . . & $\cdots$ & $\cdots$ & $\ldots$ & $\cdots$ & $\cdots$ & $\cdots$ & $\ldots$ & $\cdots$ & $\cdots$ & $\ldots$ \\
\hline Florida .......... . & 137 & 100.0 & 36 & 26.3 & 1 & 0.7 & 37 & 27.0 & 63 & 46.0 \\
\hline Georgla. . . . . . . . . . & 351 & 100.0 & 28 & 8.0 & 2 & 0.6 & 155 & 44.2 & 166 & 47.3 \\
\hline Hawall .......... & $\cdots$ & $\cdots$ & $\cdots$ & $\ldots$ & $\cdots$ & $\cdots$ & $\cdots$ & $\ldots$ & $\ldots$ & $\cdots$ \\
\hline Idaho . . . . . . . . . . & 88 & 100.0 & 11 & 12.5 & 6 & 6.8 & 40 & 45.5 & 31 & 35.2 \\
\hline Illinois. . . . . . . . . . & 305 & 100.0 & 59 & 19.3 & 10 & 3.3 & 220 & 72.1 & 16 & 5.2 \\
\hline Indlana . . . . . . . . . & 217 & 100.0 & 47 & 21.7 & 1 & 0.5 & 158 & 72.8 & 11 & 5.1 \\
\hline lowa. ............. & 466 & 100.0 & 99 & 21.2 & 16 & 3.4 & 281 & 60.3 & 70 & 15.0 \\
\hline 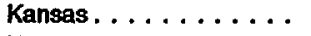 & 345 & 100.0 & 78 & 22.6 & 9 & 2.6 & 227 & 65.8 & 31 & 9.0 \\
\hline Kentucky........... & 400 & 100.0 & 64 & 16.0 & 8 & 2.0 & 162 & 40.5 & 166 & 41.5 \\
\hline Louislana. . . . . . . . . & 149 & 100.0 & 46 & 30.9 & 2 & 1.3 & 96 & 64.4 & 5 & 3.4 \\
\hline Malne. . . . . . . . . . & 126 & 100.0 & 10 & 7.9 & 6 & 4.8 & 49 & 38.9 & 61 & 48.4 \\
\hline Maryland. . ........ & 47 & 100.0 & 12 & 25.5 & 2 & 4.3 & 20 & 42.6 & 13 & 27.7 \\
\hline Massachusetts ...... & $\cdots$ & $\cdots$ & $\cdots$ & $\cdots$ & $\cdots$ & $\ldots$ & $\cdots$ & $\cdots$ & $\cdots$ & $\ldots$ \\
\hline Michigan . . . . . . . . & 682 & 100.0 & 60 & $\mathbf{8 . 8}$ & 30 & 4.4 & 117 & 17.2 & 475 & 69.6 \\
\hline Minnesota . . . . . . . & 444 & 100.0 & 98 & 22.1 & 20 & 4.5 & 209 & 47.1 & 117 & 26.4 \\
\hline Mlssissippl. . . . . . . . . . & 183 & 100.0 & 74 & 40.4 & 92 & 50.3 & 17 & 9.3 & - & - \\
\hline Mlssouri . . . . . . . . & 498 & 100.0 & 78 & 15.7 & 4 & 0.8 & 243 & 48.8 & 173 & 34.7 \\
\hline 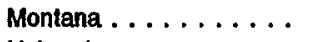 & 137 & 100.0 & 37 & 27.0 & 8 & 5.8 & 68 & 49.6 & 24 & 17.5 \\
\hline Nebraska. . . . . . . . . & 220 & 100.0 & 44 & 20.0 & 1 & 0.5 & 144 & 65.5 & 31 & 14.1 \\
\hline Nevada. . . . . . . . . . & 21 & 100.0 & 7 & 33.3 & 9 & 42.9 & 5 & 23.8 & - & - \\
\hline New Hampshire ..... . & 78 & 100.0 & 15 & 19.2 & 4 & 5.1 & 21 & 26.9 & 38 & 48.7 \\
\hline New Jersey . . . . . . . & $\cdots$ & $\cdots$ & $\cdots$ & $\cdots$ & $\cdots$ & $\cdots$ & $\cdots$ & $\cdots$ & $\cdots$ & $\cdots$ \\
\hline New Mexico. . . . . . . . . . & 82 & 100.0 & 15 & 18.3 & 21 & 25.6 & 46 & 56.1 & - & - \\
\hline New York. . . . . . . . . . & 153 & 100.0 & 27 & 17.6 & 2 & 1.3 & 40 & 26.1 & 84 & 54.9 \\
\hline North Carolina ....... & 424 & 100.0 & 60 & 14.2 & 20 & 4.7 & 101 & 23.8 & 243 & 57.3 \\
\hline North Dakota . . . . . . . . & 151 & 100.0 & 57 & 37.7 & 65 & 43.0 & 29 & 19.2 & - & - \\
\hline 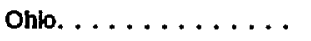 & 203 & 100.0 & 38 & 18.7 & 8 & 3.9 & 127 & 62.6 & 30 & 14.8 \\
\hline Oklahoma . . . . . . . & 255 & 100.0 & 32 & 12.5 & 2 & 0.8 & 192 & 75.3 & 29 & 11.4 \\
\hline Oregon ............ & 144 & 100.0 & 22 & 15.3 & 1 & 0.7 & 28 & 19.4 & 93 & 64.6 \\
\hline Pennsylvania . . . . . . . & 299 & 100.0 & 35 & 11.7 & 4 & 1.3 & 91 & 30.4 & 169 & 56.5 \\
\hline Fhode Istand . . . . . . & $\cdots$ & $\ldots$ & $\ldots$ & $\cdots$ & $\cdots$ & $\cdots$ & $\cdots$ & $\cdots$ & $\ldots$ & $\cdots$ \\
\hline South Carolina . . . . . . . & 125 & 100.0 & 3 & 26.4 & 3 & 2.4 & 38 & 30.4 & $\mathbf{5 1}$ & 40.8 \\
\hline South Dakota . . . . . . . . & 210 & 100.0 & 70 & 33.3 & 3 & 1.4 & 94 & $\mathbf{4 4 . 8}$ & 43 & 20.5 \\
\hline Tennessee. . . . . . . . & 277 & 100.0 & 91 & 32.9 & 2 & 0.7 & 107 & 38.6 & 77 & 27.8 \\
\hline Toxas ............ & 534 & 100.0 & 136 & 25.5 & 7 & 1.3 & 368 & 68.9 & 23 & 4.3 \\
\hline Utah. . . . . . . . . . & 46 & 100.0 & 21 & 45.7 & 2 & 4.3 & 19 & 41.3 & 4 & 8.7 \\
\hline Vermont ......... & 172 & 100.0 & 16 & 9.3 & 7 & 4.1 & 44 & 25.6 & 105 & 61.0 \\
\hline Virginia . . . . . . . . . & 239 & 100.0 & 67 & 28.0 & 5 & 2.1 & 68 & 28.5 & 99 & 41.4 \\
\hline Washington ........ & 77 & 100.0 & 8 & 10.4 & 1 & 1.3 & 36 & 46.8 & 32 & 41.6 \\
\hline West Virginla ....... & 203 & 100.0 & 30 & 14.8 & 3 & 1.5 & 55 & 27.1 & 115 & 56.7 \\
\hline Wisconsin . . . . . . & 359 & 100.0 & 55 & 15.3 & 7 & 1.9 & 160 & 44.6 & 137 & 38.2 \\
\hline Wyoming. . . . . . . & 68 & 100.0 & 27 & 39.7 & 4 & 5.9 & 23 & 33.8 & 14 & 20.6 \\
\hline
\end{tabular}

NOTES: Excludes board and care homes for the mentaly retarded. Excludes nonresponding board and care homes. A total of 85 places could not be coded urban/rural. 
Table 6. Number of residents 65 years of age and over in board and care homes: United States, 1991

\begin{tabular}{|c|c|c|c|c|}
\hline State & Residents & $\begin{array}{c}\text { Residents } \\
\text { with reported age }\end{array}$ & $\begin{array}{l}\text { Residents } \\
65 \text { years of age and over }\end{array}$ & $\begin{array}{l}\text { Percent of all residents } \\
65 \text { years of age and over }\end{array}$ \\
\hline & \multicolumn{3}{|c|}{ Number } & \\
\hline United States $\ldots \ldots \ldots \ldots \ldots \ldots \ldots$ & 302,820 & 267,514 & 182,469 & 68.2 \\
\hline Alabama $\ldots \ldots \ldots \ldots \ldots \ldots \ldots$ & 2,645 & 2,351 & 1,328 & 56.5 \\
\hline$\ldots \ldots \ldots \ldots \ldots \ldots \ldots$ & 149 & 146 & 15 & 10.3 \\
\hline Arizona $\ldots \ldots \ldots \ldots \ldots \ldots \ldots \ldots$ & 3,692 & 3,150 & 2,291 & 72.7 \\
\hline Arkansas. $\ldots \ldots \ldots \ldots \ldots \ldots \ldots \ldots$ & 1,815 & 1,681 & 1,051 & 62.5 \\
\hline 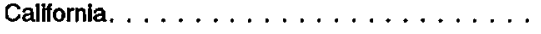 & 54,722 & 48,448 & 35,384 & 73.0 \\
\hline Colorado . . . . . . . $\ldots \ldots \ldots \ldots \ldots$ & 3,698 & $\mathbf{3 , 4 2 1}$ & 2,569 & 75.1 \\
\hline Connectlcut $\ldots \ldots \ldots \ldots \ldots \ldots \ldots$ & 2,298 & 1,981 & 1,330 & 67.1 \\
\hline Delaware. $\ldots \ldots \ldots \ldots \ldots \ldots \ldots \ldots$. & 500 & 304 & 175 & 57.6 \\
\hline District of Columbia $\ldots \ldots \ldots \ldots \ldots \ldots$ & 1,408 & 1,082 & 414 & 38.3 \\
\hline Florida $\ldots \ldots \ldots \ldots \ldots \ldots \ldots \ldots$ & 27,529 & 22,617 & 17,953 & 79.4 \\
\hline Georgla. $\ldots \ldots \ldots \ldots \ldots \ldots \ldots \ldots$ & 4,532 & 3,976 & 2,728 & 68.6 \\
\hline Hawall $\ldots \ldots \ldots \ldots \ldots \ldots \ldots$ & 2,267 & 1,289 & 849 & 65.9 \\
\hline Idaho $\ldots \ldots \ldots \ldots \ldots \ldots \ldots$ & 1,429 & 1,135 & 835 & 73.6 \\
\hline illinols. $\ldots \ldots \ldots \ldots \ldots \ldots \ldots \ldots$ & 3,064 & 2,984 & 1,685 & 56.5 \\
\hline Indiana $\ldots \ldots \ldots \ldots \ldots \ldots \ldots \ldots$ & 1,396 & 1,289 & 603 & 46.8 \\
\hline lowa. $\ldots \ldots \ldots \ldots \ldots \ldots \ldots \ldots$ & 4,340 & 3,861 & 1,637 & 42.4 \\
\hline Kansas.................. & 810 & 754 & 245 & 32.5 \\
\hline 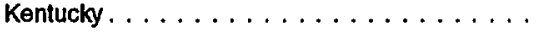 & 3,442 & 3,018 & 1,846 & 61.2 \\
\hline Louisiana. . . . . . . . . . . . . . . . & 697 & 641 & 67 & 10.5 \\
\hline Maine. . . . . . . $\ldots \ldots \ldots \ldots \ldots$ & 2,768 & 2,541 & 1,834 & $7^{\prime} 2.2$ \\
\hline 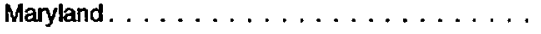 & 2,373 & 2,233 & 1,318 & 59.0 \\
\hline Massachusetts $\ldots \ldots \ldots \ldots \ldots \ldots \ldots$ & 5,767 & 5,538 & 3,023 & 54.6 \\
\hline Michigan $\ldots \ldots \ldots \ldots \ldots \ldots \ldots$ & 18,190 & 16,331 & 10,579 & 64.8 \\
\hline 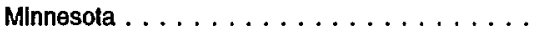 & 5,017 & 4,502 & 1,178 & 26.2 \\
\hline Mississippi. . . . . . . . . . . . . . . & 1,131 & 930 & 554 & 59.6 \\
\hline 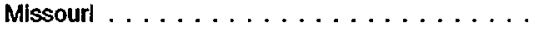 & 7,556 & 6,887 & 4,237 & 61.5 \\
\hline Montana $\ldots \ldots \ldots \ldots \ldots \ldots \ldots$ & 1,101 & 961 & 793 & 82.5 \\
\hline Nebraska. . . . . . . . . . . . . . & 2,005 & 1,942 & 1,349 & 69.5 \\
\hline Nevada. . . . . . . . . . . . . . . & 812 & 612 & 400 & 65.4 \\
\hline New Hampshire $\ldots \ldots \ldots \ldots \ldots \ldots \ldots$ & 1,329 & 1,204 & 891 & 74.0 \\
\hline New Jersey $\ldots \ldots \ldots \ldots \ldots \ldots \ldots \ldots$ & 8,174 & 6,938 & 4,186 & 60.3 \\
\hline New Mexico $\ldots \ldots \ldots \ldots \ldots \ldots \ldots$ & 1,501 & 1,412 & 520 & 36.8 \\
\hline New York. . . . . . . . . . . . . . & 27,544 & 25,145 & 16,660 & 66.3 \\
\hline North Carollna $\ldots \ldots \ldots \ldots \ldots \ldots \ldots$ & 15,321 & 13,589 & 10,099 & 74.3 \\
\hline North Dakola $\ldots \ldots \ldots$ & 910 & 793 & 683 & 86.1 \\
\hline Ohlo. $\ldots \ldots \ldots \ldots \ldots \ldots \ldots \ldots$ & 4,234 & 3,800 & 1,908 & 50.2 \\
\hline OKahoma $\ldots \ldots \ldots \ldots \ldots \ldots \ldots$ & 1,435 & 1,349 & 557 & 41.3 \\
\hline Oregon,$\ldots \ldots \ldots \ldots \ldots \ldots \ldots \ldots$ & 6,886 & 5,717 & 4,814 & 84.2 \\
\hline Pennsylvania . . . . . . . . . . . . . . . . & 23,811 & 21,210 & 16,271 & 76.7 \\
\hline Rhode Island $\ldots \ldots \ldots \ldots \ldots \ldots \ldots$ & 920 & 574 & 308 & 53.7 \\
\hline South Carolina $\ldots \ldots \ldots \ldots \ldots \ldots \ldots$ & 4,810 & 4,146 & 2,704 & 65.2 \\
\hline South Dakota . . . . . . . . . . . . . . . . . & 331 & 305 & 175 & 57.4 \\
\hline Tennessere. . . . . . . . . . . . . . . . . & $\mathbf{3 , 7 9 9}$ & 3,440 & 2,507 & 72.9 \\
\hline Texas . . . . . . . . . . . . . . . & 3,899 & 3,425 & 2,158 & 63.0 \\
\hline Utah. . . . . . . . . . . . . . . & 1,250 & 962 & 816 & 84.8 \\
\hline Vermont $\ldots \ldots \ldots \ldots \ldots \ldots \ldots \ldots$ & 1,830 & 1,679 & 1,285 & 76.5 \\
\hline Virginia $\ldots \ldots \ldots \ldots \ldots \ldots \ldots \ldots$ & 10,296 & 9,556 & 6,810 & 71.3 \\
\hline Washington $\ldots \ldots \ldots \ldots \ldots \ldots \ldots$ & 8,489 & 7,374 & 5,233 & 71.0 \\
\hline West Virginla $\ldots \ldots \ldots \ldots \ldots \ldots \ldots$ & 1,996 & 1,819 & 1,428 & 78.5 \\
\hline Wisconsin $\ldots \ldots \ldots \ldots \ldots \ldots \ldots$ & 6,464 & 6,110 & 3,913 & 64.0 \\
\hline 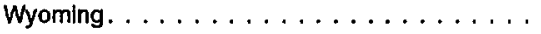 & 438 & 362 & 273 & 75.4 \\
\hline
\end{tabular}

NOTES: Excludes board and care homes for the mentaly retarded. Excludes nonresponding board and care homes. 
Table 7. Number of residents 65 years of age and over in nursing homes: United States, 1991

\begin{tabular}{|c|c|c|c|c|}
\hline State & Residents & $\begin{array}{c}\text { Residents } \\
\text { with reported age }\end{array}$ & $\begin{array}{c}\text { Residents } \\
65 \text { years of age and over }\end{array}$ & $\begin{array}{l}\text { Percent of all residents } \\
65 \text { years of age and over }\end{array}$ \\
\hline & \multicolumn{3}{|c|}{ Number } & \\
\hline United States . . . . . . . . . . . & $1,478,903$ & $1,287,279$ & $1,188,308$ & 92.3 \\
\hline Alabama . . . . . . . . . . . & 21,675 & 19,435 & 17,972 & 92.5 \\
\hline Alaska $\ldots \ldots \ldots \ldots \ldots \ldots$ & 808 & 657 & 517 & 78.7 \\
\hline Arlzona. . . . . . . . . . & 12,103 & 10,806 & 9,931 & 91.9 \\
\hline Arkansas. . . . . . . . . & 20,298 & 17,298 & 15,650 & 90.5 \\
\hline Californla. . . . . . . . . & 98,885 & 89,420 & 79,398 & 88.8 \\
\hline Colorado . . . . . . . . & 15,871 & 14,316 & 13,017 & 90.9 \\
\hline Connecticut . . . . . . . . . & 27,921 & 22,366 & 20,651 & 92.3 \\
\hline Delaware. . . . . . . . . . . & 4,308 & 3,660 & 3,378 & 92.3 \\
\hline District of Columbia $\ldots \ldots \ldots \ldots$ & 2,881 & 2,408 & 2,246 & 93.3 \\
\hline Florida $\ldots \ldots \ldots \ldots \ldots$ & 59,878 & 52,276 & 49,628 & 94.9 \\
\hline Georgla. . . . . . . . . . . & 34,728 & 31,324 & 28,407 & 90.7 \\
\hline Hawall $\ldots \ldots \ldots \ldots \ldots \ldots$ & 2,840 & 2,721 & 2,504 & 92.0 \\
\hline 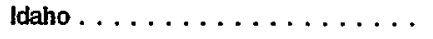 & 4,871 & 4,408 & 3,997 & 90.7 \\
\hline Illinols. . . . . . . . . . . . . & 87,540 & 74,418 & 66,207 & 89.0 \\
\hline 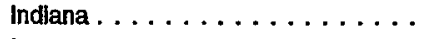 & 46,231 & 40,765 & 37,140 & 91.1 \\
\hline lowa. . . . . . . . . . & 33,214 & 29,965 & 28,524 & 95.2 \\
\hline 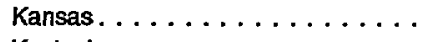 & 25,304 & 22,116 & 20,495 & 92.7 \\
\hline Kentucky. . . . . . . . . . . & 24,966 & 22,020 & 20,283 & 92.1 \\
\hline Louisiana. . . . . . . . . . . . & 32,367 & 26,863 & 23,934 & 89.1 \\
\hline Maine. . . . . . . . . . & 9,241 & $\mathbf{8 , 6 1 0}$ & 8,229 & 95.6 \\
\hline Maryland. . . . . . . . . . . & 25,977 & 20,745 & 19,081 & 92.0 \\
\hline Massachusetts . . . . . . . . & 48,276 & 42,620 & 39,848 & 93.5 \\
\hline Michigan . . . . . . . . . . & 46,198 & 40,067 & 36,643 & 91.5 \\
\hline Minnesota . . . . . . . . . & 43,298 & 36,095 & 34,054 & 94.3 \\
\hline Mississippi. . . . . . . . . . . & 14,819 & 11,145 & 10,355 & 92.9 \\
\hline Missouri $\ldots \ldots \ldots \ldots \ldots \ldots$ & 45,745 & 38,302 & 35,666 & 93.1 \\
\hline Montana . . . . . . . . . & 6,297 & 5,922 & 5,480 & 92.5 \\
\hline Nebraska. . . . . . . . . . . . & 17,779 & 16,752 & 15,738 & 93.9 \\
\hline Nevada. . . . . . . . . . . . . & 3,043 & 2,765 & 2,434 & 88.0 \\
\hline New Hampshire $\ldots \ldots \ldots \ldots$ & 7,523 & 7,202 & 6,846 & 95.1 \\
\hline New Jersey . . . . . . . . . . . & 40,068 & 34,619 & 32,325 & 93.4 \\
\hline New Mexico. . . . . . . . . . . . & 5,834 & 5,003 & 4,602 & 92.0 \\
\hline New York. . . . . . . . . . . . . & 99,372 & 88,233 & 83,060 & 94.1 \\
\hline North Carollna . . . . . . . . . & 28,546 & 24,827 & 22,907 & 92.3 \\
\hline North Dakota . . . . . . . . . . . & 6,784 & 5,965 & 5,688 & 95.4 \\
\hline Ohlo. ................. & 77,676 & 68,252 & 62,560 & 91.7 \\
\hline Oklahoma .............. & 27,456 & 24,994 & 23,206 & 92.8 \\
\hline Oregon............... & 13,392 & 11,262 & 10,473 & 93.0 \\
\hline Pennsylvania . . . . . . . . . . & 83,107 & 71,095 & 66,824 & 94.0 \\
\hline Rhode Island $\ldots \ldots \ldots \ldots \ldots$ & 9,440 & 8,524 & 8,226 & 96.5 \\
\hline South Carolina . . . . . . . . . & 13,089 & 11,907 & 11,026 & 92.6 \\
\hline South Dakota . . . . . . . . . . . & 8,192 & 7,378 & 7,064 & 95.7 \\
\hline Tennesser. . . . . . . . . . . . . & 32,304 & 28,277 & 26,211 & 92.7 \\
\hline Texas . . . . . . . . . . . . & 90,405 & 77,246 & 71,875 & 93.0 \\
\hline Utah. . . . . . . . . . . . & 5,544 & 5,037 & 4,357 & 86.5 \\
\hline Vermont $\ldots \ldots \ldots \ldots \ldots \ldots$ & 3,591 & 3,137 & 2,963 & 94.5 \\
\hline Virginla . . . . . . . . . . . . & 25,775 & 22,567 & 20,852 & 92.4 \\
\hline Washington $\ldots \ldots \ldots \ldots \ldots$ & 24,525 & 20,985 & 19,321 & 92.1 \\
\hline West Virginia . . . . . . . . . & 9,809 & 8,236 & 7,761 & 94.2 \\
\hline Wisconsin $\ldots \ldots \ldots \ldots \ldots$ & 46,898 & 40,150 & 36,772 & 91.6 \\
\hline Wyoming. . . . . . . . . . . & 2,211 & 2,118 & 1,982 & 93.6 \\
\hline
\end{tabular}




\section{Technical notes}

\section{Creating a mailing list}

The 1991 NHPI was a mail survey conducted by NCHS. The inventory's mailing list of home health care agencies and hospices contained 14,089 addresses; the mailing list of nursing homes and board and care homes (including those for the mentally retarded) contained 73,106. Both the agency and facility lists were constructed using NCHS's Agency Reporting System, which is an ongoing system designed to update periodically the NHPI listings (5).

\section{Mail survey}

The Bureau of Census under an interagency agreement with NCHS served as the data collection and data-processing agent. Three questionnaire mailouts plus a field followup were used to complete the inventory. At the end of the mail survey, refusals, postmaster returns, and nonresponses were contacted by telephone. Also contacted were places who did not respond to questionnaire items considered critical for selecting samples for the Long-Term Care Component of the National Health Care Survey (6).

Because of the large number $(17,156)$ of nonresponding board and care homes, resource constraints made it possible to follow up only one-half of these nonrespondents. As a result, nonresponding board and care homes will not be included in the data presented in this report.

\section{Results of mail survey}

\section{Home health care agencies and hospices}

Of the 14,089 agencies to which questionnaires were mailed, 7,804 responded and classified as home health agencies or hospices. Of the remaining $6,285,116$ agencies were nonrespondents, and 6,169 agencies were out of scope or not in operation (questionnaires were returned by the post office and/or field interviewers were unable to locate by telephone). The overall agency response rate was 98.5 percent. The numerator is 7,804 , the number of responding agencies. The denominator is 7,920 , the number of responding agencies plus 116 refusing agencies and nonresponding agencies.

\section{Nursing homes and board and care homes}

The facility response rate, excluding those board and care places not in the followup, was 99 percent (there were 262 refusals). If the 8,578 were counted in the calculation as in business and nonresponses, the response rate for facilities would be 84 percent. Because some of these 8,578 agencies were either out of scope or out of business, the response rate was probably somewhat higher than 84 percent.

\section{Classification system}

\section{Home health care agencies and hospices}

NCHS classified the 7,804 agencies using the questionnaire item "type of client." The client data were used because they allowed for the classification of the largest numbers of agencies into either the home health care agency category $(6,797)$ or hospice category (943), with only 64 agencies remaining in the category of agencies providing both home health and hospice care. Based upon additional information in an agency's record, 56 of the agencies providing both home health and hospice care are included with home health agencies, and 8 are included with hospices.

\section{Nursing homes and board and care homes}

Excluding the 8,578 nonresponding board and care homes and the 262 facilities that refused to participate, each of the remaining facilities was classified as either a nursing home $(15,511)$ or a board and care home $(31,431)$. For purposes of this survey, no facilities for the mentally retarded were classified as nursing homes. However, if a facility was primarily a nursing home and happened to be certified as an intermediate care facility for the mentally retarded (ICF-MR), it would have been classified as a nursing home. As a result this file contains 24 nursing homes that were also ICF-MR's, and 11,204 board and care homes that were not ICF-MR facilities for the mentally retarded. The classification system used to separate nursing homes from board and care homes relied heavily on criteria such as the respondent's categorization of the home, the home's certification, the number of beds set up and staffed for use, the employment of registered nurses or licensed practical nurses, the services provided, and the number of mentally retarded patients.

Note that a more detailed technical notes section for the 1991 NHPI is included in other reports $(1,3)$.

\section{Definitions}

Home health care agency-An agency providing health services to individuals in their homes for the purpose of (a) promoting, maintaining, or restoring health; or (b) maximizing the level of independence, while minimizing the effects of disability and illness (including terminal illness).

Hospice-An agency providing specialized services for terminally ill people and their families, including medical services, social and emotional support for patients and families, volunteer support, and bereavement services for families following the death of the patient.

Nursing homes-A nursing home is a facility with three beds or more that is either licensed as a nursing home, certified as a nursing facility under Medicare or Medicaid, identified as a nursing care unit of a retirement center, or determined to provide nursing or medical care.

Board and care homes-This generic term describes a residential setting that provides either routine general protective oversight or assistance with activities necessary for independent living to physically limited persons (excludes those for the mentally retarded).

Rural-urban continuum codes for metropolitan and nonmetropolitan counties-These codes are based on the 1990 census. Rural counties included nonmetropolitan counties that had an 
urban population fewer than 2,500 . Urban counties included counties not considered rural. Nonmetropolitan counties are those counties that are not considered metropolitan. Metropolitan areas, as defined by the Office of Management and Budget, include core counties containing a city of 50,000 or more people and a total area population of at least 100,000 . Additional contiguous counties are included in metropolitan areas if they are economically and socially integrated with the core county (7).

Geographic divisions-The U.S.

Bureau of the Census groups the 50

States plus the District of Columbia into the following divisions:

\begin{tabular}{cl} 
Division & \multicolumn{1}{c}{ States included } \\
New & $\begin{array}{l}\text { Connecticut, Maine, } \\
\text { England } \\
\text { Massachusetts, New } \\
\text { Hampshire, Rhode Island, } \\
\text { Vermont }\end{array}$ \\
Middle & New Jersey, New York, \\
Atlantic & Pennsylvania \\
East North & $\begin{array}{l}\text { Illinois, Indiana, } \\
\text { Central }\end{array}$ \\
& $\begin{array}{l}\text { Michigan, Ohio, } \\
\text { Wisconsin }\end{array}$ \\
West North & Iowa, Kansas, Minnesota, \\
Central & $\begin{array}{l}\text { Missouri, Nebraska, } \\
\text { North Dakota, South }\end{array}$ \\
& Dakota
\end{tabular}

South

Delaware, District of

Atlantic

Columbia, Florida,

Georgia, Maryland, North

Carolina, South Carolina,

Virginia, West Virginia

East South Alabama, Kentucky,

Central Mississippi, Tennessee

West South Arkansas, Louisiana,

Central Oklahoma, Texas

Mountain Arizona, Colorado, Idaho, Montana, Nevada, New Mexico, Utah, Wyoming

Pacific Alaska, California, Hawaii, Oregon, Washington 


\section{Symbols}

-. Data not available

... Category not applicable

- Quantity zero

* Figure does not meet standard of reliability or precision (more than 30-percent relative standard error in numerator of percent or rate)

* Figure does not meet standard of reliability and quantity zero 


\title{
Advance
}

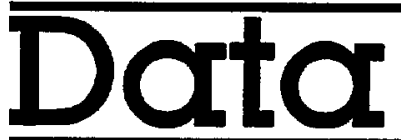

From Vital and Health Statistics of the CENTERS FOR DISEASE CONTROL AND PREVENTION/National Center for Health Statistics

\section{Office Visits to Neurologists: United States, 1991-92}

\author{
by Susan M. Schappert, M.A., Division of Health Care Statistics
}

\section{Introduction}

During 1991-92 an estimated 14.5 million visits were made in the United States to nonfederally employed, office-based physicians specializing in neurology, the diagnosis and treatment of disorders of the nervous system-an average of 7.3 million visits per year. This report summarizes data pertaining to office visits to neurologists in terms of physician practice characteristics, patient characteristics, and visit characteristics.

The information presented in this report is based on data collected by the National Ambulatory Medical Care Survey (NAMCS), a national probability sample survey conducted by the Division of Health Care Statistics of the National Center for Health Statistics, Centers for Disease Control and Prevention. This survey was conducted yearly from 1973 through 1981, again in 1985 , and has resumed an annual schedule with the 1989 survey.

The 1991 and 1992 NAMCS shared identical survey instruments, definitions, and procedures. The resulting 2 years of data have been combined to provide more reliable estimates, and the reader should note that the estimates, percent distributions, and rates presented in this report reflect average annual estimates based on the combined 1991 and 1992 data, unless otherwise stated. Figure 1 shows the Patient Record form, which is the survey instrument used by participating physicians to record information about their patients' office visits.

Only visits to the offices of nonfederally employed physicians who were classified by the American Medical Association or the American Osteopathic Association as "office-based, patient care" were included in the NAMCS sample. Visits to private nonhospitalbased clinics and health maintenance organizations were considered to be within scope of the survey, but those that took place in government-operated facilities were not. Physicians specializing in anesthesiology, pathology, or radiology were not included in the sample, nor were visits to hospital-based physicians or physicians primarily engaged in training, research, or administration. Telephone contacts and visits made outside the physician's office were also excluded. The National Hospital Ambulatory Medical Care Survey (NHAMCS) collects patient and visit data from hospital-based outpatient departments and emergency departments. Results from that survey are available in other published reports (1-4).

It is necessary to keep in mind that the estimates presented in this report are based on a sample, rather than on the entire universe of office visits, and, as such, they are subject to sampling variability. The Technical notes at the end of this report discuss briefly the sample design, sampling errors, and guidelines for judging the precision of NAMCS estimates. Additional publications summarizing NAMCS data from 1991 and 1992 are available (5-7).

\section{Physician practice characteristics}

On average, 2.9 visits per 100 persons per year were made to neurologists during 1991 and 1992 (table 1). This specialty received 1.0 percent of all office visits made to ambulatory care physicians during the 2-year period.

Visit rates did not differ by geographic region, except that the West had a higher annual visit rate ( 3.8 visits per 100 persons) than did the Northeast (2.4 visits per 100 persons). The majority of neurology visits (95.4 percent) were made to doctors of medicine; 4.6 percent were made to doctors of osteopathy (table 2).

\section{Patient characteristics}

Visits to neurologists are shown by patient's age, sex, and race in table 3. The visit rate was significantly higher for persons 25 years and over compared with those under age 25. However, no

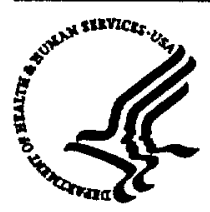

\section{U.S. DEPARTMENT OF HEALTH AND HUMAN SERVICES \\ Public Health Service \\ Centers for Disease Control and Prevention \\ National Center for Health Statistics}

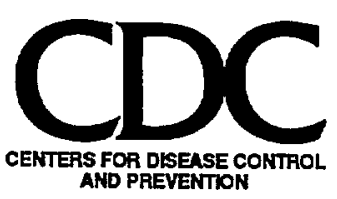




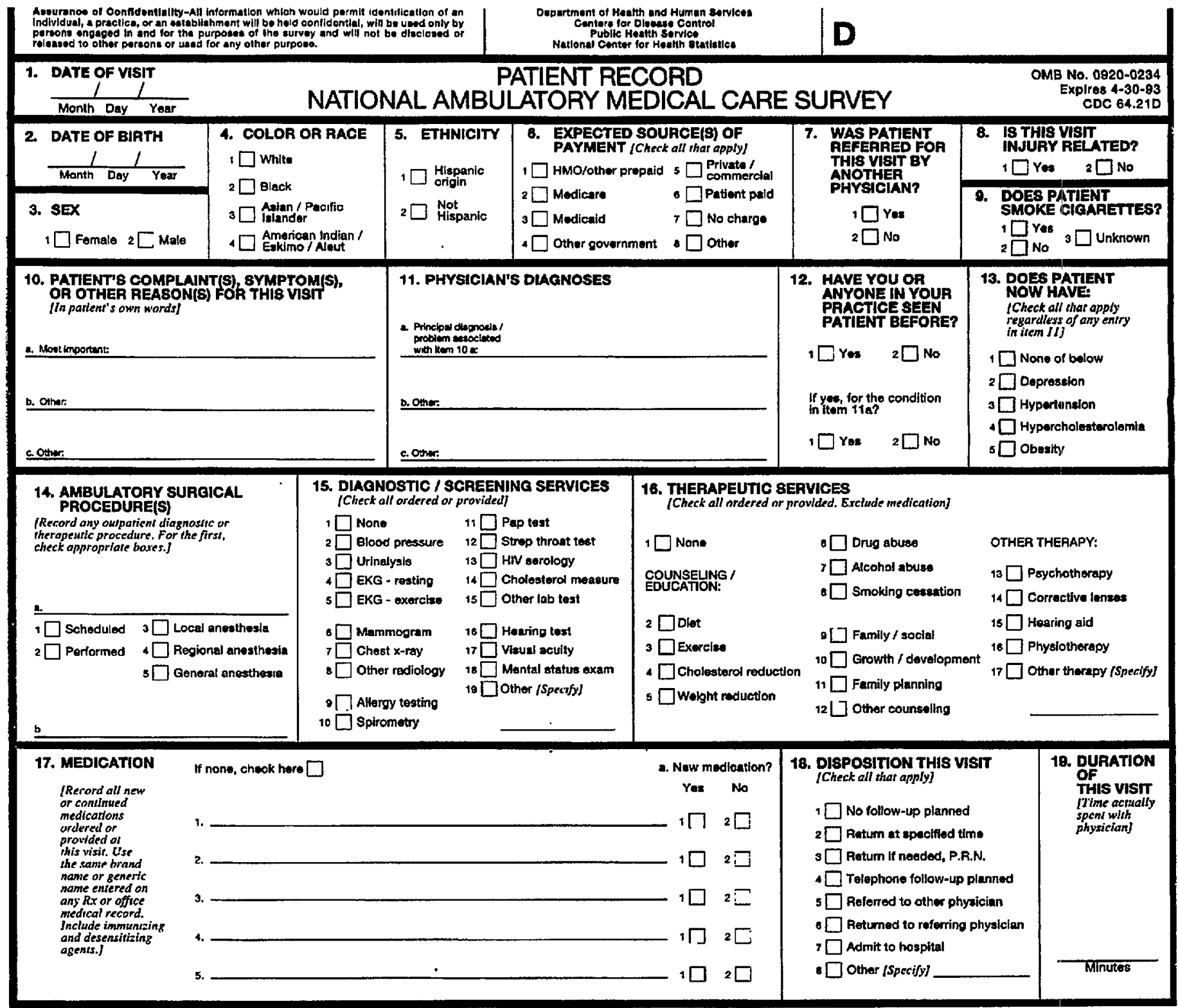

Figure 1. Patient Record form

significant differences were noted among visit rates for those in the age groups 24-44 years, 45-64 years, 65-74 years, and 75 years and over. In addition, visit rates for persons under 15 years and 15-24 years of age did not differ significantly from each other. Females had a higher visit rate to neurologists than did males (3.3 visits per 100 females per year compared with 2.5 visits per 100 males). Significant differences were confined to the age group 25-44 years, with females in that group making 4.0 visits per 100 compared with 2.3 visits per 100 males. White persons had a significantly higher rate of visits to neurologists (3.2 visits per 100 persons per year) than did black persons (1.6 visits per 100 persons per year).

Persons 25-44 years of age accounted for more than one-third (35.3 percent) of all office visits to neurologists; those 44 years and over accounted for slightly less than half of the visits (46.1 percent). Females made a higher proportion of visits to neurologists than did males, 58.0 percent and 42.0 percent, respectively. White persons made 91.1 percent of the visits to this specialty, and black persons accounted for 7.0 percent.

\section{Visit characteristics}

\section{Referral status and prior-visit status}

Nearly one-third ( 30.2 percent) of office visits to neurologists were the result of a referral by another physician compared with 6.0 percent of the visits to all other physicians (figure 2). While 60.5 percent of neurology visits were made by patients returning for care of a previously treated problem, more than one-third ( 35.8 percent) were made by new patients. In comparison, only 15.5 percent of the visits to all other 
Table 1. Annual number, percent distribution, and rate of office visits by physician specialty, averaged over a 2-year perlod: United States, 1991-92

\begin{tabular}{|c|c|c|c|}
\hline Physician specialty & $\begin{array}{l}\text { Number of } \\
\text { visits in } \\
\text { thousands }\end{array}$ & $\begin{array}{c}\text { Percent } \\
\text { alstribution }\end{array}$ & $\begin{array}{l}\text { Number of } \\
\text { visits per } \\
100 \text { persons } \\
\text { per year }\end{array}$ \\
\hline All visits $\ldots \ldots \ldots \ldots \ldots \ldots \ldots$ & 715,867 & 100.0 & 286.3 \\
\hline General and family practice . . . . . . . . . & 192,051 & 26.8 & 76.8 \\
\hline Intemal medicine . . . . . . . . . . . . & 101,598 & 14.2 & 40.6 \\
\hline Pedlatrics . . . . . . . . . . . & 85,387 & 11.9 & 34.1 \\
\hline Obstetrics and gynecology . . . . . . . . . & 62,601 & 8.7 & 25.0 \\
\hline Ophthalmology ................ & 43,884 & 6.1 & 17.5 \\
\hline Orthopedic sungery. . . . . . . . . . . . . & 36,958 & 5.2 & 14.8 \\
\hline Dermatology. . . . . . . . . . . . . & 29,179 & 4.1 & 11.7 \\
\hline General surgery . . . . . . . . . . . . & 22,797 & 3.2 & 9.1 \\
\hline Otolaryngology $\ldots \ldots \ldots \ldots \ldots$ & 21,007 & 2.9 & 8.4 \\
\hline Psychlatny . . . . . . . . . . . . & 17,769 & 2.5 & 7.1 \\
\hline Urological surgery . . . . . . . . . . . & 13,857 & 1.9 & 5.5 \\
\hline Cardiovascular diseases . . . . . . . . . . & 13,146 & 1.8 & 5.3 \\
\hline Neurology . . . . . . . . . . . . . . & 7,253 & 1.0 & 2.9 \\
\hline All other specialties . . . . . . . . . . & 68,382 & 9.6 & 27.3 \\
\hline
\end{tabular}

'Based on U.S. Bureau of the Census estimales of the civilian, noninstitutionalized population of the United States for July 1 , 1991 , and July 1, 1992, averaged over the 2-year period.

Table 2. Annual number, percent distribution, and rate of office visits to neurologists by selected physician practice characteristics, averaged over a 2-year period: United States, 1991-92

\begin{tabular}{|c|c|c|c|}
\hline $\begin{array}{l}\text { Physiclan practice } \\
\text { characteristlas }\end{array}$ & $\begin{array}{l}\text { Number of vistts } \\
\text { in thousands }\end{array}$ & $\begin{array}{l}\text { Percent } \\
\text { distribution }\end{array}$ & $\begin{array}{l}\text { Number of vistts per } \\
100 \text { persons per year }\end{array}$ \\
\hline All visits & 7,253 & 100.0 & 2.9 \\
\hline \multicolumn{4}{|l|}{ Geographic region } \\
\hline Northeast & 1,191 & 16.4 & 2.4 \\
\hline$\ldots \ldots \ldots \ldots \ldots$ & 1,815 & 25.0 & 3.0 \\
\hline$\cdots \ldots \ldots \cdots \cdots \cdots$ & 2,172 & 30.0 & 2.6 \\
\hline West $\ldots \ldots \ldots \ldots \ldots \ldots \ldots \ldots$ & 2,076 & 28.6 & 3.8 \\
\hline \multicolumn{4}{|l|}{ Professional identity } \\
\hline Doctor of medlcine. . . . . . . . . . . & 6,921 & 95.4 & 2.8 \\
\hline Doctor of osteopathy $\ldots \ldots \ldots \ldots \ldots$ & 332 & 4.6 & 0.1 \\
\hline
\end{tabular}

'Based on U.S. Bureau of the Census estimates of the civilian, noninstitutionalized population of the United States for July 1 , 1891 , and July 1, 1992, averaged over the 2-yoar period.

physicians were by new patients (figure 3). Visits by referral status and prior-visit status are summarized in table 4.

\section{Expected sources of payment}

Private insurance was an expected source of payment at nearly one-half (44.4 percent) of all visits to neurologists compared with one-third (34.1 percent) of visits to all other specialties. Medicare was an expected pay source at one-fifth of all neurology visits (21.8 percent). Data on expected sources of payment are shown in table 5. It should be noted that more than one expected source of payment could be recorded per visit.

\section{Patient's principal reason for visit}

Table 6 shows the patient's principal reason for visiting the physician, according to the eight modules, or groups of reasons, outlined in A Reason for Visit Classification for Ambulatory Care (RVC) (8). Principal reason for visit (item 10a on the Patient Record form) is the patient's most important complaint(s), symptom(s), or other reason(s) for this visit expressed in the patient's (or patient's

spokesperson's) own words. Up to three reasons per visit may be coded based upon the classification system found in the RVC.

Eight out of ten visits to this specialty ( 81.1 percent) were due to a symptomatic problem or complaint, with the largest proportion of symptoms being those referable to the nervous system (excluding sense organs) (43.3 percent). Musculoskeletal symptoms were listed at 22.9 percent of the visits.

Specific reasons for visit are listed in table 7 . The single most frequently mentioned principal reason for visiting the neurologist was headache or pain in head, accounting for 18.4 percent of the visits. A higher proportion of visits by females were for this reason (22.5 percent) compared with males (12.7 percent). Convulsions, mentioned at 9.1 percent of visits, was the second most frequent reason, followed by disturbances of sensation ( 5.5 percent). It should be noted that estimates that differ in rank order may not be significantly different from each other.

\section{Diagnostic services}

About one-third of all visits to neurologists included no diagnostic or screening services; 4 of every 10 visits (42.2 percent) included one service. The most frequently mentioned specific category was blood pressure check, which was reported at more than one-third of the visits ( 37.3 percent).

Mental status exams were more likely to be ordered or provided at visits to neurologists compared with visits to all other physicians ( 8.8 percent and 1.1 percent, respectively), as was "other radiology" (radiology other than chest $x$ ray). Unspecified diagnostic services were reported at 29.6 percent of all visits to neurologists. Table 8 displays visits by the number and type of diagnostic services ordered or provided.

\section{Principal diagnosis}

Data on principal diagnoses rendered at office visits are obtained from item 11a of the Patient Record form where physicians are asked to record the principal diagnosis associated with the patient's most important reason for visit. Diagnoses are classified and coded according to the International Classification of Diseases, 9th Revision, Clinical Modification (ICD-9-CM) (9). 
Table 3. Annual number, percent distribution, and rate of office visits to neurologists by selected patient characteristics, averaged over a 2-year period: United State8, 1991-92

\begin{tabular}{|c|c|c|c|}
\hline Patlent characteristic & $\begin{array}{l}\text { Number of } \\
\text { vistts in } \\
\text { thousands }\end{array}$ & $\begin{array}{c}\text { Percent } \\
\text { distribution }\end{array}$ & $\begin{array}{l}\text { Vist rate } \\
\text { per } 100 \\
\text { persons }\end{array}$ \\
\hline$\ldots \ldots \ldots \ldots$ & 7,253 & 100.0 & 2.9 \\
\hline \multicolumn{4}{|l|}{ Age } \\
\hline Under 15 years. . . . . . . . . . . . . & 770 & 10.6 & 1.4 \\
\hline 15-24 years. . . . . . . . . . . . & 577 & 8.0 & 1.7 \\
\hline $25-44$ years. . . . . . . . . . . . . & 2,559 & 35.3 & 3.2 \\
\hline 45-64 years. . . . . . . . . . . . . & 1,893 & 26.1 & 4.0 \\
\hline $65-74$ years. . . . . . . . . . . . & 820 & 11.3 & 4.5 \\
\hline 75 years and over $\ldots \ldots \ldots \ldots \ldots \ldots$ & 633 & 8.7 & 5.2 \\
\hline \multicolumn{4}{|l|}{ Sex and age } \\
\hline Female $\ldots \ldots \ldots \ldots \ldots \ldots$ & 4,210 & 58.0 & 3.3 \\
\hline Under 15 years $\ldots \ldots \ldots \ldots \ldots \ldots$ & 329 & 4.5 & 1.2 \\
\hline $15-24$ years $\ldots \ldots \ldots \ldots \ldots \ldots$ & 312 & 4.3 & 1.8 \\
\hline $25-44$ yөars $\ldots \ldots \ldots \ldots \ldots \ldots$ & 1,647 & 22.7 & 4.0 \\
\hline $45-64$ years $\ldots \ldots \ldots \ldots \ldots \ldots$ & 1,071 & 14.8 & 4.3 \\
\hline $65-74$ years $\ldots \ldots \ldots \ldots \ldots \ldots$ & 441 & 6.1 & 4.3 \\
\hline 75 years and over $\ldots \ldots \ldots \ldots \ldots$ & 410 & 5.6 & 5.4 \\
\hline Male $\ldots \ldots \ldots \ldots \ldots$ & 3,044 & 42.0 & 2.5 \\
\hline Under 15 years $\ldots \ldots \ldots \ldots \ldots$ & 442 & 6.1 & 1.5 \\
\hline 15-24 years $\ldots \ldots \ldots \ldots \ldots \ldots$ & 265 & 3.7 & 1.5 \\
\hline $25-44$ years $\ldots \ldots \ldots \ldots \ldots \ldots$ & 912 & 12.6 & 2.3 \\
\hline $45-64$ years $\ldots \ldots \ldots \ldots \ldots \ldots$ & 822 & 11.3 & 3.6 \\
\hline $65-74$ years $\ldots \ldots \ldots \ldots \ldots \ldots$ & 380 & 5.2 & 4.6 \\
\hline 75 years and over . . . . . . . . & 224 & 3.1 & 4.9 \\
\hline \multicolumn{4}{|l|}{ Race } \\
\hline$\ldots \ldots \ldots \ldots \ldots$ & 6,605 & 91.1 & 3.2 \\
\hline Black . . . . . . . . . . . . & 508 & 7.0 & 1.6 \\
\hline Asian/Pacific Islander $\ldots \ldots \ldots \ldots \ldots$ & 113 & 1.6 & --- \\
\hline American Indian/Eskimo/Aleut . . . . . . . . & $" 27$ & ${ }^{*} 0.4$ & --- \\
\hline
\end{tabular}

'Visit rates are based on U.S. Bureau of the Census estimates of the civilian, noninstitutionalized U.S. population for July 1 , 1991, and July 1, 1992, averaged over the 2-year period.

More than one-third (36.1 percent) of all visits to neurologists resulted in a principal diagnosis that was classifiable to a disease of the nervous system and sense organs (table 9). About one-fifth of the visits ( 21.3 percent) were recorded as "symptoms, signs, and ill-defined conditions." Diseases of the musculoskeletal system and connective tissue accounted for 14.6 percent of the visits.

The top 20 principal diagnoses at visits to neurologists are shown in table 10 . The most frequently listed specific diagnosis was "general symptoms" (ICD-9-CM code 780), occurring at 13.3 percent of visits. This category falls within the larger classification of "symptoms, signs, and ill-defined conditions" of the ICD-9$\mathrm{CM}$. This classification includes signs and symptoms for which no more specific diagnosis can be made even after investigation of the facts, transient symptoms whose causes could not be determined, provisional diagnoses, cases referred elsewhere before a diagnosis was made, cases in which a precise diagnosis was unavailable for any other reason, and certain symptoms that represent important problems in medical care and that might be desired to classify in addition to a known cause. General symptoms (ICD-9-CM code 780) may include any of the following subcategories: coma and stupor, hallucinations, syncope (fainting) and collapse, convulsions, dizziness and giddiness, sleep disturbances, pyrexia (fever) of unknown origin, malaise and fatigue, hyperhidrosis (excessive sweating), and other general symptoms. Among the neurology visits reported here, convulsions (ICD-9-CM code 780.3) accounted for more than three-quarters of the "general symptoms" diagnoses.

The second and third most frequently reported diagnoses at neurology visits were migraine (10.3 percent) and symptoms involving head and neck (5.7 percent). A higher proportion of visits by females listed diagnoses of migraine and symptoms involving head and neck than did visits by males. (Among visits with the latter diagnosis, 97.1 percent were coded to ICD-9-CM subcategory 784.0, headache). Parkinson's disease, which accounted for 4.6 percent of the visits overall, was listed at 6.6 percent of visits by males compared with 3.1 percent of visits by femalles. The most frequently reported diagnoses by age group are presented in table 11 .

Interestingly, one-fifth (19.8 percent) of visits to neurologists were reported to be injury related in item 13 of the Patient Record form compared with about one-tenth (9.1 percent) of visits to all other physicians. This is not readily apparent from an examination of the reported ICD-9-CM codes, however, as only 6.7 percent of neurology visits were classified to the "injury and poisoning" category of the ICD-9-CM.

\section{Therapeutic services}

Table 12 presents data on therapeutic services ordered or provided at visits to neurologists. Medication therapy was mentioned at nearly two-thirds of the visits (63.7 percent), and nonmedication therapy was ordered or provided at more than one-quarter of the visits ( 27.9 percent). The most frequently mentioned types of nonmedication therapy included "other counseling" ( 8.0 percent), exercise counseling or education (7.1 percent), physiotherapy (6.4 percent), and diet counseling or education (5.2 percent). Ambulatory surgery was scheduled or performed at 1.3 percent of visits to neurologists, significantly less than the corresponding 6.1 percent of visits to all other physicians.

Tables 13, 14, and 15 present more detailed drug data relating to neurology visits. As used in the NAMCS, the term "drug" is interchangeable with the term "medication" and includes all new or continued medications ordered or provided at the visit. This includes both prescription and nonprescription preparations, immunizing agents, and desensitizing agents. "Drug mentions" refer to the total number of medications 


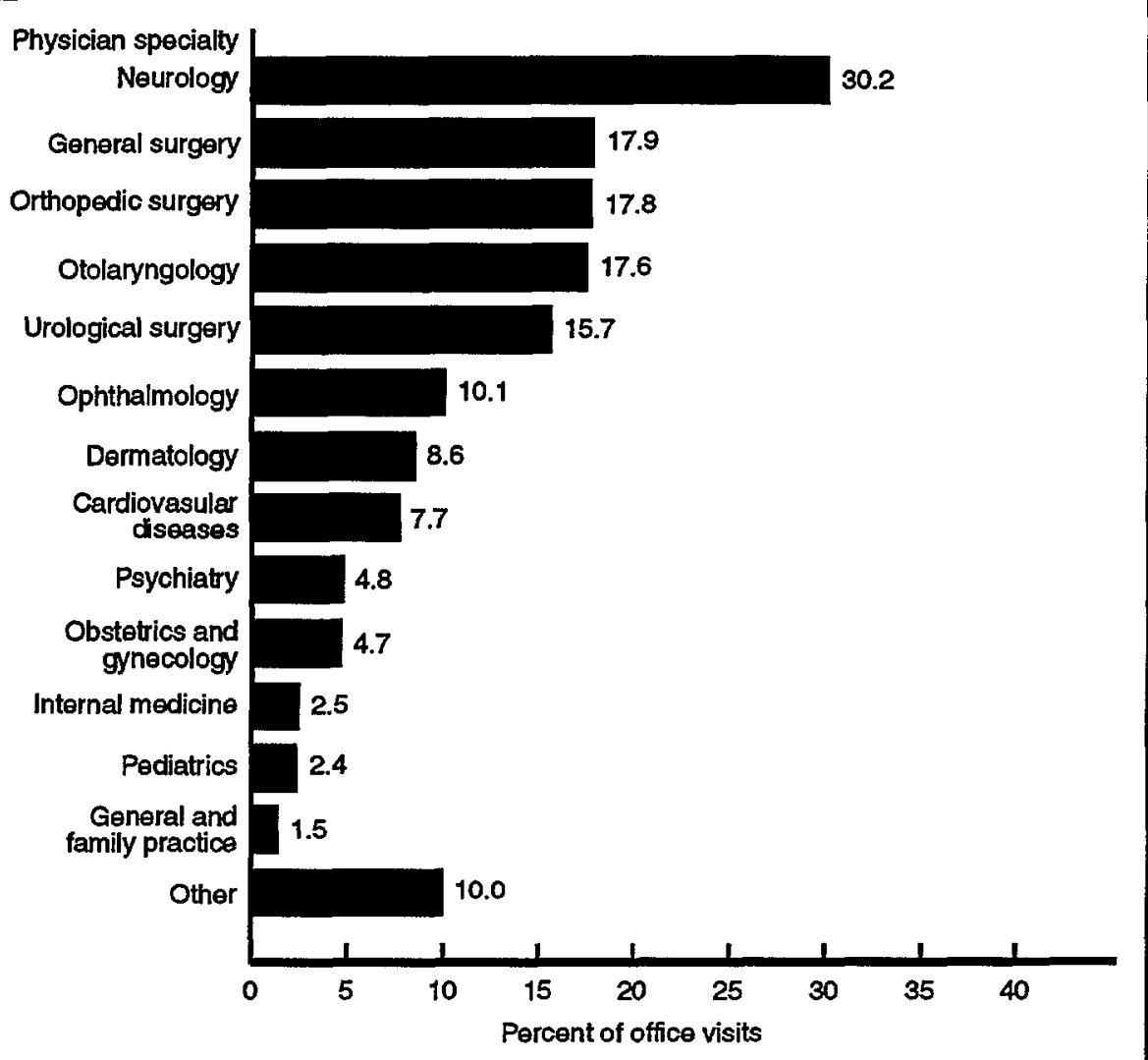

Figure 2. Percent of office visits that are referrals, according to physiclan specialty: United State8, 1991-92

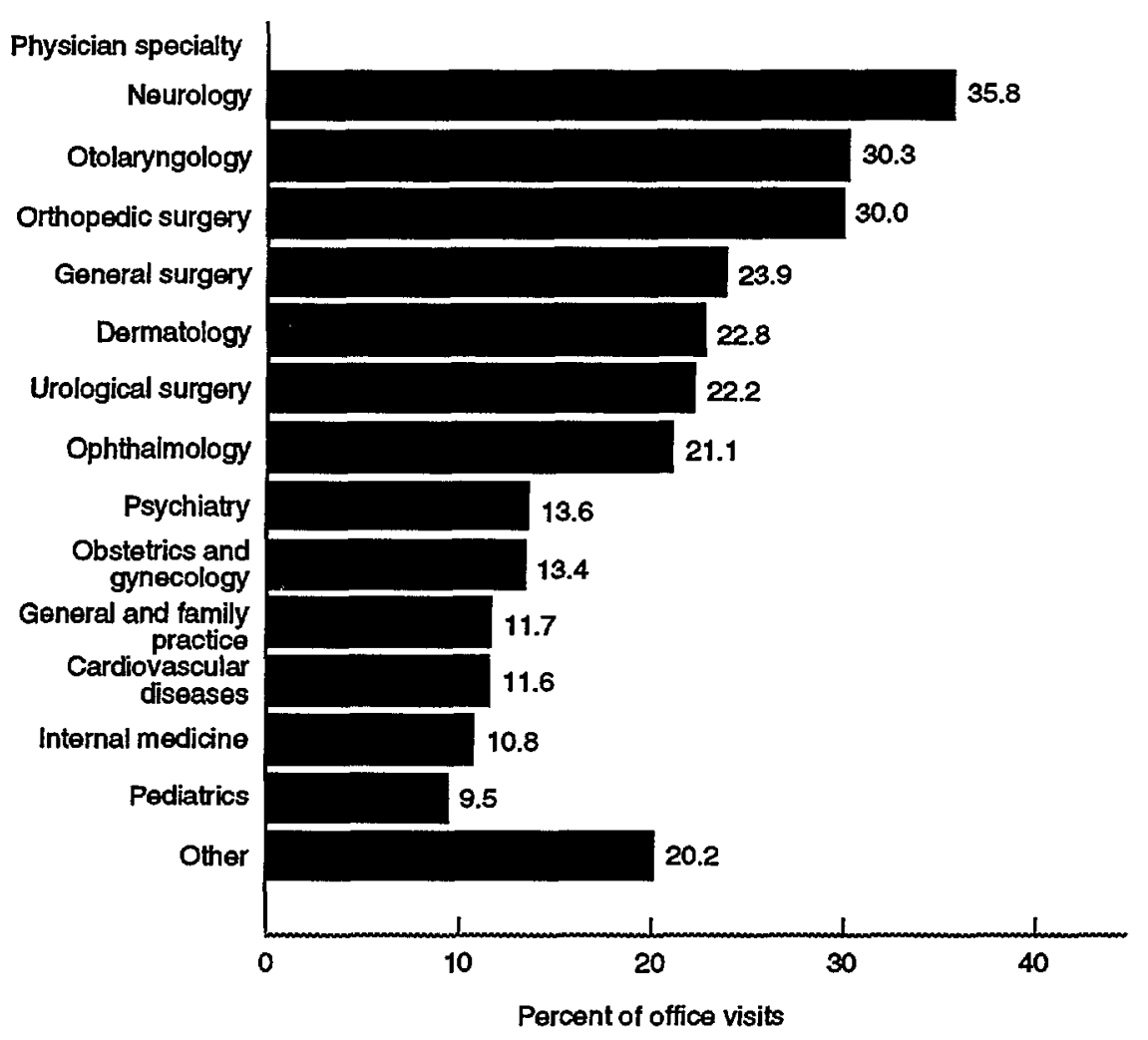

Figure 3. Percent of ofice visits made by new patients, according to physiclan specialty: United State8, 1991-92 listed in item 17 of the Patient Record form. Physicians may record more than one medication per visit, so that the total number of drug mentions may exceed the total number of visits. "Drug visits" refer to visits with at least one mention of medication ordered or provided by the physician. An earlier report describes in detail the method and instruments used in the collection and processing of NAMCS drug data (10).

Among visits to neurologists, there was an average of 8.1 million drug mentions per year for 1991 and 1992, yielding 1.8 mentions per drug visit and 1.1 mentions per visit overall. Table 13 shows the number of drug mentions by therapeutic classification, adapted from therapeutic categories used in the National Drug Code, 1985 edition (11). In cases where a particular drug was classifiable to more than one therapeutic category, it was listed under the category that occurred with the greatest frequency. Neurologic drugs (25.9 percent), drugs used for pain relief (22.4 percent), and psychopharmacologic drugs ( 20.2 percent) were reported most frequently, together accounting for about two-thirds ( 68.5 percent) of the drugs mentioned at visits to neurologists.

The generic substances used most frequently in medications ordered or provided at neurology visits are shown in table 14. Acetaminophen was the most frequently occurring substance ( 8.3 percent of mentions), followed by carbamazepine ( 6.0 percent) and amitriptyline ( 4.9 percent). It should be noted that drugs containing more than one ingredient are listed in the data for each ingredient. For example, acetaminophen with codeine would be listed both under the count for acetaminophen as well as the count for codeine.

Table 15 displays drug mentions according to entry name, that is, the name recorded by the physician in item 17 of the Patient Record form. This could be a trade name, generic name, or simply a desired therapeutic effect. Tegretol was the specific entry listed most frequently ( 6.0 percent of mentions), followed by Dilantin (4.3 percent) and Sinemet ( 3.5 percent).

\section{Disposition of visit}

Visits to neurologists were more likely to include instructions to return at 
Table 4. Annual number and percent distribution of office visits to neurologists and to all other physiclans by patient's referral status and prlor-visit status, averaged over a 2-yoar perlod: United States, 1991-92

\begin{tabular}{|c|c|c|c|c|}
\hline \multirow[b]{2}{*}{ Visit characteristic } & \multicolumn{2}{|c|}{ Visits to neurologists } & \multicolumn{2}{|c|}{ Visits to all other physicians } \\
\hline & $\begin{array}{l}\text { Number of } \\
\text { visits in } \\
\text { thousands }\end{array}$ & $\begin{array}{c}\text { Percent } \\
\text { dlstribution }\end{array}$ & $\begin{array}{l}\text { Number of } \\
\text { visits in } \\
\text { thousands }\end{array}$ & $\begin{array}{c}\text { Percent } \\
\text { distribution }\end{array}$ \\
\hline$\ldots \ldots \ldots \ldots \ldots \ldots$ & 7,253 & 100.0 & 708,614 & 100.0 \\
\hline \multicolumn{5}{|l|}{ Referral status } \\
\hline Referred by another physician. . . . . . . . & 2,189 & 30.2 & 42,598 & 6.0 \\
\hline Not referred by another physiclan. . . . . . . . & 5,064 & 69.8 & 666,016 & 94.0 \\
\hline \multicolumn{5}{|l|}{ Prior-visit status } \\
\hline$\ldots \ldots \ldots \ldots$ & 2,597 & 35.8 & 109,494 & 15.5 \\
\hline Old patient, new problem. . . . . . . & 269 & 3.7 & 159,512 & 22.5 \\
\hline Old patient, old problem & 4,388 & 60.5 & 439,608 & 62.0 \\
\hline
\end{tabular}

Table 5. Annual number and percent distribution of office visits to neurologists and to all other physlcians by patient's expected source(s) of payment, averaged over a 2-year period: United States, 1991-92

\begin{tabular}{|c|c|c|c|c|}
\hline \multirow[b]{2}{*}{ Expected source(s) of payment ${ }^{1}$} & \multicolumn{2}{|c|}{ Visits to neurologists } & \multicolumn{2}{|c|}{ Visits to all other physicians } \\
\hline & $\begin{array}{c}\text { Number of } \\
\text { vistts in } \\
\text { thousands }\end{array}$ & $\begin{array}{c}\text { Percent } \\
\text { dlstribution }\end{array}$ & $\begin{array}{c}\text { Number of } \\
\text { visits In } \\
\text { thousands }\end{array}$ & $\begin{array}{c}\text { Percent } \\
\text { distribution }\end{array}$ \\
\hline All visits $\ldots \ldots \ldots \ldots \ldots \ldots \ldots$ & 7,253 & 100.0 & 708,614 & 100.0 \\
\hline Privateicommercial insurance . . . . . . . . & 3,220 & 44.4 & 241,927 & 34.1 \\
\hline Medicare. . . . . . . . . . . . . . . & 1,581 & 21.8 & 145,086 & 20.5 \\
\hline Patient-paid . . . . . . . . . . . & 983 & 13.5 & 150,664 & 21.3 \\
\hline HMO/other prepald plan $\ldots \ldots \ldots \ldots$ & 827 & 11.4 & 122,833 & 17.3 \\
\hline Medicaid . . . . . . . . . . . . . & 523 & 7.2 & 73,231 & 10.3 \\
\hline Other government . . . . . . . . . . . . . & 221 & 3.1 & 14,795 & 2.1 \\
\hline No charge . . . . . . . . . . . . & 64 & 0.9 & 11,381 & 1.6 \\
\hline Other $\ldots \ldots \ldots \ldots \ldots \ldots \ldots \ldots$ & 736 & 10.1 & 28,123 & 4.0 \\
\hline Unknown. . . . . . . . . . . . . . & 68 & 0.9 & 15,732 & 2.2 \\
\hline
\end{tabular}

${ }^{1}$ Total may exceed total number of visits because more than one category may be reported per visit. a specific time (70.5 percent) than were visits to all other specialties (62.3 percent). Also, a higher proportion of these visits resulted in instructions for the patient to return to the referring physician ( 7.7 percent) compared with visits to all other physicians ( 0.9 percent). This is a reflection, to some degree, of the large number of referrals made to this specialty relative to other physician specialties. Data on disposition of visit are displayed in table 16.

\section{Duration of visit}

About three-quarters (73.3 percent) of visits to neurologists lasted more than 15 minutes, compared with one-third (32.3 percent) of visits to all other physicians. Average duration of neurology visits was 30.5 minutes compared with 17.2 minutes for all other visits. Average duration is based on the time spent in direct, face-to-face contact between the physician and the patient. It does not include visits of "zero" minutes duration, that is, visits in which the patient did not meet with the physician directly. Data on duration of visits are shown in table 17.

\section{Visits to neurologists between 1975 and 1992}

The overall number of visits to neurologists increased by 283.3 percent,

Table 6. Annual number and percent distribution of office visits to neurologists by patient's principal reason for visit, averaged over a 2-year period: United States, 1991-92

\begin{tabular}{|c|c|c|}
\hline Principal reason for visit and RVC code ${ }^{1}$ & $\begin{array}{l}\text { Number of visits } \\
\text { in thousands }\end{array}$ & $\begin{array}{c}\text { Percent } \\
\text { distribution }\end{array}$ \\
\hline 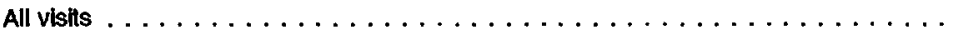 & 7,253 & 100.0 \\
\hline symptom module . . . . . . . . . . . . . . . . . . . . . . . . . . . . . . .so01-s999 & 5,881 & 81.1 \\
\hline Symptoms referable to the nervous system (excluding sense organs) . . .S200-\$259 & 3,141 & 49.3 \\
\hline Symptoms referable to the musculoskeletal system. . . . . . . . . . . . S900-S999 & 1,662 & 22.9 \\
\hline General symptoms . . . . . . . . . . . . . . . . . . . . . . . . . . . . s001-s099 & 508 & 7.0 \\
\hline Symptoms referable to psychological and mental disorders . . . . . . . . S100-\$199 & 262 & 3.6 \\
\hline symptoms referable to the eyes and ears. . . . . . . . . . . . . . . . s300-s399 & 193 & 2.7 \\
\hline Symploms referable to the resplratory system . . . . . . . . . . . . . . S400-S499 & 51 & 0.7 \\
\hline 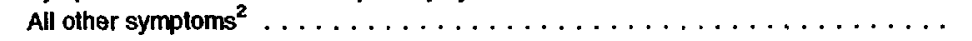 & 65 & 0.9 \\
\hline Disease module. . . . . . . . . . . . . . . . . . . . . . . . . . . Do01-D999 & 459 & 6.3 \\
\hline Diagnostlc, screening, and preventive module . . . . . . . . . . . . X100-X599 & 95 & 1.3 \\
\hline Treatment module. . . . . . . . . . . . . . . . . . . . . . . . . . . . . . T100-T899 & 382 & 5.3 \\
\hline Injury and adverse effects module . . . . . . . . . . . . . . . . . . J001-J999 & 83 & 1.1 \\
\hline Test results module. . . . . . . . . . . . . . . . . . . . . . . . . . R100-R700 & 58 & 0.8 \\
\hline Administrative module . . . . . . . . . . . . . . . . . A100-A140 & ${ }^{*} 6$ & $\star 0.1$ \\
\hline Other $^{3} \ldots \ldots \ldots \ldots \ldots \ldots \ldots \ldots \ldots \ldots \ldots \ldots \ldots$. . . . . . . . . . . . . . . . . . . . . . . . . . & 289 & 4.0 \\
\hline
\end{tabular}

Based on A Reason for Visit Classification for Ambulatory Care (RVC) (8).

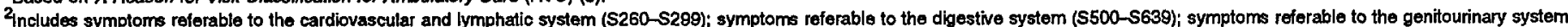
(S640-S829); and symptoms referable to the skin, hair, and nails (S830-S899).

includes blanks, problems, and complaints not elsewhere classified, ontries of "none," and illegible entres. 
Table 7. Annual number and percent distribution of office vlsits to neurologlsts by the 20 most froquently mentioned principal reasons for visit, according to patient's sex, averagod ovor a 2-year period: United States, 1991-92

\begin{tabular}{|c|c|c|c|c|}
\hline \multirow[b]{2}{*}{ Principal reason for vlsit and AVC code ${ }^{1}$} & \multirow{2}{*}{$\begin{array}{l}\text { Number of visits } \\
\text { in thousands }\end{array}$} & & \multicolumn{2}{|c|}{ Patient's sex } \\
\hline & & Total & Mals & Femals \\
\hline & & \multicolumn{3}{|c|}{ Percent distrbution } \\
\hline All visits & 7,253 & 100.0 & 100.0 & 100.0 \\
\hline Headache, paln in head . . . . . . . . . . . s210 & 1,334 & 18.4 & 12.7 & 22.5 \\
\hline Convulsions. . . . . . . . . . . . . . . . . s205 & 659 & 9.1 & 10.1 & 8.3 \\
\hline Disturbances of sensation . . . . . . . . . . . s220 & 397 & 5.5 & 5.5 & 5.4 \\
\hline Neck symptoms . . . . . . . . . . . . . . . s900 & 374 & 5.2 & 3.8 & 6.1 \\
\hline 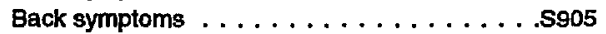 & 344 & 4.7 & 5.2 & 4.4 \\
\hline 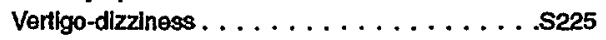 & 256 & 3.5 & 3.9 & 3.2 \\
\hline Progress visit, not otherwlse specffled. . . . . .T800 & 254 & 3.5 & 3.0 & 3.9 \\
\hline Leg symptoms . . . . . . . . . . . . . . . . . . . s920 & 251 & 3.5 & 4.0 & 3.1 \\
\hline Abnormal Involuntary movements. . . . . . . S200 & 239 & 3.3 & 4.7 & 2.3 \\
\hline Low back symptoms. . . . . . . . . . . . s910 & 198 & 2.7 & 3.2 & 2.4 \\
\hline Disorders of motor functlons. . . . . . . . . . S095 & 135 & 1.9 & 1.6 & 2.1 \\
\hline Vision dysfunctions $\ldots \ldots \ldots \ldots \ldots \ldots \ldots$. $\ldots \ldots 3305$ & 131 & 1.8 & 1.3 & 2.2 \\
\hline Hand and finger symptoms $\ldots \ldots \ldots \ldots \ldots$. . . . . . . . & 117 & 1.6 & 1.2 & 1.9 \\
\hline Arm symptoms . . . . . . . . . . . . . . S945 & 113 & 1.6 & 1.7 & 1.4 \\
\hline Dlsturbances of memory . . . . . . . . . . . . . S215 & 105 & 1.4 & 1.3 & 1.6 \\
\hline Migralne headache $\ldots \ldots \ldots \ldots \ldots \ldots$. . . D365 & 85 & 1.2 & ${ }^{*} 0.8$ & 1.4 \\
\hline Shoulder symptoms . . . . . . . . . . . . . . . . . S940 & 84 & 1.2 & 1.2 & 1.1 \\
\hline General weakness. . . . . . . . . . . . s020 & 84 & 1.2 & "0.6 & 1.6 \\
\hline Disturbances of sleep . . . . . . . . . . . . S135 & 74 & 1.0 & 1.3 & ${ }^{*} 0.8$ \\
\hline Other diseases of central nervous system . . .D370 & 69 & 1.0 & $" 1.1$ & 0.9 \\
\hline All other reasons $\ldots \ldots \ldots \ldots \ldots \ldots \ldots$ & 1,951 & 26.9 & 31.8 & 23.4 \\
\hline
\end{tabular}

'Based on A Reason for Visit Classification for Ambulatory Care (AVC) (B).

Table 8. Annual number and percent distribution of office visits to neurologists and to all other physiclans by diagnostic services ordered or performed, averaged over a 2-year perlod: United State8, 1991-92

\begin{tabular}{|c|c|c|c|c|}
\hline \multirow[b]{2}{*}{ Vsit characteristic } & \multicolumn{2}{|c|}{ Usits to neurologists } & \multicolumn{2}{|c|}{$\begin{array}{l}\text { Usits to all } \\
\text { other physickans }\end{array}$} \\
\hline & $\begin{array}{l}\text { Number of } \\
\text { visits in } \\
\text { thousands }\end{array}$ & $\begin{array}{c}\text { Percent } \\
\text { distribution }\end{array}$ & $\begin{array}{l}\text { Number of } \\
\text { visits in } \\
\text { thousands }\end{array}$ & $\begin{array}{l}\text { Percent } \\
\text { distributtion }\end{array}$ \\
\hline $\begin{array}{c}\text { All visits } \ldots \ldots \ldots \ldots \ldots \ldots \\
\begin{array}{c}\text { Number of diagnostic } \\
\text { services ordered or performed }\end{array}\end{array}$ & 7,253 & 100.0 & 708,614 & 100.0 \\
\hline$\ldots \ldots \ldots \ldots \ldots \ldots \ldots$ & 2,388 & 32.9 & 250,765 & 35.4 \\
\hline One $\ldots \ldots \ldots \ldots \ldots \ldots \ldots \ldots \ldots$ & 3,061 & 42.2 & 246,957 & 34.9 \\
\hline Two $\ldots \ldots \cdots \cdots \cdots \cdots \cdots$ & 1,154 & 15.9 & 123,726 & 17.5 \\
\hline Throo................. & 450 & 6.2 & 52,095 & 7.4 \\
\hline Four. $\ldots \ldots \ldots \ldots \ldots \ldots \ldots \ldots$ & 122 & 1.7 & 21,450 & $\mathbf{3 . 0}$ \\
\hline Five or more $\ldots \ldots \ldots \ldots \ldots \ldots \ldots$ & 78 & 1.1 & 13,621 & 1.9 \\
\hline \multicolumn{5}{|l|}{ Dlagnostic services ordered or performed ${ }^{1}$} \\
\hline None $\ldots \ldots \ldots \ldots \ldots \ldots \ldots \ldots \ldots$ & 2,388 & 32.9 & 250,765 & 35.4 \\
\hline Blood pressure check $\ldots \ldots \ldots \ldots \ldots \ldots \ldots$ & 2,702 & $\mathbf{3 7 . 3}$ & 307,770 & 43.4 \\
\hline$\ldots \ldots \ldots \ldots \ldots \ldots$ & 130 & 1.8 & 95,565 & 13.5 \\
\hline EKG-resting ${ }^{2} \ldots \ldots \ldots \ldots \ldots \ldots \ldots$ & 86 & 1.2 & 21,419 & 3.0 \\
\hline Other radiology $\ldots \ldots \ldots \ldots \ldots \ldots \ldots \ldots \ldots$ & 602 & 8.3 & 38,315 & 5.4 \\
\hline Cholesterol measure. . . . . . . . . . . . . . & 42 & 0.6 & 25,360 & 3.6 \\
\hline Other lab test $\ldots \ldots \ldots \ldots \ldots \ldots \ldots \ldots \ldots$ & 977 & 13.5 & 119,980 & 16.9 \\
\hline Hearing test $\ldots \ldots \ldots \ldots \ldots \ldots \ldots \ldots$ & 66 & 0.9 & 10,130 & 1.4 \\
\hline visual acuity. $\ldots \ldots \ldots \ldots \ldots \ldots \ldots \ldots$ & 165 & 2.3 & 41,088 & 5.8 \\
\hline Mental status exam $\ldots \ldots \ldots \ldots \ldots \ldots \ldots$ & 638 & 8.8 & 8,102 & 1.1 \\
\hline Other $^{3} \ldots \ldots \ldots \ldots \ldots \ldots \ldots \ldots \ldots$ & 2,196 & 30.3 & 143,983 & 20.3 \\
\hline
\end{tabular}

${ }^{i}$ Total may exceod total number of visits because more than one catogory may be reportod per visit.

2EKG is electrocardiogram.

${ }^{3}$ The following diagnostic service categories were not reportod at visits to neurologists: allergy testing, spirometry, and pap test. The following diagnostic service catogories were reported at visits to neurologists, but with froguencies that were too low to produco reliable estimates: EKG-exercise, mammogram, strop throat tost, chest $x$ ray, and HIV (human immunodeficioncy virus) sorology. These services have been included in the "other" calogory. Unspecified diagnostic services accounted for 29.6 percent of all reportod services at visits to neurologists. from a 2-year total of 3.8 million in 1975-76 to 14.5 million in 1991-92. The 1975-76 total represented approximately 0.3 percent of all visits to office-based physicians during that time period. The 1991-92 share, 1.0 percent, was significantly higher. According to data from the American Medical Association, there were 6,257 nonfederally employed, office-based neurologists in the United States (excluding possessions) in 1992 compared with 1,847 in 1975 , an increase of 238.8 percent $(12,13)$.

The rate of visits to neurologists increased from an average of 0.9 visits per 100 persons per year in 1975-76 to 2.9 visits per 100 persons per year in 1991-92. The age-adjusted visit rate for 1991-92 was 2.3 visits per 100 persons, using the 1975-76 U.S. population as the standard (figure 4). Visits to neurologists during 1975-92 are shown by patient's age in table 18 .

\section{References}

1. McCaig LF. National Hospital Ambulatory Medical Care Survey: 1992 emergency department summary. Advance data from vital and health statistics, no. 245 . Hyattsville, Maryland. National Center for Health Statistics. 1994.

2. McCaig LF. National Hospital Ambulatory Medical Care Survey: 1992 outpatient department summary. Advance data from vital and health statistics, no. 248. Hyattsville, Maryland. National Center for Health Statistics. 1994.

3. Nelson CR, Stussman BJ. Alcoholand drug-related visits to hospital emergency departments: 1992 National Hospital Ambulatory Medical Care Survey. Advance data from vital and health statistics, no. 251. Hyattsville, Maryland. National Center for Health Statistics. 1994.

4. Burt CW. Injury-related visits to hospital emergency departments: United States, 1992. Advance data from vital and health statistics, no. 261. Hyattsville, Maryland. National Center for Health Statistics. 1994.

5. Schappert SM. National Ambulatory Medical Care Survey: 1991 summary. Advance data from vital and bealth statistics; no. 230. Hyattsville, Maryland: National Center for Health Statistics. 1993. 
Table 9. Annual number and percent distribution of office visits to nourologlste by princlpal diagnosis, averaged over a 2-year period: Unlted States, 1991-92

\begin{tabular}{|c|c|c|}
\hline Principal dlagnosis and ICD-9-CM code' & $\begin{array}{l}\text { Number of } \\
\text { visits in } \\
\text { thousands }\end{array}$ & $\begin{array}{c}\text { Percent } \\
\text { distribution }\end{array}$ \\
\hline Ail visits & 7,253 & 100.0 \\
\hline Infectlous and parasitic diseases $\ldots \ldots \ldots \ldots \ldots \ldots \ldots \ldots$.001-139 & 65 & 0.9 \\
\hline Neoplasms . . . . . . . . . . . . . . . . . . . . . . . . .140-239 & 60 & 0.8 \\
\hline Endocrine, nutritional and metabolic diseases, and Immunity disorders. . . 240-279 & 75 & 1.0 \\
\hline Mental disorders . . . . . . . . . . . . . . . . . . . . . . . . . 290-319 & 602 & 8.3 \\
\hline Dlseases of the nervous system and sense organs $\ldots \ldots \ldots \ldots \ldots .320-389$ & 2,618 & 36.1 \\
\hline Dlseases of the circulatory system . . . . . . . . . . . . . . . . . . 390-459 & 344 & 4.7 \\
\hline Diseases of the respiratory system. . . . . . . . . . . . . . . . .460-519 & 41 & 0.6 \\
\hline Diseases of the musculoskeletal system and connective tlssue $\ldots \ldots \ldots .710-739$ & 1,060 & 14.6 \\
\hline Symptoms, signs, and ill-defined conditions . . . . . . . . . . .780-799 & 1,544 & 21.3 \\
\hline Injury and polsoning $\ldots \ldots \ldots \ldots \ldots \ldots \ldots \ldots \ldots \ldots$. $\ldots \ldots 0-999$ & 484 & 6.7 \\
\hline$\ldots \ldots \ldots \ldots \ldots \ldots \ldots$ vo1-V82 & 159 & 2.2 \\
\hline All other dlagnoses ${ }^{2} \ldots \ldots$ & 71 & 1.0 \\
\hline Unknown ${ }^{3}$. & 132 & 1.8 \\
\hline
\end{tabular}

${ }^{1}$ Based on the International Classification of Diseases, Sth Rovision, Clinical Modification (ICD-Q-CM) (9).

Includes diseases of the blood and blood-forming organs (280-289); diseases of the digestive system (520-579); diseases of the genitourinary system $(580-629)$; complications of pregnancy, childbirth, and the puerperium (630-676); diseases of the ski and subcutaneous tissue (680-709); congenital anomalies (740-759); and cortain conditions originating in the perinatal period $(760-779)$.

Includes blank diagnoses, uncodable diagnoses, and illegible diagnoses.

Table 10. Annual number and percent distribution of office visits to neurologists by the 20 most frequently mentloned principal dlagnoses, according to patient's sox, averaged over 2-year perlod: United States, 1991-92

\section{Princlpal diagnosls and ICD-9-CM code}

\begin{tabular}{cc}
$\begin{array}{c}\text { Number of } \\
\text { Vists in } \\
\text { thousands }\end{array}$ & Total Matlent's sex \\
\cline { 2 - 3 } & Male Fomale
\end{tabular}

Percent distrbution

All visits

General symptoms. . . . . . . . . . . . . . . . . . 780

Convulsions . . . . . . . . . . . . . . . . .780.3

Coma and stupor, hallucinations, syncope and collapse, dizziness and giddlness, sleep dlsturbances, malalse and taltgue, other general

symptoms. . . . . . . . . 780.0-780.2, 780.4-780.5, 780.7, 780.9

Migraine . . . . . . . . . . . . . . . . . . . . 346

Symptoms involving head and neck . . . . . . . . . . . . . .784

Headache . . . . . . . . . . . . . . . . . . . .784.0

Aphasla, other speech disturbance, other symbollc dysfunction,

epistaxis . . . . . . . . . . . . . . . 784.3, 784.5-784.7

Parkinson's dlsease . . . . . . . . . . . . . . . . . . . .382

Other and unspecified disorders of back . . . . . . . . . . . . 724

Mononeuritls of upper llmb and mononeurttis multiplex . . . . . . . .354

Multlple sclerosis. . . . . . . . . . . . . . . . . . . . . . 340

Sprains and strains of other and unspecified parts of back . . . . . .847

Other disorders of soft tlssues . . . . . . . . . . . . . . . . . . . . . . . . . . .

Other dlsorders of cervical reglon . . . . . . . . . . . . . 723

Spectal symptoms or syndromes, not elsewhere classifled . . . . . . 307

Epllepsy . . . . . . . . . . . . . . . . . . . . . . . . 345

Acute, but III-defined, cerebrovascular disease . . . . . . . . . . 436

Hyperknetic syndrome of chlldhood . . . . . . . . . . . . 314

Spondylosls and alled disorders . . . . . . . . . . . . . . .721

Intervertebral dlsc disorders . . . . . . . . . . . . . . . . . . . 722

Other extrapyramidal dlsease and abnormal movement disorders. . .333

Hereditary and Idlopathic peripheral neuropathy. . . . . . . . . 356

Mononeuritis of lower limb . . . . . . . . . . . . . . . . . . . . . . . . . . . . . . . . . . .

Neurotlc disorders . . . . . . . . . . . . . . . . . . . . . . . . . . . . . . . . . . .

All other diagnoses $\ldots \ldots \ldots \ldots \ldots \ldots$
6. Schappert SM. National Ambulatory Medical Care Survey: 1991 summary. National Center for Health Statistics. Vital and Health Stat 13(116). 1994.

7. Scbappert SM. National Ambulatory Medical Care Survey: 1992 summary. Advance data from vital and health statistics; no. 253. Hyattsville, Maryland: National Center for Health Statistics. 1994.

8. Schneider D, Appleton L, McLemore T. A reason for visit classification for ambulatory care. National Center for Health Statistics. Vital and Health Stat 2(78). 1979.

9. Public Health Service and Health Care Financing Administration. International Classification of Diseases, 9th Revision, clinical modification. Washington: Public Health Service. 1980.

10. Koch H, Campbell W. The collection and processing of drug information. National Ambulatory Medical Care Survey, 1980. National Center for Health Statistics. Vital and Health Stat 2(90). 1982.

11. Food and Drug Administration. National Drug Code Directory, 1985 Edition. Washington: Public Health Service, 1985.

12. Goodman LJ, Mason HR. Physician distribution and medical licensure in the United States, 1975. American Medical Association, Center for Health Services Research and Development. Chicago, Mlinois. 1976.

13. Roback G, Randolph L, Seidman B. Physician characteristics and distribution in the United States. American Medical Association, Department of Physician Data Services. Chicago, Illinois: 1993. 
Table 11. Annual number and percent distribution of office vlsits to neurologists by the most frequently mentioned principal diagnoses, according to patient's age, averaged over a 2-year period: United States, 1991-92

\begin{tabular}{|c|c|c|c|}
\hline Princloal dlagnosls and ICD-9-CM code' & $\begin{array}{c}\text { Number of vists } \\
\text { in thousands }\end{array}$ & $\begin{array}{c}\text { Percent } \\
\text { distribution }\end{array}$ & $\begin{array}{c}\text { Cumulathe } \\
\text { percent }\end{array}$ \\
\hline \multicolumn{4}{|l|}{ Under 15 years } \\
\hline 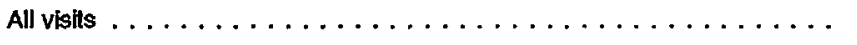 & 770 & 100.0 & $\cdots$ \\
\hline General symptoms . . . . . . . . . . . . . . . . . . . . . . .780 & 287 & 37.3 & 37.3 \\
\hline Hyperknetlc syndrome of childhood . . . . . . . . . . . . . . . .314 & 102 & 13.3 & 50.6 \\
\hline Migralne . . . . . . . . . . . . . . . . . . . .346 & 53 & 6.8 & 57.4 \\
\hline Symptoms involving head and neck . . . . . . . . . . . . . . . . 784 & 41 & 5.4 & 62.8 \\
\hline 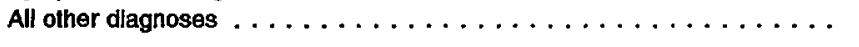 & 287 & 37.2 & 100.0 \\
\hline \multicolumn{4}{|l|}{$15-24$ years } \\
\hline 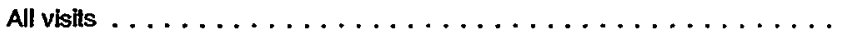 & 577 & 100.0 & $\cdots$ \\
\hline General symptoms . . . . . . . . . . . . . . . . . . . . . . . . . .780 & 120 & 20.8 & 20.8 \\
\hline Migraine . . . . . . . . . . . . . . . . . . . . . 346 & 64 & 11.1 & 31.9 \\
\hline Symptoms involving head and neck . . . . . . . . . . . . . . .784 & 57 & 9.8 & 41.7 \\
\hline Sprains and strains of other and unspeclfied parts of back . . . . . . 847 & 56 & 9.7 & 51.3 \\
\hline 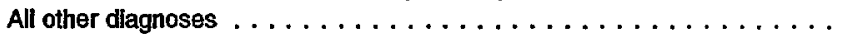 & 281 & 48.7 & 100.0 \\
\hline \multicolumn{4}{|l|}{$25-44$ years } \\
\hline 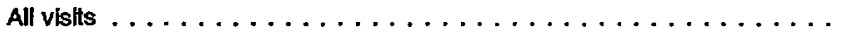 & 2,559 & 100.0 & $\cdots$ \\
\hline Migraine . . . . . . . . . . . . . . . . . . . . .346 & 421 & 16.4 & 16.4 \\
\hline General symptoms . . . . . . . . . . . . . . . . . . . . . . .780 & 256 & 10.0 & 26.4 \\
\hline Symploms involving head and neck . . . . . . . . . . . . . . . . 784 & 198 & 7.8 & 34.1 \\
\hline Multple sclerosis . . . . . . . . . . . . . . . . . . . 340 & 139 & 5.4 & 39.6 \\
\hline Sprains and strains of other and unspecifled parts of back . . . . . . . .847 & 126 & 4.9 & 44.5 \\
\hline Other and unspeclifed dlsorders of back. . . . . . . . . . . . . . . . . .724 & 120 & 4.7 & 49.2 \\
\hline Mononeuritis of upper limb and mononeurttis multiplex . . . . . . . . . .354 & 100 & 3.9 & 53.1 \\
\hline Other dlsarders of sott tissues . . . . . . . . . . . . . . . . . . .729 & 95 & 3.7 & 56.8 \\
\hline Other disorders of cenvical reglon . . . . . . . . . . . . . . .723 & 95 & 3.7 & 60.5 \\
\hline 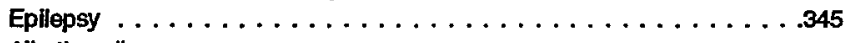 & 79 & 3.1 & 63.6 \\
\hline 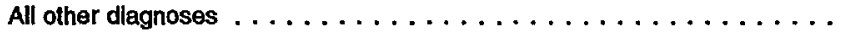 & 930 & 36.4 & 100.0 \\
\hline \multicolumn{4}{|l|}{$45-64$ years } \\
\hline 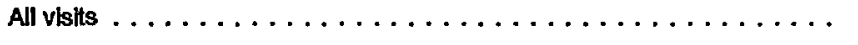 & 1,893 & 100.0 & $\cdots$ \\
\hline General symptoms . . . . . . . . . . . . . . . . . . . . . . . . . . . . & 194 & 10.2 & 10.2 \\
\hline Migraine . . . . . . . . . . . . . . . . . . . . . . . . . . . . . . . . . . . & 183 & 9.6 & 19.8 \\
\hline Other and unspecified dlsorders of back. . . . . . . . . . . . . . . . .724 & 97 & 5.1 & 25.0 \\
\hline Symptoms involving head and neck . . . . . . . . . . . . . 784 & 96 & 5.1 & 30.1 \\
\hline Multiple sclerosis . . . . . . . . . . . . . . . . . . . . . . . . . . 340 & 94 & 5.0 & 35.0 \\
\hline Mononeurtis of upper limb and mononeuritis multiplex . . . . . . . . . 354 & 86 & 4.5 & 39.6 \\
\hline Parkinson's disease . . . . . . . . . . . . . . . . . . . . 332 & 83 & 4.4 & 44.0 \\
\hline Other disorders of soft tissues . . . . . . . . . . . . . . . . 729 & 69 & 3.6 & 47.6 \\
\hline Other disorders of cenvical region . . . . . . . . . . . . . . . . .723 & 69 & 3.6 & 51.2 \\
\hline Spondylosis and allied disorders $\ldots \ldots \ldots \ldots \ldots \ldots \ldots \ldots$. . . . . . . . . . . . . & 56 & 3.0 & 54.2 \\
\hline 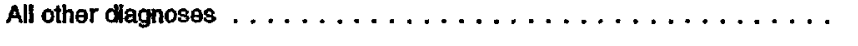 & 867 & 45.8 & 100.0 \\
\hline \multicolumn{4}{|l|}{65 years and over } \\
\hline 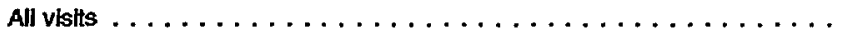 & 1,454 & 100.0 & $\cdots$ \\
\hline Parkinson's dlsease . . . . . . . . . . . . . . . . . . . . . . . . . 332 & 246 & 16.9 & 16.9 \\
\hline General symptoms . . . . . . . . . . . . . . . . . . . . . . . 780 & 108 & 7.4 & 24.3 \\
\hline Acute, but ill-defined, cerebrovascular disease . . . . . . . . . . . 436 & 86 & 5.9 & 30.2 \\
\hline Mononeuritis of upper limb and mononeurkis multiplex . . . . . . . . . .354 & 78 & 5.4 & 35.6 \\
\hline Hereditary and idlopathic peripheral neuropathy . . . . . . . . . 356 & 58 & 4.0 & 39.6 \\
\hline Jther extrapyramidal disease and abnormal movement disorders . . . . .333 & 53 & 3.7 & 43.2 \\
\hline Other and unspectiled disorders of back. . . . . . . . . . . . . 724 & 53 & 3.6 & 46.8 \\
\hline All other dlagnoses $\ldots \ldots \ldots \ldots \ldots \ldots \ldots \ldots \ldots \ldots \ldots$ & 772 & 53.1 & 100.0 \\
\hline
\end{tabular}

'Based on the Intemational Classiffeation of Disaases, 9th Rovision, Clinical Modification (ICD-9-CM) (9).

NOTE: A maximum of 10 diagnoses wero listed per age group. Only reliable eatimates were included in the table, so some categories may have tewer than 10 diagnoses. 
Table 12. Annual number and percent distribution of office visits to neurologists and to all other physiclans by therapeutic services ordered or provided, averaged over a 2-year perlod: United States, 1991-92

\begin{tabular}{|c|c|c|c|c|}
\hline \multirow[b]{2}{*}{ Therapeutic service ordered or provided } & \multicolumn{2}{|c|}{ Visits to neurologists } & \multicolumn{2}{|c|}{ Visits to all other physlclans } \\
\hline & $\begin{array}{l}\text { Number of } \\
\text { visits } \\
\text { in thousands }\end{array}$ & $\begin{array}{c}\text { Percent } \\
\text { distrlbution }\end{array}$ & $\begin{array}{l}\text { Number of } \\
\text { visits } \\
\text { in thousands }\end{array}$ & $\begin{array}{c}\text { Percent } \\
\text { distribution }\end{array}$ \\
\hline All vistts $\ldots \ldots \ldots \ldots \ldots \ldots \ldots \ldots \ldots$ & 7,253 & 100.0 & 708,614 & 100.0 \\
\hline \multicolumn{5}{|l|}{ Medication therapy } \\
\hline New or continued medication $\ldots \ldots \ldots \ldots \ldots$ & 4,624 & 63.7 & 450,237 & 63.5 \\
\hline 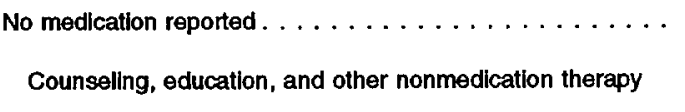 & 2,629 & 36.3 & 258,377 & 36.5 \\
\hline None . . . . . . . . . . . . . . . . . . . . & 5,230 & 72.1 & 481,938 & 68.0 \\
\hline 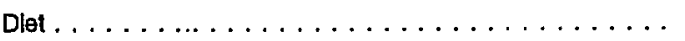 & 379 & 5.2 & 82,639 & 11.7 \\
\hline 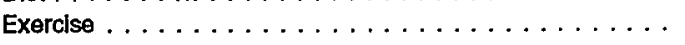 & 513 & 7.1 & 54,426 & 7.7 \\
\hline Cholesterol reduction $\ldots \ldots \ldots \ldots \ldots \ldots \ldots$ & 73 & 1.0 & 21,567 & 3.0 \\
\hline Weight reduction $\ldots \ldots \ldots \ldots \ldots \ldots \ldots$ & 174 & 2.4 & 27,773 & 3.9 \\
\hline Drug abuse . . . . . . . . . . . . . . . . & 37 & 0.5 & 1,787 & 0.3 \\
\hline Alcohol abuse. . . . . . . . . . . . . . . . . & $* 17$ & ${ }^{\star} 0.2$ & 3,157 & 0.4 \\
\hline Smokng cessatton. . . . . . . . . . . . . . . & 49 & 0.7 & 15,621 & 2.2 \\
\hline Famlly/soclal $\ldots \ldots \ldots \ldots \ldots \ldots \ldots \ldots \ldots$ & 152 & 2.1 & 13,574 & 1.9 \\
\hline Growth/development. . . . . . . . . . . . . . . . & 90 & 1.2 & 17,145 & 2.4 \\
\hline Family planning. . . . . . . . . . . . . . & $\star 14$ & ${ }^{*} 0.2$ & 6,220 & 0.9 \\
\hline Other counseling $\ldots \ldots \ldots \ldots \ldots \ldots \ldots$ & 579 & 8.0 & 58,119 & 8.2 \\
\hline Psychotherapy . . . . . . . . . . . . . . . & 163 & 2.2 & 18,970 & 2.7 \\
\hline Gorrective lenses. . . . . . . . . . . . . . . . & - & - & 7,763 & 1.1 \\
\hline Hearing ald $\ldots \ldots \ldots \ldots \ldots \ldots \ldots \ldots \ldots$ & - & - & 432 & 0.1 \\
\hline Physiotherapy. . . . . . . . . . . . . . . & 465 & 6.4 & 14,829 & 2.1 \\
\hline Other $\ldots \ldots \ldots \ldots \ldots \ldots \ldots \ldots \ldots \ldots$ & 178 & 2.5 & 20,163 & 2.8 \\
\hline \multicolumn{5}{|l|}{ Ambulatory surgery scheduled or performed } \\
\hline No procedures $\ldots \ldots \ldots \ldots \ldots \ldots \ldots \ldots \ldots$ & 7,159 & 98.7 & 665,389 & 93.9 \\
\hline One or more procedures $\ldots \ldots \ldots \ldots \ldots \ldots \ldots$ & 94 & 1.3 & 43,225 & 6.1 \\
\hline
\end{tabular}

${ }^{1}$ Total may exceed total number of visits because more than one category may be reported per visit. 
Table 13. Annual number and percent distribution of drug mentions at office visits to neurologists by therapeutic classification, averaged over a 2-year perlod: United States, 1991-92

\begin{tabular}{|c|c|c|}
\hline Therapeutic classification ${ }^{1}$ & $\begin{array}{l}\text { Number of } \\
\text { drug mentions } \\
\text { in thousands }\end{array}$ & $\begin{array}{c}\text { Percent } \\
\text { distribution }\end{array}$ \\
\hline All mentions. . . . . . . . . . . . . . . . . . . & 8,143 & 100.0 \\
\hline Neurologle drugs $\ldots \ldots \ldots \ldots \ldots \ldots$ & 2,109 & 25.9 \\
\hline Anticonvulsants $\ldots \ldots \ldots \ldots \ldots \ldots$ & 1,294 & 15.9 \\
\hline Drugs used to treat skeletal muscle hyperactivity. . . . . . & 419 & 5.1 \\
\hline Drugs used in extrapyramidal movement disorders . . . . . & $\mathbf{3 7 5}$ & 4.6 \\
\hline Drugs used in myasthenia gravls $\ldots \ldots \ldots \ldots$ & $\mathbf{2 0}$ & ${ }^{\star} 0.2$ \\
\hline Drugs used tor rellef of pain $\ldots \ldots \ldots \ldots \ldots \ldots$ & 1,825 & 22.4 \\
\hline General analgesics . . . . . . . . . . . . . . . & 910 & 11.2 \\
\hline 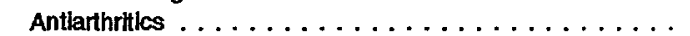 & 612 & 7.5 \\
\hline Drugs used to treat migraine and other headaches . . . . . & 280 & 3.4 \\
\hline 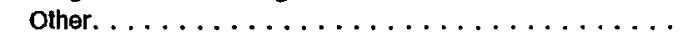 & "23 & ${ }^{*} 0.3$ \\
\hline Psychopharmacologic drugs . . . . . . . . . . . . & 1,642 & 20.2 \\
\hline 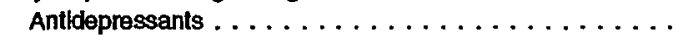 & 920 & 11.3 \\
\hline Antlanxlety drugs $\ldots \ldots \ldots \ldots \ldots \ldots \ldots \ldots$ & 269 & 3.3 \\
\hline Sedatives and hypnotics . . . . . . . . . . . . & 224 & 2.7 \\
\hline Ant'psychotic drugs $\ldots \ldots \ldots \ldots \ldots \ldots \ldots$ & 116 & 1.4 \\
\hline CNS stimulants, anorexiants $\ldots \ldots \ldots \ldots \ldots$ & 113 & 1.4 \\
\hline Cardlovascular-renal drugs. . . . . . . . . . . . . . & 929 & 11.4 \\
\hline Anthypertensive agents $\ldots \ldots \ldots \ldots \ldots$ & 435 & 5.3 \\
\hline Antlarrhythmic agents $\ldots \ldots \ldots \ldots \ldots \ldots$ & 166 & 2.0 \\
\hline 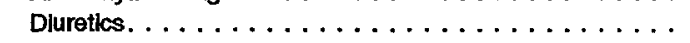 & 137 & 1.7 \\
\hline 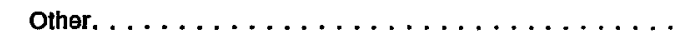 & 191 & 2.3 \\
\hline Hormones and agents affecting hormonal mechanisms .... & 310 & $\mathbf{3 . 8}$ \\
\hline Respiratory tract drugs $\ldots \ldots \ldots \ldots \ldots \ldots$ & 184 & 2.3 \\
\hline Antimlcroblal drugs. $\ldots \ldots \ldots \ldots \ldots \ldots$ & 178 & 2.2 \\
\hline Gastrointestinal drugs . . . . . . . . . . . . . . & 145 & 1.8 \\
\hline Metabollc and nutrient agents $\ldots \ldots \ldots \ldots \ldots$ & 127 & 1.6 \\
\hline Hematologlc drugs $\ldots \ldots \ldots \ldots \ldots \ldots \ldots \ldots$ & 79 & 1.0 \\
\hline Otologic drugs $\ldots \ldots \ldots \ldots \ldots \ldots \ldots \ldots$ & 70 & 0.9 \\
\hline Skln/mucous membrance & 55 & 0.7 \\
\hline Other $^{2} \ldots \ldots \ldots \ldots \ldots \ldots \ldots \ldots \ldots \ldots \ldots \ldots$ & 68 & 0.8 \\
\hline Unclassifled, miscellaneous . . . . . . . . . . . . . . & 423 & 5.2 \\
\hline
\end{tabular}

'Therapeutic classification is based on the standard drug classification used in the National Drug Code Directory, 1985 edition (NDC) (11).

Includes anesthetics, radiopharmaceuticals/contrast media, oncolytics, immunologic agents, ophthalmic drugs, and antiparasitic agents. 
Table 14. Number and percent of drug mentions at office visits to neurologists by the 20 most frequently used generic substances, averaged over a 2-year perlod: United States, 1991-92

\begin{tabular}{|c|c|c|}
\hline Generic substance & $\begin{array}{l}\text { Number of } \\
\text { occurrences in } \\
\text { thousands }^{1}\end{array}$ & $\begin{array}{c}\text { Percent of } \\
\text { all drug mentions }\end{array}$ \\
\hline All mentlons . . . . . . . . . . . . . . . & 10,186 & $\cdots$ \\
\hline Acetaminophen . . . . . . . . . . . & 673 & 8.3 \\
\hline Carbamazepine. . . . . . . . . . . . . & 491 & 6.0 \\
\hline Amitriptyline . . . . . . . . . . . . . . & 398 & 4.9 \\
\hline Phenytoin $\ldots \ldots \ldots \ldots \ldots \ldots \ldots$ & 352 & 4.3 \\
\hline Aspirin $\ldots \ldots \ldots \ldots \ldots \ldots \ldots \ldots$ & 345 & 4.2 \\
\hline Caffelne $\ldots \ldots \ldots \ldots \ldots \ldots \ldots \ldots$ & 283 & 3.5 \\
\hline Levodopa . . . . . . . . . . . . . . . . & 281 & 3.5 \\
\hline Carbldopa . . . . . . . . . . . . . & 281 & 3.5 \\
\hline Butabltal . . . . . . . . . . . . . . & 241 & 3.0 \\
\hline 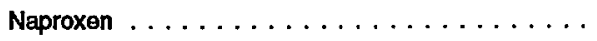 & 230 & 2.8 \\
\hline Dlvalproex sodlum . . . . . . . . . . . . . & 216 & 2.7 \\
\hline Propranolol . . . . . . . . . . . . . . & 213 & 2.6 \\
\hline Verapami . . . . . . . . . . . . . . . & 175 & 2.1 \\
\hline Dichloralantlpyrine . . . . . . . . . . . & 162 & 2.0 \\
\hline Isometheptene mucale . . . . . . . . . . . . . . & 162 & 2.0 \\
\hline Nortriptyline . . . . . . . . . . . . . . & 159 & 2.0 \\
\hline 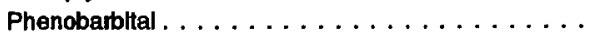 & 147 & 1.8 \\
\hline Codeine $\ldots \ldots \ldots \ldots \ldots \ldots \ldots$ & 139 & 1.7 \\
\hline 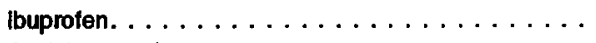 & 139 & 1.7 \\
\hline Cyclobenzaprine ................... & 125 & 1.5 \\
\hline
\end{tabular}

${ }^{1}$ Frequency of mention combines single-ingredient agents wh mentions of the agent as an ingredient in a combination drug.

${ }^{2}$ Based on an average of $8,143,000$ drug mentions per year at office visits to neurologists during 1991-92.

Table 15. Annual number, percent distrlbution, and therapeutic classification of the 20 drugs most frequently prescribed at office visits to neurologlsts by entry name, averaged over a 2-year perlod: United States, $1991-92$

\begin{tabular}{|c|c|c|c|}
\hline Entry name of arug' & $\begin{array}{l}\text { Number of } \\
\text { drug mentions } \\
\text { in thousands }\end{array}$ & $\begin{array}{c}\text { Percent } \\
\text { distribution }\end{array}$ & Therapeutic classification ${ }^{2}$ \\
\hline All mentlons. . . . . . . & 8,143 & 100.0 & $\cdots$ \\
\hline Tegretol. . . . . . . . . & 491 & 6.0 & Neurologic drugs (anticonvulsants) \\
\hline Dilantin . . . . . . . . . & 352 & 4.3 & Neurologic drugs (anticonvulsants) \\
\hline Sinemet . . . . . . . . & 281 & 3.5 & Neurologlc drugs (drugs used in extrapyramidal movement disorders) \\
\hline Elavil . . . . . . . . . & 251 & 3.1 & Psychopharmacologic drugs (antldepressants) \\
\hline Depakote. . . . . . . . . . & 216 & 2.7 & Neurologic drugs (anticonvulsanis) \\
\hline Inderal $\ldots \ldots \ldots \ldots$ & 211 & 2.6 & Cardiovascular-renal drugs (antihypertensive agents) \\
\hline Midrin. . . . . . . . . & 162 & 2.0 & Drugs used for rellef of pain (drugs used to treat migraine and other headaches) \\
\hline Pamelor . . . . . . . . . . & 145 & 1.8 & Psychopharmacologic drugs (antldepressants) \\
\hline Anaprox .......... & 131 & 1.6 & Drugs used for relief of pain (antiarthritics) \\
\hline Phonobarbltal . . . . . . . & 126 & 1.5 & Psychopharmacologic drugs (sedathes and hypnotics) \\
\hline Amitriptyline . . . . . . . & 125 & 1.5 & Psychopharmacologic drugs (antidepressants) \\
\hline Flexerll . . . . . . . . . & 125 & 1.5 & Neurologic drugs (drugs used to treat skeletal muscle hyperactivity) \\
\hline Calan. . . . . . . . . & 113 & 1.4 & Cardlovascular-renal drugs (antlarrhythmic agents) \\
\hline Prozac . . . . . . . . & 109 & 1.3 & Psychopharmacologic drugs (antidepressants) \\
\hline Naprosyn. . . . . . . . . & 98 & 1.2 & Drugs used for rellef of pain (antlarthritics) \\
\hline Mysoline . . . . . . . . . & 95 & 1.2 & Neurologic drugs (anticonvulsants) \\
\hline Aspirin $\ldots \ldots \ldots \ldots$ & 92 & 1.1 & Drugs used for rellef of paln (general analgesics) \\
\hline Piltalin. . . . . . . . . & 92 & 1.1 & Psychopharmacologic drugs (CNS stimulants, anorexianls) \\
\hline Darvocet-N ........ & 90 & 1.1 & Drugs used for relief of paln (general analgesics) \\
\hline Floricet . . . . . . . . & 85 & 1.0 & Drugs used for rellef of pain (general analgesics) \\
\hline All other $\ldots \ldots \ldots \ldots$ & 4,754 & 58.4 & $\cdots$ \\
\hline
\end{tabular}

The trade or generic name used by the physician on the prescription or other medical records.

2 Therapeutic classification is based on the standard drug classification used in the National Drug Code Directory, 1985 edition (NDC) (11). 
Table 16. Annual number and percent distrlbution of office visits to neurologists and to all other physicians by disposition of visit, averaged over a 2-year poriod: Unitod States, 1991-92

\begin{tabular}{|c|c|c|c|c|}
\hline \multirow[b]{2}{*}{ Disposittion of vistt } & \multicolumn{2}{|c|}{ Visits to nourologists } & \multicolumn{2}{|c|}{ Visits to all other physickans } \\
\hline & $\begin{array}{l}\text { Number of } \\
\text { visits in } \\
\text { thousands }\end{array}$ & $\begin{array}{c}\text { Percent } \\
\text { distribution }\end{array}$ & $\begin{array}{l}\text { Number of } \\
\text { vists in } \\
\text { thousands }\end{array}$ & $\begin{array}{l}\text { Percent } \\
\text { distribution }\end{array}$ \\
\hline All vistis & 7,253 & 100.0 & 708,614 & 100.0 \\
\hline Relum at specified time. . . . . . . & 5,117 & 70.5 & 441,353 & 62.3 \\
\hline Retum $\|$ needed $\ldots \ldots \ldots \ldots$ & 827 & 11.4 & 163,592 & 23.1 \\
\hline Return to referring physician. . . . . . & 560 & 7.7 & 6,285 & 0.9 \\
\hline No followup planned. . . . . . . . & 490 & 6.8 & 67,719 & 9.6 \\
\hline Telephone followup planned . . . . . . & 433 & 6.0 & 21,575 & $\mathbf{3 . 0}$ \\
\hline Refer to other physiclan & 276 & 3.8 & 21,838 & 3.1 \\
\hline Admit to hospital & 50 & 0.7 & 5,570 & 0.8 \\
\hline Other dlsposition . & 47 & 0.6 & 7,115 & 1.0 \\
\hline
\end{tabular}

${ }^{1}$ Total may exceed total number of visits because more than one category may be reported for each visit.

Table 17. Annual number and porcent distribution of office visits to neurologiste and to all other physicians by duration of visit, averaged over a 2-year period: United States, 1991-92

\begin{tabular}{|c|c|c|c|c|}
\hline \multirow[b]{2}{*}{ Duration of vist } & \multicolumn{2}{|c|}{ Vsits to neurologists } & \multicolumn{2}{|c|}{ Vsits to all other physlckans } \\
\hline & $\begin{array}{c}\text { Number of } \\
\text { vists hn } \\
\text { thousands }\end{array}$ & $\begin{array}{c}\text { Percent } \\
\text { olstribution }\end{array}$ & $\begin{array}{c}\text { Number of } \\
\text { vists in } \\
\text { thousands }\end{array}$ & $\begin{array}{c}\text { Percemt } \\
\text { alstribution }\end{array}$ \\
\hline All visits $\ldots \ldots \ldots \ldots \ldots \ldots \ldots$ & 7,253 & 100.0 & 708,614 & 100.0 \\
\hline o minutes ${ }^{1}$. & $" g$ & ${ }^{*} 0.1$ & 8,502 & 1.2 \\
\hline $1-5$ minutes $\ldots \ldots \ldots \ldots \ldots \ldots$ & "20 & $* 0.3$ & 57,800 & 8.2 \\
\hline 6-10 minutes . . . . . . . . . . . . & 399 & 5.5 & 186,473 & 26.3 \\
\hline $11-15$ minutes $\ldots \ldots \ldots \ldots \ldots \ldots \ldots$ & 1,502 & 20.7 & 227,145 & 32.1 \\
\hline $16-30$ minutes $\ldots \ldots \ldots \ldots \ldots \ldots$ & 2,964 & 40.9 & 179,208 & 25.3 \\
\hline $31-60$ minutes $\ldots \ldots \ldots \ldots \ldots$ & 2,088 & 28.8 & 45,710 & 6.5 \\
\hline More than 60 minutes. . . . . . . . . . . & 271 & 3.7 & 3,777 & 0.5 \\
\hline
\end{tabular}

Visits in which there was no faco-to-face contact between the physician and the patient.

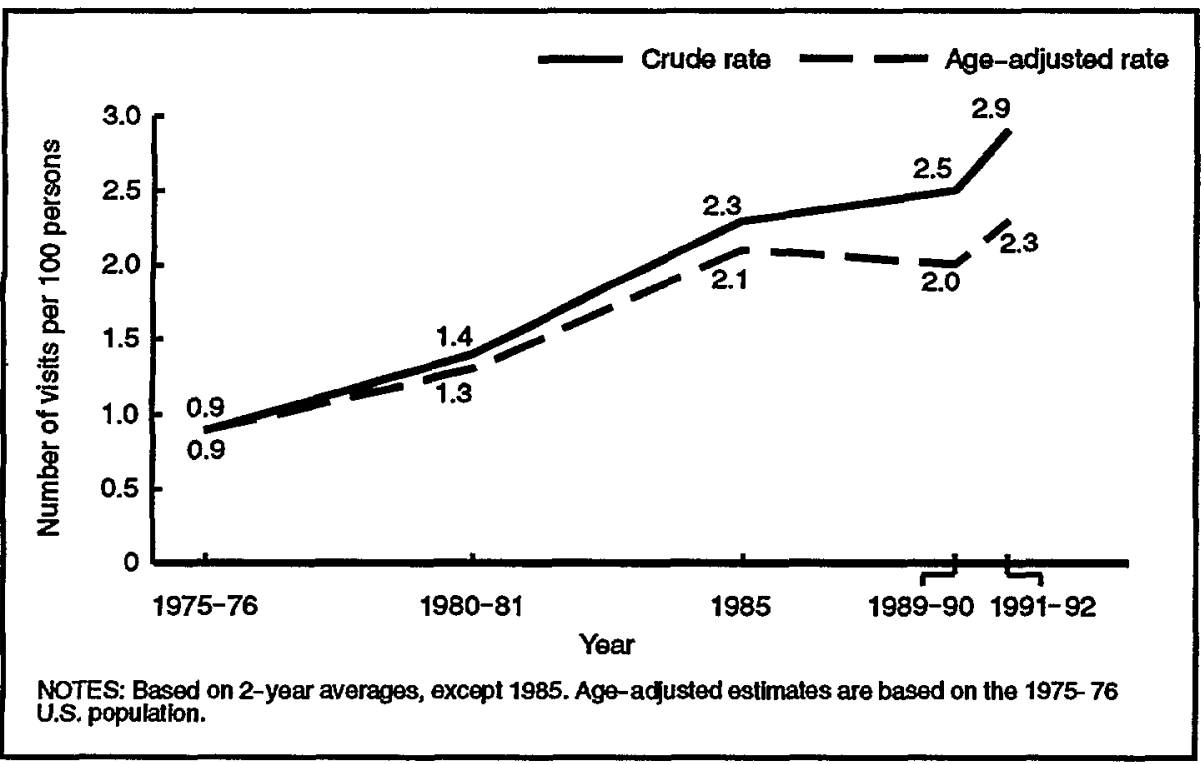

Figure 4. Annual rate of office visits to neurologists: United States, 1975-92 
Table 18. Number, percent distribution, and rate of office visits to neurologists, by patient's age: United States, 1975-92

\begin{tabular}{|c|c|c|c|c|c|}
\hline Patient's age & $1975-76$ & $1980-81$ & 1985 & $1989-90$ & $1991-92$ \\
\hline & \multicolumn{5}{|c|}{ Number of visits in thousands } \\
\hline All visits $\ldots \ldots \ldots \ldots \ldots \ldots \ldots$ & 1,892 & 3,013 & 4,992 & 6,167 & 7,253 \\
\hline Under 15 years $\ldots \ldots \ldots \ldots \ldots \ldots$ & $" 147$ & 338 & 403 & 311 & 770 \\
\hline $15-24$ years. . . . . . . . . . . . . & 248 & 311 & 500 & 542 & 577 \\
\hline $25-44$ years. . . . . . . . . . . . & 669 & 991 & 1,587 & 2,341 & 2,559 \\
\hline 45-64 years. . . . . . . . . . . . . . & 577 & 1,029 & 1,454 & 1,612 & 1,893 \\
\hline 65-74 years. . . . . . . . . . . . . . & 173 & 345 & 626 & 839 & 820 \\
\hline \multirow[t]{2}{*}{75 years and over $\ldots \ldots \ldots \ldots \ldots$} & $" 78$ & $* 176$ & 422 & 521 & 633 \\
\hline & \multicolumn{5}{|c|}{ Percent distribution } \\
\hline All visils $\ldots \ldots \ldots \ldots \ldots \ldots$ & 100.0 & 100.0 & 100.0 & 100.0 & 100.0 \\
\hline Under 15 years . . . . . . . . . . . . . & 7.8 & 11.2 & 8.1 & 5.0 & 10.6 \\
\hline 15-24 years. . . . . . . . . . . . . & 13.1 & 10.3 & 10.0 & 8.8 & 8.0 \\
\hline $25-44$ years. . . . . . . . . . . . & 35.4 & 32.9 & 31.8 & $\mathbf{3 8 . 0}$ & 35.3 \\
\hline 45-64 years. . . . . . . . . . . . . & 30.5 & 34.1 & 29.1 & 26.1 & 26.1 \\
\hline $65-74$ years . . . . . . . . . . . . . & 9.1 & 11.4 & 12.5 & 13.6 & 11.3 \\
\hline \multirow[t]{2}{*}{75 years and over . . . . . . . . . . } & 4.1 & "5.5 & 8.5 & 8.4 & 8.7 \\
\hline & \multicolumn{5}{|c|}{ Visit rate per 100 persons ${ }^{2}$} \\
\hline All visits $\ldots \ldots \ldots \ldots \ldots \ldots$ & 0.9 & 1.4 & 2.3 & 2.5 & 2.9 \\
\hline Under 15 years . . . . . . . . . . . . . . & ${ }^{\star} 0.3$ & 0.7 & 0.8 & 0.6 & 1.4 \\
\hline 15-24 years. . . . . . . . . . . . & 0.6 & 0.8 & 1.3 & 1.5 & 1.7 \\
\hline $25-44$ years. . . . . . . . . . . . & 1.3 & 1.6 & 2.7 & 2.9 & 3.2 \\
\hline 45-64 years. . . . . . . . . . . . & 1.3 & 2.4 & 3.3 & 3.5 & 4.0 \\
\hline $65-74$ years. . . . . . . . . . . & 1.3 & 2.2 & 3.8 & 4.7 & 4.5 \\
\hline 75 years and over . . . . . . . . . . & $* 1.0$ & $\approx 2.0$ & 4.1 & 4.5 & 5.2 \\
\hline
\end{tabular}

1 Numbers aro shown as 2-year averages except for 1985.

${ }^{2}$ Based on Bureau of the Census estimates of the civilian noninstitutionalized population for July 1 of each survey year. Rates for combined years are based on an average of the population estimates for July 1 of each year of the 2-year period. Survey years from 1975-1985 did not include Alaska or Hawaii. 


\section{Technical notes}

\section{Source of data and sample design}

The information presented in this report is based on data collected in the National Ambulatory Medical Care Survey (NAMCS) over the 2-year period from January 1991 through December 1992. The target universe of NAMCS includes office visits made in the United States by ambulatory patients to nonfederally employed physicians who are principally engaged in office practice, but not in the specialties of anesthesiology, pathology, or radiology. Telephone contacts and nonoffice visits are excluded.

A multistage probability sample design is used in NAMCS, involving samples of primary sampling units (PSU's), physician practices within PSU's, and patient visits within physician practices. The PSU's are counties, groups of counties, county equivalents (such as parishes or independent cities), or towns and townships (for some PSU's in New England). For 1991, a sample of 2,540 nonfederal, office-based physicians was selected from master files maintained by the American Medical Association and American Osteopathic Association. Physicians were screened at the time of the survey to ensure that they were eligible for survey participation. Of those screened, 1,887 physicians were eligible (in-scope) to participate in the survey. The remaining 653 physicians were ineligible. (out-of-scope) due to reasons of being retired, employed primarily in teaching, research, or administration, or other reasons. The physician response rate for the 1991 NAMCS was 72 percent.

For 1992, a sample of 3,000 nonfederal, office-based physicians was selected from master files maintained by the American Medical Association and American Osteopathic Association. Of those screened, 858 physicians were ruled ineligible (out-of-scope); 2,142 were in-scope for the survey. The physician response rate for the 1992 NAMCS was 71 percent.

Sample physicians were asked to complete Patient Record forms (figure 1) for a systematic random sample of office visits occurring during a randomly assigned 1-week reporting period. Responding physicians completed 33,795 Patient Record forms in 1991 and 34,606 Patient Record forms in 1992.

Characteristics of the physician's practice, such as primary specialty and type of practice, were obtained from the physicians during an induction interview. The U.S. Bureau of the Census, Housing Surveys Branch, was responsible for the survey's data collection. Processing operations and medical coding were performed by the National Center for Health Statistics, Health Care Survey Section, Research Triangle Park, North Carolina.

For 1992, several changes were made in the sample design of the NAMCS that should be considered in the interpretation of the survey results. In an effort to even the precision of estimates across each of the physician specialty strata in the sample design, the decision was made to increase the proportion in the sample of specialists in general surgery, psychiatry, otolaryngology, and neurology. Although this would result in a corresponding decrease in the sample of the larger physician specialties, most notably general and family practice, internal medicine, and pediatrics, the precision of these estimates tended to be much higher relative to the smaller specialties, and it was expected that the end result would be an acceptable balance of precision levels across all strata.

However, the reduced numbers of general practitioners, internists, and pediatricians sampled in 1992, coupled with the high percents of sampled physicians in these specialties who were determined to be ineligible (out-ofscope) for survey participation, resulted in low numbers of survey respondents in these categories and a lowering of the precision of these estimates relative to other survey years, especially when disaggregated by other variables such as race. Because visits made by black patients were often found to be clustered among the sampled physicians and were more likely to be made to general and family practitioners, which were undersampled in 1992, it is recommended that caution be exercised when interpreting differences in race data and individual physician specialties.

Despite the difference in sample sizes, the 1991 and 1992 surveys were identical in terms of survey instruments, definitions, and procedures. The resulting 2 years of data have been combined to provide more reliable estimates. All estimates, percent distributions, and rates presented here, unless otherwise noted, reflect 1991 and 1992 data that were averaged over the 2-year period.

\section{Sampling errors}

The standard error is primarily a measure of the sampling variability that occurs by chance when only a sample, rather than an entire universe, is surveyed. The relative standard error of an estimate is obtained by dividing the standard error by the estimate itself; the result is then expressed as a percent of the estimate.

Relative standard errors (RSE's) for estimated numbers of office visits, expressed as 2-year averages for the period 1991-92, are shown in table I. Relative standard errors for estimated numbers of drug mentions, also expressed as 2-year averages, are presented in table II. Standard errors for estimated percents of visits and drug mentions are displayed in tables III-VI.

Alternatively, relative standard errors for 2-year averages may be calculated using the following general formula, where $x$ is the average of interest in thousands multiplied by 2 to obtain the 2-year total, and $A$ and $B$ are the appropriate coefficients from table VII. The relative standard error obtained in this way applies to both the 2-year total and the 2-year average.

$$
\operatorname{RSE}(x)=\sqrt{A+\frac{B}{x}} \cdot 100
$$

Similarly, relative standard errors for percents may be calculated using the following general formula, where $p$ is the percent of interest and $x$ is the denominator of the percent in thousands (and the denominator is the 2-year aggregate estimate rather than the average itself), using the appropriate coefficient from table VII. (The 2-year 
Table I. Approximate relative standard errors for estimated numbers of office visits by selected physician specialties: National Ambulatory Medical Care Survey, 1991-92

\begin{tabular}{|c|c|c|}
\hline \multirow{2}{*}{$\begin{array}{c}\text { Estimated number of office visits } \\
\text { (expressed as annual average) } \\
\text { In thousands }\end{array}$} & \multicolumn{2}{|c|}{ Physician speclatty } \\
\hline & $A \|^{1}$ & Neurology ${ }^{2}$ \\
\hline & \multicolumn{2}{|c|}{ Relatlve standard error in percent } \\
\hline $25 \ldots \ldots \ldots \ldots \ldots \ldots \ldots$ & 110.8 & 34.7 \\
\hline $35 \ldots \ldots \ldots \ldots \ldots \ldots$ & 93.7 & 30.0 \\
\hline $50 \ldots \ldots \ldots \ldots \ldots \ldots \ldots$ & 78.4 & 25.9 \\
\hline 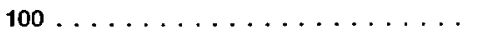 & 55.5 & 20.0 \\
\hline $250 \ldots \ldots \ldots \ldots \ldots \ldots$ & 35.2 & 15.5 \\
\hline $346 \ldots \ldots \ldots \ldots \ldots \ldots \ldots$ & 30.0 & 14.5 \\
\hline $500 \ldots \ldots \ldots \ldots \ldots \ldots \ldots$ & 25.0 & 13.6 \\
\hline $1,000 \ldots \ldots \ldots \ldots \ldots \ldots$ & 17.8 & 12.6 \\
\hline $2,500 \ldots \ldots \ldots \ldots \ldots \ldots \ldots$ & 11.6 & 11.9 \\
\hline $5,000 \ldots \ldots \ldots \ldots \ldots \ldots \ldots$ & 8.5 & 11.7 \\
\hline $10,000 \ldots \ldots \ldots \ldots \ldots \ldots$ & 6.5 & 11.6 \\
\hline $25,000 \ldots \ldots \ldots \ldots \ldots$ & 4.9 & 11.5 \\
\hline $50,000 \ldots \ldots \ldots \ldots \ldots \ldots$ & 4.2 & 11.5 \\
\hline $100,000 \ldots \ldots \ldots \ldots \ldots$ & 3.8 & 11.5 \\
\hline $250,000 \ldots \ldots \ldots \ldots \ldots \ldots$ & 3.6 & 11.5 \\
\hline $500,000 \ldots \ldots \ldots \ldots \ldots$ & 3.5 & 11.5 \\
\hline
\end{tabular}

The smallest reliable estimate for visits to aggregated specialties is 346,000 visits per year (or a 2-year total of 691,000 visits). Estimates below this figure have a relative standard error greater than 30 percent and are deemed unreliable by NCHS standards.

2The smallest reliable estimate for visits to neurologists is 35,000 visits per year (or a 2-year total of 70,000 visits). Estimates below this figure have a relative standard error greater than 30 percent and are deemed unreliable by NCHS standards.

Example of use of lable: For visits to neurologists, an estimate of 10 million visits per year has a relative standard error of 11.6 percent or a standard orror of $1,160,000$ visits (11.6 percont of 10 million).

Table II. Approximate relative standard errors for estimated numbers of drug mentions by selected physiclan specialties: National Ambulatory Medical Care Survey, 1991-92

\begin{tabular}{|c|c|c|}
\hline \multirow{2}{*}{$\begin{array}{c}\text { Estimated number of office visits } \\
\text { (expressed as annual average) } \\
\text { in thousands }\end{array}$} & \multicolumn{2}{|c|}{ Physiclan speclatty } \\
\hline & $A l^{1}$ & Neurology ${ }^{2}$ \\
\hline & \multicolumn{2}{|c|}{ Relative standard error in percent } \\
\hline $25 \ldots \ldots \ldots \ldots \ldots \ldots \ldots$ & 154.1 & 38.7 \\
\hline $46 \ldots \ldots \ldots \ldots \ldots$ & 114.3 & 30.0 \\
\hline $100 \ldots \ldots \ldots \ldots \ldots$ & 77.2 & 22.2 \\
\hline $250 \ldots \ldots \ldots \ldots \ldots \ldots$ & 48.9 & 17.1 \\
\hline $500 \ldots \ldots \ldots \ldots \ldots \ldots$ & 34.7 & 15.0 \\
\hline $674 \ldots \ldots \ldots \ldots \ldots \ldots$ & 30.0 & 14.5 \\
\hline $1,000 \ldots \ldots \ldots \ldots \ldots \ldots$ & 24.7 & 13.9 \\
\hline $2,500 \ldots \ldots \ldots \ldots \ldots \ldots$ & 16.0 & 13.1 \\
\hline $5,000 \ldots \ldots \ldots \ldots \ldots$ & 11.7 & 12.9 \\
\hline $10,000 \ldots \ldots \ldots \ldots \ldots \ldots$ & 8.8 & 12.8 \\
\hline $25,000 \ldots \ldots \ldots \ldots \ldots$ & 6.5 & 12.7 \\
\hline $50,000 \ldots \ldots \ldots \ldots \ldots$ & 5.5 & 12.6 \\
\hline $100,000 \ldots \ldots \ldots \ldots \ldots$ & 4.9 & 12.6 \\
\hline $250,000 \ldots \ldots \ldots \ldots \ldots$ & 4.6 & 12.6 \\
\hline $500,000 \ldots \ldots \ldots \ldots \ldots$ & 4.4 & 12.6 \\
\hline
\end{tabular}

\footnotetext{
${ }^{1}$ The smallest reliable estimate of drug mentions at visits to aggregated specialties is 674,000 drug mentions per year (or a 2 -year total of $1,347,000$ mentions). Estimates below this figure have a relative standard error greater than 30 percent and are deemed unreliable by NCHS standards.

2 The smallest reliable estimate of drug mentions at visits to neurologists is 46,000 drug mentions per year (or a 2-year total of 91,000 mentions). Estimates below this figure have a relative standard error greater than 30 percent and are deemed unreliable by NCHS standards.

Example of use of table: For neurologists, an estimate of 25 million drug mentions per year has a relative standard error of 12.7 percent or a standard error of $3,175,000$ drug mentions (12.7 percent of 25 million).
}

aggregate is obtained by multiplying the average estimate by 2 .)

$$
\operatorname{RSE}(p)=\frac{\sqrt{B \cdot(1-p)}}{p \cdot x} \cdot 100
$$

\section{Adjustments for nonresponse}

Estimates from NAMCS data were adjusted to account for sample physicians who were in-scope but did not participate in the study. This adjustment was calculated to minimize the impact of response on final estimates by imputing to nonresponding physicians data from visits to similar physicians. For this purpose, physicians were judged similar if they had the same specialty designation and practiced in the same PSU.

\section{Test of significance and rounding}

In this report, the determination of statistical inference is based on the two-tailed t-test. The Bonferroni inequality was used to establish the critical value for statistically significant differences ( 0.05 level of significance) based on the number of possible comparisons within a particular variable or (combination of variables) of interest. Terms relating to differences such as "greater than" or "less than" indicate that the difference is statistically significant. A lack of comment regarding the difference between any two estimates does not mean that the difference was tested and found to be not significant.

In the tables, estimates of office visits have been rounded to the nearest thousand. Consequently, estimates will not always add to totals. Rates and percents were calculated from original unrounded figures and do not necessarily agree with percents calculated from rounded data.

\section{Definition of terms}

Ambulatory patient-An ambulatory patient is an individual seeking personal health services who is not currently admitted to any health care institution on the premises.

Drug mention-A drug mention is the physician's entry on the Patient Record form of a pharmaceutical agent-by any route of administration-for prevention, diagnosis, or treatment. Generic as well as brandname drugs are included, as are nonprescription and prescription drugs. Along with all new drugs, the physician also records continued medications if the patient was specifically instructed during the visit to continue the 
Table III. Approximate standard errors of percents of estimated numbers of office visits to aggregated speclalties: National Ambulatory Medical Care Survey, 1991-92
Base of percent (visits, expressed as annual average, In thousands)
Estimated percent

1 or 995 or 9510 or 9020 or 8030 or 7040 or 6050

\begin{tabular}{|c|c|c|c|c|c|c|c|}
\hline \multirow[b]{2}{*}{ 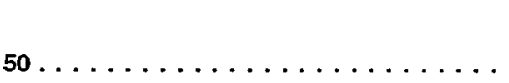 } & \multicolumn{7}{|c|}{ Standard error in percentage points } \\
\hline & 7.8 & 17.1 & 23.5 & 31.3 & 35.9 & 38.4 & 39.2 \\
\hline $100 \ldots \ldots \ldots \ldots \ldots \ldots \ldots$ & 5.5 & 12.1 & 16.6 & 22.2 & 25.4 & 27.1 & 27.7 \\
\hline $250 \ldots \ldots \ldots \ldots \ldots \ldots \ldots$ & 3.5 & 7.6 & 10.5 & 14.0 & 16.1 & 17.2 & 17.5 \\
\hline $500 \ldots \ldots \ldots \ldots \ldots \ldots \ldots$ & 2.5 & 5.4 & 7.4 & 9.9 & 11.4 & 12.1 & 12.4 \\
\hline $1,000 \ldots \ldots \ldots \ldots \ldots$ & 1.7 & 3.8 & 5.3 & 7.0 & 8.0 & 8.6 & 8.8 \\
\hline $2,500 \ldots \ldots \ldots \ldots \ldots \ldots \ldots$ & 1.1 & 2.4 & 3.3 & 4.4 & 5.1 & 5.4 & 5.5 \\
\hline $5,000 \ldots \ldots \ldots \ldots \ldots \ldots \ldots$ & 0.8 & 1.7 & 2.4 & 3.1 & 3.6 & 3.8 & 3.9 \\
\hline $10,000 \ldots \ldots \ldots \ldots \ldots \ldots \ldots$ & 0.6 & 1.2 & 1.7 & 2.2 & 2.5 & 2.7 & 2.8 \\
\hline $25,000 \ldots \ldots \ldots \ldots \ldots \ldots \ldots$ & 0.4 & 0.8 & 1.1 & 1.4 & 1.6 & 1.7 & 1.8 \\
\hline $50,000 \ldots \ldots \ldots \ldots \ldots \ldots \ldots$ & 0.3 & 0.5 & 0.7 & 1.0 & 1.1 & 1.2 & 1.2 \\
\hline $100,000 \ldots \ldots \ldots \ldots \ldots \ldots$ & 0.2 & 0.4 & 0.5 & 0.7 & 0.8 & 0.9 & 0.9 \\
\hline $250,000 \ldots \ldots \ldots \ldots \ldots \ldots \ldots$ & 0.1 & 0.2 & 0.3 & 0.4 & 0.5 & 0.6 & 0.6 \\
\hline $500,000 \ldots \ldots \ldots \ldots \ldots \ldots$ & 0.1 & 0.2 & 0.2 & 0.3 & 0.4 & 0.4 & 0.4 \\
\hline
\end{tabular}

NOTE: Example of use of table: An estimate of 20 percent based on an estimate of 25 million visits per year has a standard error of 1.4 percent or a relative standard error of 7.0 percent ( 1.4 percent divided by 20 percont).

Table IV. Approximate standard errors of percents of estimated numbers of office visits to neurologists: National Ambulatory Medlcal Care Survey, 1991-92

\begin{tabular}{|c|c|c|c|c|c|c|c|}
\hline \multirow{2}{*}{$\begin{array}{l}\text { Base of percent (visits, } \\
\text { expressed as annual } \\
\text { average, in thousands) }\end{array}$} & \multicolumn{7}{|c|}{ Estimated percent } \\
\hline & 1 or 99 & 5 or 95 & 10 or 90 & 20 or 80 & 30 or 70 & 40 or 60 & 50 \\
\hline & \multicolumn{7}{|c|}{ Standard error in percentage points } \\
\hline $50 \ldots \ldots \ldots \ldots \ldots \ldots \ldots \ldots$ & 2.3 & 5.1 & 7.0 & 9.3 & 10.6 & 11.4 & 11.6 \\
\hline $100 \ldots \ldots \ldots \ldots \ldots \ldots \ldots$ & 1.6 & 3.6 & 4.9 & 6.6 & 7.5 & 8.0 & 8.2 \\
\hline 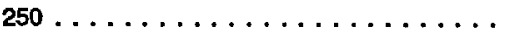 & 1.0 & 2.3 & 3.1 & 4.1 & 4.8 & 5.1 & 5.2 \\
\hline 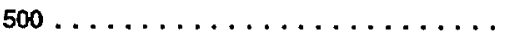 & 0.7 & 1.6 & 2.2 & 2.9 & 3.4 & 3.6 & 3.7 \\
\hline $1,000 \ldots \ldots \ldots \ldots \ldots \ldots$ & 0.5 & 1.1 & 1.6 & 2.1 & 2.4 & 2.5 & 2.6 \\
\hline 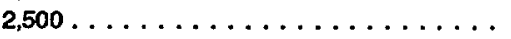 & 0.3 & 0.7 & 1.0 & 1.3 & 1.5 & 1.6 & 1.6 \\
\hline $5,000 \ldots \ldots \ldots \ldots \ldots \ldots \ldots \ldots$ & 0.2 & 0.5 & 0.7 & 0.9 & 1.1 & 1.1 & 1.2 \\
\hline $7,250 \ldots \ldots \ldots \ldots \ldots \ldots \ldots$ & 0.2 & 0.4 & 0.6 & 0.8 & 0.9 & 0.9 & 1.0 \\
\hline $10,000 \ldots \ldots \ldots \ldots \ldots \ldots$ & 0.2 & 0.4 & 0.5 & 0.7 & 0.8 & 0.8 & 0.8 \\
\hline
\end{tabular}

NOTE: Example of use of table: An estimate of 20 percent based on an estimele of 7,250,000 neurology visits per year has a standard error of 0.8 percent or a relative standard error of 4.0 percent $(0.8$ percent divided by 20 percent).

Table V. Approximate standard errors of percents of estimated numbers of drug mentions at visits to aggregated specialties: National Ambulatory Medical Care Survey, 1991-92

\begin{tabular}{|c|c|c|c|c|c|c|c|c|}
\hline & \multirow{2}{*}{$\begin{array}{l}\text { Base of percent (visits, } \\
\text { expressed as annual } \\
\text { average, in thousands) }\end{array}$} & \multicolumn{7}{|c|}{ Estimated percent } \\
\hline & & 1 or 99 & 5 or 95 & 10 or 90 & 20 or 80 & 30 or 70 & 40 or 60 & 50 \\
\hline & & \multicolumn{7}{|c|}{ Standard error in percentage points } \\
\hline $50 \ldots$ & $\ldots \ldots \ldots \ldots \ldots \ldots \ldots$ & 10,8 & 23.7 & 32.7 & 43.6 & 49.9 & 53.4 & 54.5 \\
\hline 100 & $\ldots \ldots \ldots \ldots \ldots \ldots \ldots$ & 7.7 & 16.8 & 23.1 & 30.8 & 35.3 & 37.7 & 38.5 \\
\hline 250 & 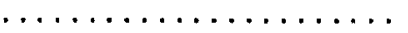 & 4.9 & 10.6 & 14.6 & 19.5 & 22.3 & 23.9 & 24.4 \\
\hline 500 & $\ldots \ldots \ldots \ldots \ldots \ldots \ldots$ & 3.4 & 7.5 & 10.3 & 13.8 & 15.8 & 16.8 & 17.2 \\
\hline $1,000 \ldots$ & 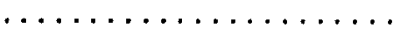 & 2.4 & 5.3 & 7.3 & 9,7 & 11.2 & 11.9 & 12.2 \\
\hline $2,500 \ldots$ & $\ldots \ldots \ldots \ldots \ldots \ldots \ldots \ldots$ & 1.5 & 3.4 & 4.6 & 6.2 & 7.1 & 7.6 & 7.7 \\
\hline $5,000 \ldots$ & $\ldots \ldots \ldots \ldots \ldots \ldots \ldots \ldots$ & 1.1 & 2.4 & 3.3 & 4.4 & 5.0 & 5.3 & 5.5 \\
\hline 10,000 . & $\ldots \ldots \ldots \ldots \ldots \ldots \ldots$ & 0.8 & 1.7 & 2.3 & 3.1 & 3.5 & 3.8 & 3.9 \\
\hline 25,000 . & $\ldots \ldots \ldots \ldots \ldots \ldots \ldots$ & 0.5 & 1.1 & 1.5 & 2.0 & 2.2 & 2.4 & 2.4 \\
\hline 50,000 . & $\ldots \ldots \ldots \ldots \ldots \ldots \ldots \ldots$ & 0.3 & 0.8 & 1.0 & 1.4 & 1.6 & 1.7 & 1.7 \\
\hline 100,000 & $\ldots \ldots \ldots \ldots \ldots \ldots \ldots \ldots$ & 0.2 & 0.5 & 0.7 & 1.0 & 1.0 & 1.2 & 1.2 \\
\hline 250,000 & $\ldots \ldots \ldots \ldots \ldots \ldots \ldots$ & 0.2 & 0.3 & 0.5 & 0.6 & 0.7 & 0.8 & 0.8 \\
\hline 500,000 & $\ldots \ldots \ldots \ldots \ldots \ldots$ & 0.1 & 0.2 & 0.3 & 0.4 & 0.5 & 0.5 & 0.6 \\
\hline
\end{tabular}

NOTE: Example of use of table: An estimate of 20 percent based on an estimate of 10 million drug mentions has a standard error of 3.1 percent or a relative standard error of 15.5 percent (3.1 percent divided by 20 percent). medication. Physicians may report up to five medications per visit.

Drug visit-A drug visit is a visit at which medication was prescribed or provided by the physician.

Neurologist-As defined in the NAMCS, a neurologist is a physician who has self-designated the practice specialty of neurology or child neurology on the American Medical Association's Physicians' Professional Activities Questionnaire. The physician's specialty is also verified during the NAMCS interview. The practice specialty of neurology is defined in the category of "other specialties" by the American Medical Association (additional categories include family/general practice, medical specialties, and surgical specialties), and the American Board of Psychiatry and Neurology certifies physicians in that specialty.

Office-An office is the space identified by a physician as a location for his or her ambulatory practice. Offices customarily include consultation, examination, or treatment spaces that patients associate with the particular physician.

Physician-A physician is a duly licensed doctor of medicine (M.D.) or doctor of osteopathy (D.O.) who is currently in office-based practice and who spends some time caring for ambulatory patients. Excluded from the NAMCS are physicians who are hospital based; who specialize in anesthesiology, pathology, or radiology; who are federally employed; who treat only institutionalized patients; or who are employed full time by an institution and spend no time seeing ambulatory patients.

Visit-A visit is a direct personal exchange between an ambulatory patient and a physician or a staff member working under the physician's supervision, for the purpose of seeking care and rendering personal health services. Excluded from the NAMCS are visits where medical care was not provided, such as visits made to drop off specimens, pay bills, make appointments, and walk-outs. 
Table VI. Approximate standard errors of percents of estimated numbers of drug mentions at office visits to neurologists: National Ambulatory Medical Care Survey, 1991-92

\begin{tabular}{|c|c|c|c|c|c|c|c|}
\hline \multirow{2}{*}{$\begin{array}{l}\text { Base of percent (vistts, } \\
\text { expressed as annual } \\
\text { average, in thousands) }\end{array}$} & \multicolumn{7}{|c|}{ Estimated percent } \\
\hline & 1 or 99 & 5 or 95 & 10 or 90 & 20 or 80 & 30 or 70 & 40 or 60 & 50 \\
\hline & \multicolumn{7}{|c|}{ Standard error in percentage points } \\
\hline 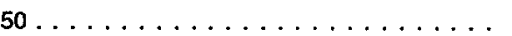 & 2.6 & 5.6 & 7.8 & 10.3 & 11.8 & 12.7 & 12.9 \\
\hline $100 \ldots \ldots \ldots \ldots \ldots \ldots \ldots \ldots$ & 1.8 & 4.0 & 5.5 & 7.3 & 8.4 & 9.0 & 9.1 \\
\hline $250 \ldots \ldots \ldots \ldots \ldots \ldots \ldots \ldots$ & 1.2 & 2.5 & 3.5 & 4.6 & 5.3 & 5.7 & 5.8 \\
\hline $500 \ldots \ldots \ldots \ldots \ldots \ldots \ldots \ldots$ & 0.8 & 1.8 & 2.5 & 3.3 & 3.7 & 4.0 & 4.1 \\
\hline $1,000 \ldots \ldots \ldots \ldots \ldots \ldots \ldots \ldots$ & 0.6 & 1.3 & 1.7 & 2.3 & 2.7 & 2.8 & 2.9 \\
\hline $2,500 \ldots \ldots \ldots \ldots \ldots \ldots \ldots$ & 0.4 & 0.8 & 1.1 & 1.5 & 1.7 & 1.8 & 1.8 \\
\hline $5,000 \ldots \ldots \ldots \ldots \ldots \ldots$ & 0.3 & 0.6 & 0.8 & 1.0 & 1.2 & 1.3 & 1.3 \\
\hline $8,000 \ldots \ldots \ldots \ldots \ldots \ldots$ & 0.2 & 0.5 & 0.6 & 0.8 & 0.9 & 1.0 & 1.0 \\
\hline $10,000 \ldots \ldots \ldots \ldots \ldots \ldots \ldots$ & 0.2 & 0.4 & 0.6 & 0.7 & 0.8 & 0.9 & 0.9 \\
\hline $25,000 \ldots \ldots \ldots \ldots \ldots \ldots$ & 0.1 & 0.3 & 0.4 & 0.5 & 0.5 & 0.6 & 0.6 \\
\hline
\end{tabular}

NOTE: Example of use of fable: An estimate of 20 percent based on an estimate of 8 million drug mentions per year at neurology visits has a standard error of 0.8 percent or a relative standard error of 4.0 percent $(0.8$ percent divided by 20 percent).

Table VII. Coefficients appropriate for determining relative standard errors by type of estimate and physician speclalty: National Ambulatory Medical Care Survey, 1991-92

\begin{tabular}{|c|c|c|}
\hline \multirow[b]{2}{*}{ Type of estimate and physician speclatty } & \multicolumn{2}{|c|}{ Coefficient for use with estimates in thousands } \\
\hline & $A$ & $B$ \\
\hline \multicolumn{3}{|l|}{ Visits } \\
\hline Overall totals $\ldots \ldots \ldots \ldots \ldots \ldots$ & 0.001157131 & 61.31199989 \\
\hline General and family practice . . . . . . . . . & 0.007330504 & 54.54704362 \\
\hline 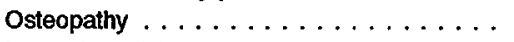 & 0.01402452 & 18.13642054 \\
\hline Intemal medicine . . . . . . . . . . . . . . & 0.008718567 & 55.2168744 \\
\hline Pediatrlos $\ldots \ldots \ldots \ldots \ldots \ldots \ldots \ldots$ & 0.007994386 & 35.33091768 \\
\hline General surgery $\ldots \ldots \ldots \ldots \ldots \ldots$ & 0.006685247 & 10.65103125 \\
\hline Obstetrics and gynecology . . . . . . . . . & 0.00919584 & 25.59962011 \\
\hline Orthopedic surgery. . . . . . . . . . . . . . & 0.005641337 & 24.20372144 \\
\hline Cardlovascular diseases . . . . . . . . . . & 0.01383253 & 12.58489271 \\
\hline Dermatology. . . . . . . . . . . . . & 0.01275351 & 10.28901849 \\
\hline Urologlcal surgery . . . . . . . . . . . & 0.008000282 & 11.92853664 \\
\hline Psychiatry . . . . . . . . . . . . & 0.009414796 & 12.88530675 \\
\hline Neurology $\ldots \ldots \ldots \ldots \ldots \ldots$ & 0.01314774 & 5.36720816 \\
\hline Ophthalomology $\ldots \ldots \ldots \ldots \ldots$ & 0.007938148 & 23.84517495 \\
\hline Otolaryngology . . . . . . . . . . . . & 0.007549396 & 8.0936265 \\
\hline All other speclalties $\ldots \ldots \ldots \ldots$ & 0.01537018 & 35.00317779 \\
\hline \multicolumn{3}{|l|}{ Drug mentlons } \\
\hline Overall totals $\ldots \ldots \ldots \ldots \ldots$ & 0.001853163 & 118.69462 \\
\hline General and family practice . . . . . . . . & 0.009085669 & 100.96778 \\
\hline Osteopathy $\ldots \ldots \ldots \ldots \ldots \ldots$ & 0.01658477 & 23.4739982 \\
\hline Intemal medicine . . . . . . . . . . . . & 0.01148498 & 103.21387 \\
\hline Pedlatrics $\ldots \ldots \ldots \ldots \ldots \ldots$ & 0.01245118 & 26.73517786 \\
\hline General surgery . . . . . . . . . . . . & 0.03935224 & 8.06806796 \\
\hline Obstetrics and gynecology . . . . . . . . . & 0.01454044 & 31.24058408 \\
\hline Orthopedic surgery. . . . . . . . . . . . & 0.01568053 & 23.3833057 \\
\hline Cardlovascular dlseases . . . . . . . . . . & 0.01575914 & 24.23751806 \\
\hline Dermatology. . . . . . . . . . . . . & 0.01299377 & 15.94507357 \\
\hline Urological surgery . . . . . . . . . . . & 0.01867719 & 10.6886669 \\
\hline Psychlatry ................ & 0.01430555 & 15.99374434 \\
\hline 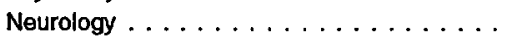 & 0.01593433 & 6.67244993 \\
\hline Ophthalomology $\ldots \ldots \ldots \ldots \ldots$ & 0.0251486 & 25.1381195 \\
\hline Otolaryngology $\ldots \ldots \ldots \ldots \ldots$ & 0.008374063 & 12.25916054 \\
\hline All other specialties $\ldots \ldots \ldots \ldots \ldots \ldots$ & 0.0226229 & 57.79950436 \\
\hline
\end{tabular}




\section{Symbols}

-. Data not available

... Category not applicable

- Quantity zero

0.0 Quantity more than zero but less than 0.05

Z Quantity more than zero but less than $\mathbf{5 0 0}$ where numbers are rounded to thousands

* Figure does not meet standard of reliability or precision (see Technical notes)

\# Figure suppressed to comply with confidentiality requirements 


\section{Suggested cltation}

Schappert SM. Office visits to neurologists: United States, 1991-92. Advance data from vital and health statistics; no 267. Hyattsville, Maryland: National Center for Health Statistics. 1995.

\section{Copyright Information}

All material appearing in this report is in the public domain and may be reproduced or copied without permission; citation as to source, however, is appreciated.

\section{Trade name dlsclaimer}

The use of trade names is for identification only and does not imply endorsement by the Public Health Service, U.S. Department of Health and Human Services.
National Center for Health Statistics

Acting Director

Jack R. Anderson

Acting Deputy Director Jennifer H. Madans, Ph.D.

\section{U.S. DEPARTMENT OF HEALTH AND \\ HUMAN SERVICES \\ Public Health Service \\ Centers for Disease Control and Prevention \\ National Center for Health Statistics \\ 6525 Belcrest Road \\ Hyattsville, Maryland 20782}

OFFICIAL BUSINESS

PENALTY FOR PRIVATE USE, $\$ \mathbf{3 0 0}$

To receive this publication regularly, contact the National Center for Health Statistics by calling 301-436-8500

E-mail:nchsquery@nch10a.em.cdc.gov Internet:http:/www.cdc.gov/nchswww/nchshome.htm

DHHS Publication No. (PHS) 95-1250

5-1394 (8/95) 


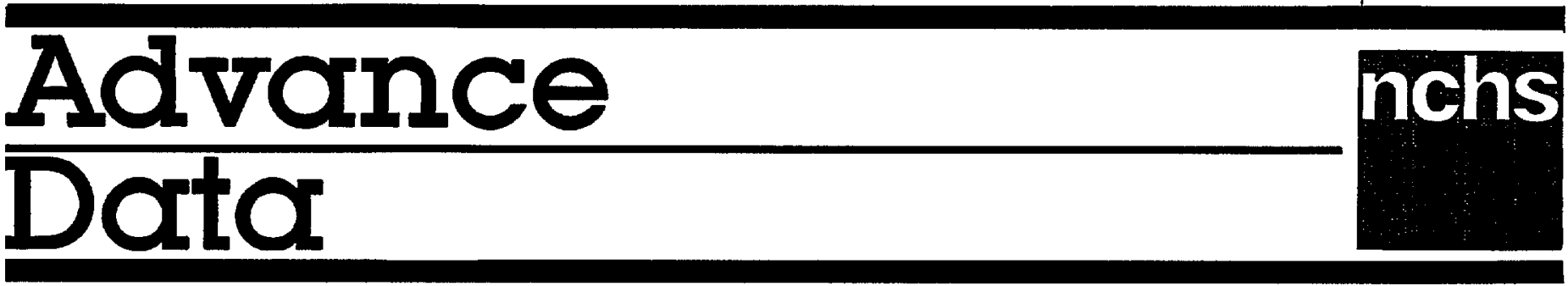

\title{
National Hospital Ambulatory Medical Care Survey: 1993 Outpatient Department Summary
}

\author{
Karen L. Lipkind, M.Ed., Division of Health Care Statistics
}

\section{Introduction}

During the 12-month period January-December 1993, an estimated 62.5 million visits were made to outpatient departments (OPD's) of non-Federal, short-stay, and general hospitals in the United States-24.6 visits per 100 persons. This was not significantly different from the 1992 rate of 22.5 visits per 100 persons.

This report presents data on OPD visits from the 1993 National Hospital Ambulatory Medical Care Survey (NHAMCS), a national probability survey conducted by the Division of Health Care Statistics, National Center for Health Statistics, Centers for Disease Control and Prevention. The survey was inaugurated in December 1991 to gather and disseminate information about the health care provided by hospital emergency and outpatient departments to the population of the United States. It is endorsed by the American Hospital Association, the Emergency Nurses Association, and the American College of Emergency Physicians.

This report presents data on OPD patient characteristics and visit characteristics. Data from the 1992 NHAMCS have been published (1-4), and a report on 1993 NHAMCS emergency department visits is

forthcoming (5).

Because the estimates presented in this report are based on a sample rather than on the entire universe of hospital OPD visits, they are subject to sampling variability. The Technical notes at the end of this report include a brief overview of the sample design used in the 1993 NHAMCS and an explanation of sampling errors. A detailed description of the NHAMCS sample design and survey methodology has been published ( 6 ).

The OPD Patient Record form is used by hospitals participating in the NHAMCS to record information about patient visits. This form (figure 1) is intended to serve as a reference for readers as they review the survey findings presented in this document.

\section{Patient characteristics}

OPD visits by patient's age, sex, and race are shown in table 1 . There were no significant differences in OPD visit rates among any of the age groups. Females made 62.3 percent of all OPD visits and had a higher visit rate (29.8 visits per 100 persons) than males (19.1 visits per 100 persons) did.

White persons made 74.1 percent of all OPD visits, with black persons and
Asian/Pacific Islanders accounting for 22.4 percent and 3.2 percent, respectively. The visit rate for black persons was significantly higher than for white persons overall and in all age categories (figure 2).

\section{Outpatient department visit characteristics}

\section{Geographic region}

By region, the largest proportion of OPD visits was made in the Northeast (34.6 percent). Visit rates in the Northeast (43.3 visits per 100 persons) and the Midwest (31.1 visits per 100 persons) were higher than those in the West (10.3 visits per 100 persons).

\section{Clinic type}

A clinic was defined as an administrative unit of the OPD where ambulatory medical care is provided under the supervision of a physician. Clinics where only ancillary services, such as radiology, renal dialysis, and pharmacy, were provided or other settings in which physician services were not typically provided, were out of scope for the survey. In addition, ambulatory surgery centers were out of scope since they are included in the National Survey of Ambulatory Surgery.

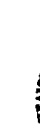

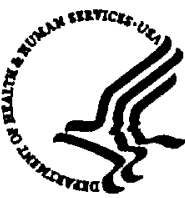

\section{U.S. DEPARTMENT OF HEALTH AND HUMAN SERVICES}

Public Health Service

Centers for Disease Control and Prevention

National Center for Health Statistics

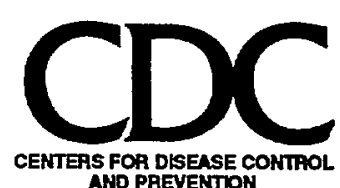


Department of Health and Human Services

Public Health Service, Centers for Disease Control

OMB No. 0920-0278

National Center for Health Statistics

Expiras: 6/30/94

CDC 64.54

NOThCE - Information contalned on this form which would permit ldentifleation of any incividual or eatablichment has been collected with a guarantes that it will be held in strict confidence, will be used only for purposes stated lor this study, and will not be disclosed or released to others without the consent of the individual ar the establishment in accordance with section 308(d) of the Publk Health Service Act (42 USC $242 \mathrm{~m}$ ). Public reporing burden for this phase of the survey is eatimated to averege 3 minutes per response. If you have any comments regarding the burden estimate or any other aspect of this aurvey, including suggestions for reducing this burden, send them to the PHS Reports Clearance Officer; Attn: PRA: HHH Qullding, Pin. 721-B; 200 Independence Ave., S.W., Washington, DC 20201, and bo the Office of Management and Budget: Papenwork Roduction Project (0920-0278); Washington, DC 20503.

\section{NATIONAL HOSPITAL AMBULATORY MEDICAL CARE SURVEY \\ OUTPATIENT DEPARTMENT PATIENT RECORD 1993-94}

1. PATIENT NAME

2. PATIENT RECORD NO.

\begin{tabular}{|c|c|c|c|c|}
\hline $\begin{array}{l}\text { 3. DATE OF VISIT } \\
\frac{l}{\text { Monih Day }} \frac{l}{\text { Year }}\end{array}$ & $\begin{array}{l}\text { 5. SEX } \\
\square \text { Female }\end{array}$ & $\begin{array}{l}\text { 6. RACE } \\
\text {, } \square \text { White } \\
2 \square \text { elack }\end{array}$ & $\begin{array}{l}\text { 7. ETHNICITY } \\
1 \square_{\text {origin }}^{\text {Hispanic }}\end{array}$ & $\begin{array}{l}\text { 8. EXPECTEL } \\
\text { PAYMENT } \\
\square \text { Private / cor }\end{array}$ \\
\hline $\begin{array}{l}\text { 4. DATE OF BIRTH } \\
\frac{1}{\text { Month } / \frac{1}{\text { Day }}} \text { Year }\end{array}$ & $2 \square$ Male & $\begin{array}{l}3 \square \\
4 \square_{\text {Islander }}^{\text {Asian I Pacific }} \\
\text { Eskimo / Aleut }\end{array}$ & $2 \square=\begin{array}{l}\text { Nol } \\
\text { Hispanic }\end{array}$ & $\begin{array}{l}2 \square \text { Medicare } \\
\square \text { Medicaid } \\
\square \text { Other gov }\end{array}$ \\
\hline \multicolumn{3}{|c|}{$\begin{array}{l}\text { 10. PATIENT'S COMPLANNT(S), SYMPTOM(S), OR } \\
\text { OTHER REASON(S) FOR THIS VISIT } \\
\text { (ln patient's own wonds) }\end{array}$} & \multicolumn{2}{|c|}{ 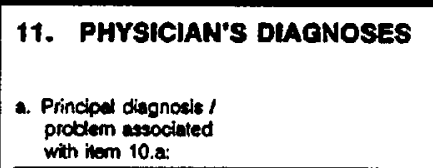 } \\
\hline \multicolumn{3}{|l|}{ b. Other: } & \multicolumn{2}{|l|}{ b. Other: } \\
\hline c. Other: & & & c. Other: & \\
\hline
\end{tabular}

- Surgeries and other

- Services in itern 13a

\section{TESTS, SURGICAL AND NONSURGICAL PROCEDUIRES, AND THERAPIES None}

a. SELECTEO SERVICES

(Check all ordered

or provided)

b. ALL OTHER SERVICES

Inctude:

- Tests - Imagings

procedures

1 DBlood pressure - Other therapies

$2 \square$ Urmalysrs

$3 \square$ Spirometry

$+\square$ Allergy testing

$5 \square$ HiV serology

(such as contact lenses.

psychotherapy.

or physiotherapy)

Exchude:

- Counseling / education

- Medications

(Record one on each lise and check performed or

ondered for each.

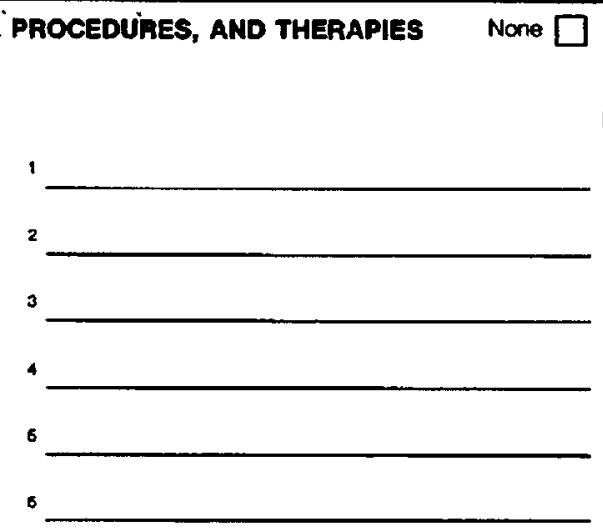

Pertormed Ordered

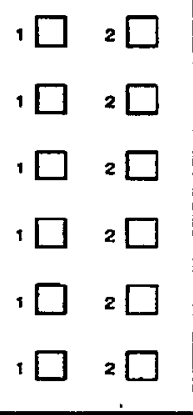
15. medications / INJECTIONS None $\square$
Include: - Rx and OTC
- Immunizations
- Altergy shots
- Meds ordered administered
- New meds
- Continuing meds
(wilh or without new orders)

16. DISPOSITION THIS VIBIT (Check all that apply)

1 $\square$ No lollow-up planned

$2 \square$ Relurn to clinic PRN

$3 \square$ Return to dinic - appointment

4 Telephone follow-up planned

5 Return to referring physkian

s $\square$ Refer to other physicianvclinic

$7 \square$ Admit to hospital

. $\square$ Other (Specify)
14. COUNGELINGIEDUCATION (Check all ordered or provided)

, $\square$ None

$\square$ Exercise

3 प Cholesterol reduction

4 Weight reduction

s $\square$ smoking cessation

- $\square$ Growth / development

$>\square$ Injury prevention

8 $\square$ HIV transmission

- $\square$ Oher STD transmission 10 $\square$ Oher 
Table 1. Number, percent distribution, and annual rate of outpatient dopartment visits with corresponding standard errors by selected patient and outpationt dopartment characteristics: United States, 1993

\begin{tabular}{|c|c|c|c|c|c|c|}
\hline Patlent and outpatlent department characterlstics & $\begin{array}{c}\text { Number of } \\
\text { visits In } \\
\text { thousands }\end{array}$ & $\begin{array}{c}\text { Standard } \\
\text { error in } \\
\text { thousands }\end{array}$ & $\begin{array}{c}\text { Percent } \\
\text { distribution }\end{array}$ & $\begin{array}{c}\text { Standard } \\
\text { error of } \\
\text { percent }\end{array}$ & $\begin{array}{l}\text { Number of } \\
\text { visits per } \\
\text { 100 persons } \\
\text { per year }\end{array}$ & $\begin{array}{c}\text { Standard } \\
\text { emor } \\
\text { of rate }\end{array}$ \\
\hline 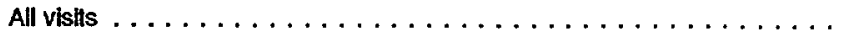 & 62,534 & 7,330 & 100.0 & $\cdots$ & 24.6 & 2.9 \\
\hline \multicolumn{7}{|l|}{ Patient characterlstic } \\
\hline \multicolumn{7}{|l|}{ Age: } \\
\hline 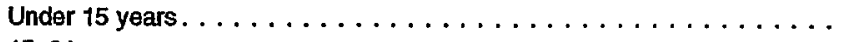 & 12,927 & 1,990 & 20.7 & 2.0 & 22.6 & 3.5 \\
\hline $15-24$ years. . . . . . . . . . . . . . . . . . . . & 8,512 & 1,248 & 13.6 & 0.9 & 24.8 & 3.6 \\
\hline $25-44$ yөars. . . . . . . . . . . . . . . . . . . . . & 18,299 & 2,248 & 29.3 & 1.0 & 22.4 & 2.8 \\
\hline $45-64$ years. . . . . . . . . . . . . . . . . . . . & 12,365 & 1,371 & 19.8 & 0.8 & 24.8 & 2.8 \\
\hline $65-74$ years. . . . . . . . . . . . . . . . . . . . & 5,865 & 893 & 9.4 & 0.7 & 31.5 & 4.8 \\
\hline 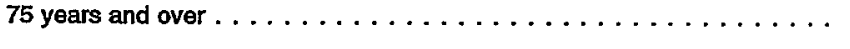 & 4,567 & 868 & 7.3 & 0.9 & 36.1 & 6.9 \\
\hline Sex and age: & & & & & $\cdot$ & \\
\hline Female . . . . . . . . . . . . . . . . . . . . . . . & 38,935 & 4,648 & 62.3 & 0.9 & 29.8 & 3.6 \\
\hline 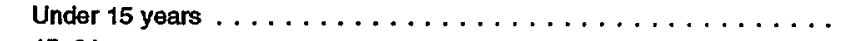 & 6,420 & 1,018 & 10.3 & 1.0 & 23.0 & 3.6 \\
\hline 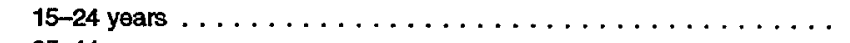 & 6,698 & 1,020 & 10.7 & 0.9 & 38.9 & 5.9 \\
\hline 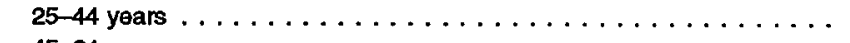 & 12,081 & 1,576 & 19.3 & 0.8 & 29.2 & 3.8 \\
\hline 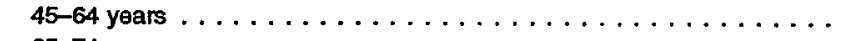 & 7,388 & 804 & 11.8 & 0.5 & 28.6 & 3.1 \\
\hline 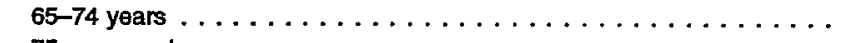 & 3,440 & 494 & 5.5 & 0.4 & 33.5 & 4.8 \\
\hline 75 years and over. $\ldots \ldots \ldots \ldots \ldots \ldots$ & 2,908 & 565 & 4.7 & 0.6 & 36.9 & 7.2 \\
\hline 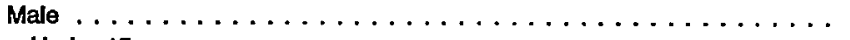 & 23,600 & 2,783 & 37.7 & 0.9 & 19.1 & 2.2 \\
\hline 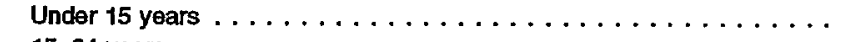 & 6,507 & 991 & 10.4 & 1.0 & 22.2 & 3.4 \\
\hline 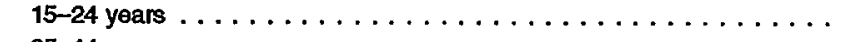 & 1,814 & 282 & 2.9 & 0.2 & 10.6 & 1.6 \\
\hline 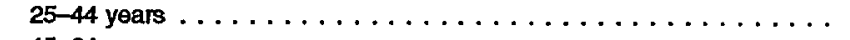 & 6,218 & 749 & 9.9 & 0.5 & 15.5 & 1.9 \\
\hline 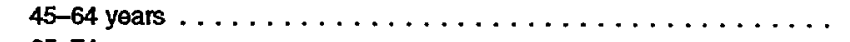 & 4,977 & 605 & 8.0 & 0.4 & 20.8 & 2.5 \\
\hline 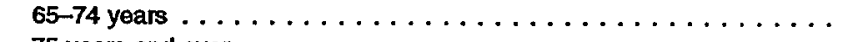 & 2,426 & 424 & 3.9 & 0.4 & 29.0 & 5.1 \\
\hline 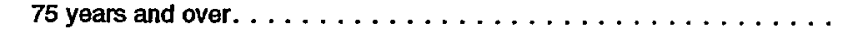 & 1,659 & 321 & 2.7 & 0.3 & 34.8 & 6.7 \\
\hline \multicolumn{7}{|l|}{ Race and age: } \\
\hline 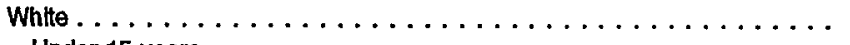 & 46,337 & 6,519 & 74.1 & 2.2 & 21.9 & 3.1 \\
\hline Under 15 years $\ldots \ldots \ldots \ldots \ldots \ldots \ldots \ldots \ldots \ldots$ & 9,026 & 1,576 & 14.4 & 1.5 & 19.9 & 3.5 \\
\hline 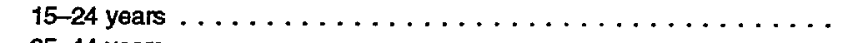 & 6,257 & 1,109 & 10.0 & 0.9 & 22.8 & 4.0 \\
\hline 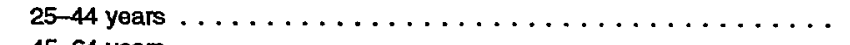 & 13,449 & 1,939 & 21.5 & 1.0 & 19.9 & 2.9 \\
\hline 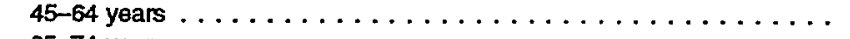 & 9,156 & 1,191 & 14.6 & 0.7 & 21.4 & 2.8 \\
\hline 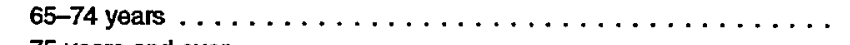 & 4,676 & 827 & 7.5 & 0.7 & 28.3 & 5.0 \\
\hline 75 years and over. $\ldots \ldots \ldots \ldots \ldots \ldots \ldots$ & 3,772 & 844 & 6.0 & 0.9 & 33.0 & 7.4 \\
\hline Black . . . . . . . . . . . . . . . . . . . . & 14,015 & 1,320 & 22.4 & 2.1 & 43.8 & 4.1 \\
\hline Under 15 years $\ldots \ldots \ldots \ldots \ldots \ldots \ldots \ldots$ & 3,398 & 534 & 5.4 & 0.8 & 37.2 & 5.8 \\
\hline 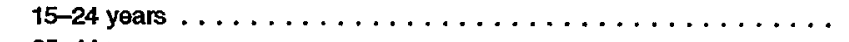 & 1,970 & 242 & 3.2 & 0.4 & 38.3 & 4.7 \\
\hline 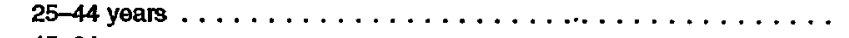 & 4,159 & 429 & 6.7 & 0.7 & 41.9 & 4.3 \\
\hline 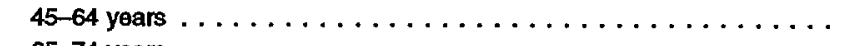 & 2,751 & 328 & 4.4 & 0.5 & 53.6 & 6.4 \\
\hline 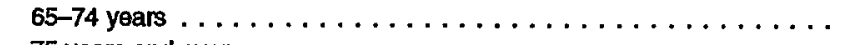 & 1,006 & 130 & 1.6 & 0.2 & 60.1 & 7.8 \\
\hline 75 years and over. $\ldots \ldots \ldots \ldots \ldots \ldots \ldots \ldots$ & 731 & 115 & 1.2 & 0.2 & 73.2 & 11.5 \\
\hline \multicolumn{7}{|l|}{ All other races: } \\
\hline 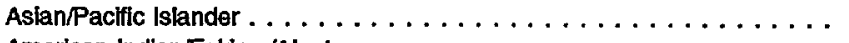 & 2,006 & 321 & 3.2 & 0.4 & $\ldots$ & $\ldots$ \\
\hline American Indian/Eskimo/Aleut. $\ldots \ldots \ldots \ldots \ldots \ldots$ & 177 & 534 & 0.3 & 0.1 & $\cdots$ & $\cdots$ \\
\hline \multicolumn{7}{|l|}{ Outpatlent department characterlstic } \\
\hline \multicolumn{7}{|l|}{ Geographic region: } \\
\hline 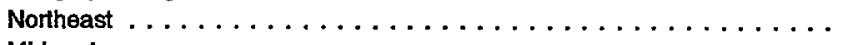 & 21,668 & 4,155 & 34.7 & 5.1 & 43.3 & 8.3 \\
\hline 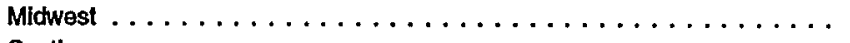 & 17,694 & 5,140 & 28.3 & 5.8 & 28.5 & 8.3 \\
\hline South . . . . . . . . . . . . . . . . . . & 14,389 & 2,328 & 23.0 & 3.6 & 16.9 & 2.7 \\
\hline 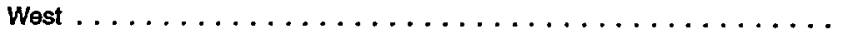 & 8,783 & 2,150 & 14.0 & 3.1 & 15.5 & 3.8 \\
\hline
\end{tabular}

1Based on U.S. Bureau of the Consus estimates of the civilian, noninstitutionalized population of the United States as of July 1, 1803.

Clinics were classified into five types as presented in table 2. About half of all OPD visits ( 47.1 percent) were made to general medicine clinics that included internal medicine and primary care clinics. Pediatric, obstetrics and gynecology, and surgery clinics accounted for 18.0 percent, 14.7 percent, and 13.4 percent of visits, respectively. The "other" clinic category, which included such clinics as psychiatry and neurology, accounted for 6.8 percent of visits. 


\section{Expected sources of payment}

Expected sources of payment were most often Medicaid (31.1 percent), private/commercial insurance (27.0 percent), and Medicare (16.9 percent) (table 3). "Patient paid" and "HMO/Other prepaid" were indicated at 12.1 and 8.8 percent of OPD visits, respectively. The patientpaid category includes the patient's contribution toward "copayments" and "deductibles."

\section{Referral status and prior visit status}

Approximately one-fifth (19.7 percent) of OPD visits were made as the result of a referral from another physician (table 4). About three-quarters (79.5 percent) of OPD visits were made by patients who had been seen in the clinic on a previous occasion, and more than half (63.7 percent) of all visits were made by persons who were returning to the clinic for care of a previously treated problem. One-fifth ( 20.5 percent) of visits were made by new patients, that is, patients who had not been seen in that clinic before.

\section{Reason for visit}

In item 10 of the Patient Record form, the patient's (or patient surrogate's) "complaint(s), symptom(s), or other reason(s) for this visit in the patient's own words" is recorded. Up to three reasons for visit are classified and coded according to A Reason for Visit Classification for Ambulatory Care (RVC) (7). The principal reason is the problem, complaint, or reason listed in item 10a of the OPD Patient Record form.

The RVC is divided into the eight modules or groups of reasons displayed in table 5 . About 4 of every 10 visits were made for reasons classified as symptoms, with the diagnostic/screening and preventive module and the treatment module each accounting for about one fifth of the visits ( 19.5 and 18.7 percent respectively). The 20 most frequently mentioned principal reasons for visit, representing 42.6 percent of all visits, are shown in table 6 . It is important to note that estimates differing in ranked

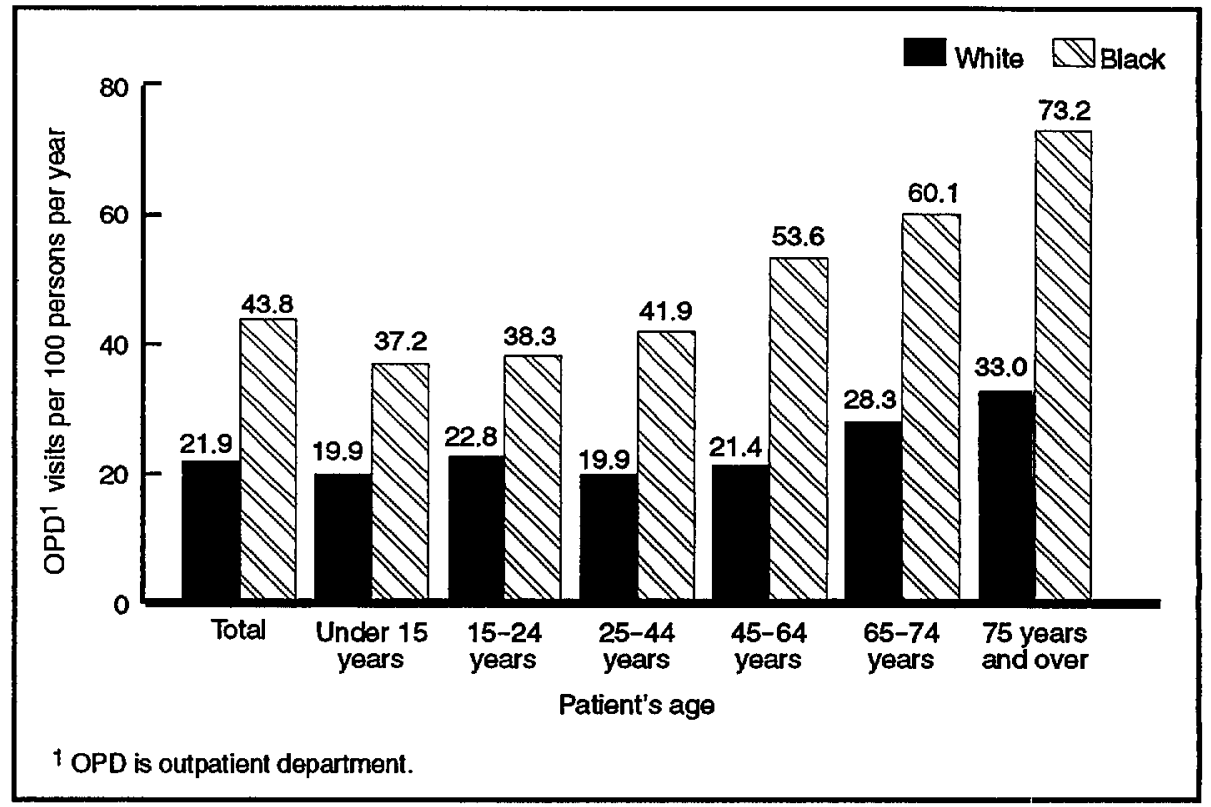

Figure 2. Annual rate of outpatient department visits by patlent's age and race: United State8, 1993

Table 2. Number and percent distribution of outpatient department visits with corresponding standard errors by type of clinic: United States, 1993

\begin{tabular}{|c|c|c|c|c|}
\hline Type of clinic & $\begin{array}{l}\text { Number of } \\
\text { visits in } \\
\text { thousands }\end{array}$ & $\begin{array}{c}\text { Standard } \\
\text { orror in } \\
\text { thousands }\end{array}$ & $\begin{array}{c}\text { Percent } \\
\text { dlstribution }\end{array}$ & $\begin{array}{r}\text { Standard } \\
\text { error of } \\
\text { percent }\end{array}$ \\
\hline All vistts $\ldots \ldots \ldots \ldots \ldots \ldots$ & 62,534 & 7,330 & 100.0 & $\ldots$ \\
\hline General medicine $\ldots \ldots \ldots \ldots$ & 29,443 & 4,265 & 47.1 & 2.9 \\
\hline Surgery. . . . . . . . . . . & 8,382 & 1,517 & 13.4 & 1.9 \\
\hline Pediatrics $\ldots \ldots \ldots \ldots \ldots \ldots$ & 11,274 & 1,728 & 18.0 & 1.8 \\
\hline Obstetrics and gynecology . . . . . . & 9,169 & 1,722 & 14.7 & 1.8 \\
\hline Other $\ldots \ldots \ldots \ldots \ldots$ & 4,266 & 808 & 6.8 & 1.2 \\
\hline
\end{tabular}

Table 3. Number and percent of outpatient department visits with corresponding standard errors by patient's expected source of payment: United States, 1993

\begin{tabular}{|c|c|c|c|c|}
\hline Expected source of payment & $\begin{array}{c}\text { Number of } \\
\text { vistls in } \\
\text { thousands }\end{array}$ & $\begin{array}{c}\text { Standard } \\
\text { error in } \\
\text { thousands }\end{array}$ & Percent & $\begin{array}{c}\text { Standard } \\
\text { error of } \\
\text { percent }\end{array}$ \\
\hline All visits $\ldots \ldots \ldots \ldots \ldots \ldots$ & 62,534 & 7,330 & $\ldots$ & $\cdots$ \\
\hline Medicald . . . . . . . . . . . & 19,442 & 2,124 & 31.1 & 2.3 \\
\hline Prlvale/commerclal. . . . . . . . . . & 16,906 & 3,390 & 27.0 & 2.6 \\
\hline Medicare . . . . . . . . . . . . & 10,542 & 1,693 & 16.9 & 1.4 \\
\hline Pallent-pald . . . . . . . . . & 7,592 & 989 & 12.1 & 1.1 \\
\hline HMO/other prepaid ${ }^{2} \ldots \ldots \ldots \ldots$ & 5,496 & 1,216 & 8.8 & 1.5 \\
\hline Other . . . . . . . . . . . & 2,835 & 605 & 4.5 & 0.7 \\
\hline Other govemment $\ldots \ldots \ldots \ldots \ldots$ & 2,755 & 557 & 4.4 & 0.7 \\
\hline No charge $\ldots \ldots \ldots \ldots \ldots$ & 1,392 & 431 & 2.2 & 0.6 \\
\hline Unspectfled . . . . . . . . . . . . . & 2,243 & 749 & 3.6 & 1.1 \\
\hline
\end{tabular}

'Numbers may exceed total number of visits because more than one source of payment may be coded for each visit. ${ }^{2} \mathrm{HMO}$ is heatth maintenance organization. 
order may not be significantly different from each other. "Progress visit" was the most frequently mentioned visit (10.5 percent), reflecting the large number of return visits for a previously treated problem. Five of the top 20 reasons for visit, which accounted for 15 percent of all OPD visits, were classified in the diagnostic screening and preventive module. The reasons were "Routine prenatal examination," "General medical examination," "Well baby examination," "Other and unspecified diagnostic tests," and "Prophylactic inoculations." "Stomach and abdominal pain, cramps and spasms," and "Cough" were the most frequently mentioned reasons for visit in the symptom module each accounting for 1.8 percent of the visits.

\section{Principal diagnosis}

The principal diagnosis or problem associated with the patient's most important reason for visit and any other significant current diagnoses are recorded in item 11. Up to three diagnoses are coded and classified according to the International Classification of Diseases, 9th Revision, Clinical Modification (ICD-9-CM) (8). Displayed in table 7 are OPD visits by principal diagnosis using the major

Table 4. Number and percent distribution of outpatient department visits with corresponding standard errors by referral status and prior visit status: United States, 1993

\begin{tabular}{|c|c|c|c|c|}
\hline Vstt characterstic & $\begin{array}{l}\text { Number of } \\
\text { vists in } \\
\text { thousands }\end{array}$ & $\begin{array}{l}\text { Standard } \\
\text { error } h \\
\text { thousands }\end{array}$ & $\begin{array}{c}\text { Percemt } \\
\text { distribution }\end{array}$ & $\begin{array}{l}\text { Standard } \\
\text { error of } \\
\text { percent }\end{array}$ \\
\hline$\ldots \ldots \ldots \ldots \ldots \ldots$ & 62,534 & 7,390 & 100.0 & ... \\
\hline \multicolumn{5}{|l|}{ Referral status } \\
\hline Not referred by another physictan $\ldots \ldots \ldots$ & 50,185 & 6,261 & 80.3 & 1.6 \\
\hline Referred by another physician $\ldots \ldots \ldots$ & 12,350 & 1,566 & 19.7 & 1.6 \\
\hline \multicolumn{5}{|l|}{ Prior visit status } \\
\hline Old pattent $\ldots \ldots \ldots \ldots \ldots \ldots \ldots \ldots$ & 49,727 & 6,061 & 79.5 & 1.1 \\
\hline old problem. . . . . $\ldots \ldots \ldots \ldots \ldots$ & 39,823 & 4,858 & 63.7 & 1.3 \\
\hline New problem . . . . . . . . . . . . . & 9,904 & 1,473 & 15.8 & 1.2 \\
\hline New patient $\ldots \ldots \ldots \ldots \ldots \ldots \ldots$ & 12,807 & 1,510 & 20.5 & 1.1 \\
\hline
\end{tabular}

disease categories specified by the ICD-9-CM. The supplementary classification is provided to deal with situations in which circumstances other than a disease or injury are recorded as diagnoses. It accounted for 22.6 percent of all OPD visits, and was followed by diseases of the respiratory system (8.7 percent).

The 20 most frequently reported principal diagnoses are shown in table 8. These are categorized at the three-digit coding level of the ICD-9-CM and accounted for more than one third (35.2 percent) of all OPD visits. The most commonly recorded diagnosis was
"Normal pregnancy," occurring at 7.9 percent of all visits.

\section{Tests, surgical and nonsurgical procedures, and therapies}

Statistics on various diagnostic tests, surgical and nonsurgical procedures, and therapies performed or ordered by hospital staff during an OPD visit are shown in table 9.

Approximately three quarters of all OPD visits included one or more diagnostic or screening service. The most frequently mentioned checkbox category (item 13a) was blood pressure check, recorded at 54.4 percent of visits. Other frequently mentioned services included

Table 5. Number and percent distributlon of outpatient department visits with corresponding standard errors by patient's principal reason for visit: United States, 1993

\begin{tabular}{|c|c|c|c|c|}
\hline Princpal reason for $v / s t t$ and $R V C$ code' & $\begin{array}{l}\text { Number of } \\
\text { vists } n \\
\text { thousands }\end{array}$ & $\begin{array}{c}\text { Standard } \\
\text { orror } n \\
\text { thousands }\end{array}$ & $\begin{array}{c}\text { Percent } \\
\text { distribution }\end{array}$ & $\begin{array}{c}\text { Standard } \\
\text { error of } \\
\text { percent }\end{array}$ \\
\hline 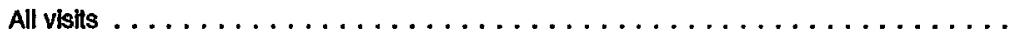 & 62,534 & 7,330 & 100.0 & $\cdots$ \\
\hline Symptom module. . . . . . . . . . . . . . . . . . . . . . . . . . . . . . . . so01-s999 & 24,846 & 3,275 & 39.7 & 1.7 \\
\hline General symptoms . . . . . . . . . . . . . . . . . . . . . . . . . . S001-S099 & 3,419 & 420 & 5.5 & 0.5 \\
\hline Symptoms relerable to psychological/mental disorders . . . . . . . . . . . S100-S199 & 2,191 & 428 & 3.5 & 0.6 \\
\hline Symptoms referable to the nervous system (excluding sense organs) . . . . . \$200-\$259 & 1,565 & 201 & 2.5 & 0.2 \\
\hline Symptoms referable to the cardiovascularfymphatlc system. . . . . . . . . \$ \$260-\$299 & 187 & 43 & 0.3 & 0.1 \\
\hline Symptoms relerable to the eyes and ears . . . . . . . . . . . . . . . . S300-S399 & 2,167 & 340 & $\mathbf{3 . 5}$ & 0.3 \\
\hline Symptoms referable to the respiratory system . . . . . . . . . . . . . . . S400-\$498 & 3,273 & 642 & 5.2 & 0.6 \\
\hline Symptoms relerable to the dlgestive system. . . . . . . . . . . . . . . . S500-s639 & 2,707 & 381 & 4.3 & 0.3 \\
\hline Symptoms reterable to the genitourinary system . . . . . . . . . . . . . . . S640-s829 & 2,837 & 629 & 4.5 & 0.7 \\
\hline Symptoms reterable to the skin, hair, and nalls . . . . . . . . . . . . . . S830-s889 & 2,318 & 522 & 3.7 & 0.5 \\
\hline Symptoms referable to the musculoskeletal system . . . . . . . . . . . . . s900-s9g9 & 4,182 & 696 & 6.7 & 0.7 \\
\hline Dlsease module . . . . . . . . . . . . . . . . . . . . . . . . . . . . D001-D999 & 6,078 & 825 & 9.7 & 0.8 \\
\hline Dlagnostc/screening and preventlve module . . . . . . . . . . . . . . . X100-X599 & 12,223 & 1,785 & 19.5 & 1.4 \\
\hline Treatment module . . . . . . . . . . . . . . . . . . . . . . . . . . . T100-T899 & 11,676 & 1,648 & 18.7 & 1.7 \\
\hline Injurles and actverse effects module . . . . . . . . . . . . . . . . . . . . . . . . J001-J999 & 2,374 & 397 & 3.8 & 0.4 \\
\hline Test results module . . . . . . . . . . . . . . . . . . . . . . . . R100-R700 & 777 & 127 & 1.2 & 0.1 \\
\hline Administratlve module. . . . . . . . . . . . . . . . . . . . . A100-A140 & 491 & 138 & 0.8 & 0.2 \\
\hline 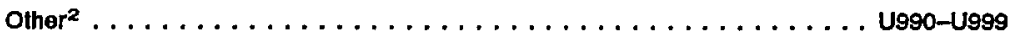 & 4,068 & 854 & 6.5 & 1.0 \\
\hline
\end{tabular}

'Based on A Reason for Visit Claseifioution for Ambulatory Care (AVC) (7).

Inciudes problems and complaints not olsowhere classified, entries of "none", blanks, and ilegible entries. 
other blood tests ( 20.5 percent) and urinalysis (13.9 percent). Readers should note that for items $8,13,14,16$, and 17 on the OPD Patient Record form, hospital staff were asked to check all of the applicable categories for that item. Therefore, multiple responses could be coded for each visit.

Up to six entries for tests, surgical and nonsurgical procedures, and therapies not listed in the checkbox categories were made in item $13 \mathrm{~b}$. Results of the open-ended responses were coded according to volume 3 of the ICD-9-CM (8). There were an estimated 33 million procedures of this type reported. Approximately two-thirds of the procedures were reported as being performed (not just ordered) during the visit. The 20 most frequently reported procedures are shown in table 10. Other individual psychotherapy, eye examinations, and Pap smears were among the most frequently mentioned procedures.

\section{Counseling/education}

Almost half ( 46.6 percent) of all OPD visits included some form of counseling or education either ordered or provided (table 11). "Other" counseling was recorded at one-third of visits ( 36.9 percent), followed by counseling on growth/development (5.3 percent of visits).

\section{Medications/injections}

Hospital staff were instructed to record all new or continued medications ordered, supplied, or administered at the visit, including prescription and nonprescription preparations, and immunizations and desensitizing agents. Up to five medications or drug mentions were coded for each visit. As used in the NHAMCS, the term "drug" is interchangeable with the term "medication". The NHAMCS drug data base permits classification by a wide range of variables, including specific drug entry name, trade name, generic class, therapeutic category, prescription or nonprescription status, federally controlled substance status, and composition status (that is, whether the drug is a single- or multiple-ingredient product). A report describing the method
Table 6. Number and percent dlstribution of outpatlent department visits with corresponding standard errors by the 20 princlpal reasons for visit most frequently mentioned by patients: United States, 1993

\begin{tabular}{|c|c|c|c|c|}
\hline Reason for vist and RVC code ${ }^{-1}$ & $\begin{array}{l}\text { Number of } \\
\text { visits in } \\
\text { thousands }\end{array}$ & $\begin{array}{l}\text { Standard } \\
\text { enror in } \\
\text { thousands }\end{array}$ & $\begin{array}{c}\text { Percent } \\
\text { distribution }\end{array}$ & $\begin{array}{l}\text { Standard } \\
\text { error of } \\
\text { percent }\end{array}$ \\
\hline All visits & 62,534 & 7,330 & 100.0 & $\cdots$ \\
\hline Progress visit . . . . . . . . . . . . . T800 & 6,593 & 1,254 & 10.5 & 1.8 \\
\hline Routine prenatal examination $\ldots \ldots \ldots \ldots$. . 205 & 3,900 & 818 & 6.2 & 1.0 \\
\hline General medical examination . . . . . . . . X X100 & 3,140 & 506 & 5.0 & 0.5 \\
\hline$\ldots \ldots \ldots \ldots$ T205 & 1,394 & 278 & 2.2 & 0.3 \\
\hline Well baby examlnation . . . . . . . . . X X105 & 1,364 & 270 & 2.2 & 0.4 \\
\hline $\begin{array}{l}\text { Stomach and abdominal pain, cramps and } \\
\text { spasms } \ldots \ldots \ldots \ldots \ldots \ldots \ldots \ldots \ldots\end{array}$ & 1,121 & 178 & 1.8 & 0.2 \\
\hline$\ldots \ldots \ldots \ldots \ldots \ldots \ldots \ldots 440$ & 1,119 & 264 & 1.8 & 0.3 \\
\hline Skin rash. . . . . . . . . . . . . . S8860 & 806 & 143 & 1.3 & 0.1 \\
\hline Fever . . . . . . . . . . . . . . . s s & 795 & 201 & 1.3 & 0.3 \\
\hline Earache or ear infection . . . . . . . . . \$355 & 728 & 131 & 1.2 & 0.1 \\
\hline Back symptoms. . . . . . . . . . . . . . . \$ \$905 & 716 & 147 & 1.1 & 0.2 \\
\hline Medication, other and unspecified . . . . . . T115 & 655 & 122 & 1.0 & 0.1 \\
\hline Headcold. . . . . . . . . . . . . . . . . . \$ \$445 & 622 & 155 & 1.0 & 0.2 \\
\hline Headache, pain in head & 620 & $\mathbf{9 3}$ & 1.0 & 0.1 \\
\hline$\ldots \ldots \ldots \ldots+s 110$ & 608 & 143 & 1.0 & 0.2 \\
\hline Counsellng, not othenwise stated . . . . . . . . T605 & 510 & 152 & 0.8 & 0.2 \\
\hline Other and unspecified dlagnostic tests $\ldots \ldots$ X370 & 500 & 215 & 0.8 & 0.3 \\
\hline Prophylactic inoculations . . . . . . . . . . X X400 & 492 & 139 & 0.8 & 0.2 \\
\hline Hypertension $\ldots \ldots \ldots \ldots \ldots \ldots \ldots$ D510 & 480 & 85 & 0.8 & 0.1 \\
\hline Knee symptoms . . . . . . . . . . . . . . . . S925 & 479 & 85 & 0.8 & 0.1 \\
\hline All other reasons. . . & 35,892 & 1,701 & 57.4 & 0.8 \\
\hline
\end{tabular}

'Based on A Reason for Visit Classification for Ambulatory Care (RVC) (7).

Table 7. Number and percent distribution of outpatient department visits with corresponding standard errors by principal diagnosis: United States, 1993

\begin{tabular}{|c|c|c|c|c|}
\hline Principal diagnos/s and $I C D-9-C M \operatorname{code}^{\top}$ & $\begin{array}{l}\text { Number of } \\
\text { visits in } \\
\text { thousands }\end{array}$ & $\begin{array}{l}\text { Standard } \\
\text { emor in } \\
\text { thousands }\end{array}$ & $\begin{array}{c}\text { Percent } \\
\text { distribution }\end{array}$ & $\begin{array}{l}\text { Standard } \\
\text { error of } \\
\text { percent }\end{array}$ \\
\hline All visils & 62,534 & 7,330 & 100.0 & $\cdots$ \\
\hline Infectious and parasitic diseases. & 1,896 & 236 & 3.0 & 0.3 \\
\hline Neoplasms. . . . . . . . . . . . . . . . 140-239 & 3,780 & 813 & 6.0 & 1.0 \\
\hline $\begin{array}{l}\text { Endocrine, nutritional and metabollc diseases } \\
\text { and Immunity dlsorders } \ldots \ldots \ldots \ldots 240-279\end{array}$ & 2,275 & 382 & 3.6 & 0.4 \\
\hline $\begin{array}{l}\text { Mental disorders . . . . . . . . . . . 290-319 } \\
\text { Diseases of the nervous system and sense }\end{array}$ & 4,489 & 795 & 7.2 & 1.1 \\
\hline organs . . . . . . . . . . . . . . . & 3,781 & 542 & 6.0 & 0.5 \\
\hline Diseases of the circulatory system . . . . . 390-459 & 3,595 & 531 & 5.7 & 0.5 \\
\hline Diseases of the respiratory system. . . . . 460-519 & 5,461 & 993 & 8.7 & 0.9 \\
\hline Diseases of the dlgestive system. & 2,091 & 299 & 3.3 & 0.4 \\
\hline $\begin{array}{l}\text { Diseases of the genitourinary system . . . 580-629 } \\
\text { Diseases of the skin and subcutaneous }\end{array}$ & 3,678 & 727 & 5.9 & 0.7 \\
\hline 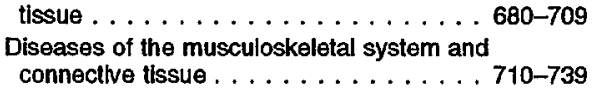 & 2,411 & 580 & 3.9 & 0.6 \\
\hline $\begin{array}{l}\text { Symptoms, signs, and III-defined } \\
\text { conditlons } \ldots \ldots \ldots \ldots \ldots\end{array}$ & 3,590 & 386 & 5.7 & 0.3 \\
\hline Injury and poisoning $\ldots \ldots \ldots$ & 3,498 & 537 & 5.6 & 0.5 \\
\hline Supplementary classification. . . . . . . . . V01-V82 & 14,156 & 1,784 & 22.6 & 1.5 \\
\hline All other dlagnoses ${ }^{2} \ldots \ldots \ldots \ldots \ldots \ldots \ldots$ & $" 1,530$ & 563 & 2.4 & 0.4 \\
\hline$\ldots \ldots \ldots \ldots \ldots$ & 2,902 & 373 & 4.6 & 0.5 \\
\hline
\end{tabular}

'Based on the Intemational Classification of Diseases, 9th Revision, Clinical Modification (ICD-9-CM) (8).

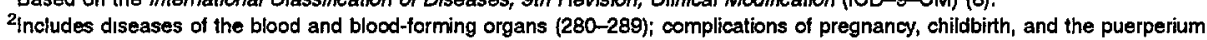
(630-676); congental anomalıes (740-759); and certain conditions originating in the perinatal period (760-779).

${ }^{3}$ includes blank diagnoses, uncodable diagnoses, and illegible diagnoses. 
and instruments used to collect and process drug information has been published (9).

Medication was used at 57.1 percent of the outpatient department visits (table 12). Hospital staff were instructed to record all new or continued medications ordered or provided at the visit, including prescription and nonprescription preparations, and immunizing and desensitizing agents. As many as five medications or drug mentions could be coded per visit.

There was a total of 75.7 million drug mentions, or an average of 1.2 drug mentions, per OPD visit. The 20 medications most frequently prescribed at OPD visits are shown in table 13 by drug entry name and therapeutic classification. The therapeutic classification is based on the therapeutic categories used in the National Drug Code Directory, 1985 edition (10). The top 20 medications account for 23 percent of all drug mentions. Tylenol was the medication most frequently prescribed, with 2.2 million mentions, or 2.9 percent of the total. It was followed by prenatal vitamins ( 2.5 percent) and amoxicillin (1.7 percent).

\section{Disposition of this visit}

Approximately two-thirds of OPD visits (65.6 percent) resulted in an appointment being made to return to the clinic. This and the previously mentioned finding that most OPD patients had been seen in the clinic before are indications of the continuous nature of care provided in the OPD setting. For 17.9 percent of visits, the disposition was "Return to clinic PRN" (as needed) (table 14). Only 1.5 percent of OPD visits resulted in hospital admission.

\section{Providers seen this visit}

A staff physician was seen at two-thirds of OPD visits ( 66.8 percent). Conversely, one-third of the visits had patients who were NOT seen by a staff physician. Registered nurses were seen at 41.8 percent of visits and residents/ interns were seen at one-quarter (24.3 percent) of visits (table 15 ).

Table 8. Number and percent distribution of outpatient department visits by the 20 princlpal diagnoses most frequently rendered by hospital staff: United States, 1993

\begin{tabular}{|c|c|c|c|c|}
\hline Principal diagnosis and ICD-9-CM code" & $\begin{array}{c}\text { Number of } \\
\text { visits in } \\
\text { thousands }\end{array}$ & $\begin{array}{c}\text { Standard } \\
\text { error in } \\
\text { thousands }\end{array}$ & $\begin{array}{l}\text { Percent } \\
\text { distribution }\end{array}$ & $\begin{array}{c}\text { Standard } \\
\text { error of } \\
\text { percent }\end{array}$ \\
\hline All visits $\ldots \ldots \ldots \ldots \ldots \ldots \ldots$ & 62,534 & 7,330 & 100.0 & $\cdots$ \\
\hline Normal pregnancy . . . . . . . . . . . . . . . . v22 & 4,923 & 913 & 7.9 & 1.1 \\
\hline Health supervision of intant or child . . . . . . V V20 & 1,876 & 345 & $\mathbf{3 . 0}$ & 0.5 \\
\hline Essenttal hypertension . . . . . . . . . . . 401 & 1,732 & 239 & 2.8 & 0.3 \\
\hline General medical examination . . . . . . . . . . V70 & 1,286 & 242 & 2.1 & 0.3 \\
\hline Suppurative and unspecified otlitis medla . . . . 382 & 1,242 & 220 & 2.0 & 0.3 \\
\hline $\begin{array}{l}\text { Acute upper respiratory infectlons of multiple or } \\
\text { unspeclified sites } \ldots \ldots \ldots \ldots \ldots \ldots \ldots \ldots\end{array}$ & 1,234 & 309 & 2.0 & 0.3 \\
\hline Diabetes mellitus. . . . . . . . . . . . . . . 250 & 1,103 & 191 & 1.8 & 0.2 \\
\hline Asthma . . . . . . . . . . . . . . . . . . . . 493 & 1,022 & 267 & 1.6 & 0.3 \\
\hline Neurotlc disorders . . . . . . . . . . . . . . 300 & 914 & 219 & 1.5 & 0.3 \\
\hline Other postsurglcal states . . . . . . . . . . . . . V45 & 900 & 219 & 1.4 & 0.3 \\
\hline Affecthe psychoses . . . . . . . . . . . . . . 296 & 803 & 201 & 1.3 & 0.3 \\
\hline Malignant neoplasm of female breast . . . . . 174 & 648 & 190 & 1.0 & 0.3 \\
\hline General symptoms . . . . . . . . . . . . . . . . . 780 & 631 & 97 & 1.0 & 0.1 \\
\hline Other and unspecifled dksorders of back . . . . . 724 & 572 & 155 & 0.9 & 0.2 \\
\hline Acute pharyngitts. . . . . . . . . . . . . . . 462 & 572 & 127 & 0.9 & 0.2 \\
\hline Alcohol dependence syndrome . . . . . . . . 303 & "565 & 233 & 0.9 & 0.3 \\
\hline Follow-up examination . . . . . . . . . . . . V67 & 525 & 109 & 0.8 & 0.2 \\
\hline Chronic sinusitis . . . . . . . . . . . 473 & 503 & 127 & 0.8 & 0.1 \\
\hline Other disorders of urethra and urinary tract. . . . 599 & 494 & 112 & 0.8 & 0.2 \\
\hline Bronchitts, not specified as acute or chronic . . . 490 & 492 & 106 & 0.8 & 0.1 \\
\hline All other dlagnoses $\ldots \ldots \ldots \ldots \ldots \ldots$ & 40,497 & 4,762 & 64.8 & 1.5 \\
\hline
\end{tabular}

'Based on the Intemational Classification of Dissases, 9th Revision, Clinical Modification (ICD-O-CM) (8).

Table 9. Number and percent of outpatlent department vlsits with corresponding standard orrors by selocted diagnostic services performed or ordered: United States, 1993.

\begin{tabular}{|c|c|c|c|c|}
\hline $\begin{array}{c}\text { Selected dkagnostic services performed or } \\
\text { ordered by hospltal staff }\end{array}$ & $\begin{array}{c}\text { Number of } \\
\text { vistts in } \\
\text { thousands }\end{array}$ & $\begin{array}{c}\text { Standard } \\
\text { orror in } \\
\text { thousands }\end{array}$ & Percent & $\begin{array}{c}\text { Standard } \\
\text { error of } \\
\text { percent }\end{array}$ \\
\hline All visits $\ldots \ldots \ldots \ldots \ldots \ldots$ & 62,534 & 7,390 & $\cdots$ & $\cdots$ \\
\hline Blood pressure $\ldots \ldots \ldots \ldots \ldots \ldots$ & 34,013 & 4,012 & 54.4 & 1.9 \\
\hline Other blood test $\ldots \ldots \ldots \ldots \ldots \ldots$ & 12,843 & 1,515 & 20.5 & 1.0 \\
\hline Urinalysis $\ldots \ldots \ldots \ldots \ldots \ldots$ & 8,719 & 1,238 & 13.9 & 1.2 \\
\hline HIV serology ${ }^{2} \ldots \ldots \ldots \ldots \ldots \ldots \ldots$ & 488 & 139 & 0.8 & 0.2 \\
\hline Splrometry . . . . . . . . . . . . . & $* 375$ & 122 & 0.6 & 0.2 \\
\hline Allergy testing. . . . . . . . . . . & ${ }^{*} 182$ & 75 & 0.3 & 0.1 \\
\hline None . . . . . . . . . . . . . . . . . . & 14,627 & 1,821 & 23.4 & 1.3 \\
\hline
\end{tabular}

'Numbers may exceed total number of visits because more than one service may be reported per visit.

${ }^{2} \mathrm{HIV}$ is human immunodeficiency virus.

Additional reports utilizing NHAMCS data are forthcoming in the Advance Data from Vital and Health Statistics series. In addition, survey data will be available on computer tape and CD-Rom from the National Technical Information Service in early 1996. Questions regarding this report, future reports, or the NHAMCS may be directed to the Ambulatory Care Statistics Branch by calling (301)436-7132.

\section{References}

1. McCaig LF. National Hospital Ambulatory Medical Care Survey: 1992 emergency department summary. Advance data from vital and health statistics; no. 245. Hyattsville, Maryland: National Center for Health Statistics. 1994.

2. McCaig LF. National Hospital Ambulatory Medical Care Survey: 1992 outpatient department summary. Advance data from vital and bealth statistics; no. 248. Hyattsville, 
Table 10. Number and percent of outpatient department visits by the top write-in diagnostic tests, surgical and non-surgical procedures, or therapies most often performed or ordered in hospital outpatient departments: United States, 1993

\begin{tabular}{|c|c|c|c|c|c|c|c|c|}
\hline \multirow{2}{*}{$\begin{array}{l}\text { Tests, surgical and non-surgical procedures } \\
\text { and therapies and ICD-9-CM code }{ }^{1}\end{array}$} & \multirow{2}{*}{$\begin{array}{l}\text { Number of } \\
\text { visits in } \\
\text { thousands }\end{array}$} & \multirow{2}{*}{$\begin{array}{l}\text { Standard } \\
\text { error in } \\
\text { thousands }\end{array}$} & \multirow[b]{2}{*}{ Percent } & \multirow{2}{*}{$\begin{array}{l}\text { Standard } \\
\text { error of } \\
\text { percent }\end{array}$} & \multicolumn{4}{|c|}{ Percent distribution } \\
\hline & & & & & Total & Performed & Ordered & Unknown ${ }^{3}$ \\
\hline All visits $\ldots \ldots \ldots \ldots \ldots \ldots \ldots \ldots$ & 62,534 & 7,330 & $\cdots$ & $\cdots$ & $\cdots$ & $\cdots$ & $\cdots$ & $\cdots$ \\
\hline All visits with procedures written in $\ldots \ldots \ldots \ldots \ldots$ & 33,023 & 1,570 & 52.8 & 0.9 & 100.0 & 69.4 & 22.2 & 8.5 \\
\hline Other individual psychotherapy . . . . . . . . . . 94.39 & 2,045 & 563 & 3.3 & 5.3 & 100.0 & 89.2 & 0.9 & 9.9 \\
\hline Eye examination not otherwise specifled . . . . . 95.09 & 1,793 & 608 & 2.9 & 5.7 & 100.0 & 90.0 & 1.6 & 8.4 \\
\hline Pap smear. . . . . . . . . . . . . . . . . . 91.46 & 1,744 & 379 & 2.8 & 5.7 & 100.0 & 83.0 & 9.1 & 7.9 \\
\hline $\begin{array}{l}\text { Other nonoperative measurements and } \\
\text { examinations } \ldots \ldots \ldots \ldots \ldots \ldots \ldots \ldots \ldots\end{array}$ & 1,548 & 526 & 2.5 & 6.1 & 100.0 & 93.3 & 2.2 & 4.5 \\
\hline 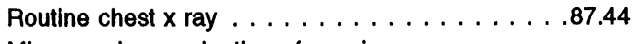 & 1,386 & 216 & 2.2 & 6.5 & 100.0 & 55.3 & 34.5 & 10.2 \\
\hline $\begin{array}{l}\text { Microscopic examination of specimen } \\
\text { from female genital tract - culture . . . . . . . 91.42 }\end{array}$ & 1,074 & 209 & 1.7 & 7.4 & 100.0 & 87.1 & 7.4 & 5.6 \\
\hline Other mammography . . . . . . . . . . . . . . .87.37 & 1,024 & 213 & 1.6 & 7.5 & 100.0 & 29.8 & 64.3 & 6.0 \\
\hline Electrocardiogram . . . . . . . . . . . . . . . . 89.52 & 948 & 123 & 1.5 & 7.8 & 100.0 & 65.2 & 25.7 & 9.1 \\
\hline Diagnostlc ultrasound of gravid uterus . . . . . . 88.78 & 825 & 185 & 1.3 & 8.4 & 100.0 & 52.6 & 43.5 & 3.9 \\
\hline $\begin{array}{l}\text { Microscopic examination of specimen } \\
\text { from ear, nose, throat and larynx-culture. . . . . .90.32 }\end{array}$ & 574 & 130 & 0.9 & 10.1 & 100.0 & 85.9 & 10.8 & 3.3 \\
\hline Other dlagnostic ultrasound $\ldots \ldots \ldots$. . . . . . . . . . . & 557 & 185 & 0.9 & 10.3 & 100.0 & 55.5 & 39.3 & 5.2 \\
\hline Fetal monltoring, not otherwise specified . . . . . . .75.34 & 531 & 267 & 0.8 & 10.5 & 100.0 & 94.9 & 2.1 & 3.0 \\
\hline Other physical therapy $\ldots \ldots \ldots$. . . . . . . . . . . . . & 463 & 102 & 0.7 & 11.3 & 100.0 & 28.5 & 53.3 & 18.1 \\
\hline Skeletal $x$ ray of ankle and foot $\ldots \ldots \ldots$. . . . . . $\ldots 8$ & 375 & 71 & 0.6 & 12.5 & 100.0 & 73.3 & 19.2 & 7.5 \\
\hline Skeletal $x$ ray of thigh, knee and lower leg . . . . 88.27 & 357 & 93 & 0.6 & 12.8 & 100.0 & 74.5 & 19.0 & 6.4 \\
\hline Skeletal $x$ ray of wrist and hand. . . . . . . . . 88.23 & 353 & 115 & 0.6 & 12.9 & 100.0 & 89.0 & 8.5 & 2.5 \\
\hline Gynecological examination . . . . . . . . . . . 89.26 & 321 & 89 & 0.5 & 13.6 & 100.0 & 85.0 & 10.6 & 4.4 \\
\hline$X$ ray, other and unspecified $\ldots \ldots \ldots$. . . . . . . . . . & 310 & 60 & 0.5 & 13.8 & 100.0 & 73.9 & 19.7 & 6.5 \\
\hline 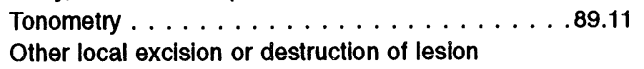 & 307 & 201 & 0.5 & 13.9 & 100.0 & 97.4 & 0.0 & 2.6 \\
\hline or tissue of skin and subcutaneous tissue $\ldots \ldots 86.3$ & 306 & 63 & 0.5 & 13.9 & 100.0 & 88.6 & 6.5 & 4.9 \\
\hline
\end{tabular}

1Based on the International Classification of Diseases, 9th Revision, Clinical Modification (ICD-9-CM) (8).

${ }^{2}$ Numbers may exceed total number of visits because more than one procedure may be reported per visit.

${ }^{3}$ Not known whether ordered or performed.

Maryland: National Center for Health Statistics. 1994.

3. Nelson CR, Stussman BJ. Alcoholand drug-related visits to hospital emergency departments: 1992 National Hospital Ambulatory Medical Care Survey. Advance data from vital and health statistics; no. 251. Hyattsville, Maryland: National Center for Health Statistics. 1994.

4. Burt CW. Injury-related visits to hospital emergency departments: United States 1992. Advance data from vital and health statistics; no. 261. Hyattsville, Maryland: National Center for Health Statistics. 1995.

5. Stussman BJ. National Hospital Ambulatory Medical Care Survey: 1993 emergency department summary. Advance data from vital and health statistics. Hyattsville, Maryland: National Center for Health Statistics. 1995. To be published.

6. McCaig LF, McLemore T. Plan and operation of the National Hospital Ambulatory Medical Care Survey. National Center for Health Statistics. Vital Health Stat 1(34). 1994.

Table 11. Number and percent of outpatient department visits with corresponding standard errors by counseling/education services: United States, 1993

\begin{tabular}{|c|c|c|c|c|}
\hline $\begin{array}{c}\text { Counselingleducation ordered or } \\
\text { provided by hospital staff }\end{array}$ & $\begin{array}{l}\text { Number of } \\
\text { visits in } \\
\text { thousands }\end{array}$ & $\begin{array}{l}\text { Standard } \\
\text { error in } \\
\text { thousands }\end{array}$ & Percent & $\begin{array}{c}\text { Standard } \\
\text { error of } \\
\text { percent }\end{array}$ \\
\hline All visits $\ldots \ldots \ldots \ldots \ldots$ & 62,534 & 7,330 & $\cdots$ & $\cdots$ \\
\hline Growth/development. . . . . . . . . . . & 3,291 & 777 & 5.3 & 1.1 \\
\hline Exercise . . . . . . . . . & 2,954 & 521 & 4.7 & 0.5 \\
\hline Injury prevention $\ldots \ldots \ldots \ldots$ & $* 1,594$ & 489 & 2.5 & 0.6 \\
\hline Weight reduction . . . . . . . . . . & 1,487 & 227 & 2.4 & 2.0 \\
\hline Smoking cessation. . . . . . . . . . & 1,112 & 186 & 1.8 & 0.2 \\
\hline Cholesterol reduction ......... & 790 & 136 & 1.3 & 0.2 \\
\hline Other STD transmission $\ldots \ldots \ldots$ & 761 & 157 & 1.2 & 0.2 \\
\hline HIV transmission . . . . . . . . . . & 722 & 166 & 1.2 & 0.2 \\
\hline Other $\ldots \ldots \ldots \ldots \ldots \ldots \ldots$ & 23,051 & 3,121 & 36.9 & 1.7 \\
\hline None $\ldots \ldots \ldots \ldots \ldots \ldots$ & 33,397 & 3,937 & 53.4 & 2.0 \\
\hline
\end{tabular}

${ }^{1}$ Numbers may exceed total number of visits because more than one service may be reported per visit.

7. Schneider D, Appleton L, McLemore T. A reason for visit classification for ambulatory care. National Center for Health Statistics. Vital and Health Stat 2(78). 1979.

8. Public Health Service and Health Care Financing Administration. International Classification of Diseases, 9th Revision, clinical modification. Washington: Public Health Service. 1980.

9. Koch H, Campbell W. The collection and processing of drug information. National Ambulatory Medical Care Survey, 1980. National Center for Health Statistics. Vital Health Stat 2(90). 1982. 
Table 12. Number and percent distribution of outpatient department visits with corresponding standard errors by number of medication codes this visit: Unlted States, 1993

\begin{tabular}{|c|c|c|c|c|}
\hline $\begin{array}{c}\text { Number of } \\
\text { medlcation codes }\end{array}$ & $\begin{array}{c}\text { Number of } \\
\text { visits } n \\
\text { thousands }\end{array}$ & $\begin{array}{l}\text { Standard } \\
\text { emor in } \\
\text { thousands }\end{array}$ & $\begin{array}{c}\text { Percent } \\
\text { distribution }\end{array}$ & $\begin{array}{c}\text { Standard } \\
\text { emor of } \\
\text { percent }\end{array}$ \\
\hline All visils $\ldots \ldots \ldots \ldots \ldots \ldots$ & 62,534 & 7330 & 100.0 & .. \\
\hline None . . . . . . . . . . . . . . . & 26,812 & 3716 & 42.9 & 1.9 \\
\hline $1 \ldots \ldots \ldots \ldots \ldots \ldots \ldots$ & 16,002 & 1983 & 25.6 & 1.0 \\
\hline $2 \ldots \ldots \ldots \ldots \ldots \ldots \ldots \ldots$ & 9,118 & 1080 & 14.6 & 0.7 \\
\hline $3 \ldots \ldots \ldots \ldots \ldots \ldots$ & 4,456 & 530 & 7.1 & 0.4 \\
\hline $4 \ldots \ldots \ldots \ldots \ldots \ldots \ldots \ldots$ & 2,628 & 318 & 4.2 & 0.4 \\
\hline 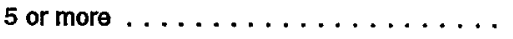 & 3,518 & 489 & 5.6 & 0.6 \\
\hline
\end{tabular}

10. Food and Drug Administration. National Drug Code Directory, 1985 Edition. Washington: Public Health Service, 1985.

11. Shah BV, Barnwell BG, Hunt PN, La Vange LM. SUDAAN user's manual release 5.50. Research Triangle Park, North Carolina: Research Triangle Institute. 1991.

Table 13. Number and percent distribution for the 20 drugs most frequently prescribed at outpatient department visits with corresponding standard errors, by entry name of drug: United States, 1993

\begin{tabular}{|c|c|c|c|c|c|}
\hline Entry name of drug ${ }^{t}$ & $\begin{array}{l}\text { Number of } \\
\text { druge mentions } \\
\text { in thousands }\end{array}$ & $\begin{array}{c}\text { Standard } \\
\text { error in } \\
\text { thousands }\end{array}$ & $\begin{array}{l}\text { Percent } \\
\text { distribution }\end{array}$ & $\begin{array}{l}\text { Standard } \\
\text { error of } \\
\text { percent }\end{array}$ & Therapeutic classification ${ }^{2}$ \\
\hline All drug mentlons. . . . . . . . . . . . & 75,710 & $\mathbf{3 , 7 8 1}$ & 100.0 & $\cdots$ & \\
\hline Tylenol . . . . . . . . . . . . . . . & 2,191 & 326 & 2.9 & 0.4 & General analgesics \\
\hline Prenatal vitamins. . . . . . . . . . . . & 1,879 & 586 & 2.5 & 0.8 & Vitamins, minerais \\
\hline $\begin{array}{l}\text { Amoxicillin } \ldots \ldots \ldots \ldots \ldots \ldots \\
\text { Diphlheria \& tetanus toxoids }\end{array}$ & 1,307 & 227 & 1.7 & 0.3 & Penicillins \\
\hline whth pertussis vaccine . . . . . . . . . & 934 & 173 & 1.2 & 0.3 & Vaccines and antiserums \\
\hline Motrin. . . . . . . . . . . . . . . . & 879 & 148 & 1.2 & 0.2 & Antiarthritics \\
\hline Poliomyelitis vaccine. . . . . . . . . . & 873 & 170 & 1.2 & 0.3 & Vaccines and antiserums \\
\hline Laslx $\ldots \ldots \ldots \ldots \ldots \ldots \ldots$ & 795 & 126 & 1.1 & 0.1 & Diuretics \\
\hline Prednisone . . . . . . . . . . . & 743 & 119 & 1.0 & 0.2 & Adrenal corticosteroids \\
\hline Zantac . . . . . . . . . . . . & 739 & 109 & 1.0 & 0.1 & Agents used in disorders of upper Gi tract \\
\hline Bactrim. . . . . . . . . . . . & 721 & 163 & 1.0 & 0.2 & Antimicrobials \\
\hline Procardia. . . . . . . . . . . . & 698 & 84 & 0.9 & 0.1 & Antlanginal agents \\
\hline Iron preparation. . . . . . . . . . . & 685 & 211 & 0.9 & 0.3 & Agents used to treat defictency anemlas \\
\hline Ventolin. . . . . . . . . . . . . & 682 & 129 & 0.9 & 0.2 & Bronchodllators, antiasthmatics \\
\hline Proventil . . . . . . . . . . . . . . & 657 & 141 & 0.9 & 0.2 & Bronchodilators, antlasthmatics \\
\hline Hepatitis B. . . . . . . . . . . . . . . . & 657 & 127 & 0.9 & 0.2 & Vaccines and antiserums \\
\hline Haemophilus B conjugate vaccine . . . . . . & 656 & 139 & 0.9 & 0.2 & Vaccines and antiserums \\
\hline Vasotec. . . . . . . . . . . . . . & 616 & 85 & 0.8 & 0.1 & Anthypertensive agents \\
\hline Influenza virus vaccine $\ldots \ldots \ldots \ldots$ & 597 & 161 & 0.8 & 0.2 & Vaccines and antiserums \\
\hline Synthroid. . . . . . . . . . . . . & 555 & 92 & 0.7 & 0.1 & Agents used to treat thyroid disease \\
\hline Vitamins $\ldots \ldots \ldots \ldots \ldots \ldots \ldots$ & 550 & 229 & 0.7 & 0.3 & Vitamins, minerals \\
\hline All other $\ldots \ldots \ldots \ldots \ldots \ldots \ldots$ & 58,296 & 2,930 & 77.0 & 0.6 & \\
\hline
\end{tabular}

'The entry made by the hospital staff on the prescription or other medical records. This may be a trade name, generic name, or desired therapeutic effect.

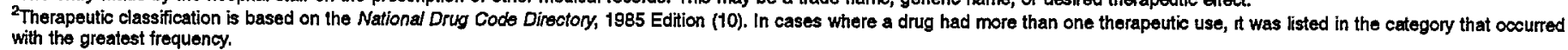


Table 14. Number and percent of outpatient department vlsits with corresponding standard errors by dlsposition of visit: United States, 1993

\begin{tabular}{|c|c|c|c|c|}
\hline Diposition & $\begin{array}{l}\text { Number of } \\
\text { visits in } \\
\text { thousands }\end{array}$ & $\begin{array}{c}\text { Standard } \\
\text { error in } \\
\text { thousands }\end{array}$ & Percent & $\begin{array}{c}\text { Standard } \\
\text { error of } \\
\text { percent }\end{array}$ \\
\hline 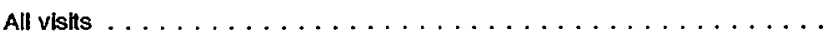 & 62,534 & 7,330 & $\cdots$ & $\cdots$ \\
\hline Retum to clinlc - appointment $\ldots \ldots \ldots \ldots \ldots \ldots$ & 41,017 & 4,746 & 65.6 & 1.9 \\
\hline Retum to clinic $\mathrm{PRN}^{2} \ldots \ldots \ldots \ldots \ldots \ldots \ldots \ldots \ldots$ & 11,183 & 1,933 & 17.9 & 1.4 \\
\hline 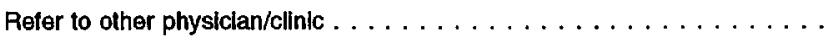 & 4,625 & 595 & 7.4 & 0.8 \\
\hline 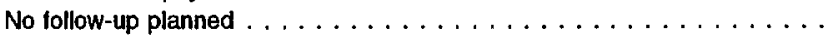 & 2,805 & 483 & 4.5 & 0.5 \\
\hline 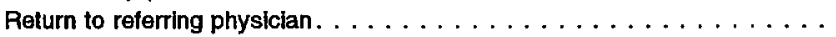 & 2,463 & 559 & 3.9 & 0.8 \\
\hline Telephone follow-up planned. $\ldots \ldots \ldots \ldots \ldots \ldots$ & 2,079 & 584 & 3.3 & 0.8 \\
\hline 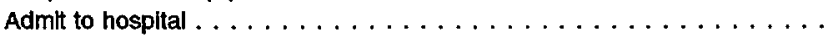 & 921 & 179 & 1.5 & 0.2 \\
\hline 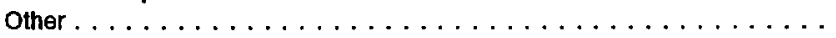 & 2,058 & 334 & 3.3 & 0.4 \\
\hline
\end{tabular}

${ }_{1}^{1}$ Numbers may exceed total number of visits because more than one disposition may be reported per visit. 2PAN is as needed.

Table 15. Number and percent of outpatient department visits with corresponding standard errors by type of provider seen: United States, 1993

\begin{tabular}{|c|c|c|c|c|}
\hline Type of provider & $\begin{array}{l}\text { Number of } \\
\text { visits } / n \\
\text { thousands }\end{array}$ & $\begin{array}{c}\text { standard } \\
\text { orror in } \\
\text { thousands }\end{array}$ & Percent & $\begin{array}{c}\text { Standard } \\
\text { error of } \\
\text { percent }\end{array}$ \\
\hline 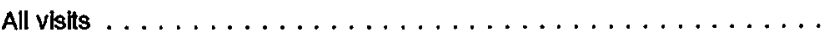 & 62,534 & 7,330 & $\cdots$ & $\cdots$ \\
\hline Staft physician $\ldots \ldots \ldots \ldots \ldots \ldots \ldots \ldots \ldots \ldots \ldots$ & 41,786 & 5,720 & 66.8 & 2.4 \\
\hline Registered nurse . . . . . . . . . . . . . . . . . & 26,155 & 2,567 & 41.8 & 3.3 \\
\hline 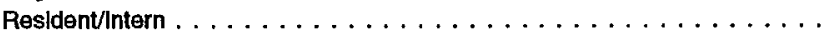 & 15,223 & 2,383 & 24.3 & 2.2 \\
\hline Licensed practical nurse $\ldots \ldots \ldots \ldots \ldots \ldots \ldots \ldots$ & ${ }^{*} 6,770$ & 2,186 & 10.8 & 2.6 \\
\hline Nurse's aide. . . . . . . . . . . . . . . . . . . . . . . & 5,254 & 998 & 8.4 & 1.3 \\
\hline $\begin{array}{l}\text { Physician assistant/Nurse } \\
\text { practitloner } \ldots \ldots \ldots \ldots \ldots \ldots \ldots \ldots \ldots \ldots \ldots\end{array}$ & 4,547 & 892 & 7.3 & 1.0 \\
\hline 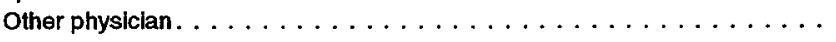 & 2,145 & 555 & 3.4 & 0.8 \\
\hline 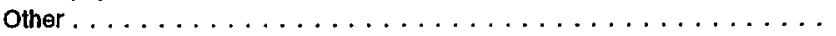 & 11,053 & 2106 & 17.7 & 2.2 \\
\hline
\end{tabular}

${ }^{1}$ Numbers may exceed total number of visits because more than one provider may be reported per visit.

\section{Symbols}

- - Data not available

.. Category not applicable

- Quantity zero

0.0 Quantity more than zero but less than 0.05

Z Quantity more than zero but less than 500 where numbers are rounded to thousands

* Figure does not meet standard of reliability or precision 


\section{Technical notes}

\section{Source of data and sample design}

The information presented in this report is based on data collected in the 1993 National Hospital Ambulatory Medical Care Survey (NHAMCS) from December 28, 1992 through December 26,1993 . The data were adjusted to produce annual estimates.

The target universe of NHAMCS includes in-person visits made in the United States by patients to emergency departments (ED's) and outpatient departments (OPD's) of non-Federal, short-stay hospitals (hospitals with average length of stays for all patients of fewer than 30 days) or those whose specialty is general (medical or surgical) or children's general. The NHAMCS sampling frame consists of hospitals listed in the April 1991 SMG Hospital Database.

A four-stage probability sample design is used in NHAMCS. The design includes samples of primary sampling units (PSU's), hospitals within PSU's, ED's within hospitals and/or clinics within outpatient departments (OPD's), and patient visits within ED's and/or clinics. The PSU sample consists of 112 PSU's comprising a probability subsample of the PSU's used in the 1985-94 National Health Interview Survey. The hospital sample for 1993 consisted of 489 hospitals. Of this group, 445 hospitals had either an ED or OPD in 1993 to make them in scope or eligible for the survey. During this period, 94 percent of the in-scope hospitals participated. Based on the induction interview, 228 of the sample hospitals had OPD's. Hospital staff were asked to complete Patient Record forms (figure 1) for a systematic random sample of patient visits occurring during a randomly assigned 4-week reporting period. The number of Patient Record forms completed for OPD's was 28,357.

Characteristics of the hospital, such as ownership and expected number of OPD visits, were obtained from the hospital administrator during an induction interview. The U.S. Bureau of the Census, Housing Surveys Branch, was responsible for the survey's data collection. Data processing operations and medical coding were performed by Analytical Sciences Inc., Durham, North Carolina.

\section{Sampling errors}

The standard error is primarily a measure of the sampling variability that occurs by chance when only a sample, rather than an entire universe, is surveyed. The standard error also reflects part of the measurement error, but does not measure any systematic biases in the data. The chances are 95 out of 100 that an estimate from the sample differs from the value that would be obtained from a complete census by less than twice the standard error.

The standard errors used in this report (including tests of significance) were approximated using SUDAAN software. SUDAAN computes standard errors by using a first-order Taylor approximation of the deviation of estimates from their expected values. A description of the software and the approach it uses has been published (11). Standard errors for all estimates are presented in each table. The relative standard error (RSE) of an estimate is obtained by dividing the standard error by the estimate itself. The result is then expressed as a percent of the estimate.

Approximate relative standard errors for aggregate estimates may be calculated using the following general formula, where $x$ is the aggregate of interest in thousands, and $A$ and $B$ are the appropriate coefficients from table I.

$$
\operatorname{RSE}(x)=\sqrt{A+\frac{B}{x}} \cdot 100
$$

Similarly, relative standard errors for an estimate of a percent may be calculated using the following general formula, where $p$ is the percent of interest, expressed as a proportion, and $x$ is the denominator of the percent in thousands, using the appropriate coefficients from table I.

$$
\operatorname{RSE}(x)=\sqrt{\frac{B \cdot(1-p)}{p \cdot x}} \cdot 100
$$

The standard error for a rate may be obtained by multiplying the relative standard error of the total estimate by the rate.
Table I. Coofficients appropriate for detormining relative standards errors: National Hospltal Ambulatory Medlcal Care Survey, 1993

\begin{tabular}{lcc}
\hline & \multicolumn{2}{c}{$\begin{array}{c}\text { Coefficient for use } \\
\text { with estimates } \\
\text { In thousands }\end{array}$} \\
\cline { 2 - 3 } Type of estimate & A & $B$ \\
\hline Visits . . . . . . . . & 0.02082 & 5.924262 \\
Drug mentions ... & 0.02387 & 8.128418 \\
\hline
\end{tabular}

\section{Adjustments for hospital nonresponse}

Estimates from NHAMCS data were adjusted to account for sample hospitals that were in scope but did not participate in the study. This adjustment was calculated to minimize the impact of response on final estimates by imputing to nonresponding hospitals data from visits to similar hospitals. For this purpose, hospitals were judged similar if they were in the same region, ownership control group, and metropolitan statistical area control group.

\section{Adjustments for ED/clinic nonresponse}

Estimates from NHAMCS data were adjusted to account for ED's and sample clinics that were in scope but did not participate in the study. This adjustment was calculated to minimize the impact of nonresponse on final estimates by imputing to nonresponding ED's or clinics data from visits to similar ED's or clinics. For this purpose, ED's or clinics were judged similar if they were in the same ED or clinic group.

\section{Test of significance and rounding}

The determination of statistical inference is based on the $t$-test. The Bonferroni inequality was used to establish the critical value for statistically significant differences $(0.05$ level of significance over all analyses performed on estimates contained in a table). Terms relating to differences such as "higher than" indicate that the difference is statistically significant. A lack of comment regarding the 
difference between any two estimates does not mean that the difference was tested and found to be not significant.

In the tables, estimates of OPD visits have been rounded to the nearest thousand. Consequently, estimates will not always add to totals. Rates and percents were calculated from original unrounded figures and do not necessarily agree with percents calculated from rounded data.

\section{Definition of terms}

Patient_An individual seeking personal health services who is not currently admitted to any health care institution on the premises.

Hospital-All hospitals with an average length of stay for all patients of less than 30 days (short-stay) or hospital whose specialty is general (medical or surgical) or children's general. Federal hospitals, hospital units of institutions, and hospitals with less than six beds staffed for patient use are excluded.

Emergency department-Hospital facility for the provision of unscheduled outpatient services to patients whose conditions require immediate care and that is staffed 24 hours a day. If an ED provided emergency services in different areas of the hospital, then all these areas were selected with certainty into the sample. Off-site emergency departments open less than 24 hours are included if staffed by the hospital's emergency department.

Outpatient department-Hospital facility where nonurgent ambulatory medical care is provided under the supervision of a physician.

Clinic-An administrative unit of the outpatient department where ambulatory medical care is provided under the supervision of a physician. The following are examples of the types of clinics excluded from the NHAMCS: ambulatory surgical centers, chemotherapy, employee health service, renal dialysis, methadone maintenance, and radiology.

Visit-A direct, personal exchange between a patient and a physician or other health care provider working under the physician's supervision, for the purpose of seeking care and receiving personal health services.

\section{Suggested cltation}

Lipkind KL. National hospital ambulatory medical care survey: 1993 outpatient department summary. Advance data from vital and health statistics; no 268. Hyattsville, Maryland: National Center for Health Statistics. 1995.

\section{Copyright Information}

All material appearing in this report is in the public domain and may be reproduced or copied without permission; citation as to source, however, is appreciated.

\section{Trade name disclaimer}

The use of trade names is for identification only and does not imply endorsement by the Public Health Service, U.S. Department of Health and Human Services.
National Center for Health Statistics

Acting Director

Jack R. Anderson

Acting Deputy Director
Jennifer H. Madans, Ph.D.

\author{
U.S. DEPARTMENT OF HEALTH AND \\ HUMAN SERVICES \\ Public Health Service \\ Centers for Disease Control and Prevention \\ National Center for Health Statistics \\ 6525 Belcrest Road \\ Hyattsville, Maryland 20782
}

BULK RATE

POSTAGE \& FEES PAID

PHSNCHS

PERMIT NO. G-281

OFFICIAL BUSINESS

PENALTY FOR PRIVATE USE, $\$ 300$

To recelve this publication regularly, contact the National Center for Health Statistics by calling $301-436-8500$

DHHS Publication No. (PHS) 95-1250

5-1596 (10/95) 


\section{Advance Data No. 268 Errata Sheet}

Please insert this sheet in your copy of National Hospital Ambulatory Medical Care Survey: 1993 outpatient department summary.

\section{Page Correction}

2 Delete items 1 and 2 from the Patient Record form.

3 Table 1:

American Indian/Eskimo/Aleut, Standard error in thousands: 53 [not 534]

9 Table 13:

Vitamins, Number of drug mentions in thousands: *550 [not 550]

11 Paragraph 3 under "Source of data ...":

Change "Based on the induction interview, [228] of the sample hospitals . .." to "Based on the induction interview, 255 of the sample hospitals ..."

\section{Suggested cltation}

Lipkind KL. National Hospital Ambulatory Medical Care Survey: 1993 outpatient department summary. Advance data from vital and health statistics; no 268. Hyattsville, Maryland: National Center for Health Statistics. 1995.

\section{Copyright information}

All material appearing in this report is in the public domain and may be reproduced or copied without permission; citation as to source, however, is appreciated.

Trade name disclaimer

The use of trade names is for identification only and does not imply endorsement by the Public Health Service, U.S. Department of Health and Human Services.
National Center for Hoalth Statistics

Acting Director Jack R. Anderson

Acting Deputy Director Jennifer H. Madans, Ph.D.
BULK RATE

POSTAGE \& FEES PAID PHSNCHS

PERMIT NO. G-281

Centers for Disease Control and Prevention

National Center for Health Statistics

6525 Belcrest Road

OFFICIAL BUSINESS

PENALTY FOR PRIVATE USE, $\$ 300$

To receive this publication regularly, contact the National Center for Health Statistics by calling 301-436-8500

DHHS Publication No. (PHS) 95-1250-E 6-0020 (10/95) 


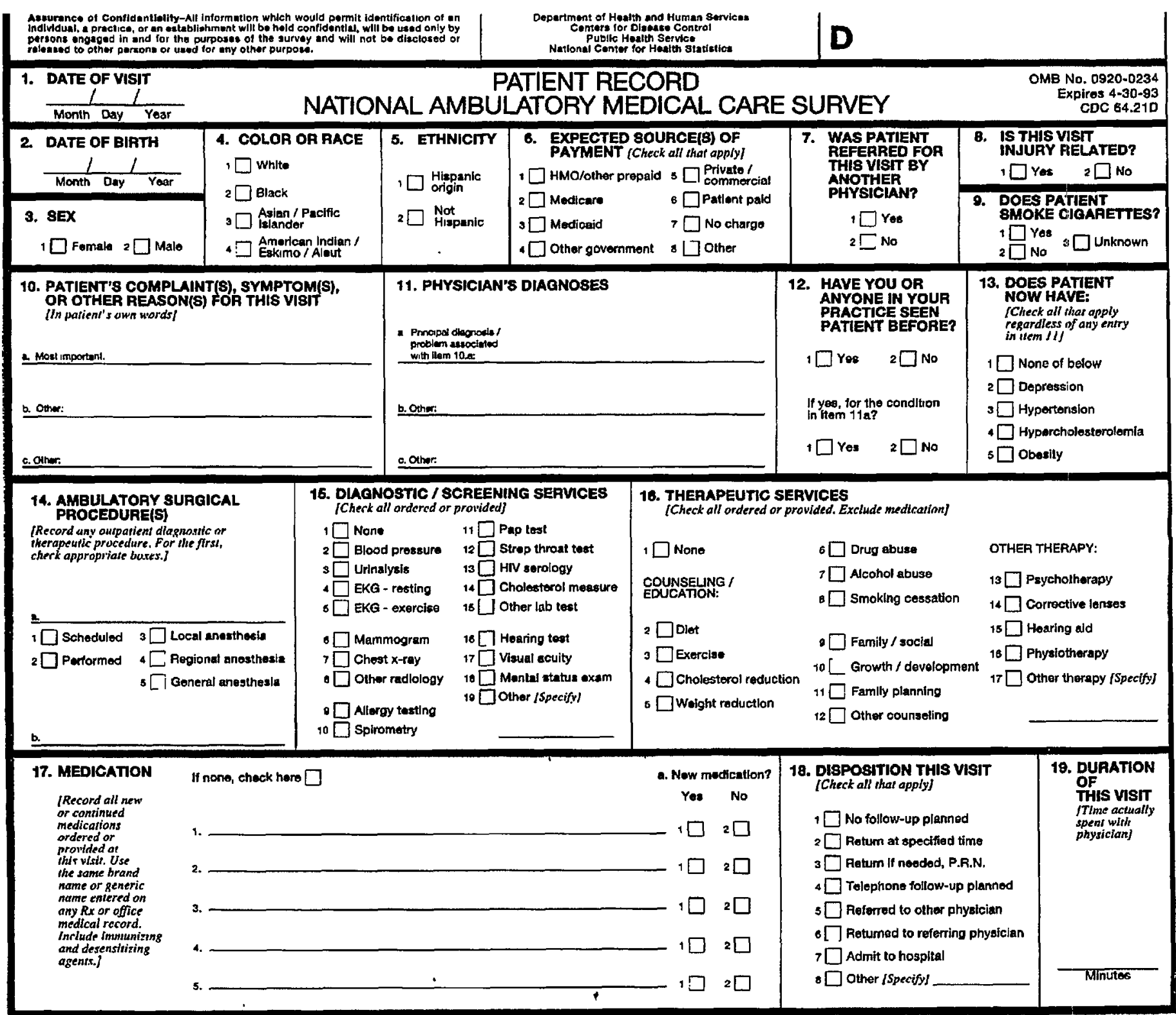

Flgure 1. Patlent record form

expected source of payment is possible. In addition, a patient who has insurance may have a visit with "patient paid" designated as the expected source of payment because of copayments or deductibles. For these reasons all nonprepaid visits have been combined into one category. Second, the prepaid plan visits that are the subject of this report cannot be analyzed according to the type of prepaid plan because all prepaid plans were grouped together into a single category on the survey instrument.

\section{Highlights}

- Between 1985 and 1991 the proportion of physician office visits by persons under 65 years of age that had an expected source of payment of "prepaid plan" almost doubled (10 and 18 percent, respectively) with a higher proportion of 1991 visits to nonprimary care specialties.

- A higher proportion of prepaid than nonprepaid visits were to office-based physicians in the primary care specialties of general and family practice, internal medicine, pediatrics, and obstetrics and gynecology (70 and 62 percent, respectively).

- A higher proportion of prepaid visits than nonprepaid visits to nonprimary care specialties were referrals from another physician (19 and 12 percent, respectively).
- A higher proportion of prepaid than nonprepaid visits by new patients were referrals (39 and 27 percent, respectively).

- The West represented 33 percent of all prepaid plan visits and 21 percent of nonprepaid visits. Less than 18 percent of prepaid visits were made in the South compared with 31 percent of nonprepaid visits.

- Prepaid and nonprepaid visits were similar with respect to the principal reason for visit, physicians' principal diagnosis, and medications prescribed.

- Excluding visits in which there was no face-to-face contact between patient and physician, the average 
duration of prepaid plan visits was similar to that of nonprepaid visits (16 minutes vs. 17 minutes, respectively).

- After the exclusion of patients 65 years of age and older, the average age of patients making prepaid plan visits was 29.2 years compared with 31.4 years for patients making nonprepaid visits.

- A significantly higher proportion of prepaid plan visits had at least one diagnostic or screening test ordered or performed ( 76 percent) compared with nonprepaid visits (70 percent) for patients $45-64$ years of age.

\section{Physician characteristics}

In 1991, there were an estimated 91.8 million visits to nonfederally employed office-based physicians at which a prepaid plan was an expected source of payment, not including visits made by patients 65 years of age and older. This is a significant increase from 1985 in which there were an estimated 51.4 million visits with an expected source of payment of a prepaid plan. In 1991, about 18 percent of the visits by persons under 65 years of age had a prepaid plan as an expected source of payment compared with 10 percent in 1985.

Of the 91.8 million prepaid plan office visits in 1991, about 70 percent were to primary care physiciansincluding general and family practitioners, internists, pediatricians, and obstetricians and gynecologists (table 2). The percent of prepaid visits to primary care physicians was down from 77 percent in 1985; the first year data were collected on an expected source of payment. The decrease in the proportion of prepaid visits to primary care physicians was due to a decline in the percent of visits to general and family practitioners from 35 percent in 1985 to 26 percent in 1991. The proportion of prepaid visits to obstetricians and gynecologists increased from 7.6 percent in 1985 to 11.0 percent in 1991. Among nonprepaid visits, general and family practitioners also dropped as a percent of visits, from 30 percent in 1985 to 25 percent in 1991. Internists increased as a percent of

Table 1. Number and percent of visits by expected sources of payment: United Stato8, 1991

\begin{tabular}{|c|c|c|}
\hline Expected sources of payment & $\begin{array}{l}\text { Number of } \\
\text { visits in } \\
\text { thousands }\end{array}$ & Percent \\
\hline All visits ${ }^{2} \ldots \ldots \ldots \ldots \ldots \ldots$ & 513,819 & 100.0 \\
\hline Prepaid plans . . . . . . . . . . . & 91,824 & 17.9 \\
\hline Nonprepard plans $\ldots \ldots \ldots \ldots \ldots$ & 421,995 & 82.1 \\
\hline All nonprepald plan visits $\ldots \ldots \ldots \ldots$ & 421,995 & 100.0 \\
\hline Medicare $\ldots \ldots \ldots \ldots \ldots \ldots$ & 15,736 & 3.7 \\
\hline Medlcald . . . . . . . . . . . & 51,055 & 12.1 \\
\hline Other government. . . . . . . . . & 12,078 & 2.7 \\
\hline Private/commercial insurance $\ldots \ldots \ldots$ & 197,046 & 46.7 \\
\hline Patient pald $\ldots \ldots \ldots \ldots \ldots \ldots$ & 132,453 & 31.4 \\
\hline No charge $\ldots \ldots \ldots \ldots \ldots \ldots$ & 8,635 & 2.0 \\
\hline Other $\ldots \ldots \ldots \ldots \ldots \ldots \ldots$ & 22,742 & 5.4 \\
\hline
\end{tabular}

Numbers do not add to totals because more than one source of payment may be reported per visit.

${ }^{2}$ An additional $155,870,000$ visits were for patients 65 years of age and over and 10,964,000 visits had no expected source of payment indicated. These visits have been excluded from this report.

Table 2. Number and percent distribution of prepaid plan visits and percent distribution of nonprepaid visits by physician specialty: United States, 1991

\begin{tabular}{|c|c|c|c|}
\hline \multirow[b]{2}{*}{ Physiclan specialty } & \multicolumn{2}{|c|}{ Prepaid pkan vistts } & \multirow{2}{*}{$\begin{array}{c}\text { Nonprepald visits } \\
\text { Percent } \\
\text { distribution }\end{array}$} \\
\hline & $\begin{array}{l}\text { Number of } \\
\text { vislts in } \\
\text { thousands }\end{array}$ & $\begin{array}{c}\text { Percent } \\
\text { distribution }\end{array}$ & \\
\hline All visits $\ldots \ldots \ldots \ldots \ldots$ & 91,824 & 100.0 & 100.0 \\
\hline \multicolumn{4}{|l|}{ Primary care: } \\
\hline General and family practice . . . . . . & 24,204 & 26.4 & 25.1 \\
\hline Intemal medlcine. . . . . . . . & 14,395 & 15.7 & 11.9 \\
\hline Pedkatrics . . . . . . . . . . . & 15,247 & 16.6 & 14.4 \\
\hline Obstetrics and gynecology. . . . . . & 10,095 & 11.0 & 10.6 \\
\hline \multicolumn{4}{|l|}{ Other than primary care: } \\
\hline Orthopedic surgery. . . . . . . . . & 5,931 & 6.5 & 5.6 \\
\hline Dermatology. . . . . . . . . . & 3,061 & 3.3 & 4.2 \\
\hline Otolaryngology . . . . . . . . & 2,774 & 3.0 & 3.1 \\
\hline General surgery . . . . . . . . & 1,875 & 2.0 & 3.0 \\
\hline Urological surgery . . . . . . . . & 1,533 & 1.7 & 1.2 \\
\hline Cardiovascular diseases . . . . . . & 1,282 & 1.4 & 0.9 \\
\hline Ophthalmology . . . . . . . . . . & 1,067 & 1.2 & 4.1 \\
\hline Psychlatry . . . . . . . . . & 1,340 & 1.5 & 3.2 \\
\hline Neurology . . . . . . . . . . . & 481 & $* 0.5$ & 1.2 \\
\hline All other specialtles $\ldots \ldots \ldots \ldots$ & 8,540 & 9.3 & 11.6 \\
\hline
\end{tabular}

nonprepaid visits from 8 percent in 1985 to 12 percent in 1991. Sixty-two percent of the nonprepaid visits were to primary care physicians.

One possible explanation for the increasing percent of prepaid plan visits to nonprimary care specialties is the increased use of network models and point-of-service/open-ended plans in 1991 as compared with traditional HMO's, which dominated the prepaid plan market in $1985(11,12)$. Traditional HMO's limit visits to specialists by requiring the patient to obtain a referral by their primary care specialist whereas the requirements for a patient enrolled in an open-ended plan are not as stringent.

Table 3 shows that the geographical distribution of prepaid plan visits and nonprepaid visits differ. The West accounted for the largest proportion of prepaid plan visits ( 33.0 percent) while the South accounted for the smallest proportion (17.9 percent). The distribution of nonprepaid visits was the 
Table 3. Number and percent distribution of prepaid plan visits and percent distribution of nonprepald visits by geographical reglon: United States, 1991

\begin{tabular}{|c|c|c|c|}
\hline \multirow[b]{2}{*}{ Region } & \multicolumn{2}{|c|}{ Prepald plan visits } & Nonprepaid visits \\
\hline & $\begin{array}{c}\text { Number of } \\
\text { visits in } \\
\text { thousands }\end{array}$ & $\begin{array}{c}\text { Percent } \\
\text { distribution }\end{array}$ & $\begin{array}{c}\text { Percent } \\
\text { distribution }\end{array}$ \\
\hline All visits $\ldots \ldots \ldots \ldots$ & 91,824 & 100.0 & 100.0 \\
\hline Northeast . . . . . . . & 19,199 & 20.9 & 24.2 \\
\hline Midwest . . . . . . . . . & 25,930 & 28.2 & 24.0 \\
\hline South . . . . . . . . & 16,420 & 17.9 & 31.1 \\
\hline West . . . . . . . . . . & 30,276 & 33.0 & 20.8 \\
\hline
\end{tabular}

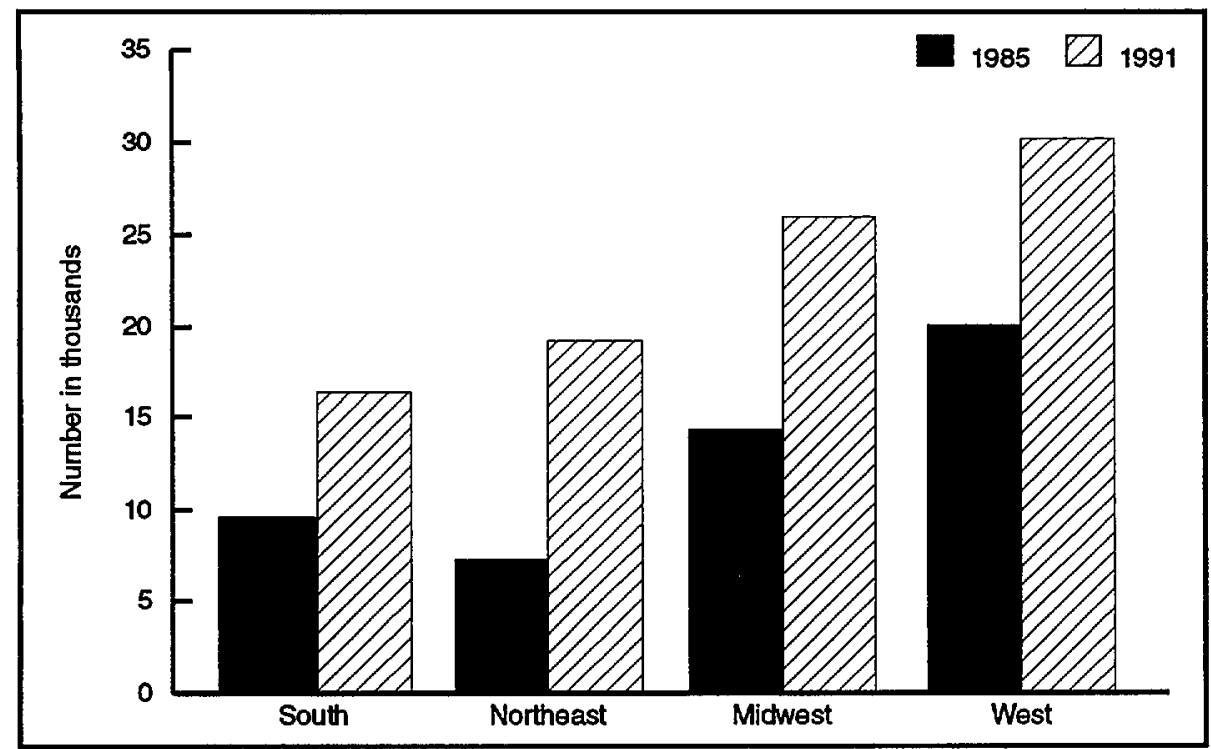

Figure 2. Number of prepaid plan visits, by geographical region: United States, 1985 and 1991

Table 4. Number and percent distribution of prepald plan visits and percent distribution of nonprepald visits by patient's age and sex: United States, 1991

\begin{tabular}{|c|c|c|c|}
\hline \multirow[b]{2}{*}{$A g e$ and sex } & \multicolumn{2}{|c|}{ Propald plan vishts } & \multirow{2}{*}{$\begin{array}{c}\text { Nonprepald visits } \\
\text { Percent } \\
\text { distribution }\end{array}$} \\
\hline & $\begin{array}{l}\text { Number of } \\
\text { visits in } \\
\text { thousands }\end{array}$ & $\begin{array}{c}\text { Percent } \\
\text { dlstribution }\end{array}$ & \\
\hline$\ldots \ldots \ldots \ldots \ldots$ & 91,824 & 100.0 & 100.0 \\
\hline Under 15 years $\ldots \ldots \ldots \ldots \ldots \ldots$ & 24,058 & 26.2 & 24.4 \\
\hline 15-24 yoars $\ldots \ldots \ldots \ldots \ldots \ldots \ldots$ & 11,009 & 12.0 & 12.1 \\
\hline $25-44$ years $\ldots \ldots \ldots \ldots \ldots \ldots$ & 36,659 & 39.9 & 35.4 \\
\hline $45-64$ years $\ldots \ldots \ldots \ldots \ldots \ldots$ & 20,099 & 21.9 & 28.1 \\
\hline Male $\ldots \ldots \ldots \ldots \ldots \ldots \ldots$ & 37,226 & 40.5 & 40.2 \\
\hline Under 15 years $\ldots \ldots \ldots \ldots \ldots \ldots$ & 12,964 & 14.1 & 12.5 \\
\hline 15-24 years $\ldots \ldots \ldots \ldots \ldots \ldots$ & 3,588 & 3.9 & 4.2 \\
\hline $25-44$ years $\ldots \ldots \ldots \ldots \ldots \ldots$ & 12,235 & 13.3 & 12.0 \\
\hline 45-64 years $\ldots \ldots \ldots \ldots \ldots \ldots$ & 8,439 & 9.2 & 11.5 \\
\hline Female. & 54,599 & 59.5 & 59.8 \\
\hline Under 15 years $\ldots \ldots \ldots \ldots \ldots$ & 11,094 & 12.1 & 11.9 \\
\hline 15-24 years . . . . . . . . . . & 7,421 & 8.1 & 8.0 \\
\hline $25-44$ years $\ldots \ldots \ldots \ldots \ldots \ldots$ & 24,424 & 26.6 & 23.3 \\
\hline $45-64$ years $\ldots \ldots \ldots \ldots \ldots \ldots \ldots$ & 11,660 & 12.7 & 16.6 \\
\hline
\end{tabular}

reverse, with 31.1 percent occurring in the South and 20.8 percent in the West. Comparing the geographical distribution of prepaid plan visits over time, NAMCS data show that in 1985 the West had the largest number of prepaid plan visits (20.1 million visits) and the Northeast had the smallest number of prepaid plan visits (7.4 million visits) (figure 2).

\section{Patient characteristics}

Approximately two-fifths (39.9 percent) of prepaid plan visits were made by patients $25-44$ years of age, and about one-quarter ( 26.2 percent) were made by patients under 15 years of age. This pattern holds true for visits for nonprepaid sources of payment as well (table 4). However, patients 25-44 years of age made up a relatively larger portion of prepaid plan visits compared with nonprepaid visits (39.9 vs. 35.4 percent). Correspondingly, patients 45-64 years of age made up a relatively smaller portion of prepaid plan visits compared with nonprepaid visits ( 21.9 vs. 28.1 percent). The majority of visits by persons with both prepaid and nonprepaid sources of payment were made by females, who accounted for a higher percent of visits than males in all age categories except under 15 years.

As shown in table 5, the majority of prepaid plan visits were made by white persons ( 80.2 percent). Black persons made 15.7 percent of these visits, with all other races accounting for the remaining 4.0 percent. In all race categories, females made a higher percent of visits than did males. A greater proportion of office-based prepaid plan visits were made by black persons ( 15.7 percent) compared with office-based nonprepaid visits (8.5 percent). However, data from 1992 show that the percent of office-based visits by black persons were similar for prepaid and nonprepaid visits based on that year (see Discussion section).

\section{Visit characteristics}

Item 7 on the Patient Record form asks if the patient was referred by another physician for this visit. In 
Table 5. Number and percent distribution of prepaid plan visits and percent distribution of nonpropald visits by pationt's race and sex: United States, 1991

\begin{tabular}{|c|c|c|c|}
\hline \multirow[b]{2}{*}{ Race and sex } & \multicolumn{2}{|c|}{ Prepaid plan vishts } & \multirow{2}{*}{$\begin{array}{c}\text { Nonprepaid vishts } \\
\text { Percent } \\
\text { distribution }\end{array}$} \\
\hline & $\begin{array}{l}\text { Number of } \\
\text { visits in } \\
\text { thousands }\end{array}$ & $\begin{array}{c}\text { Percent } \\
\text { distribution }\end{array}$ & \\
\hline Total visits $\ldots \ldots \ldots \ldots \ldots \ldots$ & 91,824 & 100.0 & 100.0 \\
\hline Black . . . . . . . . . . . & 14,462 & 15.7 & 8.5 \\
\hline 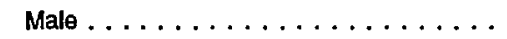 & 5,176 & 5.6 & 3.1 \\
\hline Female $\ldots \ldots \ldots \ldots \ldots \ldots$ & 9,286 & 10.1 & 5.4 \\
\hline White $\ldots \ldots \ldots \ldots \ldots \ldots$ & 73,687 & 80.2 & 87.7 \\
\hline Male $\ldots \ldots \ldots \ldots \ldots \ldots$ & 30,596 & 33.3 & 35.5 \\
\hline 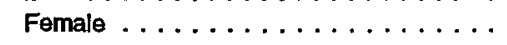 & 43,091 & 46.9 & 52.2 \\
\hline Other ${ }^{-1}$ & 3,675 & 4.0 & 3.8 \\
\hline Male $\ldots \ldots \ldots \ldots \ldots \ldots \ldots$ & 1,454 & 1.6 & 1.6 \\
\hline Female $\ldots \ldots \ldots \ldots \ldots \ldots \ldots$ & 2,222 & 2.4 & 2.2 \\
\hline
\end{tabular}

Iincludes Asian/Pacific Islander and American Indian/Eskimo/Alout.

8.2 percent of prepaid plan visits the patient had been referred compared with 6.0 percent of the nonprepaid visits (table 6). There was a significant difference in the referral rate for primary care physicians compared with other specialists. Patients were referred in 3.4 percent of the prepaid plan visits for primary care physicians but were referred in 19.2 percent of visits for other specialists. Note that only 11.5 percent of the nonprepaid visits to other specialists were referrals.

The majority of prepaid plan visits were made by patients who had seen the physician previously for the same problem (54.9 percent). In addition, over one-quarter of the prepaid plan visits were made by "old patients" with a new problem (28.6 percent). New patients made up less than one-fifth of the visits (16.5 percent). These patterns were also observed for nonprepaid visits. However, a higher proportion of prepaid than nonprepaid visits by new persons were referrals ( 38.8 and 26.5 percent, respectively).

Patients that had an expected source of payment of a prepaid plan were less likely to smoke cigarettes compared with those patients that had another expected source of payment. As shown in table 7, about two-thirds of the visits with a prepaid plan source were made by patients who did not smoke cigarettes (68.5 percent), 8.4 percent were by patients who did smoke. In comparison, one-tenth of nonprepaid visits were made by patients who smoke (11.7 percent) and three-fifths were made by patients who did not smoke (62.1 percent). Smoking status of the patient was not specified for about one-quarter of the visits.
Prepaid plan visits were less likely to be associated with injuries compared with nonprepaid visits (table 7). Over all age groups, about 9 out of every 100 prepaid plan visits were injury related (9.4 percent) compared with 12 out of every 100 for nonprepaid visits (12.2 percent). The only age group to show a statistically significant difference, however, was for persons 25-44-years-old. For persons in this age group, nonprepaid visits were 50 percent more likely to be injury related compared with prepaid plan visits.

Item 10 of the Patient Record form asks for the patient's (or patient's surrogate) "complaint(s), symptom(s), or other reason(s) for this visit (In patient's own words)." Up to three reasons for visit are coded and classified according to $A$ Reason for Visit Classification for Ambulatory Care (RVC) (13). The principal reason is the problem, complaint, or reason listed on item 10a of the Patient Record form.

Approximately 61.0 percent of prepaid plan visits were classified in the symptom module of the RVC, one of the eight modules that makes up the classification (table 8). Of these symptoms, 14.0 percent were classifiable to the respiratory system and 11.2 percent were classifiable to the musculoskeletal system. Nonprepaid visits followed the same pattern.

Table 9 shows the 20 most frequently mentioned principal reasons for visit, accounting for almost 47 percent of the prepaid plan visits. Note that estimates that differ in ranked order may not be significantly different

Table 6. Number, percent distribution, and percent of visits referred by another physiclan by physican specialty and visit status for propaid plan and nonprepaid visits, United States, 1991

\begin{tabular}{|c|c|c|c|c|c|c|}
\hline \multirow[b]{2}{*}{ Vsit characteristias } & \multicolumn{4}{|c|}{ Propald plan vistis } & \multicolumn{2}{|c|}{ Nonprepaid vistis } \\
\hline & $\begin{array}{l}\text { Number } \\
\text { of vistts }\end{array}$ & $\begin{array}{c}\text { Percent } \\
\text { dfstrlbution }\end{array}$ & $\begin{array}{l}\text { Number } \\
\text { of vishts } \\
\text { reterred }\end{array}$ & $\begin{array}{l}\text { Percent } \\
\text { referted }\end{array}$ & $\begin{array}{c}\text { Percemt } \\
\text { distribution }\end{array}$ & $\begin{array}{l}\text { Percent } \\
\text { referred }\end{array}$ \\
\hline All visits $\ldots \ldots \ldots \ldots \ldots \ldots \ldots$ & 91,824 & 100.0 & 7,505 & 8.2 & 100.0 & 6.0 \\
\hline Primary care ${ }^{1} \ldots \ldots \ldots \ldots \ldots \ldots \ldots$ & 63,941 & 69.6 & 2,151 & 3.4 & 61.7 & 2.5 \\
\hline Other specialties $\ldots \ldots \ldots \ldots \ldots \ldots \ldots$ & 27,884 & 30.4 & 5,354 & 19.2 & 38.3 & 11.5 \\
\hline Now patlent $\ldots \ldots \ldots \ldots \ldots \ldots \ldots \ldots$ & 15,111 & 16.5 & 5,860 & 38.8 & 18.8 & 26.5 \\
\hline Old patient-new problem $\ldots \ldots \ldots \ldots \ldots$ & 26,277 & 28.6 & 1,644 & 6.3 & 22.5 & 4.5 \\
\hline Old patlent-old problem ${ }^{2} \ldots \ldots \ldots \ldots \ldots$ & 50,436 & 54.9 & 0.0 & 0.0 & 58.7 & 0.0 \\
\hline
\end{tabular}

1Primary care spocialtios include general and family practico, internal modicine, pediatrics, and obstetrics/gynecology.

2Survey edit specifications did not allow reforrals for old pationt-old problem cases. 
Table 7. Number and percent distrlbution of prepald plan visits and percent distribution of nonprepaid visits by patient's cigarette-smoking status and whether vlsit is injury related: United States, 1991

\begin{tabular}{|c|c|c|c|}
\hline \multirow[b]{2}{*}{ Visit characteristios } & \multicolumn{2}{|c|}{ Prepald plan vistts } & Nonprepald visits \\
\hline & $\begin{array}{c}\text { Number of } \\
\text { visits in } \\
\text { thousands }\end{array}$ & $\begin{array}{c}\text { Percent } \\
\text { distribution }\end{array}$ & $\begin{array}{c}\text { Percent } \\
\text { distribution }\end{array}$ \\
\hline All visits ... & 91,824 & 100.0 & 100.0 \\
\hline \multicolumn{4}{|l|}{ Does patient smoke cigareltes? } \\
\hline Yes... & 7,709 & 8.4 & 11.7 \\
\hline No $\ldots \ldots \ldots \ldots \ldots$ & 62,878 & 68.5 & 62.1 \\
\hline Unknown $\ldots \ldots \ldots \ldots \ldots \ldots$ & 21,238 & 23.1 & 26.2 \\
\hline \multicolumn{4}{|l|}{ Is visit Injury related? } \\
\hline Yes $\ldots \ldots \ldots \ldots \ldots \ldots$ & 8,613 & 9.4 & 12.2 \\
\hline No $\ldots \ldots \ldots \ldots \ldots \ldots \ldots$ & 83,212 & 90.6 & 87.8 \\
\hline
\end{tabular}

from each other. A general medical exam, accounting for about 5.2 million visits, or 5.7 percent, was most frequently mentioned at prepaid plan visits. Cough ( 4.5 percent), symptoms referable to throat ( 3.7 percent), and prenatal examination ( 3.3 percent) followed, all similar to the top principal reasons for visits from other expected payment sources.

Data on the principal diagnoses rendered by physicians are shown in tables 10 and 11. The principal diagnosis is the first-recorded diagnosis in item 11 of the Patient Record form and is associated with the principal reason for visit as recorded in item 10a. The principal diagnosis was coded and classified according to the International Classification of Diseases, 9 th Revision, Clinical Modification (ICD-9-CM) (14). The ICD-9-CM is organized into broad categories, most relating to the major systems of the body as shown in table 10. Diseases of the respiratory system and the supplementary classification (for diagnoses that are not illness or injury related), each accounted for about 18.5 percent of prepaid plan visits, followed by injury and poisoning and diseases of the nervous system and sense organs each representing about 8.0 percent of the visits. Nonprepaid visits followed the same pattern.

Table 11 lists the 20 most frequently mentioned principal diagnoses rendered by the physician at the three-digit coding level of the ICD-9-CM. Approximately 40 percent of the visits are accounted for by the top 20 principal diagnoses. Health supervision of infant or child was the most frequent principal diagnosis, accounting for 4.9 percent of the prepaid plan visits. Health supervision of infant or child was followed by normal pregnancy and acute upper respiratory infections of multiple or unspecified sites, accounting for 4.5 and 3.4 percent, respectively. Comparing the principal diagnoses of the two types of visits, there is a difference in the percent of visits for health supervision of infant or child $(4.9$ vs. 3.0 percent). This diagnosis represented 18.8 percent of the prepaid plan visits for children under age 15 , which was significantly higher than the corresponding 12.5 percent of nonprepaid visits. However, this difference was not found in the 1992 NAMCS data (see Discussion section). On item 13 of the Patient Record form, the physician was asked to

Table B. Number and percent distribution of prepaid plan visits and percent distribution of nonprepaid visits by patient's prineipal reason for visit: United States, 1991

\begin{tabular}{|c|c|c|c|}
\hline \multirow[b]{2}{*}{ Princlpal reason for vistt module and RVC code ${ }^{1}$} & \multicolumn{2}{|c|}{ Prepald plan vistts } & \multirow{2}{*}{$\begin{array}{c}\text { Nonprepaid visits } \\
\text { Percent } \\
\text { distribution }\end{array}$} \\
\hline & $\begin{array}{l}\text { Number of } \\
\text { vislts in } \\
\text { thousands }\end{array}$ & $\begin{array}{c}\text { Percent } \\
\text { distribution }\end{array}$ & \\
\hline All visits & 91,824 & 100.0 & 100.0 \\
\hline$\ldots \ldots \ldots \ldots \ldots \ldots \ldots \ldots \ldots \ldots \ldots \ldots \ldots$. . . . . . . . & 55,980 & 61.0 & 59.4 \\
\hline General symptoms . . . . . . . . . . . . . . . . . . . . . s001-s099 & 6,356 & 6.9 & 6.9 \\
\hline Symptoms referable to psychological and mental dlsorders . . . . . . . S100-S199 & 1,798 & 2.0 & 3.3 \\
\hline Symploms reterable to the nervous system (excluding sense organs) . . . . S200-S259 & 2,923 & 3.2 & 3.1 \\
\hline Symploms reterable to the cardlovascular and lymphatic system . . . . . S260-\$299 & "487 & *0.5 & 0.4 \\
\hline Symploms referable to the eyes and ears . . . . . . . . . . . . . s300-s399 & 6,046 & 6.6 & 6.5 \\
\hline Symptoms referabie to the respiratory system $\ldots \ldots \ldots \ldots \ldots \ldots$ S400-S499 & 12,866 & 14.0 & 12.4 \\
\hline Symptoms referable to the digestive system. . . . . . . . . . . . S500-\$639 & 3,500 & 3.8 & 4.0 \\
\hline Symptoms referable to the genitourinary system . . . . . . . . . S640-\$829 & 5,340 & 5.8 & 4.6 \\
\hline Systems referable to the skin, nails, and hair . . . . . . . . . . . . S830-\$8999 & 6,337 & 6.9 & 6.6 \\
\hline Symptoms referable to the musculoskeletal system . . . . . . . . . . s900-s999 & 10,328 & 11.2 & 11.7 \\
\hline Disease module . . . . . . . . . . . . . . . . . . . . . . . . D001-D999 & 7,314 & 8.0 & 7.5 \\
\hline Dlagnostlc, screening, and preventive module . . . . . . . . . . . X100-X599 & 15,593 & 17.0 & 16.0 \\
\hline Treatment module . . . . . . . . . . . . . . . . . . . . . . . . . T100-T899 & 7,102 & 7.7 & 8.6 \\
\hline Injury and adverse effects module . . . . . . . . . . . . . . . . J J001-J999 & 3,041 & 3.3 & 3.6 \\
\hline 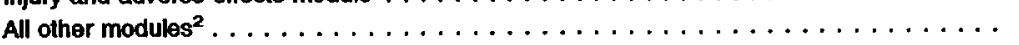 & 2,795 & 3.0 & 4.9 \\
\hline
\end{tabular}

'Based on A Rasen for Visit Claseification for Ambulatory Caro (RVC) (13).

Includes test results module, administrative module, uncodable and blank ontries. 
Table 9. Number, and percent distribution of prepaid plan visits and percent distribution of nonprepald visits by the $\mathbf{2 0}$ principal reasons for visit most frequently mentioned by patients: United States, 1991

\begin{tabular}{|c|c|c|c|}
\hline \multirow[b]{2}{*}{ Principal reason for vistt and RVC code' } & \multicolumn{2}{|c|}{ Prepald plan vistts } & \multirow{2}{*}{$\frac{\text { Nonprepald vists }}{\begin{array}{c}\text { Percent } \\
\text { distribution }\end{array}}$} \\
\hline & $\begin{array}{c}\text { Number of vists } \\
\text { in thousands }\end{array}$ & $\begin{array}{c}\text { Percent } \\
\text { distribution }\end{array}$ & \\
\hline All visits $\ldots \ldots \ldots \ldots \ldots \ldots \ldots \ldots \ldots$ & 91,824 & 100.0 & 100.0 \\
\hline General medical examination . . . . . . . X100 & 5,247 & 5.7 & 4.3 \\
\hline Cough $\ldots \ldots \ldots \ldots \ldots \ldots \ldots \ldots \ldots$ \$440 & 4,158 & 4.5 & 3.9 \\
\hline Symptoms referable to throat $\ldots \ldots \ldots \ldots \ldots$ S455 & 3,377 & 3.7 & 3.2 \\
\hline Prenatal examination, routine $\ldots \ldots \ldots \ldots \ldots$ X205 & 3,064 & $\mathbf{3 . 3}$ & 3.9 \\
\hline Well-baby examination $\ldots \ldots \ldots \ldots \ldots \ldots$ X105 & 2,966 & 3.2 & 2.4 \\
\hline Progress visit . . . . . . . . . . . . . . . .T800 & 2,528 & 2.8 & 2.8 \\
\hline Earache or ear infectlon . . . . . . . . \$355 & 2,511 & 2.7 & 2.4 \\
\hline Skin rash. . . . . . . . . . . . . . . . . . . . . S8860 & 2,175 & 2.4 & 1.9 \\
\hline Knev symptoms . . . . . . . . . . . . . . . . . S925 & 1,839 & 2.0 & 1.2 \\
\hline Postoperative visit . . . . . . . . . . . . . . . . . . .T205 & 1,777 & 1.9 & 2.0 \\
\hline Stomach pain, cramps, and spasms . . . . . S545 & 1,776 & 1.9 & 1.6 \\
\hline 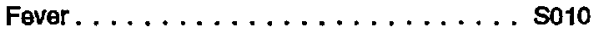 & 1,726 & 1.9 & 2.0 \\
\hline Headache, pain in head . . . . . . . . . . . S210 & 1,703 & 1.9 & 1.7 \\
\hline Nasal congestion. . . . . . . . . . . . . . . s400 & 1,514 & 1.6 & 1.5 \\
\hline Head cold, upper resplratory infection . . . . . S445 & 1,283 & 1.4 & 1.3 \\
\hline 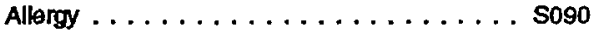 & 1,277 & 1.4 & 0.8 \\
\hline Back symptoms. . . . . . . . . . . . . . . . . s905 & 1,223 & 1.3 & 2.0 \\
\hline 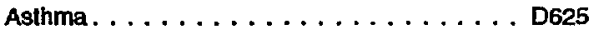 & 1,000 & 1.1 & 0.4 \\
\hline Shoulder symptoms . . . . . . . . . . . . . . . S940 & 992 & 1.1 & 1.0 \\
\hline Sinus problems. . . . . . . . . . . . . . S410 & 933 & 1.0 & 0.7 \\
\hline All other reasons. . & 48,755 & 53.2 & 59.0 \\
\hline
\end{tabular}

'Based on A Aouson for Visit Classifiantion for Ambelatory Gars (AVC) (13).

indicate if the patient, at the time of visit, was afflicted with any of the chronic conditions listed, despite what was reported as the patient diagnosis in item 11. Obesity and hypertension were checked most frequently, at 7.8 and
6.5 percent of prepaid plan visits, respectively. In contrast, nonprepaid visits recorded a significantly higher percent of visits by patients suffering from depression, 6.3 percent, as compared with 4.7 percent for prepaid plans (table 12). However, when examining the differences at various age groups, only the patients between the ages of 15 and 24 years were more likely to have depression in the nonprepaid visits ( 3.5 percent) compared with prepaid plan visits (1.0 percent). Past analysis of this question has shown that physicians seem to underreport chronic conditions as diagnoses on item 11 of the Patient Record form (15). The same would hold true for visits to prepaid and nonprepaid sources of payment.

Diagnostic services performed or ordered at the time of visit are shown in table 13. The most common service recorded at prepaid plan visits was a blood pressure test, 43.0 percent. A urinalysis was performed or ordered in 13.9 percent while "all other diagnostic services" accounted for 34.7 percent. Diagnostic services utilization rates in nonprepaid visits followed the same pattern (table 14). Looking at age

Table 10. Number and percent distribution of prepaid plan visits and percent dlstribution of nonprepaid visits by principal diagnosis: United States, 1991

\begin{tabular}{|c|c|c|c|}
\hline \multirow[b]{2}{*}{ Princlpal dkagnosis and ICD-g-CM code' } & \multicolumn{2}{|c|}{ Propald pkan vksits } & \multirow{2}{*}{ 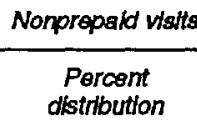 } \\
\hline & $\begin{array}{c}\text { Number of visits } \\
\text { in thousands }\end{array}$ & $\begin{array}{c}\text { Percent } \\
\text { dkstribution }\end{array}$ & \\
\hline 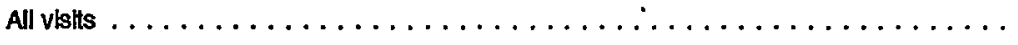 & 91,824 & 100.0 & 100.0 \\
\hline Infectlous and parasitic diseases . . . . . . . . . . . . . . . . . . . . . 001-139 & 4,476 & 4.9 & 4.2 \\
\hline $\begin{array}{l}\text { Neoplasms. } \ldots \ldots \ldots \ldots \ldots \ldots \ldots \ldots \ldots \ldots \ldots \ldots \ldots \ldots \ldots \ldots \ldots \\
\text { Endocrine, nutrilional, and metabolic }\end{array}$ & 2,438 & 2.7 & 2.5 \\
\hline diseases and immunity disorders . . . . . . . . . . . . . . . . . . . . . . . 240-279 & 2,664 & 2.9 & 3.3 \\
\hline Mental disorders . . . . . . . . . . . . . . . . . . . . . . . . . . . . . . . 290-319 & 2,880 & 3.1 & 4.8 \\
\hline Dlseases of the nervous system and sense organs. . . . . . . . . . . . . . . 320-389 & 7,445 & 8.1 & 10.7 \\
\hline Diseases of the circulatory system . . . . . . . . . . . . . . . . . . . . . . . . 390-459 & 3,577 & 3.9 & 4.3 \\
\hline Dlseases of the respiratory system. . . . . . . . . . . . . . . . . . . . . 460-519 & 16,977 & 18.5 & 14.9 \\
\hline Dlseases of the dlgestlve system. . . . . . . . . . . . . . . . . . . . . . . 520-579 & 2,673 & 2.9 & 3.3 \\
\hline Diseases of the genitourinary system . . . . . . . . . . . . . . . . . . 580-629 & 5,486 & 6.0 & 6.0 \\
\hline Diseases of the skin and subcutaneous tissue. . . . . . . . . . . . . . . . . 680-709 & 5,320 & 5.8 & 6.0 \\
\hline Dlseases of the musculoskeletal system and connective tissue . . . . . . . . . 710-739 & 6,608 & 7.2 & 6.5 \\
\hline Symptoms, slgns, and Ill-defined condltlons . . . . . . . . . . . . . . . 780-799 & 3,235 & 3.5 & 3.7 \\
\hline Injury and polsoning . . . . . . . . . . . . . . . . . . . . . . . . . . . . 800-999 & 7,870 & 8.6 & 9.3 \\
\hline Supplementany classlfication. . . . . . . . . . . . . . . . . . vo1-v82 & 16,839 & 18.3 & 17.5 \\
\hline 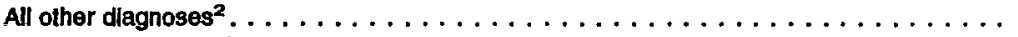 & 1,700 & 1.9 & 1.4 \\
\hline 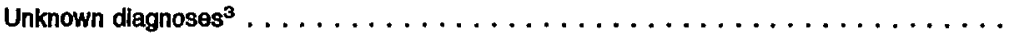 & 1,639 & 1.8 & 1.7 \\
\hline
\end{tabular}

${ }^{1}$ Based on the Intomational Classification of Diseases, 9th Rovision, Clinical Modification (ICD-8-CM) (14).

Includes diseases of the blood-forming organs (280-289); complications of pregnancy, childbirth, and the puerperium (630-676); congenital anomalies (740-759); and cortain conditions originating in the perinatal period $(760-779)$.

includes blank diagnoses, uncodable diagnoses, and illogible diagnoses. 
Table 11. Number and percent of propald plan visits by 20 principal dlagnoses most frequently rendered by physiclans: United States, 1991

\begin{tabular}{|c|c|c|c|}
\hline \multirow[b]{2}{*}{ Principal dlagnosls and $1 C D-9-C M$ code $e^{\dagger}$} & \multicolumn{2}{|c|}{ Prepald plan vishts } & \multirow{2}{*}{$\begin{array}{c}\text { Nonprepald visits } \\
\text { Percent } \\
\text { distribution }\end{array}$} \\
\hline & $\begin{array}{l}\text { Number of } \\
\text { vistts In } \\
\text { thousands }\end{array}$ & $\begin{array}{l}\text { Percent } \\
\text { distribution }\end{array}$ & \\
\hline 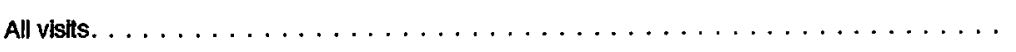 & 91,824 & 100.0 & 100.0 \\
\hline Health supervision of infant or child $\ldots \ldots \ldots \ldots \ldots \ldots \ldots \ldots \ldots$ & 4,531 & 4.9 & 3.0 \\
\hline 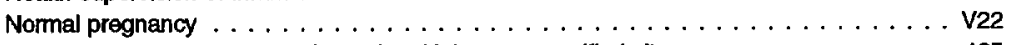 & 4,091 & 4.5 & 3.9 \\
\hline Acute upper respiratory infections of multiple or unspeclfled siles. . . . . . . . . . . . 465 & 3,160 & 3.4 & 3.0 \\
\hline Suppurative and unspeclfied ottis media $\ldots \ldots \ldots \ldots \ldots \ldots \ldots \ldots$ & 2,917 & 3.2 & 3.0 \\
\hline Allergic rhlnitis . . . . . . . . . . . . . . . . . . . . . . . 477 & 2,670 & 2.9 & 1.5 \\
\hline 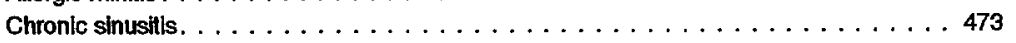 & 2,659 & 2.9 & 1.9 \\
\hline General medical examination . . . . . . . . . . . . . . . . . . . . V70 & 2,404 & 2.6 & 3.5 \\
\hline Essentlal hypertension . . . . . . . . . . . . . . . . . . . . . . . . . 401 & 2,150 & 2.3 & 2.3 \\
\hline 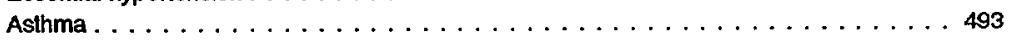 & 1,983 & 2.2 & 1.3 \\
\hline 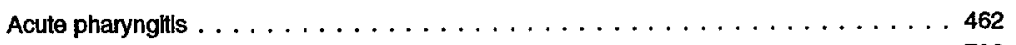 & 1,802 & 2.0 & 2.1 \\
\hline 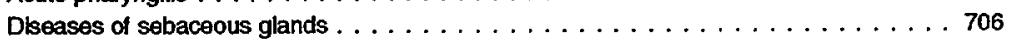 & 1,496 & 1.6 & 1.7 \\
\hline Bronchitis, not speclfied as acule or chronic . . . . . . . . . . . . . . . . 490 & 1,392 & 1.5 & 1.6 \\
\hline Contact dermatitis and other eczema $\ldots \ldots \ldots \ldots \ldots \ldots$ & 1,221 & 1.3 & 1.1 \\
\hline Dlabetes melltus . . . . . . . . . . . . . . . . . . . . 250 & 1,098 & 1.2 & 1.2 \\
\hline Other dlsorders of synovium, tendon, and bursa. $\ldots \ldots \ldots \ldots \ldots \ldots \ldots$ & 989 & 1.1 & 0.6 \\
\hline 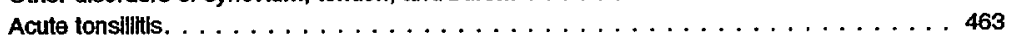 & 940 & 1.0 & 0.7 \\
\hline Certain adverse effects not elsewhere classified. . . . . . . . . . . . . . . . 995 & 930 & 1.0 & 0.5 \\
\hline Peripheral enthesopathies and allied syndromes. . . . . . . . . . . . . . 726 & 907 & 1.0 & 0.8 \\
\hline Spralns and strains of other.and unspecifled parts of back. . . . . . . . . . . 847 & 846 & 0.9 & 1.1 \\
\hline Personal history of certain other diseases $\ldots \ldots \ldots \ldots \ldots \ldots \ldots \ldots \ldots$ & 782 & 0.9 & 0.6 \\
\hline 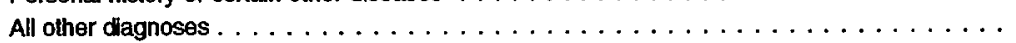 & 52,856 & 57.6 & 64.6 \\
\hline
\end{tabular}

'Based on the international Classification of Diseases, 9th Revision, Clinical Modification (ICD-9-CM) (14).

differences, children under 15 years of age were less likely to receive diagnostic tests compared with older age groups in both types of visits (40 vs. 72 percent for prepaid plan visits and 37 vs. 65 percent in nonprepaid visits). Persons 45-64 years of age were more likely to receive diagnostic tests in prepaid plan visits compared with nonprepaid visits (76 vs. 70 percent, respectively).

Visits at which at least one nonmedication therapeutic service was ordered or provided represented one-third of the total prepaid plan visits, as shown in table 15 . The most frequently checked therapeutic service was diet counseling/education, reported at 10.7 percent of the visits. Exercise and growth development counseling/ education followed with percents of 8.6 and 3.8, respectively. "All other therapeutic services" ordered or provided accounted for 14.8 percent of the visits. No significant differences were found between prepaid and nonprepaid visits.

The majority of both types of visits were drug visits in which the patient was given, prescribed, and/or continued on at least one medication (table 16).

Table 12. Number and percent of prepaid plan visits and percent of nonprepaid visits by selected medical conditions: United States, 1991

\begin{tabular}{|c|c|c|c|}
\hline \multirow[b]{2}{*}{ Medical condition ${ }^{1} .2$} & \multicolumn{2}{|c|}{ Prepaid plan vistts } & \multirow[t]{2}{*}{ Nonprepaid visits } \\
\hline & $\begin{array}{l}\text { Number of } \\
\text { visits in } \\
\text { thousands }\end{array}$ & Percent & \\
\hline All visits & 91,824 & $\cdots$ & $\cdots$ \\
\hline Depression & 4,298 & 4.7 & 6.3 \\
\hline Hypertension $\ldots \ldots \ldots \ldots \ldots \ldots$ & 5,987 & 6.5 & 7.0 \\
\hline Hypercholesterolemla . . . . . . . . . . . & 3,791 & 4.1 & 3.4 \\
\hline Obesity . . . . . . . . . . . & 7,194 & 7.8 & 7.5 \\
\hline
\end{tabular}

iRefers to question 13 on the Patient Record form.

${ }^{2}$ Numbers do not add to totals because more than one medical condition may be reported per visit and not all categories are shown.

Physicians were asked to record all new or continued medications provided at the visit, including prescription and nonprescription preparations and immunizing and desensitizing agents. About one-third of both prepaid and nonprepaid visits included only one drug mention (35.4 and 34.6 percent, respectively). The drugs entered on item 17 of the Patient Record form are classified based on the therapeutic categories used in the National Drug Code Directory, 1985 edition (16). The reader should understand that some drugs have more than one therapeutic application and in these cases, each drug was assigned to the category that occurred with the greatest frequency.

As shown in table 17, antimicrobial agents represented the largest share of the 95.1 million drug mentions in prepaid plan visits, 21.2 percent. Of these, penicillins were the largest group (7.9 percent). Of the drug mentions, 14.7 percent were respiratory tract drugs, and drugs used for relief of pain accounted for 12 percent. Two significant differences were found between the prepaid and nonprepaid 
visits in the distribution of drug mentions by therapeutic class.

Psychopharmacologic drugs represented a larger percent of drug mentions at nonprepaid visits compared with prepaid plan visits (7.0 percent and 4.5 percent, respectively). This strengthened the earlier finding that physicians involved in nonprepaid visits recorded a higher percent of visits with a diagnosis of depression than did those involved with prepaid plan visits (item 13 ). The only age group to show a significant difference, however, was the 25-44 years group. On the other hand, respiratory tract drugs represented a larger portion of drug mentions at prepaid plan visits compared with nonprepaid visits for persons in this same age group (15.2 vs. 9.9 percent).

Table 18 shows the most frequently occurring generic ingredients of the drug mentions at prepaid plans visits during 1991. Note that drug products containing more than one ingredient are included in the data for each ingredient. For example, acetaminophen with codeine is included in both the count for acetaminophen and the count for codeine. Amoxicillin was the most frequently occurring generic ingredient, with 7.1 million mentions; it represented 7.4 percent of the total. The second and third listed generic ingredients were acetaminophen and erythromycin representing 4.3 and 2.7 percent, respectively. A report describing the method and instructions used to collect and process drug information for the NAMCS is available (17).

More than one-half ( 57.7 percent) of prepaid plan visits resulted in instructions for the patient to return at a specific time (table 19), and about one-quarter of the visits resulted in instructions to return if needed (27.0 percent). These percents are not significantly different from the 1985 estimates for prepaid plan visits (55.3 percent and 26.6 percent, respectively) and follow the same pattern as the nonprepaid visits.

Table 19 also shows the duration of visit. Of the prepaid plan visits, 61.7 percent lasted between 6 and 15 minutes, 21.6 percent lasted 16 to 30
Table 13. Number and percent of prepaid plan visits and percent of nonprepaid visits by diagnostic and screening services ordered or provided: United States, 1991

\begin{tabular}{|c|c|c|c|}
\hline \multirow[b]{2}{*}{$\begin{array}{l}\text { Diagnostic and screening } \\
\text { services ordered or provided }\end{array}$} & \multicolumn{2}{|c|}{ Prepaid plan visits } & \multirow[t]{2}{*}{ Nonprepaid visits } \\
\hline & $\begin{array}{l}\text { Number of } \\
\text { visits in } \\
\text { thousands }\end{array}$ & Percent & \\
\hline Total visits $^{1} \ldots \ldots \ldots \ldots \ldots$ & 91,824 & $\cdots$ & $\cdots$ \\
\hline Blood pressure . . . . . . . . . . . & 39,485 & 43.0 & 39.9 \\
\hline Urinalysis $\ldots \ldots \ldots \ldots \ldots \ldots$ & 12,771 & 13.9 & 13.3 \\
\hline EKG resting $\ldots \ldots \ldots \ldots \ldots$ & 1,807 & 2.0 & 1.9 \\
\hline EKG exercise . . . . . . . . . . . . . & $* 197$ & "0.2 & 0.4 \\
\hline Mammogram . . . . . . . . . . & 1,636 & 1.8 & 1.7 \\
\hline Chest $x$ ray $\ldots \ldots \ldots \ldots \ldots$ & 1,740 & 1.9 & 1.8 \\
\hline Pap test $\ldots \ldots \ldots \ldots \ldots \ldots \ldots$ & 4,737 & 5.2 & 5.0 \\
\hline Strep throat test $\ldots \ldots \ldots \ldots \ldots \ldots$ & 2,710 & 3.0 & 2.5 \\
\hline Cholesterol measure. . . . . . . . . . . . & 3,389 & 3.7 & 3.2 \\
\hline Hearing test . . . . . . . . . . . . & 1,723 & 1.9 & 1.6 \\
\hline Visual acuity. . . . . . . . . . . . & 2,030 & 2.2 & 4.8 \\
\hline Mental status exam $\ldots \ldots \ldots \ldots \ldots$ & 1,254 & 1.4 & 1.6 \\
\hline All other dlagnostic services ${ }^{2} \ldots \ldots \ldots$ & 31,832 & 34.7 & 30.1 \\
\hline None . . . . . . . . . . . . . . . . . & 33,350 & 36.3 & 39.6 \\
\hline
\end{tabular}

1 Numbers do not add to totals because more than one service may be reported per visit.

Includes other radiology, allergy testing, spirometry, HIV serology, other lab tests, and other.

Table 14. Number and percent distribution of prepaid plan visits and percent distribution of nonprepaid visits by number of diagnostic services ordered or provided: United States, 1991

\begin{tabular}{|c|c|c|c|}
\hline \multirow[b]{2}{*}{$\begin{array}{c}\text { Number of diagnostic senvices } \\
\text { ordered or provided }\end{array}$} & \multicolumn{2}{|c|}{ Prepald plan visits } & \multirow[t]{2}{*}{ Nonprepaid vishts } \\
\hline & $\begin{array}{l}\text { Number of } \\
\text { vists in } \\
\text { thousands }\end{array}$ & $\begin{array}{c}\text { Percent } \\
\text { distribution }\end{array}$ & \\
\hline All visits $\ldots \ldots \ldots \ldots \ldots \ldots$ & 91,824 & 100.0 & 100.0 \\
\hline 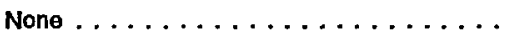 & 33,350 & 36.3 & 39.6 \\
\hline $1 \ldots \ldots \ldots \ldots \ldots \ldots \ldots$ & 31,754 & 34.6 & 32.1 \\
\hline 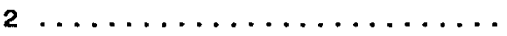 & 14,739 & 16.1 & 16.4 \\
\hline 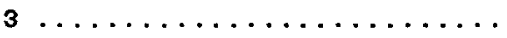 & 7,142 & 7.8 & 7.5 \\
\hline 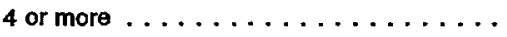 & 4,839 & 5.3 & 4.5 \\
\hline
\end{tabular}

Table 15. Number and percent of prepaid plan visits and percent of nonprepald visits by nonmedication therapy ordered or provided: Unitod States, 1991

\begin{tabular}{|c|c|c|c|}
\hline \multirow[b]{2}{*}{ Nonmedication therapy } & \multicolumn{2}{|c|}{ Prepald pkan visits } & \multirow[t]{2}{*}{ Nonprepaid vists } \\
\hline & $\begin{array}{c}\text { Number of } \\
\text { Wistis in } \\
\text { thousands }\end{array}$ & Percent & \\
\hline All visils ${ }^{1} \ldots \ldots \ldots \ldots \ldots \ldots$ & 91,824 & $\cdots$ & $\cdots$ \\
\hline Dlet $\ldots \ldots \ldots \ldots \ldots \ldots \ldots$ & 9,855 & 10.7 & 11.2 \\
\hline Exercise . . . . . . . . . . . . . & 7,888 & 8.6 & 8.1 \\
\hline Cholesterol reduction $\ldots \ldots \ldots \ldots \ldots \ldots$ & 2,620 & 2.9 & 2.5 \\
\hline Welght reduction . . . . . . . . . . . & 3,090 & 3.4 & 3.9 \\
\hline Alcohol abuse. . . . . . . . . . . . . & $\star 424$ & ${ }^{*} 0.5$ & 0.6 \\
\hline Smoking cessation. . . . . . . . . . . & 1,406 & 1.5 & 2.3 \\
\hline Family/social . . . . . . . . . . & 1,944 & 2.1 & 2.3 \\
\hline Growth development. . . . . . . . . . . . & 3,450 & 3.8 & 4.2 \\
\hline Family planning. . . . . . . . . . . . & 877 & 1.0 & 1.1 \\
\hline Psychotherapy ... . . . . . . . . & 1,644 & 1.8 & 3.4 \\
\hline Physiotherapy. . . . . . . . . . . . & 1,913 & 2.1 & 3.0 \\
\hline All other therapeutic services ${ }^{2} \ldots \ldots \ldots \ldots$ & 13,574 & 14.8 & 13.0 \\
\hline None . . . . . . . . . . . . . . . . & 61,495 & 67.0 & 65.2 \\
\hline
\end{tabular}

'Numbers do not add to totals because more than one type of nonmedication therapy may be reported per visit. ${ }^{2}$ Includes drug abuse, other counseling, corrective lenses, hearing aid, and other therapy. 
Table 16. Number and percent distribution of prepaid plan visits and percent distribution of nonprepaid vleits by number of medlcations provided or prescrlbed: United States, 1991

\begin{tabular}{|c|c|c|c|}
\hline \multirow[b]{2}{*}{ Medication therapy ${ }^{1}$} & \multicolumn{2}{|c|}{ Prepald plan vists } & \multirow{2}{*}{$\begin{array}{c}\text { Nonprepald visits } \\
\text { Percent } \\
\text { distribution }\end{array}$} \\
\hline & $\begin{array}{l}\text { Number of } \\
\text { visits in } \\
\text { thousands }\end{array}$ & $\begin{array}{c}\text { Percent } \\
\text { distribution }\end{array}$ & \\
\hline All visits & 91,824 & 100.0 & 100.0 \\
\hline \multicolumn{4}{|l|}{ Type of visit: } \\
\hline Nondrug visit (0 medlcations) . & 35,176 & 38.3 & 38.3 \\
\hline Drug visit ${ }^{2} \ldots \ldots \ldots \ldots$ & 56,648 & 61.7 & 61.7 \\
\hline \multicolumn{4}{|l|}{ Number of medications: } \\
\hline $1, \ldots \ldots \ldots \ldots \ldots \ldots \ldots \ldots \ldots \ldots$ & 32,514 & 35.4 & 34.6 \\
\hline $2 \ldots \ldots \ldots \ldots \ldots \ldots \ldots \ldots \ldots$ & 14,890 & 16.2 & 16.3 \\
\hline$\ldots \ldots \ldots \ldots \ldots \ldots$ & 9,244 & 10.1 & 10.9 \\
\hline
\end{tabular}

Includes prescription drugs, over-the-counter preparations, immunizing agents, and desensitizing agents.

${ }^{2}$ Visits at which one or more drugs were provided or prescribed by the physician.

Table 17. Number and percent distribution of prepaid plan visits and percent distribution of nonpropaid visits for drug mentions by therapeutic classification: United States, 1991

\begin{tabular}{|c|c|c|c|}
\hline \multirow[b]{2}{*}{ Therapeutic classification ${ }^{1}$} & \multicolumn{2}{|c|}{ Prepaid plan visits } & \multirow{2}{*}{$\begin{array}{c}\text { Nonprepaid vistts } \\
\text { Percent } \\
\text { dlstrlbution }\end{array}$} \\
\hline & $\begin{array}{l}\text { Number of } \\
\text { drug mentions } \\
\text { in thousands }\end{array}$ & $\begin{array}{c}\text { Percent } \\
\text { distribution }\end{array}$ & \\
\hline All drug mentions. $\ldots \ldots \ldots \ldots \ldots \ldots$ & 95,104 & 100.0 & 100.0 \\
\hline Antimlcroblal agents. & 20,191 & 21.2 & 18.8 \\
\hline Peniclitins $\ldots \ldots \ldots \ldots \ldots \ldots \ldots$ & 7,546 & 7.9 & 6.1 \\
\hline Cephalosporins $\ldots \ldots \ldots \ldots \ldots \ldots$ & 3,645 & 3.8 & 3.8 \\
\hline Enythromycins and lincosamides . . . . . . & 3,126 & 3.3 & 3.2 \\
\hline Cardlovascular-renal drugs. . . . . . . . . & 7,181 & 7.6 & 8.9 \\
\hline Psychopharmacologic drugs. . . . . . . . & 4,242 & 4.5 & 7.0 \\
\hline Radiopharmacouticals/contrast medla & 2,371 & 2.5 & 1.4 \\
\hline Gastrointestinal agents & 2,912 & 3.1 & 3.9 \\
\hline Metabolic and nutrlent agents $\ldots \ldots \ldots$ & 3,798 & 4.0 & 4.3 \\
\hline $\begin{array}{l}\text { Hormones and agents aflecting } \\
\text { hormonal mechanisms. . . . }\end{array}$ & 7,942 & 8.4 & 9.5 \\
\hline Immunologic agents $\ldots \ldots \ldots \ldots \ldots \ldots$ & 5,390 & 5.7 & 4.5 \\
\hline Skin/mucous membrane $\ldots \ldots \ldots \ldots \ldots$ & 6,024 & 6.3 & 6.7 \\
\hline Nourologic drugs $\ldots \ldots \ldots \ldots \ldots \ldots$ & 1,716 & 1.8 & 2.5 \\
\hline Ophthalmics. $\ldots \ldots \ldots \ldots \ldots \ldots \ldots$ & 1,750 & 1.8 & 2.6 \\
\hline Drugs used for relief of paln $\ldots$ & 11,187 & 11.8 & 10.9 \\
\hline General analgesics $\ldots \ldots \ldots \ldots \ldots$ & 5,384 & 5.7 & 5.5 \\
\hline Antiarthritics & 5,448 & 5.7 & 5.1 \\
\hline Respiratory tract drugs $\ldots \ldots \ldots \ldots \ldots$ & 13,956 & 14.7 & 11.3 \\
\hline Nasal decongestants $\ldots \ldots \ldots \ldots \ldots$ & 3,823 & 4.0 & 3.1 \\
\hline$\ldots \ldots \ldots \ldots \ldots$ & 3,600 & 3.8 & 2.4 \\
\hline Unclassified and miscellaneous. . . & 3,797 & 4.0 & 4.5 \\
\hline All others ${ }^{2} \ldots \ldots$ & 2,479 & 2.6 & 2.7 \\
\hline
\end{tabular}

'Based on the standard drug classification used in the National Drug Code Dinectory, 1985 edition (16).

Includes anesthetic drugs, antidotes, hematologic agents, oncolytics, ofologic drugs, and antiparasitic agents.

minutes. Visits with a duration of "zero" minutes are those in which there was no face-to-face contact between the patient and physician. In 1991, 1.7 percent of the visits had a duration of zero minutes. No significant difference was found between the average durations of prepaid and nonprepaid visits (16 minutes vs. 17 minutes, respectively).

\section{Discussion}

In describing the patient and visit characteristics of $\mathrm{HMO} / \mathrm{other}$ prepaid plan visits found in the 1991 NAMCS, it appears that such visits are generally similar to nonprepaid visits for patients under 65 years of age with respect to the principal reason for visit, physicians' diagnosis, medications prescribed, and duration of visit. This report focused only on visits made by patients under the age of 65 to reduce the confounding effects of age and health conditions on the characteristics examined. Prepaid visits were found to differ from nonprepaid visits as follows:

- relative to nonprepaid visits, a higher proportion of prepaid visits were to physicians in the primary care specialties

- HMO/other prepaid plans tend to have a higher proportion of visits with diagnostic tests performed or ordered but especially for persons between the ages of 45 and 64 years

- a higher proportion of prepaid plan visits to nonprimary care specialties were referrals

- a lower proportion of prepaid plan visits were for patients over 65 years of age

- a higher proportion of prepaid plan visits were in the West

The comparisons of visit and patient characteristics between prepaid plan and nonprepaid visits based on the 1991 NAMCS must be interpreted with caution. This report focused on describing characteristics of prepaid plan visits. For comparison purposes, the corresponding statistics for visits from other expected sources of payment were presented. However, nonprepaid visits are for a very diverse set of people with respect to expected sources of payment. For example, 12 percent of the nonprepaid visits had an expected source of payment identified as "Medicaid." Thirty-one percent were identified as "patient paid." Only 47 percent were identified as "private/ commercial." In comparing the statistics presented in this report, one must consider how the diversity of coverage in the nonprepaid group may influence prepaid and nonprepaid visit comparisons. The results should not be interpreted as a straight comparison between HMO/other prepaid plans and fee-for-service plans. The reader must also consider that this report focuses on 
describing patient and visit characteristics of prepaid plan visits to office-based physicians and does not represent characteristics of all visits by persons who are insured with a prepaid health plan. The variable that differentiates the two comparison groups in this report is an expected source of payment for the visit. Persons insured in an HMO may pay out-of-pocket expenses to seek health care from a provider other than the HMO to obtain either noncovered health care or care from a provider that is not associated with the prepaid plan.

This report does not include all possible providers of physician services. Physicians in hospital-based practices are not in-scope for the NAMCS, therefore, hospital-based managed care offices may not be included if the physician indicated that he/she was employed by a hospital. Similarly, visits to hospital outpatient clinics are not included in this report. For example, women seeking mammograms may use mobile units associated with radiology clinics of hospitals. Such sources would not be included in the NAMCS. Data from population-based surveys may obtain different estimates of health care resource use compared with event-based surveys. The reader is encouraged to examine data from the National Health Interview Survey for population-based estimates of use of cancer screening by women insured by an HMO or other prepaid health insurance plans (18).

Examining data from the 1992 NAMCS for cross-validation purposes we found that some differences between the 1991 prepaid plan and nonprepaid plan visits were not significant. These differences were noted where applicable. All of the findings presented in the Highlights section were replicated using results from the 1992 NAMCS.

Examining 1992 data also allows us to look at visits to hospital outpatients and emergency departments, which make up approximately 17 percent of the ambulatory care visits for persons under the age of 65 . The National Hospital Ambulatory Medical Care Survey (NHAMCS) first collected data in 1992 from hospital providers to help round out the description of ambulatory care visits (19). Considering all three

Table 18. Number and percent of drug mentions for prepald plan visits and percent of drug mentions for nonprepaid visits for the 20 most frequently used generic substances: United Statos, 1991

\begin{tabular}{|c|c|c|c|}
\hline \multirow[b]{2}{*}{ Generfc substance } & \multicolumn{2}{|c|}{ Prepald plan vistis } & \multirow{2}{*}{$\begin{array}{c}\text { Nonprepaid visits } \\
\text { Percent of } \\
\text { atf drug } \\
\text { mentions }\end{array}$} \\
\hline & $\begin{array}{l}\text { Number of } \\
\text { orug mentions } \\
\text { in thousands }\end{array}$ & $\begin{array}{c}\text { Percant of } \\
\text { all drug } \\
\text { mentions }\end{array}$ & \\
\hline All drug mentions. . . . . . . . . . . & 95,104 & $\cdots$ & $\cdots$ \\
\hline Amoxicillin . . . . . . . . . . . & 7,085 & 7.4 & 5.4 \\
\hline Acetaminophen . . . . . . . . . . . & 4,099 & 4.3 & 4.2 \\
\hline Enythromycin . . . . . . . . . . . & 2,566 & 2.7 & 2.7 \\
\hline Phenylephrine. . . . . . . . . . . . & 2,489 & 2.6 & 1.9 \\
\hline Ibuproten. . . . . . . . . . . . . . . . & 2,325 & 2.4 & 1.9 \\
\hline Phenylpropanolamine . . . . . . . . . & 2,110 & 2.2 & 1.9 \\
\hline Pseudoephedrine. . . . . . . . . . & 2,056 & 2.2 & 1.0 \\
\hline Gualfenesin . . . . . . . . . . . . . & 1,934 & 2.0 & 1.5 \\
\hline Albuterol . . . . . . . . . . . . . . & 1,840 & 1.9 & 1.5 \\
\hline Alcohol . . . . . . . . . . . . . . . . & 1,827 & 1.9 & 1.2 \\
\hline 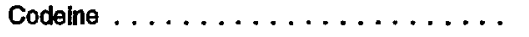 & 1,722 & 1.8 & 2.0 \\
\hline Diph pertussis tetanus vaccine . . . . . . . & 1,716 & 1.8 & 1.2 \\
\hline Vitamin A. . . . . . . . . . . & 1,685 & 1.8 & 1.7 \\
\hline Trimethoprim . . . . . . . . . . . . & 1,668 & 1.8 & 1.4 \\
\hline Sulfamethoxazole .............. & 1,665 & 1.7 & 1.4 \\
\hline Ergocalciferol . . . . . . . . . . . & 1,614 & 1.7 & 1.5 \\
\hline Naproxen .................. & 1,597 & 1.7 & 1.5 \\
\hline Terfenadine . . . . . . . . . . . . & 1,442 & 1.5 & 0.8 \\
\hline Rboflavin . . . . . . . . . . . . & 1,390 & 1.5 & 1.5 \\
\hline Cefactor . . . . . . . . . . . . & 1,388 & 1.5 & 1.5 \\
\hline
\end{tabular}

'Frequency of mention combines single-ingredient agents with mentions of the agent as an ingredient in a combination drug.

Table 19. Number and percent of prepaid plan visits and percent of nonprepaid visits by disposition and duration: United States, 1991

\begin{tabular}{|c|c|c|c|}
\hline \multirow[b]{2}{*}{ Vist characteristic } & \multicolumn{2}{|c|}{ Prepald plan vishs } & \multirow[t]{2}{*}{ Nonprepakd vishts } \\
\hline & $\begin{array}{c}\text { Number of } \\
\text { visits in } \\
\text { thousands }\end{array}$ & Pencent & \\
\hline All visits $\ldots \ldots \ldots \ldots \ldots \ldots$ & 91,824 & $\cdots$ & $\cdots$ \\
\hline \multicolumn{4}{|l|}{ Dispostlion: ${ }^{2}$} \\
\hline No tollowup planned. . . . . . . . . . & 7,797 & 8.5 & 11.4 \\
\hline Retum at spectilc time $\ldots \ldots \ldots \ldots \ldots$ & 53,001 & 57.7 & 59.5 \\
\hline Return if needed $\ldots \ldots \ldots \ldots \ldots$ & 24,791 & 27.0 & 23.8 \\
\hline Telephone followup planned . . . . . . . . . . . & 3,357 & $\mathbf{3 . 7}$ & 3.6 \\
\hline Referred to other physician $\ldots \ldots \ldots$ & 4,308 & 4.7 & 2.6 \\
\hline Referred to referting physician $\ldots . . .$. & 693 & 0.8 & 0.7 \\
\hline Admit to hospltal . . . . . . . . . . . & 635 & 0.7 & 0.8 \\
\hline other $\ldots \ldots \ldots \ldots \ldots \ldots \ldots$ & 906 & 1.0 & 1.2 \\
\hline \multicolumn{4}{|l|}{ Duration: } \\
\hline 0 minutes ${ }^{2} \ldots \ldots \ldots \ldots \ldots \ldots$ & 1,595 & 1.7 & 1.1 \\
\hline 1-5 minutes . . . . . . . . . . . . & 8,943 & 9.7 & 9.4 \\
\hline $6-10$ minutes $\ldots \ldots \ldots \ldots \ldots \ldots \ldots$ & 27,786 & 30.3 & 27.4 \\
\hline 11-15 minutes . . . . . . . . . . . & 28,815 & 31.4 & 30.9 \\
\hline $16-30$ minutes $\ldots \ldots \ldots \ldots \ldots$ & 19,859 & 21.6 & 23.7 \\
\hline $31-60$ minules $\ldots \ldots \ldots \ldots \ldots$ & 4,665 & 5.1 & 7.0 \\
\hline 60 minutes and over. . . . . . . . . . . & $* 161$ & ${ }^{*} 0.2$ & 0.5 \\
\hline
\end{tabular}

Numbers may not add to totals because more than one disposition may be reported per visit

2 Visits in which there was no face-to-face contact between patient and physician.

types of ambulatory care providers (physician offices, hospital outpatients, and emergency departments), black persons comprised the same proportion of prepaid and nonprepaid visits (11.6 vs. 13.5 percent, respectively) $(20,21)$. Figure 3 shows the 1992 distributions of both prepaid plan and nonprepaid visits 


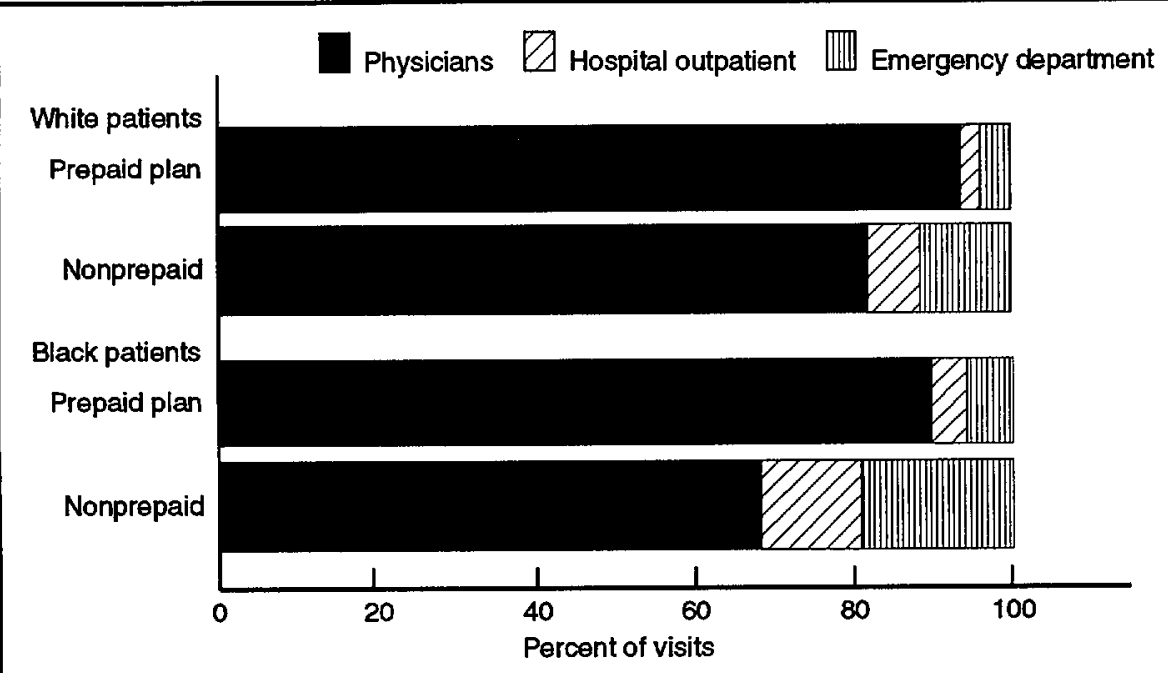

NOTE: Excludes visits for patients 65 years of age and over.

Figure 3. Percent distribution of visits to various providers for prepaid plan and nonprepaid visits by patient's race: United States, 1992

to various providers. More of the prepaid plan visits were to officebased physicians rather than hospital settings. Approximately 9 out of 10 prepaid plan visits made by black patients were to office-based physicians whereas the corresponding number for nonprepaid visits is 7 out of 10 . For both races, the proportion of total prepaid plan visits to emergency departments are lower than for nonprepaid visits. The proportion of visits to emergency departments are approximately three times higher for nonprepaid visits compared with prepaid plan visits. The reader should note that the nonprepaid visits include visits made by people who have no health insurance and it has been shown that such populations receive more primary care from emergency settings (22-24).

In summary, results from this study indicate that after controlling for age differences between prepaid plan and nonprepaid visits, prepaid plan visits differ from nonprepaid visits on referral status, physician specialty, and regional distribution. The visits are similar with respect to reason for visit, diagnosis, treatments ordered or provided, and duration. The proportion of office-based physicians' visits that has an expected source of payment as "prepaid plan" has increased since 1985.

\section{References:}

1. Roghmann KJ, Gavett JW, Sorensen AA, Wells S, Wersinger R. Who chooses prepaid medical care: Survey results from two marketings of three new prepayment plans. Public Health Rep 90:516-27. 1975.

2. Nycz GR, Wenzel FJ, Lohrenz FN, Mitchell JH. Composition of the subscribers in a rural prepaid group practice plan. Public Health Rep 91:504-7. 1976.

3. Jackson-Beeck M, Kleinman JH. Evidence for self-selection among bealth maintenance organization enrollees. J Am Med Assoc 250:2826-29. 1983.

4. Lairson DR, Herd JA. The role of health practices, health status, and prior health care claims in HMO selection bias. Inquiry 24:276-84. 1987.

5. Lichtenstein R, Thomas JW, Watkins B, Puto C, Lepkowski J, AdamsWatson J, Simone B, Vest D. HMO marketing and selection bias. Med

6. Ware Jr, JE, Rogers WH, Davies AR, Goldberg GA, et al. Comparison of health outcomes at a health maintenance organization with those of fee-for-service care. Lancet 1017-22. 1986.

7. Stern RS, Juhn PI, Gertler PJ, Epstein AM. A comparison of length of stay and costs for health maintenance organization and Care 30:329-45. 1992. fee-for-service patients. Arch Intern Med 149:1185-8. 1989.

8. Young GJ, Cohen BB. Inequities in hospital care, the Massachusetts experience. Inquiry 28:255-62. 1991.

9. Carlisle DM, Siu AL, Keeler EB, McGlynn EA, Kahn KL, Rubenstein LV, Brook RH. HMO vs fee-forservice care of older persons with acute myocardial infarction. Am J Public Health 82:1626-30. 1992.

10. Greenwald HP, Henke CJ. HMO membership, treatment, and mortality risk among prostatic cancer patients. Am J Public Health 82:1099-1104. 1992.

11. Szilagyi PG, Roghmann KJ, Foye HR, Parks C, et al. Increased ambulatory utilization in IPA plans among children receiving hyposensitization therapy. Inquiry 29:467-75. 1992.

12. Gemson DH, Freudenheim E, Senie RT, Elinson J, Fink R. Health promotion and disease prevention in HMOs: A survey of newly established IPAs in New York City. Am J Prev Med 6:333-8. 1990.

13. Schneider D, Appleton L, McLemore $T$. A reason for visit classification for ambulatory care. National Center for Health Statistics. Vital and Health Stat 2(78). 1979.

14. Public Health Service and Health Care Financing Administration. International Classification of Diseases, 9th Revision, clinical modification. Washington: Public Health Service. 1980.

15. Schappert SM. National Ambulatory Medical Care Survey: 1991

Summary. Advance data from vital and health statistics; no 230. Hyattsville, Maryland: National Center for Health Statistics. 1993.

16. Food and Drug Administration. National Drug Code Directory, 1985 Edition. Washington: Public Health Service. 1985.

17. Koch H, Campbell W. The collection and processing of drug information. National Ambulatory Medical Care Survey, 1980. National Center for Health Statistics. Vital Health Stat 2(90). 1982.

18. Makuc D, Freid VM, Parsons PE. Health insurance and cancer screening among women. Advance data from vital and health statistics; no 254. Hyattsville, Maryland: National Center for Health Statistics. 1994. 
19. McCaig LF, McLemore T. Plan and operation of the National Hospital Ambulatory Medical Care Survey. National Center for Health Statistics. Vital Health Stat 1(34). 1994.

20. Unpublished data from the National Ambulatory Medical Care Survey, 1992.

21. Unpublished data from the National Hospital Ambulatory Medical Care Survey, 1992.
22. Grumbach K, Keane D, Bindman A. Primary care and public emergency overcrowding. Am J Public Health 83(3):372-8. 1993.

23. Kellerman AL. Nonurgent emergency department visits: Meeting an unmet need. J Am Med Assoc 271(24):1953-4. 1994.
24. Schappert $S$. Race differences in hospital emergency department use. Stat Bull Vol 76 No 3. 1995.

25. Shah BV, Barnwell BG, Hunt PN, LaVange LM. SUDAAN user's manual, release 5.50. Research Triangle Park, North Carolina: Research Triangle Institute. 1991. 


\section{Technical notes}

\section{Source of data and sample design}

The information presented in this report is based on data collected by means of the National Ambulatory Medical Care Survey (NAMCS) from January 1991 through December 1991. The target universe of NAMCS includes office visits made in the United States by ambulatory patients to nonfederally employed physicians who are principally engaged in office practice, but not in the specialties of anesthesiology, pathology, or radiology. Telephone contacts and nonoffice visits are excluded.

A multistage probability sample design is used in NAMCS, involving samples of primary sampling units (PSU's), physician practices within PSU's, and patient visits within physician practices. The PSU's are counties, groups of counties, county equivalents (such as parishes or independent cities), or towns and townships (for PSU's in New England). For 1991, a sample of 2,540 nonfederal, office-based physicians was selected from the master files maintained by the American Medical Association and American Osteopathic Association. Physicians were screened at the time of the survey to ensure that they were eligible for survey participation. Of those screened, 653 physicians were ruled ineligible (out-of-scope). The remaining 1,887 physicians were in-scope or eligible to participate in the survey. The physician response rate for the 1991 NAMCS was 72 percent. Sample physicians were asked to complete Patient Records (see figure 1) for a systematic random sample of office visits occurring during a randomly assigned 1-week reporting period. Responding physicians completed 33,795 patient records.

Characteristics of the physician's practice, such as primary specialty and type of practice, were obtained from the physicians during an induction interview. The U.S. Bureau of the Census, Housing Surveys Branch, was responsible for the survey's data collection. Processing operations and medical coding were performed by the
National Center for Health Statistics, Health Care Survey Section, Research Triangle Park, North Carolina.

\section{Sampling errors}

The standard error is primarily a measure of the sampling variability that occurs by chance when only a sample, rather than an entire universe, is surveyed. The standard error also reflects part of the measurement error but does not measure any systematic biases in the data. The chances are 95 out of 100 that an estimate from the sample differs from the value that would be obtained from a complete census by less than twice the standard error.

The standard errors that were used in tests of significance for this report were calculated using generalized linear models for predicting the relative standard error for estimates based on the linear relationship between the actual standard error, as approximated using SUDAAN software, and the size of the estimate. SUDAAN computes standard errors by using a first-order Taylor approximation of the deviation of estimates from their expected values. A description of the software and the approach it uses has been published (25). The relative standard error (RSE) of an estimate is obtained by dividing the standard error by the estimate itself. The result is then expressed as a percent of the estimate.

Relative standard errors for emergency department estimates are shown in tables I and II. Standard errors for estimates in percents of visits and drug mentions are shown in tables III and IV. Multiplying the estimate by the RSE will provide an estimate of the standard error for the estimate.

Alternatively, relative standard errors for aggregate estimates may be calculated using the following general formula, where $x$ is the aggregate of interest in thousands, and $A$ and $B$ are the appropriate coefficients from table V.

$$
\operatorname{RSE}(x)=\sqrt{A+\frac{B}{x}} \cdot 100
$$

Similarly, relative standard errors for an estimate of a percent may be calculated using the following general formula, where $p$ is the percent of
Table I. Approximate relative standard errors for estimated numbers of ofince visits: National Ambulatory Medical Care Survey, 1991

\begin{tabular}{|c|c|}
\hline $\begin{array}{l}\text { Estimated number of } \\
\text { office visits in thousands }\end{array}$ & $\begin{array}{l}\text { Relative standard } \\
\text { error in percent }\end{array}$ \\
\hline $100 \ldots \ldots \ldots \ldots$ & 72.1 \\
\hline $200 \ldots \ldots \ldots \ldots \ldots$ & 51.1 \\
\hline $500 \ldots \ldots \ldots \ldots$ & 32.5 \\
\hline$\ldots \ldots \ldots \ldots \ldots$ & 30.0 \\
\hline $1,000 \ldots \ldots \ldots \ldots \ldots$ & 23.1 \\
\hline $2,000 \ldots \ldots \ldots \ldots \ldots$ & 16.6 \\
\hline $5,000 \ldots \ldots \ldots \ldots \ldots$ & 11.0 \\
\hline $10,000 \ldots \ldots \ldots \ldots$ & 8.3 \\
\hline $20,000 \ldots \ldots \ldots \ldots \ldots$ & 6.6 \\
\hline $50,000 \ldots \ldots \ldots \ldots \ldots$ & 5.3 \\
\hline $100,000 \ldots \ldots \ldots \ldots$ & 4.8 \\
\hline $200,000 \ldots \ldots \ldots \ldots$ & 4.5 \\
\hline $500,000 \ldots \ldots \ldots \ldots$ & 4.3 \\
\hline $700,000 \ldots \ldots \ldots \ldots$ & 4.3 \\
\hline
\end{tabular}

NOTES: The smallest reliable estimate for visits to aggregated specialties is 588,000 visits. Estimates below this figure have a relative standard error greater than 30 percent and are deemed unreliable by NCHS standards.

Example of use of table: An aggregate estimate of 50 million visits has a relative standard error of 5.3 percent or a standard error of $2,650,000$ visits ( 5.3 percent of 50 million).

Tablo il. Approximate relative standard errors for estimated numbers of drug mentions: National Ambulatory Medlcal Caro Survoy, 1991

\begin{tabular}{|c|c|}
\hline $\begin{array}{l}\text { Estimated number of } \\
\text { drug mentions } / \mathrm{n} \text { thousands }\end{array}$ & $\begin{array}{l}\text { Relative standarc } \\
\text { error in percent }\end{array}$ \\
\hline $100 \ldots \ldots \ldots$ & 78.1 \\
\hline $200 \ldots \ldots \ldots \ldots$ & 68.8 \\
\hline $500 \ldots \ldots \ldots \ldots$ & 43.7 \\
\hline $1,000 \ldots \ldots \ldots$ & 31.2 \\
\hline $1,083 \ldots \ldots \ldots \ldots$ & 30.0 \\
\hline $2,000 \ldots \ldots \ldots \ldots$ & 22.4 \\
\hline $5,000 \ldots \ldots \ldots \ldots$ & 14.8 \\
\hline $10,000 \ldots \ldots \ldots$ & 11.2 \\
\hline $20,000 \ldots \ldots \ldots \ldots$ & 8.9 \\
\hline $50,000 \ldots \ldots \ldots$ & 7.1 \\
\hline $100,000 \ldots \ldots \ldots$ & 6.5 \\
\hline $200,000 \ldots \ldots \ldots$ & 6.1 \\
\hline $600,000 \ldots \ldots \ldots$ & 5.8 \\
\hline $800,000 \ldots \ldots \ldots$ & 5.8 \\
\hline
\end{tabular}

NOTES: The smallest reliable estimate for drug mentions is $1,083,000$ mentions. Estimates below this figure have a relative standard error greater than 30 percent and are deemed unreliable by NCHS standards.

Example of use of table: An aggregate estimate of 50 million drug mentions has a relative standard error of 7.1 percent or a standard error of $3,550,000$ mentions $(7.1$ percent of 50 million).

interest, expressed as a proportion, and $x$ is the denominator of the percent in thousands, using the appropriate coefficients from table $\mathrm{V}$.

$$
\operatorname{RSE}(x)=\sqrt{\frac{B \cdot(1-p)}{p \cdot x}} \cdot 100
$$


Table III. Approximate standard errors for percents of estimated number of office visits: National Ambulatory Medical Care Survey: United States, 1991

\begin{tabular}{|c|c|c|c|c|c|c|c|}
\hline \multirow{2}{*}{$\begin{array}{l}\text { Base of percent } \\
\text { (vlsts in thousands) }\end{array}$} & \multicolumn{7}{|c|}{ Estimated percent } \\
\hline & 1 or 99 & 5 or 95 & 10 or 90 & 20 or 80 & 30 or 70 & 40 or 60 & 50 \\
\hline & \multicolumn{7}{|c|}{ Standard error in percentage points } \\
\hline $100 \ldots \ldots \ldots \ldots \ldots \ldots \ldots \ldots \ldots \ldots$ & 7.2 & 15.7 & 21.6 & 28.8 & 33.0 & 35.3 & 36.0 \\
\hline $200 \ldots \ldots \ldots \ldots \ldots \ldots \ldots \ldots \ldots \ldots$ & 5.1 & 11.1 & 15.3 & 20.4 & 23.3 & 24.9 & 25.5 \\
\hline 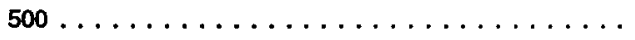 & 3.2 & 7.0 & 9.7 & 12.9 & 14.8 & 15.8 & 16.1 \\
\hline $1,000 \ldots \ldots \ldots \ldots \ldots \ldots \ldots \ldots \ldots \ldots \ldots$ & 2.3 & 5.0 & 6.8 & 9.1 & 10.4 & 11.2 & 11.4 \\
\hline $2,000 \ldots \ldots \ldots \ldots \ldots \ldots \ldots \ldots \ldots \ldots$ & 1.6 & 3.5 & 4.8 & 6.4 & 7.4 & 7.9 & 8.1 \\
\hline 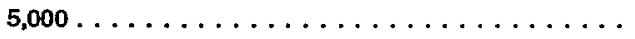 & 1.0 & 2.2 & 3.1 & 4.1 & 4.7 & 5.0 & 5.1 \\
\hline $10,000 \ldots \ldots \ldots \ldots \ldots \ldots \ldots \ldots \ldots$ & 0.7 & 1.6 & 2.2 & 2.9 & 3.3 & 3.5 & 3.6 \\
\hline $20,000 \ldots \ldots \ldots \ldots \ldots \ldots \ldots \ldots \ldots \ldots$ & 0.5 & 1.1 & 1.5 & 2.0 & 2.3 & 2.5 & 2.6 \\
\hline $50,000 \ldots \ldots \ldots \ldots \ldots \ldots \ldots \ldots \ldots$ & 0.3 & 0.7 & 1.0 & 1.3 & 1.5 & 1.6 & 1.6 \\
\hline $100,000 \ldots \ldots \ldots \ldots \ldots \ldots \ldots \ldots$ & 0.2 & 0.5 & 0.7 & 0.9 & 1.0 & 1.1 & 1.1 \\
\hline $200,000 \ldots \ldots \ldots \ldots \ldots \ldots \ldots \ldots$ & 0.2 & 0.4 & 0.5 & 0.6 & 0.7 & 0.8 & 0.8 \\
\hline $500,000 \ldots \ldots \ldots \ldots \ldots \ldots \ldots \ldots$ & 0.1 & 0.2 & 0.3 & 0.4 & 0.5 & 0.5 & 0.5 \\
\hline $1,000,000 \ldots \ldots \ldots \ldots \ldots \ldots \ldots \ldots \ldots \ldots \ldots$ & 0.1 & 0.2 & 0.2 & 0.3 & 0.3 & 0.4 & 0.4 \\
\hline
\end{tabular}

Example of use of table: An estimate of 30 percent based on an aggregate estimale of 10 million visits has a standard error of 3.3 percent or a relative standard error of 11.0 percent (3.3 percent divided by 30).

Table IV. Approximate standard errors of percents of estimated numbers of drug mentions: National Ambulatory Medical Care Survey: United State8, 1991

\begin{tabular}{|c|c|c|c|c|c|c|c|}
\hline \multirow{2}{*}{$\begin{array}{l}\text { Base of percent } \\
\text { (drug mentions in thousands) }\end{array}$} & \multicolumn{7}{|c|}{ Estimated percent } \\
\hline & ior 99 & 5 or 95 & 10 or 90 & 20 or 80 & 30 or 70 & 40 or 60 & 50 \\
\hline & \multicolumn{7}{|c|}{ Standard error in percentage points } \\
\hline $100 \ldots \ldots \ldots \ldots \ldots \ldots \ldots \ldots \ldots \ldots$ & 9.6 & 21.1 & 29.1 & 38.8 & 44.4 & 47.5 & 48.5 \\
\hline $200 \ldots \ldots \ldots \ldots \ldots \ldots \ldots \ldots \ldots$ & 6.8 & 14.9 & 20.6 & 27.4 & 31.4 & 33.6 & 34.3 \\
\hline 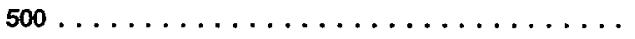 & 4.3 & 9.5 & 13.0 & 17.3 & 19.9 & 21.2 & 21.7 \\
\hline $1,000 \ldots \ldots \ldots \ldots \ldots \ldots \ldots \ldots \ldots \ldots \ldots$ & 3.1 & 6.7 & 9.2 & 12.3 & 14.0 & 15.0 & 15.3 \\
\hline $2,000 \ldots \ldots \ldots \ldots \ldots \ldots \ldots \ldots \ldots \ldots$ & 2.1 & 4.7 & 6.5 & 8.7 & 9.9 & 10.6 & 10.8 \\
\hline $5,000 \ldots \ldots \ldots \ldots \ldots \ldots \ldots \ldots \ldots$ & 1.4 & 3.0 & 4.1 & 5.5 & 6.3 & 6.7 & 6.9 \\
\hline 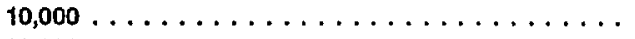 & 1.0 & 2.1 & 2.9 & 3.9 & 4.4 & 4.8 & 4.9 \\
\hline $20,000 \ldots \ldots \ldots \ldots \ldots \ldots \ldots \ldots \ldots$ & 0.7 & 1.5 & 2.1 & 2.7 & 3.1 & 3.4 & 3.4 \\
\hline 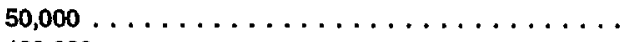 & 0.4 & 0.9 & 1.3 & 1.7 & 2.0 & 2.1 & 2.2 \\
\hline 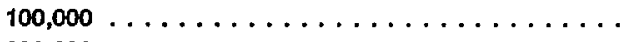 & 0.3 & 0.7 & 0.9 & 1.2 & 1.4 & 1.5 & 1.5 \\
\hline $200,000 \ldots \ldots \ldots \ldots \ldots \ldots \ldots \ldots \ldots \ldots$ & 0.2 & 0.5 & 0.7 & 0.9 & 1.0 & 1.1 & 1.1 \\
\hline $500,000 \ldots \ldots \ldots \ldots \ldots \ldots \ldots \ldots \ldots$ & 0.1 & 0.3 & 0.4 & 0.6 & 0.6 & 0.7 & 0.7 \\
\hline $1,000,000 \ldots \ldots \ldots \ldots \ldots \ldots \ldots \ldots \ldots$ & 0.1 & 0.2 & 0.3 & 0.4 & 0.4 & 0.5 & 0.5 \\
\hline
\end{tabular}

Example of use of table: An estimate of 20 percent based on an aggregate estimate of 10 million visits has a standard error of 3.9 percent or a relative standard error of 19.5 percent (3.9 percent divided by 20 percent).

\section{Adjustments for nonresponse}

Estimates from NAMCS data were adjusted to account for sample physicians who were in-scope but did not participate in the study. This adjustment was calculated to minimize the impact of response on final estimates by imputing to nonresponding physicians data from visits to similar physicians. For this purpose, physicians were judged similar if they had the same specialty designation and practiced in the same PSU.

\section{Test of significance and rounding}

In this report, the determination of statistical inference is based on the t-test. The Bonferroni inequality was used to establish the critical value for statistical significant differences $(0.05$ level of confidence). Terms relating to differences such as "greater than" or "less than" indicate that the difference is statistically significant.

In the tables, estimates of office visits have been rounded to the nearest thousand. Consequently, estimates will not always add to totals. A lack of comment regarding any two estimates does not mean that the difference was tested and found not to be significant. Rates and percents were calculated from original unrounded figures and do not necessarily agree with percents calculated from rounded data.

\section{Definition of terms}

Ambulatory patient-An ambulatory patient is an individual seeking personal health services who is not currently admitted to any health care institution on the premises.

Physician-A physician is a duly licensed doctor of medicine (MD) or doctor of osteopathy (DO) who is currently in office-based practice and who spends some time caring for ambulatory patients. Excluded from the NAMCS are physicians who are hospital based; who specialize in anesthesiology, pathology, or radiology; who are federally employed; who treat only institutionalized patients; or who are 
Table V. Coeffleients appropriate for dotermining relative standard orrors by type of estimate and physician specialty: National Ambulatory Medlcal Care Survey, 1991

\begin{tabular}{|c|c|c|}
\hline \multirow[b]{2}{*}{ Type of estimate and physiclan speclatty } & \multicolumn{2}{|c|}{ Coofliclent for use with estimates in thousands } \\
\hline & $A$ & $B$ \\
\hline \multicolumn{3}{|l|}{ Visits: } \\
\hline Overall total $\ldots \ldots \ldots \ldots \ldots \ldots$ & 0.001744284 & 51.82697927 \\
\hline General and family practice $\ldots \ldots \ldots \ldots$ & 0.006617364 & 33.29640705 \\
\hline Osteopathy . . . . . . . . . . . . . . & 0.0163602 & 10.90230286 \\
\hline Intermal medicine . . . . . . . . . . . . . . & 0.01573396 & 45.10067385 \\
\hline Pedlatrics . . . . . . . . . . . . . . & 0.0163602 & 10.90230286 \\
\hline General surgery . . . . . . . . . . . . . . . . & 0.0163602 & 10.90230286 \\
\hline Obstetrles and gynecology $\ldots \ldots \ldots \ldots \ldots$ & 0.0163602 & 10.90230286 \\
\hline Orthopedlc surgery. . . . . . . . . . . . . . & 0.0163602 & 10.90230286 \\
\hline Cardlovascular diseases . . . . . . . . . . . . & 0.0163602 & 10.90230286 \\
\hline Dermatology. . . . . . . . . . . . . . . & 0.0163602 & 10.90230286 \\
\hline Urological surgery . . . . . . . . . . . . . & 0.0163602 & 10.90230286 \\
\hline Psychlatry . . . . . . . . . . . . . . & 0.0163602 & 10.90230286 \\
\hline Neurology . . . . . . . . . . . . . . . & 0.0163602 & 10.90230286 \\
\hline Ophthalmotogy . . . . . . . . . . . . . . & 0.0163602 & 10.90230286 \\
\hline Otolaryngology . . . . . . . . . . . . . & 0.0163602 & 10.90230286 \\
\hline All other speclaltles $\ldots \ldots \ldots \ldots \ldots \ldots$ & 0.03340709 & 29.631108 \\
\hline \multicolumn{3}{|l|}{ Drug mentions: } \\
\hline Overall total $\ldots \ldots \ldots \ldots \ldots \ldots \ldots \ldots$ & 0.003224617 & 93.92631687 \\
\hline General and family practice . . . . . . . . . . & 0.0122584 & 57.64543271 \\
\hline Osteopathy . . . . . . . . . . . . . . & 0.02784109 & 11.55212504 \\
\hline Intemal medicine . . . . . . . . . . . . . . & 0.0122584 & 57.64543271 \\
\hline Pedlatrlos . . . . . . . . . . . . . & 0.0122584 & 57.64543271 \\
\hline General surgery . . . . . . . . . . . . . . . . & 0.0122584 & 57.64543271 \\
\hline Obstetrics and gynecology $\ldots \ldots \ldots \ldots \ldots$ & 0.0122584 & 57.64543271 \\
\hline Orthopedic surgery. . . . . . . . . . . . . & 0.0122584 & 57.64543271 \\
\hline Cardlovascular diseases . . . . . . . . . . . & 0.0122584 & 57.64543271 \\
\hline Dermatology. . . . . . . . . . . . . . . & 0.0122584 & 57.64543271 \\
\hline Urological surgeny $\ldots \ldots \ldots \ldots \ldots \ldots \ldots$ & 0.0122584 & 57.64543271 \\
\hline Psychiatry . . . . . . . . . . . . . . & 0.0122584 & 57.64543271 \\
\hline Neurology . . . . . . . . . . . . . . & 0.0122584 & 57.64543271 \\
\hline Ophthalmology . . . . . . . . . . . . . . . & 0.0122584 & 57.64543271 \\
\hline Otolaryngology $\ldots \ldots \ldots \ldots \ldots \ldots$ & 0.0122584 & 57.64543271 \\
\hline All other speclalties . . . . . . . . . . . . & 0.0483582 & 46.53697419 \\
\hline
\end{tabular}

employed full time by an institution and spend no time seeing ambulatory patients.

Office-Offices are the premises physicians identify as locations for their ambulatory practice; these customarily include consultation, examination, or treatment spaces that patients associate with the particular physician.

Visit-A visit is a direct personal exchange between an ambulatory patient and a physician (or a staff member working under the physician's supervision), for the purpose of seeking care and rendering personal health services.

Drug mention-A drug mention is the physician's entry of a pharmaceutical agent - by any route of administration - for prevention,
(HMO's), independent practice organizations (IPA's), and all other prepaid health care plans.

Nonprepaid visit-A nonprepaid visit is a visit for which any expected source of payment with the exception of "HMO/other prepaid" was checked on item 6 of the Patient Record form. diagnosis, or treatment. Generic as well as brand-name drugs are included, as are nonprescription and prescription drugs. Along with all new drugs, the physician also records continued medications if the patient was specifically instructed during the visit to continue the medication.

Drug visit-A drug visit is a visit in which medication was prescribed or provided by the physician.

Prepaid plan visit-A prepaid plan visit is one for which "HMO/other prepaid plan" was checked as an expected source of payment in item 6 of the Patient Record form. Instructions for completing this item on the 1991 Patient Record form defines "HMO/other prepaid" as including visits covered under heath maintenance associations 


\section{Symbols}

-. Data not available

.. Category not applicable

- Quantity zero

0.0 Quantity more than zero but less than 0.05

Z Quantity more than zero but less than 500 where numbers are rounded to thousands

* Figure does not meet standard of reliability or precision 


\section{Suggested cltation}

Woodwell DA. Characteristics of prepaid plan visits to office-based physicians: United States, 1991. Advance data from vital and health statistics; no. 269. Hyattsville, Maryland: National Center for Health Statistics. 1995.

\section{Trade name disclalmer}

The use of trade names is for identification only and does not imply endorsement by the Public Health Service, U.S. Department of Health and Human Services.

\section{Copyright information}

All material appearing in this report is in the public domain and may be reproduced or copied without permission; citation as to source, however, is appreciated.
National Center for Health Statistics

Acting Director

Jack R. Anderson

Acting Deputy Director Jennifer H. Madans, Ph.D.

\section{U.S. DEPARTMENT OF HEALTH AND HUMAN SERVICES \\ Public Health Service \\ Centers for Disease Control and Prevention \\ National Center for Health Statistics \\ 6525 Belcrest Road \\ Hyattsville, Maryland 20782}

\section{OFFICIAL BUSINESS \\ PENALTY FOR PRIVATE USE, $\$ 300$}

To receive this publication regularly, contact the National Center for Health Statistics by calling 301-436-8500

E-mail: nchsquery nch10a.em.cdc.gov

Internet: http://www.cdc.gov/nchswww/nchshome.htm

DHHS Publication No. (PHS) 96-1250

5-1604 (11/95) 


\title{
National Ambulatory Medical Care Survey: 1993 Summary
}

\author{
by David A. Woodwell and Susan M. Schappert, M.A., Division of Health Care Statistics
}

\section{Introduction}

During the 12-month period from January 1993 through December 1993, an estimated 717.2 million visits were made to nonfederally employed, office-based physicians in the United States, or 2.8 visits per person. This rate is not significantly different from office visit rates observed since 1975 (1-5).

This report presents data highlights from the 1993 National Ambulatory Medical Care Survey (NAMCS), a national probability sample survey conducted by the Division of Health Care Statistics of the National Center for Health Statistics, Centers for Disease Control and Prevention. Statistics are presented on physician, patient, and visit characteristics.

Only visits to the offices of nonfederally employed physicians (excluding those in the specialties of anesthesiology, radiology, and pathology) who were classified by the American Medical Association or the American Osteopathic Association as "office-based, patient care" were included in the NAMCS. Visits to private, nonhospital-based clinics and health maintenance organizations were within the scope of the survey, but those occurring in government-operated facilities and hospital-based outpatient departments were not. Telephone contacts and visits made outside the physician's office were also excluded.

Because the estimates presented in this report are based on a sample rather than on the entire universe of office visits, they are subject to sampling variability. The Technical notes at the end of this report include an overview of the sample design used in the 1993 NAMCS, an explanation of sampling errors, and guidelines for judging the precision of the estimates.

The Patient Record form is used by physicians participating in the NAMCS to record information about their patients' office visits. This form is reproduced in figure 1 and is intended to serve as a reference for readers as they review the survey findings presented in this document.

The physician sample for the NAMCS was selected with the cooperation of the American Medical Association and the American Osteopathic Association. Their contribution to this effort is gratefully acknowledged.

\section{Physician characteristics}

The distribution of office visits according to physician specialty is presented in table 1 . The largest share of visits was made to physicians in general and family practice (27.6 percent). Visit rates to each of the physician specialty groups did not differ significantly from 1992 visit rates with the exception of otolaryngologists. The rate of visits to this specialty decreased from 9.1 visits per 100 persons in 1992 to 6.0 visits per 100 persons in 1993. However, the 1993 figure is not significantly different from the corresponding rate of 7.7 visits per 100 in 1991. In fact, the visit rate to otolaryngologists has ranged between 6.5 and 7.0 visits per 100 persons between 1975 and 1990, so the 1992 figure appears to be an anomaly.

Doctors of osteopathy received 44.9 million visits during 1993, or 6.3 percent of all office visits. Visits to this specialty occurred at a rate of $\mathbf{1 7 . 7}$ per 100 persons, which was not significantly different from the 1992 visit rate.

Visits according to geographic characteristics of the physician's practice are also displayed in table 1. Visit rates by region-Northeast, Midwest, South, and West-did not differ from each other in 1993, except that the Northeast rate was higher than the South and Midwest. Regional rates were not significantly different than the corresponding 1992 rates.

\section{Patient characteristics}

Office visits by patient's age, sex, and race are shown in table 2 . Females made 60.0 percent of all office visits

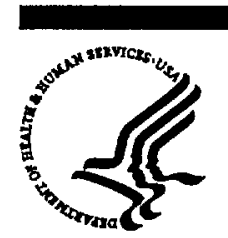

\section{U.S. DEPARTMENT OF HEALTH AND HUMAN SERVICES \\ Public Health Service \\ Centers for Disease Control and Prevention National Center for Health Statistics}

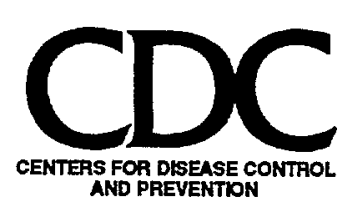




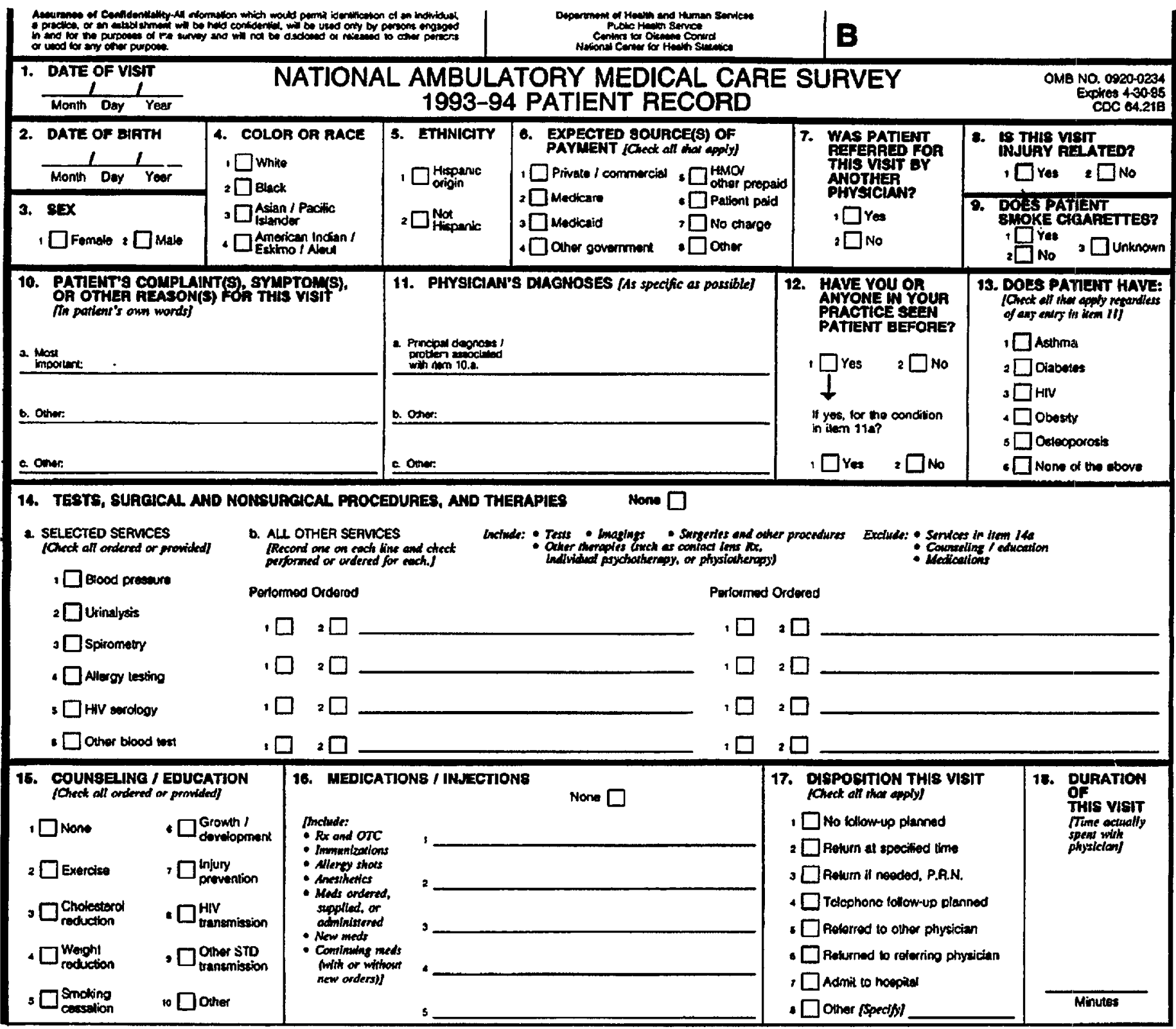

Figure 1. Patient Record form

during 1993 and accounted for a higher percent of visits than males in all age categories except the youngest (under 15 years). Females also had significantly higher visit rates than males in each age category with the exception of the youngest group (under 15 years) and the two oldest groups (65-74 years and 75 years and over). These patterns were also observed in the 1990-92 National Ambulatory Medical Care Surveys.

Visit rates were found to increase with age after the age of 24. Persons aged 75 years and over had the highest visit rate of the six age categories analyzed, at 6.1 visits per person. The pattern, however, was found to be slightly different for males and females. Among males, the visit rate for the age group 15-24 years was significantly lower than for those under 15 years. But males in the age group 25-44 years had a higher rate than those 15-24 years. The rate increased with each successive age group, with males aged 75 years and over having the highest rate of 6.2 visits per person.

There was no significant difference in the visit rates for females under 15 years and those 15-24 years. However, the rate was higher for females $25-44$ years than for those 15-24, and increased again for those $45-64$ years and 65-74 years of age. There was no significant difference in visit rates between females in the two oldest age groups, 65-74 years and 75 years and over.

The visit rate for the white population was significantly higher (3.0 visits per person) than the rate for the black population (1.8 visits per person) in 1993. Visit rates were higher for white persons in each age group compared with black persons, with the 
exception of those 75 years and over. White persons made 88.2 percent of all office visits, with black persons and Asians/Pacific Islanders accounting for 8.1 percent and 3.3 percent, respectively.

The visit rate for the black population in 1993 was significantly lower than the 1992 rate (2.6 visits per person), but was not significantly different from the 1991 rate (1.9 visits per person). The higher rates in 1992 may be the result of sampling variability rather than a true increase, as discussed in a previous report (5).

\section{Visit characteristics}

\section{Referral status and prior-visit status}

Table 3 shows data on office visits categorized by patient's referral status and prior-visit status. The distribution of visits by referral status and prior-visit status according to physician specialty is shown in table 4. It is important to note that, in previous years, several data items were used to determine referral status. Return visits made for treatment of an "old" problem were not considered referral visits even if the referral item on the Patient Record form had been checked "yes" by the physician. This edit procedure was instituted on the assumption that if the physician had seen the patient previously for treatment of the same problem (defined as the current episode of care), that patient could not have been referred for the current visit.

However, in recent years, increasing numbers of physicians in the NAMCS sample have characterized visits as referrals and, at the same time, as being made by "old" patients for "old" problems. This apparent inconsistency may have a number of possible explanations: some physicians may be reporting referred patients as referred visits; changes in referral patterns may have occurred related to changes in insurance coverage; or physicians may be including patients seen before for past episodes of care, rather than current episodes of care.

Beginning with the 1993 survey year, only data from the referral status

Table 1. Annual number, percent dletrlbution, and rate of office vislts by selected physiclan practice characteristics: United States, 1993

\begin{tabular}{|c|c|c|c|}
\hline Physiclan practlce characteristic & $\begin{array}{l}\text { Number of } \\
\text { visits in } \\
\text { thousands }\end{array}$ & $\begin{array}{c}\text { Percent } \\
\text { distribution }\end{array}$ & $\begin{array}{l}\text { Number of } \\
\text { visits per } \\
100 \text { persons } \\
\text { per yeart }\end{array}$ \\
\hline $\begin{array}{c}\text { All visits } \ldots \ldots \ldots \ldots \ldots \ldots \ldots \ldots \ldots \ldots \\
\text { Physician specialty }\end{array}$ & 717,191 & 100.0 & 282.0 \\
\hline General and famlly practice . . . . . . . . . . . & 197,605 & 27.6 & 77.7 \\
\hline Intemal medicine $\ldots \ldots \ldots \ldots \ldots \ldots \ldots$ & 102,436 & 14.3 & 40.3 \\
\hline Pedlatrios $\ldots \ldots \ldots \ldots \ldots \ldots \ldots$ & 76,982 & 10.7 & 30.3 \\
\hline Obstetrics and gynecology $\ldots \ldots \ldots \ldots \ldots$ & 64,030 & 8.9 & 27.2 \\
\hline 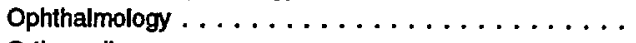 & 39,373 & 5.5 & 15.5 \\
\hline Orthopedic surgery. . . . . . . . . . . . . . & 33,638 & 4.7 & 13.2 \\
\hline Dermatology. . . . . . . . . . . . . . . . & 31,469 & 4.4 & 12.4 \\
\hline General surgery . . . . . . . . . . . . . . . . & 21,703 & 3.0 & 8.5 \\
\hline Psychlatry . . . . . . . . . . . . . . & 20,469 & 2.9 & 8.0 \\
\hline Urology . . . . . . . . . . . . . . . . & 15,690 & 2.2 & 6.2 \\
\hline Otolaryngology . . . . . . . . . . . . . & 15,380 & 2.1 & 6.0 \\
\hline Cardlovascular dlseases . . . . . . . . . . . & 12,178 & 1.7 & 4.8 \\
\hline Allergy and immunology ${ }^{3} \ldots \ldots \ldots \ldots$ & 10,605 & 1.5 & 4.2 \\
\hline Neurology . . . . . . . . . . . . . . . . & 8,393 & 1.2 & $\mathbf{3 . 3}$ \\
\hline Pulmonary diseases $^{3} \ldots \ldots \ldots \ldots \ldots$ & 4,251 & 0.6 & 1.7 \\
\hline $\begin{array}{l}\text { All other speclalties } \ldots \ldots \ldots \ldots \ldots \ldots \ldots \ldots \\
\qquad \text { Professlonal identity }\end{array}$ & 62,991 & 8.8 & 24.8 \\
\hline Doctor of medicine. . . . . . . . . . . . . . & 672,306 & 93.7 & 264.4 \\
\hline $\begin{array}{l}\text { Doctor of osteopathy } \ldots \ldots \ldots \ldots \\
\text { Geographic region }\end{array}$ & 44,885 & 6.3 & 17.7 \\
\hline Northeast $\ldots \ldots \ldots \ldots \ldots \ldots \ldots \ldots$ & 168,438 & 23.5 & 336.6 \\
\hline Midwest . . . . . . . . . . . . . . . & 169,035 & 23.6 & 272.5 \\
\hline 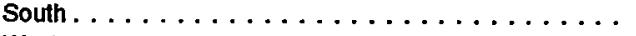 & 213,356 & 29.7 & 250.0 \\
\hline West $\ldots \ldots \ldots \ldots \ldots \ldots \ldots \ldots$ & 166,363 & 23.2 & 292.7 \\
\hline
\end{tabular}

'Based on U.S. Bureau of the Census estimates of the civilian noninstitutionalized population of the United States as of July $1,1993$.

2The visit rate is 48.5 per 100 females.

${ }^{3}$ These specialties were sampled separately in 1993 only as part of a supplemental data collection project.

item on the Patient Record form will be used to determine referral status. The definition of a referred visit consistent with past usage can be recreated using information available on the public use data file. Recent changes in the health care system may have altered the way referral status is conceptualized and interpreted.

Research is under way to improve the collection of this information in the NAMCS.

When referred visits are restricted to those made by new patients and those made by old patients for new problems, their share of total visits is 6.6 percent, not significantly different than the 1992 NAMCS figure of 6.2 percent. Using the number of referred visits reported by physicians (which includes visits made by old patients for old problems), the percent of referred visits is 13.7 (table 3).
Also shown in table 3 are office visits by prior-visit status. Eight out of ten office visits ( 84.4 percent) were made by patients who had seen the physician on a previous occasion, and more than half of all visits (63.1 percent) were made by persons returning to the physician for care of a previously treated problem.

As expected, the percent of referred visits reported by primary care specialties was relatively low, 10 percent or less of the total visits to general and family practitioners, internists, pediatricians, and obstetriciansgynecologists. In contrast, about half of all visits to neurologists (50.7 percent) were reported to be referrals (table 4).

\section{Expected sources of payment}

Data on expected sources of payment are shown in table 5. 
Table 2. Annual number, percent distribution, and rate of office visits by patient's age, sex, and race: United States, 1993

\begin{tabular}{|c|c|c|c|}
\hline Patlent's age, sex, and race & $\begin{array}{l}\text { Number of } \\
\text { visits in } \\
\text { thousands }\end{array}$ & $\begin{array}{c}\text { Percent } \\
\text { distribution }\end{array}$ & $\begin{array}{l}\text { Number of } \\
\text { visits per } \\
\text { persons } \\
\text { per year }\end{array}$ \\
\hline All visits $\ldots \ldots \ldots \ldots \ldots \ldots \ldots \ldots$ & 717,191 & 100.0 & 2.8 \\
\hline \multicolumn{4}{|l|}{ Age } \\
\hline Under 15 years $\ldots \ldots \ldots \ldots \ldots \ldots$ & 129,279 & 18.0 & 2.3 \\
\hline 15-24 years. $\ldots \ldots \ldots \ldots \ldots \ldots \ldots \ldots$ & 62,346 & 8.7 & 1.8 \\
\hline $25-44$ years. $\ldots \ldots \ldots \ldots \ldots \ldots \ldots \ldots \ldots$ & 193,914 & 27.0 & 2.4 \\
\hline $45-64$ years. $\ldots \ldots \ldots \ldots \ldots \ldots \ldots \ldots$ & 160,146 & 22.3 & 3.2 \\
\hline 65-74 years. $\ldots \ldots \ldots \ldots \ldots \ldots \ldots$ & 93,873 & 13.1 & 5.0 \\
\hline 75 years and over $\ldots \ldots \ldots \ldots \ldots \ldots$ & 77,633 & 10.8 & 6.1 \\
\hline \multicolumn{4}{|l|}{ Sex and age } \\
\hline Female $\ldots \ldots \ldots \ldots \ldots \ldots \ldots$ & 430,170 & 60.0 & 3.3 \\
\hline Under 15 years $\ldots \ldots \ldots \ldots \ldots \ldots$ & 60,664 & 8.5 & 2.2 \\
\hline 15-24 years $\ldots \ldots \ldots \ldots \ldots \ldots \ldots$ & 41,408 & 5.8 & 2.4 \\
\hline $25-44$ years $\ldots \ldots \ldots \ldots \ldots \ldots \ldots \ldots$ & 128,854 & 18.0 & 3.1 \\
\hline 45-64 years $\ldots \ldots \ldots \ldots \ldots \ldots \ldots$ & 96,011 & 13.4 & 3.7 \\
\hline 65-74 years $\ldots \ldots \ldots \ldots \ldots \ldots \ldots$ & 55,215 & 7.7 & 5.4 \\
\hline 75 years and over $\ldots \ldots \ldots \ldots \ldots$ & 48,017 & 6.7 & 6.1 \\
\hline$\ldots \ldots \ldots \ldots \ldots \ldots \ldots$ & 287,021 & 40.0 & 2.3 \\
\hline Under 15 years $\ldots \ldots \ldots \ldots \ldots \ldots$ & 68,615 & 9.6 & 2.3 \\
\hline 15-24 years $\ldots \ldots \ldots \ldots \ldots \ldots \ldots \ldots$ & 20,938 & 2.9 & 1.2 \\
\hline 25-44 years $\ldots \ldots \ldots \ldots \ldots \ldots \ldots$ & 65,060 & 9.1 & 1.6 \\
\hline 45-64 years $\ldots \ldots \ldots \ldots \ldots \ldots \ldots$ & 64,135 & 8.9 & 2.7 \\
\hline $65-74$ years $\ldots \ldots \ldots \ldots \ldots \ldots \ldots$ & 38,658 & 5.4 & 4.6 \\
\hline 75 years and over $\ldots \ldots \ldots \ldots \ldots$ & 29,616 & 4.1 & 6.2 \\
\hline \multicolumn{4}{|l|}{ Race and age } \\
\hline$\ldots \ldots \ldots \ldots \ldots$ & 632,500 & 88.2 & 3.0 \\
\hline Under 15 years $\ldots \ldots \ldots \ldots \ldots \ldots \ldots$ & 113,506 & 15.8 & 2.5 \\
\hline $15-24$ years $\ldots \ldots \ldots \ldots \ldots \ldots \ldots \ldots$ & 53,650 & 7.5 & 2.0 \\
\hline $25-44$ years $\ldots \ldots \ldots \ldots \ldots \ldots \ldots \ldots$ & 167,026 & 23.3 & 2.5 \\
\hline $45-64$ years $\ldots \ldots \ldots \ldots \ldots \ldots \ldots$ & 140,231 & 19.6 & $\mathbf{3 . 3}$ \\
\hline $65-74$ years $\ldots \ldots \ldots \ldots \ldots \ldots \ldots$ & 86,204 & 12.0 & 5.2 \\
\hline 75 years and over $\ldots \ldots \ldots \ldots \ldots$ & 71,884 & 10.0 & 6.3 \\
\hline${ }_{\ldots \ldots \ldots \ldots \ldots \ldots \ldots}$ & 58,154 & 8.1 & 1.8 \\
\hline Under 15 years $\ldots \ldots \ldots \ldots \ldots \ldots \ldots$ & 10,328 & 1.4 & 1.1 \\
\hline $15-24$ yoars $\ldots \ldots \ldots \ldots \ldots \ldots \ldots \ldots$ & 6,308 & 0.9 & 1.2 \\
\hline $25-44$ years $\ldots \ldots \ldots \ldots \ldots \ldots \ldots \ldots$ & 16,946 & 2.4 & 1.7 \\
\hline $45-64$ years $\ldots \ldots \ldots \ldots \ldots \ldots$ & 14,399 & 2.0 & 2.8 \\
\hline $65-74$ years $\ldots \ldots \ldots \ldots \ldots \ldots \ldots$ & 5,381 & 0.8 & 3.2 \\
\hline 75 years and over $\ldots \ldots \ldots \ldots \ldots$ & 4,793 & 0.7 & 4.8 \\
\hline \multicolumn{4}{|l|}{ All other races } \\
\hline Aslan/Paclfic Islander & 23,377 & 3.3 & - - \\
\hline American Indlan/Eskimo/Aleut $\ldots \ldots \ldots \ldots$ & 3,160 & 0.4 & - - \\
\hline
\end{tabular}

Physicians were asked to check all of the applicable payment categories for this survey item, with the result that multiple payment sources could be coded for each visit. The patient-paid category includes the patient's contribution toward "co-payments" and "deductibles."

Expected sources of payment were most often private/commercial insurance (38.7 percent of visits), Medicare (22.1 percent of visits), $\mathrm{HMO} /$ other prepaid (19.3 percent), and patient-paid (15.0 percent). Medicaid was listed as an expected source of payment at 10.4 percent of visits.

\section{Injury-related visits}

Injury-related office visits are presented in terms of patient's age, sex, and race in table 6 . Based on data collected in item 8 of the Patient Record form, there were an estimated 84.0 million injury-related office visits in 1993, representing 11.7 percent of all office visits. Corresponding figures for 1992 were 65.6 million and 8.6 percent of visits, respectively. About half of the injury visits (51.0 percent) were made by males, and 38.8 percent were made by persons $25-44$ years old.

The injury visit rate for males was not significantly higher than the rate for females in 1993 (34.6 visits per 100 males compared with 31.5 visits per 100 females), nor were there any differences noted between males and females by age.

Among females, injury visit rates were not significantly different for women in the age groups $25-44$, $45-64,65-74$, and 75 years and over. However, the rates for these groups were significantly higher than for females under 15 years and 15-24 years of age. Males in the age group 25-44 years had an injury visit rate higher than those aged under 15 years and 15-24 years. However, the rate was not statistically different for males in the $25-44,45-64,65-74$, and 75 years and over groups.

The injury visit rate for black persons was 19.1 visits per 100 persons in 1993, significantly lower than the rate of 35.6 injury visits per 100 white persons. Rates were not significantly different between white males ( 36.8 per $100)$ and white females ( 34.3 per 100$)$, or between black males (20.9 per 100) and black females (17.5 per 100) (data not shown).

\section{Pationt's cigarette-smoking status}

Results from the 1993 survey showed that 67.7 million office visits, or 9.4 percent of the total, were made by patients who smoke cigarettes. However, patient's smoking status was not reported for 27.0 percent of office visits. Data on visits according to patient's cigarette-smoking status are presented in tables 7 and 8.

\section{Patient's principal reason for visit}

Item 10 of the Patient Record form asks the physician to record the patient's (or patient surrogate's) "complaint(s), 
Table 3. Number and percent distribution of office visits by patient's roferral status, according to prior-visit status: United States, 1993

\begin{tabular}{|c|c|c|c|c|}
\hline \multirow[b]{2}{*}{ Referral status } & \multicolumn{4}{|c|}{ Prlor-vish status } \\
\hline & All visits & $\underset{\text { pationt }}{\text { Now }}$ & $\begin{array}{l}\text { Old patient, } \\
\text { new problem }\end{array}$ & $\begin{array}{l}\text { Old pattent, } \\
\text { old problem }\end{array}$ \\
\hline & \multicolumn{4}{|c|}{ Number of visits in thousands } \\
\hline All vislis & 717,191 & 111,922 & 152,898 & 452,372 \\
\hline Referred for this visit & 98,159 & 37,068 & 10,063 & 51,028 \\
\hline \multirow[t]{2}{*}{ Not referred for thls vist $\ldots \ldots \ldots \ldots \ldots \ldots \ldots$} & 619,032 & 74,854 & 142,835 & 401,344 \\
\hline & \multicolumn{4}{|c|}{ Percent distrbution } \\
\hline All visits $\ldots \ldots \ldots \ldots \ldots \ldots \ldots$ & 100.0 & 100.0 & 100.0 & 100.0 \\
\hline Relerred for this visit & 13.7 & 33.1 & 6.6 & 11.3 \\
\hline Not referred for thls visit $\ldots \ldots \ldots \ldots \ldots$ & 86.3 & 66.9 & 93.4 & 88.7 \\
\hline
\end{tabular}

NOTE; Numbers may not add to totals because of rounding.

Table 4. Number and percent distrlbution of ofice visits by physician specialty, according to referral status and prior-visit status: United Statee, 1993

\begin{tabular}{|c|c|c|c|c|c|c|c|c|}
\hline \multirow[b]{2}{*}{ Physiclan spectally } & \multirow{2}{*}{$\begin{array}{l}\text { Number of } \\
\text { visits in } \\
\text { thousands }\end{array}$} & \multirow[b]{2}{*}{ Total } & \multicolumn{3}{|c|}{ Referred for this vist } & \multicolumn{3}{|c|}{ Not referred for this visit } \\
\hline & & & $\begin{array}{c}\text { New } \\
\text { pattont }\end{array}$ & $\begin{array}{l}\text { Old patient, } \\
\text { new problem }\end{array}$ & $\begin{array}{l}\text { Odd patient, } \\
\text { old problem }\end{array}$ & $\underset{\text { patlent }}{\text { New }}$ & $\begin{array}{l}\text { Odd patient, } \\
\text { new problem }\end{array}$ & $\begin{array}{l}\text { Odd pationt, } \\
\text { old problem }\end{array}$ \\
\hline & & \multicolumn{7}{|c|}{ Percent distribution } \\
\hline All visits $\ldots \ldots \ldots \ldots \ldots \ldots \ldots$ & 717,191 & 100.0 & 5.2 & 1.4 & 7.1 & 10.4 & 19.9 & 56.0 \\
\hline General and famlly practlce . . . . . . . . & 197,605 & 100.0 & 0.9 & 1.1 & 1.9 & 11.3 & 30.3 & 54.6 \\
\hline Internal medicine . . . . . . . . . . . & 102,436 & 100.0 & 2.2 & 2.0 & 3.4 & 7.1 & 24.9 & 60.4 \\
\hline Pedlatrics $\ldots \ldots \ldots \ldots \ldots \ldots \ldots$ & 76,982 & 100.0 & 0.9 & 1.0 & 1.5 & 5.1 & 35.8 & 55.7 \\
\hline Obstetrics and gynecology . . . . . . . . & 64,030 & 100.0 & 3.0 & 1.5 & 5.9 & 13.1 & 15.0 & 61.5 \\
\hline 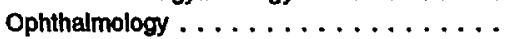 & 39,373 & 100.0 & 5.3 & 1.5 & 5.7 & 12.1 & 7.6 & 67.8 \\
\hline Orthopedlc surgery. . . . . . . . . & 33,638 & 100.0 & 15.3 & 2.6 & 16.6 & 12.1 & 7.3 & 46.2 \\
\hline Dermatology. . . . . . . . . . . . . . & 31,469 & 100.0 & 6.1 & 1.4 & 8.5 & 17.3 & 12.1 & 54.5 \\
\hline General surgery $\ldots \ldots \ldots \ldots \ldots \ldots$ & 21,703 & 100.0 & 16.3 & 3.0 & 18.7 & 9.9 & 9.1 & 42.9 \\
\hline Psychiatry ............... & 20,469 & 100.0 & 5.0 & ${ }^{\star} 0.2$ & 15.4 & 10.7 & ${ }^{*} 0.2$ & 68.6 \\
\hline Urology. . . . . . . . . . . . . . & 15,690 & 100.0 & 14.6 & 1.8 & 17.4 & 6.5 & 3.4 & 56.2 \\
\hline Otolanyngology . . . . . . . . . . . & 15,380 & 100.0 & 16.0 & 2.1 & 14.2 & 17.6 & 5.3 & 44.7 \\
\hline Cardlovascular dlseases $\ldots \ldots \ldots \ldots \ldots$ & 12,178 & 100.0 & 10.2 & -1.4 & 16.9 & 6.4 & 4.7 & 60.4 \\
\hline Allergy and immunology $\ldots \ldots \ldots \ldots$ & 10,605 & 100.0 & 6.7 & 0.3 & 13.6 & 8.3 & 2.8 & 68.3 \\
\hline Nourology . . . . . . . . . . . & 8,393 & 100.0 & 28.9 & 2.1 & 19.7 & 7.6 & 3.5 & 38.2 \\
\hline Puknonary diseases' $\ldots \ldots \ldots \ldots \ldots$ & 4,251 & 100.0 & 9.9 & 1.1 & 9.1 & 11.2 & 6.3 & 62.4 \\
\hline All other specialties . . . . . . . . . & 62,991 & 100.0 & 11.5 & ${ }^{*} 0.8$ & 16.9 & 12.6 & 9.7 & 48.4 \\
\hline
\end{tabular}

${ }^{1}$ These speciallies were sampled separately in 1893 only as part of a supplemental data collection project.

symptom(s), or other reason(s) for this visit in the patient's own words." Up to three reasons for visit are classified and coded from the survey according to the Reason for Visit Classification for Ambulatory Care (RVC) (6). The principal reason for visit is the problem, complaint, or reason listed in item 10a.

The RVC is divided into the eight modules or groups of reasons displayed in table 9. More than half of all visits were made for reasons classified as symptoms (57.7 percent). Respiratory symptoms accounted for 11.6 percent of all visits, and musculoskeletal symptoms accounted for 10.7 percent.
The 20 most frequently mentioned principal reasons for visit, representing 42.2 percent of all visits, are shown in table 10. General medical examination was the most frequently mentioned reason for visit (5.3 percent of the total), while cough was the most frequently mentioned reason related to illness or injury ( 3.4 percent). Nineteen of the top 20 reasons for office visits in 1993 were also listed among the 20 most frequently mentioned reasons in 1992, albeit in slightly different order. It should be noted that estimates that differ in ranked order may not be significantly different from each other.

\section{Tests, procedures, and therapies}

Statistics on tests, procedures, and therapies scheduled or performed by the physician during the office visit are displayed in tables 11-13. The 1993 NAMCS Patient Record form combined tests, surgical and nonsurgical procedures, and therapies (except counseling/education and medication therapy) into a single item, with six checkboxes for commonly performed services and space to record up to eight additional services. Results of the open-ended part of the item were coded according to the International 
Table 5. Number and percent of office vielts by patient's expected source(e) of payment Unlted States, 1993

\begin{tabular}{|c|c|c|}
\hline Expected source(s) of payment' & $\begin{array}{c}\text { Number of } \\
\text { visits in } \\
\text { thousands }\end{array}$ & $\begin{array}{c}\text { Percont } \\
\text { of all } \\
\text { vists }\end{array}$ \\
\hline All visits $\ldots \ldots \ldots \ldots \ldots \ldots \ldots \ldots$ & 717,191 & $\cdots$ \\
\hline Prlvate/commercial insurance . . . . . . . . . . & 277,596 & 38.7 \\
\hline Medicare. . . . . . . . . . . . . . . & 158,804 & 22.1 \\
\hline HMO/other prepald ${ }^{2} \ldots \ldots \ldots \ldots \ldots \ldots$ & $+38,387$ & 19.3 \\
\hline Patient pald . . . . . . . . . . . . . . . & 107,629 & 15.0 \\
\hline Medicald . . . . . . . . . . . . . . . . & 74,712 & 10.4 \\
\hline Other govemment $\ldots \ldots \ldots \ldots \ldots \ldots$ & 11,946 & 1.7 \\
\hline No charge . . . . . . . . . . . . . . . . . & 9,623 & 1.3 \\
\hline Other $\ldots \ldots \ldots \ldots \ldots \ldots \ldots \ldots \ldots$ & 25,618 & 3.6 \\
\hline 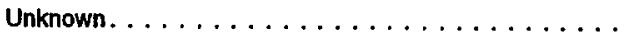 & 14,054 & 2.0 \\
\hline
\end{tabular}

'Numbers may not add to totals because more than one expected source of payment may be reported per visit.

${ }^{2} \mathrm{HMO}$ is health maintenance organization.

Table 6. Number, percent dlstribution, and annual rate of Injury-related office vlsits by patlent's age, sex, and race: United States, 1993

\begin{tabular}{|c|c|c|c|}
\hline Patlent's age, sex, and race & $\begin{array}{l}\text { Number of } \\
\text { visits in } \\
\text { thousands }\end{array}$ & $\begin{array}{c}\text { Percent } \\
\text { distribution }\end{array}$ & $\begin{array}{l}\text { Number of } \\
\text { visits per } \\
\text { too persons } \\
\text { per year }\end{array}$ \\
\hline $\begin{array}{c}\text { All injury-related visits . . . . . . . . . . } \\
\text { Age }\end{array}$ & 83,980 & 100.0 & $\mathbf{3 3 . 0}$ \\
\hline Under 15 years $\ldots \ldots \ldots \ldots \ldots$ & 11,018 & 13.1 & 19.2 \\
\hline 15-24 years. . . . . . . . . . . . . & 8,489 & 10.1 & 24.7 \\
\hline $25-44$ years. . . . . . . . . . . . . & 32,552 & 38.8 & 39.9 \\
\hline $45-64$ years. . . . . . . . . . . . & 18,148 & 21.6 & 36.5 \\
\hline $65-74$ years. . . . . . . . . . . . . . & 7,297 & 8.7 & 39.2 \\
\hline $\begin{array}{l}75 \text { years and over } \ldots \ldots \ldots \ldots \ldots \\
\qquad \text { Sex and age }\end{array}$ & 6,476 & 7.7 & 51.2 \\
\hline Female $\ldots \ldots \ldots \ldots \ldots \ldots \ldots$ & 41,156 & 49.0 & 31.5 \\
\hline Under 15 years $\ldots \ldots \ldots \ldots \ldots \ldots$ & 4,798 & 5.7 & 17.2 \\
\hline 15-24 years $\ldots \ldots \ldots \ldots \ldots \ldots \ldots$ & 3,667 & 4.4 & 21.3 \\
\hline $25-44$ years $\ldots \ldots \ldots \ldots \ldots \ldots$ & 14,979 & 17.8 & 36.2 \\
\hline 45-64 years $\ldots \ldots \ldots \ldots \ldots \ldots$ & 8,982 & 10.7 & 34.8 \\
\hline $65-74$ years . . . . . . . . . . . . & 4,078 & 4.9 & 39.8 \\
\hline 75 years and over $\ldots \ldots \ldots \ldots$ & 4,653 & 5.5 & 59.0 \\
\hline Male $\ldots \ldots \ldots \ldots \ldots \ldots \ldots$ & 42,824 & 51.0 & 34.6 \\
\hline Under 15 years $\ldots \ldots \ldots \ldots \ldots \ldots$ & 6,220 & 7.4 & 21.2 \\
\hline $15-24$ years $\ldots \ldots \ldots \ldots \ldots \ldots \ldots$ & 4,822 & 5.7 & 28.2 \\
\hline $25-44$ years $\ldots \ldots \ldots \ldots \ldots \ldots$ & 17,573 & 20.9 & 43.7 \\
\hline $45-64$ years $\ldots \ldots \ldots \ldots \ldots \ldots \ldots$ & 9,166 & 10.9 & 38.3 \\
\hline $65-74$ years $\ldots \ldots \ldots \ldots \ldots \ldots \ldots$ & 3,219 & 3.8 & 38.5 \\
\hline $\begin{array}{l}75 \text { years and over } \ldots \ldots \ldots \ldots \ldots \\
\qquad \text { Race }\end{array}$ & 1,823 & 2.2 & 38.2 \\
\hline White . . . . . . . . . . . . . . . & 75,140 & 89.5 & 35.6 \\
\hline Black . . . . . . . . . . . . . . . & 6,102 & 7.3 & 19.1 \\
\hline Asian/Paclfic Islander . . . . . . . . . . . & 2,299 & 2.7 & $\cdots$ \\
\hline American Indian/Eskimo/Aleut. . . . . . . . & $\star 439$ & 0.5 & -- \\
\hline
\end{tabular}

'Based on U.S. Bureau of the Census estimates of the civilian noninstitutionalized population of the United States as of July 1, 1993.

Classification of Diseases, 9 th revision, Clinical Modification, Volume 3,

Procedures Classification (ICD-9-CM)

(7). It was hoped that allowing

physicians to record services in this way would result in greater specificity of responses, thereby clarifying the large number of services generally recorded in the "other" checkbox category in previous versions of the survey. Data are shown separately for the checkbox items (part a of item 14) and the open-ended response categories (part b) in keeping with the format used on the Patient Record form.

Slightly less than three-quarters (73.0 percent) of all office visits included one or more tests, procedures or therapies (excluding counseling/ education and medication therapy that are collected in separate data items) (table 11). Blood pressure check was the most frequently mentioned checkbox category, recorded at half ( 49.8 percent) of the visits. Blood pressure checks were ordered or provided at a significantly higher proportion of visits by females ( 54.3 percent) than at visits by males (43.1 percent).

Other frequently mentioned services were "other" blood test ( 16.0 percent of visits) and urinalysis ( 13.5 percent). HIV serology was ordered or provided at 0.3 percent of office visits.

The top 25 diagnostic and therapeutic services (other than those reported in the checkbox categories on the Patient Record form) are shown in table 12. Pap smear, electrocardiogram, eye examinations, and routine chest $x$ rays were among the most frequently mentioned procedures. Table 13 presents data on additional procedures that, while not among the top 25 , were also of interest.

\section{Physician's principal diagnosis}

Item 11 of the Patient Record form asks the physician to record the principal diagnosis or problem associated with the patient's most important reason for the current visit as well as any other significant current diagnoses. Up to three diagnoses are coded and classified according to the ICD-9-CM (7). Displayed in table 14 are office visits by principal diagnosis using the major disease categories specified by the ICD-9-CM. The supplementary classification, used for diagnoses that are not classifiable to injury or illness (for example, general medical examination, routine prenatal examination, and health supervision of an infant or child), accounted for 15.6 percent of all office visits. Diseases of the respiratory system (13.8 percent) and diseases of the nervous system and 
Table 7. Number and percent distribution of offlce vlsits mado by patients who smoke clgarettes by pationt's age, sox, and race: United States, 1993

\begin{tabular}{|c|c|c|}
\hline Patient's age, sex, and race & $\begin{array}{l}\text { Number of } \\
\text { visits in } \\
\text { thousands }\end{array}$ & $\begin{array}{c}\text { Percent } \\
\text { dlstribution }\end{array}$ \\
\hline All vislls by patlents who smake clgarettes . . . . . . . . & 67,720 & 100.0 \\
\hline \multicolumn{3}{|l|}{ Age } \\
\hline Under 15 years . . . . . . . . . . . . . . . . . . . & *117 & ${ }^{*} 0.2$ \\
\hline$\ldots \ldots \ldots \ldots \ldots \ldots$ & 6,121 & 9.0 \\
\hline $25-44$ years. $\ldots \ldots \ldots \ldots \ldots \ldots \ldots \ldots \ldots \ldots$ & 27,692 & 40.9 \\
\hline $45-64$ years. $\ldots \ldots \ldots \ldots \ldots \ldots \ldots \ldots \ldots \ldots$ & 22,541 & 33.3 \\
\hline $65-74$ years. $\ldots \ldots \ldots \ldots \ldots \ldots \ldots \ldots \ldots \ldots$ & 8,357 & 12.3 \\
\hline 75 years and over $\ldots \ldots \ldots \ldots \ldots \ldots \ldots$ & 2,891 & 4.3 \\
\hline \multicolumn{3}{|l|}{ Sex } \\
\hline$\ldots \ldots \ldots \ldots \ldots$ & 39,928 & 59.0 \\
\hline Male $\ldots \ldots \ldots \ldots \ldots \ldots \ldots \ldots \ldots \ldots$ & 27,792 & 41.0 \\
\hline \multicolumn{3}{|l|}{ Race } \\
\hline White $\ldots \ldots \ldots \ldots \ldots \ldots \ldots \ldots \ldots \ldots$ & 59,282 & 87.5 \\
\hline 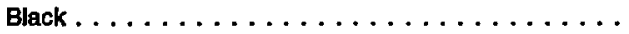 & 6,389 & 9.4 \\
\hline Asian/Paclicic lstander $\ldots \ldots \ldots \ldots \ldots \ldots \ldots \ldots$ & 1,498 & 2.2 \\
\hline American Indlan/Eskima/Alaut. $\ldots \ldots \ldots \ldots \ldots$ & "551 & 0.8 \\
\hline
\end{tabular}

sense organs (10.8 percent) were also prominent on the list.

The 20 most frequently reported principal diagnoses for 1993 are shown in table 15. These are categorized at the three-digit coding level of the ICD-9$\mathrm{CM}$, and accounted for 35.5 percent of all office visits made during the year. The most frequent diagnosis rendered by physicians at office visits in 1993 was essential hypertension, occurring at 3.9 percent of all visits. Essential hypertension has been the most frequently reported morbidity-related diagnosis in every survey year since the NAMCS began in 1973. (Morbidityrelated diagnoses are those classifiable to illness or injury. Nonmorbidity related diagnoses include routine prenatal examination, health supervision of an infant or child, and general medical examination, among others.) Of the 20 diagnoses shown in table 15,18 also appeared on the list of the 20 most frequent diagnoses for 1992.

\section{Physician's checklist of medical conditions}

In addition to the diagnostic data reported in item 11 of the Patient Record form, selected information on the patient's current health status was collected in item 13. Physicians were given a list of common conditions and asked to record whether the patient now has any of them, regardless of what was recorded as the current diagnosis in item 11. The list of conditions was modified for the 1993 NAMCS and will be expanded in the 1995 NAMCS. Results from item 13 are shown in table 16.

Slightly less than one-fifth (18.9 percent) of the visits were made by patients who were reported to have one or more of the five conditions listed on the survey form. Obesity was checked at 8.7 percent of the total, or 62.7 million office visits. Diabetes (5.6 percent), asthma ( 4.9 percent), and osteoporosis (2.5 percent) were all recorded at a greater proportion of visits in this item than as a diagnosis in item

Table 8. Number and percent dletribution of office visits by physician specialty, according to patient's cigarette-8moking status: Unlted States, 1993

\begin{tabular}{|c|c|c|c|c|c|}
\hline \multirow[b]{2}{*}{ Physician specialty } & \multirow{2}{*}{$\begin{array}{c}\text { Number of } \\
\text { visits in } \\
\text { thousands }\end{array}$} & \multicolumn{4}{|c|}{ Does patient smoke clgarettes? } \\
\hline & & Total & Yes & No & Unknown' \\
\hline & & \multicolumn{4}{|c|}{ Percent distribution } \\
\hline All visits $\ldots \ldots \ldots \ldots \ldots \ldots \ldots \ldots \ldots$ & 717,191 & 100.0 & 9.4 & 63.5 & 27.0 \\
\hline General and family practice $\ldots \ldots \ldots \ldots \ldots$ & 197,605 & 100.0 & 10.4 & 61.2 & 28.5 \\
\hline Intemal medicine. . . . . . . . . . . . . . . & 102,436 & 100.0 & 13.8 & 70.9 & 15.3 \\
\hline Pedlatrics $\ldots \ldots \ldots \ldots \ldots \ldots \ldots \ldots$ & 76,982 & 100.0 & ${ }^{\star 0} 0.6$ & 94.1 & 5.3 \\
\hline Obstetrics and gynecology . . . . . . . . . . . . . & 64,030 & 100.0 & 10.1 & 65.3 & 24.7 \\
\hline Ophthalmology ................. & 39,373 & 100.0 & 3.4 & 40.5 & 56.1 \\
\hline Orthopedic surgery. . . . . . . . . . . . . . . & 33,638 & 100.0 & 11.7 & 40.0 & 48.4 \\
\hline Dermatology. . . . . . . . . . . . . . . . . & 31,469 & 100.0 & 4.7 & 41.2 & 54.1 \\
\hline General surgery . . . . . . . . . . . . . . . . . . & 21,703 & 100.0 & 11.7 & 51.3 & 36.9 \\
\hline Psychiatry . . . . . . . . . . . . . . . & 20,469 & 100.0 & 19.3 & 60.1 & 20.5 \\
\hline Urology . . . . . . . . . . . . . . . . . . & 15,690 & 100.0 & 9.4 & 48.3 & 42.4 \\
\hline Otolaryngology $\ldots \ldots \ldots \ldots \ldots \ldots \ldots$ & 15,380 & 100.0 & 8.3 & 71.9 & 19.8 \\
\hline Cardlovascular diseases . . . . . . . . . . . . . . & 12,178 & 100.0 & 9.1 & 66.3 & 24.6 \\
\hline Allergy and immunology ${ }^{2} \ldots \ldots \ldots \ldots$ & 10,605 & 100.0 & 4.5 & 73.5 & 22.0 \\
\hline Neurology . . . . . . . . . . . . . . . . . . & 8,393 & 100.0 & 10.8 & 59.6 & 29.6 \\
\hline Pulmonary diseases ${ }^{2} \ldots \ldots \ldots \ldots \ldots \ldots$ & 4,251 & 100.0 & 12.7 & 69.1 & 18.3 \\
\hline All other speciallies . . . . . . . . . . . . . . & 62,991 & 100.0 & 11.3 & 62.7 & 26.0 \\
\hline
\end{tabular}

Includes entries of "unknown" and blank entries.

${ }^{2}$ These speciakties were sampled separately in 1993 only as part of a supplemental data collection project. 
Table 9. Number and percent distribution of office visits by patient's princlpal reason for visit: United States, 1993

\begin{tabular}{|c|c|c|}
\hline Principal reason for visti and RVC code ${ }^{1}$ & $\begin{array}{c}\text { Number of } \\
\text { vistts in } \\
\text { thousands }\end{array}$ & $\begin{array}{l}\text { Percent } \\
\text { distribution }\end{array}$ \\
\hline 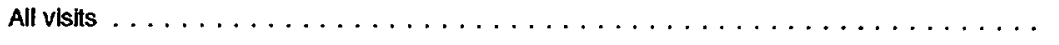 & 717,191 & 100.0 \\
\hline symptom module. . . . . . . . . . . . . . . . . . . . . . . . . . . . s001-s999 & 414,163 & 57.7 \\
\hline General symptoms. . . . . . . . . . . . . . . . . . . . . . . . . . so01-sos9 & 46,990 & 6.6 \\
\hline Symptoms referable to psychological/mental disorders . . . . . . . . . . . . . s100-s199 & 22,256 & 3.1 \\
\hline Symptoms referable to the nervous system (excluding sense organs). . . . . . $\$ 200-\$ 259$ & 22,556 & 3.1 \\
\hline Symptoms referable to the cardiovascularflymphatic system. . . . . . . . . . S260-\$299 & 3,748 & 0.5 \\
\hline Symptoms referable to the eyes and ears . . . . . . . . . . . . . . . . . S300-S399 & 51,514 & 7.2 \\
\hline Symptoms referable to the respiratory system . . . . . . . . . . . . . . S400 s499 & 83,482 & 11.6 \\
\hline symptoms referable to the dlgestive system . . . . . . . . . . . . . s500-s639 & 32,454 & 4.5 \\
\hline Symptoms referable to the genitourinary system . . . . . . . . . . . . . . . . S640-\$829 & 31,370 & 4.4 \\
\hline Symptoms referable to the skin, hair, and nalls. . . . . . . . . . . . . . . . S830-s899 & 43,130 & 6.0 \\
\hline symptoms referable to the musculoskeletal system . . . . . . . . . . . . . s900-\$s999 & 76,664 & 10.7 \\
\hline Disease module . . . . . . . . . . . . . . . . . . . . . . . . . D001-D999 & 63,981 & 8.9 \\
\hline Diagnostic/screening and preventive module. . . . . . . . . . . . . X100-X599 & 115,728 & 16.1 \\
\hline Treatment module . . . . . . . . . . . . . . . . . . . . . . . . . T100-T899 & 67,537 & 9.4 \\
\hline Injurles and adverse effects module $\ldots \ldots \ldots \ldots \ldots \ldots \ldots \ldots \ldots$. . . . . . . . . . . . . J001999 & 23,248 & 3.2 \\
\hline 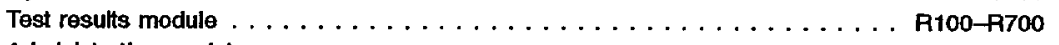 & 9,141 & 1.3 \\
\hline Administrattve module. . . . . . . . . . . . . . . . . . . A A100-A140 & 7,939 & 1.1 \\
\hline 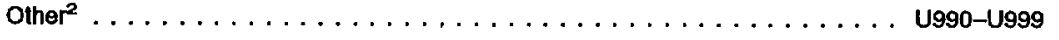 & 15,455 & 2.2 \\
\hline
\end{tabular}

'Based on A Reason for Visit Classification for Ambulatory Care (RVC) (6).

Includes problems and complaints not elsewhero classified, entries of "none," blanks, and illegible entries.

Table 10. Number and percent distribution of office visits by the 20 principal reasons for visit most frequently mentloned by patients, according to patient's sex: United States, 1993

\begin{tabular}{|c|c|c|c|c|}
\hline \multirow[b]{2}{*}{ Princlpal reason for visit and AVC code' } & \multirow{2}{*}{$\begin{array}{c}\text { Number of } \\
\text { visits in } \\
\text { thousands }\end{array}$} & \multicolumn{3}{|c|}{ Patient's sex } \\
\hline & & Total & Female & Male \\
\hline & & \multicolumn{3}{|c|}{ Percent distribution } \\
\hline 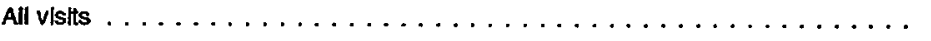 & 717,191 & 100.0 & 100.0 & 100.0 \\
\hline General medical examination $\ldots \ldots \ldots \ldots \ldots \ldots \ldots \ldots \ldots$. . . . . . . . . . . . . . . . . . & 38,185 & 5.3 & 5.7 & 4.7 \\
\hline Routine prenatal examinatlon . . . . . . . . . . . . . . . X205 & 25,893 & 3.6 & 6.0 & $\cdots$ \\
\hline 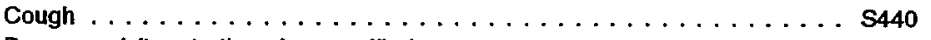 & 24,642 & 3.4 & 2.9 & 4.3 \\
\hline Progress visit, not otherwise specifled . . . . . . . . . . . . . . . . . . T800 & 20,836 & 2.9 & 2.5 & 3.6 \\
\hline Postoperathe visit . . . . . . . . . . . . . . . . . . . . . . T205 & 18,129 & 2.5 & 2.4 & 2.7 \\
\hline 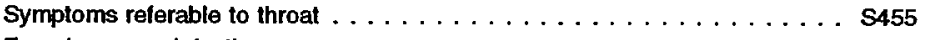 & 17,263 & 2.4 & 2.5 & 2.3 \\
\hline Earache or ear infection $\ldots \ldots \ldots \ldots \ldots \ldots \ldots \ldots$ & 16,130 & 2.2 & 2.0 & 2.6 \\
\hline Welt baby examination $\ldots \ldots \ldots \ldots \ldots \ldots \ldots \ldots \ldots$ X105 & 14,023 & 2.0 & 1.7 & 2.3 \\
\hline Stomach pain, cramps, and spasms . . . . . . . . . . . . . . . . . . \$ \$545 & 13,027 & 1.8 & 2.0 & 1.6 \\
\hline Back symptoms. . . . . . . . . . . . . . . . . . . . . . . . . . . s & 12,768 & 1.8 & 1.5 & 2.2 \\
\hline Vision dysfunctions $\ldots \ldots \ldots \ldots \ldots \ldots \ldots$. . . . . . . . . . . . . S305 & 12,416 & 1.7 & 1.9 & 1.4 \\
\hline skin rash. . . . . . . . . . . . . . . . . . . . . . . . . . s860 & 12,138 & 1.7 & 1.5 & 1.9 \\
\hline Headache, pain in head $\ldots \ldots \ldots \ldots \ldots \ldots \ldots$. . . . . . . . . . . . . . . . . . . . . . & 10,736 & 1.5 & 1.8 & 1.0 \\
\hline Head cold, upper resplratory infection (coryza) . . . . . . . . . . . . . . \$ \$445 & 10,160 & 1.4 & 1.3 & 1.5 \\
\hline Fever . . . . . . . . . . . . . . . . . . . . . . s010 & 10,006 & 1.4 & 1.2 & 1.7 \\
\hline Nasal congestlon. . . . . . . . . . . . . . . . . . . . . S400 & 9,872 & 1.4 & 1.3 & 1.5 \\
\hline Chest pain and related symptoms . . . . . . . . . . . . . . . . . so50 & 9,535 & 1.3 & 1.2 & 1.5 \\
\hline Hypertension . . . . . . . . . . . . . . . . . . . . D510 & 9,503 & 1.3 & 1.2 & 1.5 \\
\hline Knes symptoms . . . . . . . . . . . . . . . . . . . . . . . . . . S925 & 8,824 & 1.2 & 1.1 & 1.5 \\
\hline 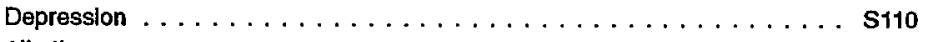 & 8,758 & 1.2 & 1.3 & 1.0 \\
\hline 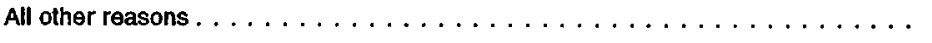 & 414,347 & 57.8 & 57.0 & 59.2 \\
\hline
\end{tabular}

'Based on A Reason for Visit Classification for Ambulatory Caro (RVC) (6).

11. It should be noted that in item 11, physicians are instructed to record up to two additional diagnoses, if any (in addition to the principal diagnosis), whether or not they are of direct concern to the current visit. The fact that higher estimates were produced by item 13 than by item 11 may indicate that chronic conditions are underreported in item 11.

\section{Medication therapy}

In item 16, physicians were instructed to record all new or continued medications ordered, supplied, or administered at the visit, including prescription and nonprescription preparations, immunization and desensitizing agents, and anesthetics. As used in the NAMCS, the term "drug" is interchangeable with the term 
Table 11. Number and percent distribution of office visits by tests, surgical and nonsurgical procedures, and theraples ordered or provided, according to patient's sex: United States, 1993

\begin{tabular}{|c|c|c|c|c|}
\hline \multirow[b]{2}{*}{ Visit characteristic } & \multirow{2}{*}{$\begin{array}{l}\text { Number of } \\
\text { visits in } \\
\text { thousands }\end{array}$} & \multicolumn{3}{|c|}{ Patient's sex } \\
\hline & & Total & Female & Male \\
\hline & & \multicolumn{3}{|c|}{ Percent dlstrbution } \\
\hline All visils $\ldots \ldots \ldots \ldots \ldots \ldots \ldots$ & 717,191 & 100.0 & 100.0 & 100.0 \\
\hline \multicolumn{5}{|l|}{ Number of services ordered or provided' } \\
\hline None . . . . . . . . . . . . . . . . . . & 191,891 & 26.8 & 24.0 & 30.9 \\
\hline 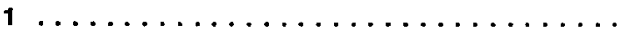 & 265,483 & 37.0 & 36.4 & 37.9 \\
\hline 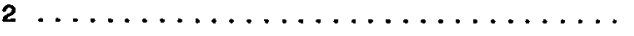 & 154,344 & 21.5 & 22.7 & 19.8 \\
\hline 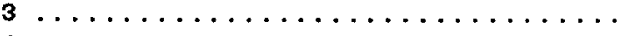 & 64,331 & 9.0 & 10.1 & 7.2 \\
\hline $4 \ldots \ldots \ldots \ldots \ldots \ldots \ldots \ldots \ldots$ & 25,400 & 3.5 & 4.2 & 2.6 \\
\hline 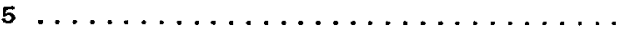 & 10,956 & 1.5 & 1.8 & 1.1 \\
\hline 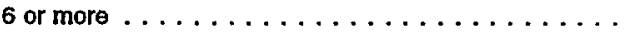 & 4,787 & 0.7 & 0.8 & 0.4 \\
\hline
\end{tabular}

\section{Selected services ${ }^{2}$}

Blood pressure . . . . . . . . . . . . .

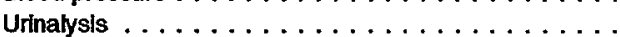

Splrometry $\ldots \ldots \ldots \ldots \ldots \ldots \ldots \ldots \ldots$

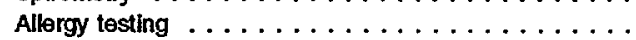

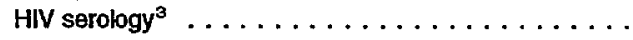

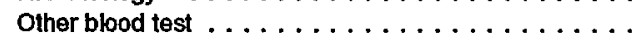

0.7

Percent of visits

'Includes the six checkbox categories for selected services and up to oight other services recorded by the physician in the spaces provided on the Patient Record form. These include tests, imagings, surgeries and other procedures, and therapies with the excoption of education/counseling and medication.

${ }^{2}$ Numbers may not add to totals because more than one service may be reported per visit.

HFIV is human immunodeficioncy virus.

Table 12. Number and percent of office visits by the 25 write-in diagnostic and therapeutic procedures most often ordered or performed: United States, 1993

\begin{tabular}{|c|c|c|}
\hline $\begin{array}{l}\text { Diagnostic and therapeutic procedures } \\
\text { ordered or performed and ICD-9-CM code }\end{array}$ & $\begin{array}{l}\text { Number of } \\
\text { visits in } \\
\text { thousands }\end{array}$ & $\begin{array}{c}\text { Percent } \\
\text { of all } \\
\text { visits }\end{array}$ \\
\hline All visits & 717,191 & $\cdots$ \\
\hline$\ldots \ldots \ldots 91.46$ & 19,613 & 2.7 \\
\hline Electrocardlogram . . . . . . . . . . . . . . . . . 89.52 & 18,539 & 2.6 \\
\hline Other nonoperative measurements and examinations . . 89.39 & 18,268 & 2.5 \\
\hline Eye examination, not otherwise specified $\ldots \ldots \ldots .95 .09$ & 17,179 & 2.4 \\
\hline 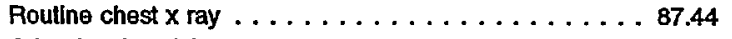 & 14,015 & 2.0 \\
\hline $\begin{array}{l}\text { Other local excision or destruction of lesion or tissue of } \\
\text { skin and subcutaneous tlssue } \ldots \ldots \ldots \ldots \ldots \ldots \ldots\end{array}$ & 13,881 & 1.9 \\
\hline $\begin{array}{l}\text { Microscopic examination of specimen from ear, nose, } \\
\text { throat, and larynx-culture } \ldots \ldots \ldots \ldots \ldots \ldots \ldots \ldots .32\end{array}$ & 12,392 & 1.7 \\
\hline Other indlvidual psychotherapy . . . . . . . . . . . . . 994.39 & 11,570 & 1.6 \\
\hline Tonometry . . . . . . . . . . . . . . . . 89.11 & 10,267 & 1.4 \\
\hline Limited eye examination . . . . . . . . . . . . . . . . 95.01 & 9,659 & 1.3 \\
\hline Other mammography . . . . . . . . . . . . . . 87.37 & 9,363 & 1.3 \\
\hline Other physical therapy . . . . . . . . . . . . . . . . . 93.39 & 7,313 & 1.0 \\
\hline General physical examination . . . . . . . . . . . . 89.7 & 6,562 & 1.0 \\
\hline Gynecological examination $\ldots \ldots \ldots \ldots \ldots . \ldots . . .29$ & 5,650 & 0.8 \\
\hline 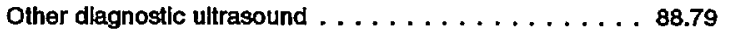 & 4,506 & 0.6 \\
\hline Audiometry $\ldots \ldots \ldots \ldots \ldots \ldots \ldots \ldots \ldots \ldots \ldots 9.41$ & 4,218 & 0.6 \\
\hline Dlagnostic ultrasound of gravid uterus $\ldots \ldots \ldots \ldots$. . . 88.78 & 4,198 & 0.6 \\
\hline Skeletal $x$ ray of wrist and hand $\ldots \ldots \ldots \ldots$. . . . 88.23 & 4,149 & 0.6 \\
\hline Skeletal $x$ ray of thigh, knee, and lower leg $\ldots \ldots \ldots .88 .27$ & 3,978 & 0.6 \\
\hline 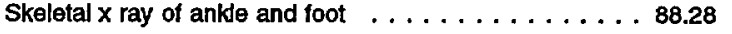 & 3,881 & 0.5 \\
\hline Removal of other therapeutic device & 3,355 & 0.5 \\
\hline$\ldots \ldots \ldots \ldots \ldots$ & 3,331 & 0.5 \\
\hline Manual examination of breast & 2,964 & 0.4 \\
\hline Fetal monitoring, not otherwise specified $\ldots \ldots \ldots$ & 2,943 & 0.4 \\
\hline$X$ ray, other and unspeciffed $\ldots \ldots \ldots \ldots$ & 2,873 & 0.4 \\
\hline
\end{tabular}

Based on the Internationa/ Classification of Diseases, Sth Revision, Clinical Modification (ICD-9-CM) (7). "medication," and the term

"prescribing" is used broadly to mean ordering or providing any medication, whether prescription or over-the-counter. Visits with one or more drug mentions are termed "drug visits" in the NAMCS. Up to five medications, or drug mentions, were coded per drug visit.

The NAMCS drug data base permits classification by a wide range of variables, including specific product name, generic class, entry form chosen by the physician (that is, brand name, generic name, or the desired therapeutic effect), prescription status (that is, whether the product is prescription or nonprescription), federally controlled substance status, composition status (that is, single or multiple ingredient product), and therapeutic category. A report describing the method and instruments used to collect and process drug information for the NAMCS is available (8).

Data on medication therapy are shown in tables 17-21 and figure 2. Medication therapy was the most commonly mentioned therapeutic service 
Table 13. Number and percent of office visits by selected diagnostic and therapeutic procedures: Unlted States, 1993

\begin{tabular}{|c|c|c|}
\hline $\begin{array}{l}\text { Selected procedures and } \\
\qquad I C D-9-C M \text { code }\end{array}$ & $\begin{array}{c}\text { Number of } \\
\text { visits in } \\
\text { thousands }\end{array}$ & $\begin{array}{c}\text { Percent } \\
\text { of all } \\
\text { visits }\end{array}$ \\
\hline 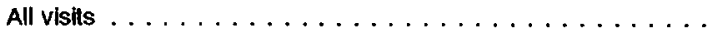 & 717,191 & $\ldots$ \\
\hline$\ldots \ldots \ldots \ldots \ldots \ldots \ldots \ldots . \ldots \ldots .21$ & 1,690 & 0.2 \\
\hline Other endoscopy of small intestine $\ldots \ldots \ldots \ldots . \ldots 4.13$ & 1,709 & 0.2 \\
\hline Colonoscopy . . . . . . . . . . . . . . . . . . 45.23 & 955 & 0.1 \\
\hline Flexible sigmoldoscopy $\ldots \ldots \ldots$. . . . . . . . . . 45.24 & 1,446 & 0.2 \\
\hline Other cystoscopy . . . . . . . . . . . . . . 57.32 & 1,730 & 0.2 \\
\hline Closed blopsy of uterus $\ldots \ldots \ldots \ldots \ldots . \ldots \ldots$ & 1,259 & 0.2 \\
\hline $\begin{array}{l}\text { Vaginoscopy } \ldots \ldots \ldots \ldots \ldots \ldots \ldots . \ldots \ldots \ldots \\
\text { Injection of therapeutic substance into joint or }\end{array}$ & 1,057 & 0.1 \\
\hline $\begin{array}{l}\text { ligament } \ldots \ldots \ldots \\
\text { Other inclsion with drainage of skin and subcutaneous }\end{array}$ & 1,368 & 0.2 \\
\hline tlssue . . . . . . . . . . . . . . . . . . . . 886.04 & 1,340 & 0.2 \\
\hline Biopsy of skin and subcutaneous tissue ........ 86.11 & 2,687 & 0.4 \\
\hline Application of other cast . . . . . . . . . . . 93.53 & 1,323 & 0.2 \\
\hline Application of splint . . . . . . . . . . . . . . 93.54 & 1,514 & 0.2 \\
\hline Irrigation of ear $\ldots \ldots \ldots \ldots \ldots \ldots . \ldots \ldots \ldots$ & 2,745 & 0.4 \\
\hline
\end{tabular}

${ }^{1}$ Based on the Intomational Classification of Diseases, Sth Rovision, Clinical Modification (ICD-9-CM) (7).

Table 14. Number and percent distribution of office visits by physician's princlpal dlagnosis: United States, 1993

\begin{tabular}{|c|c|c|}
\hline $\begin{array}{l}\text { Princlpal dlagnosis and } \\
\text { ICD-9-CM code }\end{array}$ & $\begin{array}{c}\text { Number of } \\
\text { visits in } \\
\text { thousands }\end{array}$ & $\begin{array}{c}\text { Percent } \\
\text { distrlbution }\end{array}$ \\
\hline 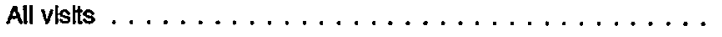 & 717,191 & 100.0 \\
\hline Infectious and paraslllc diseases $\ldots \ldots \ldots \ldots$. . . . 001-139 & 21,828 & 3.0 \\
\hline $\begin{array}{l}\text { Neoplasms } \ldots \ldots \ldots \ldots \ldots \ldots \ldots \ldots \text {. . . . . . . . . . 140-239 } \\
\text { Endocrine, nutrltional and metabolic dlseases and }\end{array}$ & 21,876 & 3.1 \\
\hline immunity dlsorders $\ldots \ldots \ldots \ldots \ldots \ldots$. . . . . . . . . . & 25,428 & 3.5 \\
\hline $\begin{array}{l}\text { Mental disorders . . . . . . . . . . . . . . 290-319 } \\
\text { Dlseases of the nervous system and sense }\end{array}$ & 33,613 & 4.7 \\
\hline organs . . . . . . . . . . . . . . . . 320-389 & 77,737 & 10.8 \\
\hline Diseases of the clrculatory system ... . . . . . 390-459 & 57,564 & 8.0 \\
\hline Diseases of the respiratory system . . . . . . . . 460-519 & 99,114 & 13.8 \\
\hline Diseases of the digestive system & 27,651 & 3.9 \\
\hline $\begin{array}{l}\text { Diseases of the genitourinary system . . . . . . . . 580-629 } \\
\text { Diseases of the skin and subcutaneous }\end{array}$ & 41,281 & 5.8 \\
\hline 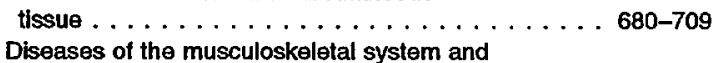 & 42,771 & 6.0 \\
\hline connectlve tissue $\ldots \ldots \ldots \ldots \ldots \ldots \ldots \ldots \ldots$ & 51,910 & 7.2 \\
\hline Symptoms, signs, and ill-defined conditions . . . . . . 780-799 & 32,503 & 4.5 \\
\hline Injury and poisoning . . . . . . . . . . . 800-999 & 46,161 & 6.4 \\
\hline Supplementary classification $\ldots \ldots \ldots$. . . . . V01-V82 & 112,087 & 15.6 \\
\hline All other diagnoses ${ }^{2} \ldots \ldots \ldots \ldots \ldots \ldots$ & 8,554 & 1.2 \\
\hline Unknown $^{3} \ldots \ldots \ldots \ldots \ldots \ldots$ & 17,112 & 2.4 \\
\hline
\end{tabular}

'Based on the Intemational Classification of Diseases, 9th Revision, Clinical Modification (ICD-9-CM) (7).

Includes diseases of the blood and blood-forming organs (280-289); complications of pregnancy, childbirth, and the puerperium (630-676); congenital anomalies (740-759); and cortain conditions originating in the perinatal period (760-779).

${ }^{3}$ Includes blank diagnoses, uncodable diagnoses, and illegible diagnoses.

in 1993, reported at 467.3 million office visits or 65.2 percent of the total (table 17).

There were 913.5 million drug mentions at visits to office-based physicians during 1993. This yields an average of 1.3 drug mentions per office visit, or 2.0 drug mentions per drug visit.

Data on number of drug visits and drug mentions by physician specialty are shown in table 18 . Nine of every 10 visits to allergists and immunologists included at least one drug mention, as did 8 of every 10 visits to internists.

Drug mentions are displayed by therapeutic class in figure 2 and table 19. This classification is based on the therapeutic categories used in the National Drug Code Directory, 1985 edition (NDC) (9). It should be noted that some drugs have more than one therapeutic application. In these cases, the drug was listed under the NDC classification that occurred with the greatest frequency.

Cardiovascular-renal drugs (14.0 percent), antimicrobial agents (13.9 percent), and drugs used for pain relief (11.0 percent) were listed most frequently. About one-third (31.6 percent) of all mentions of antimicrobial agents were at visits made by persons under 15 years, and about two-thirds ( 69.4 percent) of the mentions of immunologic agents were at visits by this age group. Four of every 10 neurologic drug mentions (41.1 percent) occurred at visits by persons $25-44$ years.

The 20 most frequently used generic substances for 1993 are shown in table 20. Drug products containing more than one ingredient (combination products) are included in the data for each ingredient. For example, acetaminophen with codeine is included in both the count for acetaminophen and the count for codeine. Amoxicillin was the generic ingredient most frequently used in drugs ordered or provided by the physician at office visits in 1993 (as well as in 1990-92), occurring in 3.9 percent of drug mentions.

Table 21 presents the 20 medications most frequently mentioned by physicians in the NAMCS, according to the entry name of drug. Entry name refers to the actual designation used by the physician on the Patient Record form and may be a trade name, generic name, or simply a desired therapeutic effect. Amoxicillin was the medication most frequently reported by physicians, with 19.2 million mentions (2.1 percent of the total). It was followed by Tylenol, Premarin, Lasix, Amoxil, and Prednisone, each accounting for 1.2 percent of the total. All of these were among the top 10 drug entry names mentioned in 1992.

\section{Counseling and education}

Data on counseling and education services ordered or provided at physicians' office visits were collected 
Table 15. Number and percent distribution of office visits by the 20 principal dlagnoses most frequently rendered by phyelcians, accordlng to patient's sex: Unitod States, 1993

\begin{tabular}{|c|c|c|c|c|}
\hline \multirow[b]{2}{*}{ Princlpal dlagnosis and ICD-9-CM code ${ }^{1}$} & \multirow{2}{*}{$\begin{array}{l}\text { Number of } \\
\text { visits in } \\
\text { thousands }\end{array}$} & \multicolumn{3}{|c|}{ Patient's sax } \\
\hline & & Total & Female & Male \\
\hline & & \multicolumn{3}{|c|}{ Percent distribution } \\
\hline 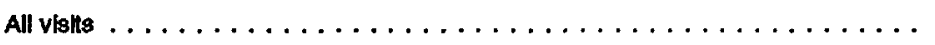 & 717,191 & 100.0 & 100.0 & 100.0 \\
\hline$\ldots \ldots \ldots \ldots \ldots \ldots 4$ & 28,124 & 3.9 & 3.9 & 3.9 \\
\hline Normal pregnancy $\ldots \ldots \ldots \ldots \ldots \ldots \ldots \ldots \ldots \ldots$ va2 & 26,489 & 3.7 & 6.2 & $\ldots$ \\
\hline Suppurative and unspectifled otitls medla $\ldots \ldots \ldots \ldots \ldots \ldots \ldots$ & 19,309 & 2.7 & 2.0 & 3.7 \\
\hline General medical examination $\ldots \ldots \ldots \ldots \ldots \ldots \ldots \ldots \ldots$. . . . . . . . & 19,065 & 2.7 & 2.4 & 3.0 \\
\hline Health supervision of intant or child $\ldots \ldots \ldots \ldots \ldots \ldots \ldots \ldots$ V20 & 18,508 & 2.6 & 2.2 & 3.2 \\
\hline Acute upper respiratory infections of mulliple or unspecifled sittes $\ldots \ldots \ldots 465$ & 17,557 & 2.4 & 2.2 & 2.8 \\
\hline 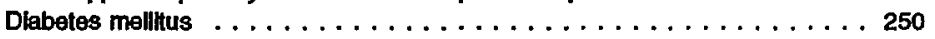 & 12,997 & 1.8 & 1.7 & 2.0 \\
\hline Chronlc sinusitts $\ldots \ldots \ldots \ldots \ldots \ldots \ldots \ldots \ldots \ldots \ldots \ldots \ldots$ & 11,594 & 1.6 & 1.8 & 1.3 \\
\hline Asthma $\ldots \ldots \ldots \ldots \ldots \ldots \ldots \ldots \ldots \ldots \ldots \ldots \ldots \ldots \ldots \ldots \ldots, 496$ & 11,340 & 1.6 & 1.6 & 1.5 \\
\hline 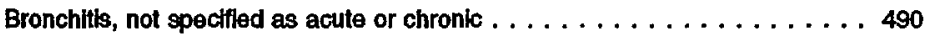 & 10,093 & 1.4 & 1.3 & 1.5 \\
\hline Allergle rhinttls $\ldots \ldots \ldots \ldots \ldots \ldots \ldots \ldots \ldots \ldots \ldots \ldots \ldots$ & 9,637 & 1.3 & 1.4 & 1.3 \\
\hline Acute pharyngitts $\ldots \ldots \ldots \ldots \ldots \ldots \ldots \ldots \ldots \ldots \ldots \ldots \ldots$ & 9,576 & 1.3 & 1.3 & 1.3 \\
\hline 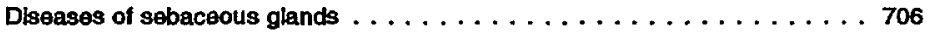 & 9,193 & 1.3 & 1.3 & 1.3 \\
\hline Neurotle dlsorders . . . . . . . . . . . . . . . . . . . . . 300 & 8,532 & 1.2 & 1.1 & 1.3 \\
\hline Other postsurgical states $\ldots \ldots \ldots \ldots \ldots \ldots \ldots \ldots \ldots \ldots$ V45 & 7,880 & 1.1 & 1.2 & 1.0 \\
\hline Affective psychoses $\ldots \ldots \ldots \ldots \ldots \ldots \ldots \ldots \ldots \ldots \ldots \ldots \ldots \ldots \ldots 296$ & 7,351 & 1.0 & 1.1 & 0.9 \\
\hline Spectal Investigations and examinations $\ldots \ldots \ldots \ldots \ldots \ldots \ldots \ldots \ldots$ v72 & 7,111 & 1.0 & 1.5 & 0.3 \\
\hline 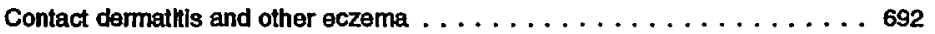 & 6,919 & 1.0 & 0.8 & 1.2 \\
\hline Osteoarthrosis and allied dlsorders $\ldots \ldots \ldots \ldots \ldots \ldots \ldots \ldots \ldots 715$ & 6,890 & 1.0 & 1.0 & 0.9 \\
\hline 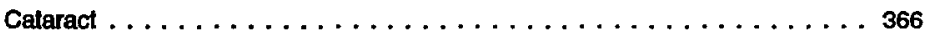 & 6,739 & 0.9 & 1.0 & 0.8 \\
\hline All other dlagnoses $\ldots \ldots \ldots \ldots \ldots \ldots \ldots \ldots \ldots \ldots \ldots$ & 462,287 & 64.5 & 63.0 & 66.8 \\
\hline
\end{tabular}

'Based on the Intemational Classification of Disoasos, 9th Revision, Clinical Modification (ICD-O-CM) (7).

Table 16. Number and percent of office visits by selected medical conditions, according to patient's age and sex: United States, 1993

\begin{tabular}{|c|c|c|c|c|c|c|c|c|c|}
\hline \multirow[b]{2}{*}{ Medical condttion 1} & \multirow[b]{2}{*}{$\begin{array}{c}\text { All ages, } \\
\text { both sexes }\end{array}$} & \multicolumn{6}{|c|}{ Patient's age } & \multicolumn{2}{|c|}{ Patient's sex } \\
\hline & & $\begin{array}{l}\text { Under } \\
15 \text { years }\end{array}$ & $\begin{array}{l}15-24 \\
\text { years }\end{array}$ & $\begin{array}{l}25-44 \\
\text { years }\end{array}$ & $\begin{array}{l}45-64 \\
\text { years }\end{array}$ & $\begin{array}{l}65-74 \\
\text { years }\end{array}$ & $\begin{array}{l}75 \text { years } \\
\text { and over }\end{array}$ & Femala & Mals \\
\hline & \multicolumn{9}{|c|}{ Number of visits in thousands } \\
\hline All vishs $\ldots \ldots \ldots \ldots \ldots \ldots \ldots$ & 717,191 & 129,279 & 62,346 & 193,914 & 160,146 & 93,873 & 77,633 & 430,170 & 287,021 \\
\hline Obesty $\ldots \ldots \ldots \ldots \ldots \ldots \ldots \ldots$ & 62,707 & 2,286 & 3,580 & 16,896 & 23,508 & 11,145 & 5,291 & 43,171 & 19,536 \\
\hline Diabetes $\ldots \ldots \ldots \ldots \ldots \ldots \ldots$ & 40,358 & 2229 & 765 & 4,692 & 14,366 & 12,496 & 7,810 & 22,992 & 17,366 \\
\hline Asthma $\ldots \ldots \ldots \ldots \ldots \ldots \ldots$ & 35,154 & 9,098 & 2,771 & 8,946 & 7,214 & 4,258 & 2,865 & 20,634 & 14,520 \\
\hline Osteoporosis & 17,752 & "262 & "6 & *399 & 2,388 & 5,931 & 8,766 & 15,049 & 2,703 \\
\hline$\ldots \ldots \ldots \ldots \ldots \ldots$ & 1,343 & $\ldots$ & ${ }^{*} 120$ & 939 & \#212 & 73 & $\ldots$ & *503 & 840 \\
\hline \multirow[t]{2}{*}{ None of the above } & 581,632 & 117,672 & 55,416 & 164,986 & 120,028 & 67,012 & 56,518 & 343,225 & 238,407 \\
\hline & \multicolumn{9}{|c|}{ Percent of vislts } \\
\hline$\ldots \ldots \ldots$ & $\cdots$ & $\cdots$ & $\ldots$ & $\cdots$ & $\cdots$ & $\cdots$ & $\cdots$ & .. & $\cdots$ \\
\hline Obeshy .. & 8.7 & 1.8 & 5.7 & 8.7 & 14.7 & 11.9 & 6.8 & 10.0 & 6.8 \\
\hline Dlabetes $\ldots \ldots \ldots \ldots \ldots \ldots \ldots$ & 5.6 & ${ }^{*} 0.2$ & $" 1.2$ & 2.4 & 9.0 & 13.3 & 10.1 & 5.3 & 6.1 \\
\hline Asthma $\ldots \ldots \ldots \ldots \ldots \ldots \ldots$ & 4.9 & 7.0 & 4.4 & 4.6 & 4.5 & 4.5 & 3.7 & 4.8 & 5.1 \\
\hline Osteoporosis $\ldots \ldots \ldots \ldots \ldots \ldots \ldots$ & 2.5 & 0.2 & 0.0 & ${ }^{*} 0.2$ & 1.5 & 6.3 & 11.3 & 3.5 & 0.9 \\
\hline$H_{V}{ }^{2} \ldots \ldots \ldots \ldots \ldots \ldots \ldots$ & 0.2 & $\ldots$ & "0.2 & 0.5 & ${ }^{\star} 0.1$ & ${ }^{\star 0} 0.1$ & $\ldots$ & ${ }^{*} 0.1$ & 0.3 \\
\hline None of the above $\ldots \ldots \ldots \ldots \ldots \ldots$ & 81.1 & 91.0 & 88.9 & 85.1 & 74.9 & 71.4 & 72.8 & 79.8 & 83.1 \\
\hline
\end{tabular}

'Numbers may not add to totale becausa moro than one condition may be reported per visit.

2HIV is human immunodeficiency virus.

in item 15 of the Patient Record form. As shown in table 22, counseling and education services were recorded at about half ( 48.5 percent) of all office visits during 1993. Exercise
( 9.0 percent), weight reduction

(5.7 percent), and growth/development (4.2 percent) were mentioned most frequently. One-third of visits $(34.2$ percent) included "other" counseling not included in one of the nine checkbox categories.

The counseling and education categories of injury prevention, HIV transmission, and other STD 
Table 17. Number and percent distribution of office visits by medication therapy and number of medications provided or preseribed, according to patlent's sex: United States, 1993

\begin{tabular}{|c|c|c|c|c|}
\hline \multirow[b]{2}{*}{ Visit characteristic } & \multirow{2}{*}{$\begin{array}{l}\text { Number of } \\
\text { visits in } \\
\text { thousands }\end{array}$} & \multicolumn{3}{|c|}{ Patient's sex } \\
\hline & & Total & Female & Male \\
\hline & & \multicolumn{3}{|c|}{ Percent dlstrbution } \\
\hline \multicolumn{5}{|l|}{ Medlcatlon therapy ${ }^{1}$} \\
\hline$\ldots \ldots \ldots \ldots$ & 717,191 & 100.0 & 100.0 & 100.0 \\
\hline Drug visits ${ }^{2}$ & 467,301 & 65.2 & 65.0 & 65.5 \\
\hline Visits without mention of medication . . . . . . & 249,890 & 34.8 & 35.0 & 34.5 \\
\hline \multicolumn{5}{|l|}{$\begin{array}{l}\text { Number of medications } \\
\text { provided or prescribed by physictan }\end{array}$} \\
\hline All visits $\ldots \ldots \ldots \ldots \ldots \ldots \ldots \ldots \ldots$ & 717,191 & 100.0 & 100.0 & 100.0 \\
\hline None & 249,890 & 34.8 & 35.0 & 34.5 \\
\hline 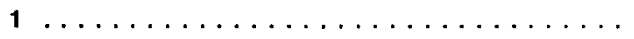 & 226,541 & 31.6 & 30.8 & 32.8 \\
\hline $2 \ldots \ldots \ldots \ldots \ldots \ldots \ldots \ldots \ldots$ & 124,634 & 17.4 & 17.4 & 17.4 \\
\hline 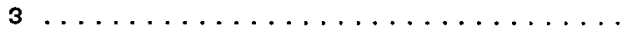 & 56,803 & 7.9 & 7.9 & 7.9 \\
\hline 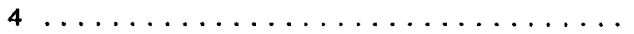 & 29,329 & 4.1 & 4.5 & 3.5 \\
\hline 5 or more $\ldots \ldots \ldots \ldots \ldots \ldots \ldots \ldots \ldots$ & 29,994 & 4.2 & 4.4 & 3.9 \\
\hline
\end{tabular}

Includes prescription drugs, over-the-counter preparations, immunizing agents, and desensitizing agants.

Visits at which one or more drugs were provided or proscribed by the physician.

Table 18. Number and percent distribution of drug visits and drug mentions by physiclan speclalty: United States, 1993

\begin{tabular}{|c|c|c|c|c|c|}
\hline Physician specialty & $\begin{array}{c}\text { Number of } \\
\text { drug visits } \\
\text { in thousands }{ }^{1}\end{array}$ & $\begin{array}{c}\text { Percent } \\
\text { distribution }\end{array}$ & $\begin{array}{l}\text { Number of } \\
\text { drug mentions } \\
\text { in thousands }\end{array}$ & $\begin{array}{c}\text { Percent } \\
\text { distilbution }\end{array}$ & $\begin{array}{l}\text { Percent } \\
\text { of ding } \\
\text { visits }^{2}\end{array}$ \\
\hline All specialtles $\ldots \ldots \ldots \ldots \ldots \ldots \ldots \ldots$ & 467,301 & 100.0 & 913,503 & 100.0 & 65.2 \\
\hline General and famlly practice . . . . . . . . . . . . & 147,257 & 31.5 & 296,201 & 32.4 & $\mathbf{7 4 . 5}$ \\
\hline Intemal medicine $\ldots \ldots \ldots \ldots \ldots \ldots$ & 81,874 & 17.5 & 187,379 & 20.5 & 79.9 \\
\hline Pedlatrics . . . . . . . . . . . . . . . & 54,773 & 11.7 & 89,594 & 9.8 & 71.2 \\
\hline Obstetrics and gynecology $\ldots \ldots \ldots \ldots$ & 29,736 & 6.4 & 44,818 & 4.9 & 46.4 \\
\hline Dermatology . . . . . . . . . . . . . . . & 21,255 & 4.5 & 38,635 & 4.2 & 67.5 \\
\hline Ophthalmology $\ldots \ldots \ldots \ldots \ldots \ldots \ldots$ & 19,230 & 4.1 & 33,686 & 3.7 & 48.8 \\
\hline Psychlatry . . . . . . . . . . . . . . & 15,161 & 3.2 & 30,379 & 3.3 & 74.1 \\
\hline Orthopedic surgery $\ldots \ldots \ldots \ldots \ldots$ & 11,783 & 2.5 & 17,656 & 1.9 & $\mathbf{3 5 . 0}$ \\
\hline Allergy and immunology ${ }^{3} \ldots \ldots \ldots \ldots \ldots$ & $\mathbf{9 , 8 6 1}$ & 2.1 & 20,738 & 2.3 & 93.0 \\
\hline Cardlovascular diseases $\ldots \ldots \ldots \ldots \ldots$ & 8,614 & 1.8 & 24,800 & 2.7 & 70.7 \\
\hline Otolaryngology $\ldots \ldots \ldots \ldots \ldots \ldots \ldots$ & 7,949 & 1.7 & 12,945 & 1.4 & 51.7 \\
\hline General surgery $\ldots \ldots \ldots \ldots \ldots \ldots$ & 7,189 & 1.5 & 12,908 & 1.4 & 33.1 \\
\hline Urology $\ldots \ldots \ldots \ldots \ldots \ldots \ldots$ & 6,350 & 1.4 & 8,611 & 0.9 & 40.5 \\
\hline Neurology . . . . . . . . . . . . . . & 4,953 & 1.1 & 9,356 & 1.0 & 59.0 \\
\hline 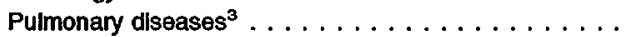 & 3,312 & 0.7 & 9,743 & 1.1 & 77.9 \\
\hline All other specialties $\ldots \ldots \ldots \ldots \ldots \ldots \ldots$ & 38,006 & 8.1 & 76,054 & 8.3 & 60.3 \\
\hline
\end{tabular}

${ }^{1}$ Visits at which one or more drugs were provided or prescribed by the physician.

${ }^{2}$ Number of drug visits divided by number of office visits multiplled by 100 .

${ }^{3}$ These specialties were sampled separately in 1993 only as part of a supplemental data collection project.

transmission were added to the 1993 Patient Record form. Such services were ordered or provided at 2.6 percent, 1.3 percent, and 1.4 percent of visits, respectively.

\section{Disposition of visit}

Two-thirds of office visits (66.7 percent) included a scheduled followup visit or telephone call in 1993.
One-quarter (23.3 percent) of office visits included instructions to return if needed. Less than 1 percent of visits resulted in a hospital admission. Table 23 displays data on disposition of office visits.

\section{Duration of visit}

Data on the duration of office visits is presented in table 24. Duration of visit refers to the amount of time spent in face-to-face contact between the physician and the patient. This time is estimated and recorded by the physician and does not include time spent waiting to see the physician, time spent receiving care from someone other than the physician without the presence of the physician, or time spent by the physician in reviewing patient records 
Table 19. Number and percent distrlbution of drug mentions by patient's age, according to therapeutic classification: United States, 1993

\begin{tabular}{|c|c|c|c|c|c|c|c|c|}
\hline \multirow[b]{2}{*}{ Therapeutic classsffication ${ }^{1}$} & \multirow{2}{*}{$\begin{array}{l}\text { Number of } \\
\text { drug mentions } \\
\text { in thousands }\end{array}$} & \multirow[b]{2}{*}{ Total } & \multicolumn{6}{|c|}{ Patient's age } \\
\hline & & & $\begin{array}{c}\text { Under } 15 \\
\text { years }\end{array}$ & $\begin{array}{l}15-24 \\
\text { years }\end{array}$ & $\begin{array}{l}25-44 \\
\text { years }\end{array}$ & $\begin{array}{l}45-64 \\
\text { years }\end{array}$ & $\begin{array}{l}65-74 \\
\text { years }\end{array}$ & $\begin{array}{l}75 \text { years } \\
\text { and over }\end{array}$ \\
\hline & & \multicolumn{7}{|c|}{ Percent distrbution } \\
\hline All drug mentlons & 913,503 & 100.0 & 15.1 & 6.7 & 22.8 & 24.7 & 15.9 & 14.9 \\
\hline Cardlovascular-renal drugs & 127,549 & 100.0 & 0.7 & 0.7 & 7.7 & 29.3 & 28.2 & 33.4 \\
\hline Antimicroblal agents $\ldots \ldots \ldots \ldots \ldots \ldots \ldots$ & 127,190 & 100.0 & 31.6 & 10.6 & 25.7 & 18.6 & 8.0 & 5.6 \\
\hline Drugs used for rellef of pain $\ldots \ldots \ldots \ldots \ldots \ldots \ldots \ldots$ & 100,898 & 100.0 & 9.0 & 5.2 & 29.9 & 26.6 & 15.7 & 13.6 \\
\hline Respiratory tract drugs $\ldots \ldots \ldots \ldots \ldots \ldots \ldots$ & 87,751 & 100.0 & 26.3 & 8.7 & 26.6 & 21.2 & 10.1 & 7.2 \\
\hline Hormones and agenls affecting hormonal mechanisms $\ldots \ldots \ldots$ & 85,421 & 100.0 & 4.2 & 5.2 & 21.3 & 36.6 & 20.1 & 12.7 \\
\hline Psychopharmacologk drugs $\ldots \ldots \ldots \ldots \ldots \ldots \ldots \ldots$ & 62,592 & 100.0 & 5.6 & 4.9 & 33.1 & 31.0 & 14.5 & 10.9 \\
\hline Skin/mucous membrane $\ldots \ldots \ldots \ldots \ldots \ldots \ldots$ & 54,551 & 100.0 & 14.5 & 16.5 & 29.7 & 20.9 & 10.1 & 8.2 \\
\hline Metabollc and nutrlent agents $\ldots \ldots \ldots \ldots \ldots \ldots$ & 43,427 & 100.0 & 8.3 & 11.2 & 25.8 & 19.5 & 17.1 & 18.2 \\
\hline Immunologic agents $\ldots \ldots \ldots \ldots \ldots \ldots \ldots \ldots \ldots \ldots$ & 39,732 & 100.0 & 69.4 & 2.3 & 6.2 & 6.8 & 7.6 & 7.7 \\
\hline Gastrointestinal agents $\ldots \ldots \ldots \ldots \ldots \ldots \ldots \ldots \ldots \ldots$ & $\mathbf{3 8 , 6 5 8}$ & 100.0 & 4.3 & 4.3 & 23.5 & 28.4 & 21.4 & 18.1 \\
\hline Ophthalmic drugs $\ldots \ldots \ldots \ldots \ldots \ldots \ldots \ldots \ldots$ & 31,320 & 100.0 & 8.9 & "3.6 & 13.9 & 19.0 & 23.9 & 30.6 \\
\hline Neurologic drugs $\ldots \ldots \ldots \ldots \ldots \ldots \ldots \ldots \ldots \ldots$ & 20,418 & 100.0 & *3.5 & ${ }^{\star 5.0}$ & 41.1 & 27.5 & 10.9 & 12.0 \\
\hline Hematologle agents $\ldots \ldots \ldots \ldots \ldots \ldots \ldots \ldots \ldots \ldots$ & 16,219 & 100.0 & “3.2 & 12.2 & 18.6 & 18.6 & 20.6 & 26.7 \\
\hline Other and unclassified $d^{2} \ldots \ldots \ldots \ldots \ldots \ldots \ldots \ldots \ldots$ & 77,777 & 100.0 & 16.6 & 7.6 & 23.8 & 26.2 & 13.7 & 12.0 \\
\hline
\end{tabular}

Gased on the standard drug classification used in the National Drug Codo Directory, 1985 edition (NDC) (9).

Inciudes anesthestics, antidotes, radiopharmaceuticala/contrast media, oncolytics, otologics, antiparasitics, and unclassified/miscellaneous drugs.

and/or test results. In cases where the patient received care from a member of the physician's staff but did not actually see the physician during the visit, duration was recorded as " 0 " minutes.

Nearly two-thirds (63.5 percent) of physicians' office visits had a duration of 15 minutes or less in 1993. The mean duration time for all visits was $\mathbf{1 8 . 4}$ minutes. Corresponding numbers for 1992 were 66.6 percent and 17.6 minutes, respectively.

Additional reports utilizing 1993 NAMCS data are forthcoming in the Advance data from Vital and Health Statistics series. Data from the 1993 NAMCS will be available on computer tape and CD-ROM from the National Technical Information Service in early 1996. Questions regarding this report, future reports, or the NAMCS may be directed to the Ambulatory Care Statistics Branch by calling (301) 436-7132.

\section{References}

1. Nelson C, McLemore T. The National Ambulatory Medical Care Survey. United States, 1975-81 and 1985 trends. National Center for Health Statistics. Vital Health Stat 13(93). 1988.

2. Schappert, SM. National Ambulatory Medical Care Survey: 1989

Table 20. The 20 most frequently occurring generic substances in drug mentions at offlce visits by number of occurrences and percent of all drug mentions: United State6, 1993

\begin{tabular}{|c|c|c|}
\hline Generic substance & $\begin{array}{l}\text { Number of } \\
\text { occurrences } \\
\text { in thousands' }\end{array}$ & $\begin{array}{l}\text { Percent of } \\
\text { all drug } \\
\text { mentions }\end{array}$ \\
\hline All generlc substances $\ldots \ldots \ldots \ldots \ldots \ldots \ldots$ & $1,080,968$ & $\cdots$ \\
\hline$\ldots \ldots \ldots \ldots \ldots \ldots \ldots \ldots \ldots$ & 35,234 & 3.9 \\
\hline Acetaminophen $\ldots \ldots \ldots \ldots \ldots \ldots \ldots \ldots$ & 34,277 & 3.8 \\
\hline Hydrochlorolhlazide $\ldots \ldots \ldots \ldots \ldots \ldots \ldots \ldots$ & 15,217 & 1.7 \\
\hline Albutemol $\ldots \ldots \ldots \ldots \ldots \ldots \ldots \ldots \ldots$ & 14,943 & 1.6 \\
\hline Ibuprofen $\ldots \ldots \ldots \ldots \ldots \ldots \ldots \ldots \ldots \ldots$ & 14,405 & 1.6 \\
\hline Multivitamins-general $\ldots \ldots \ldots \ldots \ldots \ldots \ldots$ & 14,064 & 1.5 \\
\hline Erythromycin $\ldots \ldots \ldots \ldots \ldots \ldots \ldots \ldots$ & 13,459 & 1.5 \\
\hline Asplrin $\ldots \ldots \ldots \ldots \ldots \ldots \ldots \ldots \ldots$ & 13,293 & 1.5 \\
\hline Phenylephrine $\ldots \ldots \ldots \ldots \ldots \ldots \ldots \ldots$ & 12,568 & 1.4 \\
\hline Gualfenesin $\ldots \ldots \ldots \ldots \ldots \ldots \ldots \ldots$ & 11,727 & 1.3 \\
\hline Estrogens $\ldots \ldots \ldots \ldots \ldots \ldots \ldots \ldots \ldots$ & 11,660 & 1.3 \\
\hline Furosemide $\ldots \ldots \ldots \ldots \ldots \ldots \ldots \ldots \ldots$ & 11,212 & 1.2 \\
\hline Prednisone $\ldots \ldots \ldots \ldots \ldots \ldots \ldots \ldots \ldots \ldots$ & 10,833 & 1.2 \\
\hline Codein ${ } \ldots \ldots \ldots \ldots \ldots \ldots \ldots \ldots \ldots$ & 10,153 & 1.1 \\
\hline Digoxin $\ldots \ldots \ldots \ldots \ldots \ldots \ldots \ldots \ldots$ & 9,964 & 1.1 \\
\hline Trimethoprim $\ldots \ldots \ldots \ldots \ldots \ldots \ldots \ldots$ & 9,886 & 1.1 \\
\hline Ditliazem $\ldots \ldots \ldots \ldots \ldots \ldots \ldots \ldots \ldots \ldots$ & 9,541 & 1.0 \\
\hline Hydrocortisone $\ldots \ldots \ldots \ldots \ldots \ldots \ldots \ldots \ldots$ & 9,516 & 1.0 \\
\hline Phenylpropanolamine $\ldots \ldots \ldots \ldots \ldots \ldots \ldots$ & 9,485 & 1.0 \\
\hline Ranittdine $\ldots \ldots \ldots \ldots \ldots \ldots \ldots \ldots \ldots$ & $\mathbf{9 , 3 2 5}$ & 1.0 \\
\hline
\end{tabular}

'Frequency of mention combines single-ingredient agents with montions of the agent as an ingredient in a combination drug. ${ }^{2}$ Based on an estimated $813,503,000$ drug mentions in 1983.

Summary. National Center for Health Statistics. Vital and Health Stat 13(110). 1992.

3. Schappert, SM. National Ambulatory Medical Care Survey: 1990 Summary. Advance data from vital and health statistics; no. 213.
Hyattsville, Maryland: National Center for Health Statistics. 1992.

4. Schappert, SM. National Ambulatory Medical Care Survey: 1991

Summary. National Center for Health Statistics. Vital and Health Stat 13(116). 1994. 
Table 21. Number, percent distribution, and therapeutic claseification for the $\mathbf{2 0}$ drugs most frequently prescribed at office visite, by ontry name of drug: United States, 1993

\begin{tabular}{|c|c|c|c|}
\hline Entry name of drug & $\begin{array}{l}\text { Number of } \\
\text { drug mentlons } \\
\text { in thousands }\end{array}$ & $\begin{array}{c}\text { Percent } \\
\text { dlstribution }\end{array}$ & Therapeutic classiffication ${ }^{2}$ \\
\hline All drug mentlons $\ldots \ldots \ldots \ldots \ldots \ldots$ & 913,503 & 100.0 & $\cdots$ \\
\hline Amoxicilln $\ldots \ldots \ldots \ldots \ldots \ldots \ldots$ & 19,212 & 2.1 & Penlcillins \\
\hline Tylenol . . . . . . . . . . . . . . . . . . & 11,225 & 1.2 & General analgesics \\
\hline Premarin $\ldots \ldots \ldots \ldots \ldots \ldots \ldots \ldots$ & 10,675 & 1.2 & Estrogens and progestins \\
\hline $\operatorname{Lasix} \ldots \ldots \ldots \ldots \ldots \ldots \ldots \ldots \ldots$ & 10,578 & 1.2 & Dluretics \\
\hline 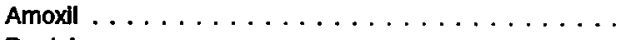 & 10,569 & 1.2 & Penicillins \\
\hline Prednisone $\ldots \ldots \ldots \ldots \ldots \ldots \ldots$ & 10,562 & 1.2 & Adrenal corticosteroids \\
\hline Zantac . . . . . . . . . . . . . . . & 9,303 & 1.0 & Agents used in disorders of upper Gl tract \\
\hline Cardizem $\ldots \ldots \ldots \ldots \ldots \ldots \ldots \ldots \ldots$ & 8,977 & 1.0 & Antlanginal agents \\
\hline Allergy rellef or shots $\ldots \ldots \ldots \ldots \ldots \ldots$ & 8,029 & 0.9 & Dlagnostics, nonradioactlve and radiopaque \\
\hline Influenza virus vaccine $\ldots \ldots \ldots \ldots \ldots \ldots$ & 7,685 & 0.8 & Vaccines and antiserums \\
\hline Procardia $\ldots \ldots \ldots \ldots \ldots \ldots \ldots \ldots \ldots$ & 7,575 & 0.8 & Antianginal agents \\
\hline 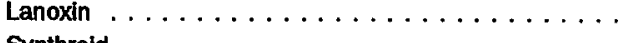 & 7,177 & 0.8 & Cardiac glycosides \\
\hline Synthroid $\ldots \ldots \ldots \ldots \ldots \ldots \ldots \ldots$ & 7,169 & 0.8 & Agents used to treat thyrold disease \\
\hline Vasotec $\ldots \ldots \ldots \ldots \ldots \ldots \ldots \ldots \ldots$ & 7,032 & 0.8 & Antihypertensive agents \\
\hline Diphtheria/Tetanus Toxoids/Pertussis $\ldots \ldots \ldots \ldots$ & 6,994 & 0.8 & Vaccines and antiserums \\
\hline Ventolin . . . . . . . . . . . . . . . & 6,940 & 0.8 & Bronchodilators, antlasthmatics \\
\hline Prenatal formula (vitamins) $\ldots \ldots \ldots \ldots$ & 6,902 & 0.8 & Vitamins, minerals \\
\hline Naprosyn $\ldots \ldots \ldots \ldots \ldots \ldots \ldots \ldots$ & 6,769 & 0.7 & Antiarthritics \\
\hline Proventil . . . . . . . . . . . . . . . & 6,626 & 0.7 & Bronchodilators, antiasthmatics \\
\hline Prozac $\ldots \ldots \ldots \ldots \ldots \ldots \ldots \ldots \ldots$ & 6,462 & 0.7 & Antldepressants \\
\hline All other $\ldots \ldots \ldots \ldots \ldots \ldots \ldots \ldots \ldots$ & 737,042 & 80.7 & $\cdots$ \\
\hline
\end{tabular}

The entry made by the physician on the prescription or other medical records. This may be a trade name, generic name, or desired therapeutic effect.

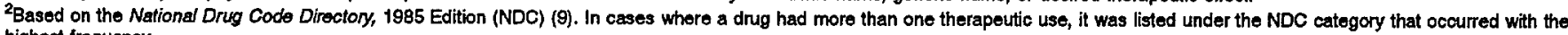
highest frequency.

5. Schappert, SM. National Ambulatory Medical Care Survey: 1992

Summary. Advance data from vital and health statistics; no. 253.

Hyattsville, Maryland: National

Center for Health Statistics. 1994.

6. Schneider D, Appleton L, McLemore T. A reason for visit classification for ambulatory care. National Center for Health Statistics. Vital and Health Stat 2(78). 1979.

7. Public Health Service and Health Care Financing Administration. International Classification of Diseases, 9th Revision, clinical modification. Washington: Public Health Service. 1980.

8. Koch H, Campbell W. The collection and processing of drug information. National Ambulatory Medical Care Survey, 1980. National Center for Health Statistics. Vital Health Stat 2(90). 1982.

9. Food and Drug Administration. National Drug Code Directory, 1985 Edition. Washington: Public Health Service. 1985.

10. Shah BV, Barnwell BG, Hunt PN, La Vange LM. SUDAAN User's Manual, Release 5.50. Research Triangle Institute. Research Triangle Park, NC. 1991.

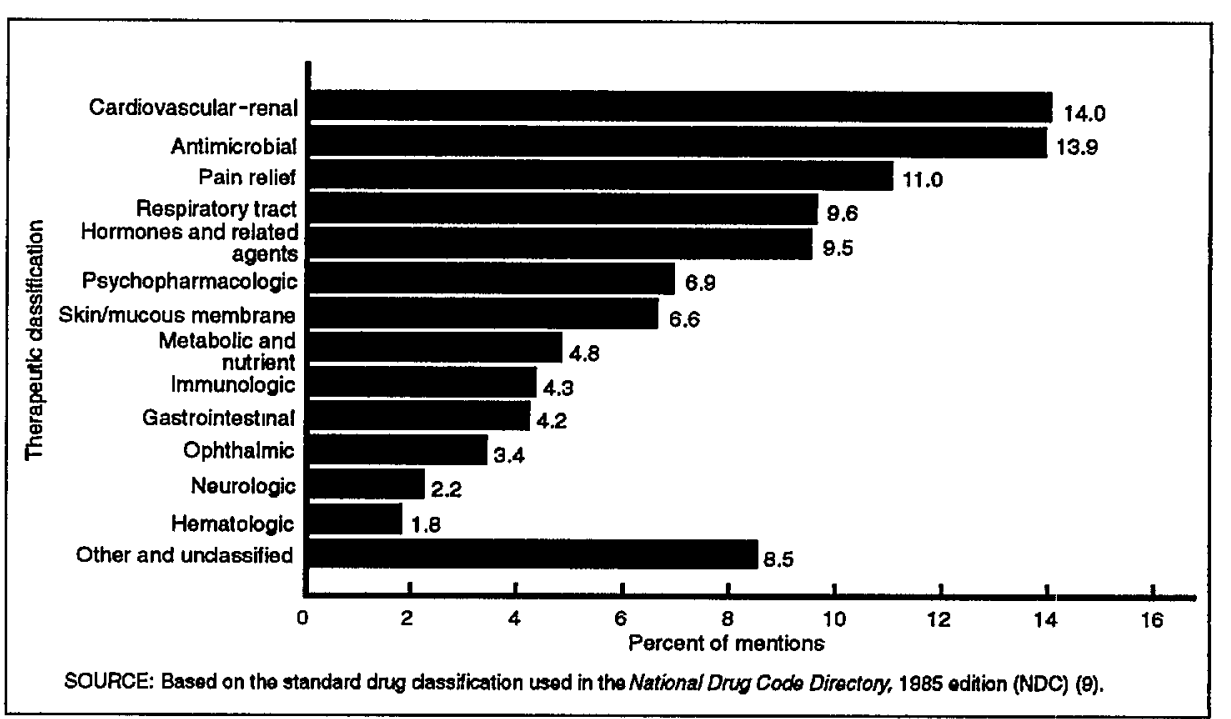

Figure 2. Percent distribution of drug mentions at office visits by therapeutic classification: United States, 1993 
Table 22. Number and percent of office visits by counseling/education ordered or provided: United State8, 1993

\begin{tabular}{|c|c|c|c|c|}
\hline \multirow[b]{2}{*}{ Counseling/education ordered or provided' } & \multirow{2}{*}{$\begin{array}{c}\text { Number of } \\
\text { visits in } \\
\text { thousands }\end{array}$} & \multicolumn{3}{|c|}{ Patient's sex } \\
\hline & & Total & Female & Mate \\
\hline & & \multicolumn{3}{|c|}{ Percent of visits } \\
\hline All vislts $\ldots \ldots \ldots \ldots \ldots \ldots \ldots \ldots \ldots$ & 717,191 & $\cdots$ & $\cdots$ & $\cdots$ \\
\hline 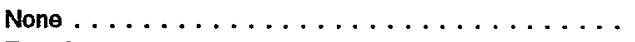 & 369,494 & 51.5 & 50.4 & 53.2 \\
\hline Exercise $\ldots \ldots \ldots \ldots \ldots \ldots \ldots \ldots \ldots$ & 64,257 & 9.0 & 8.9 & 9.0 \\
\hline Welght reductlon $\ldots \ldots \ldots \ldots \ldots \ldots$ & 40,715 & 5.7 & 5.9 & 5.3 \\
\hline Growth/development $\ldots \ldots \ldots \ldots \ldots \ldots$ & 30,255 & 4.2 & 4.2 & 4.3 \\
\hline Cholesterol reduction $\ldots \ldots \ldots \ldots \ldots \ldots$ & 27,063 & 3.8 & 3.7 & 3.9 \\
\hline Smoking cessation $\ldots \ldots \ldots \ldots \ldots \ldots$ & 22,674 & 3.2 & 3.1 & 3.3 \\
\hline Injury prevention $\ldots \ldots \ldots \ldots \ldots \ldots$ & 21,786 & 3.0 & 2.6 & 3.8 \\
\hline STD transmission (except HIV) $)^{2,3} \ldots \ldots \ldots \ldots$ & 10,216 & 1.4 & 1.8 & 0.9 \\
\hline HW transmission ${ }^{3} \ldots \ldots \ldots \ldots \ldots \ldots$ & 9,114 & 1.3 & 1.5 & 1.0 \\
\hline 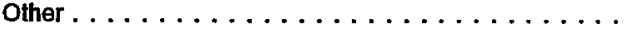 & 245,261 & 34.2 & 35.4 & 32.5 \\
\hline
\end{tabular}

'Numbers may not add to totals because more than one type of counseling/education may be reported per visit.

2STD is sexually transmitted diseases.

3HV is human immunodeficiency virus.

Table 23. Number and percent of office visits by disposition of vlsit: United States, 1993

\begin{tabular}{|c|c|c|}
\hline Disposition ${ }^{1}$ & $\begin{array}{l}\text { Number of } \\
\text { vists } h \\
\text { thousands }\end{array}$ & $\begin{array}{c}\text { Percent } \\
\text { of all } \\
\text { visits }\end{array}$ \\
\hline All visits $\ldots \ldots \ldots \ldots \ldots \ldots \ldots \ldots$ & 717,191 & $\cdots$ \\
\hline Retum at specified time $\ldots \ldots \ldots \ldots \ldots$ & 447,169 & 62.4 \\
\hline Retum if needed . . . . . . . . . . . . . . . & 166,947 & 23.3 \\
\hline No followup planned $\ldots \ldots \ldots \ldots \ldots$ & 61,687 & 8.6 \\
\hline Telephone followup planned $\ldots \ldots \ldots \ldots \ldots$ & 30,937 & 4.3 \\
\hline Referred to other physician $\ldots \ldots \ldots \ldots$ & 26,411 & 3.7 \\
\hline Admit to hospital . . . . . . . . . . . . . & 6,022 & 0.8 \\
\hline Returned to reterring physiclan . . . . . . . . . . & 8,960 & 1.2 \\
\hline Other $\ldots \ldots \ldots \ldots \ldots \ldots \ldots \ldots \ldots \ldots$ & 13,954 & 1.9 \\
\hline
\end{tabular}

${ }^{1}$ Numbers may not add to totals because more than one disposition may be reported per visit.

Table 24. Number and percent distribution of office visits by duration of visit: Unlted States, 1993

\begin{tabular}{|c|c|c|}
\hline Duration & $\begin{array}{c}\text { Number of } \\
\text { Visits in } \\
\text { thousands }\end{array}$ & $\begin{array}{c}\text { Percemt } \\
\text { distribution }\end{array}$ \\
\hline All vists $\ldots \ldots \ldots \ldots \ldots \ldots$ & 717,191 & 100.0 \\
\hline 0 minutes $^{1} \ldots \ldots \ldots \ldots \ldots \ldots \ldots$ & 17,484 & 2.4 \\
\hline $1-5$ minutes $\ldots \ldots \ldots \ldots \ldots \ldots \ldots$ & 40,611 & $\mathbf{5 . 7}$ \\
\hline 6-10 minutes $\ldots \ldots \ldots \ldots \ldots \ldots$ & 177,841 & 24.8 \\
\hline $11-15$ minutes $\ldots \ldots \ldots \ldots \ldots \ldots$ & 219,418 & 30.6 \\
\hline $16-30$ minutes $\ldots \ldots \ldots \ldots \ldots \ldots$ & 204,296 & 28.5 \\
\hline 31 minutes and over $\ldots \ldots \ldots \ldots \ldots$ & 57,540 & 8.0 \\
\hline
\end{tabular}

Visits in which there was no face-to-face contact between patient and physician. 


\section{Technical notes}

\section{Source of data and sample design}

The information presented in this report is based on data collected by means of the National Ambulatory Medical Care Survey (NAMCS) from January 4, 1993, through January 2, 1994. The target universe of NAMCS includes office visits made in the United States by ambulatory patients to nonfederally employed physicians who are principally engaged in office practice, but not in the specialties of anesthesiology, pathology, or radiology. Telephone contacts and nonoffice visits are excluded.

A multistage probability sample design is used in NAMCS, involving samples of primary sampling units (PSU's), physician practices within PSU's, and patient visits within physician practices. The PSU's are counties, groups of counties, county equivalents (such as parishes or independent cities), or towns and townships (for some PSU's in New England). For 1993, a sample of 3,400 nonfederal, office-based physicians was selected from master files maintained by the American Medical Association and American Osteopathic Association. Physicians were screened at the time of the survey to ensure that they were eligible for survey participation. Of those screened, 936 physicians were ruled ineligible (out-of-scope) due to reasons of being retired, employed primarily in teaching, research, or administration, or other reasons. The remaining 2,464 physicians were in-scope, or eligible to participate in the survey. The physician response rate for the 1993 NAMCS was 73.0 percent.

Sample physicians were asked to complete Patient Record forms (figure 1) for a systematic random sample of office visits occurring during a randomly assigned 1-week reporting period. Responding physicians completed 35,978 Patient Record forms.

Characteristics of the physician's practice, such as primary specialty and type of practice, were obtained from the physicians during an induction interview. The U.S. Bureau of the Census, Housing Surveys Branch, was responsible for the survey's data collection. Processing operations and medical coding were performed by the National Center for Health Statistics, Health Care Survey Section, Research Triangle Park, North Carolina.

\section{Sampling errors}

The standard error is primarily a measure of the sampling variability that occurs by chance when only a sample, rather than an entire universe, is surveyed. The standard error also reflects part of the measurement error, but does not measure any systematic biases in the data. The chances are 95 out of 100 that an estimate from the sample differs from the value that would be obtained from a complete census by less than twice the standard error.

The standard errors used in tests of significance for this report were calculated using generalized linear models for predicting the relative standard error for estimates based on the linear relationship between the actual standard error, as approximated using SUDAAN software, and the size of the estimate. SUDAAN computes standard errors by using a first-order Taylor approximation of the deviation of estimates from their expected values. A description of the software and the approach it uses has been published (10). The relative standard error (RSE) of an estimate is obtained by dividing the standard error by the estimate itself. The result is then expressed as a percent of the estimate.

Relative standard errors (RSE's) for estimated numbers of office visits in 1993 are shown in table I; relative standard errors for estimated numbers of drug mentions are presented in table II. Standard errors for estimated percents of visits and drug mentions are displayed in tables III and IV. Multiplying the estimate by the RSE will provide an approximation of the standard error for the estimate.

Alternatively, relative standard errors for aggregate estimates may be calculated using the following general formula, where $x$ is the aggregate of
Table I. Approximate relative standard errors for estlmated numbers of ofilce visits: National Ambulatory Medical Care Survey, 1993

\begin{tabular}{|c|c|}
\hline $\begin{array}{l}\text { Estimated } \\
\text { number of } \\
\text { office visits } \\
\text { in thousands }\end{array}$ & $\begin{array}{l}\text { Relative } \\
\text { standard } \\
\text { enor in } \\
\text { percent }\end{array}$ \\
\hline $100 \ldots \ldots \ldots \ldots \ldots$ & 83.2 \\
\hline $200 \ldots \ldots \ldots \ldots \ldots$ & 58.9 \\
\hline $500 \ldots \ldots \ldots \ldots \ldots \ldots$ & 37.4 \\
\hline $781 \ldots \ldots \ldots \ldots \ldots \ldots$ & 30.0 \\
\hline $1,000 \ldots \ldots \ldots \ldots \ldots$ & 26.6 \\
\hline $2,000 \ldots \ldots \ldots \ldots \ldots \ldots$ & 19.0 \\
\hline $5,000 \ldots \ldots \ldots \ldots \ldots \ldots$ & 12.3 \\
\hline $10,000 \ldots \ldots \ldots \ldots \ldots$ & 9.1 \\
\hline $20,000 \ldots \ldots \ldots \ldots \ldots \ldots$ & 7.0 \\
\hline $50,000 \ldots \ldots \ldots \ldots \ldots$ & 5.3 \\
\hline $100,000 \ldots \ldots \ldots \ldots$ & 4.6 \\
\hline $200,000 \ldots \ldots \ldots \ldots \ldots$ & 4.2 \\
\hline $500,000 \ldots \ldots \ldots \ldots$ & 3.9 \\
\hline $1,000,000 \ldots \ldots \ldots \ldots$ & 3.8 \\
\hline
\end{tabular}

NOTE: The smallest reliable estimate for visits to aggregated specialties is 781,000 visits. Estimates below this figure have a relative standard error greater than 30 percent and are deemed unreliable by NCHS standards.

Example of use of table: An aggregate estimate of 10 million visits has a relative standard error of 9.1 percent or a standard error of 910,000 visits $(9,1$ percent of 10 million).

Table II. Approximate relative standard errors for estimated numbers of drug mentions: National Ambulatory Medical Care Survey, 1993

\begin{tabular}{|c|c|}
\hline $\begin{array}{l}\text { Estimated } \\
\text { number of } \\
\text { drug mentions } \\
\text { in thousands }\end{array}$ & $\begin{array}{c}\text { Relative } \\
\text { standard } \\
\text { error in } \\
\text { percent }\end{array}$ \\
\hline $100 \ldots \ldots \ldots \ldots \ldots$ & 114.4 \\
\hline $200 \ldots \ldots \ldots \ldots \ldots \ldots$ & 81.0 \\
\hline $500 \ldots \ldots \ldots \ldots \ldots$ & 51.4 \\
\hline $1,000 \ldots \ldots \ldots \ldots \ldots$ & 36.5 \\
\hline $1,496 \ldots \ldots \ldots \ldots \ldots \ldots$ & 30.0 \\
\hline $2,000 \ldots \ldots \ldots \ldots \ldots$ & 26.1 \\
\hline $5,000 \ldots \ldots \ldots \ldots$ & 17.0 \\
\hline $10,000 \ldots \ldots \ldots \ldots \ldots$ & 12.5 \\
\hline $20,000 \ldots \ldots \ldots \ldots \ldots \ldots$ & 9.6 \\
\hline $50,000 \ldots \ldots \ldots \ldots \ldots$ & 7.3 \\
\hline $100,000 \ldots \ldots \ldots \ldots$ & 6.3 \\
\hline $200,000 \ldots \ldots \ldots \ldots$ & 5.8 \\
\hline $500,000 \ldots \ldots \ldots \ldots$ & 5.4 \\
\hline $1,000,000 \ldots \ldots \ldots$ & 5.3 \\
\hline
\end{tabular}

NOTE: The smallest reliable estimate of drug mentions for aggregated specialties is 1,496,000 mentions. Estimates below this figure have a relative standard error greater than 30 percent and are deemed unreliable by NCHS standarcs. Example of use of table: An aggregate estimate of 100 million drug mentions has a relative standard error of 6.3 percent or a standard error of $6,300,000$ mentions $(6.3$ percent of 100 million).

interest in thousands, and $A$ and $B$ are the appropriate coefficients from table $\mathrm{V}$.

$$
\operatorname{RSE}(x)=\sqrt{A+\frac{B}{x}} \cdot 100
$$

Similarly, relative standard errors for percents may be calculated using the 
Table III. Approximate standard errors of percents of estimated numbers of office visits: National Ambulatory Medical Care Survey, 1993

\begin{tabular}{|c|c|c|c|c|c|c|c|}
\hline \multirow{2}{*}{$\begin{array}{l}\text { Base of percent } \\
\text { (vistts in thousands) }\end{array}$} & \multicolumn{7}{|c|}{ Estimated percent } \\
\hline & 1 or 99 & 5 or 95 & 10 or 90 & 20 or 80 & 30 or 70 & 40 or 60 & 50 \\
\hline & \multicolumn{7}{|c|}{ Standard error in percentage points } \\
\hline $100 \ldots \ldots \ldots \ldots \ldots \ldots$ & 8.3 & 18.1 & 25.0 & 33.3 & 38.1 & 40.7 & 41.6 \\
\hline $200 \ldots \ldots \ldots \ldots \ldots$ & 5.9 & 12.8 & 17.6 & 23.5 & 27.0 & 28.8 & 29.4 \\
\hline $500 \ldots \ldots \ldots \ldots \ldots \ldots$ & 3.7 & 8.1 & 11.2 & 14.9 & 17.0 & 18.2 & 18.6 \\
\hline $1,000 \ldots \ldots \ldots \ldots \ldots \ldots$ & 2.6 & 5.7 & 7.9 & 10.5 & 12.1 & 12.9 & 13.2 \\
\hline $2,000 \ldots \ldots \ldots \ldots \ldots \ldots$ & 1.9 & 4.1 & 5.6 & 7.4 & 8.5 & 9.1 & 9.3 \\
\hline $5,000 \ldots \ldots \ldots \ldots \ldots \ldots$ & 1.2 & 2.6 & 3.5 & 4.7 & 5.4 & 5.8 & 5.9 \\
\hline $10,000 \ldots \ldots \ldots \ldots \ldots \ldots$ & 0.8 & 1.8 & 2.5 & 3.3 & 3.8 & 4.1 & 4.2 \\
\hline $20,000 \ldots \ldots \ldots \ldots \ldots \ldots$ & 0.6 & 1.3 & 1.8 & 2.4 & 2.7 & 2.9 & 2.9 \\
\hline $50,000 \ldots \ldots \ldots \ldots \ldots$ & 0.4 & 0.8 & 1.1 & 1.5 & 1.7 & 1.8 & 1.9 \\
\hline $100,000 \ldots \ldots \ldots \ldots \ldots$ & 0.3 & 0.6 & 0.8 & 1.1 & 1.2 & 1.3 & 1.3 \\
\hline $200,000 \ldots \ldots \ldots \ldots \ldots$ & 0.2 & 0.4 & 0.6 & 0.7 & 0.9 & 0.9 & 0.9 \\
\hline $500,000 \ldots \ldots \ldots \ldots \ldots$ & 0.1 & 0.3 & 0.4 & 0.5 & 0.5 & 0.6 & 0.6 \\
\hline $1,000,000 \ldots \ldots \ldots \ldots \ldots \ldots$ & 0.1 & 0.2 & 0.3 & 0.3 & 0.4 & 0.4 & 0.4 \\
\hline
\end{tabular}

NOTE: Example of use of table: An estimate of 30 percent based on an aggregate estimate of 10 million visits has a standard error of 3.5 percent or a relative standard error of 11.7 percent ( 3.5 percont divided by 30 percent).

Table IV. Approximate standard errors of percents of estimated numbers of drug mentions: National Ambulatory Medical Care Survey, 1993

\begin{tabular}{|c|c|c|c|c|c|c|c|}
\hline \multirow{2}{*}{$\begin{array}{l}\text { Base of percent } \\
\text { (vists in thousands) }\end{array}$} & \multicolumn{7}{|c|}{ Estimated percent } \\
\hline & 1 or 99 & 5 or 95 & 10 or 90 & 20 or 80 & 30 or 70 & 40 or 60 & 50 \\
\hline & \multicolumn{7}{|c|}{ Standard error in percentage points } \\
\hline $100 \ldots \ldots \ldots \ldots \ldots \ldots$ & 11.4 & 24.9 & 34.3 & 45.7 & 52.4 & 56.0 & 57.1 \\
\hline $200 \ldots \ldots \ldots \ldots \ldots \ldots$ & 8.0 & 17.6 & 24.2 & 32.3 & 37.0 & 39.6 & 40.4 \\
\hline $500 \ldots \ldots \ldots \ldots \ldots \ldots$ & 5.1 & 11.1 & 15.3 & 20.4 & 23.4 & 25.0 & 25.6 \\
\hline $1,000 \ldots \ldots \ldots \ldots \ldots \ldots$ & 3.6 & 7.9 & 10.8 & 14.5 & 16.6 & 17.7 & 18.1 \\
\hline $2,000 \ldots \ldots \ldots \ldots \ldots \ldots$ & 2.5 & 5.6 & 7.7 & 10.2 & 11.7 & 12.5 & 12.8 \\
\hline $5,000 \ldots \ldots \ldots \ldots \ldots$ & 1.6 & 3.5 & 4.9 & 6.5 & 7.4 & 7.9 & 8.1 \\
\hline $10,000 \ldots \ldots \ldots \ldots \ldots$ & 1.1 & 2.5 & 3.4 & 4.6 & 5.2 & 5.6 & 5.7 \\
\hline $20,000 \ldots \ldots \ldots \ldots \ldots \ldots$ & 0.8 & 1.8 & 2.4 & 3.2 & 3.7 & 4.0 & 4.0 \\
\hline $50,000 \ldots \ldots \ldots \ldots \ldots$ & 0.5 & 1.1 & 1.5 & 2.0 & 2.3 & 2.5 & 2.6 \\
\hline $100,000 \ldots \ldots \ldots \ldots \ldots \ldots$ & 0.4 & 0.8 & 1.1 & 1.5 & 1.7 & 1.8 & 1.8 \\
\hline $200,000 \ldots \ldots \ldots \ldots \ldots$ & 0.3 & 0.6 & 0.8 & 1.0 & 1.2 & 1.3 & 1.3 \\
\hline $500,000 \ldots \ldots \ldots \ldots \ldots$ & 0.2 & 0.4 & 0.5 & 0.7 & 0.7 & 0.8 & 0.8 \\
\hline $1,000,000 \ldots \ldots \ldots \ldots \ldots$ & 0.1 & 0.3 & 0.3 & 0.5 & 0.5 & 0.6 & 0.6 \\
\hline
\end{tabular}

NOTE: Example of use of table: An estimate of 30 percent based on an aggregate estimate of 100 million drug mentions has a standard error of 1.7 percent or a relative standard error of 5.7 percent (1.7 percont divided by 30 percent).

following general formula, where $p$ is the percent of interest expressed as a proportion, and $x$ is the denominator of the percent in thousands, using the appropriate coefficient from table V.

$$
\operatorname{RSE}(x)=\sqrt{\frac{B \cdot(1-p)}{p \cdot x}} \cdot 100
$$

\section{Adjustments for nonresponse}

Estimates from NAMCS data were adjusted to account for sample physicians who were in-scope but did not participate in the study. This adjustment was calculated to minimize the impact of response on final estimates by imputing to nonresponding physicians data from visits to similar physicians. For this purpose, physicians were judged similar if they had the same specialty designation and practiced in the same PSU.

\section{Test of significance and rounding}

In this report, the determination of statistical inference is based on the two-tailed $t$-test. The Bonferroni inequality was used to establish the critical value for statistically significant differences ( 0.05 level of significance) based on the number of possible comparisons within a particular variable (or combination of variables) of interest. Terms relating to differences such as "greater than" or "less than" indicate that the difference is statistically significant. A lack of comment regarding the difference between any two estimates does not mean that the difference was tested and found to be not significant.

In the tables, estimates of office visits have been rounded to the nearest thousand. Consequently, estimates will not always add to totals. Rates and percents were calculated from original unrounded figures and do not necessarily agree with percents calculated from rounded data. 
Table V. Coefficlents appropriate for determining relative standard errors by type of estimate and physiclan specialty: National Ambulatory Medical Care Survey, 1993

\begin{tabular}{|c|c|c|}
\hline \multirow{2}{*}{$\begin{array}{l}\text { Type of estimate and } \\
\text { physician specialty }\end{array}$} & \multicolumn{2}{|c|}{ Coefficient for use with estimates in thousands } \\
\hline & $A$ & $B$ \\
\hline \multicolumn{3}{|l|}{ Visits } \\
\hline$\ldots \ldots \ldots \ldots \ldots$ & 0.001402906 & 69.14991889 \\
\hline General and family practice & 0.00967743 & 82.86427569 \\
\hline$\ldots \ldots \ldots \ldots \ldots \ldots \ldots$ & 0.009694146 & 21.982539 \\
\hline$\ldots \ldots \ldots \ldots \ldots$ & 0.009613634 & 66.93051288 \\
\hline Pedlatrics $\ldots \ldots \ldots \ldots \ldots \ldots \ldots \ldots \ldots \ldots$ & 0.01497736 & 43.04423624 \\
\hline General surgery $\ldots \ldots \ldots \ldots \ldots \ldots \ldots \ldots \ldots$ & 0.004562476 & 6.18923111 \\
\hline Obstetrics and gynecology $\ldots \ldots \ldots \ldots \ldots \ldots \ldots$ & 0.01215906 & 45.17522836 \\
\hline Orthopedlc surgery. $\ldots \ldots \ldots \ldots \ldots \ldots \ldots \ldots$ & 0.01847372 & 30.1373659 \\
\hline Cardiovascular dlseases $\ldots \ldots \ldots \ldots \ldots \ldots \ldots$ & 0.01842725 & 13.33081384 \\
\hline Dermatology. $\ldots \ldots \ldots \ldots \ldots \ldots \ldots \ldots \ldots$ & 0.01300847 & 14.22174725 \\
\hline Urology. $\ldots \ldots \ldots \ldots \ldots \ldots \ldots \ldots$ & 0.01482425 & 10.21006093 \\
\hline Psychlatry $\ldots \ldots \ldots \ldots \ldots \ldots \ldots \ldots \ldots \ldots$ & 0.01111663 & 8.36850241 \\
\hline Neurology $\ldots \ldots \ldots \ldots \ldots \ldots \ldots \ldots \ldots \ldots$ & 0.01082749 & 4.46207203 \\
\hline Ophthalomology $\ldots \ldots \ldots \ldots \ldots \ldots \ldots \ldots$ & 0.01380671 & 23.79909861 \\
\hline Otolaryngology $\ldots \ldots \ldots \ldots \ldots \ldots \ldots \ldots \ldots$ & 0.01594593 & 7.10113491 \\
\hline Allergy and immunology ${ }^{\prime} \ldots \ldots \ldots \ldots \ldots \ldots$ & 0.02015721 & 3.35915068 \\
\hline Pulmonary diseases $^{1} \ldots \ldots \ldots \ldots \ldots \ldots$ & 0.01604307 & 2.76807823 \\
\hline All other specialttes $\ldots \ldots \ldots \ldots \ldots \ldots \ldots$ & 0.01185348 & 45.14667587 \\
\hline \multicolumn{3}{|l|}{ Drug mentions } \\
\hline Overall total ... & 0.002655818 & 130.60816 \\
\hline General and famlly practice $\ldots \ldots \ldots \ldots \ldots \ldots$ & 0.01454036 & 153.42208 \\
\hline Osteopathy $\ldots \ldots \ldots \ldots \ldots \ldots \ldots$ & 0.01482355 & 34.91826215 \\
\hline Intemal medicine $\ldots \ldots \ldots \ldots \ldots \ldots \ldots \ldots$ & 0.01501777 & 127.67927 \\
\hline Pediatrics $\ldots \ldots \ldots \ldots \ldots \ldots \ldots \ldots \ldots \ldots$ & 0.02139038 & 29.86328192 \\
\hline General surgery $\ldots \ldots \ldots \ldots \ldots \ldots \ldots \ldots$ & 0.02674708 & 6.25993055 \\
\hline Obstetrics and gynecology . . . . . . . . . . . & 0.02833093 & 47.78172168 \\
\hline Orthopedic surgery. . . . . . . . . . . . . & 0.03190595 & 31.27018391 \\
\hline Cardiovascular diseases $\ldots \ldots \ldots \ldots \ldots \ldots \ldots$. . . & 0.02412645 & 28.653378 \\
\hline Dermatology. . . . . . . . . $\ldots \ldots \ldots \ldots \ldots$ & 0.02064188 & 14.49471796 \\
\hline Urology. $\ldots \ldots \ldots \ldots \ldots \ldots \ldots \ldots \ldots \ldots \ldots$ & 0.03026505 & 10.1235506 \\
\hline Psychlatry $\ldots \ldots \ldots \ldots \ldots \ldots \ldots \ldots \ldots \ldots$ & 0.02554631 & 11.76240189 \\
\hline Nourology,$\ldots \ldots \ldots \ldots \ldots \ldots \ldots$ & 0.01978151 & 5.29800076 \\
\hline Ophthalomology $\ldots \ldots \ldots \ldots \ldots \ldots \ldots \ldots \ldots$ & 0.02642952 & 39.03224396 \\
\hline$\ldots \ldots \ldots \ldots \ldots$ & 0.03147744 & 6.68505135 \\
\hline Allergy and immunology $y^{1} \ldots \ldots \ldots \ldots \ldots \ldots \ldots$ & 0.02579988 & 6.30451913 \\
\hline Pulmonary diseases ${ }^{1} \ldots$ & 0.02283295 & 5.93833904 \\
\hline All other speclalties $\ldots \ldots \ldots \ldots \ldots \ldots \ldots \ldots \ldots$ & 0.02135922 & 59.19073373 \\
\hline
\end{tabular}

TPhysician strata added as a supplemont to the 1993 NAMCS only. who spends some time caring for ambulatory patients. Excluded from the NAMCS are physicians who are hospital based; who specialize in anesthesiology, pathology, or radiology; who are federally employed; who treat only institutionalized patients; or who are employed full time by an institution and spend no time seeing ambulatory patients.

Visit-A visit is a direct personal exchange between an ambulatory patient and a physician or a staff member working under the physician's supervision, for the purpose of seeking care and rendering personal health services. Excluded from the NAMCS are visits where medical care was not provided, such as visits made to drop off specimens, pay bills, make appointments, and walk-outs.

\section{Definition of terms}

Ambulatory patient-An ambulatory patient is an individual seeking personal health services who is not currently admitted to any health care institution on the premises.

Drug mention-A drug mention is the physician's entry on the Patient Record form of a pharmaceutical agent-by any route of administrationfor prevention, diagnosis, or treatment. Generic as well as brand-name drugs are included, as are nonprescription and prescription drugs. Along with all new drugs, the physician also records continued medications if the patient was specifically instructed during the visit to continue the medication. Physicians may report up to five medications per visit.

Drug visit-A drug visit is a visit at which medication was prescribed or provided by the physician.

Office-An office is the space identified by a physician as a location for his or her ambulatory practice. Offices customarily include consultation, examination, or treatment spaces that patients associate with the particular physician.

Physician-A physician is a duly licensed doctor of medicine (M.D.) or doctor of osteopathy (D.O.) who is currently in office-based practice and 


\section{Symbols}

- - Data not available

... Category not applicable

- Quantity zero

0.0 Quantity more than zero but less than 0.05

Z Quantity more than zero but less than $\mathbf{5 0 0}$ where numbers are rounded to thousands

* $\quad$ Figure does not meet standard of reliability or precision 


\section{Suggested citation}

Woodwell DA, Schappert SM. National Ambulatory Medical Care Survey: 1993 summary. Advance data from vital and health statistics; no. 270. Hyaltsville, Maryland: National Center for Health Statistics. 1995.

\section{Trade name dlsclaimer}

The use of trade names is for identification only and does not imply endorsement by the Public Health Service, U.S. Department of Health and Human Services.

\section{Copyright information}

All material appearing in this report is in the public domain and may be reproduced or copied without permission; citation as to source, however, is appreciated.
National Center for Health Statistics

Acting Director

Jack R. Anderson

Acting Deputy Director Jennifer H. Madans, Ph.D.

\section{U.S. DEPARTMENT OF HEALTH AND HUMAN SERVICES \\ Public Health Service \\ Centers for Disease Control and Prevention \\ National Center for Health Statistics \\ 6525 Belcrest Road \\ Hyattsville, Maryland 20782}

OFFICIAL BUSINESS

PENALTY FOR PRIVATE USE, $\$ 300$

To receive this publication regularly, contact the National Center for Health Statistics by calling 301-436-8500

E-mail: nchsquery@nch10a.em.cdc.gov

Internet: http://www.cdc.gov/nchswww/nchshome.htm

DHHS Publication No. (PHS) 96-1250

6-0029 (12/95) 


\section{Vital and Health Statistics series descriptions}

SERIES 1. Programs and Collection Procedures-These reports describe the data collection programs of the National Center for Health Statistics. They include descriptions of the methods used to collect and process the data, definitions, and other material necessary for understanding the data.

SERIES 2. Data Evaluation and Methods Research-These reports are studies of new statistical methods and include analytical techniques, objective evaluations of reliability of collected data, and contributions to statistical theory. These studies also include experimental tests of new survey methods and comparisons of U.S. methodology with those of other countries.

SERIES 3. Analytical and EpIdemiological Studies-These reports present analytical or interpretive studies based on vital and health statistics. These reports carny the analyses further than the expository types of reports in the other series.

SERIES 4. Documents and Committee Reports-These are final reports of major committees concerned with vital and health statistics and documents such as recommended model vital registration laws and revised birth and death certificates.

SERIES 5. International Vital and Health Statistics Reports-These reports are analytical or descriptive reports that compare U.S. vital and health statistics with those of other countries or present other international data of relevance to the health statistics system of the United States.

JERIES 6. Cognition and Survey Measurement-These reports are from the National Laboratory for Collaborative Research in Cognition and Survey Measurement. They use methods of cognitive science to design, evaluate, and test survey instruments.

3ERIES 10. Data From the National Health Interview Survey-These reports contain statistics on illness; unintentional injuries; disability; use of hospital, medical, and other health services; and a wide range of special current health topics covering many aspects of health behaviors, health status, and health care utilization. They are based on data collected in a continuing national household interview survey.

SERIES 11. Data From the National Health Examination Survey, the National Health and Nutrition Examination Surveys, and the Hispanic Health and Nutrition Examination SurveyData from direct examination, testing, and measurement on representative samples of the civilian noninstitutionalized population provide the basis for (1) medically defined total prevalence of specific diseases or conditions in the United States and the distributions of the population with respect to physical, physiological, and psychological characteristics, and (2) analyses of trends and relationships among various measurements and between survey periods.

SERIES 12. Data From the Institutlonalized Population SurveysDiscontinued in 1975. Reports from these surveys are included in Series 13.

SERIES 13. Data From the National Health Care Survey-These reports contain statistics on health resources and the public's use of health care resources including ambulatory, hospital, and long-term care services based on data collected directly from health care providers and provider records.
SERIES 14. Data on Health Resources: Manpower and FacilitiesDiscontinued in 1990. Reports on the numbers, geographic distribution, and characteristics of health resources are now included in Series 13.

SERIES 15. Data From Special Surveys-These reports contain statistics on health and health-related topics collected in special surveys that are not part of the continuing data systems of the National Center for Health Statistics.

SERIES 16. Compilations of Advance Data From Vital and Health Statistics-Advance Data Reports provide early release of information from the National Center for Health Statistics' health and demographic surveys. They are compiled in the order in which they are published. Some of these releases may be followed by detailed reports in Series 10-13.

SERIES 20. Data on Mortality-These reports contain statistics on mortality that are not included in regular, annual, or monthly reports. Special analyses by cause of death, age, other demographic variables, and geographic and trend analyses are included.

SERIES 21. Data on Natality, Marriage, and Divorce-These reports contain statistics on natality, marriage, and divorce that are not included in regular, annual, or monthly reports. Special analyses by health and demographic variables and geographic and trend analyses are included.

SERIES 22. Data From the National Mortality and Natality SurveysDiscontinued in 1975. Reports from these sample surveys, based on vital records, are now published in Series 20 or 21.

SERIES 23. Data From the National Survey of Family GrowthThese reports contain statistics on factors that affect birth rates, including contraception, infertility, cohabitation, marriage, divorce, and remarriage; adoption; use of medical care for family planning and infertility; and related maternal and infant health topics. These statistics are based on national surveys of childbearing age.

SERIES 24. Compilations of Data on Natality, Mortality, Marriage, Divorce, and Induced Terminations of PregnancyThese include advance reports of births, deaths, marriages, and divorces based on final data from the National Vital Statistics System that were published as supplements to the Monthly Vital Statistics Report (MVSR). These reports provide highlights and summaries of detailed data subsequently published in Vital Statistics of the United States. Other supplements to the MVSR published here provide selected findings based on final data from the National Vital Statistics System and may be followed by detailed reports in Series 20 or 21.

For answers to questions about this report or for a list of reports published in these series, contact:

Data Dissemination Branch

National Center for Health Statistics

Centers for Disease Control and Prevention

Public Health Service

6525 Belcrest Road, Room 1064

Hyattsville, MD 20782

(301) 436-8500

E-mail: nchsquery@nch10a.em.cdc.gov

Internet: http:/www.cdc.gov/nchswww/nchshome.htm 
Public Health Service

Centers for Disease Control and Prevention

POSTAGE \& FEES PAID

National Center for Health Statistics

PHS/NCHS

6525 Belcrest Road

PERMIT NO. G-281

OFFICIAL BUSINESS

PENALTY FOR PRIVATE USE, $\$ 300$ 